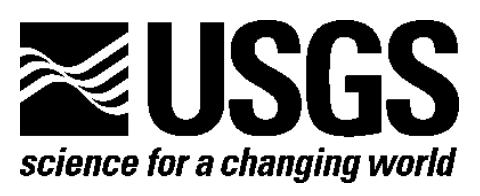

\title{
Efficacy of Pseudomonas fluorescens Strain CL145A Spray Dried Powder for Controlling Zebra Mussels Adhering to Native Unionid Mussels Within Field Enclosures
}

By James A. Luoma, Kerry L. Weber, Todd J. Severson, and Denise A. Mayer

Open-File Report 2015-1051

U.S. Department of the Interior

U.S. Geological Survey 


\section{U.S. Department of the Interior \\ SALLY JEWELL, Secretary}

\section{U.S. Geological Survey \\ Suzette M. Kimball, Acting Director}

U.S. Geological Survey, Reston, Virginia: 2015

For more information on the USGS—-the Federal source for science about the Earth, its natural and living resources, natural hazards, and the environment-visit http://www.usgs.gov or call 1-888-ASK-USGS (1-888-275-8747)

For an overview of USGS information products, including maps, imagery, and publications, visit http://www.usgs.gov/pubprod

To order this and other USGS information products, visit http://store.usgs.gov

Any use of trade, firm, or product names is for descriptive purposes only and does not imply endorsement by the U.S. Government.

Although this information product, for the most part, is in the public domain, it also may contain copyrighted materials as noted in the text. Permission to reproduce copyrighted items must be secured from the copyright owner.

Suggested citation:

Luoma, J.A., Weber, K.L., Severson, T.J., and Mayer, D.A., 2015, Efficacy of Pseudomonas fluorescens strain CL145A spray dried powder for controlling zebra mussels adhering to native unionid mussels within field enclosures: U.S. Geological Survey Open-File Report 2015-1051, 301 p., http://dx.doi.org/10.3133/ofr20151051.

ISSN 2331-1258 (online) 


\section{Acknowledgments}

This study was funded through a combination of a U.S. Environmental Protection Agency Great Lakes Restoration Initiative Grant and U.S. Geological Survey appropriated funds. Michelle R. Bartsch, Jeremy K. Wise, and Hugh E. McMath (Upper Midwest Environmental Sciences Center) assisted with data collection. Mark Gaikowski (Upper Midwest Environmental Sciences Center) assisted in study design, data analysis, and report preparation. Joe Eisterhold and Mark Ranweiler (Minnesota Department of Natural Resources) assisted with field logistics and project permitting. Special thanks are extended to Carl Towley and Mike Bump for logistical assistance and for allowing us to use their private property for equipment storage and project operations. 


\section{Contents}

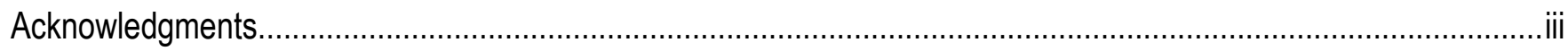

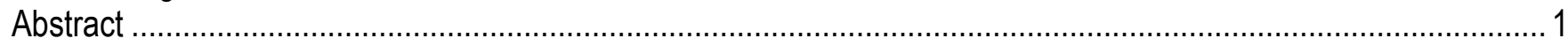

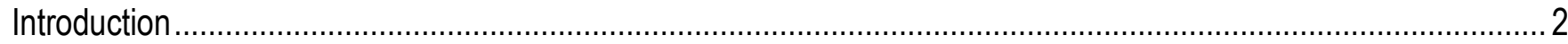

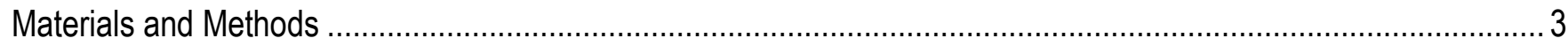

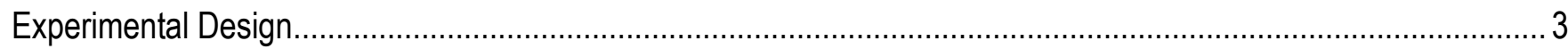

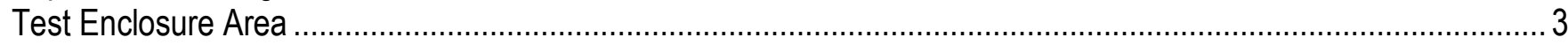

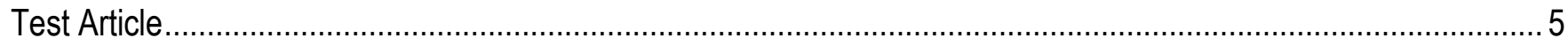

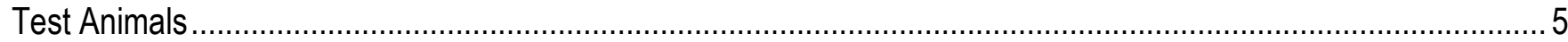

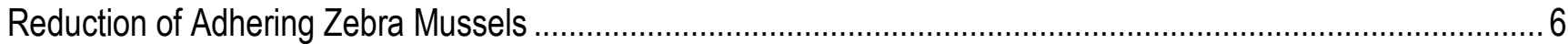

Estimation of Initial Number of Adhering Zebra Mussels (Group 1)............................................................ 6

Estimation of the Number of Adhering Zebra Mussels (Group 2) …….......................................................

Calculation of Zebra Mussel Body Weight Burden (Group 2) ......................................................................

Calculation of Adhering Zebra Mussels Reduction (Group 2) .....................................................................

Calculation of SDP Reduction Coefficient and Efficiency (Group 2) ........................................................... 8

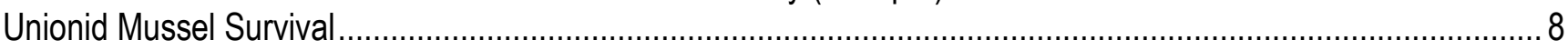

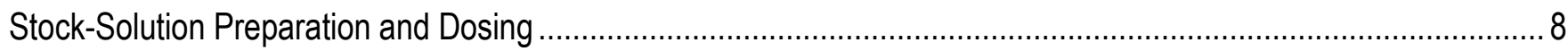

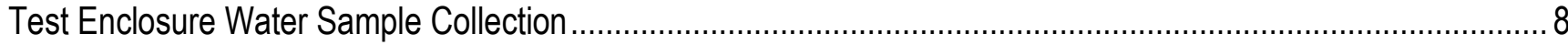

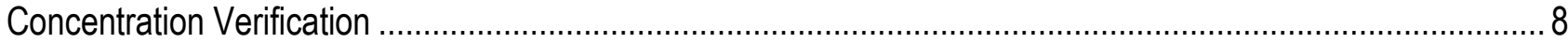

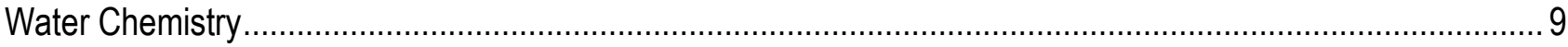

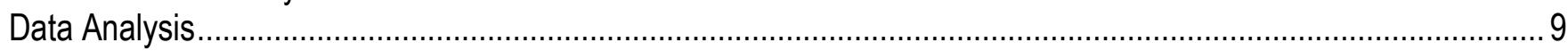

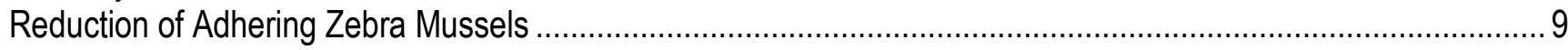

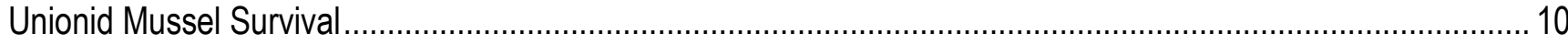

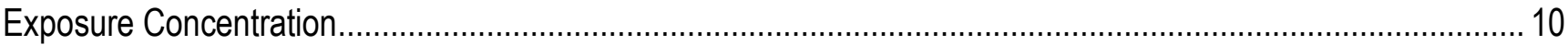

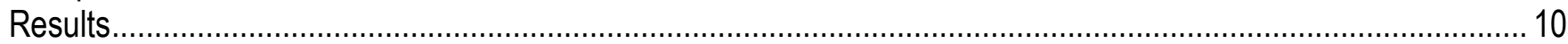

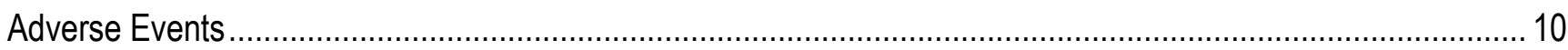

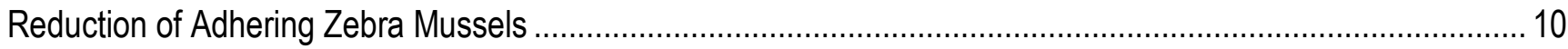

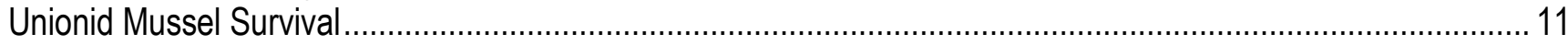

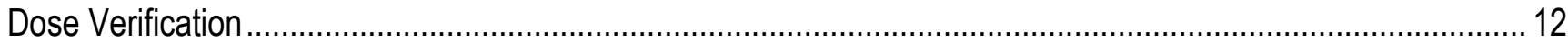

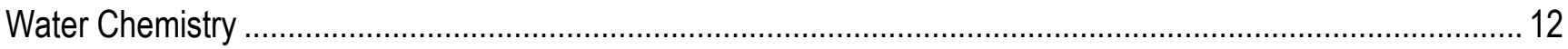

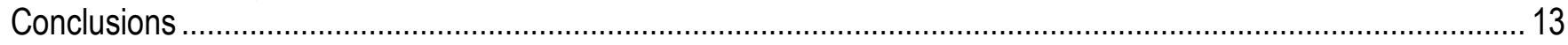

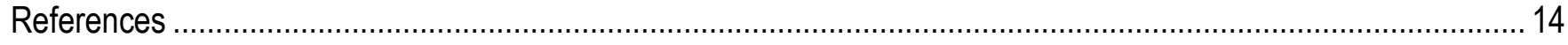

Appendix 1.Study Protocol With Data Forms ………………

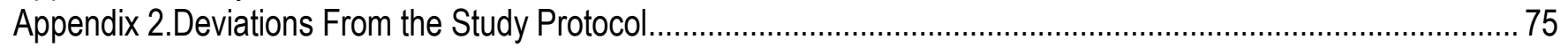

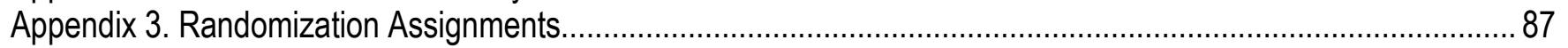

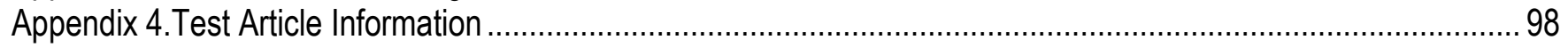

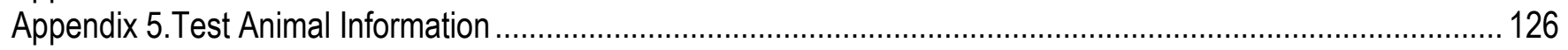

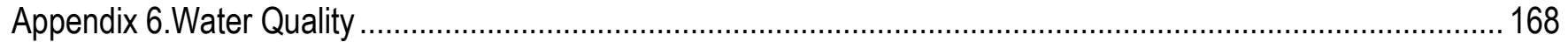

Appendix 7.Spectrophotometric Summary and SAS Output, Program, and Log ............................................ 181

Appendix 8.Zebra Mussel Density Association Summary Data Analysis ...........................................................201

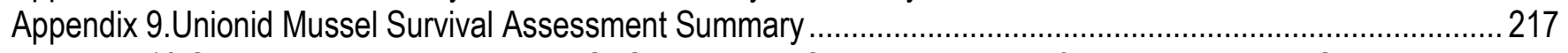

Appendix 10.Statistical Analysis, Including SAS Programs, Outputs, and Logs for Unionid Mussel Survival, Zebra

Mussel Colonization Density Associations, and Zebra Mussel Test Animal Lengths..........................................223 


\section{Figures}

Figure 1. Test enclosure site and test animal collection locations.

Figure 2. Example of 1-square-meter $\left(\mathrm{m}^{2}\right)$ expanded metal mussel retention barrier (left) and 2.25-m² impermeable test enclosure (right).

Figure 3. Representative unionid mussel with adhering zebra mussels preexposure (top left), postexposure (top right) and with zebra mussels removed (bottom).....

\section{Tables}

Table 1. Universal Transverse Mercator (UTM) centroid location of each test enclosure.

Table 2. Mean (standard deviation) number of adhering zebra mussels per unionid mussel before and after exposure, percent change of zebra mussel reduction, zebra mussel burden (as percent unionid body weight), and spray dried powder (SDP) reduction coefficient for number of adhering zebra mussels for each treatment group.

Table 3. Mean (standard deviation; number measured) length of adhering zebra mussels (live) by treatment group.

Table 4. Mean (standard deviation) percent survival of Group 1 and 2 unionid mussels.

Table 5. Mean (standard deviation) spray dried powder exposure concentration in surface water samples collected from test enclosures during the exposure period.

Table 6. Mean (standard deviation) water chemistry (dissolved oxygen, temperature) and $\mathrm{pH}$ range of surface water samples collected from each treatment group during the study period

Table 7. Mean (standard deviation) alkalinity, hardness, conductivity, and ammonia content of surface water samples collected from each treatment group during the study period 


\section{Conversion Factors}

International System of Units to Inch/Pound

\begin{tabular}{lcl}
\hline & Multiply & Bo obtain \\
\hline micrometer $(\mu \mathrm{m})$ & Length & \\
millimeter $(\mathrm{mm})$ & $3.937 \times 10^{-5}$ & inch (in.) \\
meter $(\mathrm{m})$ & $0.03937 \quad$ inch (in.) & \\
nanometer $(\mathrm{nm})$ & $3.281 \quad$ foot (ft) \\
\hline & $3.937 \times 10^{-8}$ & inch (in.) \\
\hline square meter $\left(\mathrm{m}^{2}\right)$ & Area & \\
\hline & 10.76 & square foot $\left(\mathrm{ft}^{2}\right)$ \\
\hline liter $(\mathrm{L})$ & Volume & \\
\hline & 0.2642 & gallon (gal) \\
\hline gram $(\mathrm{g})$ & Mass \\
\hline
\end{tabular}

Conductivity is given in microsiemens per centimeter at 25 degrees Celsius $\left(\mu \mathrm{S} / \mathrm{cm}\right.$ at $\left.25^{\circ} \mathrm{C}\right)$.

Concentrations of chemical constituents in water are given in milligrams per liter (mg/L).

Temperature in degrees Celsius $\left({ }^{\circ} \mathrm{C}\right)$ may be converted to degrees Fahrenheit $\left({ }^{\circ} \mathrm{F}\right)$ as ${ }^{\circ} \mathrm{F}=\left(1.8 \times{ }^{\circ} \mathrm{C}\right)+32$. 


\section{Abbreviations}

$\begin{array}{ll}\mathrm{Al} & \text { active ingredient } \\ \mathrm{CaCO}_{3} & \text { calcium carbonate } \\ \mathrm{DO} & \text { dissolved oxygen } \\ \mathrm{MBI} & \text { Marrone Bio Innovations } \\ \mathrm{mE} & \text { meters East } \\ \mathrm{mN} & \text { meters North } \\ \mathrm{NH}_{3} & \text { un-ionized ammonia } \\ \mathrm{OR} & \text { odds ratio } \\ \mathrm{SAS} & \text { Statistical Analysis System } \\ \mathrm{SD} & \text { standard deviation } \\ \mathrm{SDP} & \text { spray dried powder } \\ \text { TAN } & \text { total ammonia nitrogen } \\ \text { Pf-CL145A } & \text { Pseudomonas fluorescens strain CL145A } \\ \text { USGS } & \text { U.S. Geological Survey } \\ \text { UTM } & \text { Universal Transverse Mercator } \\ \text { w/w } & \text { weight to weight ratio }\end{array}$




\title{
Efficacy of Pseudomonas fluorescens Strain CL145A Spray Dried Powder for Controlling Zebra Mussels Adhering to Native Unionid Mussels Within Field Enclosures
}

\author{
By James A. Luoma, ${ }^{1}$ Kerry L. Weber, ${ }^{1}$ Todd J. Severson, ${ }^{1}$ and Denise A. Mayer ${ }^{2}$
}

\begin{abstract}
The efficacy of a commercially prepared spray dried powder (SDP) formulation of Pseudomonas fluorescens (strain CL145A) was evaluated for removing zebra mussels (Dreissena polymorpha) adhering to a population of unionid mussels in Lake Darling (Alexandria, Minnesota). Two groups of unionid mussels were used in the study. Unionid mussels were collected near the test area, weighed, photographed, individually tagged, and randomly allocated to one of nine test enclosures in equal proportions and then divided into two groups. The first group of unionid mussels (Group 1, $n=5$ per test enclosure) were indiscriminately selected from each test enclosure and used to estimate the number of zebra mussels adhering to unionid mussels prior to exposure. The second group of unionid mussels (Group 2, $n=22$ per test enclosure) were used to evaluate the efficacy of SDP for removal of adhering zebra mussels. Both Group 1 and Group 2 mussels were used to evaluate the effects of SDP exposure on unionid mussel survival.

Treatment was assigned to each test enclosure by using a randomized block design. The three treatment groups were tested in triplicate and included an untreated control group and groups that received a single application of 50 or 100 milligrams per liter (mg/L) of SDP based on active ingredient. All treatment concentrations are reported as active ingredient of SDP. Test enclosures were removed at the 8-hour exposure termination. Both Group 1 and Group 2 mussels remained in their assigned exposure location during the postexposure holding period. The number of zebra mussels adhering to Group 2 mussels (live and dead) was assessed 18 to 20 days postexposure in addition to assessing the survival of Group 1 and Group 2 unionid mussels.

SDP, administered as a single treatment, significantly $(p<0.01)$ reduced the number of adhering zebra mussels when compared to the untreated controls. The number of zebra mussels adhering to unionid mussels (Group 2) was reduced 53 percent in the 50-mg/L treatment group and 68 percent in the $100-\mathrm{mg} / \mathrm{L}$ treatment group. The number of adhering zebra mussels did not differ $(p=0.79)$ between the 50- and 100-mg/L treatment groups after exposure. When standardized to the amount of SDP applied per square meter, each gram (g) of SDP applied in the $50-\mathrm{mg} / \mathrm{L}$ treatment reduced the number of adhering zebra mussel 59.8 percent more than the $100-\mathrm{mg} / \mathrm{L}$ treatment group.

\footnotetext{
${ }^{1}$ U.S. Geological Survey.

${ }^{2}$ New York State Education Department.
} 
Group 1 mussel survival did not differ between treatment groups ( $p>0.05)$; however, a difference was detected $(p<0.01)$ in the survival of Group 2 mussels. The survival of Group 2 mussels did not differ $(p>0.23)$ between control and treated groups. A difference in Group 2 mussel survival was detected $(p=0.03$; odds ratio $[\mathrm{OR}]=0.290$ ) between the 50 - and $100-\mathrm{mg} / \mathrm{L}$ treatment groups (that is, the survival was highest in the $50-\mathrm{mg} / \mathrm{L}$ treatment group and lowest in the $100-\mathrm{mg} / \mathrm{L}$ treatment group), however, the biological significance of the difference is indeterminate.

\section{Introduction}

Freshwater mussel populations of North America were historically considered the most diverse in the world, but diversity is declining rapidly in response to a variety of anthropogenic influences such as habitat degradation and alteration, pollution, and overharvest (Williams and others, 1993; Neves and others, 1997). Master (1990) found that 55 percent of North American mussel species were either extinct or imperiled. At least 127 imperiled mussel species are predicted to reach extinction within the next 100 years; however, the 6.4 percent decadal extinction rate does not factor in extirpations related to invasions by dreissenid mussels (zebra, Dreissena polymorpha and quagga, D. rostriformis bugensis) (Ricciardi and Rasmussen, 1999).

Because of their high reproductive capacity and their planktonic lifestage, dreissenid mussels can quickly disperse and inundate aquatic environments (Mackie, 1991; Birnbaum, 2011). Since their introduction into the United States and establishment in the Great Lakes in the 1980s, zebra mussels have been identified in 680 lakes, not including impoundments and connected waterways, within 27 states (Benson and others, 2015). In a power plant canal in Lake Erie, Schloesser and Kovalak (1991) estimated zebra mussel colonization as high as approximately 10,700 zebra mussels per unionid mussel, with a mean estimated density of approximately 6,800 zebra mussels per unionid mussel. Adhering zebra mussel colonies may contain in excess of 10,000 individuals, which could weigh two to five times as much as the unionid mussel (Hebert and others, 1991; Mackie, 1991). Prediction models estimate that a colonization density of as few as 100 zebra mussels per unionid can result in unionid mussel mortality (Ricciardi and others, 1995).

Colonization by zebra mussels may interfere with unionid mussel locomotion, feeding, reproduction, and respiration (Mackie, 1991; Schloesser and Kovalak, 1991). Heavy zebra mussel colonization may contribute to starvation of the unionid through reduced intake of food and increased metabolic costs (Baker and Hornbach, 1997; Strayer and Malcom, 2007). Heavily colonized unionid mussels may lack the energy stores required to survive winter, and their ability to burrow into the sediment to avoid winter or other environmental stressors is impeded (Nalepa, 1994; Schloesser and Nalepa, 1994). Colonization by zebra mussels causes declines in unionid mussel populations and is dependent upon zebra mussel density, biomass, time since invasion, and substrate type (Burlakova and others, 2000).

Federal and state management agencies have implemented recovery and propagation programs for threatened and endangered unionid species coinciding with legislation and programs for control and removal of introduced and invasive species such as zebra mussels (Neves, 2004; Nalepa and Schloesser, 2014). However, there is currently a lack of access to environmentally safe and effective tools to control dreissenid mussel populations in open-water environments. A potential tool for limited open-water control of dreissenid mussels is a commercially formulated spray dried powder (SDP) product, which contains the killed cells of a specific strain (CL145A) of the common soil bacterium Pseudomonas fluorescens. The goal of this study was to determine the potential use of SDP in unionid mussel recovery and restoration efforts by controlling zebra mussel populations. The study objectives were (1) to determine the efficacy of SDP to control zebra mussels adhering to unionid mussels, and (2) to 
evaluate the effects of SDP exposure on survival of unionid mussels compromised by zebra mussel colonization. This report summarizes a single field trial completed from July 18, 2013, to November 18, 2013. The exposures were completed on July 26, 2013, and the postexposure assessments were completed August 13-15, 2013.

\section{Materials and Methods}

The protocol for this study entitled "Efficacy of Pseudomonas fluorescens (Pf-CL145A) SDP for controlling zebra mussels within field enclosures” is contained in appendix 1, item 1 . All methods and materials follow the written protocol except those instances that were identified in a note to file (appendix 1, item 2) and in study deviations (appendix 2, items 1-5).

\section{Experimental Design}

The study was done in Lake Darling (Alexandria, Minnesota) and consisted of a twofold assessment to evaluate the reduction in the number of zebra mussels adhering to unionid mussels and to evaluate unionid mussel survival following exposure to SDP. Zebra mussel reduction was assessed by comparing the estimated number of zebra mussels adhering to unionid mussels prior to SDP exposure to the enumerated number of zebra mussels adhering to mussels after SDP exposure. Unionid mussel survival was assessed after SDP exposure by observing foot movement or resistance to valve pressure.

Unionid mussels were collected, individually tagged, and randomly assigned (appendix 3, item 4 ) to test enclosures in equal proportions ( $n=243 ; 27$ per test enclosure). A group of unionid mussels (Group 1; $n=45 ; 5$ per test enclosure) were removed and used to estimate the number of adhering zebra mussels prior to SDP exposure (that is, adhering zebra mussels were removed and enumerated). After zebra mussel removal, Group 1 unionid mussels were returned to their assigned treatment enclosure for the remainder of the study. The undisturbed unionid mussels (Group 2; $n=198$; 22 per test enclosure) remained in the assigned test enclosure area for the entire study duration and were used to evaluate the efficacy of SDP for removal of adhering zebra mussels. Both groups were exposed concurrently in the test enclosures.

Treatments were administered in triplicate as a single SDP exposure according to a randomized block design (appendix 3, item 1). The treatment levels were (1) an untreated control group, (2) a group that received an application of 50 milligrams per liter $(\mathrm{mg} / \mathrm{L})$ of SDP active ingredient (AI) for 8 hours, and (3) a group that received an application of $100 \mathrm{mg} / \mathrm{L}$ of SDP AI for 8 hours. The experimental unit for the trial was the individual test enclosure.

\section{Test Enclosure Area}

Nine test enclosure areas were identified near the north shore of Lake Darling (fig. 1), and the Universal Transverse Mercator (UTM) coordinates (test area centroid) were recorded (table 1). Each test area was characterized as predominately sandy substrate with minimal algae and macrophyte growth. The areas selected were approximately 1.4 meter $(\mathrm{m})$ deep and were spaced at least $6 \mathrm{~m}$ apart (by center point). Unionid mussel retention barriers (fig. 2) were placed in each test enclosure area approximately 48 hours prior to exposure. The expanded metal 1-square-meter $\left(\mathrm{m}^{2}\right)$ retention barriers were used to confine the unionid mussels to the test enclosure area for the duration of the study. After unionid mussel allocation, a 2.25- $\mathrm{m}^{2}$ test enclosure (fig. 2) was placed around each retention barrier and secured to fenceposts driven into the lakebed. The bottom sealing flaps of each test enclosure were secured to the lakebed with sandbags. 


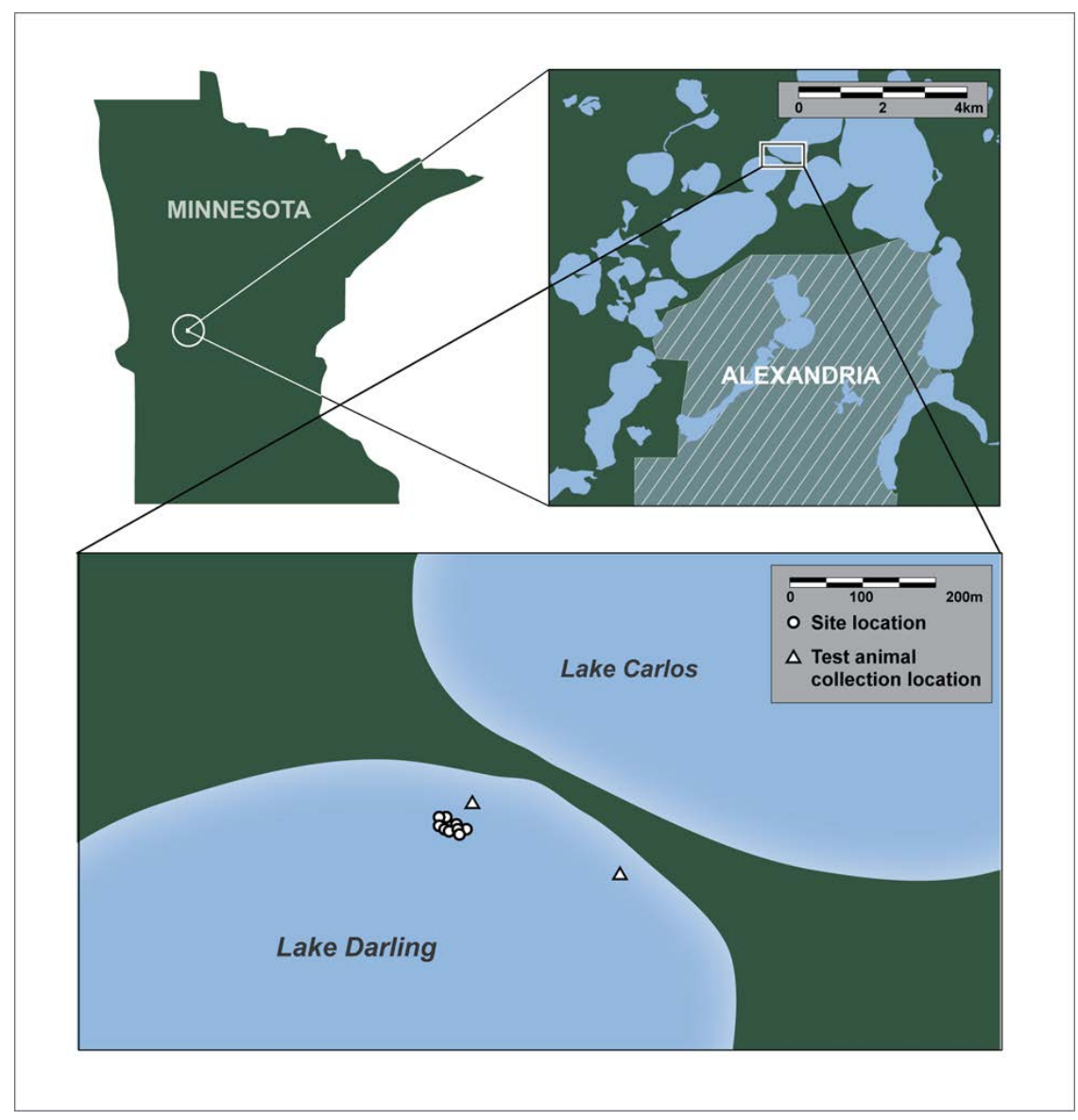

Figure 1. Test enclosure site and test animal collection locations.

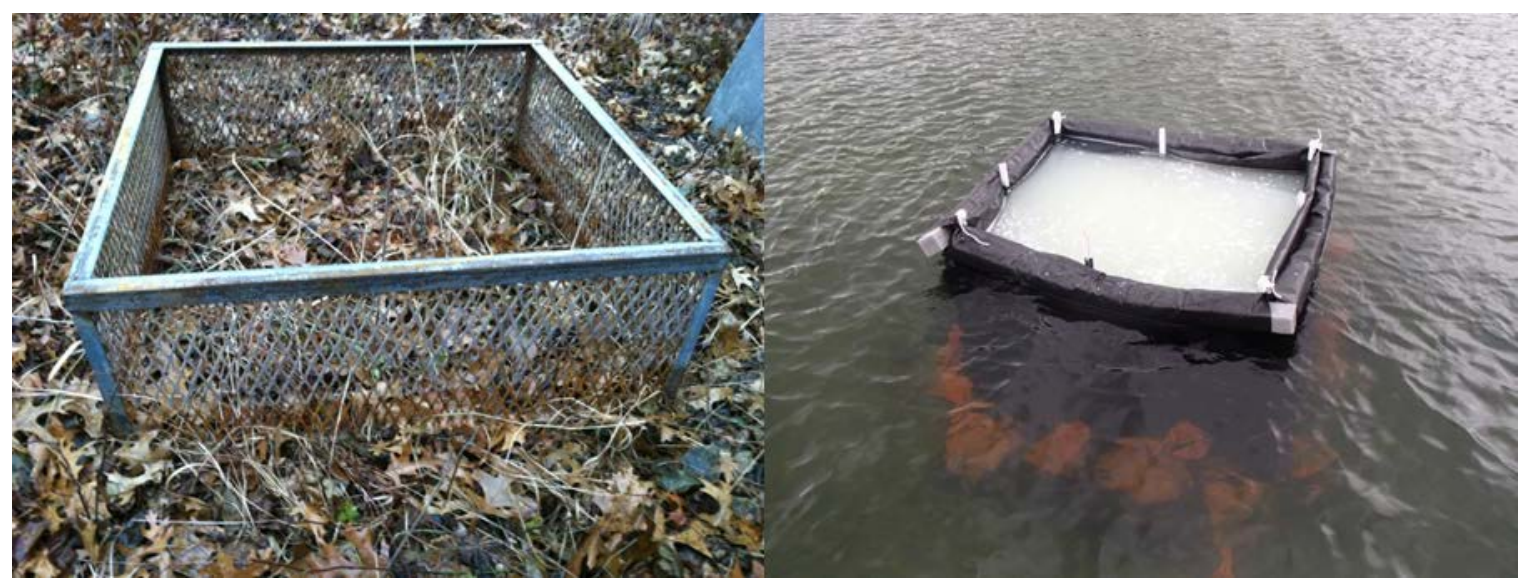

Figure 2. Example of 1-square-meter $\left(\mathrm{m}^{2}\right)$ expanded metal mussel retention barrier (left) and $2.25-\mathrm{m}^{2}$ impermeable test enclosure (right). 
Table 1. Universal Transverse Mercator (UTM) centroid location of each test enclosure.

[mg/L, milligrams per liter; mE, meters East; mN, meters North]

\begin{tabular}{cccccc}
\hline Enclosure & $\begin{array}{c}\text { Treatment } \\
\text { group } \\
(\mathrm{mg} / \mathrm{L})\end{array}$ & Zone & Row & $\begin{array}{c}\text { Easting } \\
(\mathrm{mE})\end{array}$ & $\begin{array}{c}\text { Northing } \\
(\mathrm{mN})\end{array}$ \\
\hline 1 & 100 & 15 & $\mathrm{~T}$ & 315318 & 5089217 \\
2 & 50 & 15 & $\mathrm{~T}$ & 315305 & 5089224 \\
3 & 0 & 15 & $\mathrm{~T}$ & 315292 & 5089230 \\
4 & 50 & 15 & $\mathrm{~T}$ & 315281 & 5089231 \\
5 & 50 & 15 & $\mathrm{~T}$ & 315279 & 5089221 \\
6 & 100 & 15 & $\mathrm{~T}$ & 315290 & 5089218 \\
7 & 0 & 15 & $\mathrm{~T}$ & 315294 & 5089215 \\
8 & 0 & 15 & $\mathrm{~T}$ & 315302 & 5089211 \\
9 & 100 & 15 & $\mathrm{~T}$ & 315307 & 5089205 \\
\hline
\end{tabular}

\section{Test Article}

The test article was a commercially prepared SDP formulation of Pseudomonas fluorescens, strain CL145A containing 50 percent AI (weight to weight ratio [w/w] P. fluorescens, strain CL145A). The test article (lot number TR 4669-4-3) was obtained from Marrone Bio Innovations, Inc. (MBI; Davis, California; Certificate of Analysis, appendix 4, item 2). Test article use was documented in the test chemical logbooks (appendix 4, items 5 and 6). Verification of test article biological activity was determined on a sample of test article by the New York State Museum Field Research Laboratory (Cambridge, New York), using their standard dreissenid mussel bioassay (appendix 4, item 7). Biological activity was confirmed as demonstrated by a mean zebra mussel mortality in the treated group of $96.0 \pm 6.9$ percent compared to $1.3 \pm 2.2$ percent in the untreated group.

\section{Test Animals}

Adult fatmucket unionid mussels (Lampsilis siliquoidea) with adhering zebra mussels (fig. 3) were collected 3 days prior to exposure from an existing population located within $300 \mathrm{~m}$ of the test enclosures. An incidental number of fragile papershell unionid mussels (Leptodea fragilis, $n=0-3$ per enclosure) were inadvertently included. The $L$. fragilis were treated and analyzed the same as $L$. siliquoidea. Unionid mussels were confined within a $1-\mathrm{m}^{2}$ retention barrier in approximately $0.75 \mathrm{~m}$ of water until distributed to the test enclosures. A Hallprint ${ }^{\circledR}$ shellfish tag with a unique alphanumeric code was fixed to each mussel shell with cyanoacrylate glue, and an initial wet weight with adhering zebra mussels was obtained for each unionid mussel.

Groups of unionid mussels $(n=4-5)$ with adhering zebra mussels were randomly allocated to each test enclosure (appendix 3, item 4) and placed within a retention barrier until each test enclosure/retention barrier received a total of 27 unionid mussels.

At exposure termination, the test enclosures were removed and both Group 1 and Group 2 unionid mussels remained in the retention barriers at the treatment location for a postexposure holding period. At 18-20 days postexposure, the unionid mussels were removed from the retention barriers, and 
wet weights were measured before and after removal of adhering zebra mussels. Additionally, each unionid mussel was photographed, assessed for survival, and measured for shell length. Unionid mussels were euthanized according to permit requirements (appendix 5, items 1-3). The unionid mussel shells were retained and used for confirmative species identification. All zebra mussels removed were sorted into categories of dead or alive and were enumerated; a subsample from each group was measured for shell length.

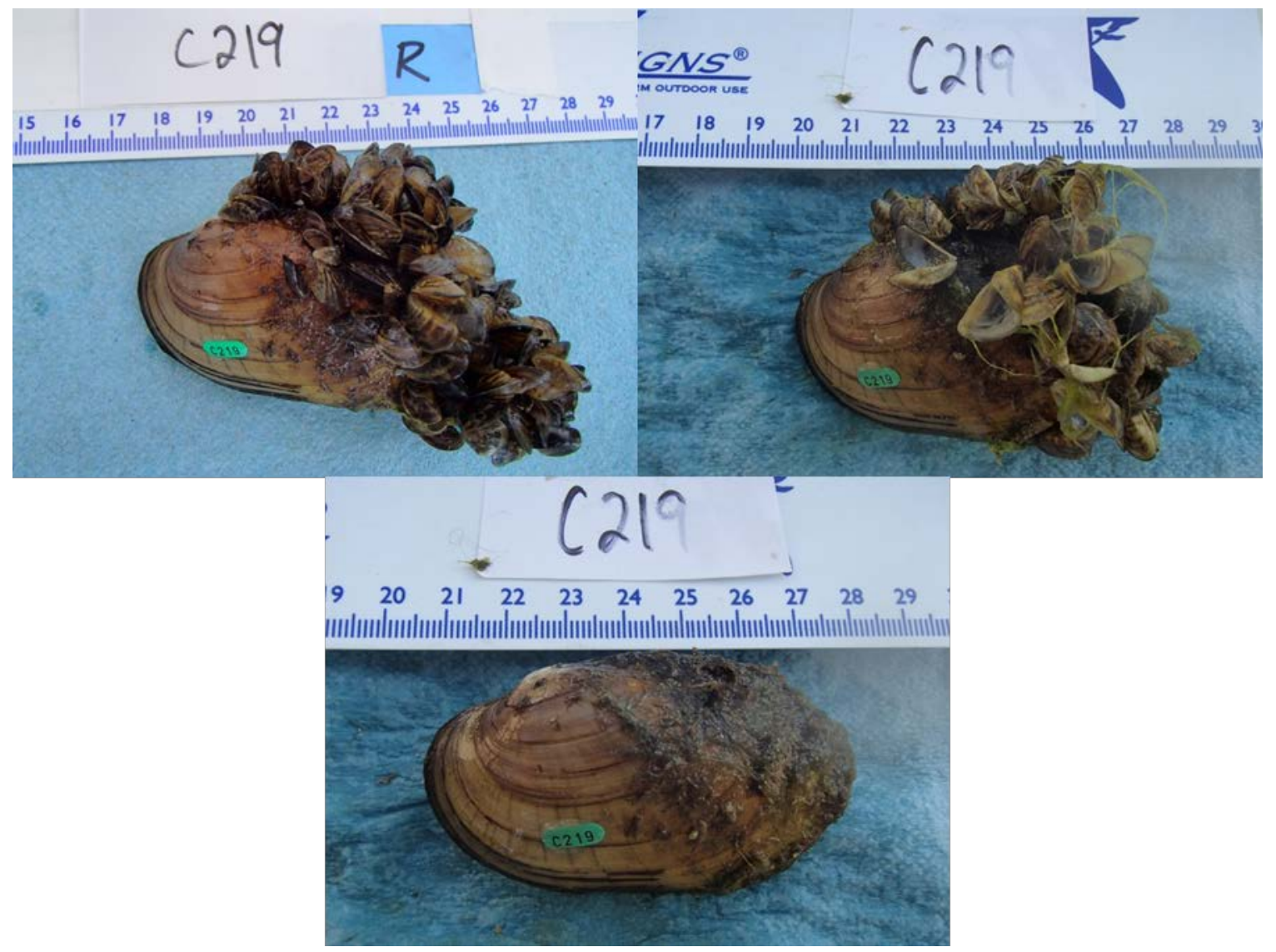

Figure 3. Representative unionid mussel with adhering zebra mussels preexposure (top left), postexposure (top right) and with zebra mussels removed (bottom).

\section{Reduction of Adhering Zebra Mussels}

\section{Estimation of Initial Number of Adhering Zebra Mussels (Group 1)}

After distribution, five unionid mussels (Group 1) were indiscriminately removed from each test enclosure and used to estimate the initial preexposure biomass of adhering zebra mussels for each test enclosure (that is, the initial number of adhering zebra mussels per gram of zebra mussels [equation 1]):

$$
\text { en }=\frac{n_{z m}}{\left[W_{1}-W_{2}\right]}
$$

where en is the estimated number of adhering zebra mussels per gram of zebra mussels, $n_{\mathrm{zm}} \quad$ is the number of live adhering zebra mussels removed from Group 1 mussels (preexposure),

$W_{1} \quad$ is the group 1 mussel wet weight with adhering zebra mussels, in grams (g), and 
$W_{2} \quad$ is the group 1 mussel wet weight after zebra mussel removal (g).

The average number of adhering zebra mussels, per gram, from each test enclosure was used to estimate the initial number of adhering zebra mussels on Group 2 mussels (equation 2).

\section{Estimation of the Number of Adhering Zebra Mussels (Group 2)}

For each test enclosure, the number of zebra mussel adhering to Group 2 mussels prior to exposure was estimated (equation 2 ) by using the mean estimated number $\left(\overline{\mathrm{x}}_{e n}\right)$ of adhering zebra mussels, per gram, of Group 1 mussels (derived from equation 1). Initial Group 2 mussel wet weight with adhering zebra mussels before exposure and Group 2 mussel wet weight after zebra mussel removal were used to determine the weight of adhering zebra mussels (equation 2);

$$
e n_{2}=\left[W_{1}-W_{2}\right] \times \overline{\mathrm{x}}_{\text {en }}
$$

where $e n_{2} \quad$ is the estimated number of zebra mussels adhering to Group 2 mussel prior to exposure,

$W_{1} \quad$ is the preexposure wet weight of Group 2 mussel with adhering zebra mussels (g),

$W_{2} \quad$ is the postexposure wet weight Group 2 mussel after zebra mussel removal (g), and

$\overline{\mathrm{x}}_{\text {en }} \quad$ is the mean estimated number of adhering zebra mussels per gram of zebra mussels for each test enclosure (derived from equation 1 ).

\section{Calculation of Zebra Mussel Body Weight Burden (Group 2)}

The percent body weight burden of adhering zebra mussels was calculated for each individual unionid mussel by comparing the estimated weight of adhering zebra mussels to the weight of the unionid mussel without zebra mussels (equation 3). A mean percent body weight burden was calculated for each treatment group:

where

$$
Z M \text { Burden }(\text { percent })=\left(W_{1} / W_{2}\right) \times 100
$$

ZM Burden is the adhering zebra mussel burden expressed as percent of unionid mussel body weight,

$W_{1} \quad$ is the estimated preexposure wet weight of zebra mussels (g), and

$W_{2} \quad$ is the postexposure wet weight unionid mussel without zebra mussels (g).

\section{Calculation of Adhering Zebra Mussels Reduction (Group 2)}

For each test enclosure, the reduction in the number of zebra mussels adhering to Group 2 mussels was estimated by using the mean estimated number $\left(\overline{\mathrm{x}}_{\mathrm{en}_{2}}\right)$ of adhering zebra mussels (derived from equation 2) and the number of adhering zebra mussels postexposure (equation 4). A mean percent reduction was calculated for each treatment group:

$$
\text { Percent } \Delta=\left(\left[\frac{\left\{\overline{\mathrm{x}}_{e n_{2}}-n_{z m}\right\}}{\overline{\mathrm{x}}_{e n_{2}}}\right] \times 100\right)
$$

where $\Delta \quad$ is the percent reduction in the number of adhering zebra mussels on Group 2 unionids,

$$
\begin{array}{ll}
\overline{\mathrm{x}}_{e n_{2}} & \text { is the mean estimated number of zebra mussels adhering to Group } 2 \text { mussel prior to } \\
\text { exposure (derived from equation 2), } \\
n_{\mathrm{zm}} & \text { is the number of live zebra mussels removed from Group } 2 \text { mussels (postexposure). }
\end{array}
$$




\section{Calculation of SDP Reduction Coefficient and Efficiency (Group 2)}

A reduction coefficient of SDP was calculated for each test enclosure by comparing the reduction in the number of adhering zebra mussels to the amount of SDP applied to the test enclosure area (equation 5). A mean reduction coefficient was calculated for each treatment group:

$$
R C=\left(\frac{\Delta}{[P / A}\right)
$$

where $R C \quad$ (reduction coefficient) is the percent reduction of adhering zebra mussels per gram of SDP applied,

$\Delta \quad$ is the percent reduction in the number of adhering zebra mussels on Group 2 unionids (derived from equation 4),

$P \quad$ is the amount of SDP applied (g), and

A is the area treated $\left(=2.25 \mathrm{~m}^{2}\right)$.

\section{Unionid Mussel Survival}

Each unionid mussels was assessed for survival 18-20 days after SDP exposure. Survival was defined as foot or valve movement in response to tactile stimuli or resistance to valve pressure by adductor muscle contraction. Unrecovered unionid mussels were treated as mortalities within the data analysis.

\section{Stock-Solution Preparation and Dosing}

A separate SDP stock solution was prepared and immediately applied to each test enclosure. The appropriate amount of SDP required to treat each test enclosure (appendix 4, item 4) was added to approximately 10 liters (L) of unfiltered lake water and mixed. Immediately after mixing, the stock was poured through a strainer, and clumps of test article were macerated with a pestle and rinsed into the stock solution with unfiltered lake water, bringing the final stock volume to approximately $15 \mathrm{~L}$.

The prepared stock solution was transported in a 19.4-L screw-top bucket to the assigned test enclosure. Treatments were individually applied in the following sequence: control, 50-, and 100-mg/L SDP. The 50- and 100-mg/L SDP treatments were applied by adding the stock solution to four areas of the test enclosure. The water and stock solution within the test enclosure was then mixed by using a boat paddle. The control treatments involved the same application technique used for the SDP-treated groups, except that untreated Lake Darling water was applied in place of SDP stock solution.

\section{Test Enclosure Water Sample Collection}

Water samples were collected by submersing a 1-L screw-top container below the surface of each test enclosure. The water samples were used to verify exposure concentration and to measure water-quality parameters (that is, hardness, alkalinity, conductivity, dissolved oxygen, temperature, $\mathrm{pH}$, and ammonia).

\section{Concentration Verification}

Exposure water SDP concentrations were determined at 1, 4, and 8 hours by comparing surface water samples collected from each test enclosure to a linear regression curve created from known concentrations (25, 50, 100 and $200 \mathrm{mg} / \mathrm{L}$ ) and absorbance of the test article. Sample absorbance was measured on a Barnstead-Turner SP-830 Plus (model SM110215) spectrophotometer at 660 nanometers $(\mathrm{nm})$. Linear regression equations were fit by using the SAS ${ }^{\circledR}$ software Proc Reg procedure (SAS ${ }^{\circledR}$ 
version 9.3; SAS Institute Inc., Cary, North Carolina), and the exposure sample concentrations were predicted from the regression analysis (appendix 7, items 2 and 3).

Water Chemistry

Hardness, alkalinity, and conductivity were measured in surface water samples collected from each test enclosure before administering the test article. Dissolved oxygen, $\mathrm{pH}$, and temperature were measured in surface water samples collected from each test enclosure prior to exposure as well as 4 and 8 hours after exposure initiation. Immediately before exposure period termination, water samples were collected, filtered (0.45 micrometer $[\mu \mathrm{m}])$, acidified with 10 percent sulfuric acid to $\leq \mathrm{pH} 2.5$, and stored at approximately 4 degrees Celsius $\left({ }^{\circ} \mathrm{C}\right.$ ) until analyzed for total ammonia nitrogen (TAN) content by using the automated phenate method (Standard Method 4500G; American Public Health Association and others, 2012). The un-ionized ammonia fractions were calculated by using the sample $\mathrm{pH}$ and temperatures measured at the time of sample collection according to the formula identified by Emerson and others (1975). Temperature loggers (Onset, Bourne, Massachusetts, HOBO ${ }^{\circledR}$ Pendent Temperature/Light Data Logger) were attached to the retention barriers and used to measure water temperature every 3 hours during the postexposure period. Dissolved oxygen, $\mathrm{pH}$, and temperature of the lake water were measured daily during the assessment period (18-20 days postexposure) near the retention barriers.

\section{Data Analysis}

Water chemistry data analyses were limited to simple descriptive statistics; comparative statistics were not generated. Statistical comparisons of zebra mussel density, zebra mussel length, and unionid mussel survival were performed by using SAS ${ }^{\circledR}$ software version 9.3 (SAS Institute, Inc., Cary, N.C.). Significance for all analyses was declared at $p \leq 0.05$. Exposure concentrations were determined by using SAS $^{\circledR}$ software version 9.3.

\section{Reduction of Adhering Zebra Mussels}

A general linear model created with the $\mathrm{SAS}^{\circledR}$ software Proc GLM procedure was used to analyze the number of zebra mussels adhering to unionid mussels before and after exposure (appendix 10, item 5). The mean number of zebra mussels adhering to unionid mussels in each treatment group before and after exposure was modeled with the "no intercept" and "solution” options specified. The assumptions of normal distribution and homogeneity of variance were assessed by using the univariate procedure with the "normal” option specified and the Bartlett's test. The mean number of zebra mussels adhering to unionid mussels of each treatment group (before and after exposure) was individually compared to the number of zebra mussels adhering to unionid mussels of the untreated control group by using a two-sided means comparison test.

By using the procedures previously described (SAS ${ }^{\circledR}$ software Proc GLM; appendix 10, item 8), the mean length of zebra mussels removed from unionid mussels collected from each treatment replicate before (Group 1) and after (Group 2) SDP exposure was analyzed to determine whether a correlation existed between zebra mussel length and zebra mussel survival within each treatment assignment. The analysis of test animal length was limited to adhering live zebra mussels because dead zebra mussel shells could not be retained. 


\section{Unionid Mussel Survival}

A generalized linear mixed model (SAS ${ }^{\circledR}$ software Proc GLIMMIX) was used to analyze the survival of unionid mussels in each treatment (appendix 10, item 2). The proportion of mortalities (number of dead unionid mussels compared to the total number unionid mussels) was modeled with a binomial distribution and a logit link function. A scale parameter was added to the model using the "random_residual” statement. Unionid mussel survival of each treatment group was individually compared to the survival in the untreated control group by using a two-sided means comparison test with a Tukey post hoc adjustment.

\section{Exposure Concentration}

The mean exposure concentrations were determined for individual test enclosures, for each sampling time, and for each treatment group using the SAS ${ }^{\circledR}$ software Proc Means statement (appendix 7, item 3).

\section{Results}

\section{Adverse Events}

There were no observable adverse effects observed in the unionid mussels or zebra mussels within the test enclosures. There was a significant algal bloom within Lake Darling (dominated by Mougeotia and Spirogyra species) during the postexposure period. The study was terminated approximately 10 days earlier than planned because of concerns that the algae bloom may impact native or zebra mussel survival (appendix 2, items 1 and 6).

\section{Reduction of Adhering Zebra Mussels}

Statistical analysis output can be found in appendix 10 (item 4), and the data summary can be found in appendix 8 (item 1). The number of zebra mussels adhering to unionid mussels before and after treatment is summarized in table 2 . No difference $(p>0.74)$ was detected in the number of zebra mussels adhering to unionid mussels in each treatment group prior to exposure. The observed percent body weight burden of adhering zebra mussels from each treatment group ranged from 125.9 to 130.0 percent of unionid mussel body weight. The mean number of adhering zebra mussels before and after exposure differed in the 50- and 100-mg/L treatment groups (160 versus 74 [ $p<0.01$ ] and 180 versus $59[p<0.01]$, respectively). The number of adhering zebra mussels was reduced on average by 53 and 68 percent in the 50 - and $100-\mathrm{mg} / \mathrm{L}$ treatment groups, respectively. The number of adhering zebra mussels did not differ ( $p=0.79$ ) between the 50- and 100-mg/L treatment groups after exposure. When standardized to the amount of SDP applied per square meter, the 50-mg/L treatment was more efficient than the $100-\mathrm{mg} / \mathrm{L}$ treatment at reducing the number of adhering zebra mussels $(0.44$ percent reduction per gram applied versus 0.28 percent reduction per gram applied, respectively).

Statistical analysis output can be found in appendix 10 (item 7), and the data summaries can be found in appendix 5 (items 6-7). Mean length of adhering zebra mussels is summarized in table 3. Mean length did not differ ( $p>0.89$ ), indicating that zebra mussel length was not a predictor of mortality. 
Table 2. Mean (standard deviation) number of adhering zebra mussels per unionid mussel before and after exposure, percent change of zebra mussel reduction, zebra mussel burden (as percent unionid body weight), and spray dried powder (SDP) reduction coefficient for number of adhering zebra mussels for each treatment group. [mg/L, milligrams per liter; ZM, zebra mussel; SDP, spray dried powder; NA, not applicable. Means with the same letter are not significantly different $(p>0.05)$.]

\begin{tabular}{lllclc}
\hline \multirow{2}{*}{$\begin{array}{c}\text { Treatment group } \\
(\mathbf{m g} / \mathrm{L})\end{array}$} & \multicolumn{3}{c}{ Zebra mussels per unionid mussel } & ZM burden ${ }^{1}$ & $\begin{array}{c}\text { SDP reduction coefficient }{ }^{2} \\
\text { (percent) }\end{array}$ \\
\cline { 2 - 5 } & Preexposure & Postexposure & $\begin{array}{c}\text { Colonization change } \\
\text { (percent) }\end{array}$ & & \\
\hline Control (0) & $165^{\mathrm{a}}(15)$ & $179^{\mathrm{a}}(9)$ & $9(9)$ & $130.0(4.4)$ & NA \\
50 & $160^{\mathrm{a}}(11)$ & $74^{\mathrm{b}}(9)$ & $-53(8)$ & 125.9 & $0.441(0.043)$ \\
& & & & $(12.5)$ & \\
100 & $180^{\mathrm{a}}(13)$ & $59^{\mathrm{b}}(23)$ & $-68(10)$ & $\begin{array}{c}127.1 \\
(11.7)\end{array}$ & $0.276(0.033)$ \\
\hline
\end{tabular}

${ }^{1}$ Expressed as a percentage of unionid mussel body weight.

${ }^{2}$ Expressed as percent reduction in the number of adhering zebra mussels per gram of SDP applied.

Table 3. Mean (standard deviation; number measured) length of adhering zebra mussels (live) by treatment group. [mg/L, milligrams per liter; mm, millimeters. Means with the same letter are not significantly different $(p>0.05)]$

\begin{tabular}{lll}
\hline \multirow{2}{*}{ Treatment group $(\mathrm{mg} / \mathrm{L})$} & \multicolumn{2}{c}{ Length $(\mathrm{mm})$} \\
\cline { 2 - 3 } & Preexposure & Postexposure \\
\hline Control $(0)$ & $12.0^{\mathrm{a}}(0.1 ; 1,453)$ & $11.2^{\mathrm{a}}(2.3 ; 1,366)$ \\
50 & $12.1^{\mathrm{a}}(0.5 ; 1,266)$ & $11.8^{\mathrm{a}}(0.5 ; 590)$ \\
100 & $11.2^{\mathrm{a}}(0.4 ; 1,359)$ & $12.0^{\mathrm{a}}(0.5 ; 584)$ \\
\hline
\end{tabular}

\section{Unionid Mussel Survival}

Statistical analysis output can be found in appendix 10 (item 1), and the data summary can be found in appendix 9 (item 1). Survival of unionid mussels is summarized in table 4. Treatment did not significantly affect Group 1 mussel survival $(p>0.05)$ but did significantly affect Group 2 mussel survival $(p<0.01)$ and Group 1 and Group 2 combined mussel survival $(p<0.01)$. No difference $(p>0.23)$ was detected in survival between the Group 2 control group and the 50- or 100-mg/L treatment groups. A difference ( $p=0.03$ ) was detected in the mean survival between the 50 - and the $100-\mathrm{mg} / \mathrm{L}$ treatment groups (96.8 and 85.1 percent, respectively), however, the biological significance of the difference is indeterminate. The log odds of unionid mussel survival in the $100-\mathrm{mg} / \mathrm{L}$ treatment was 0.290 times the odds of survival of unionid mussels assigned to the $50-\mathrm{mg} / \mathrm{L}$ treatment. 
Table 4. Mean (standard deviation) percent survival of Group 1 and 2 unionid mussels.

$[\mathrm{mg} / \mathrm{L}$, milligrams per liter. Means within columns with the same letter are not significantly different $(p>0.05)]$

\begin{tabular}{lcl}
\hline \multirow{2}{*}{ Treatment group (mg/L) } & \multicolumn{2}{c}{ Survival (percent) } \\
\cline { 2 - 3 } & Group 1 & Group 2 \\
\hline Control (0) & $100.0^{\mathrm{a}}(0.0)$ & $89.4^{\mathrm{ab}}(6.9)$ \\
50 & $100.0^{\mathrm{a}}(0.0)$ & $96.8^{\mathrm{a}}(3.9)$ \\
100 & $80.0^{\mathrm{a}}(20.0)$ & $85.1^{\mathrm{b}}(13.8)$ \\
\hline
\end{tabular}

\section{Dose Verification}

The linear regression, exposure concentrations, and data summary can be found in appendix 7 (items 1-2). Exposure concentrations for each treatment group are summarized in table 5. The measured exposure concentrations were lower than the target concentration. The mean SDP exposure concentrations measured throughout the exposure period were $35.3 \pm 3.3 \mathrm{mg} / \mathrm{L}$ and $74.2 \pm 3.4 \mathrm{mg} / \mathrm{L}$ for the 50- and $100-\mathrm{mg} / \mathrm{L}$ treatment groups, respectively.

Table 5. Mean (standard deviation) spray dried powder exposure concentration in surface water samples collected from test enclosures during the exposure period.

[mg/L, milligrams per liter; ND, not detectable/below detection limit]

\begin{tabular}{lccc}
\hline \multirow{2}{*}{ Treatment group (mg/L) } & \multicolumn{3}{c}{ Time (hours) } \\
\cline { 2 - 4 } & \multicolumn{1}{c}{$\mathbf{4}$} & $\mathbf{8}$ \\
\hline Control (0) & ND & ND & ND \\
50 & $38.6(1.7)$ & $35.5(2.7)$ & $31.9(1.0)$ \\
100 & $76.9(1.3)$ & $75.5(2.4)$ & $70.3(1.3)$ \\
\hline
\end{tabular}

\section{Water Chemistry}

The water-chemistry data summaries are presented in appendix 6 (items 1-5). Water-chemistry parameters (dissolved oxygen, $\mathrm{pH}$, and temperature) in the test enclosures are summarized in table 6 . Dissolved oxygen concentration remained above the minimum level recommended $(4 \mathrm{mg} / \mathrm{L})$ in the ASTM International guide for conducting laboratory tests with freshwater mussels (ASTM International, 2013). Hardness, alkalinity, conductivity, and ammonia are summarized in table 7. The alkalinity ranged from 174 to $176 \mathrm{mg} / \mathrm{L}$ as calcium carbonate $\left(\mathrm{CaCO}_{3}\right)$, hardness from 189 to $193 \mathrm{mg} / \mathrm{L}$ as $\mathrm{CaCO}_{3}$, and conductivity from 322 to 324 microsiemens per centimeter $(\mu \mathrm{S} / \mathrm{cm})$ (automatic temperature corrected to $25^{\circ} \mathrm{C}$ ); un-ionized ammonia was $\leq 0.01 \mathrm{mg} / \mathrm{L}$. Data collected by the temperature data loggers during the postexposure period indicated a water temperature range of $22.3^{\circ} \mathrm{C}$ to $23.9^{\circ} \mathrm{C}$. Mean (standard deviation [SD]) water-chemistry parameters measured 18-20 days postexposure were dissolved oxygen, $8.60 \mathrm{mg} / \mathrm{L}(0.40)$; temperature, $21.9^{\circ} \mathrm{C}(0.3)$; and $\mathrm{pH}$ range, $8.54-$ 8.63. 
Table 6. Mean (standard deviation) water chemistry (dissolved oxygen, temperature) and $\mathrm{pH}$ range of surface water samples collected from each treatment group during the study period.

[mg/L, milligrams per liter; ${ }^{\circ} \mathrm{C}$; degrees Celsius]

\begin{tabular}{|c|c|c|c|}
\hline Sample time & $\mathrm{pH}$ & Dissolved oxygen (mg/L) & Temperature $\left({ }^{\circ} \mathrm{C}\right)$ \\
\hline \multicolumn{4}{|c|}{ Control (0 mg/L) } \\
\hline Preexposure & $8.45-8.58$ & $7.03(0.12)$ & $20.6(0.5)$ \\
\hline 4 hours & $8.45-8.50$ & $6.49(0.12)$ & $22.9(0.2)$ \\
\hline 8 hours & $8.40-8.49$ & $6.36(0.39)$ & $23.0(0.0)$ \\
\hline \multicolumn{4}{|c|}{$50 \mathrm{mg} / \mathrm{L}$} \\
\hline Preexposure & $8.47-8.56$ & $7.22(0.48)$ & $20.4(0.2)$ \\
\hline 4 hours & $8.35-8.38$ & $5.95(0.17)$ & $23.1(0.1)$ \\
\hline 8 hours & $8.25-8.36$ & $5.63(0.06)$ & $23.1(0.1)$ \\
\hline \multicolumn{4}{|c|}{$100 \mathrm{mg} / \mathrm{L}$} \\
\hline Preexposure & $8.53-8.56$ & $6.86(0.24)$ & $19.6(0.6)$ \\
\hline 4 hours & $8.16-8.28$ & $5.85(0.19)$ & $23.0(0.2)$ \\
\hline 8 hours & $8.18-8.23$ & $5.36(0.17)$ & $23.1(0.2)$ \\
\hline
\end{tabular}

Table 7. Mean (standard deviation) alkalinity, hardness, conductivity, and ammonia content of surface water samples collected from each treatment group during the study period.

[mg/L, milligrams per liter; $\mu \mathrm{S} / \mathrm{cm}$, microsiemens per centimeter; TAN, total ammonia nitrogen; $\mathrm{mg} \mathrm{NH}_{3}-\mathrm{N} / \mathrm{L}$, milligrams unionized ammonia nitrogen per liter; $\mathrm{NH}_{3}$, un-ionized ammonia; <, less-than; $\mathrm{CaCO}_{3}$, calcium carbonate; ${ }^{\circ} \mathrm{C}$, degrees Celsius]

\begin{tabular}{|c|c|c|c|c|c|}
\hline \multirow{2}{*}{$\begin{array}{l}\text { Treatment group } \\
(\mathrm{mg} / \mathrm{L})\end{array}$} & \multicolumn{3}{|c|}{ Preexposure } & \multicolumn{2}{|c|}{ Exposure termination } \\
\hline & $\begin{array}{l}\text { Alkalinity } \\
(\mathrm{mg} / \mathrm{L})^{1}\end{array}$ & $\begin{array}{c}\text { Hardness } \\
(\mathrm{mg} / \mathrm{L})^{1}\end{array}$ & $\begin{array}{l}\text { Conductivity } \\
(\mu \mathrm{S} / \mathrm{cm})^{2}\end{array}$ & $\begin{array}{c}\text { TAN (mg NH }{ }^{-}- \\
\mathrm{N} / \mathrm{L})\end{array}$ & $\mathrm{NH}_{3}$ (mg/L) \\
\hline Control (0) & $175(0)$ & $189(1)$ & $323(5)$ & $0.04(0.02)$ & $<0.01(<0.01)$ \\
\hline 50 & $174(2)$ & $190(2)$ & $322(3)$ & $0.10(0.02)$ & $<0.01(<0.01)$ \\
\hline 100 & $176(3)$ & $193(4)$ & $324(4)$ & $0.13(0.01)$ & $0.01(<0.01)$ \\
\hline
\end{tabular}

${ }^{1}$ Reported as milligrams per liter $\mathrm{CaCO}_{3}$.

${ }^{2}$ Temperature compensated to $25^{\circ} \mathrm{C}$.

\section{Conclusions}

The application of a single dose of spray dried powder (SDP) formulation of Pseudomonas fluorescens (strain CL145A, 50 or 100 milligrams per liter [mg/L]) for 8 hours to open-water test enclosures significantly reduced the number of zebra mussels adhering to unionid mussels. The number of adhering zebra mussels after exposure did not differ $(p=0.79)$ between the $50-\mathrm{mg} / \mathrm{L}$ and the $100-\mathrm{mg} / \mathrm{L}$ treatment groups. When standardized to the amount of SDP applied, the 50-mg/L treatment 
was more efficient than the $100-\mathrm{mg} / \mathrm{L}$ treatment at reducing the number of zebra mussels adhering to zebra mussels ( 0.44 percent reduction per gram of SDP applied versus 0.28 percent reduction per gram of SDP applied, respectively). Multiple 50-mg/L applications may be more efficient at reducing zebra mussels than a single 100-mg/L application of SDP. The use of a subsurface injection application technique to deliver SDP only to the benthic zone may further reduce the amount required to achieve control. Treatment success was not dependent upon zebra mussel length $(p>0.89)$. No difference was detected in unionid mussel survival between the treated and control groups. A difference $(p=0.03$; OR $=0.290$ ) in unionid mussel survival was detected between the Group 2 50-mg/L and Group 2 100-mg/L treatment groups. Though statistically different, the biological significance of the slight difference in observed mortality between the treatment groups is indeterminate. Unionid mussel mortality may have been related to poor initial condition, as indicated by the high initial burden of adhering zebra mussels (> 125 percent of unionid mussel body weight).

\section{References}

American Public Health Association, American Water Works Association, and Water Environment Federation, 2012, Standard methods for examination of water and wastewater (22d ed): Washington, D.C., American Public Health Association, 1,360 p.

ASTM International, 2013, ASTM Standard E2455-06-Standard guide for conducting laboratory toxicity tests with freshwater mussels: West Conshohocken, PA, ASTM International, 52 p.

Baker, S.M., and Hornbach, D.J., 1997, Acute physiological effects of zebra mussel (Dreissena polymorpha) infestation on two unionid mussels, Actinonaias ligamentina and Amblema plicata: Canadian Journal of Fisheries and Aquatic Sciences, v. 54, p. 512-519.

Benson, A.J., Raikow, D., Larson, J., Fusaro, A., and Bogdanoff, A.K., 2015, Dreissena polymorpha (fact sheet, revision date June 26, 2014): Gainesville, Fla., USGS Nonindigenous Aquatic Species Database, accessed February 27, 2015 at http://nas.er.usgs.gov/queries/FactSheet.aspx?speciesID=5.

Birnbaum, Christina, 2011, NOBANIS—Invasive alien species fact sheet-Dreissena polymorpha: Online Database of the European Network on Invasive Alien Species, NOBANIS, accessed February 13, 2014, at http://www.nobanis.org/files/factsheets/Dreissena_polymorpha.pdf.

Burlakova, L.E., Karatayev, A.Y., and Padilla, D.K., 2000, The impact of Dreissena polymorpha (PALLAS) invasion on unionid bivalves: International Review of Hydrobiology, v. 85, no. 5-6, p. 529-541.

Emerson, K., Russo, R.C., Lund, R.E., and Thurston, R.V., 1975, Aqueous ammonia equilibrium calculations-Effect of $\mathrm{pH}$ and temperature: Journal of the Fisheries Research Board of Canada, v. 32, p. 2379-2383.

Hebert, P.D.N., Wilson, C.C., Murdoch, M.H., and Lazar, R., 1991, Demography and ecological impacts of the invading mollusc Dreissena polymorpha: Canadian Journal of Zoology, v. 69, p. 405409.

Mackie, G.L., 1991, Biology of the exotic zebra mussel, Dreissena polymorpha, in relation to native bivalves and its potential impact in Lake St. Clair: Hydrobiologia, v. 219, p. 251-268.

Master, L., 1990, The imperiled status of North American aquatic animals: Biodiversity Network News, v. 3, no. 3, p. 5-8.

Nalepa, T.F., 1994, Decline of native unionids in Lake St. Clair after infestation by the zebra mussel, Dreissena polymorpha: Canadian Journal of Fisheries and Aquatic Sciences, v. 51, p. 2227-2233. Nalepa, T.F., and Schloesser, D.W., eds., 2014, Response, management, and mitigation, Part III of Quagga and zebra mussels-Biology, impacts, and control (2d ed.): Boca Raton, Fla. CRC Press, Taylor \& Francis Group, p. 116-284. 
Neves, R.J., 2004, Propagation of endangered freshwater mussels in North America: Journal of Conchology, special publication 3, p. 69-80.

Neves, R.J., Bogan, A.E., Williams, J.D., Ahlstedt, S.A., and Hartfield, P.W., 1997, Status of aquatic mollusks in the southeastern United States-A downward spiral of diversity, in Benz, G.W., and Collins, D.E., eds., Aquatic fauna in peril-The southeastern perspective: Decatur, Ga., Southeastern Aquatic Research Institute, Lenz Design and Communications, p. 43-85.

Ricciardi, A., and Rasmussen, J.B., 1999, Extinction rates of North American freshwater fauna: Conservation Biology, v. 13, no. 5, p. 1220-1222.

Ricciardi, A., Whoriskey, F.G., and Rasmussen, J.B., 1995, Predicting the intensity and impact of Dreissena infestation on native unionid bivalves from Dreissena field density: Canadian Journal of Fisheries and Aquatic Sciences, v. 52, p. 1449-1461.

Schloesser, D.W., and Kovalak, W.P., 1991, Infestation of unionids by Dreissena polymorpha in a power plant canal in Lake Erie: Journal of Shellfish Research, v. 10, no. 2, p. 355-359.

Schloesser, D.W., and Nalepa, T.F., 1994, Dramatic decline of unionid bivalves in offshore waters of western Lake Erie after infestation by the zebra mussel, Dreissena polymorpha: Canadian Journal of Fisheries and Aquatic Sciences, v. 51, p. 2234-2242.

Strayer, D.L., and Malcom, H.M., 2007, Effects of zebra mussels (Dreissena polymorpha) on native bivalves - The beginning of the end or the end of the beginning?: Journal of the North American Benthological Society, v. 26, no. 1, p. 111-122.

Williams, J.D., Warren, M.L., Jr., Cummings, K.S., Harris, J.L., and Neves, R.J., 1993, Conservation status of freshwater mussels of the United States and Canada: Fisheries, v. 18, no. 9, p. 6-22. 


\section{Appendix 1. Study Protocol With Data Forms}

\begin{tabular}{rlrc}
\hline $\begin{array}{c}\text { Item } \\
\text { number }\end{array}$ & \multicolumn{1}{c}{ Item description } & $\begin{array}{c}\text { Number } \\
\text { of } \\
\text { pages }\end{array}$ & $\begin{array}{c}\text { Report } \\
\text { Page } \\
\text { number }\end{array}$ \\
\hline 1 & $\begin{array}{l}\text { Protocol: “Efficacy of Pseudomonas fluorescens (Pf-CL145A) SDP for controlling } \\
\text { zebra mussels within field enclosures” }\end{array}$ & 32 & 17 \\
2 & Note to File 1: Foot biopsy collection and resulting data use & 1 & 49 \\
3 & Test Chemical Stock Preparation & 1 & 50 \\
4 & Mussel Species Identification & 9 & 51 \\
5 & Mussel Distribution Data & 1 & 60 \\
6 & Water Quality & 1 & 61 \\
7 & Alkalinity & 1 & 62 \\
8 & Conductivity and Hardness & 1 & 63 \\
9 & HOBO Data & 1 & 64 \\
10 & Spectrophotometric Data & 2 & 65 \\
11 & Initial Assessment of Native and Zebra Mussels & 1 & 67 \\
12 & Mussel Initial Assessment & 1 & 68 \\
13 & Mussel Weight and Length Assessment & 1 & 69 \\
14 & Mussel Survival Assessment & 1 & 70 \\
15 & Mussel Final Survival Assessment & 1 & 71 \\
16 & Zebra Mussel Lengths & 1 & 72 \\
17 & Assessment Zebra Mussel Lengths & 1 & 73 \\
18 & Mussel Volume Displacement Assessment - Not Used in Study & 1 & 74 \\
\hline
\end{tabular}


Protocol Title:

Efficacy of Pseudomonas fluorescens (Pf-CL145A) SDP for controlling zebra mussels within field enclosures

Study Number: AEH-13-PSEUDO-05

Test Facilities and Study Sponsor

Upper Midwest Environmental Sciences Center (UMESC)

US Geological Survey

2630 Fanta Reed Rd.

La Crosse, Wisconsin 54603

Field Study Location

Lake Darling, Douglas County, MN

Proposed Experimental Start Date: July 2013

Proposed Experimental Termination Date: March 2014

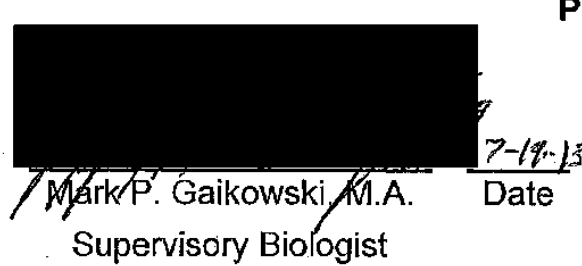

Supervisory Biologist
Protocol Approval

Reviewed by:

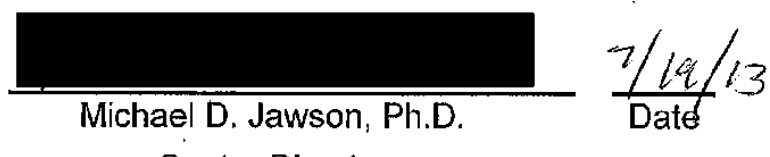

Center Director
Approved by:
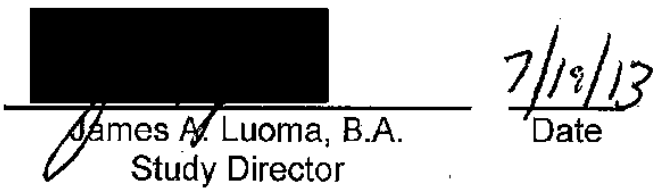

File Folder:

3

Item Number:

Page 1 of 32 


\section{TABLE OF CONTENTS}

1. INTRODUCTION

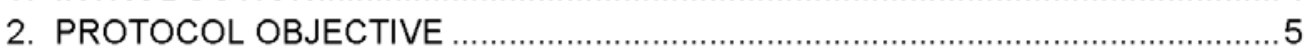

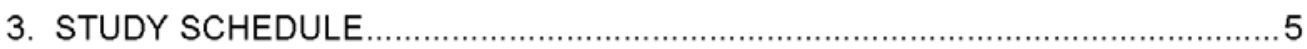

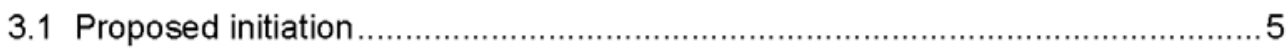

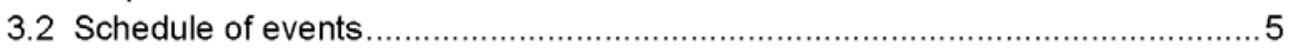

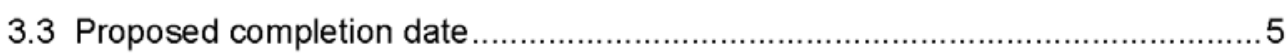

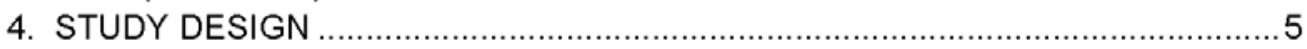

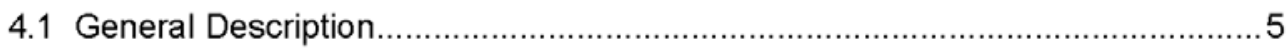

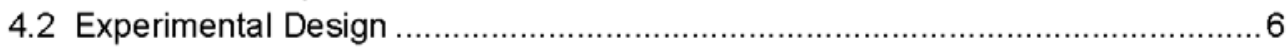

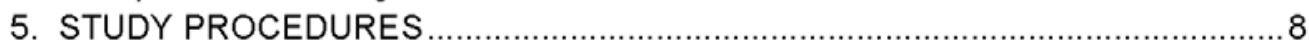

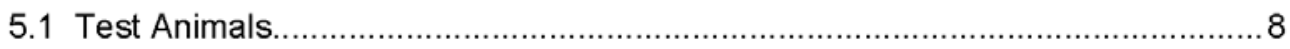

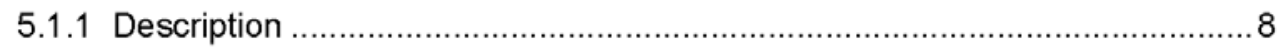

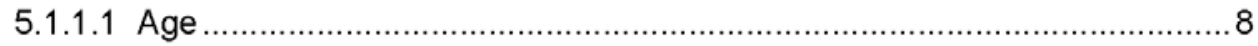

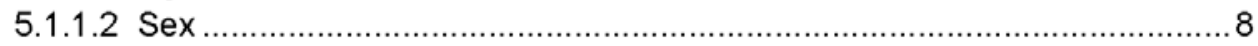

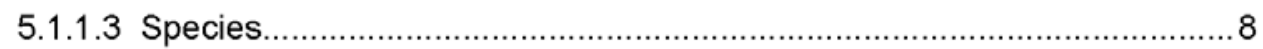

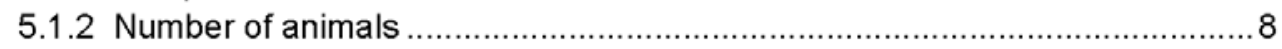

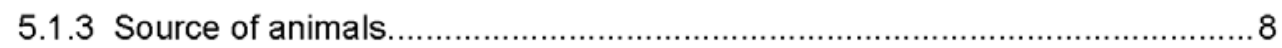

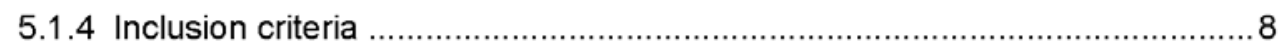

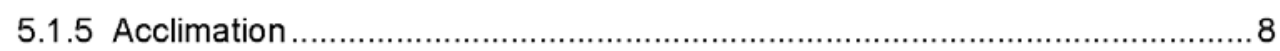

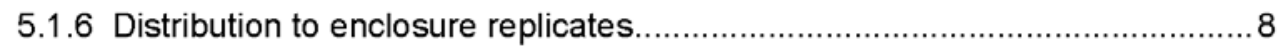

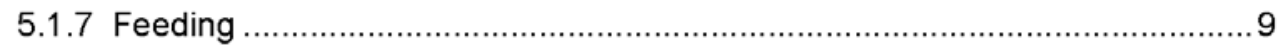

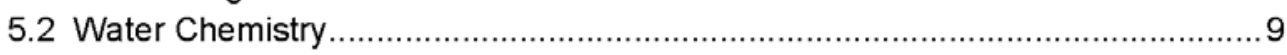

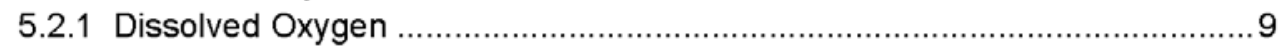

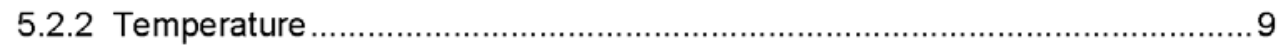

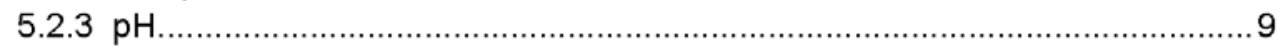

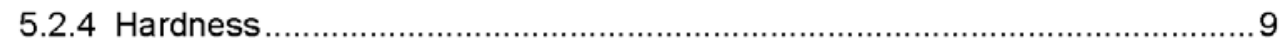

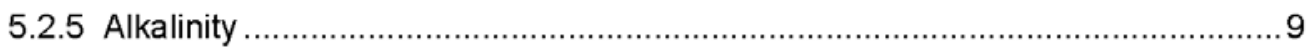

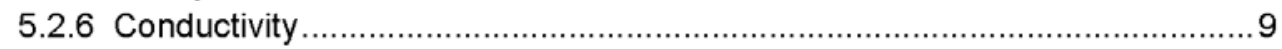

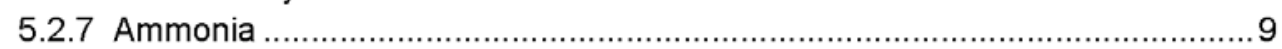

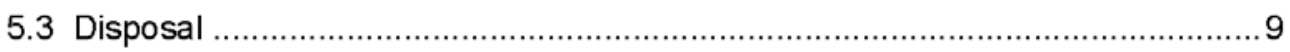

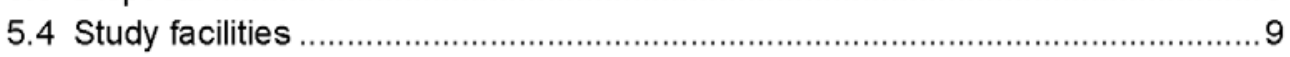

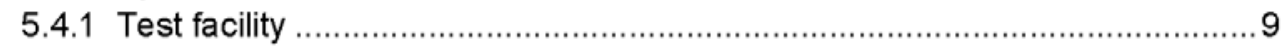

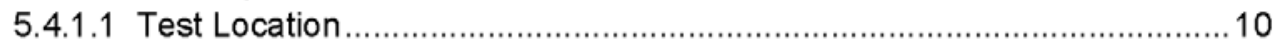

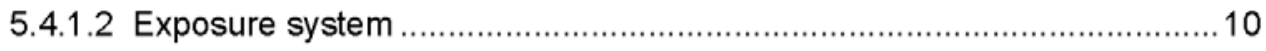

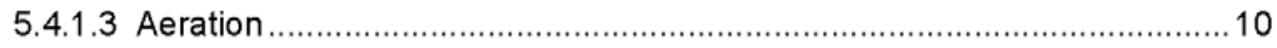

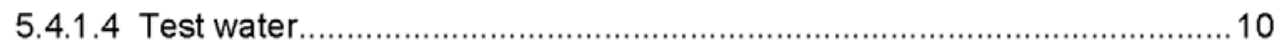

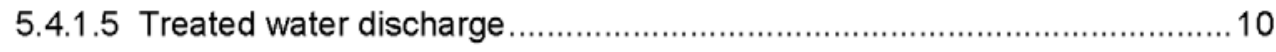

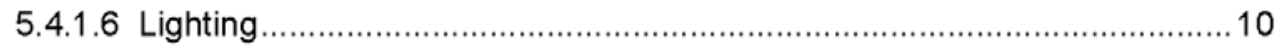

Page 2 of 32 


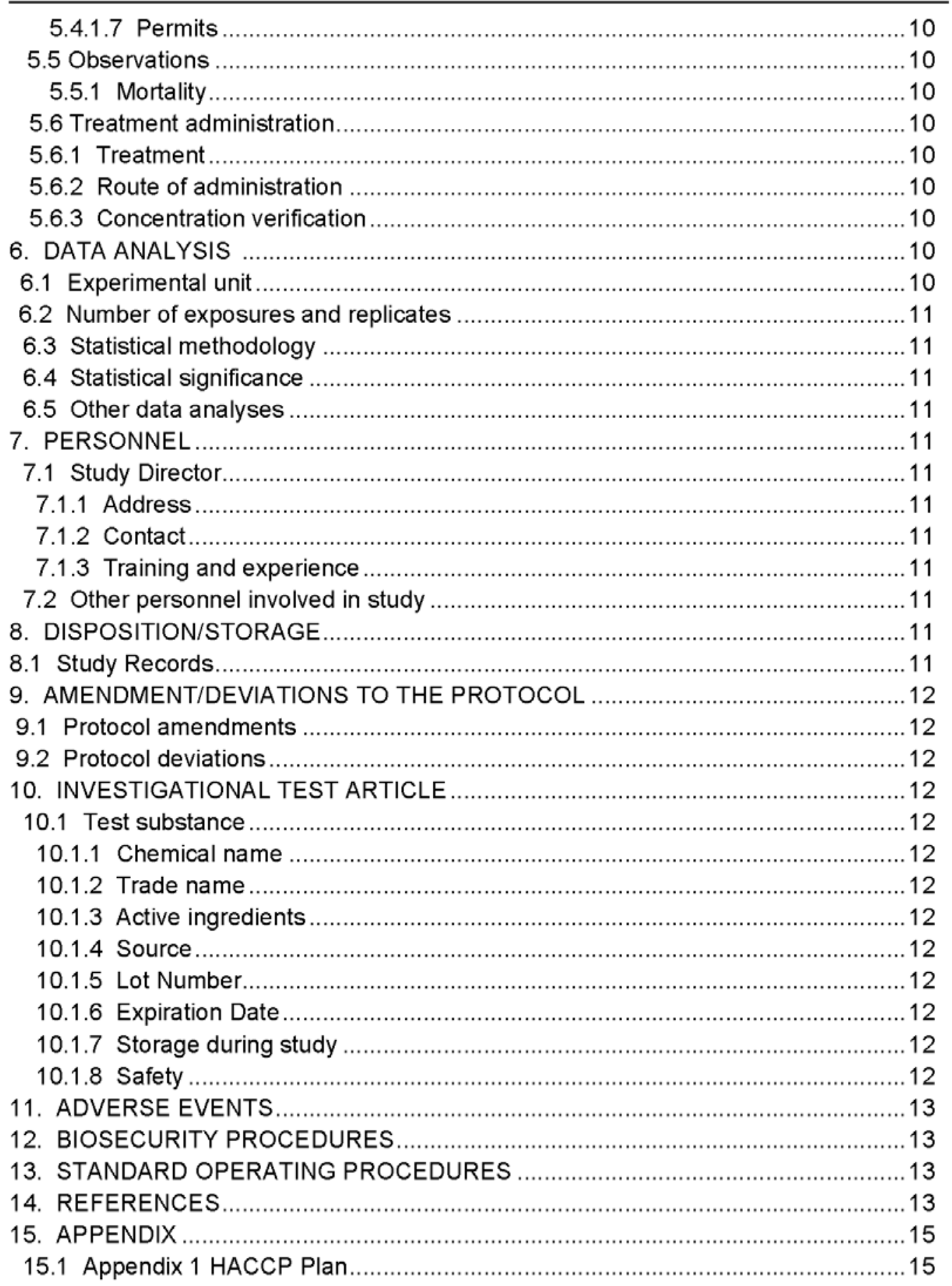

Page 3 of 32 


\section{INTRODUCTION:}

Native freshwater mussel populations of North America were historically considered the most diverse in the world with about 297 recognized taxa consisting of 281 species and 16 subspecies (Williams et al., 1993). Mussels are largely sedentary in nature, relying on movement of host fish during glochidial attachment as means of transport. They are thus particularly vulnerable to a variety of anthropogenic influences including habitat degradation and alteration, pollution and overharvest. A Nature Conservancy survey (Master, 1990) found 55\% of North America's mussels as extinct or imperiled compared to $7 \%$ of terrestrial species, even though terrestrial species traditionally receive far greater attention. Projections in 1999 (Ricciadi and Rasmussen, 1999) suggested that at least 127 imperiled mussel species will be lost in the next 100 years - a conservative extinction rate of $6.4 \%$ per decade given it did not take into account extirpations caused by invasive dreissenid mussels (zebra mussel Dreissena polymorpha and quaqqa mussel $D$. bugensis).

Concerns for native mussels in the Southeast are potentially even greater given that only $25 \%$ of the 269 species historically present are reported as stable compared to the $13 \%$ presumed extinct and the 28,14 and $18 \%$ listed, respectively, as endangered, threatened or of special concern. (Neves et al., 1997)

Many unionid mussels in North America were imperiled prior to epizoic colonization by zebra and quaqqa mussels though the introduction of dreissenid mussels have dramatically heightened concerns for the continued survival of native mussels. Zebra mussels were reported to be responsible for the extirpation of unionids from waters in Europe as early as 1937 (Sebestyen, 1937). Severe declines in unionid abundance in Europe (Karatayev and Burlakova, 1995; Burlakova, 1998) and North America (Haag et al., 1993; Nalepa, 1994; Ricciardi et al., 1996) have since been well documented in the literature.

The 1973 Endangered Species Act (ESA) brought forth the need to recognize, protect and recover rare mussels in the United States. The United States Fish and Wildlife Service (USFWS) develops recovery plans for threatened and endangered species which utilize a range of tools to promote recovery of the species including restoring and acquiring critical habitat, removing introduced or invasive species and captive propagation and release into historic ranges.

Biologists at the New York State Museum (NYSM) Field Research Laboratory have been researching dreissenid mussel control techniques since 1991. They discovered that cell components of a strain of common bacterium isolated from soils (Pseudomonas fluorescens [PfCL145A]) are capable of causing mortality when ingested by dreissenid mussels by degrading the epithelial cells lining the dreissenid digestive system. The NYSM also demonstrated that that efficacy for Pf-CL145A to induce dreissenid mussel mortality was retained when using dead vs. live cells. 
Marrone Bio Innovations (MBI; Davis, $\mathrm{CA}$ ) has developed a dead cell, spray dried formulation (SDP) of this bacterium called Zequanox $\AA$. Zequanox $®$ is currently registered with the USEPA for use within closed systems such as power generating plant cooling systems. The NYSM, USFWS (Genoa NFH) and United States Geological Survey (USGS) Upper Midwest Environmental Sciences Center (UMESC) were awarded a grant from the US Environmental Protection Agency Great Lakes Restoration Initiative entitled "Safe Dreissena Control: Promise for Unionid Restoration". The goal of this grant is to determine the suitability of this product for limited open water zebra mussel control applications including treatment of native mussel propagation cages or native mussel beds. Reference to any specific commercial products, process, or service by trade name, trademark, manufacturer, or otherwise, does not constitute or imply its endorsement, recommendation, or favoring by the United States government. Throughout the remainder of this document the formulated Pf-CL145A product (Zequanox®) will be referred to as sprayed dried powder (SDP).

Naturally occurring surface waters may be unique in their chemical and biological properties which may affect the efficacy of applied control agents such as SDP. The research to be completed according to this protocol will assess the efficacy as well as the non-target unionid mussel safety of SDP treatments for controlling settled zebra mussels ( $D$. polymorpha) in open water enclosures.

\section{PROTOCOL OBJECTIVE:}

To assess the efficacy and non-target unionid mussel safety of two exposure concentrations of SDP for controlling settled zebra mussels in open water enclosures.

\section{STUDY SCHEDULE:}

3.1 Proposed initiation: July 2013

3.2 Schedule of events: A proposed schedule of events is provided in Table 1.

3.3 Proposed completion date: March 2014

Table 1. Proposed Schedule of Events

\begin{tabular}{cc}
\hline Date & Activity \\
\hline July 2013 & SDP field exposures \\
August 2013 & Efficacy/safety assessment \\
March 2014 & final report submission \\
\hline
\end{tabular}

\section{STUDY DESIGN:}

\subsection{General Description:}

Native mussels with attached zebra mussels will be collected from Lake Darling, MN individually tagged and assessed for zebra mussel colonization. The mussels will then be placed into retention barriers situated in areas within the lake that will be enclosed

Page $\mathbf{5}$ of $\mathbf{3 2}$ 
(enclosure area) by an impermeable membrane barrier (enclosures) during a SDP treatment period (exposure period). One of three SDPtreatment levels (untreated control, $50 \mathrm{mg} / \mathrm{L} \mathrm{A.I}$ and $100 \mathrm{mg} / \mathrm{L} \mathrm{A.I)} \mathrm{will} \mathrm{be} \mathrm{applied} \mathrm{to} \mathrm{each} \mathrm{enclosure} \mathrm{for} \mathrm{an} \mathrm{8-h}$ exposure period. After the exposure period the enclosures will be removed. Approximately $30-d$ after exposure, the native mussels will be assessed for survival and zebra mussel colonization.

\subsection{Experimental Design:}

Major study activities are summarized in Figure 1. Approximate locations for the nine enclosure areas were previously identified in Lake Darling, WI. Final enclosure area selection will be completed upon arrival at the test location. The enclosure areas will be $<2 \mathrm{~m}$ deep and will have sandy to a sandy/silt substrate, be similar in substrate composition and be spatially separated as far as feasibly possible within approximately $50 \mathrm{~m}$ of shoreline. Each enclosure area selected will be marked with a numbered buoy and a perforated metal retention barrier $\left(1-\mathrm{m}^{2}\right)$ will be installed at each location to contain the test animals for the duration of the study. The $35-\mathrm{cm}$ high retention barrier will be driven into the lake bed approximately $10 \mathrm{~cm}$ and the coordinates of each barrier will be determined using a GPS.

Native mussels with attached zebra mussels will be collected from Lake Darling, MN by wading or SCUBA. A minimum of 8 mussels and up to 25 mussels of each species will be placed in each enclosure for a total of 72 to 225 mussels per species ( 8 mussels $\times 9$ enclosures $=72 ; 25$ mussels $\times 9$ enclosures $=225$ ). If a single species of native mussels is collected, 25 mussels will be placed in each enclosure, if available. If two species of native mussels are collected, 15 mussels of each species will be placed in each enclosure, if available. If multiple species are used, they will be distributed to the enclosure areas in balanced numbers (i.e. 15 of each species) or equal ratios (i.e. 10 mussels of species $X ; 15$ mussels of species $Y$, etc.) as determined by the number of animals collected. The exact number of animals collected and distributed to the enclosure areas will be documented in the study records. Native mussels will be consolidated in large coolers with frequent $(<1 \mathrm{~h})$ water exchanges until distributed to the enclosures. Prior to distribution, individual mussels will be tagged with a Hallprint tag, photographed on each side, weighed and their water displacement measured to assist in individual tracking and estimating pre-treatment zebra mussel colonization.

Initial zebra mussel colonization on native mussels will be determined prior to treatment on 3 to 5 mussels per species selected at random from each enclosure. Individual native mussel weight and water displacement will be measured pre- and post-zebra mussel removal on the native mussels selected for initial zebra mussel colonization determination and the zebra mussels removed from each individual mussel will be enumerated. Estimates of initial zebra mussel colonization for the remaining native mussels will be completed by comparison to data collected from the initial colonization determinations (using individual weight and water displacement data collected prior to

Page 6 of 32 
treatment and at the termination of the study). Exact measures used to assess pretreatment zebra mussels colonization will be documented in the study records.

If a single species of native mussels are collected, up to 25 mussels (if available) will be randomly distributed to each enclosure area in groups of 5 mussels in five distributions rounds (ie: 5 mussels placed in a bucket and transferred to a randomly selected enclosure area). If multiple species of native mussels are collected, equal ratios of mussels will be equally distributed to each enclosure area in multiple distribution rounds with up to 5 mussels of each species per round. The exact procedures used to distribute the mussels will be recorded in the study records.

Figure 1. Flow chart of major study activities

Identify enclosure areas, place retention barriers and determine location cooridinates.

Collect and randomly distribute native mussels with attached zebra mussels. Native mussels will be individually tagged or marked with a dremel tool. If possible, the number of zebra mussel on each native mussel will be enumerated. Photographic records, wet weight and water displacement will be recorded for each mussel before placement in an enclosure.

Impermeable containment membranes are placed around each retention barrier. SDP exposures (8-h duration, static) are initated 12-24 h after native mussel placement in retention barriers. Treatment (control, 50 or $100 \mathrm{mg} \mathrm{Al} / \mathrm{L}$ ) will be randomly assigned to 3 replicate enclosures. Containment membranes are removed at the conclusion of the exposure period

Native mussels will be assessed for zebra mussel colonization, wet weight, displacement and survival at 3-4 weeks post exposure then returned to their approximate collection location. Decontaminated all equipment prior to removal from the test site

Decontaminated all equipment prior to removal from the test site

Enclosures containing native mussels with attached zebra mussels will be assigned to one of three treatments, a non-exposed control, a $50 \mathrm{mg} / \mathrm{L} \mathrm{A} . \mathrm{I} . / \mathrm{L}$ treatment and a 100 $\mathrm{mg} / \mathrm{L}$ A.I./L treatment. There will be 3 replicates per treatment level and enclosures will be assigned a treatment according to a completely randomized assignment scheme. An exposure duration of 8 hours will be assigned for all treatment groups. The treatment exposure period will be initiated by the addition of an appropriate amount of SDP stock solution. The treatment concentration will be verified by comparison to a standard curve 
prepared from a known mass of SDP. Exposures will be terminated 8-h after treatment application by removal of the enclosure membranes.

Approximately 30 days following treatment, the native mussels will be assessed for survival and for zebra mussel colonization by the measuring individual weight and water displacement pre- and post-zebra mussel removal. All zebra mussels removed will be preserved for enumeration to determine the number zebra mussels attached to each native mussel. Native mussel survival and the change in weight and water displacement along with the number of zebra mussels removed from each mussel will be compared between treatment groups to determine treatment related effects.

After assessment, all native mussels will be returned to their approximate collection location.

\section{STUDY PROCEDURES}

\subsection{Test Animals}

\subsubsection{Description:}

5.1.1.1 Age - Native mussels $>2$ yrs

Zebra Mussels < 2yrs

5.1.1.2 Sex - Test animals will be used without regard to sex.

5.1.1.3 Species - Fatmucket, (Lampsilis siliquoidea); Fragile Papershell (Leptodea fragilis); Giant floater, (Pyganodon grandis); zebra mussel, (Dreissena polymorpha) The exact native unionid species will be determined at the time of collection. If multiple species are used, native species will be equally distributed in equal ratios to the treatment enclosures. During the final assessment, the mussels will be cleaned of any remaining zebra mussels and photographed for identification confirmation by an experienced USGS, USFWS or DNR malacologist.

5.1.2 Number of animals: No more than 360 native mussels will be used and no more than 225 of a single species will be used. This number is consistent with the objective of the study and contemporary scientific standards. Approximately 9,000 to 36,000 zebra mussels will be used ( 360 native mussels $X 25-100$ attached zebra mussels on each native $=9,000-36,000)$.

5.1.3 Source of animals: Animals will be collected from Lake Darling, Douglas County, $\mathrm{MN}$.

5.1.4 Inclusion criterion: Native mussels with $\geq 25$ attached zebra mussels.

5.1.5 Acclimation: Mussels will be held in the assigned retention barrier $\geq 12 \mathrm{~h}$ prior to exposure initiation.

5.1.6 Distribution to enclosure area: Up to 25 mussels will be distributed to each enclosure area according to a predetermined randomization scheme. Five mussels will be placed in each enclosure replicate in each distribution round.

Page 8 of 32 
5.1.7 Feeding: Mussels (native and zebra mussels) will have access to natural food in the water column supplemental feeds will not be provided.

\subsection{Water Chemistry}

5.2.1 Dissolved oxygen: Dissolved oxygen will be measured (UMESC SOP AEH 394 or equivalent) and recorded in each enclosure replicate during the acclimation and exposure periods. Dissolved oxygen will be measured and recorded at least once during the pre-exposure period and at least twice during the exposure period with the last measurement observed $\leq 30$ minutes prior to exposure termination. The location of the measurements will be documented in the study records.

5.2.2 Temperature: Temperature will be monitored throughout the pre-exposure, exposure and post-exposure periods using submersible $\mathrm{HOBO} \circledast$ data loggers placed in each enclosure. Additionally, temperature will be measured and recorded at least twice during the exposure period with the last measurement observed $\leq 30$ minutes prior to exposure termination using a handheld digital thermometer. The location of the measurements will be documented in the study records.

5.2.3 pH: $\mathrm{pH}$ will be measured (UMESC SOP AEH 335 or equivalent) and recorded in each enclosure replicate during the pre-exposure and exposure periods. $\mathrm{pH}$ will be measured and recorded at least once during the pre-exposure period and at least twice during the exposure with the last measurement observed $\leq 30$ minutes prior to exposure termination. The location of the measurements will be documented in the study records.

5.2.4 Hardness: Hardness will be measured (UMESC SOP AEH 712) and recorded prior to exposure initiation.

5.2.5 Alkalinity: Alkalinity will be measured (UMESC SOP AEH 706) and recorded prior to exposure initiation.

5.2.6 Conductivity: Conductivity will be measured (UMESC SOP AEH 188 or equivalent) and recorded prior to exposure initiation.

5.2.7 Ammonia: Samples for total ammonia-nitrogen will be collected in $50-\mathrm{mL}$ centrifuge tubes from the surface of each enclosure at the termination of the exposure period for each exposure replicate. Ammonia samples will be filtered through a $0.45-\mu \mathrm{m}$ syringe filter, acidified ( $\sim \mathrm{pH} 2.5)$ with sulfuric acid and then stored at $\sim 4^{\circ} \mathrm{C}$ until analyzed by the UMESC Long Term Resources Monitoring (LTRM) Water Quality Laboratory using the automated phenate method.

5.3 Disposal: All live native mussels at the end of the post-exposure observation period will be euthanized by MS-222 overdose and disposed of as required by MN-DNR permit.

5.4 Study facilities:

5.4.1 Test Facility

U.S. Geological Survey, Upper Midwest Environmental Sciences Center 


\section{Fanta Reed Rd}

La Crosse, Wisconsin 54603

\subsubsection{Test location: Lake Darling, Douglas County, MN}

5.4.1.2 Exposure system: The test system consists of nine replicate $1.5 \mathrm{~m}^{2}$ enclosures positioned in $<2 \mathrm{~m}$ of water. The enclosures will be spatially placed are far as feasibly possible along $\sim 50 \mathrm{~m}$ of shoreline to reduce cross contamination. Each enclosure area location will have similar substrate and water depth. Each enclosure area will be uniquely identified and coding procedures and the treatment assignment will be documented in the study records.

5.4.1.3 Aeration: Supplemental aeration will not be provided.

5.4.1.4 Test Water: Exposures will be conducted using natural lake water. Exposures will be conducted within an impermeable membrane suspended around the mussel retention barrier.

5.4.1.5 Treated water discharge: The SDP will be immediately mixed with raw lake water at the termination of the exposure period when the impermeable membranes are withdrawn. The SDP concentrations are expected to rapidly decrease during mixing with the lake water.

5.4.1.6 Lighting: Native mussels will be exposed to a natural photoperiod with no manipulation of the photoperiod.

5.4.1.7 Permits: This study has been verified exempt and does not require an EPA or State of Minnesota Experimental Use Permit.

\subsection{Observations:}

5.5.1 Mortality: Mussels that are gapping and do not respond to tactile stimuli by shell closure will be coded as a mortality.

5.6 Exposure administration:

5.6.1 Treatment: There will be three treatment groups: 0 [control], 50 or $100 \mathrm{mg} / \mathrm{L} \mathrm{A.I.} \mathrm{A}$ single exposure duration (8-h) will be applied. Each treatment group will have three replicate enclosures.

5.6.2 Route of administration: Exposure will be initiated by addition of an appropriate amount of SDP stock solution followed by a gently mixing to uniformly distribute the SDP.

5.6.3 Concentration verification: Concentration will be determined spectrophotometrically. A standard curve will be prepared using a known mass of SDP. The absorbance of exposure solutions will be compared to the standard curve to determine the exposure concentration. Absorbance will be determined using a Barnstead/Thermolyne Corporation Model Turner SP-830 Plus Beckman spectrophotometer (UMESC SOP AEH 302) or equivalent.

6. DATA ANALYSIS

6.1 Experimental unit: The experimental unit will be the enclosure.

Page 10 of $\mathbf{3 2}$ 
6.2 Number of exposures and replicates: There will be a total of 3 treatment levels $(0$ [control], 50 and $100 \mathrm{mg} \mathrm{SDP/L}$ ) and 1 treatment duration (8-h). There will be a total of 3 enclosures for each treatment level which will serve as the replicates. All treatment levels and replicates will be run concurrently.

6.3 Statistical methodology:

Post treatment zebra mussel density associations with treatments types will be analyzed using a count model that addresses nesting of density data within enclosures. Pairwise comparison tests will be completed to compare each treatment group to the control group. Count models will be fitted using a log link and $S A S^{\circledR}$ generalized linear mixed modeling procedure (PROC GLIMMIX; SAS ${ }^{\circledR}$ 9.3).

Native Mussel survival data will be analyzed using a binary assumption that addresses nesting of the enclosures. Models will be fitted using $S S^{\mathbb{B}}$ generalized linear mixed modeling procedure (PROC GLIMMIX; SAS ${ }^{\oplus}$ 9.3). Pairwise comparison tests will be completed to compare each treatment group to the control group.

Change in weight and displacement pre- and post-treatment with treatment types will be analyzed using pairwise comparison tests to compare each treatment group to the control group. Models will be fitted using SAS ${ }^{\oplus}$ generalized linear mixed modeling procedure (PROC GLIMMIX; SAS ${ }^{\oplus}$ 9.3).

6.4 Statistical significance: Statistical significance will be declared at $p<0.05$.

6.5 Other data analyses: Statistical methods for other study data collected will include calculation of means, standard deviations and coefficients of variation. The statistical procedures used will be described in detail in the final study report.

\section{PERSONNEL}

7.1 Study Director: James A. Luoma, B.A.

7.1.1 Address: Upper Midwest Environmental Sciences Center, US Geological Survey, 2630 Fanta Reed Rd., La Crosse, Wisconsin 54603

7.1.2 Contact: Tel: (608) 781-6391, Fax: (608) 783-6066; jluoma@usgs.gov

7.1.3 Training and experience: $\mathrm{CV}$ on file at UMESC.

7.2 Other personnel involved in study: Technical staff involved in the study will be identified in the study raw data to include study function. UMESC technical staff training and experience will be documented in CVs included in the study raw data.

\section{DISPOSITION/STORAGE}

8.1 Study Records: All data generated in the study will be recorded in bound laboratory notebooks, electronic files or kept in file folders. All data sheets, file folders, laboratory notebooks and computer disks will be encoded with the study number when the data are generated. Raw data, laboratory notebooks and electronic files (including a CDROM containing the annotated SAS program used for the statistical analysis, the data

Page 11 of 32 
files, SAS log and SAS output files) generated by UMESC and contract laboratory reports will be filed in the UMESC archives (SOP No. GEN 007) of the Upper Midwest Environmental Sciences Center, La Crosse Wisconsin, before the final report is signed by the Study Director. The final report will then be signed and archived.

\section{AMENDMENT/DEVIATIONS TO THE PROTOCOL}

9.1 Protocol amendments: A signed copy of the Study Protocol will be retained on-site. Proposed amendments to the protocol shall be brought to the attention of UMESC Management. When the Study Director and Management agree verbally, the study can proceed with the change. As soon as possible, the Study Director will then prepare a written protocol amendment that is signed by the Study Director and Branch Chief. The amendment then becomes an official part of the protocol.

9.2 Protocol deviations: All deviations from this approved protocol will be documented and reviewed by the Study Director. The Study Director will make a judgment on the impact of the deviations. The Study Director will notify Management, as soon as possible, of any deviations to the protocol, including their impact on the study.

\section{INVESTIGATIONAL TEST ARTICLE}

10.1 Test Substance(s): Pseudomonas fluorescens (Pf-CL145A) SDP formulation

10.1.1 Chemical name: Pseudomonas fluorescens (Pf-CL145A)

10.1.2 Trade name: Zequanox

10.1.3 Active ingredients: Pseudomonas fluorescens (Pf-CL145A), 50\% active ingredient (A.I.) by weight.

10.1.4 Source: Marrone Bio Innovations (MBI); Davis, CA

10.1.5 Lot number: Lot number(s) will be included in the test chemical log books, lab notebook, and study files.

10.1.6 Expiration date: As determined by the manufacturer. An aliquot of each lot tested will be returned to the NYSM or MBI at the conclusion of exposures for post-exposure zebra mussel bioassay tests (the standard testing protocol to assess Pseudomonas fluorescens [Pf-CL145A] formulation activity). Results of these confirmation bioassays will be used to validate the retention of activity of the Pseudomonas fluorescens ( $P f$-CL145A) SDP and will be included in the study files when available.

10.1.7 Storage during study: The test chemical will be stored refrigerated. Test material will be transported in a cooler with ice packs to maintain proper storage temperature $\left(4-10^{\circ} \mathrm{C}\right)$

10.1.8 Safety: A NIOSH approved respirator will be used when preparing stock solutions to avoid inhalation. Protective eyewear, gloves and lab coats will be worn at all times when working with the test substance.

Page 12 of 32 
11. ADVERSE EVENTS: Any adverse event will be recorded in the study logbook and the Study Director will be notified.

12. BIOSECURITY PROCEDURES: All personnel involved in the study will review the UMESC biosecurity (UMESC SOP APP 075) and project HACCP plans. Testing will be conducted in a field setting. All equipment will be thoroughly cleaned and disinfected prior to return to UMESC. Waste generated will be placed in plastic garbage bags and disposed of in accordance with local and state regulations. See Appendix 1 for the HACCP plan for this project.

\section{STANDARD OPERATING PROCEDURES}

A complete list of the standard operating procedures used in the study will be included in the study guide. The follow SOP's were cited in this protocol:

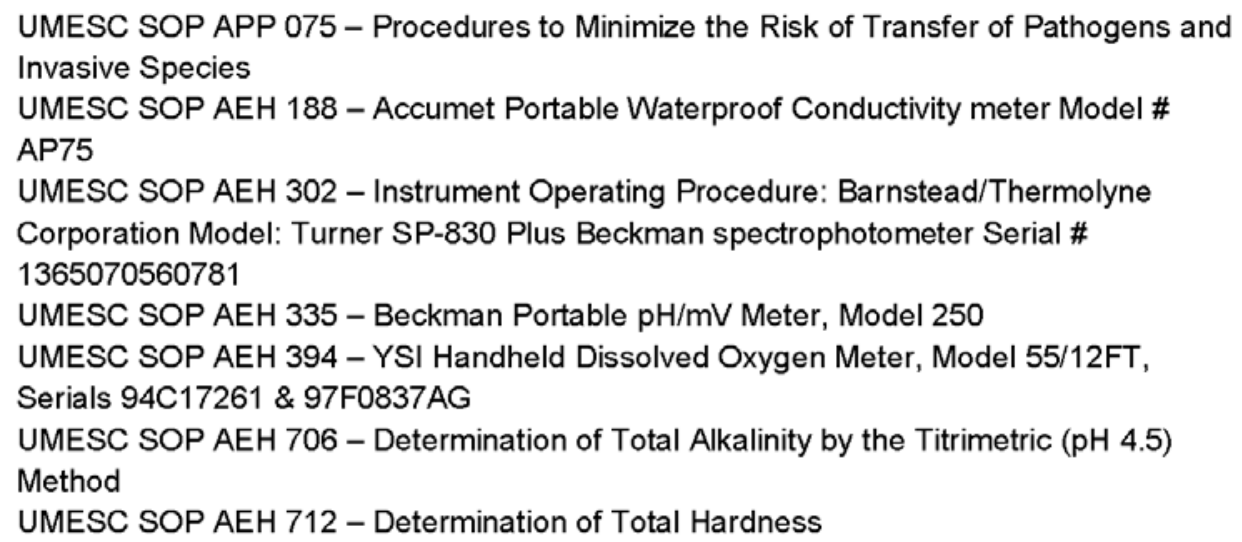

\section{REFERENCES.}

Burlakova, L.E., 1998. Ecology of Dreissena polymorpha (PALLAS) and its role in the structure and function of aquatic ecosystems. Candidate dissertation, Zoology Institute of the Academy of Science Republic Belarus, 168 p. (in Russian) in Burlakova L.E., A.Y. Karatayev and D. K. Padilla. 2000. The impact of Dreissena polymorpha (PALLAS) invasion on Unionid bivalves. Internat. Rev. hydrobiol. 85 (5-6):529-541.

Haag, W.R., D.L. Berg, D.W. Garton, and J.L. Farris, 1993. Reduced survival and fitness in native bivalves in response to fouling by the introduced zebra mussel (Dreissena polymorpha) in western Lake Erie. Canadian Journal of Fisheries and Aquatic Sciences 50(1):13-19.

Karatayev, A.Y. and L.E. Burlakova, 1995. Present and future patterns in Dreissena population development in the Narochanskaya lakes system. Vestisi Akad. Navuk Belarusi. Ser. Biyol. Navuk 3: 95098 (in Belarussian) in Burlakova L.E., A.Y. Karatayev and D. K. Padilla. 2000. 
The impact of Dreissena polymorpha (PALLAS) invasion on Unionid bivalves. Internat. Rev. hydrobiol. 85 (5-6):529-541.

Master, L., 1990. The imperiled status of North American aquatic animals. Biodiversity Network News 3(3):5-8.

Napela, T.F., 1994. Decline of native unionid bivalves in Lake St. Clair after infestation by the zebra mussel, Dreissena polymorpha. Can. J. Fish. Aquat. Sci. 51:2227-2233.

Neves R. J., 2004. Propagation of endangered freshwater mussels in North America. Journal of Conchology, special publication 3:69-80.

Neves, R.J., A.E. Bogan, J.D. Williams, S.A. Ahlstedt, and P.W. Hartfield, 1997. Status of aquatic mollusks in the southeastern United States: a downward spiral of diversity. Pages 4385 in G.W. Benz and D.E. Collins, editors. Aquatic fauna in peril: the southeastern perspective. Southeastern Aquatic Research Institute, Lenz Design and communications, Decatur, Georgia.

Ricciardi, A. F.G. Whoriskey, and J.B. Rasamussen, 1996. Impact of the Dreissena invasion on native unionid bivalves in the upper St. Lawerance River. Can. J. Fish. Aquat. Sci. 53:14341444.

Ricciardi A., and J.B. Rasmussen, 1999. Extinction rates of North American freshwater fauna. Conservation Biology. Vol. 13 (5):1220-1222.

Sebestyn, O., 1937. Colonization of two new fauna-elements of Pontus-origin (Dreissena polymorpha Pall. And Corophuim curvispinum G.O. Sars forma devium Wundsch) in Lake Balaton, Verh. Int. Ver. Theor. Angew. Limnol. 8:169-182 in Burlakova L.E., A.Y. Karatayev and D. K. Padilla. 2000. The impact of Dreissena polymorpha (PALLAS) invasion on Unionid bivalves. Internat. Rev. hydrobiol. 85 (5-6):529-541.

Williams, J.D., M.L. Warren Jr., K.S. Cummings, J.L. Harris, and R.J. Neves. 1993. Conservation status of freshwater mussels of the United States and Canada. Fisheries 18(9):622.

Zequanox: EPA Reg. No.: 84059-15. MSDS [Online]: Marrone Bio Innovations: Davis, CA. http://wnw.marronebioinnovations.com/products/labelsmsds/zequanox-labelsmsds (accessed Jan. 3, 2013). 


\section{APPENDIX.}

15.1 Appendix 1. HACCP PLAN for the study Efficacy of Pseudomonas fluorescens (PfCL145A) SDP for controlling zebra mussels within field enclosures

\section{Step 1 - Activity Description}

\begin{tabular}{|l|c|}
\hline $\begin{array}{l}\text { Facility: US Geological Survey-Upper Midwest Environmental Seiences } \\
\text { Center }\end{array}$ & Site: Various - Keyes Lake, Florence, WI \\
\hline Site Coordinator: Jim Luoma & $\begin{array}{r}\text { Activity: Efficacy of Pseudomonas fluorescens (Pf-CL145A) SDP for } \\
\text { controlling zebra mussels within field enclosures }\end{array}$ \\
\hline $\begin{array}{l}\text { Site Manager: Mark Gaikowski } \\
\text { Address: 2630 Fanta Reed Road } \\
\text { La Crosse WI, 54601 }\end{array}$ & \\
\hline $\begin{array}{c}\text { Phone: 608-781-6322 } \\
\end{array}$ & \\
\hline
\end{tabular}

\section{Project Description}

The objective of this study is to assess the efficacy and non-target native mussel safety of two exposure concentrations of SDP for controlling settled zebra mussels $(D$. polymorpha) in open water enclosures

Step 2 - Potential Hazards: Species which may potentially be moved/introduced Vertebrates:

Multiple species of freshwater fish, eggs and gametes found in the Great Lakes Basin.

Invertebrates:

Zebra mussel (Dreissena polymorpha)

Multiple endemic species found in the Great Lakes Basin

Plants:

Multiple endemic species found in the Great Lakes Basin

Other biologicals (disease, pathogen, parasite):

Largemouth Bass Virus

Spring Viremia of Carp Virus

Bluegill Virus

Infectious Pancreatic Necrosis Virus

Viral Hemorrhagic Septicemia

Furunculosis Aeromonas saimonicido

Enteric Redmouth Disease Yersinia ruckeri

Bacterial Kidney Disease Renibacterium salmoninarum

Other Assorted parasites/pathogens found in the Great Lakes Basin

Other: 
Flow diagram outlining sequential tasks to complete activity/project

\begin{tabular}{|c|c|}
\hline Task 1 & Enclosure test sites identified and retention barriers placed in Keyes Lake, WI \\
\hline \multirow{2}{*}{\multicolumn{2}{|c|}{ Native mussels with attached zabro mussels are obtained via SCUBA or wading from Keves Lake WI }} \\
\hline & Native mussels with attached zebra mussels are obtained via SCUBA or wading from Keyes Lake, WI \\
\hline \\
\hline Task 3 & Native mussels are place in enclosures and acclimated to test conditions with impermeable barriers lowered \\
\hline$\frac{1}{\text { Task } 4}$ & Native mussels are exposed to test article for 8-h \\
\hline \multicolumn{2}{|r|}{ (2) } \\
\hline Task 5 & Impermeable barriers are removed and native mussels left undisturbed for post-exposure holding \\
\hline \multicolumn{2}{|r|}{ 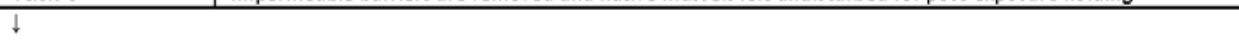 } \\
\hline Task 6 & Equipment is decontaminated and returned to UMESC \\
\hline \multicolumn{2}{|r|}{ 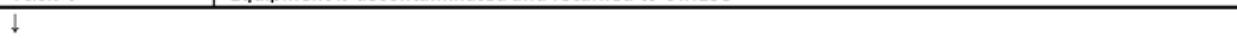 } \\
\hline Task 7 & Native mussels are removed from enclosures and assessed for zebra mussel colonization and survival \\
\hline \multicolumn{2}{|r|}{ 舟 } \\
\hline Task 8 & Native mussels are cleaned of zebra mussels and returned to the approximate collection location \\
\hline \multicolumn{2}{|l|}{$\downarrow$} \\
\hline Task 9 & All remaining equipment, retention barriers, etc. are decontaminated and returned to UMESC \\
\hline
\end{tabular}

\begin{tabular}{|c|c|c|c|c|c|}
\hline $\begin{array}{c}1 \\
\text { Tasks } \\
\text { (from HACCP Step 3 } \\
\text { - Flow Diagram) }\end{array}$ & $\begin{array}{c}\text { Potential hazards } \\
\text { identified in HACCP } \\
\text { Step 2 }\end{array}$ & $\begin{array}{c}\text { Are any potential } \\
\text { hazards probable? } \\
\text { (yes/no) }\end{array}$ & $\begin{array}{c}\text { Justify evaluation for } \\
\text { column 3 }\end{array}$ & $\begin{array}{c}\text { What control } \\
\text { measures can be } \\
\text { applied to prevent } \\
\text { undesirable results? }\end{array}$ & $\begin{array}{c}\text { Is this task a critical } \\
\text { control point? } \\
\text { (yes/no) }\end{array}$ \\
\hline
\end{tabular}

\begin{tabular}{|l|l|l|l|l|l|}
\hline Task 1 & Vertebrates & yes & $\begin{array}{l}\text { Surface water } \\
\text { contains multiple } \\
\text { vertebrate species } \\
\text { identified and } \\
\text { retention barriers } \\
\text { placed in Lake } \\
\text { Darling, MN }\end{array}$ & & $\begin{array}{l}\text { Assure the thorough } \\
\text { decontamination all } \\
\text { equipment with } \\
\text { steam, } \\
\text { chemosterilants, or } \\
\text { other approved } \\
\text { methods prior to } \\
\text { removal from location, } \\
\text { unless impractical, } \\
\text { then decontamination } \\
\text { shall be done as soon } \\
\text { as possible and must } \\
\text { be done before } \\
\text { equipment is deployed } \\
\text { again. Inspect all } \\
\text { equipment prior to set } \\
\text { up and repeat } \\
\text { decontamination if } \\
\text { warranted. }\end{array}$ \\
\hline
\end{tabular}

Page 16 of 32 


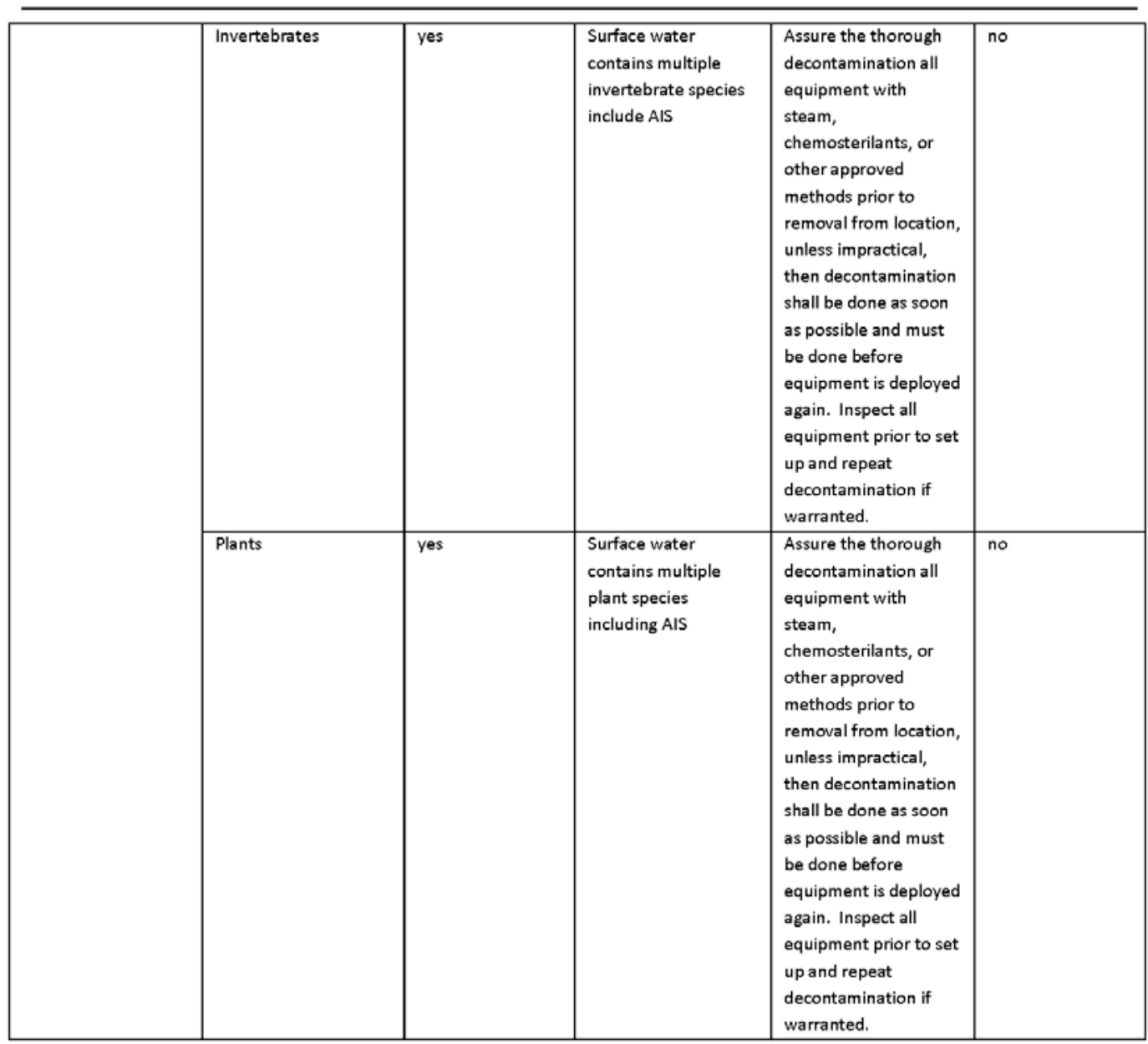

Page $\mathbf{1 7}$ of $\mathbf{3 2}$ 


\begin{tabular}{|l|l|l|l|l|l|}
\hline & Others & yes & $\begin{array}{l}\text { Surface water has } \\
\text { potential to transfer } \\
\text { fish diseases }\end{array}$ & $\begin{array}{l}\text { Assure the thorough } \\
\text { decontamination all } \\
\text { equipment with } \\
\text { steam, } \\
\text { chemosterilants, or } \\
\text { other approved } \\
\text { methods prior to } \\
\text { removal from location, } \\
\text { unless impractical, } \\
\text { then decontamination } \\
\text { shall be done as soon } \\
\text { as possible and must } \\
\text { be done before } \\
\text { equipment is deployed } \\
\text { again. Inspect all } \\
\text { equipment prior to set } \\
\text { up and repeat } \\
\text { decontamination if } \\
\text { warranted. Do not } \\
\text { proceed if any fish } \\
\text { disease outbreak (i.e.: } \\
\text { VHS) is documented in } \\
\text { the waterbody }\end{array}$ \\
\hline
\end{tabular}

\begin{tabular}{|c|c|c|c|c|c|}
\hline $\begin{array}{l}\text { Task } 2 \\
\text { Native mussels with } \\
\text { attached zebra } \\
\text { mussels are obtained } \\
\text { via SCUBA or wading } \\
\text { from Keyes Lake, WI }\end{array}$ & Vertebrates & yes & $\begin{array}{l}\text { Surface water } \\
\text { contains multiple } \\
\text { vertebrate species }\end{array}$ & $\begin{array}{l}\text { Assure the thorough } \\
\text { decontamination all } \\
\text { equipment including } \\
\text { all potentially } \\
\text { wetted surfaces with } \\
\text { steam, } \\
\text { chemosterilants, or } \\
\text { other approved } \\
\text { methods prior to } \\
\text { removal from } \\
\text { location, unless } \\
\text { impractical, then } \\
\text { decontamination } \\
\text { shall be done as } \\
\text { soon as possible and } \\
\text { must be done before } \\
\text { equipment is } \\
\text { deployed again. } \\
\text { Inspect all } \\
\text { equipment prior to } \\
\text { set up and repeat } \\
\text { decontamination if } \\
\text { warranted. }\end{array}$ & no \\
\hline
\end{tabular}

Page 18 of $\mathbf{3 2}$ 


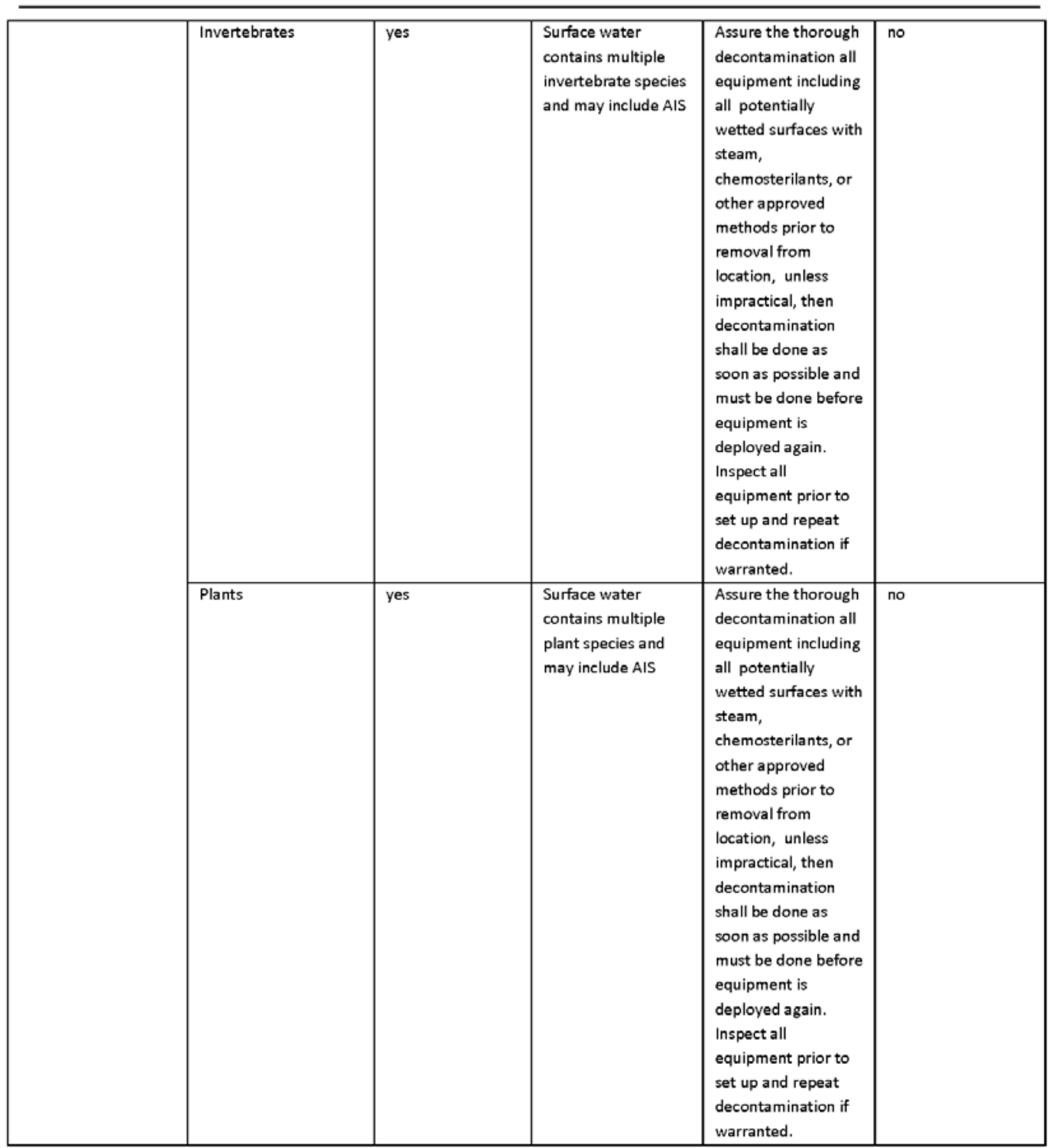

Page 19 of 32 


\begin{tabular}{|c|c|c|c|c|c|c|}
\hline & Others & yes & $\begin{array}{l}\text { Surface water has } \\
\text { potential to transfer } \\
\text { fish diseases }\end{array}$ & $\begin{array}{l}\text { Assure the thorough } \\
\text { decontamination all } \\
\text { equipment including } \\
\text { all potentially } \\
\text { wetted surfaces with } \\
\text { steam, } \\
\text { chemosterilants, or } \\
\text { other approved } \\
\text { methods prior to } \\
\text { removal from } \\
\text { location, unless } \\
\text { impractical, then } \\
\text { decontamination } \\
\text { shall be done as } \\
\text { soon as possible and } \\
\text { must be done before } \\
\text { equipment is } \\
\text { deployed again. } \\
\text { Inspect all } \\
\text { equipment prior to } \\
\text { set up and repeat } \\
\text { decontamination if } \\
\text { warranted. Do not } \\
\text { proceed if any fish } \\
\text { disease outbreak } \\
\text { (i.e.: VHS) is } \\
\text { documented in the } \\
\text { waterbody }\end{array}$ & no & \\
\hline $\begin{array}{l}\text { Task } 3 \\
\text { Native mussels are } \\
\text { place in enclosures } \\
\text { and acclimated to test } \\
\text { conditions with } \\
\text { impermeable barriers } \\
\text { lowered }\end{array}$ & Vertebrates & yes & $\begin{array}{l}\text { Surface water contai } \\
\text { multiple vertebrate } \\
\text { species }\end{array}$ & \multicolumn{2}{|c|}{$\begin{array}{l}\text { Assure the thorough } \\
\text { decontamination all } \\
\text { equipment including all } \\
\text { potentially wetted } \\
\text { surfaces with steam, } \\
\text { chemosterilants, or } \\
\text { other approved } \\
\text { methods prior to } \\
\text { removal from location, } \\
\text { unless impractical, } \\
\text { then decontamination } \\
\text { shall be done as soon } \\
\text { as possible and must } \\
\text { be done before } \\
\text { equipment is deployed } \\
\text { again. Inspect all } \\
\text { equipment prior to set } \\
\text { up and repeat } \\
\text { decontamination if } \\
\text { warranted. }\end{array}$} & no \\
\hline
\end{tabular}

Page $\mathbf{2 0}$ of $\mathbf{3 2}$ 


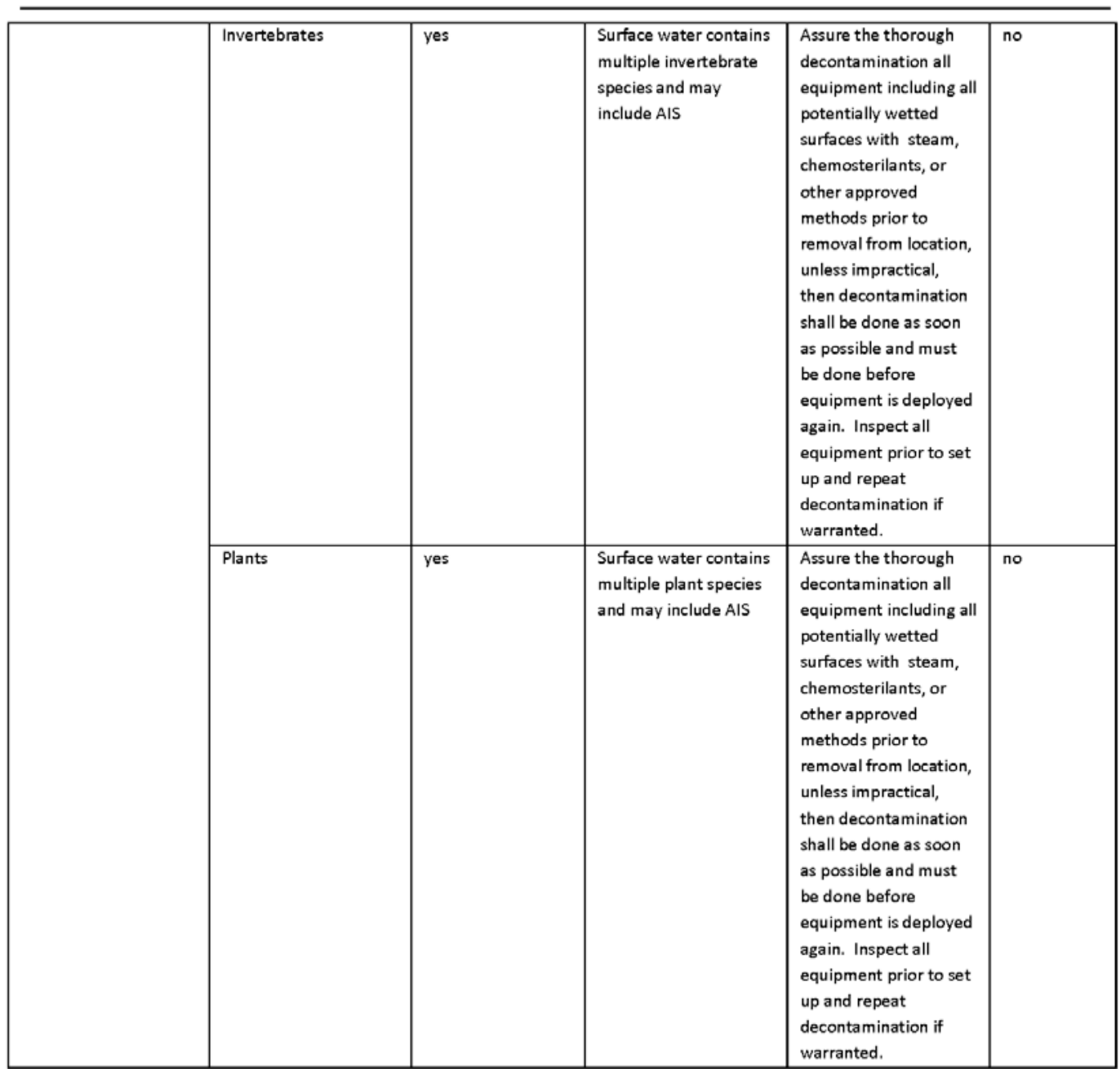

Page $\mathbf{2 1}$ of $\mathbf{3 2}$ 


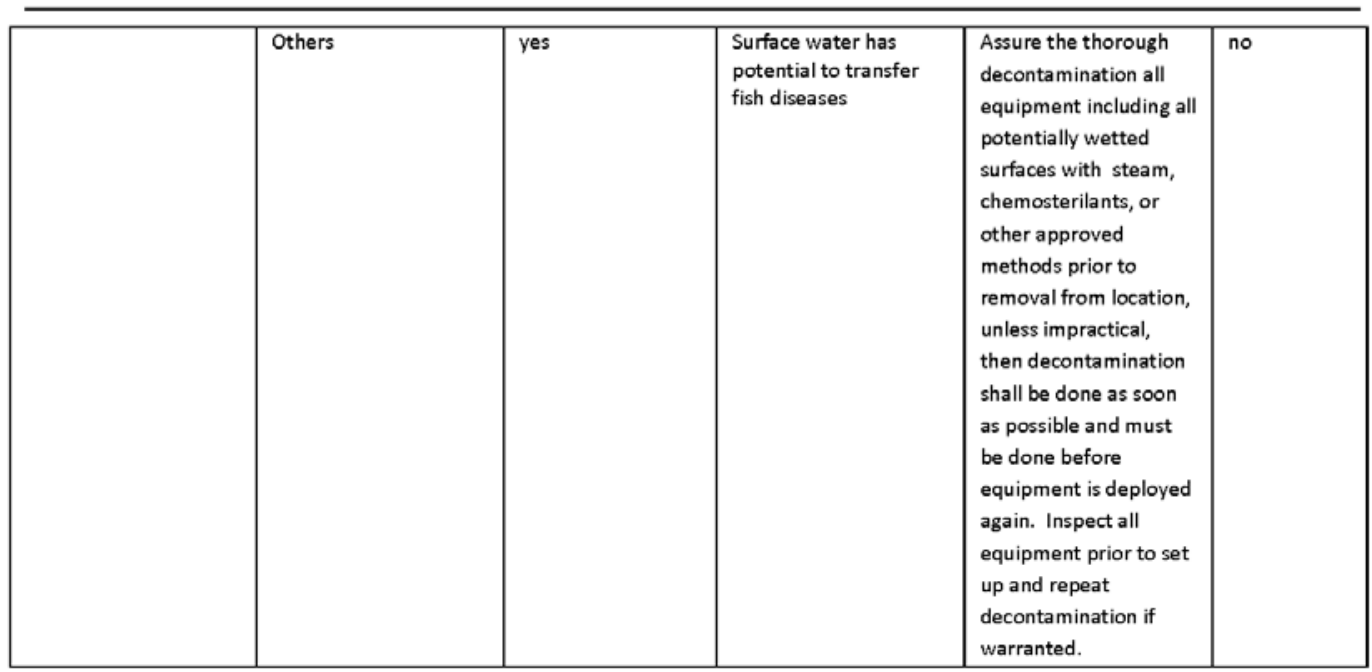

\begin{tabular}{|c|c|c|c|c|c|}
\hline $\begin{array}{l}\text { Task } 4 \\
\text { Native mussels are } \\
\text { exposed to test article } \\
\text { for } 8 \text {-h }\end{array}$ & Vertebrates & yes & $\begin{array}{l}\text { Surface water contains } \\
\text { multiple vertebrate } \\
\text { species }\end{array}$ & $\begin{array}{l}\text { Assure the thorough } \\
\text { decontamination all } \\
\text { equipment including all } \\
\text { potentially wetted } \\
\text { surfaces with steam, } \\
\text { chemosterilants, or } \\
\text { other approved } \\
\text { methods prior to } \\
\text { removal from location, } \\
\text { unless impractical, } \\
\text { then decontamination } \\
\text { shall be done as soon } \\
\text { as possible and must } \\
\text { be done before } \\
\text { equipment is deployed } \\
\text { again. Inspect all } \\
\text { equipment prior to set } \\
\text { up and repeat } \\
\text { decontamination if } \\
\text { warranted. }\end{array}$ & No \\
\hline
\end{tabular}

Page $\mathbf{2 2}$ of $\mathbf{3 2}$ 


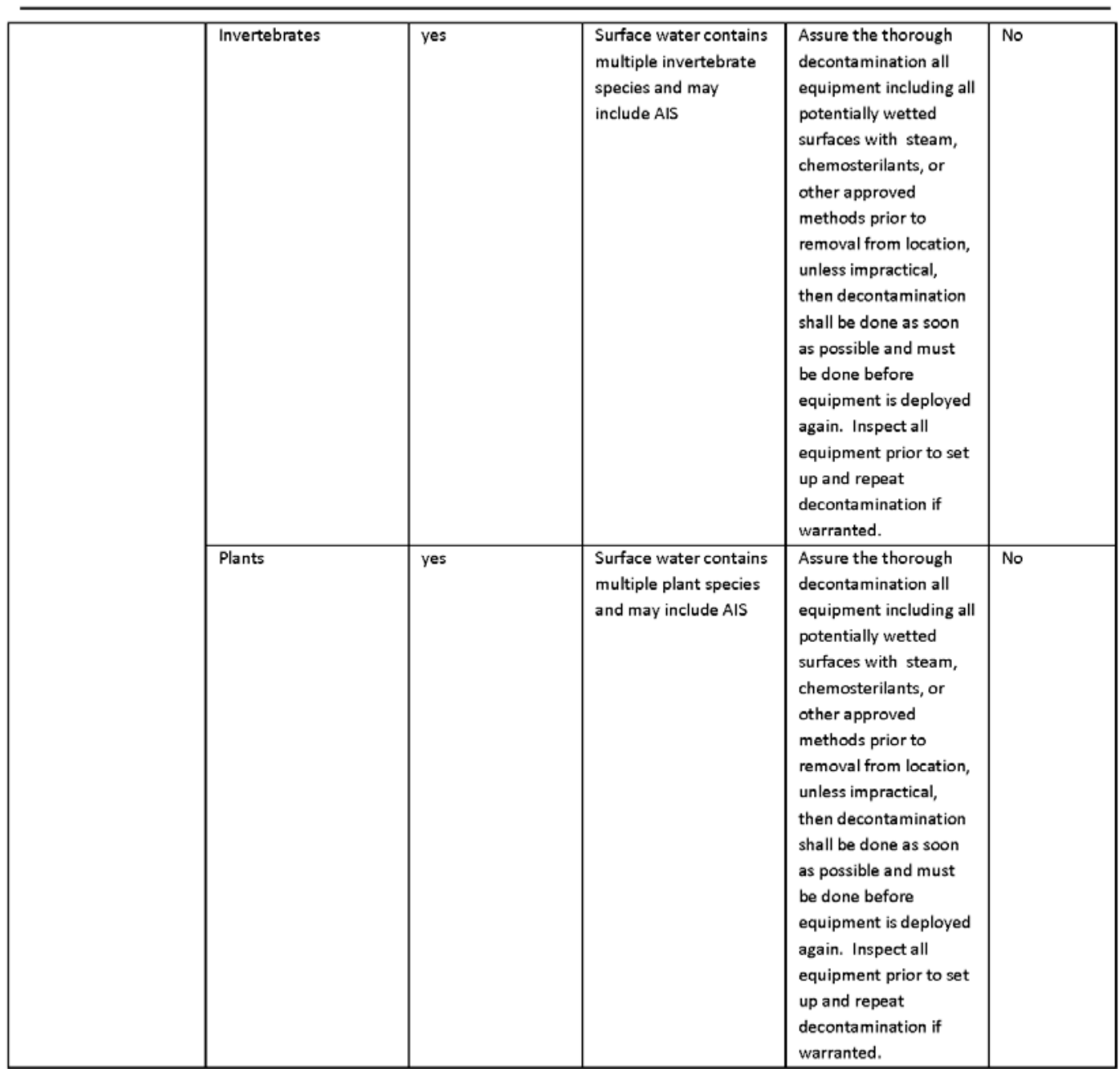

Page $\mathbf{2 3}$ of $\mathbf{3 2}$ 


\begin{tabular}{|l|l|l|l|l|}
\hline & Others & yes & $\begin{array}{l}\text { Surface water has } \\
\text { potential to transfer } \\
\text { fish diseases }\end{array}$ & $\begin{array}{l}\text { Assure the thorough } \\
\text { decontamination all } \\
\text { equipment including all } \\
\text { potentially wetted } \\
\text { surfaces with steam, } \\
\text { chemosterilants, or } \\
\text { other approved } \\
\text { methods prior to } \\
\text { removal from location, } \\
\text { unless impractical, } \\
\text { then decontamination } \\
\text { shall be done as soon } \\
\text { as possible and must } \\
\text { be done before } \\
\text { equipment is deployed } \\
\text { again. Inspect all } \\
\text { equipment prior to set } \\
\text { up and repeat } \\
\text { decontamination if } \\
\text { warranted. }\end{array}$ \\
\hline
\end{tabular}

\begin{tabular}{|c|c|c|c|c|c|}
\hline $\begin{array}{l}\text { Task } 5 \\
\text { Impermeable barriers } \\
\text { are removed and } \\
\text { native mussels left } \\
\text { undisturbed for post- } \\
\text { exposure holding }\end{array}$ & Vertebrates & yes & $\begin{array}{l}\text { Surface water contains } \\
\text { multiple vertebrate } \\
\text { species }\end{array}$ & $\begin{array}{l}\text { Assure the thorough } \\
\text { decontamination all } \\
\text { equipment including all } \\
\text { potentially wetted } \\
\text { surfaces with steam, } \\
\text { chemosterilants, or } \\
\text { other approved } \\
\text { methods prior to } \\
\text { removal from location, } \\
\text { unless impractical, } \\
\text { then decontamination } \\
\text { shall be done as soon } \\
\text { as possible and must } \\
\text { be done before } \\
\text { equipment is deployed } \\
\text { again. Inspect all } \\
\text { equipment prior to set } \\
\text { up and repeat } \\
\text { decontamination if } \\
\text { warranted. }\end{array}$ & No \\
\hline
\end{tabular}

Page $\mathbf{2 4}$ of $\mathbf{3 2}$ 


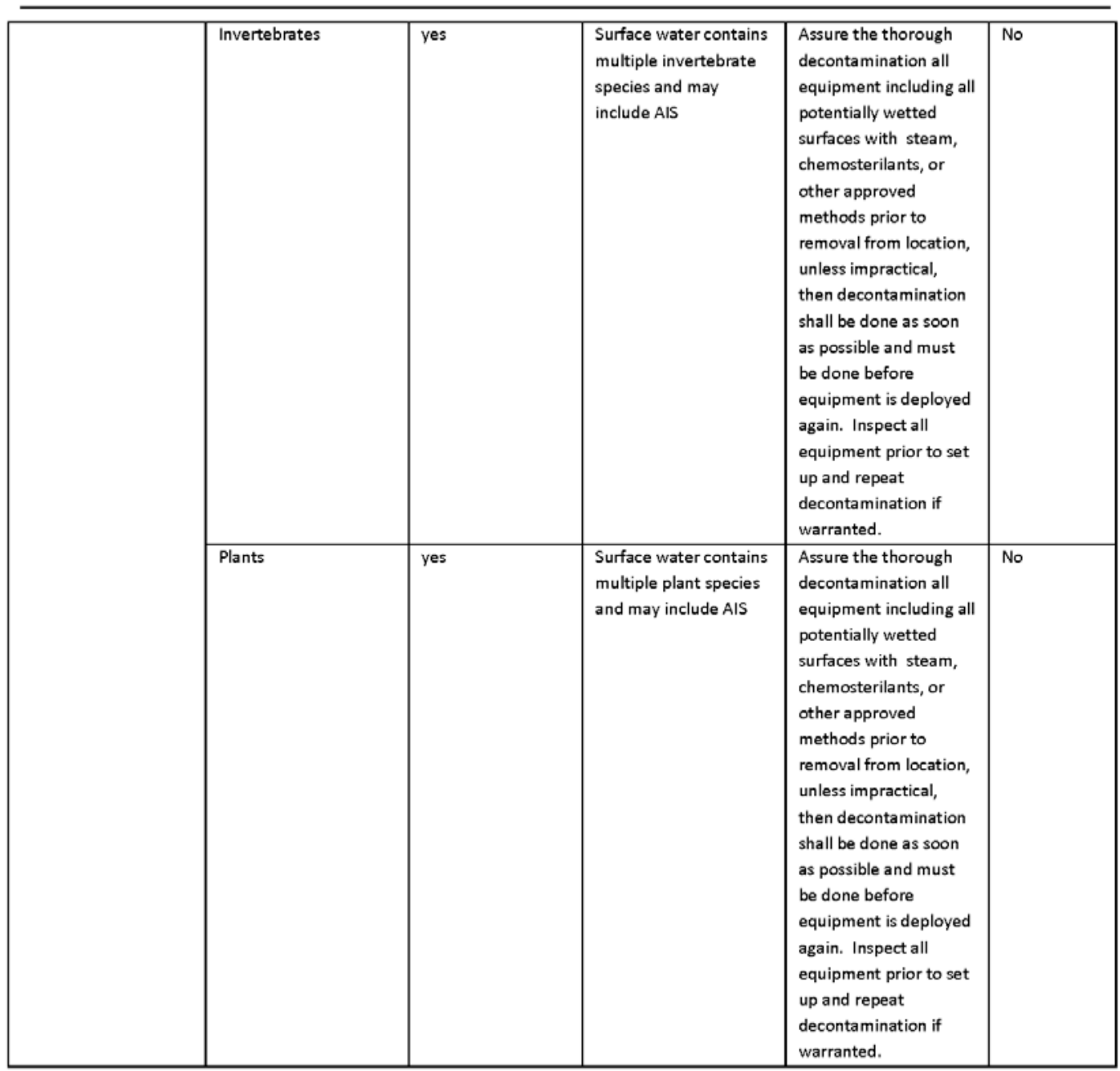

Page $\mathbf{2 5}$ of $\mathbf{3 2}$ 


\begin{tabular}{|c|c|c|c|c|c|}
\hline & Others & yes & $\begin{array}{l}\text { Surface water has } \\
\text { potential to transfer } \\
\text { fish diseases }\end{array}$ & $\begin{array}{l}\text { Assure the thorough } \\
\text { decontamination all } \\
\text { equipment including all } \\
\text { potentially wetted } \\
\text { surfaces with steam, } \\
\text { chemosterilants, or } \\
\text { other approved } \\
\text { methods prior to } \\
\text { removal from location, } \\
\text { unless impractical, } \\
\text { then decontamination } \\
\text { shall be done as soon } \\
\text { as possible and must } \\
\text { be done before } \\
\text { equipment is deployed } \\
\text { again. Inspect all } \\
\text { equipment prior to set } \\
\text { up and repeat } \\
\text { decontamination if } \\
\text { warranted. }\end{array}$ & no \\
\hline \multirow[t]{2}{*}{$\begin{array}{l}\text { Task } 6 \\
\text { Equipment is } \\
\text { decontaminated and } \\
\text { returned to UMESC }\end{array}$} & Vertebrates & yes & $\begin{array}{l}\text { Surface water contains } \\
\text { multiple vertebrate } \\
\text { species }\end{array}$ & $\begin{array}{l}\text { Physical removal of all } \\
\text { visible vertebrates. } \\
\text { Assure the thorough } \\
\text { decontamination all } \\
\text { equipment including all } \\
\text { internal and external } \\
\text { potentially wetted } \\
\text { surfaces with steam, } \\
\text { chemosterilants, or } \\
\text { other approved } \\
\text { methods prior to } \\
\text { removal from location, } \\
\text { unless impractical, } \\
\text { then decontamination } \\
\text { shall be done as soon } \\
\text { as possible and must } \\
\text { be done before } \\
\text { equipment is deployed } \\
\text { again. }\end{array}$ & Yes \\
\hline & Invertebrates & yes & $\begin{array}{l}\text { Surface water contains } \\
\text { multiple invertebrate } \\
\text { species and may } \\
\text { include AIS }\end{array}$ & $\begin{array}{l}\text { Physical removal of all } \\
\text { visible invertebrates. } \\
\text { Assure the thorough } \\
\text { decontamination all } \\
\text { equipment including all } \\
\text { internal and external } \\
\text { potentially wetted } \\
\text { surfaces with steam, } \\
\text { chemosterilants, or } \\
\text { other approved } \\
\text { methods prior to } \\
\text { removal from location, } \\
\text { unless impractical, } \\
\text { then decontamination } \\
\text { shall be done as soon } \\
\text { as possible and must } \\
\text { be done before } \\
\text { equipment is deployed } \\
\text { again. }\end{array}$ & Yes \\
\hline
\end{tabular}

Page $\mathbf{2 6}$ of $\mathbf{3 2}$ 


\begin{tabular}{|c|c|c|c|c|c|}
\hline & Plants & yes & $\begin{array}{l}\text { Surface water contains } \\
\text { multiple plant species } \\
\text { and may include AIS }\end{array}$ & $\begin{array}{l}\text { Physical removal of all } \\
\text { visible plants. Assure } \\
\text { the thorough } \\
\text { decontamination all } \\
\text { equipment including all } \\
\text { internal and external } \\
\text { potentially wetted } \\
\text { surfaces with steam, } \\
\text { chemosterilants, or } \\
\text { other approved } \\
\text { methods prior to } \\
\text { removal from location, } \\
\text { unless impractical, } \\
\text { then decontamination } \\
\text { shall be done as soon } \\
\text { as possible and must } \\
\text { be done before } \\
\text { equipment is deployed } \\
\text { again. }\end{array}$ & Yes \\
\hline & Others & yes & $\begin{array}{l}\text { Surface water has } \\
\text { potential to transfer } \\
\text { fish diseases }\end{array}$ & $\begin{array}{l}\text { Assure the thorough } \\
\text { decontamination all } \\
\text { equipment including all } \\
\text { internal and external } \\
\text { potentially wetted } \\
\text { surfaces with steam, } \\
\text { chemosterilants, or } \\
\text { other approved } \\
\text { methods prior to } \\
\text { removal from location, } \\
\text { unless impractical, } \\
\text { then decontamination } \\
\text { shall be done as soon } \\
\text { as possible and must } \\
\text { be done before } \\
\text { equipment is deployed } \\
\text { again. }\end{array}$ & Yes \\
\hline $\begin{array}{l}\text { Task } 7 \\
\text { Native mussels are } \\
\text { removed from } \\
\text { enclosures and } \\
\text { assessed for zebra } \\
\text { mussel colonization } \\
\text { and survival }\end{array}$ & Vertebrates & yes & $\begin{array}{l}\text { Surface water contains } \\
\text { multiple vertebrate } \\
\text { species }\end{array}$ & $\begin{array}{l}\text { Assure the thorough } \\
\text { decontamination all } \\
\text { equipment including all } \\
\text { internal and external } \\
\text { potentially wetted } \\
\text { surfaces with steam, } \\
\text { chemosterilants, or } \\
\text { other approved } \\
\text { methods prior to } \\
\text { removal from location, } \\
\text { unless impractical, } \\
\text { then decontamination } \\
\text { shall be done as soon } \\
\text { as possible and must } \\
\text { be done before } \\
\text { equipment is deployed } \\
\text { again. }\end{array}$ & no \\
\hline
\end{tabular}

Page $\mathbf{2 7}$ of $\mathbf{3 2}$ 


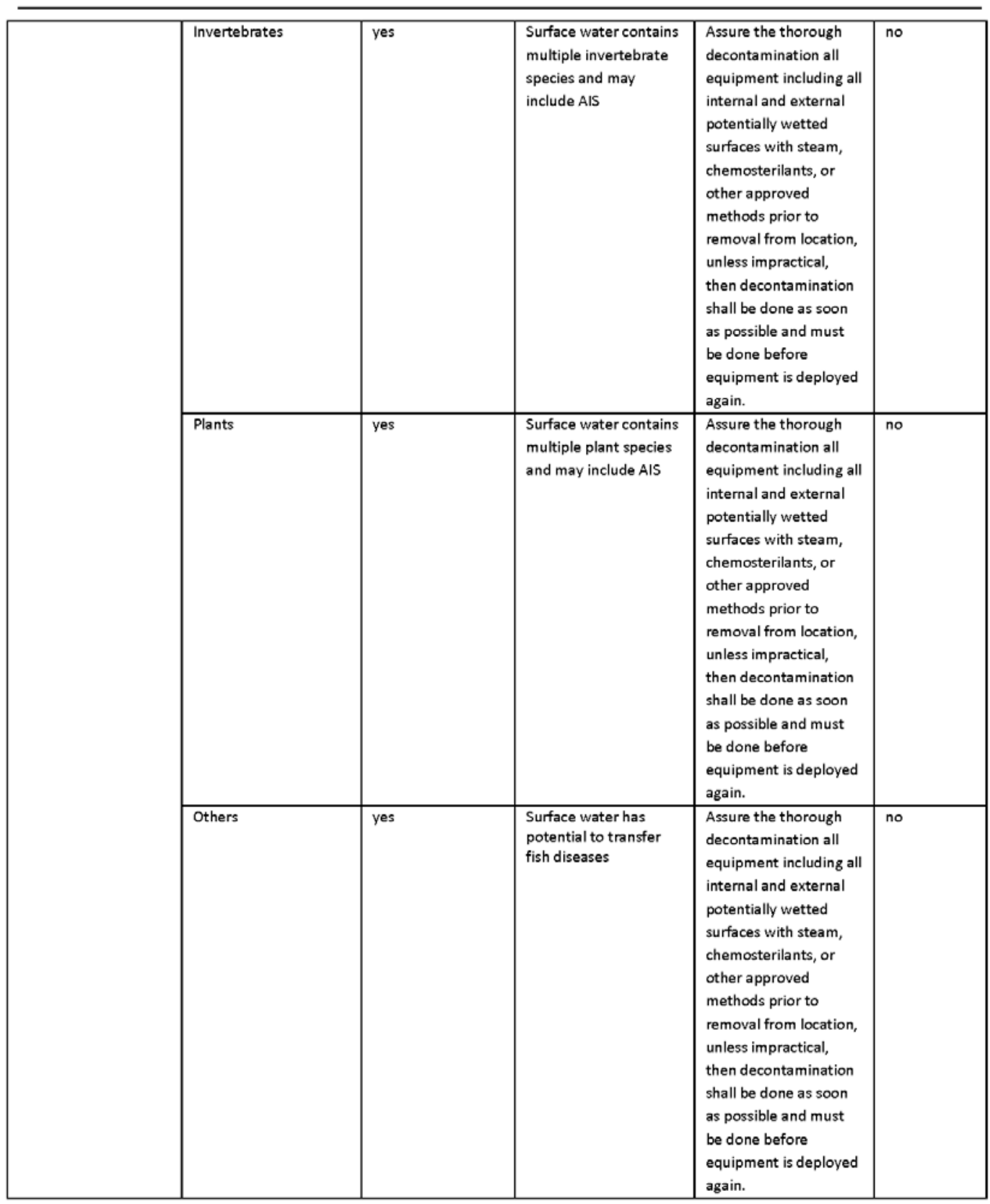

Page $\mathbf{2 8}$ of $\mathbf{3 2}$ 


\begin{tabular}{|c|c|c|c|c|c|}
\hline \multirow[t]{3}{*}{$\begin{array}{l}\text { Task } 8 \\
\text { Native mussels are } \\
\text { cleaned of zebra } \\
\text { mussels and returned } \\
\text { to their approximate } \\
\text { place of collection }\end{array}$} & Vertebrates & yes & $\begin{array}{l}\text { Surface water } \\
\text { contains multiple } \\
\text { vertebrate species }\end{array}$ & $\begin{array}{l}\text { Assure the thorough } \\
\text { decontamination all } \\
\text { equipment including all } \\
\text { internal and external } \\
\text { potentially wetted } \\
\text { surfaces with steam, } \\
\text { chemosterilants, or other } \\
\text { approved methods prior } \\
\text { to removal from location, } \\
\text { unless impractical, then } \\
\text { decontamination shall be } \\
\text { done as soon as possible } \\
\text { and must be done before } \\
\text { equipment is deployed } \\
\text { again. }\end{array}$ & no \\
\hline & Invertebrates & yes & $\begin{array}{l}\text { Surface water } \\
\text { contains multiple } \\
\text { invertebrate } \\
\text { species and may } \\
\text { include AIS }\end{array}$ & $\begin{array}{l}\text { Assure the thorough } \\
\text { decontamination all } \\
\text { equipment including all } \\
\text { internal and external } \\
\text { potentially wetted } \\
\text { surfaces with steam, } \\
\text { chemosterilants, or other } \\
\text { approved methods prior } \\
\text { to removal from location, } \\
\text { unless impractical, then } \\
\text { decontamination shall be } \\
\text { done as soon as possible } \\
\text { and must be done before } \\
\text { equipment is deployed } \\
\text { again. }\end{array}$ & no \\
\hline & Plants & yes & $\begin{array}{l}\text { Surface water } \\
\text { contains multiple } \\
\text { plant species and } \\
\text { may include AIS }\end{array}$ & $\begin{array}{l}\text { Assure the thorough } \\
\text { decontamination all } \\
\text { equipment including all } \\
\text { internal and external } \\
\text { potentially wetted } \\
\text { surfaces with steam, } \\
\text { chemosterilants, or other } \\
\text { approved methods prior } \\
\text { to removal from location, } \\
\text { unless impractical, then } \\
\text { decontamination shall be } \\
\text { done as soon as possible } \\
\text { and must be done before } \\
\text { equipment is deployed } \\
\text { again. }\end{array}$ & no \\
\hline
\end{tabular}

Page $\mathbf{2 9}$ of $\mathbf{3 2}$ 


\begin{tabular}{|c|c|c|c|c|c|}
\hline & Others & yes & $\begin{array}{l}\text { Surface water has } \\
\text { potential to } \\
\text { transfer fish } \\
\text { diseases }\end{array}$ & $\begin{array}{l}\text { Assure the thorough } \\
\text { decontamination all } \\
\text { equipment including all } \\
\text { internal and external } \\
\text { potentially wetted } \\
\text { surfaces with steam, } \\
\text { chemosterilants, or other } \\
\text { approved methods prior } \\
\text { to removal from location, } \\
\text { unless impractical, then } \\
\text { decontamination shall be } \\
\text { done as soon as possible } \\
\text { and must be done before } \\
\text { equipment is deployed } \\
\text { again. }\end{array}$ & no \\
\hline $\begin{array}{l}\text { Task } 9 \\
\text { All remaining } \\
\text { equipment, retention } \\
\text { barriers, etc. are } \\
\text { decontaminated and } \\
\text { returned to UMESC }\end{array}$ & Vertebrates & yes & $\begin{array}{l}\text { Surface water } \\
\text { contains multiple } \\
\text { vertebrate species }\end{array}$ & $\begin{array}{l}\text { Physical removal of all } \\
\text { visible vertebrates. } \\
\text { Assure the thorough } \\
\text { decontamination all } \\
\text { equipment including all } \\
\text { internal and external } \\
\text { potentially wetted } \\
\text { surfaces with steam, } \\
\text { chemosterilants, or other } \\
\text { approved methods prior } \\
\text { to removal from location, } \\
\text { unless impractical, then } \\
\text { decontamination shall be } \\
\text { done as soon as possible } \\
\text { and must be done before } \\
\text { equipment is deployed } \\
\text { aga Physical removal of } \\
\text { all visible vertebrates. } \\
\text { Assure the thorough } \\
\text { decontamination all } \\
\text { equipment including all } \\
\text { internal and external } \\
\text { potentially wetted } \\
\text { surfaces with steam, } \\
\text { chemosterilants, or other } \\
\text { approved methods prior } \\
\text { to removal from location, } \\
\text { unless impractical, then } \\
\text { decontamination shall be } \\
\text { done as soon as possible } \\
\text { and must be done before } \\
\text { equipment is deployed } \\
\text { again. }\end{array}$ & Yes \\
\hline
\end{tabular}

Page $\mathbf{3 0}$ of $\mathbf{3 2}$ 


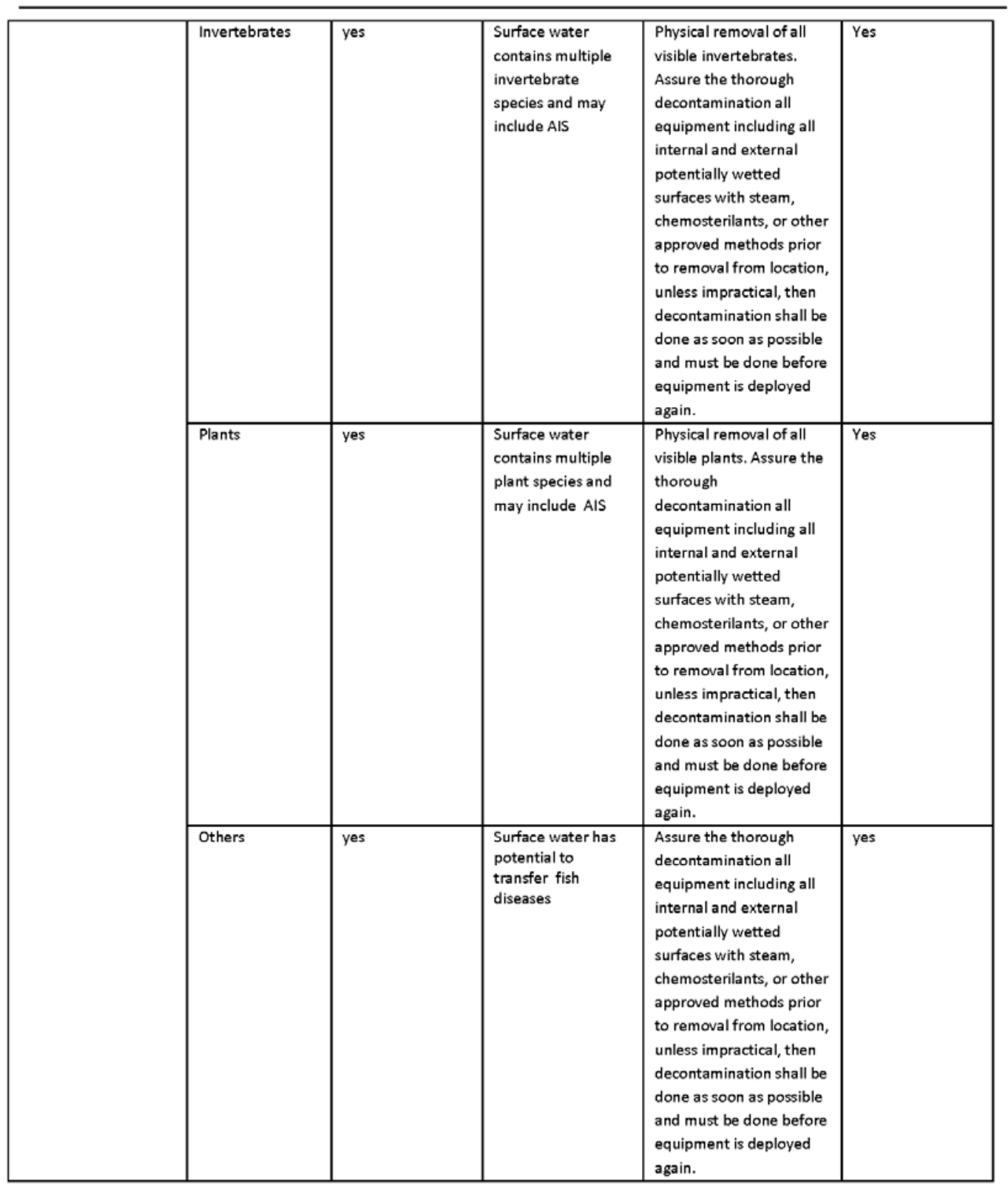

Page $\mathbf{3 1}$ of $\mathbf{3 2}$ 


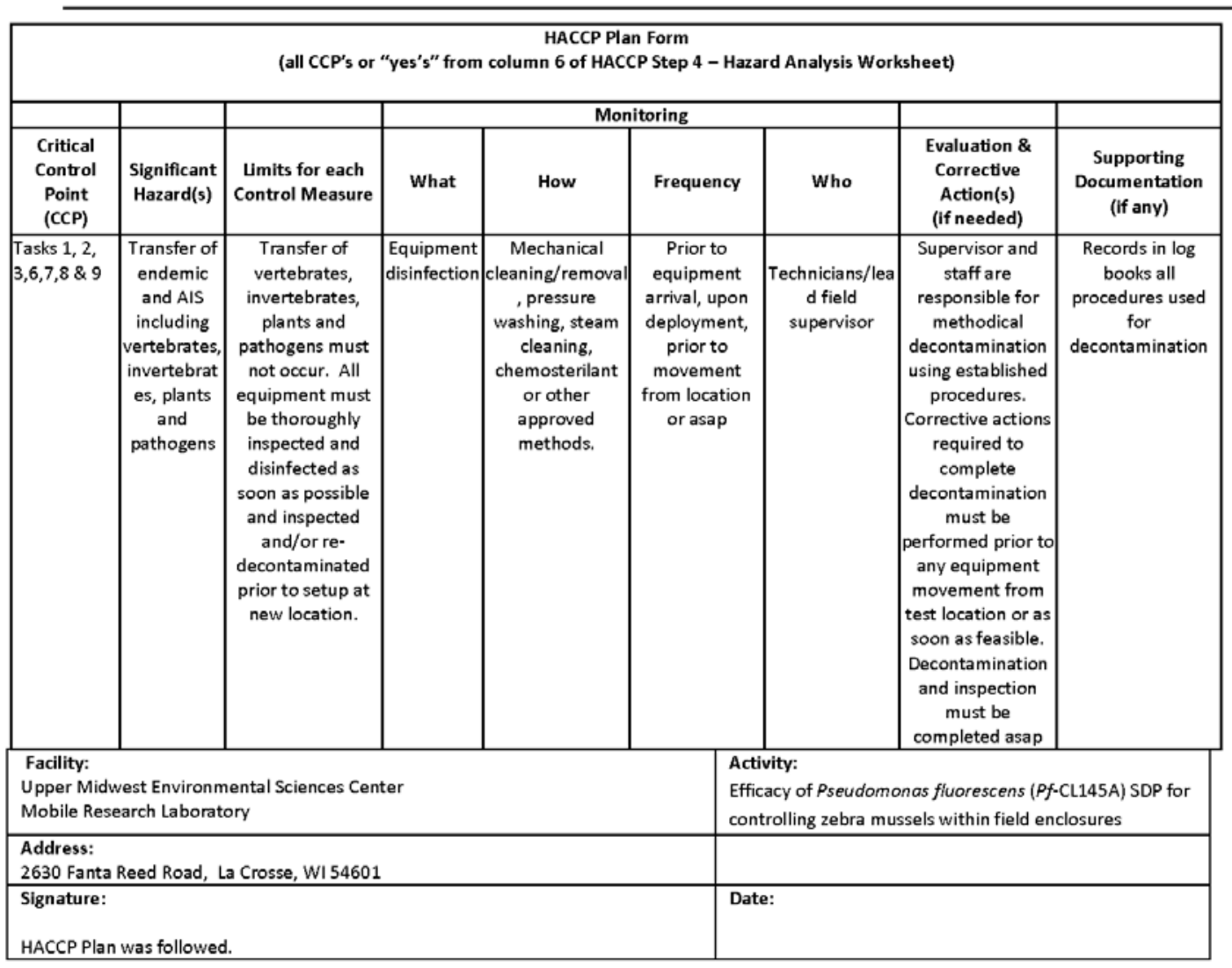

Page $\mathbf{3 2}$ of $\mathbf{3 2}$ 


\section{Uniled States Department of the Interior}

\section{U.S. GEOLOGICAL SURVIT}

Upper Midwest Fnvironmental Sciences Center

2630 Fanta Reed Road

I a Crosse, Wisconsin 54603

Date: February 19, 2014

To: The Record Study Number AFH-13-PSFUDO-0S

Subject: Note To File \# 1; Study number AFH-13-PSEUDO-05

This note is to document and clarify foot biopsy collection and resulting data use.

A 10-mg foot sample was collected from each unionid test animal using a biopsy needle during the postexposure assessments conducted August 13-15, 2013. The biopsy samples were placed in individually labeled cryogenic vials and then stored in liquid nitrogen. After the assessment, the unionids were consolidated into retention barriers for an additional holding period of 26 days. A second foot biopsy sample was taken from each surviving mussel unionid on September 10,2013.

Pending funding, the foot biopsy samples will be analyzed for glycogen content and will be compared to unionid survival and treatment. If completed, a separate report will be generated for these data.

Due to confound factors (i.e. additional handling stress, biopsy stress) the data collected at the termination of the second holding period (i.e. survival) will be retained but not used during analysis.

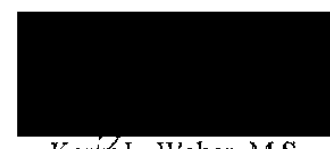

Kerry L. Weber, M.S.

Principal Investigator, UMESC

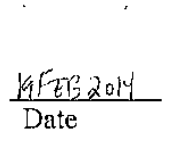

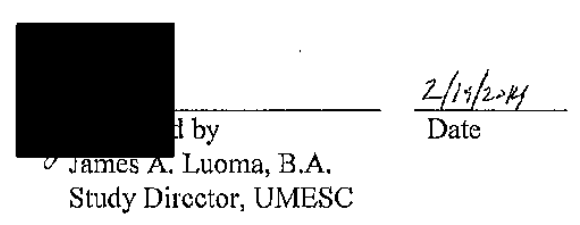

Flle Folder: $\quad 3$

Page 1 of 1 
Study Number: AEH-13-PSEUDO-05

File Folder:

Lab book/pgs:
Reviewed by: __. _..____ Date:

Verilled by:

Date:
Test Chemical Stock Preparation

Test Chemical: Pseudomonas fluroescens strain 145A

Test Chemical Lot \#:

Test Location:

Date Rec'd:

Instruments Used:

Exp. Date

Weights of Chemical Samples:

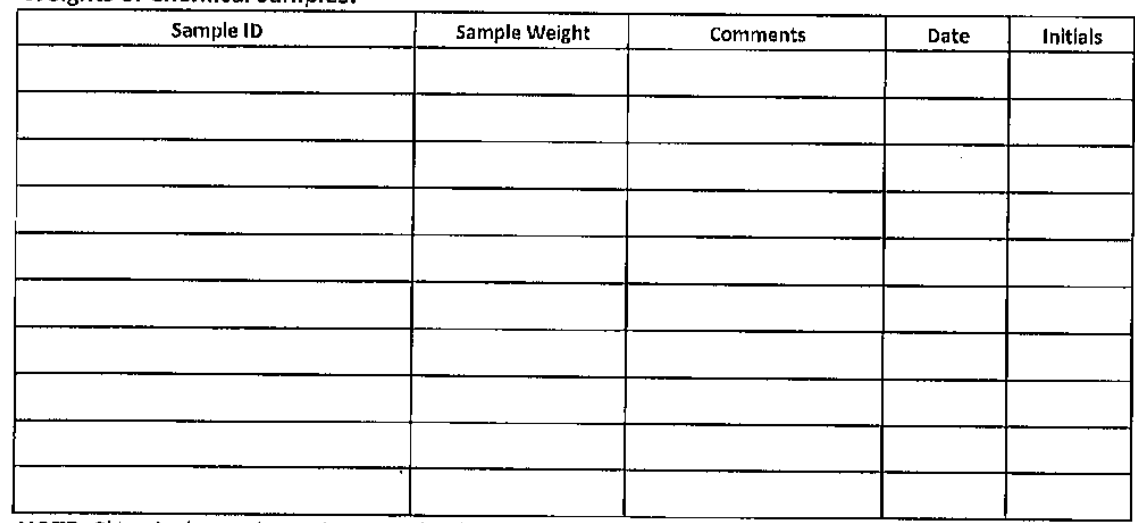

NOTE: Chemical samples to be stored refrigerated until used for stock preparation.

Stock Solution Preparation:

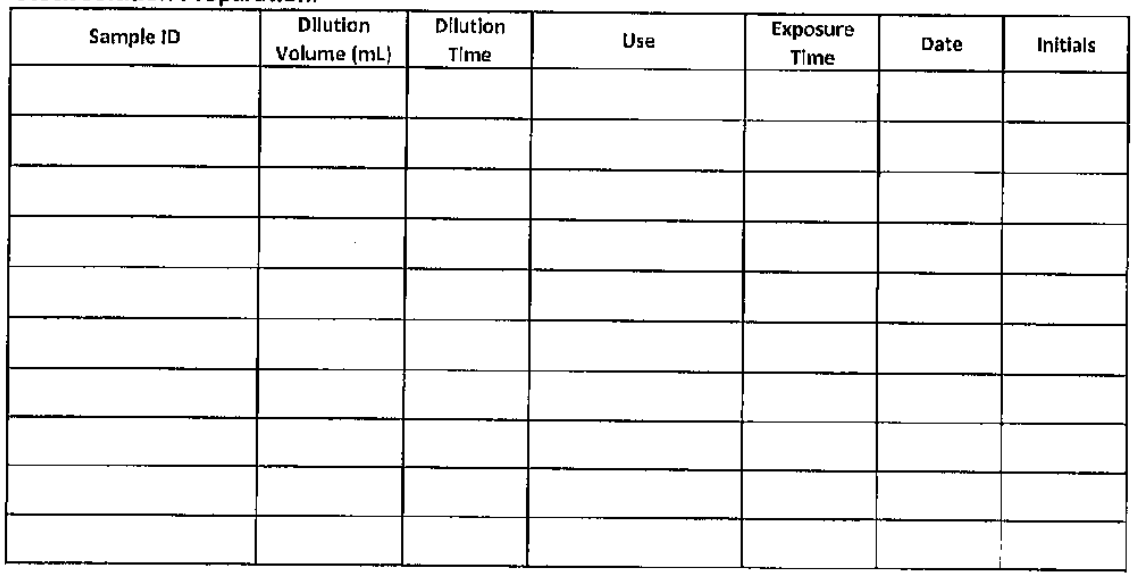

FF

Item No. I

$\mathrm{Pg} \mathrm{Pg}_{\mathrm{L}}$ of 1 
Study Nu mber: AEH-13-PSEUDO-0S File Folder:

Lab book/pgs:
Revlewed by:

verifled by:

Date:

Date:

\section{Mussel Species Identification}

\begin{tabular}{|c|c|c|c|c|c|}
\hline \multicolumn{3}{|c|}{$\begin{array}{l}\text { Exposure Date: } 26 \text { JUL } 2013 \\
\text { Test Chemical: Pf-CL145A }\end{array}$} & \multicolumn{3}{|c|}{$\begin{array}{l}\text { Test Location: Lake Darling, Alexandria, MN } \\
\text { Lot \#: TR4669-4-\{3\} }\end{array}$} \\
\hline $\begin{array}{c}\text { Enclosure } \\
\text { ID }\end{array}$ & Tag ID & $\begin{array}{l}\text { Mussel } \\
\text { Species }\end{array}$ & Comments & Date & Initials \\
\hline 1 & C379 & & & & \\
\hline 1 & C358 & & & & \\
\hline 1 & C414 & & & & \\
\hline 1 & $\mathrm{C} 237$ & & & & \\
\hline 1 & $\mathrm{C} 221$ & & & & \\
\hline 1 & C312 & & & & \\
\hline 1. & $\mathrm{C} 292$ & & & & \\
\hline 1 & $\mathrm{C} 350$ & & & & \\
\hline 1 & $\mathrm{C} 380$ & & & & \\
\hline 1 & $\mathrm{C} 233$ & & & & \\
\hline 1 & C377 & & & & \\
\hline 1 & C424 & & & & \\
\hline 1 & C314 & & & & \\
\hline 1 & $\mathrm{C} 263$ & & & & \\
\hline 1 & C363 & & & & \\
\hline 1 & С309 & & & & \\
\hline 1 & C208 & & & & \\
\hline 1 & C228 & & & & \\
\hline 1 & $\mathrm{C} 266$ & & & & \\
\hline 1 & C195 & & & & \\
\hline 1 & C 360 & & & & \\
\hline 1 & C359 & & & & \\
\hline 1 & $\mathrm{C} 302$ & & & & \\
\hline 1 & $\mathrm{C} 207$ & & & & \\
\hline 1 & C345 & & & & \\
\hline 1 & $\mathrm{C} 245$ & & & & \\
\hline 1 & C329 & & & & \\
\hline
\end{tabular}

$F F \# 17$

Item No. $\Rightarrow$

$\mathrm{Pg} \perp$ of 9 
Study Number: AEH-13-PSEUDO-C5

File Folder:

Lab book/pgs:
Reviewed by:

Verified by:

Date:

Date:

\section{Mussel Species Identification}

\begin{tabular}{|c|c|c|c|c|c|}
\hline \multicolumn{3}{|c|}{$\begin{array}{l}\text { Exposure Date: } 26 \text { JUL } 2013 \\
\text { Test Chemical: Pf-CL.145A }\end{array}$} & \multicolumn{3}{|c|}{$\begin{array}{l}\text { Test Location: Lake Darling, Alexandria, MN } \\
\text { Lot \#: TR4669-4-(3) }\end{array}$} \\
\hline \begin{tabular}{|c|} 
Enclosure \\
ID
\end{tabular} & Tag ID & $\begin{array}{l}\text { Mussel } \\
\text { Species }\end{array}$ & Comments & Date & Initials \\
\hline 2 & C321 & & & & \\
\hline 2 & $\mathrm{C} 276$ & & & & \\
\hline 2 & C249 & & & & \\
\hline 2 & C310 & & & & \\
\hline 2 & $\mathrm{C} 242$ & & & & \\
\hline 2 & $\mathrm{C} 406$ & & & & \\
\hline 2 & $\mathrm{C} 375$ & & & & \\
\hline 2 & $\mathrm{C} 232$ & & & & \\
\hline 2 & $\mathrm{C} 436$ & & & & \\
\hline 2 & $\mathrm{C} 293$ & & & & \\
\hline 2 & C419 & & & & \\
\hline 2 & C342 & & & & \\
\hline 2 & C304 & & & & \\
\hline 2 & C187 & & & & \\
\hline 2 & $\mathrm{C} 332$ & & & & \\
\hline 2 & $\mathrm{C} 217$ & & & & \\
\hline 2 & $\mathrm{C} 203$ & & & & $\cdot$ \\
\hline 2 & C331 & & & & \\
\hline 2 & C298 & & & & \\
\hline 2 & C317 & & & & \\
\hline 2 & $\mathrm{C} 408$ & & & & \\
\hline 2 & $\mathrm{C} 1.88$ & & & & \\
\hline 2 & C395 & & & & \\
\hline 2 & $\mathrm{C} 410$ & & & & \\
\hline 2 & $\mathrm{C} 278$ & & & & \\
\hline 2. & $\mathrm{C} 219$ & & & & \\
\hline 2 & $\mathrm{C} 235$ & & & & \\
\hline
\end{tabular}

Page 2 of 9 
Study Number; AEH-13-PSEUDO-05

Flle Folder:

Lab book/pgs:
Reviewed oy:

Verlffed by:
Date:

Date:

\section{Mussel Species Identification}

\begin{tabular}{|c|c|c|c|c|c|}
\hline \multicolumn{3}{|c|}{$\begin{array}{l}\text { Exposure Date: } 26 \text { JUL } 2013 \\
\text { Test Chemical: Pf-CL145A }\end{array}$} & \multicolumn{3}{|c|}{$\begin{array}{l}\text { Test Location: Lake Darling, Alexandria, MN } \\
\text { Lot H: TR4669-4-(3) }\end{array}$} \\
\hline \begin{tabular}{|c|} 
Enclosure \\
ID \\
\end{tabular} & Tag ID & $\begin{array}{l}\text { Mussel } \\
\text { Species } \\
\end{array}$ & Comments & Date & Initials \\
\hline 3 & $\mathrm{C} 434$ & & & & \\
\hline 3 & $\mathrm{C} 374$ & & & & \\
\hline 3 & C197 & & & & \\
\hline 3 & C382 & & & & \\
\hline 3 & $\mathrm{C} 418$ & & & & \\
\hline 3 & C194 & & & & \\
\hline 3 & $C 210$ & & & & \\
\hline 3 & $\mathrm{C} 325$ & & & & \\
\hline 3 & $\mathrm{C} 218$ & & & & \\
\hline 3 & $\mathrm{C} 243$ & & & & \\
\hline 3 & $\mathrm{C} 405$ & & & & \\
\hline 3 & C354 & & & & \\
\hline 3 & $\mathrm{C} 437$ & & & & \\
\hline 3 & C311 & & & & \\
\hline 3 & $\mathrm{C} 427$ & & & & \\
\hline 3 & $\mathrm{C} 326$ & & & & \\
\hline 3 & C364 & & & & \\
\hline 3 & C193 & & & & \\
\hline 3 & $\mathrm{C} 204$ & & & & \\
\hline 3 & C384 & & & & \\
\hline 3 & C393 & & & & \\
\hline 3 & C231 & & & & \\
\hline 3 & $\mathrm{C} 254$ & & & & \\
\hline 3 & $\mathrm{C} 373$ & & & & \\
\hline 3 & $\mathrm{C} 390$ & & & & \\
\hline 3 & $\mathrm{C} 246$ & & & & \\
\hline 3 & $\mathrm{C} 215$ & & & & \\
\hline
\end{tabular}

Page 3 of 9 
Study Number: AEH-13-PSEUDO-05

File Folder:

iab book/pgs;
Revlewed by:

Verified by:

Date:

Date

\section{Mussel Species Identification}

\begin{tabular}{|c|c|c|c|c|c|}
\hline \multicolumn{3}{|c|}{$\begin{array}{l}\text { Exposure Date: } 26 \text { JUL } 2013 \\
\text { Test Chemical: Pf-CL145A }\end{array}$} & \multicolumn{3}{|c|}{$\begin{array}{l}\text { Test Location: Lake Darling, Alexandria, MN } \\
\text { Lot \#: TR4669-4-(3) }\end{array}$} \\
\hline $\begin{array}{c}\text { Enclosure } \\
\text { ID }\end{array}$ & Tag ID & $\begin{array}{l}\text { Mussel } \\
\text { Species }\end{array}$ & Comments & Date & Initials \\
\hline 4 & $\mathrm{C} 282$ & & & & \\
\hline 4 & $\mathrm{C} 255$ & & & & \\
\hline 4 & $\mathrm{C} 296$ & & & & \\
\hline 4 & $\mathrm{C} 412$ & & & & \\
\hline 4 & C239 & & & & \\
\hline 4 & $\mathrm{C} 283$ & & & & \\
\hline 4 & C285 & & & & \\
\hline 4 & $\mathrm{C} 225$ & & & & \\
\hline 4 & C351 & & & & \\
\hline 4 & $\mathrm{C} 388$ & & & & \\
\hline 4 & $\mathrm{C} 339$ & & & & \\
\hline 4 & $\mathrm{C} 429$ & & & & \\
\hline 4 & $\mathrm{C} 403$ & & & & \\
\hline 4 & $C 404$ & & & & \\
\hline 4 & $\mathrm{C} 270$ & & & & \\
\hline 4 & C279 & & & & \\
\hline 4 & .0286 & & & & \\
\hline 4 & $\mathrm{C} 421$ & & & & \\
\hline 4 & C353 & & & & \\
\hline 4 & $\mathrm{C} 398$ & & & & \\
\hline 4. & $\mathrm{C} 333$ & & & & \\
\hline 4 & $\mathrm{C} 348$ & & & & \\
\hline 4 & $\mathrm{C} 223$ & & & & \\
\hline 4 & $\mathrm{C} 271$ & & & & \\
\hline 4 & $\mathrm{C} 303$ & & & & \\
\hline 4 & C389 & & & & \\
\hline 4 & C 334 & & & & \\
\hline
\end{tabular}

Page 4 of 9 
Study Number: AEH-:3-PSEUDO-05 Flle Folder:

Lab book/pgs:
Revlewed by:

Verlfled by:

Date:

Date:

\section{Mussel Species Identification}

\begin{tabular}{|c|c|c|c|c|c|}
\hline \multicolumn{3}{|c|}{$\begin{array}{l}\text { Fxposure Date: } 26 \text { JUL } 2013 \\
\text { Test Chemical: Pf-CL145A }\end{array}$} & \multicolumn{3}{|c|}{$\begin{array}{l}\text { Test Location: Lake Darling, Alexandria, MN } \\
\text { Lot H: TR4669-4-(3) }\end{array}$} \\
\hline \begin{tabular}{|c|} 
Enclosure \\
ID \\
\end{tabular} & Tag 10 & $\begin{array}{l}\text { Mussel } \\
\text { Species }\end{array}$ & Comments & Date & Initials \\
\hline 5 & $\mathrm{C} 256$ & & & & \\
\hline 5 & $\mathrm{C} 407$ & & & & \\
\hline 5 & $\mathrm{C} 190$ & & & & \\
\hline 5 & C284 & & & & \\
\hline 5 & C238 & & & & \\
\hline 5 & $\mathrm{C} 240$ & & & & \\
\hline 5 & $\mathrm{C} 244$ & & & & \\
\hline 5 & $\mathrm{C} 253$ & & & & \\
\hline 5 & C335 & & & & \\
\hline 5 & C300 & & & & \\
\hline 5 & C352 & & & & \\
\hline 5 & $\mathrm{C} 294$ & & & & \\
\hline 5 & $\mathrm{C} 320$ & & & & \\
\hline 5 & $\mathrm{C} 433$ & & & & \\
\hline 5 & C330 & & & & \\
\hline 5 & $\mathrm{C} 368$ & & & & \\
\hline 5 & C392 & & & & \\
\hline 5 & $\mathrm{C} 236$ & & & & \\
\hline 5 & C397 & & & & \\
\hline 5 & $\mathrm{C} 259$ & & & & \\
\hline 5 & C365 & & & & \\
\hline 5 & $\mathrm{C} 290$ & & & & \\
\hline 5 & $\mathrm{CA} 25$ & & & & \\
\hline 5 & C343 & & & & \\
\hline 5 & C214 & & $\cdot$ & & \\
\hline 5 & $\mathrm{C} 431$ & & & & \\
\hline 5 & C265 & & & & \\
\hline
\end{tabular}

Page 5 of 9 
StLdy Number: AEH-13-PSEUDO-05

Fille folder:

Lab book/pgs:
Revlewed by:

Veriflediby;

Date:

Date:

\section{Mussel Species Identification}

\begin{tabular}{|c|c|c|c|c|c|}
\hline \multicolumn{3}{|c|}{$\begin{array}{l}\text { Exposure Date: } 26 \text { JUL } 2013 \\
\text { Test Chemlcal: Pf-CL145A }\end{array}$} & \multicolumn{3}{|c|}{$\begin{array}{l}\text { Test Location: Lake Darling, Alexandria, MN } \\
\text { Lot \#: TR4669-4- (3) }\end{array}$} \\
\hline $\begin{array}{c}\begin{array}{c}\text { Enclosure } \\
\text { ID }\end{array} \\
\end{array}$ & Tag ID & $\begin{array}{l}\text { Mussel } \\
\text { Specles }\end{array}$ & Comments & Date & Initials \\
\hline 6 & $\mathrm{C} 297$ & & & & \\
\hline 6 & $\mathrm{C} 362$ & & & & \\
\hline 6 & $\mathrm{C} 385$ & & & & \\
\hline 6 & $\mathrm{C} 250$ & & & & \\
\hline 6 & $\mathrm{C} 280$ & & & & \\
\hline 6 & $\mathrm{C} 423$ & & & & \\
\hline 6 & C356 & & & & \\
\hline 6 & $\mathrm{C} 200$ & & & & \\
\hline 6 & $\mathrm{C} 396$ & & & & \\
\hline 6 & $\mathrm{C} 281$ & & & & \\
\hline 6 & $\mathrm{C} 322$ & & & & \\
\hline 6 & $\mathrm{C} 251$ & & & & \\
\hline 6 & C313 & & & & \\
\hline 6 & $\mathrm{C} 252$ & & & & \\
\hline 6 & C308 & & & & \\
\hline 6 & $\mathrm{C} 277$ & & - & & \\
\hline 6 & C336 & & . & & \\
\hline 6 & $\mathrm{C} 402$ & & & & \\
\hline 6 & $\mathrm{C} 315$ & & & & \\
\hline 6 & $\mathrm{C} 213$ & & & & \\
\hline 6 & $\mathrm{C} 205$ & & & & \\
\hline 6 & $\mathrm{C} 426$ & & & & \\
\hline 6 & C378 & & & & \\
\hline 6 & $\mathrm{C} 383$ & & & & \\
\hline 6 & $\mathrm{C} 372$ & & . & & \\
\hline 6 & $\mathrm{C} 307$ & & & & \\
\hline 6 & $\mathrm{C} 316$ & & & & \\
\hline
\end{tabular}

Page 6 드 of 
Study Number: AEH-13-PSEUDO-05

تle Folder:

Lab book/pgs
Revlewed by:

Verlfied by:

Date:

Date

\section{Mussel Species Identification}

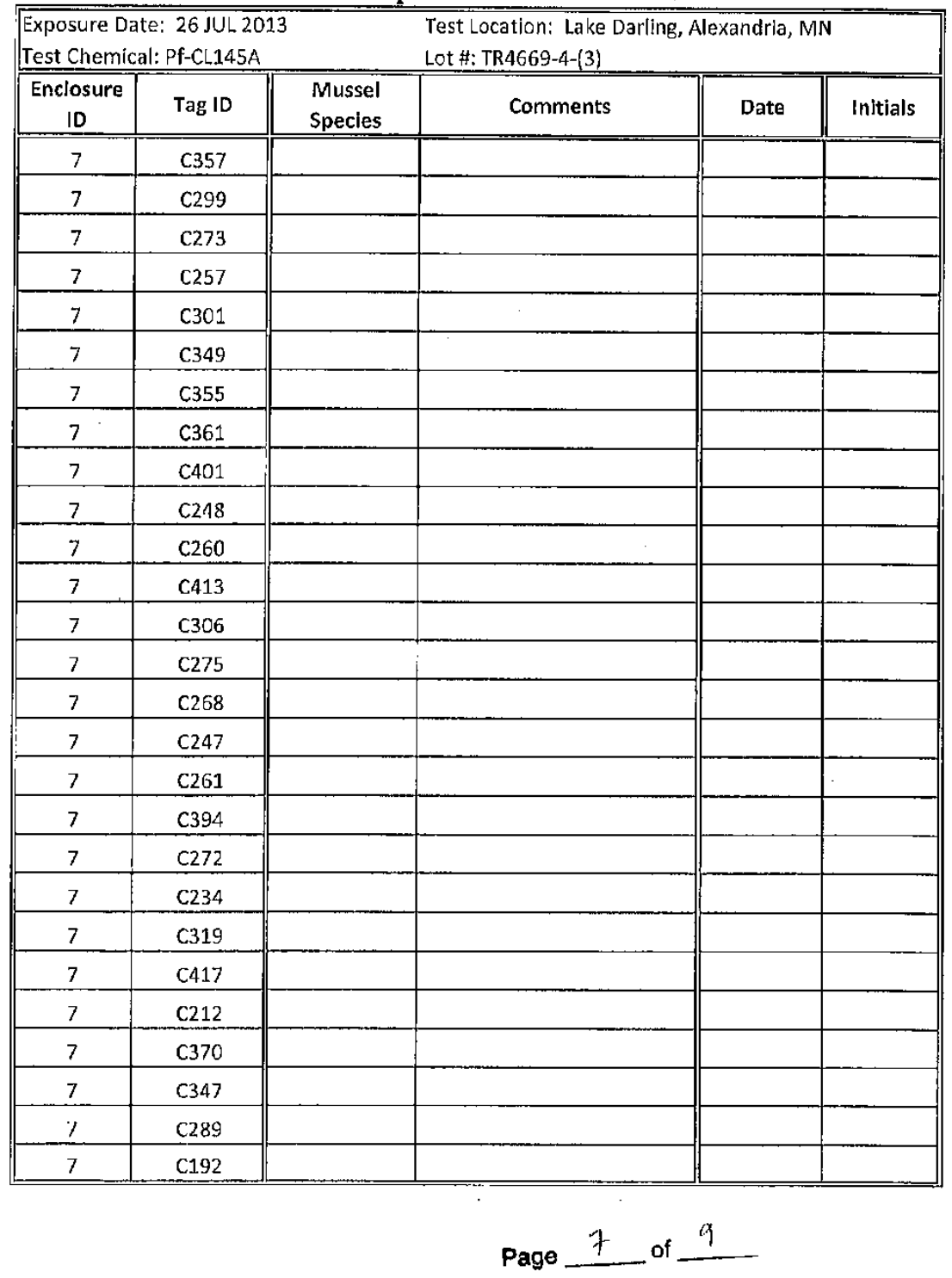


St.udy Number: AEH-13-PSEUDO-05

Flle Folder:

Lab book/pgs:
Revlewed by:

Verified by:

Date:

Date:

\section{Mussel Species Identification}

\begin{tabular}{|c|c|c|c|c|c|}
\hline \multicolumn{3}{|c|}{$\begin{array}{l}\text { Exposure Date: } 26 \text { JUL } 2013 \\
\text { Test Chemical: Pf-CL145A }\end{array}$} & \multicolumn{3}{|c|}{$\begin{array}{l}\text { Test Location: Lake Darling, Alexandria, MN } \\
\text { Lot \#: TR4669-4-(3) }\end{array}$} \\
\hline $\begin{array}{c}\text { Enclosure } \\
\text { ID }\end{array}$ & Tag ID & $\begin{array}{l}\text { Mussel } \\
\text { Species }\end{array}$ & Comments & Date & Initlals \\
\hline 8 & $C 369$ & & & & \\
\hline 8 & C189 & & & & \\
\hline 8 & $\mathrm{C} 415$ & & & & \\
\hline 8 & $\mathrm{C} 269$ & & & & \\
\hline 8 & C274 & & & & \\
\hline 8 & C.191. & & & & \\
\hline 8 & C186 & & & & \\
\hline 8 & $\mathrm{C} 211$ & & & & \\
\hline 8 & C337 & & & & \\
\hline 8 & C399 & & & & \\
\hline 8 & C366 & & & & \\
\hline 8 & $\mathrm{C} 435$ & & & & \\
\hline 8 & C196 & & & & \\
\hline 8 & C428 & & & & \\
\hline 8 & $\mathrm{C} 222$ & & & & \\
\hline 8 & C391 & & & & \\
\hline 8 & C198 & & & & \\
\hline 8 & C230 & & & & \\
\hline 8 & C199 & & & & \\
\hline 8 & $\mathrm{C} 216$ & & & & \\
\hline 8 & C.264 & & & & \\
\hline 8 & $\mathrm{C} 241$ & & & & \\
\hline 8 & C324 & & & & \\
\hline 8 & C387 & & & & \\
\hline 8 & C340 & & & & \\
\hline 8 & C209 & & & & \\
\hline 8 & $\mathrm{C} 220$ & & & & \\
\hline
\end{tabular}

Page _ 8_ of 9 
Study Number: AEH-13-PSEUDO-05

File iolder:

Lab book/pgs:
Revlewed by:

Verified by:

Date:

Date:

\section{Mussel Species Identification}

\begin{tabular}{|c|c|c|c|c|c|}
\hline \multicolumn{3}{|c|}{$\begin{array}{l}\text { Exposure Date: } 26 \text { JUL } 2013 \\
\text { Test Chemical: Pf-CL145A }\end{array}$} & \multicolumn{3}{|c|}{$\begin{array}{l}\text { Test Location: Lake Darling, Nlexandria, MN } \\
\text { Lot \#: TR.4669-4- } 3 \text { \} }\end{array}$} \\
\hline $\begin{array}{c}\text { Enclosure } \\
\text { ID }\end{array}$ & Tag ID & $\begin{array}{l}\text { Mussel } \\
\text { Species }\end{array}$ & Comments & Date & Initials \\
\hline 9 & C327 & & & & \\
\hline 9 & C338 & & & & \\
\hline 9 & C323 & & & & \\
\hline 9 & C305 & & & & \\
\hline 9 & C227 & & & & \\
\hline 9 & C229 & & & & \\
\hline 9 & C376 & & & & \\
\hline 9 & C206 & & & & \\
\hline 9 & C411 & & & & \\
\hline 9 & $\mathrm{C} 262$ & & & & \\
\hline 9 & $\mathrm{C} 22.26$ & & & & \\
\hline 9 & C344 & & & & \\
\hline 9 & C346 & & & & \\
\hline 9 & $\mathrm{C} 422$ & & & & \\
\hline 9 & $\mathrm{C} 420$ & & & & \\
\hline 9 & $\mathrm{C} 202$ & & & & \\
\hline 9 & C430 & & & & \\
\hline 9 & $C 409$ & & & & \\
\hline 9 & C295 & & & & \\
\hline 9 & $\mathrm{C} 287$ & & & & \\
\hline 9 & $\mathrm{C} 258$ & & & & \\
\hline 9 & C367 & & & & \\
\hline 9 & C386 & & & & \\
\hline 9 & C328 & & & & \\
\hline 9 & $\mathrm{C} 416$ & & & & \\
\hline 9 & C381 & & & & \\
\hline 9 & $\mathrm{C} 432$ & & & & \\
\hline
\end{tabular}

FF \# 17

Lem No. 2

$\mathrm{Pg} .9$ of 4 


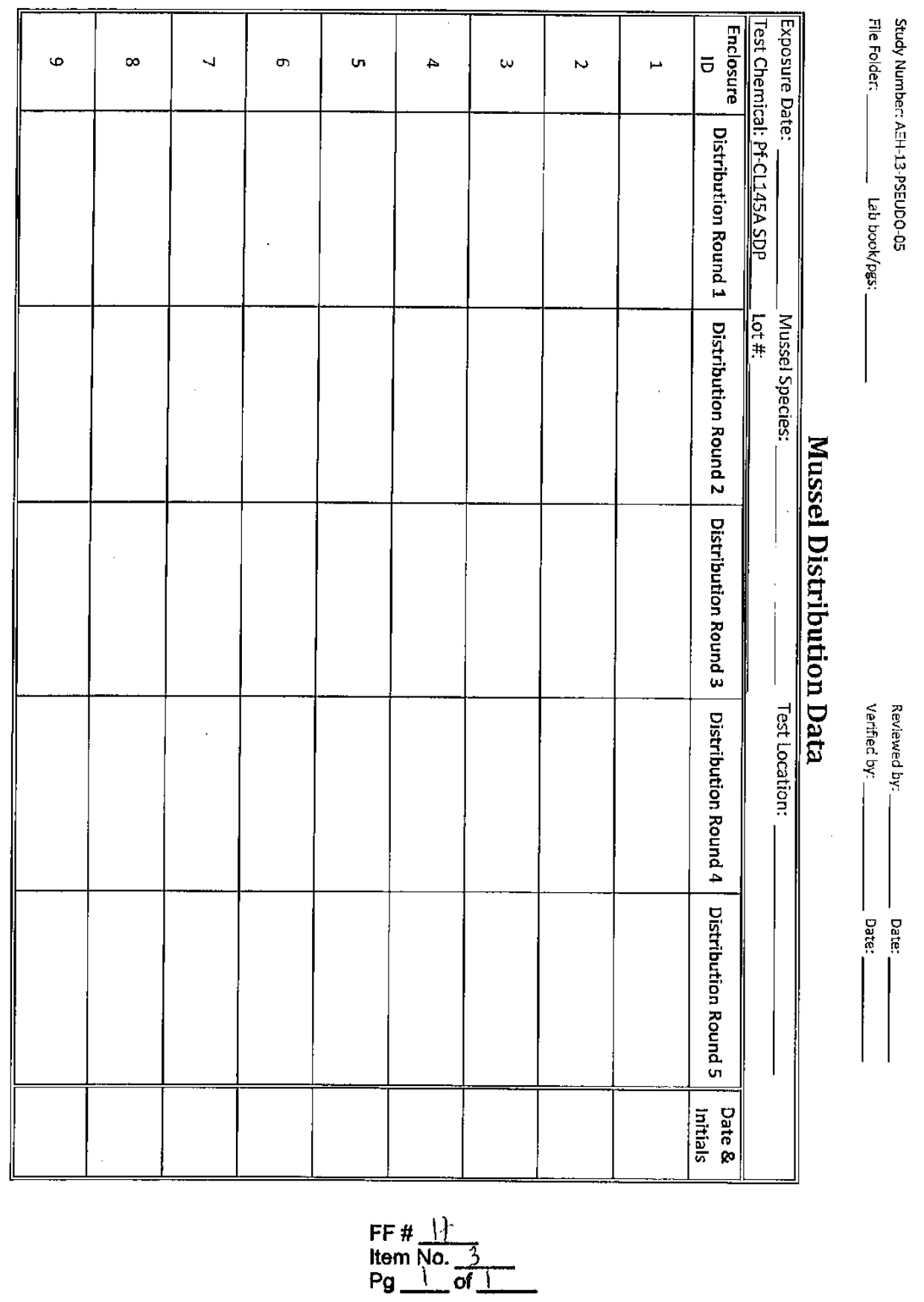




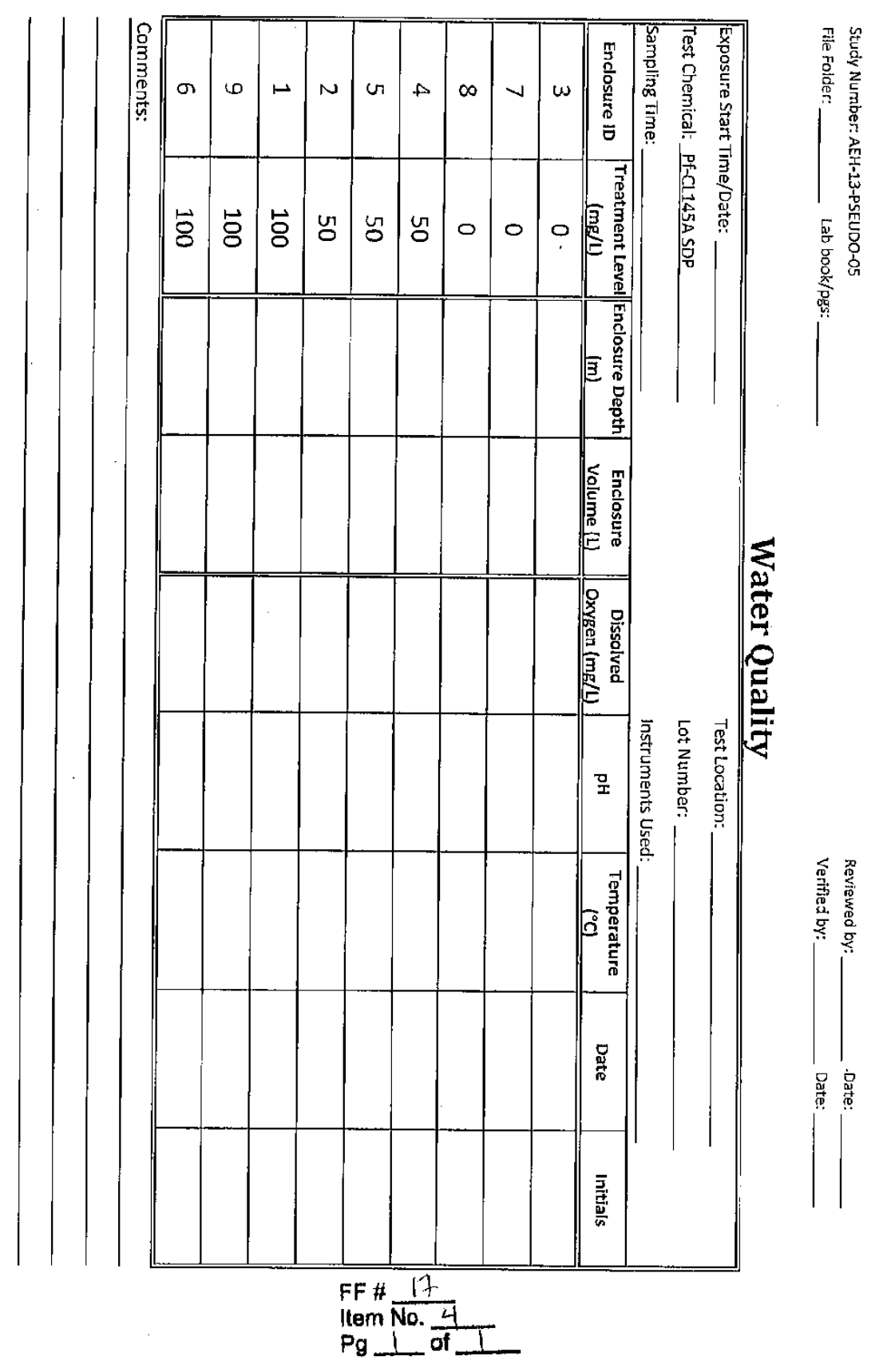




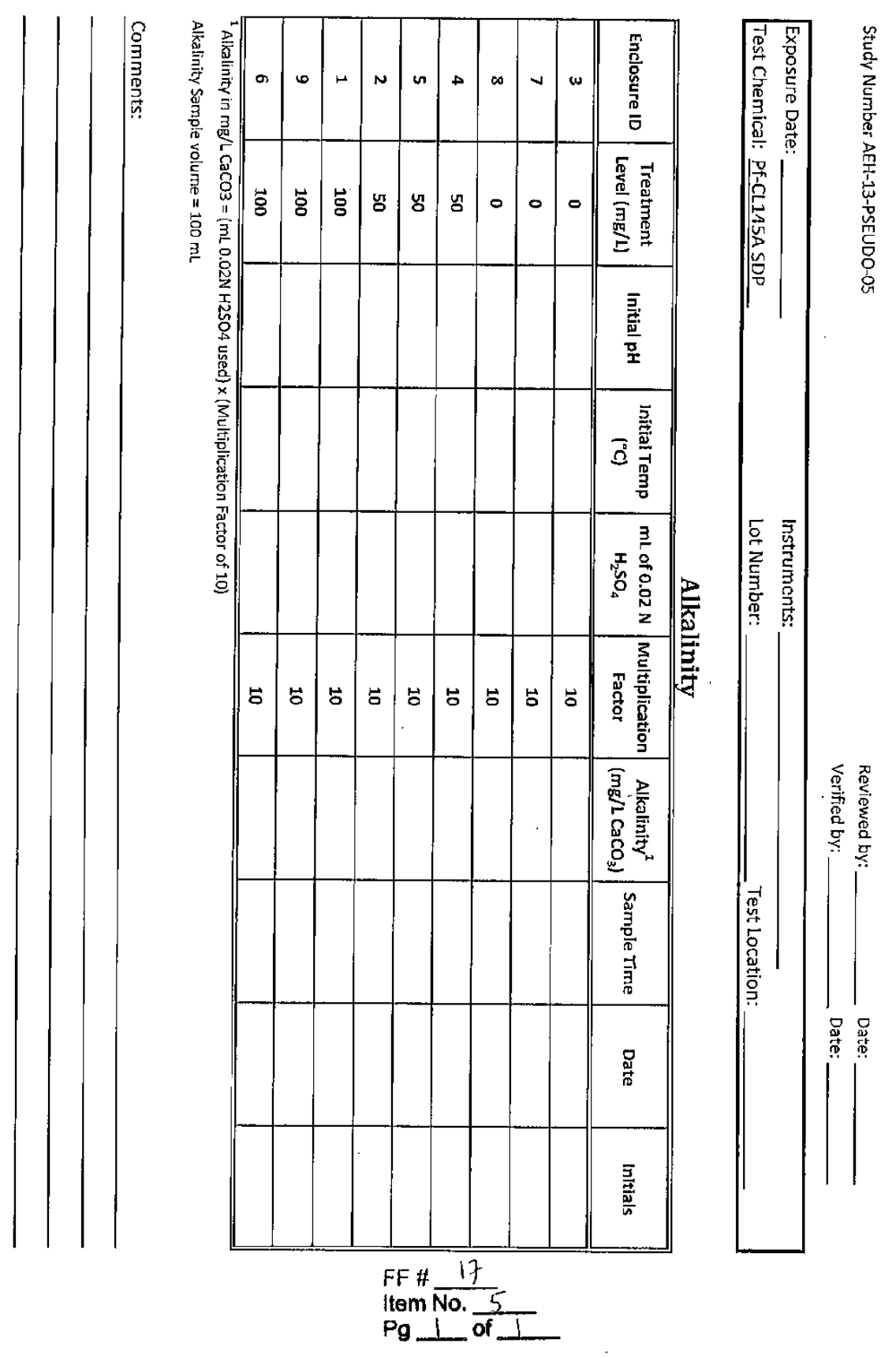




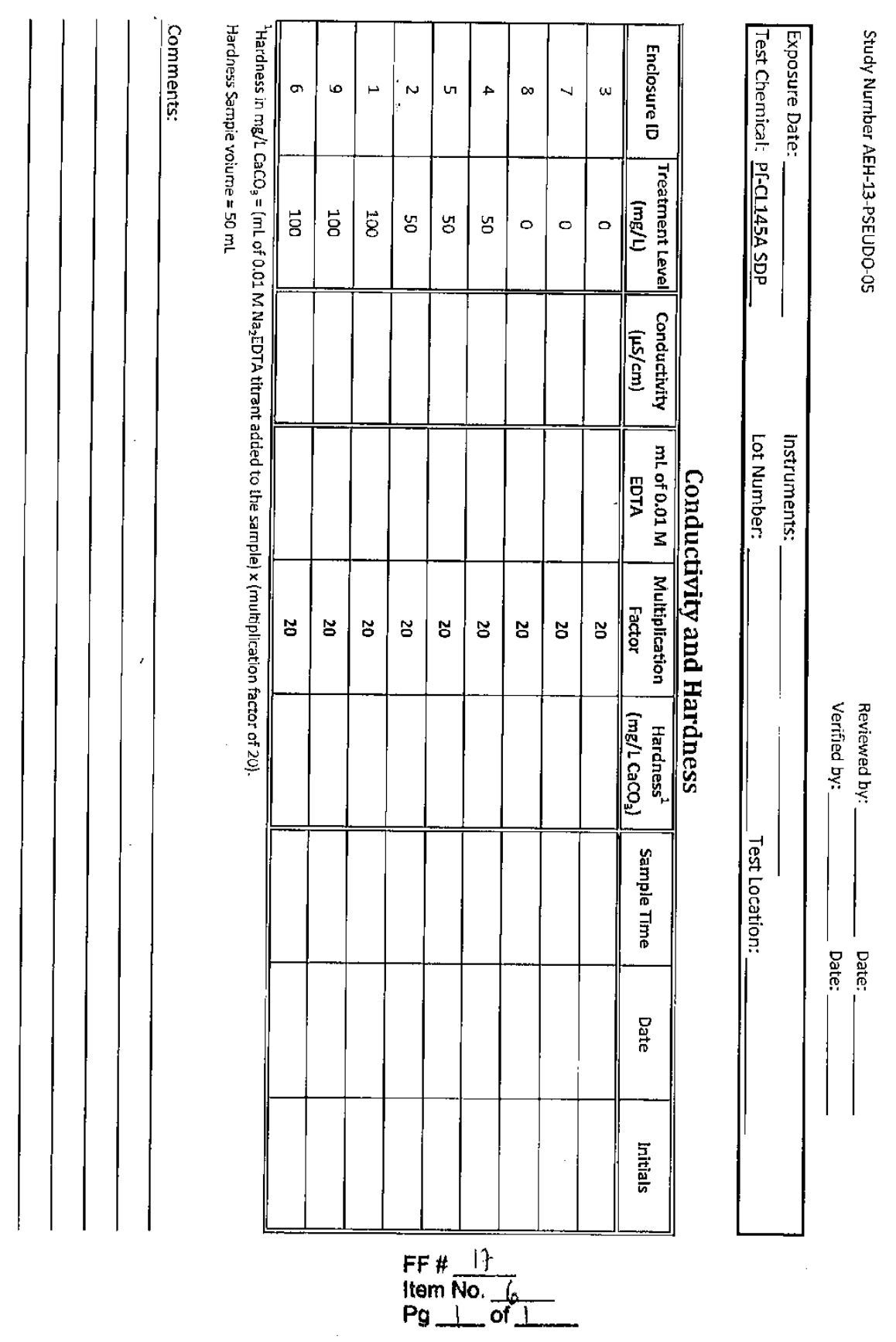




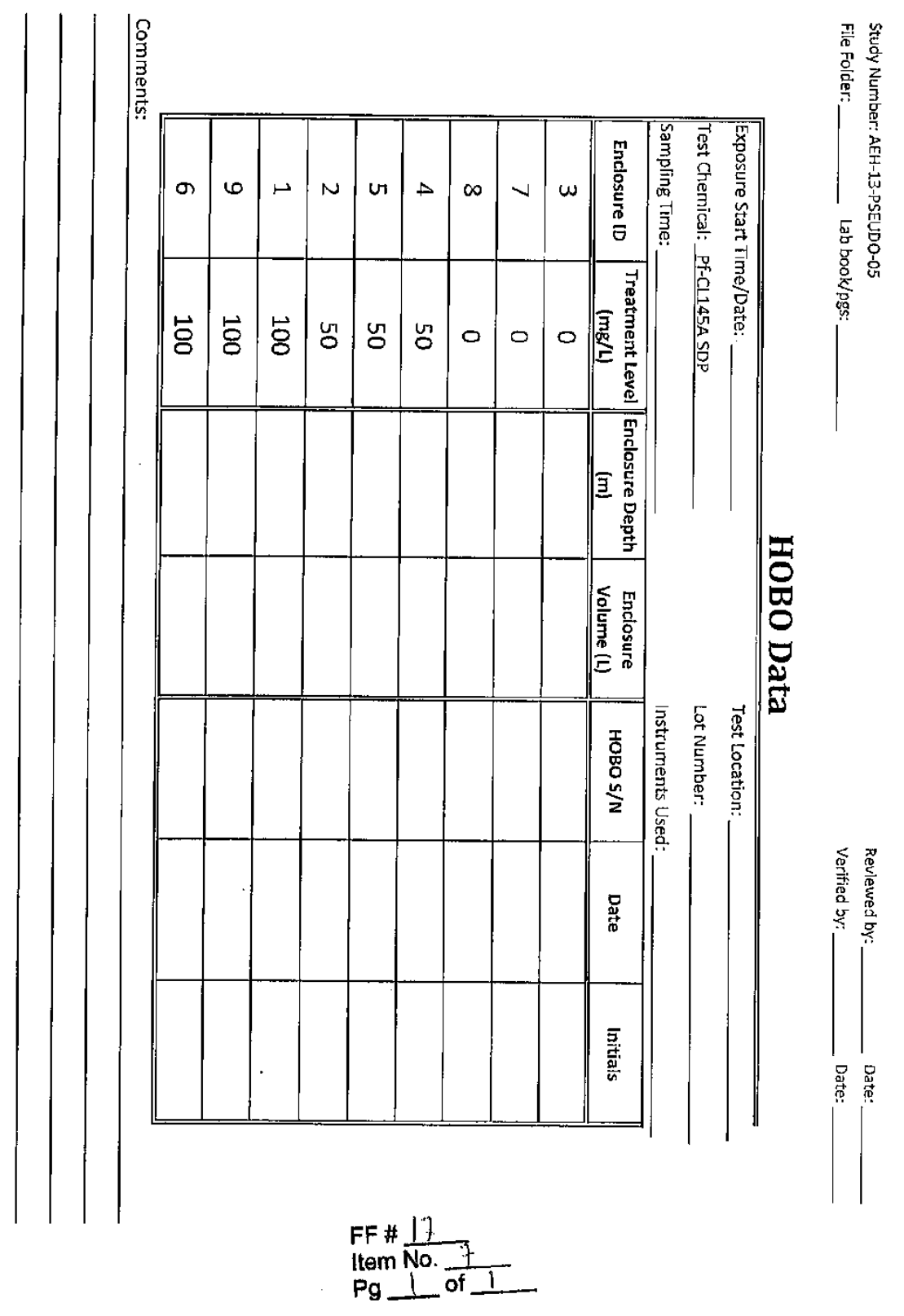


Study Number: AEH-13-PSEUDO-05

File Folcer:

Lab book/pgs:
Fevlewed by

Verifled by:

Date

Date:

\section{Spectrophotometric Data}

\begin{tabular}{||ll|}
\hline Exposure Start Time/Date: & Test Location: \\
Test Chemical: $\mathrm{P}^{\mathrm{E}}$-CL145A SDP & Lot Number: \\
Sampling Time: & instruments Used: \\
Wavelength: & Analyst:
\end{tabular}

Calculation of Regression Coefficient for a standard curve

\begin{tabular}{|c|c|}
\hline Std Conc. (mg/L) & Spec abs \\
\hline 25 & \\
\hline 25 & \\
\hline 25 & \\
\hline 50 & \\
\hline 50 & \\
\hline 50 & \\
\hline 100 & \\
\hline 100 & \\
\hline 100 & \\
\hline 200 & \\
\hline 200 & \\
\hline 200 & \\
\hline
\end{tabular}

Pf-CL145A SDP Treatment Concenteration Verification

\begin{tabular}{||c|c|c|c|c|c||c||}
\hline \hline Sample ID & $\begin{array}{c}\text { Enclosure } \\
\text { Number }\end{array}$ & $\begin{array}{c}\text { Treatment Level } \\
(\mathrm{mg} / \mathrm{L})\end{array}$ & $\begin{array}{c}\text { Sample } \\
\text { Time (h) }\end{array}$ & $\begin{array}{c}\text { Sample } \\
\text { Location }\end{array}$ & $\begin{array}{c}\text { Observed } \\
\text { Spec abs }\end{array}$ & $\begin{array}{c}\text { Date \& } \\
\text { Initials }\end{array}$ \\
\hline & & & & & & \\
\hline & & & & & & \\
\hline & & & & & & \\
\hline & & & & & & \\
\hline & & & & & & \\
\hline
\end{tabular}

FF \# B

Item No. 8

$\mathrm{Pg} \_$of 2 
Study Number: AEH-13-PSEUDO-05

Flle Folder:

Lab hook/ogs:

Revlewed by:

Date:

verified by:

Date:

\section{Spectrophotometric Data}

\begin{tabular}{|ll|}
\hline Exposure Start Time/Date: & Test Location: \\
Test Chemical: Pf-CL145ASDP' & Lot Number: \\
Sampling Time: & Instruments Used: \\
Wavelength: & Analyst: \\
\hline
\end{tabular}

Pf-CL145A SDP Treatment Concenteration Verification

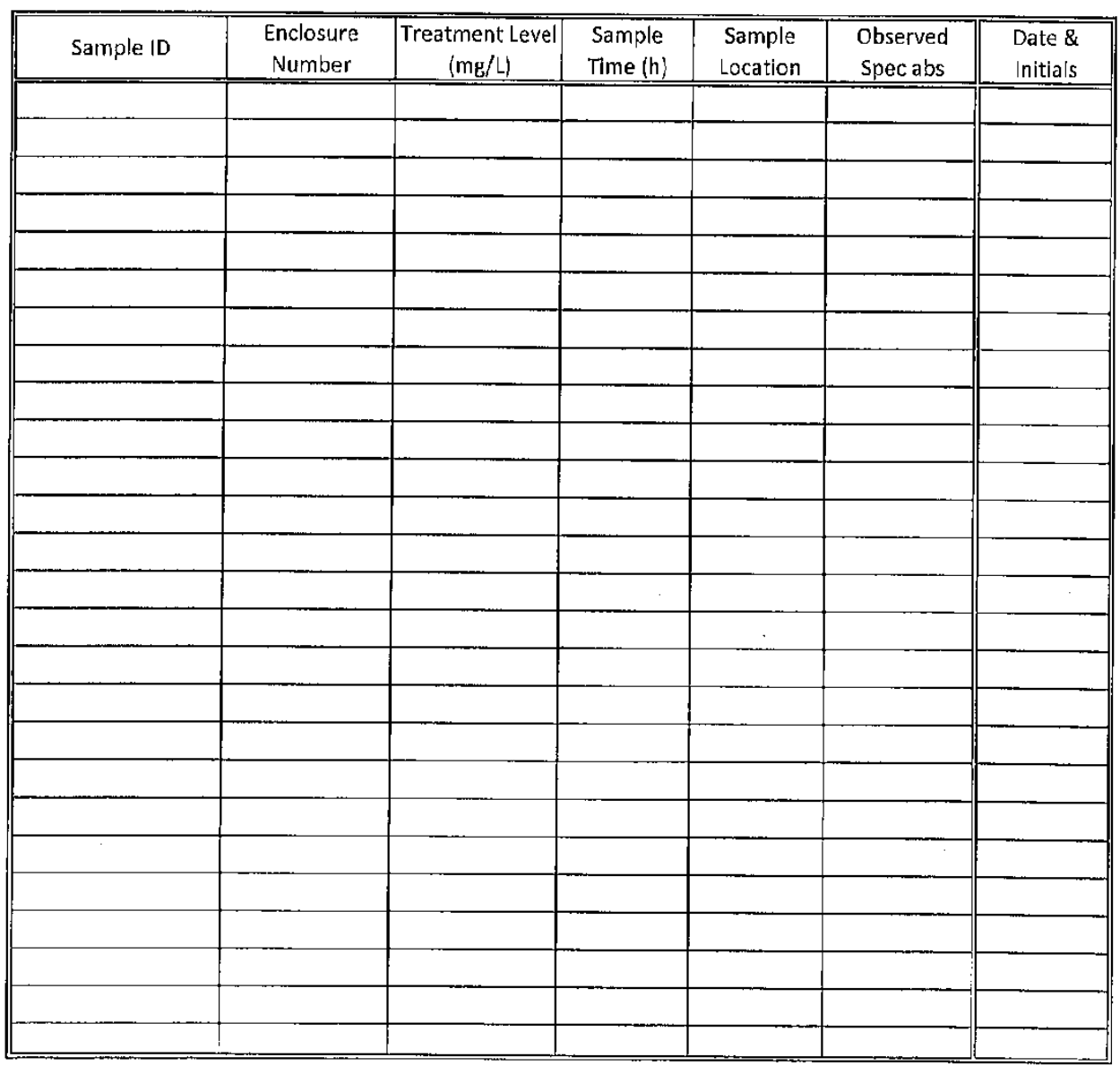

FF \# $\#$ 1.

Item No. 8

$\mathrm{Pg} \partial$ of 


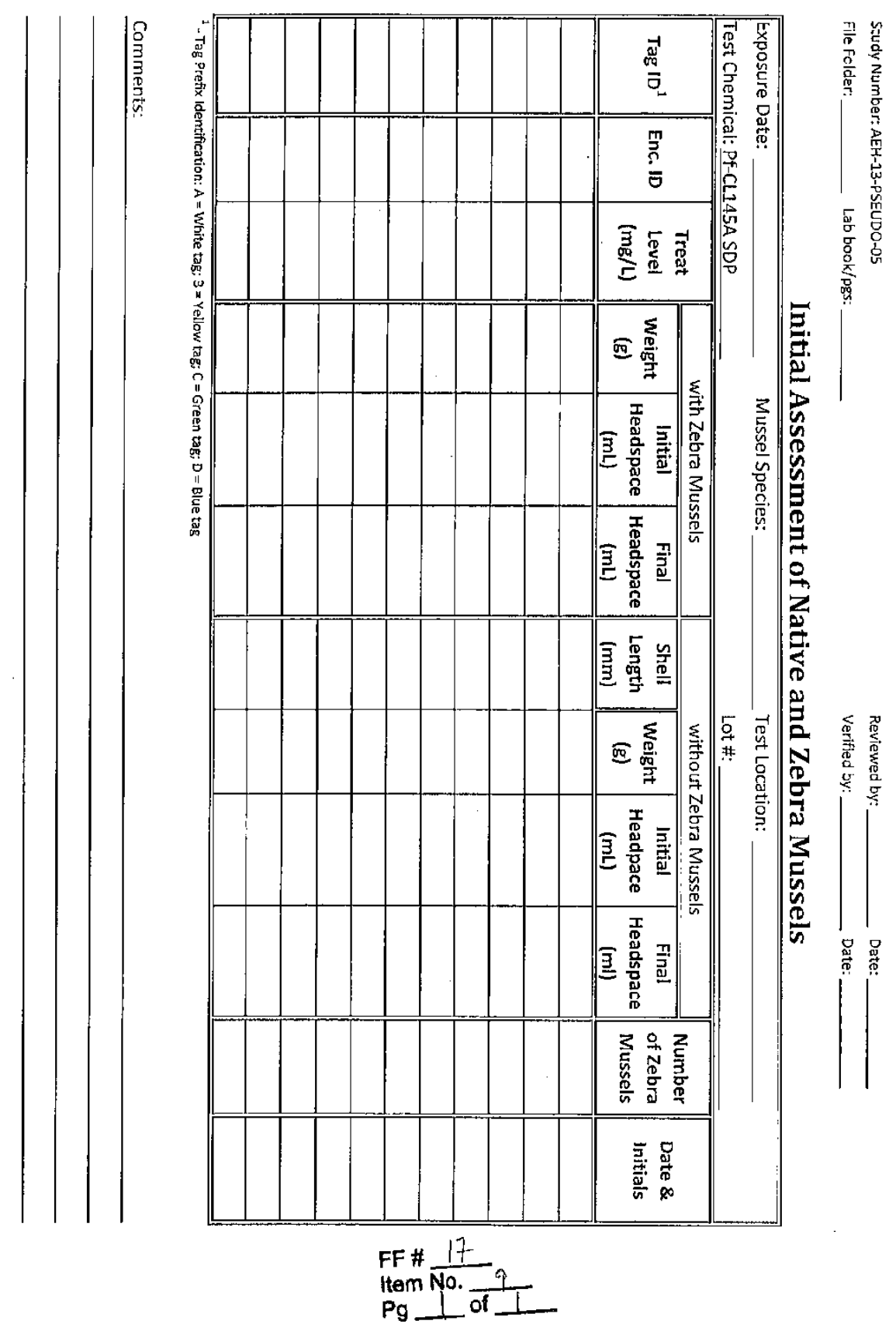


Study Number: AEH-13-PSEUDO-05

File Folder:

Lab book/pg
Revlewed by: _____ Date:

Verified by

Date:

\section{Mussel Initial Assessment}

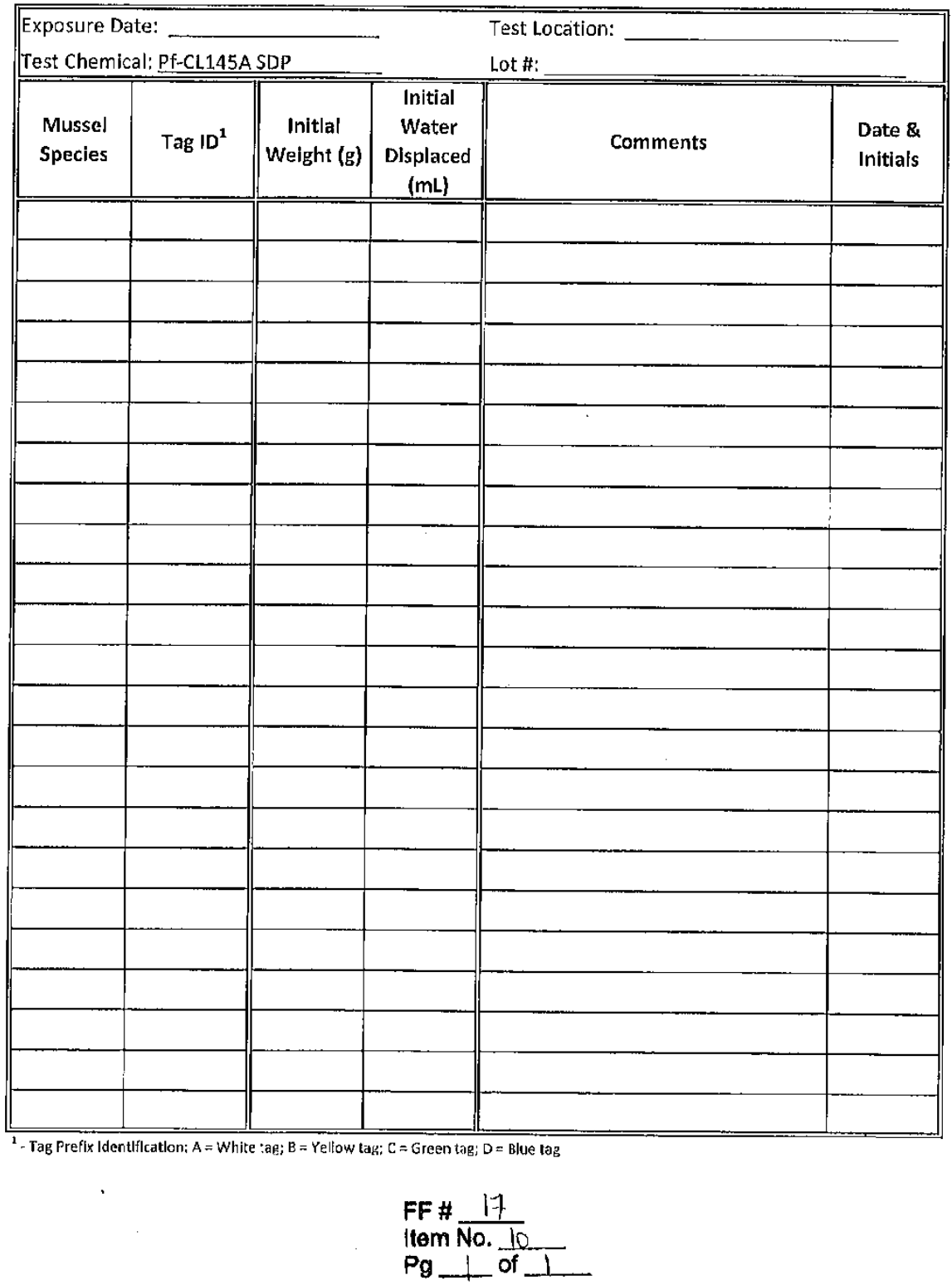




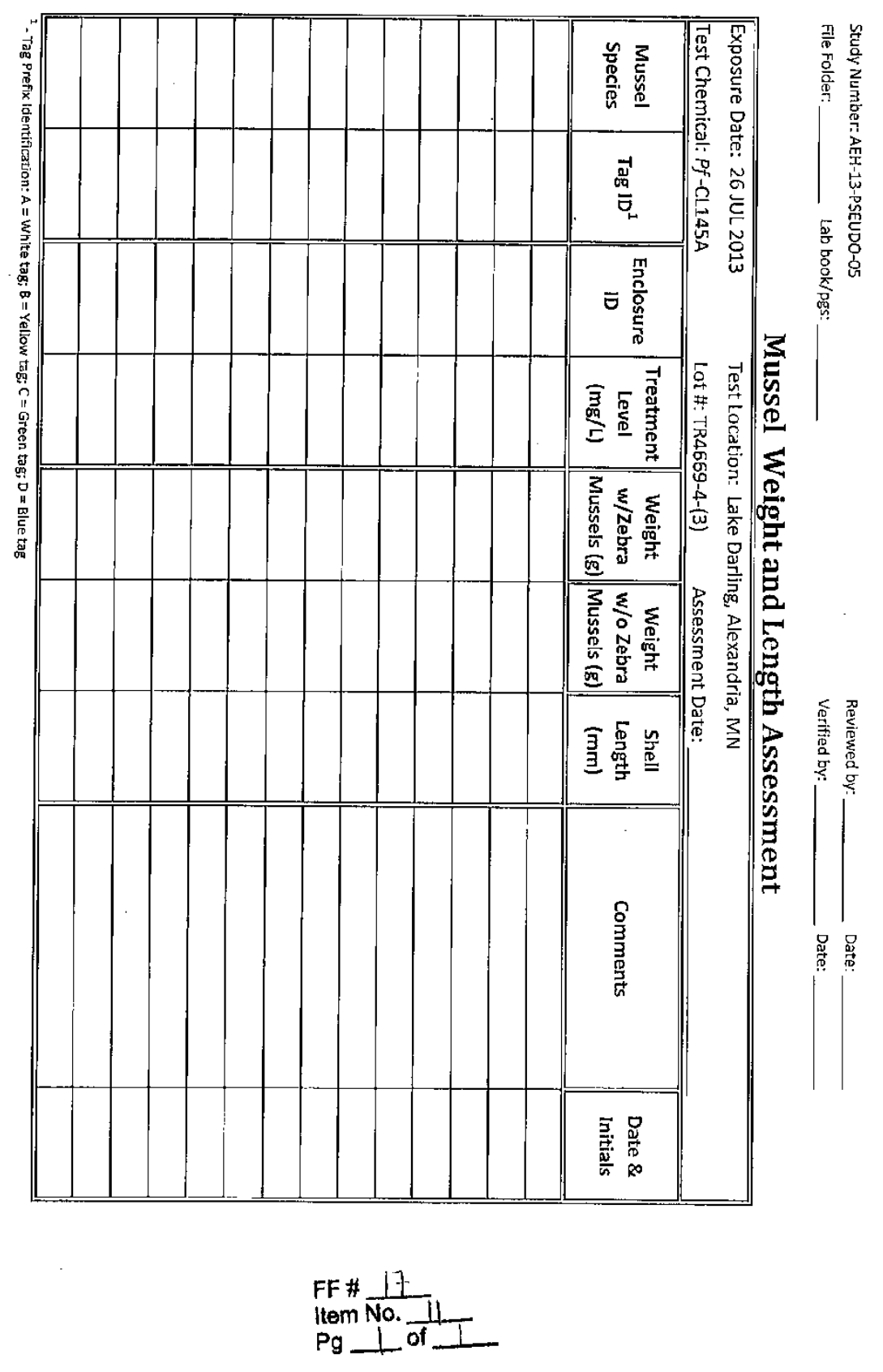




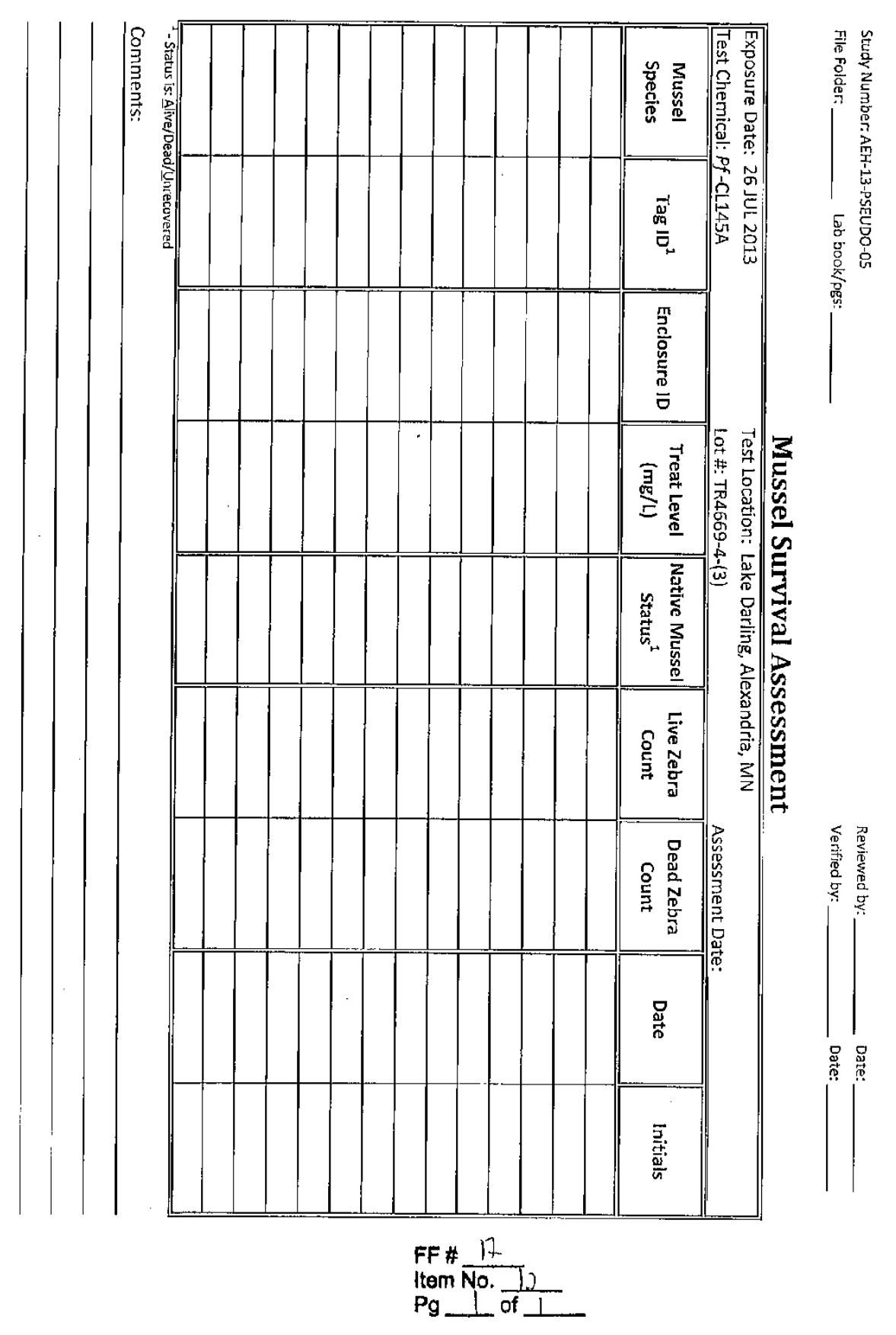


Study Number: AEH-13-PSEUDO-05 Flle Folder:

Lab book/pgs
Reviewed by:

Verffled by:

Date:

Date:

\section{Mussel Final Survival Assessment}

\begin{tabular}{|c|c|c|c|c|c|}
\hline \multicolumn{2}{|c|}{$\begin{array}{l}\text { Exposure Date: } 26 \text { JUL } 2013 \\
\text { Test Chemical: Pf-CL145A } \\
\text { Final Assessmen: Date: Septer }\end{array}$} & \multicolumn{4}{|c|}{$\begin{array}{l}\text { Test Location: Lake Darling, Alexandria, MN } \\
\text { Lot ff: TR4669-4-(3) }\end{array}$} \\
\hline Tag ID & $\begin{array}{c}\text { Native Mussel } \\
\text { Status }^{1}\end{array}$ & $\begin{array}{c}\text { Number of } \\
\text { Translocated } \\
\text { Zebra Mussels }\end{array}$ & Comments & Date & Initials \\
\hline & & & . & & \\
\hline & & & & & \\
\hline & & & & & \\
\hline & & & & & \\
\hline & & & & & \\
\hline & & & & & \\
\hline & & & & & \\
\hline & & & & & \\
\hline & & & & & \\
\hline & & & & & \\
\hline & & & & & \\
\hline & & & & & \\
\hline & & & & & \\
\hline & & & & & \\
\hline & & & & & \\
\hline & & & & & \\
\hline & & & & & \\
\hline & & & & & \\
\hline & & & & & \\
\hline & & & & & \\
\hline & & & & & \\
\hline & & & & & \\
\hline & & & & & \\
\hline & & & & & \\
\hline & & & & & \\
\hline
\end{tabular}

FF \# 17

Item No. 13

$\mathrm{Pg} \_$of $\ldots$ 
Study Number: AEH-13-PSEUDO-05

File Folder:

Lab book/pgs
Revlewed by:

Verlfied by:

Date:

Date:

\section{Zebra Mussel Lengths}

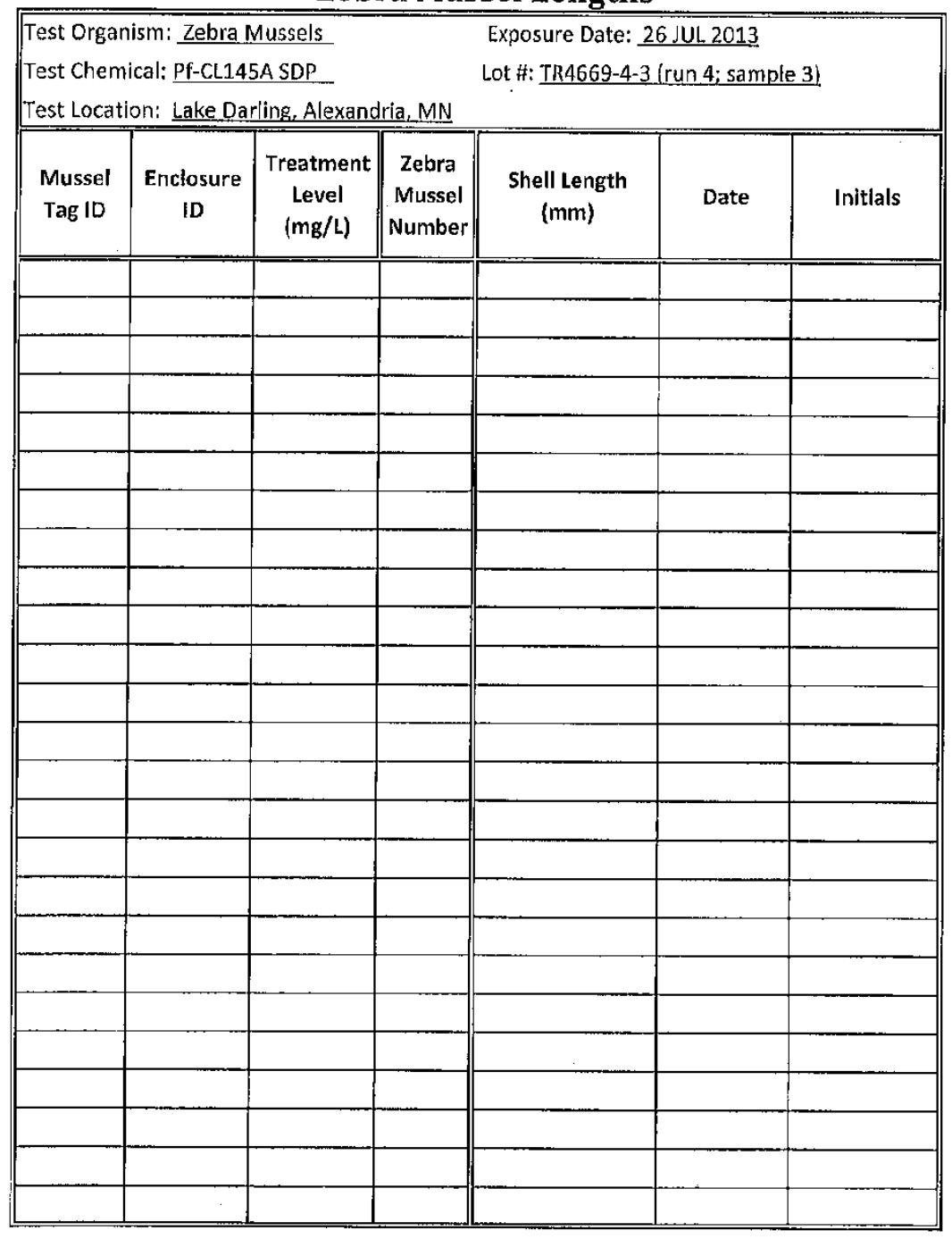

FF \# 17.

Item No. 14

$\mathrm{Pg} \_$of 1 
Study Number: AEH-13-PSEUDO-05

Revlewed by:

Date:

File Folder:

Lab book/pgs:

Verifled by:

Date:

\section{Assessment Zebra Mussel Lengths}

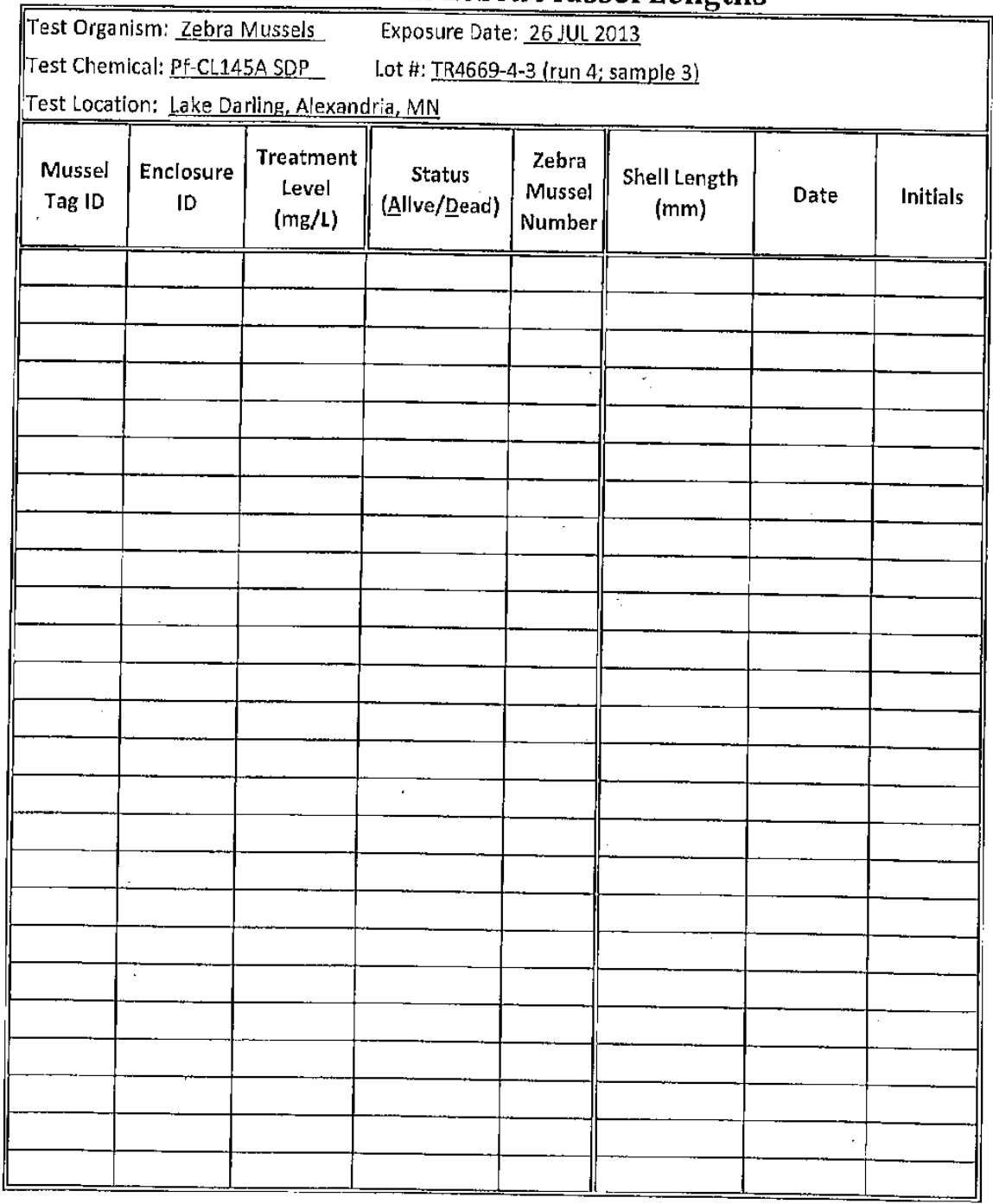

FF \# 17

Item No. 15

$\mathrm{Pg} \ldots$ 


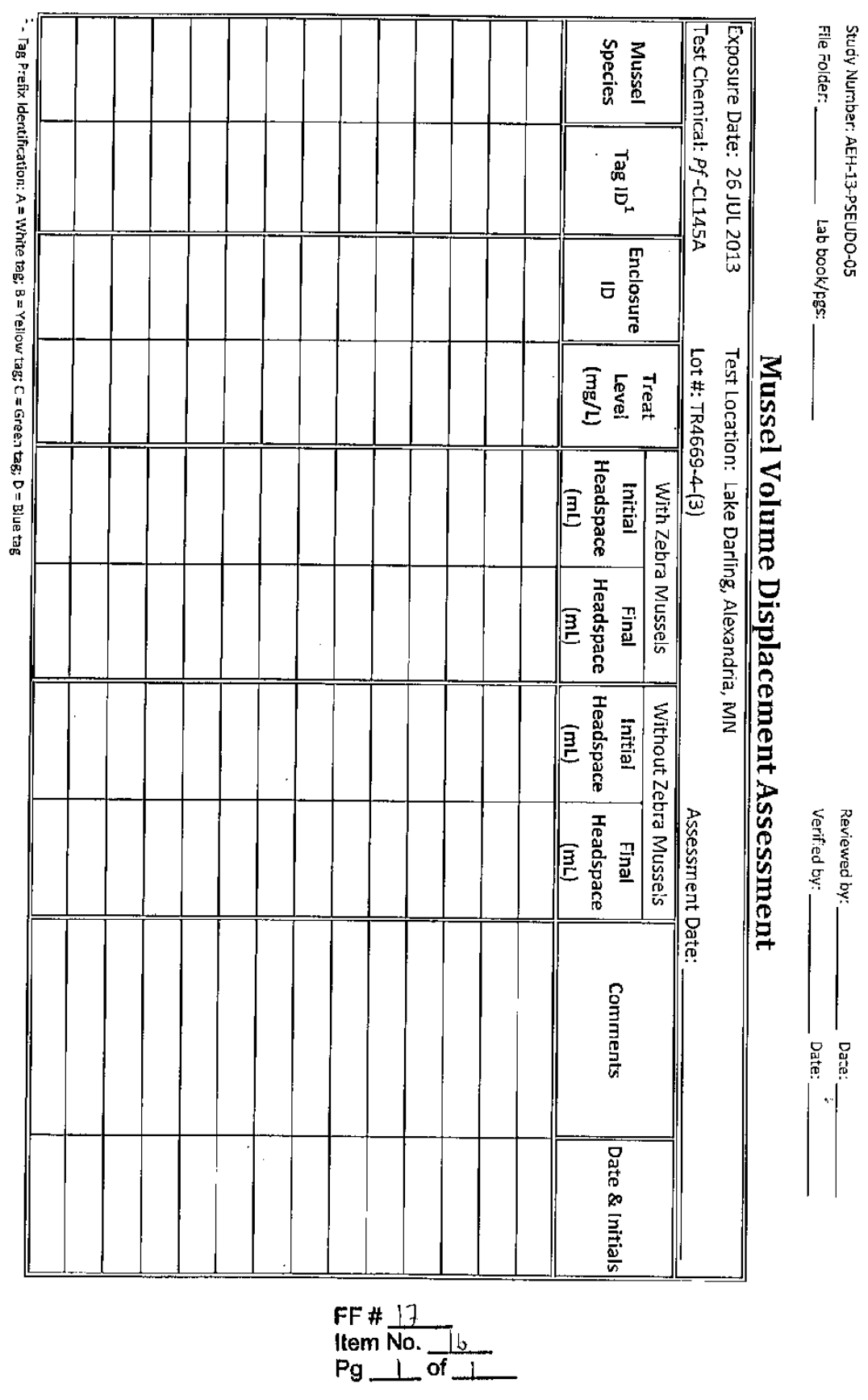




\section{Appendix 2. Deviations From the Study Protocol}

\begin{tabular}{|c|c|c|c|}
\hline $\begin{array}{c}\text { Item } \\
\text { number }\end{array}$ & Item description & $\begin{array}{c}\text { Number } \\
\text { of } \\
\text { pages }\end{array}$ & $\begin{array}{l}\text { Report } \\
\text { page } \\
\text { number }\end{array}$ \\
\hline 1 & Deviation 1: Early termination of holding period & 1 & 76 \\
\hline 2 & Deviation 2: Unrecovered unionid mussels during post-exposure assessment & 1 & 77 \\
\hline 3 & Deviation 3: Mussel \#C331 assessed twice & 1 & 78 \\
\hline 4 & Deviation 4: Discontinuation of volume displacement & 1 & 79 \\
\hline 5 & $\begin{array}{l}\text { Deviation 5: Unequal ratios of Fragile Papershell (Leptodea fragilis) mussels in the } \\
\text { study }\end{array}$ & 2 & 80 \\
\hline 6 & Filamentous algae report from John Manier & 5 & 82 \\
\hline
\end{tabular}




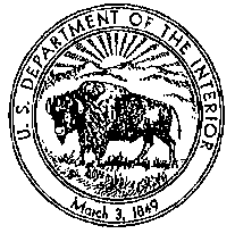

United States Department of the Interior

U.S. GEOLOGICAL SURVEY

Biological Resources Division

Upper Midwest Environmental Sciences Center

2630 Fanta Reed Road

La Crosse, Wisconsin 54603

\section{MEMORANDUM}

Date: February 18, 2014

To: The Record Study Number AEH-13-PSEUDO-05

Subject: Deviation 1 to study AEH-13-PSEUDO-05

Deviation \#1 - Early termination of holding period

Sections 3.3, 4.1, and 4.2 of study protocol (study number AEH-13-PSEUDO-05; study title "Efficacy of Pseudomonas fluorescens (Pf-CL145A) SDP for controlling zebra mussels within field enclosures") state that $\sim 30 \mathrm{~d}$ after exposure, the native mussels will be assessed for survival and zebra mussel colonization.

Twelve days post-exposure (August 7, 2013), the status of the mussel retention barriers was assessed due to concerns with filamentous algae within Lake Darling. Heavy infestations of filamentous algae were observed and subsequently removed from each retention barrier. Due to potential for suffocation and poor water quality related to the filamentous algae bloom, the study was terminated early at $\sim 18$ days post-exposure on Auguist $13-15,2013$.

Samples of the algae were collected between August 13 and 15, 2013 and were characterized as being dominated by Mougeotia and Spirogyra. Other genera were present, but to a lesser extent, including Oedogonium, Zygnema, and Lyngbya. A copy of the report can be found in the study records (FF 4: Correspondence).

The effects of the filamentous algae bloom are indeterminate, however, zebra mussel survival in the controls was excellent $>91.4 \%$ ) indicating that observed zebra mussel mortality was likely treatment related. The potential impacts to the study will be evaluated and any impacts to the study will be noted in the final report.

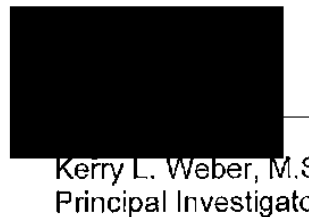

UMESC

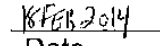

Date

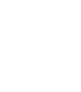

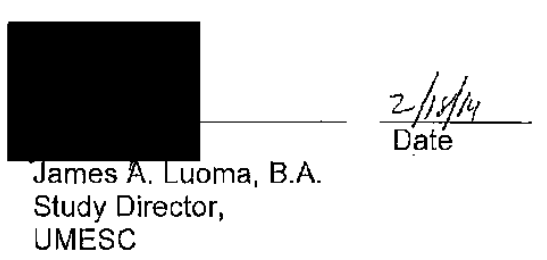

File Folder: 3

Page 1 of 1

Item Number: 3 


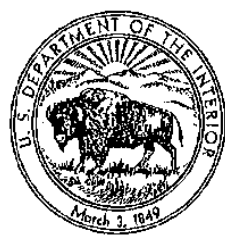

United States Department of the Interior

U.S. GEOLOGICAL SURVEY

Biological Resources Division

Upper Midwest Environmental Sciences Center

2630 Fanta Reed Road

La Crosse, Wisconsin 54603

\section{MEMORANDUM}

Date: February 18, 2014

To: The Record Study Number AEH-13-PSEUDO-05

Subject: Deviation 2 to study AEH-13-PSEUDO-05

Deviation \#2 - Unrecovered unionid mussels during post-exposure assessment

During the post-exposure assessment, unionid mussels from each treatment replicate (i.e. enclosure) were assessed for survival and zebra mussel coionization. Two unionid mussels (C356 from enclosure 6 [100 $\mathrm{mg} / \mathrm{L}]$; C323 from enclosure $9[100 \mathrm{mg} / \mathrm{L}]$ ) were not recovered.

Mussels C356 and C323 may have been inadvertently removed from the enclosures during algae removal on August 7, 2013 (see study deviation \#1). The unionid mussels will be coded as mortalities within the survival analysis. The initial zebra mussel colonization data may be removed from analysis. The exact details of how these data are treated will be noted in the data summaries.

No adverse impacts to the study are anticipated as a result of this deviation. Any impacts to the study will be noted in the final report.

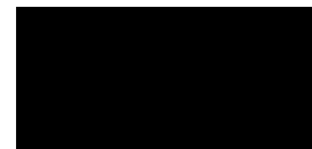

KerryL. Weber, M.S. Principal Investigator. UMESC

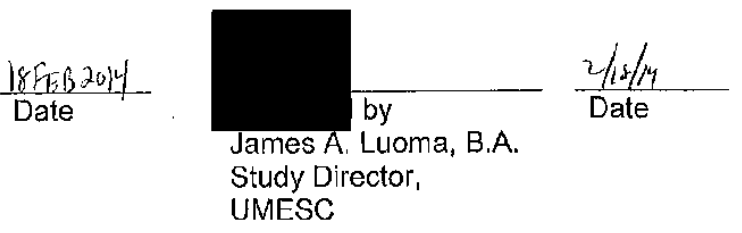

File Folder: $\quad 3$

Page 1 of 1 


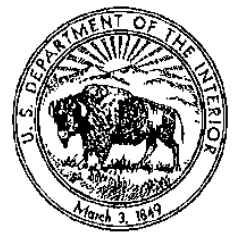

United States Department of the Interior

U.S. GEOLOGICAL SURVEY

Biological Resources Division

Upper Midwest Environmental Sciences Center

2630 Fanta Reed Road

La Crosse, Wisconsin 54603

\section{MEMORANDUM}

Date: February 18, 2014

To: The Record Study Number AEH-13-PSEUDO-05

Subject: Deviation 3 to study AEH-13-PSEUDO-05

Deviation \#3-Mussel \#C331 assessed twice

During the post-exposure unionid mussel assessment, native mussel C331 (Enclosure 2 [50 mg $/ L$ ]) was assessed for survival and zebra mussel colonization following the procedure outlined in the laboratory notebook on August 13, 2013. Mussel C331 was inadvertently left in a retention barrier used to temporarily hold the mussels prior to assessment and mussels from Enclosure $9(100 \mathrm{mg} / \mathrm{L})$ were placed in the same retention barrier as mussel C331. As a result, mussel C331was assessed for a second time with the mussels from Enclosure 9. The error was discovered during data analysis and data collected for mussel C331 during the Enclosure 9 assessment will be removed from analysis.

This error also clarifies the logbook entry made on August 15,2013, which indicated that a single mussel from the retention barrier containing the native mussels assessed on August 13,2013 was not recovered before being placed into the new holding area.

There are no adverse impacts to the study as the necessary data was collected for mussel C331.

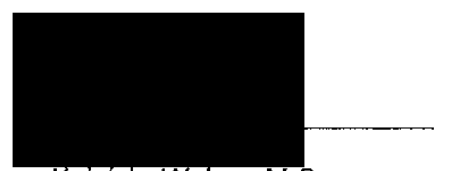

Keryy L. Weber, M.S.

Principal Investigator, UMESC

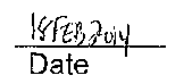$$
\frac{14 \sqrt{2} 2}{\text { Date }}
$$

$$
\text { (2) }
$$

Page 1 of 1
Flle Folder: $\quad 3$<smiles>[AlH2][18N]C[SiH3]</smiles> 


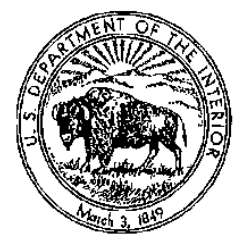

\author{
United States Department of the Interior \\ U.S. GEOLOGICAL SURVEY \\ Biological Resources Division \\ Upper Midwest Environmental Sciences Center \\ 2630 Fanta Reed Road \\ La Crosse, Wisconsin $\mathbf{5 4 6 0 3}$
}

\title{
MEMORANDUM
}

Date: February 18, 2014

To: $\quad$ The Record Study Number AEH-13-PSEUDO-05

Subject: Deviation 4 to study AEH-13-PSEUDO-05

Deviation \#4 - Discontinuation of volume displacement

Section 4.2 of the study protocol (study number AEH-13-PSEUDO-05) states that individual mussels will be tagged, photographed on each side, weighed and their water displacement measured to assist with individual tracking and estimating pre- and posttreatment zebra mussel colonization.

The apparatus constructed and initially used to measure the water displacement of the unionids leaked and was found to be inconsistent. Therefore, during the post-exposure assessment, water displacement measurements were discontinued. The data will be retained but will not be used during analysis.

There are no adverse impacts to the study anticipated from the discontinuation of water displacement as the wet weights will be used to estimate the pre-exposure zebra mussel colonization. Any impacts to the study will be noted in the final report.

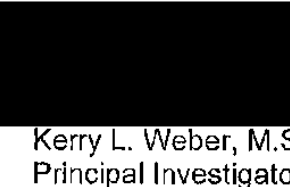

Principal Investigator UMESC

$$
\frac{\mid \gamma F \text { F } 20 y \mid}{\text { Date }}
$$

d by

James A. Luoma, B.A.

Study Director,

UMESC

File Folder:

Item Number:

Page 1 of 1 


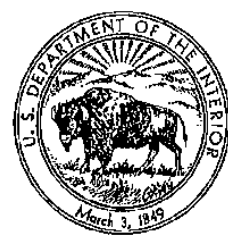

United States Department of the Interior

U.S. GEOLOGICAL SURVEY Biological Resources Division

Upper Mldwest Envlronmental Sclences Center

2630 Fanta Reed Road

La Crosse, Wisconsin 54603

\section{MEMORANDUM}

Date: February 18, 2014

To: The Record Study Number AEH-13-PSEUDO-05

Subject: Deviation 5 to study AEH-13-PSEUDO-05

Deviation \#5 - Unequal ratios of Fragile Papershell (Leptodea fragilis) mussels in the study

Sections 4.2 and 5.1.1.3 of the protocol states that "if multiple species are used, native species will be equally distributed in equal ratios to the treatment enclosures". A total of 243 unionid mussels were included in the study. During collection and tagging of test animals for inclusion in the study, fatmucket (Lampsilis siliquoidea) was the dominate species, however, positive identification to species was difficult due to heavy zebra mussel colonization. Therefore, the mussels were equally distributed to enclosures regardless of species.

At the conclusion of the study, the unionid mussel shells (with their associated identification tag) were retained for species confirmation. Species confirmation was completed based on the structure of the pseudocardinal teeth, umbo, and shell thickness. Fatmucket (FAM) mussels were the dominate species while fragile papershell (FPS) mussels were rare (i.e., 231 FAM versus 12 FPS). Enclosure, treatment level and tag numbers of fragile papershell mussels test animals are summarized in Table 1. Data collected for fragile papershell will be used for analysis.

File Folder: 3

Item Number: 7

Page 1 of 2 
Table 1. Fragile papershell mussels present in the study

\begin{tabular}{ccc}
\hline Enc. & $\begin{array}{c}\text { Treatment } \\
\text { Level }(\mathrm{mg} / \mathrm{L})\end{array}$ & $\begin{array}{c}\text { Musse } \\
\text { Tag }\end{array}$ \\
\hline 1 & 100 & $\begin{array}{c}\text { C363 } \\
\text { C207 }\end{array}$ \\
2 & 50 & C342 \\
& & C331 \\
& & C398 \\
4 & 50 & C335 \\
5 & 50 & C362 \\
6 & 100 & C336 \\
& & C307 \\
& & C394 \\
& & C344 \\
7 & Control & \\
9 & 100 & C386 \\
\hline
\end{tabular}

There are no adverse impacts to the study anticipated as the primary study objective was to determine the efficacy of formulated Pf-CL145a for removal of zebra mussels adhering to unionid mussels. Any impacts to the study will be noted in the final report.
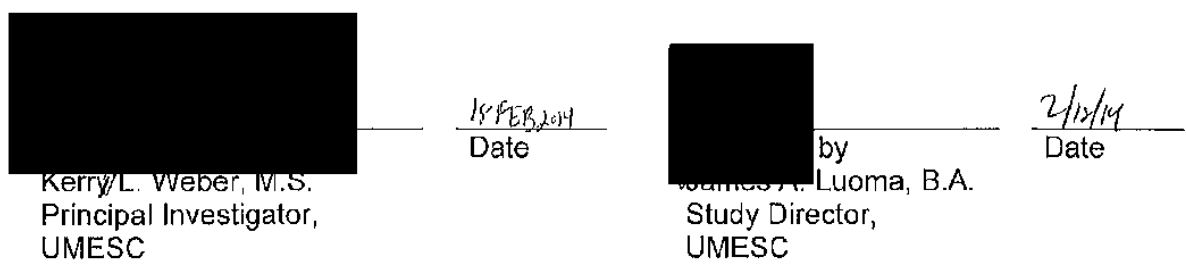

File Folder: 3

Item Number: 1

Page 2 of 2 
Lab Technician: John Manier

Sample ID: AEH-13-PSUEDO-05

Sample Date: August $13^{\text {th }}$ and $15^{\text {th }}, 2013$

AEH-13-PSEUDO-05

Location: Lake Darling

Preservation: Two samples $(1000 \mathrm{~mL})$ were preserved with Lugol's Solution; two samples (1000 $\mathrm{mL}$ ) were un-preserved but refrigerated.

Method: A wet mount was created using a sub-sample (approx. $1 \mathrm{~mL}$ ). The sub-sample was then analyzed using a Nikon Eclipse E600 microscope with attached camera (Q Imaging, Micropublisher 3.3 RTV). The microscope was also equipped with 10,20 , and $40 \mathrm{X}$ magnifications, as well as phase contrast.

Results: This is a typical periphyton community, consisting of filamentous green algae, attached diatoms, and protozoa. The algal community was dominated by Mougeotia and Spirogyra (Figures 1-2). Other genera were present, but to a lesser extent, including Oedogonium , Zygnema, and Lyngbya (Figures 3-6). The diatoms commonly seen were Gomphonema and Cymbella (Figures 7-8).

FF \# 4

Item No. 4

$\mathrm{Pg} \perp$ of 5 


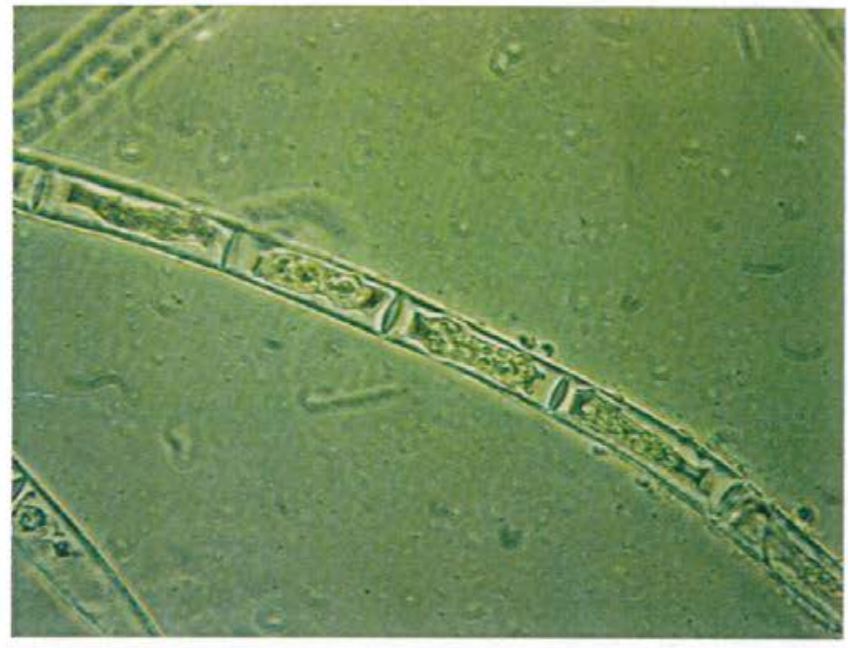

AEH-13-PSEUDO-05

Figure 1. Mougeotia-Contains a plate-like chloroplast, which rotates within the cell to gain better access to light. Widely known to create large, nuisance growths, especially in waters affected by acidification.

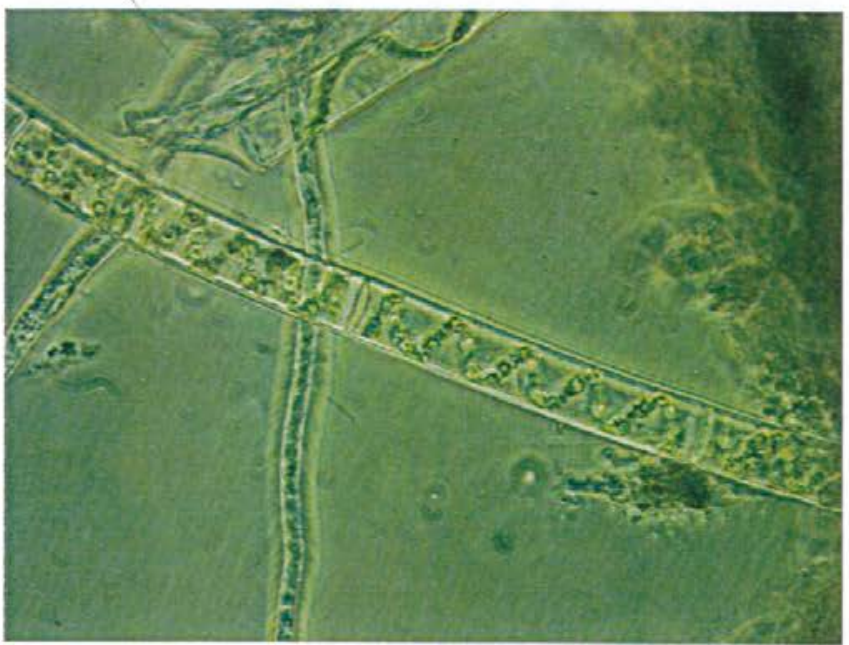

Figure 2. Spirogyra-Identified by the spiral-shaped chloroplast. They are very common within periphytic mats.

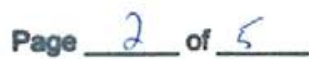




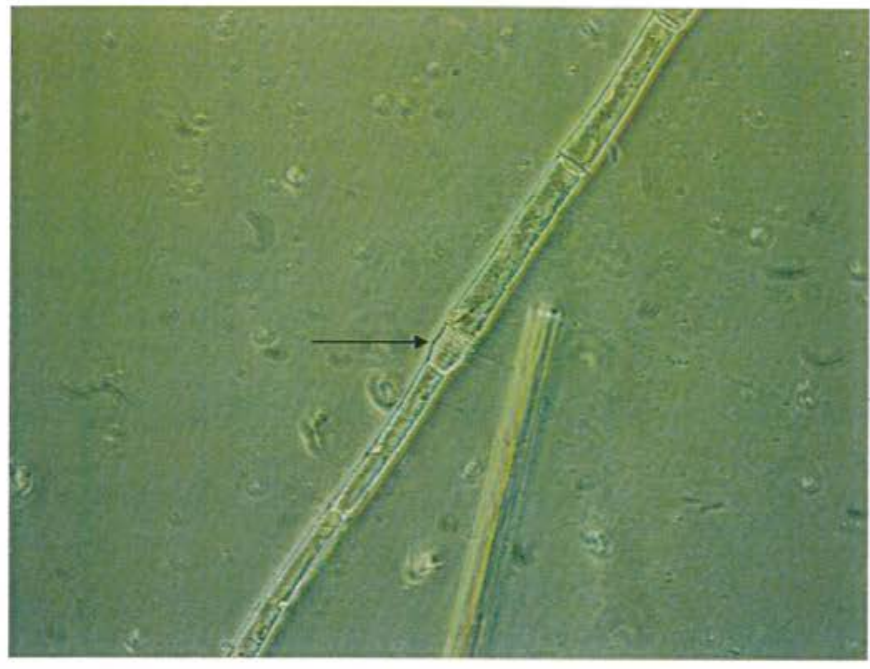

AEH-13-PSEUDO-05

Figure 3. Oedogonium- Distinguished by the annular "rings", which denote the number of times it has reproduced.

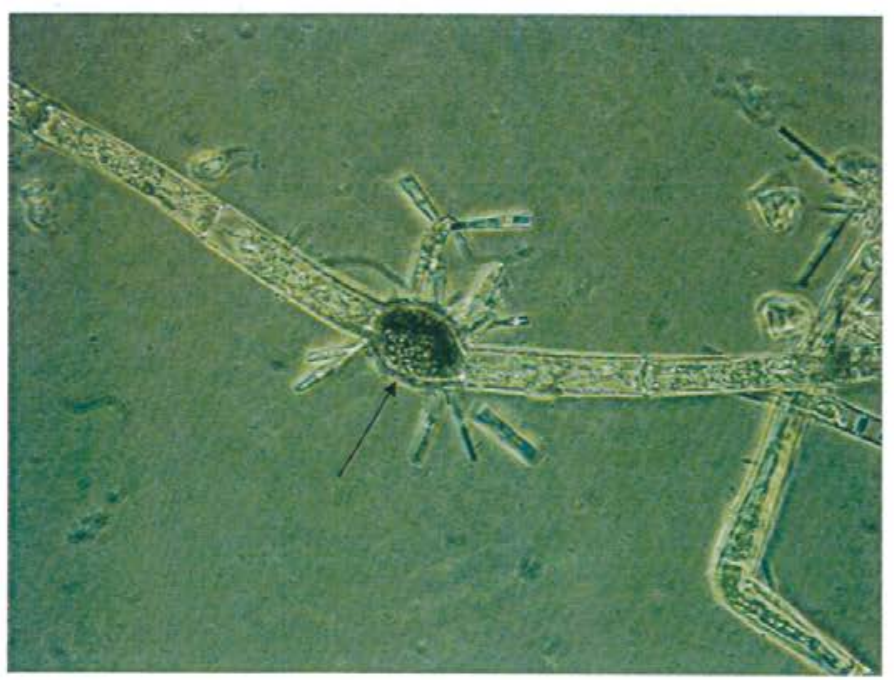

Figure 4. Oedogonium filament with attached zygote, formed during reproduction. The zygote is covered with stalked diatoms.

Page 3 of 5 


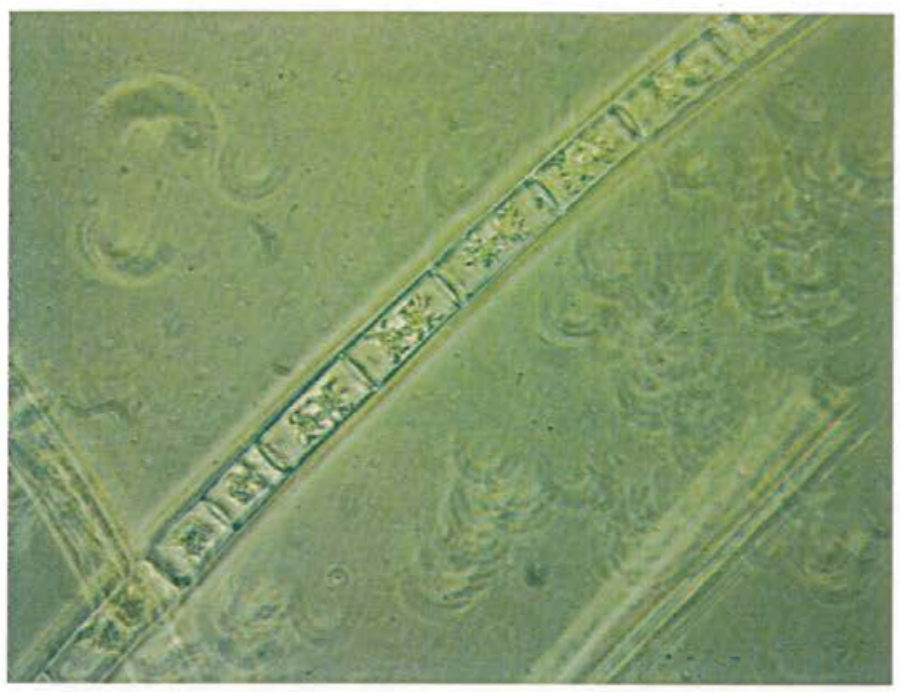

Figure 5. Zygnema- Distinguished by the axial, stellate, and paired chloroplasts.

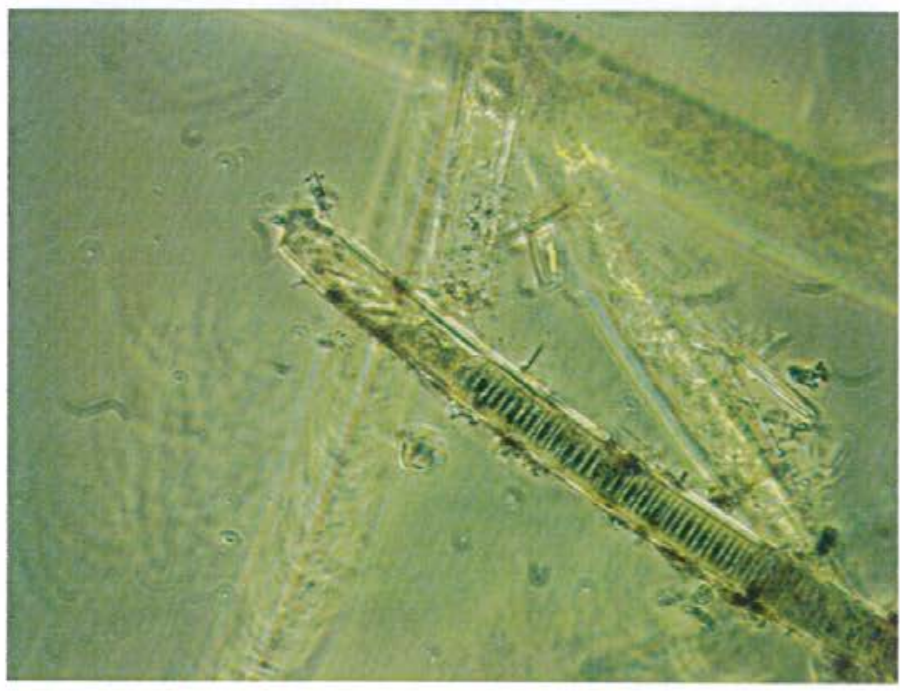

Figure 6. Lyngbya-Members of this genus have long, unbranching filaments with a mucilage sheath. They are widely known to create large, benthic mats.

Page 4 of 5 


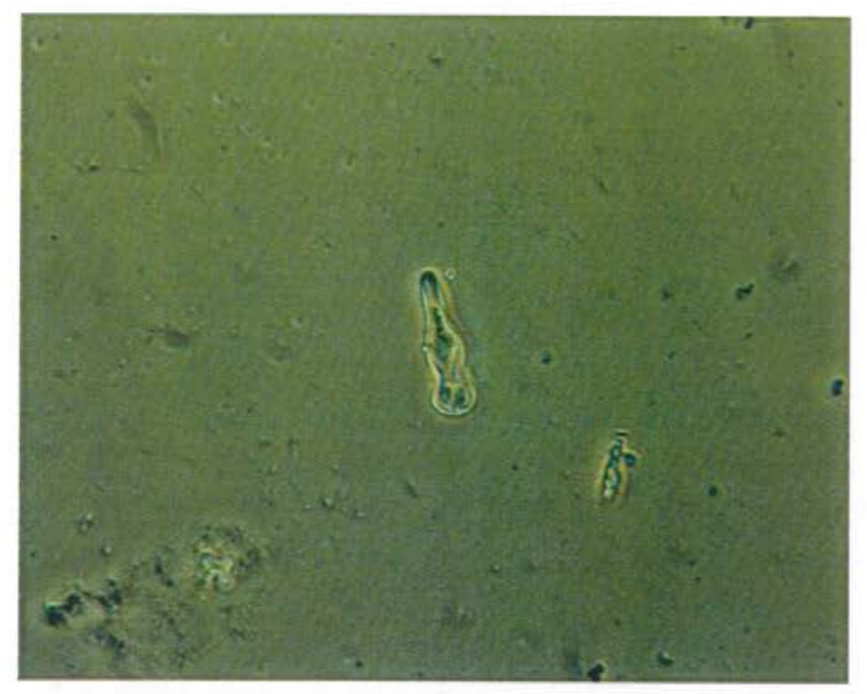

AEH-13-PSEUDO-05

Figure 7. Gomphonema- All species are symmetrical to the apical axis and asymmetrical to the transapical axis. Many attach themselves to the periphyton using a stalk.

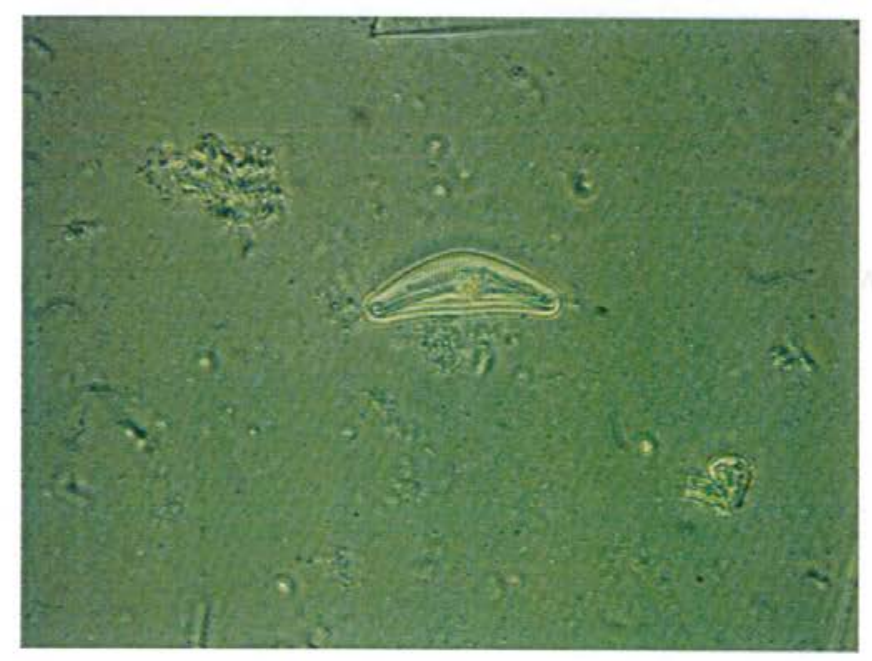

Figure 8. Cymbella-Members of this genus usually grow in benthic habitats via an attached stalk.

FF \# 4

Item No. 4

$\mathrm{Pg} 5$ of 5 


\section{Appendix 3. Randomization Assignments}

\begin{tabular}{clcc}
\hline $\begin{array}{c}\text { Item } \\
\text { number }\end{array}$ & \multicolumn{1}{c}{ Item description } & $\begin{array}{c}\text { Number } \\
\text { of } \\
\text { pages }\end{array}$ & $\begin{array}{c}\text { Report } \\
\text { page } \\
\text { number }\end{array}$ \\
\hline 1 & SAS output for random assignment of treatment to experimental enclosures & 1 & 88 \\
2 & SAS program for random assignment of treatment to experimental enclosures & 2 & 89 \\
3 & SAS log for random assignment of treatment to experimental enclosures & 2 & 91 \\
4 & SAS output for random assignment of mussels to enclosures & 2 & 93 \\
5 & SAS program for random assignment of mussels to enclosures & 1 & 95 \\
6 & SAS log for random assignment of mussels to enclosures & 2 & 96 \\
\hline
\end{tabular}




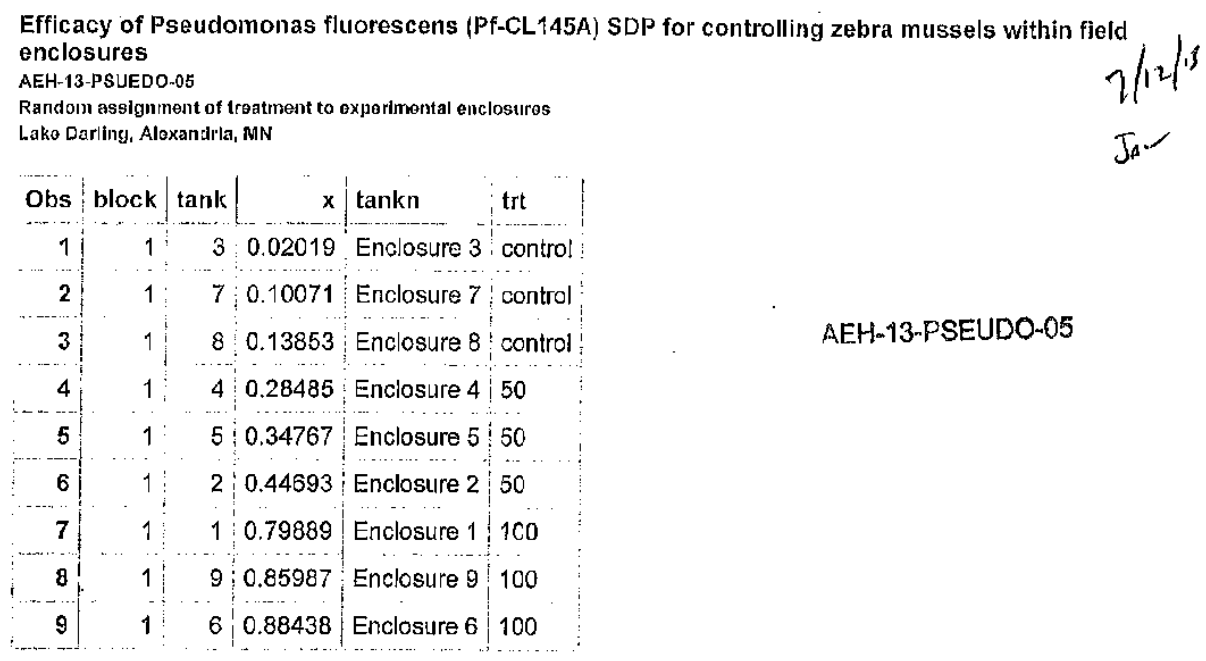

Analysis performed by J. Lucma SAS version 9.3 12:28 12JUL.13

$F F \# 9$

Item No.

$\mathrm{Pg} \perp$ of 1 


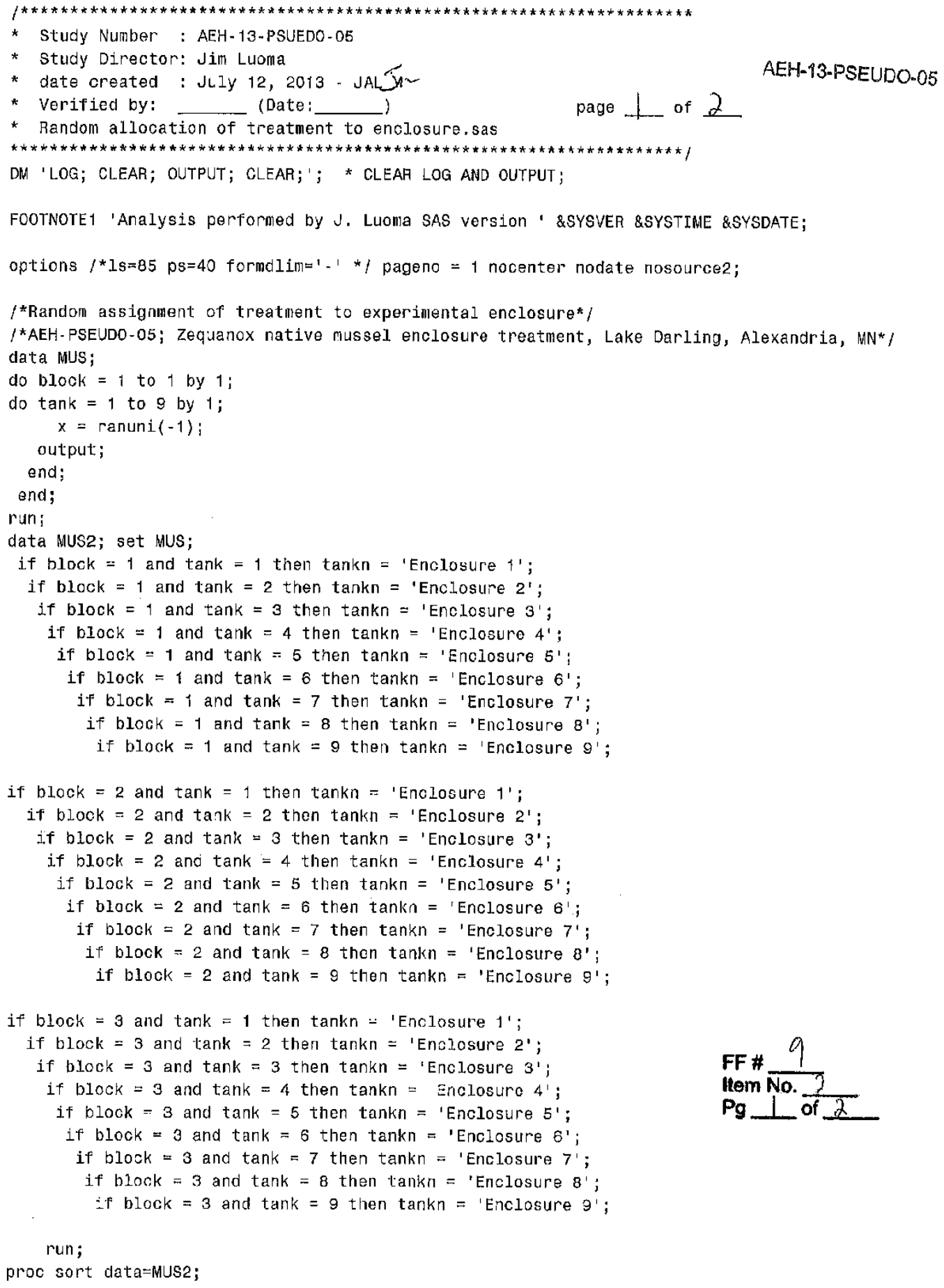




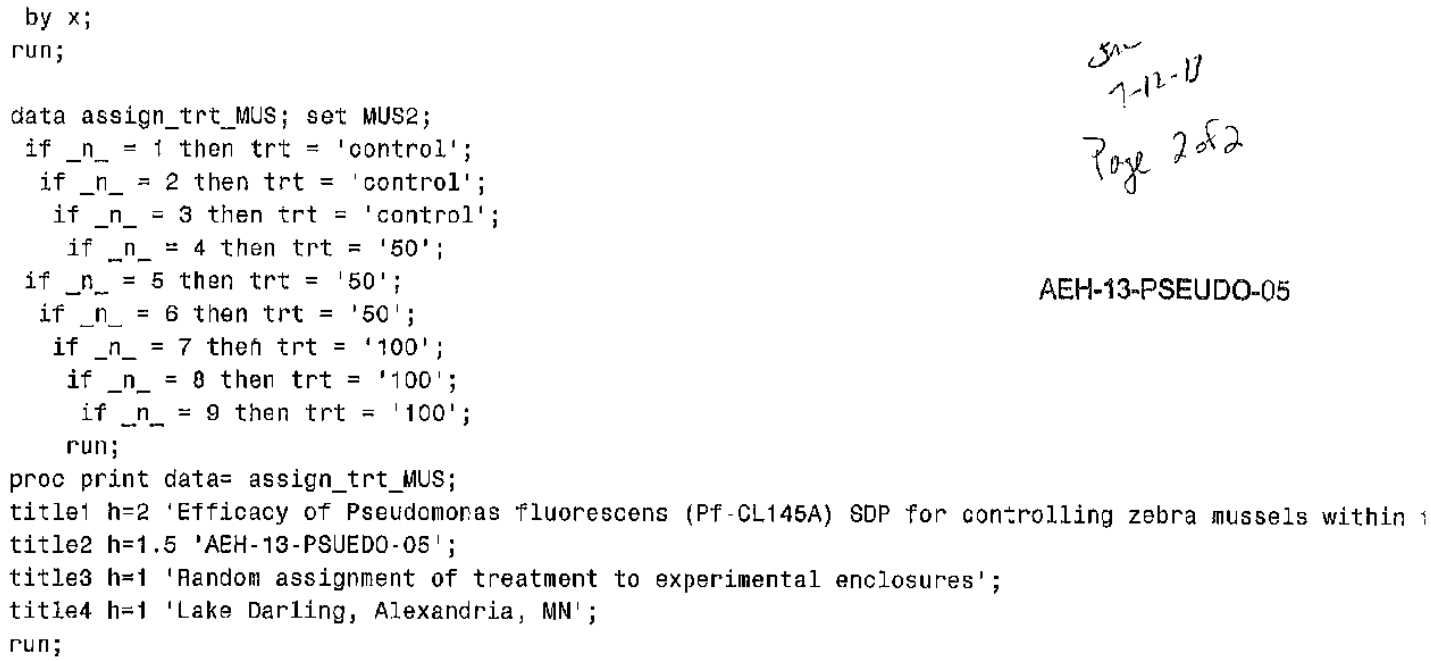

$$
\begin{aligned}
& 5 n^{2}-11 \\
& 70 \times 20 \times 2
\end{aligned}
$$

AEH-13-PSEUDO-05 


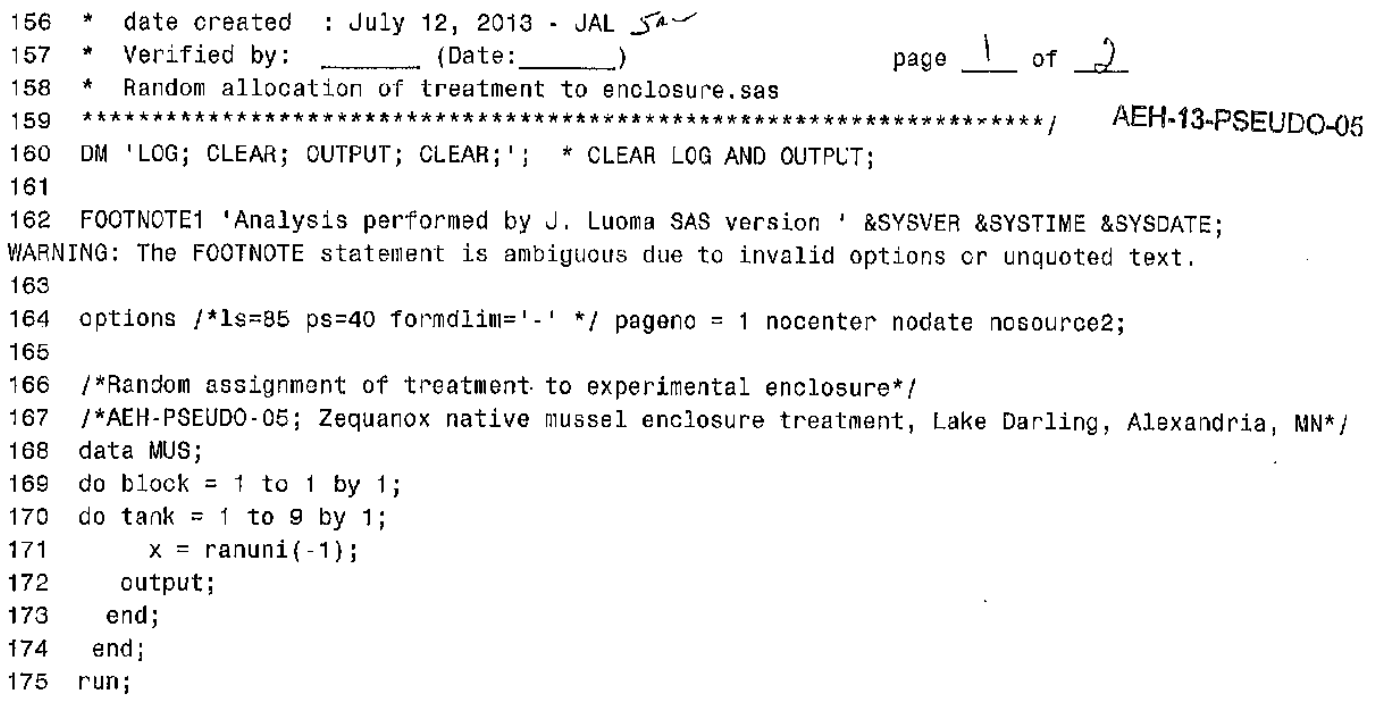

NOTE: The data set WORK, MUS has 9 observations and 3 variables. NOTE: DATA statement used (Total process time):

real time $\quad 0.01$ seconds

cpu time $\quad 0.01$ seconds

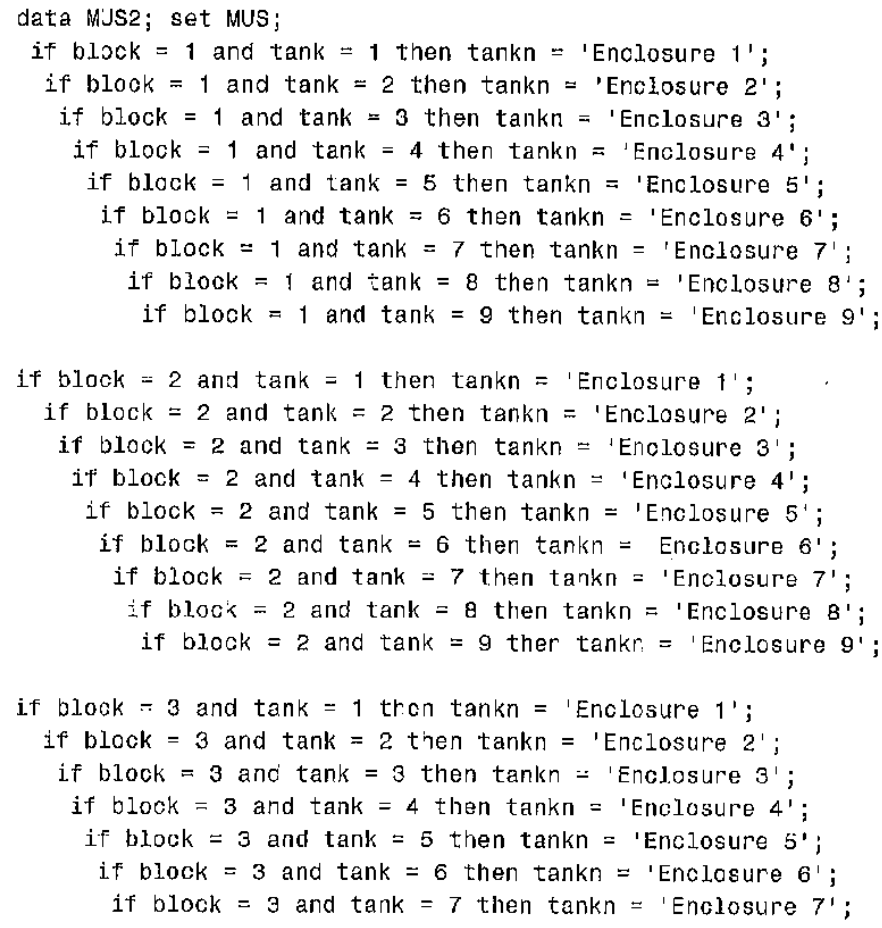

FF \# 9

Item $\overline{\text { No. } 3}$

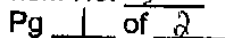




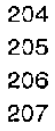

NOTE: There were $\theta$ observations read from the data set WORK.MUS2. NOTE: The data set WORK.MUS2 has 9 observations and 4 variables.

NOTE: PROCEDURE SORT used (Total process time):
real time
0.01 seconds
cpl time
0.01 seconds

data assign_trt_MUS; set MUS2;

if $n_{-}=1$ then trt = 'control';

if $\bar{n}_{-}=2$ then trt = 'control';

if $n_{-}=3$ then trt $=$ 'control'; if $\bar{n}_{-}=4$ then trt $={ }^{\prime} 50^{\prime}$;

if $n_{-}=5$ then trt $=150^{\prime}$;

if $n_{-}=6$ then trt $={ }^{\prime} 50^{\prime}$;

if $n_{-}=7$ then $\operatorname{trt}=1100^{\prime}$; if $\bar{n}_{-}=8$ then trt $=1100 '$; if $\bar{n}_{-}=9$ then trt $=1100^{\prime}$; run;

$$
\begin{aligned}
& \text { Jan } \\
& p^{2}-13 \\
& \text { page } 20+2
\end{aligned}
$$

AEH-13-PSEUDO-05

222

NOTE: There were 9 observations read from the data set WORK.MUS2.

NOTE: The data set WORK.ASSIGN_TRT_MUS has 9 observations and 5 variables.

NOTE: DATA statement used (Total process time):

real time $\quad 0.01$ seconds

cpu time $\quad 0.01$ seconds

223 proc.print data= assign_trt_MUS;

NOTE: Writing HTML Body fi.le: sashtml1.htm

224 title1 h=2 'Efficacy of Pseudomonas fluorescens (Pf-CLi45A) SDP for controlling zebra mussels

224 ! within field enclosures';

225 title2 $h=1.5$ 'AEH-13..PSUEDO-05';

226 title3 $h=1$ 'Random assignment of treatment to experimental enclosures';

227 title4 $h=1$ 'Lake Darling, Alexandria, MN';

228 run;

NOTE: There were 9 observations read from the data set WORK.ASSIGN TRT MUS.

NOTE: PROCEDURE PRINT used (Total process time):
real time
0.36 soconds
cpu time
0.25 seconds

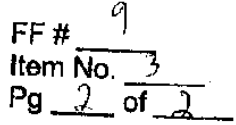

Item No. $\mathrm{Pg} 2$ of 


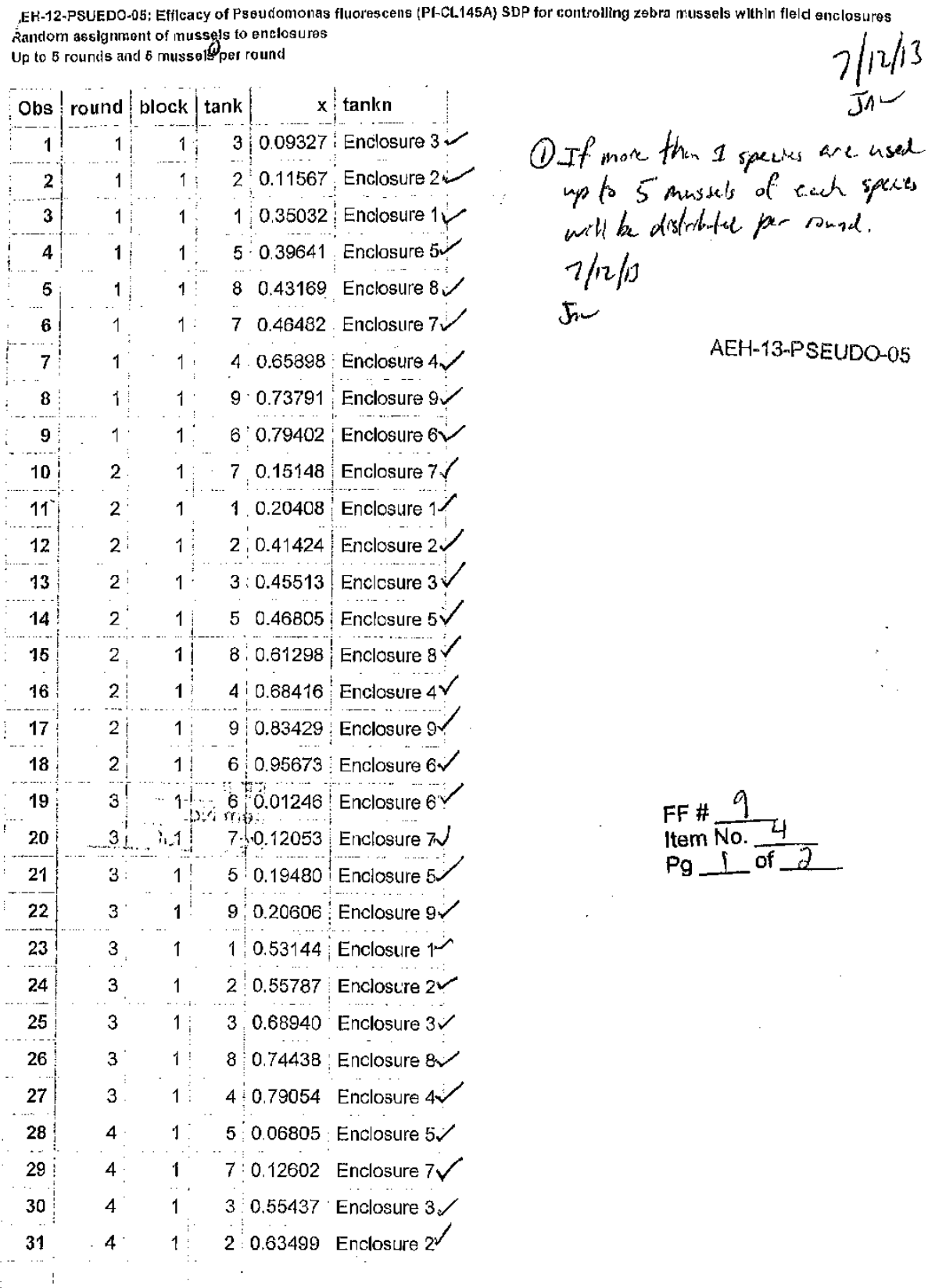




\begin{tabular}{|c|c|c|c|c|c|}
\hline 32 & 4 & 1 & 8 & 0.67644 & Enclosure $8 \checkmark$ \\
\hline 33 & 4 & 1 & 4 & 0.68682 & Enclosure \\
\hline 34 & 4 & í & 9 & 0.70878 & Enclosure $9 \mathrm{~V}$ \\
\hline 35 & 4 & 1 & $1:$ & 0.78054 & Enclosure \\
\hline 36 & 4 & $1 !$ & 6 & 0.87768 & Enclosure 6 \\
\hline 37 & 5 & 1 & 9 & 0.02835 & Enclosure 98 \\
\hline 38 & 5 & 1 & $6^{!}$ & 0.03358 & Enclosure 6 \\
\hline 39 & $5 !$ & 1 & 1 & C. 10315 & Enclosure 1 \\
\hline & 5 & 11 & 8 & 0.12591 & Enclosure 8 \\
\hline & 5 & 1 & 2 & 0.27132 & Enclosure \\
\hline 2 & 5 & 1 & 5 & 0.41851 & Enclosure 5 \\
\hline & 5 & 1 & 3 & 0.42010 & Enclosure $3 \gamma$ \\
\hline & 5 & 1 & 4 & 0.88894 & Enclosure 4 \\
\hline & 5 & 1 & 7 & 0.97724 & Enclosure 7 \\
\hline
\end{tabular}

Analysis performed by $\mathrm{J}$. Luoma SAS version $9.313: 53$ 12JUL13

\section{AEH-13-PSEUDO-05}

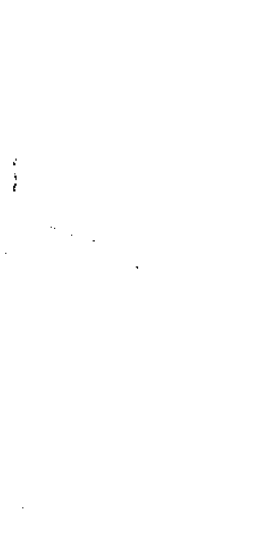

FF \# 9

Item No. 4

$\mathrm{Pg} 2$ of 2 


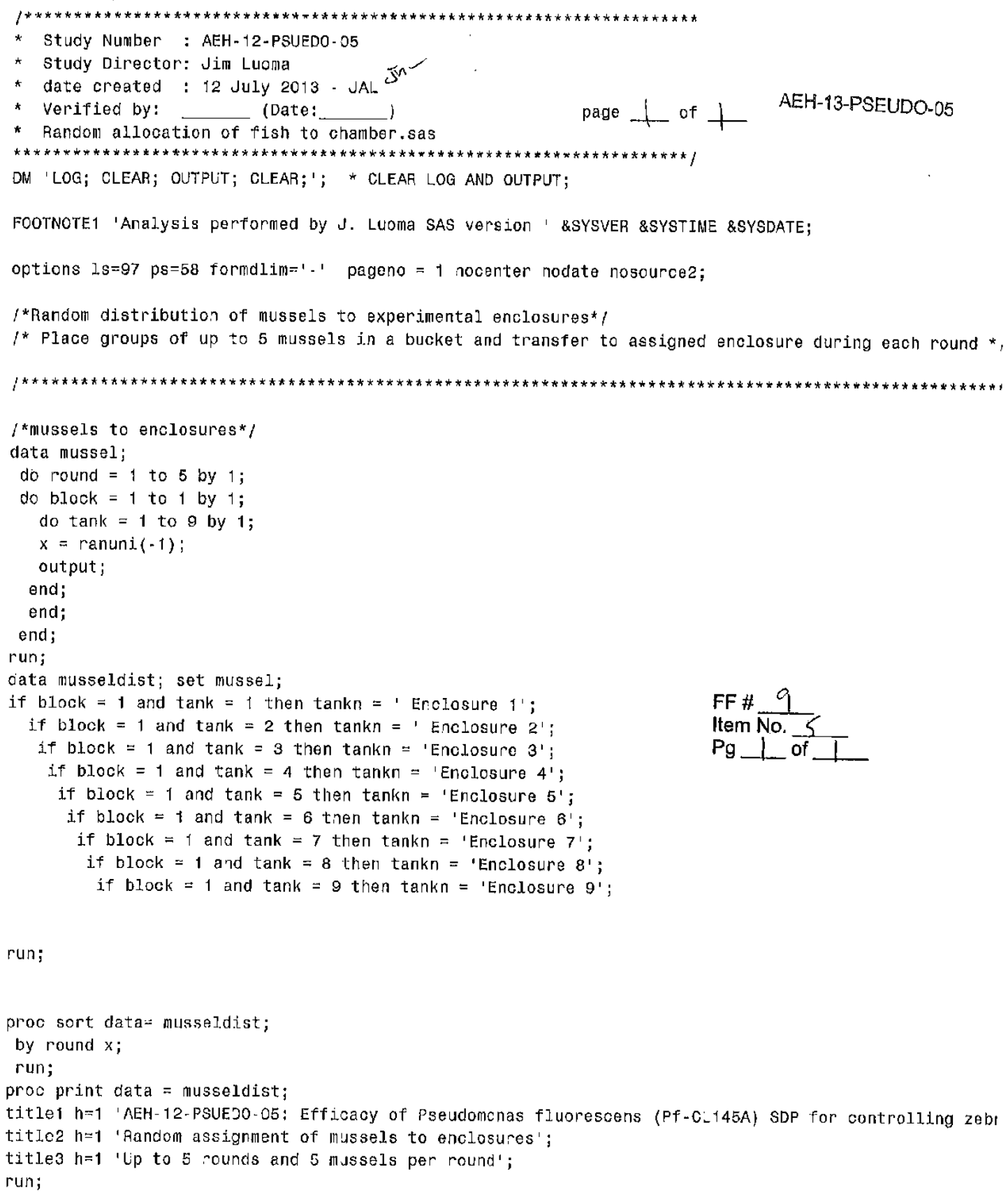

FF \# 9

Item No. 5

run;

proc sort data = musseldist;

by round $x$;

run;

proc print data = musseldist;

titlei $h=1$ 'AEH-12-PSUEכ0-05: Efficacy of Pseudomenas fluorescens (Pf-C_145A) SDP for controlling zebi title2 $h=1$ 'Random assignment of mussels to enclosures';

titles $h=1$ 'lp to 5 rounds and 5 mussels per round';

run; 


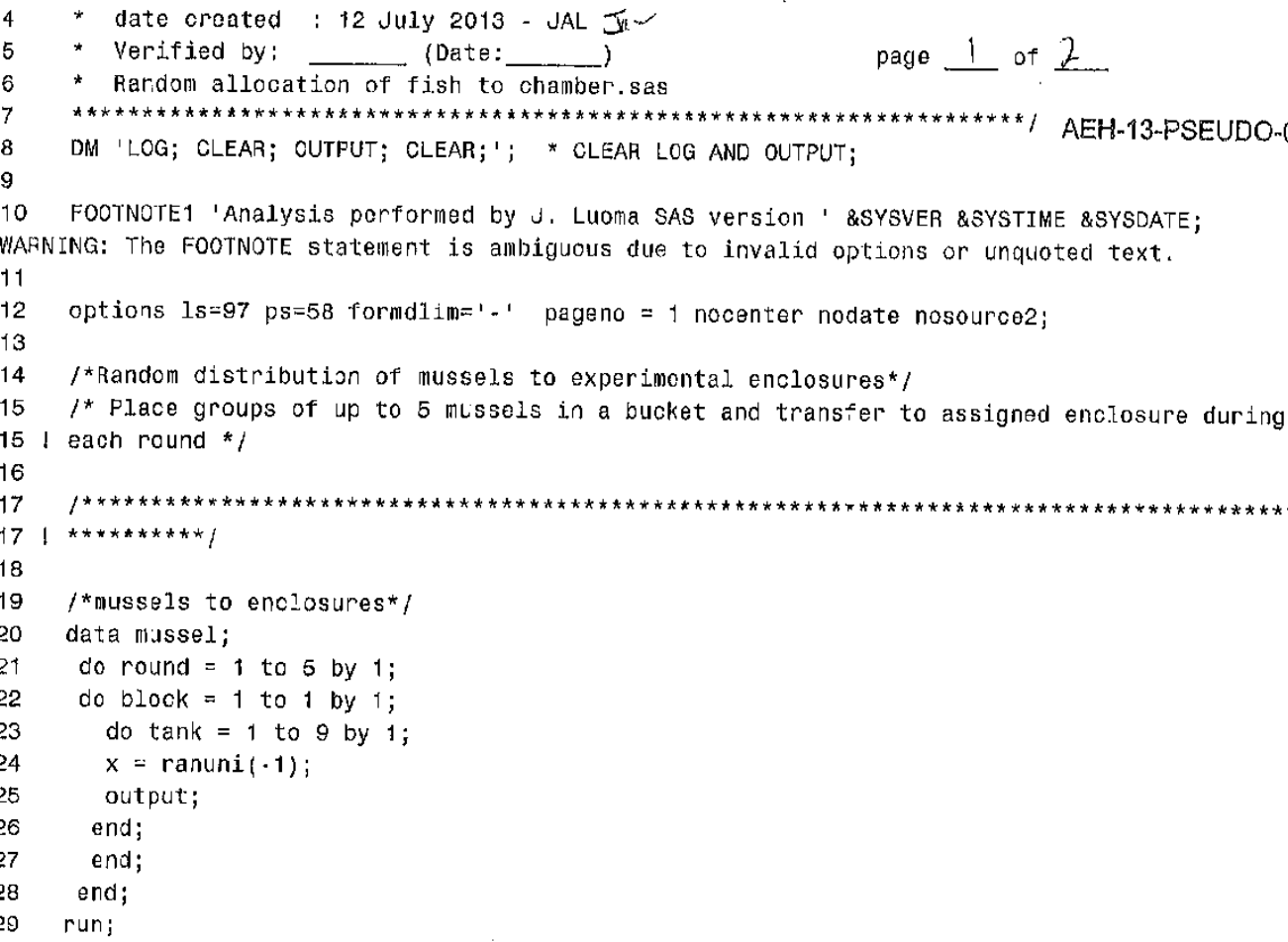

NOTE: The data set WORK.MUSSEL has 45 observations and 4 variables.

NOTE: DATA statement used (Total process time):
real time
0.01 seconds
cpu time
0.01 seconds

data musseldist; set mussel;

if $b_{-o c k}=1$ and tank $=1$ then tankn = 'Enclosure 1 ';

if block $=1$ and tank $=2$ then tankn $=1$ Enclosure 2 '

if block $=1$ and tank $=3$ then tankn = 'Enclosure $3^{\prime}$;

if block $=1$ and tank $=4$ then tankn = 'Enclosure $4 '$ ';

if block $=1$ and tank $=5$ then tankn $=$ 'Enclosure 5 ';

if block $=1$ and tank $=6$ then tankn = 'Enclosure $6^{\prime}$;

if block $=1$ and tank $=7$ then $\operatorname{tankn}=$ 'Enclosure 7 '

if block $=1$ and $\tan k=8$ then $\operatorname{tankn}=$ 'Enclosure 8 ';

if block $=1$ and tank $=9$ then tankn $=$ 'Enclosure 9 ';

run;

FF \# 9

Item No. $\frac{1}{4}$

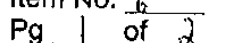

NOTE: There were 45 observations read from the data set WORK.MUSSEL.

NOTE: The data sot WORK.MUSSELDIST has 45 observations and 5 variables,

NOTE: DATA statement used (Total process time):
real time
0.01 seconds
cpu time
0.00 seconds 


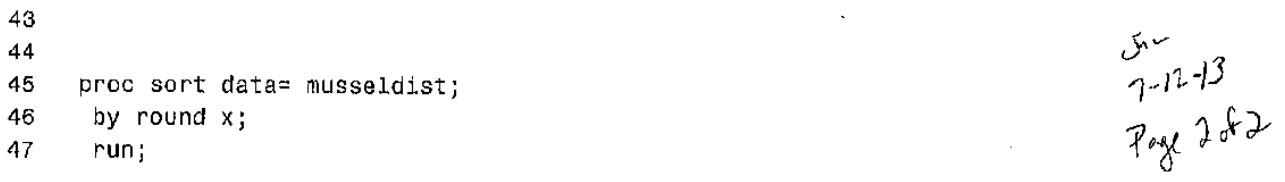

NOTE: There were 45 observations read from the data set WORK.MUSSELDIST.

NOTE: The data set WORK.MUSSELDIST has 45 observations and 5 variables.

NOTE: PROCECURE SORT used (Total process time)
real time
0.00 seconds

cpu time

0.01 seconds

AEH-13-PSEUDO-05

48 proc print data $=$ musseldist;

NOTE: Writing HTML Body fj.le: sashtml.htm

49 title1 h=1 'AEH-12-PSUED0-05: Efficacy of Pseudomonas fluorescens (Pf-CL145A) SOP for

49 ! controlling zebra mussels within field enclosures ';

50 title2 $h=1$ 'Random assignment of mussels to enclosures';

51 titles $\mathrm{h}=1$ 'Up to 5 rounds and 5 mussels per round';

52 runi

NOTE: There were 45 observations read from the data set wORK.NUSSELDIST,

NOTE: PROCEDURE PRINT used (Total process time):
real time
0.34 seconds
cpu time
0.25 seconds

FF \# 9

Item No. 6

$\mathrm{Pg} \_2$ of 2 


\section{Appendix 4. Test Article Information}

\begin{tabular}{|c|c|c|c|}
\hline $\begin{array}{l}\text { Item } \\
\text { number }\end{array}$ & Item description & $\begin{array}{c}\text { Number } \\
\text { of } \\
\text { pages }\end{array}$ & $\begin{array}{c}\text { Report } \\
\text { page } \\
\text { number }\end{array}$ \\
\hline 1 & Material Safety Data Sheet: MBI-401 Spray Dried Powder & 2 & 99 \\
\hline 2 & MBI-401 SDP (lot \# TR-4669-4-3) Test Article: Certificate of Analysis & 1 & 101 \\
\hline 3 & Test Material Requirements for Enclosure treatments & 1 & 102 \\
\hline 4 & Test Chemical Stock Preparation & 1 & 103 \\
\hline 5 & $\begin{array}{l}\text { Copy of test article information from test article log book for MBI-401 SDP; lot } \\
\text { number TR4669-4-3; Container } 1 \text { of } 2 \text { - Used for enclosure treatment at Lake } \\
\text { Darling }\end{array}$ & 6 & 104 \\
\hline 6 & $\begin{array}{l}\text { Copy of test article information from test article log book for MBI-401 SDP; lot } \\
\text { number TR4669-4-3; Container } 2 \text { of } 2 \text { - Used for enclosure treatment at Lake } \\
\text { Darling }\end{array}$ & 6 & 110 \\
\hline 7 & Post-treatment product validation assay for test article (lot \# TR4669-4-3) & 2 & 116 \\
\hline 8 & $\begin{array}{l}\text { MBI-401 SDP (lot \# 401P120724C) Test Article: Certificate of Analysis - Not Used } \\
\text { for Treatment }\end{array}$ & 1 & 118 \\
\hline 9 & Test Chemical Stock Preparation - Not Used for Treatment & 1 & 119 \\
\hline 10 & Pre-treatment product validation assay for test article (lot \# 401P120724C) - FAILED & 2 & 120 \\
\hline 11 & $\begin{array}{l}\text { Copy of test article information from test article log book for MBI-401 SDP; lot } \\
\text { number 401P120724C; Container } 1 \text { of } 1 \text { - Not used in dosing (FAILED pre-test } \\
\text { validation) }\end{array}$ & 4 & 122 \\
\hline
\end{tabular}


AEH-13-PSEUDO-O5

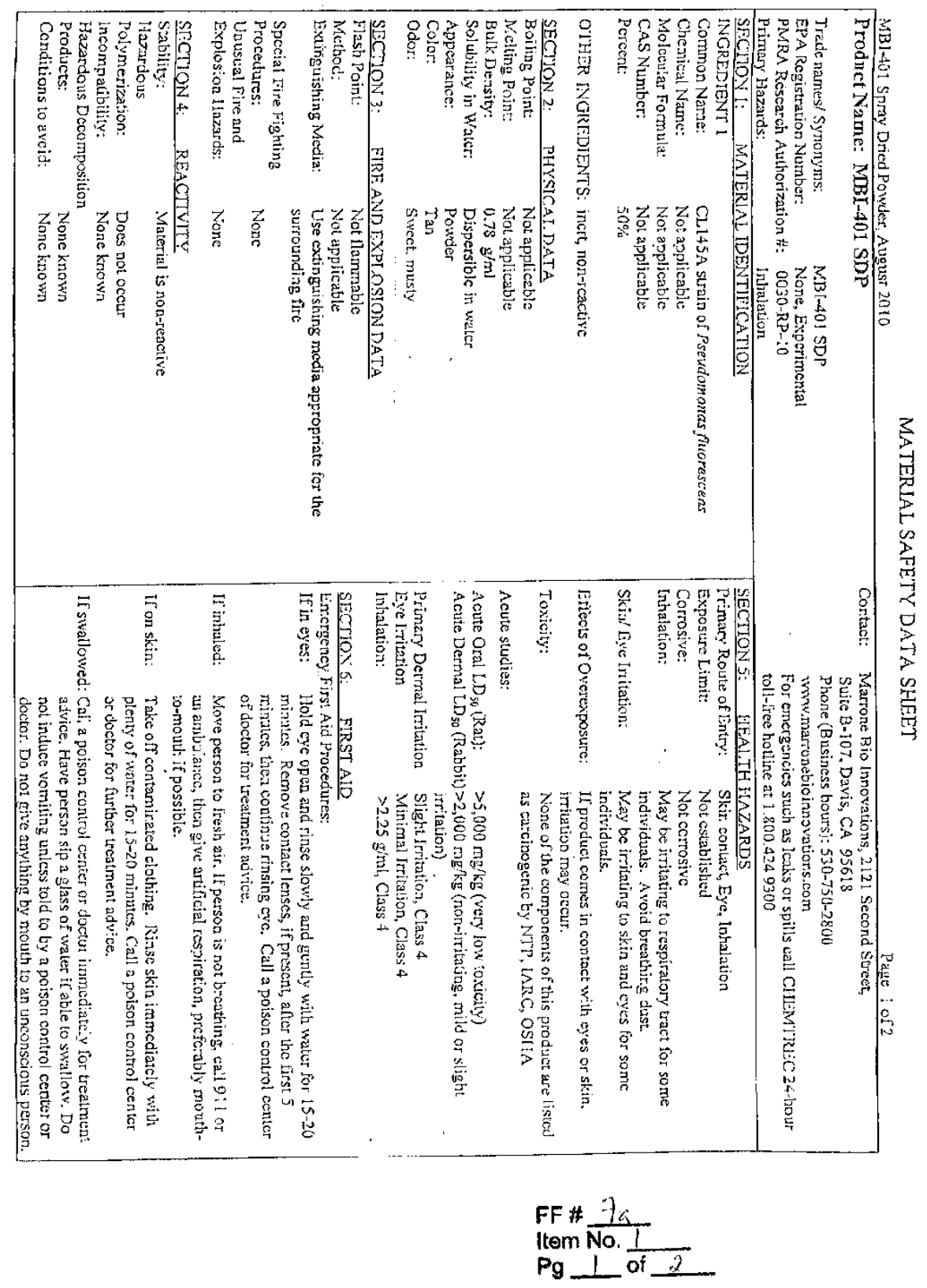


AEH-13 PSEUDO-05

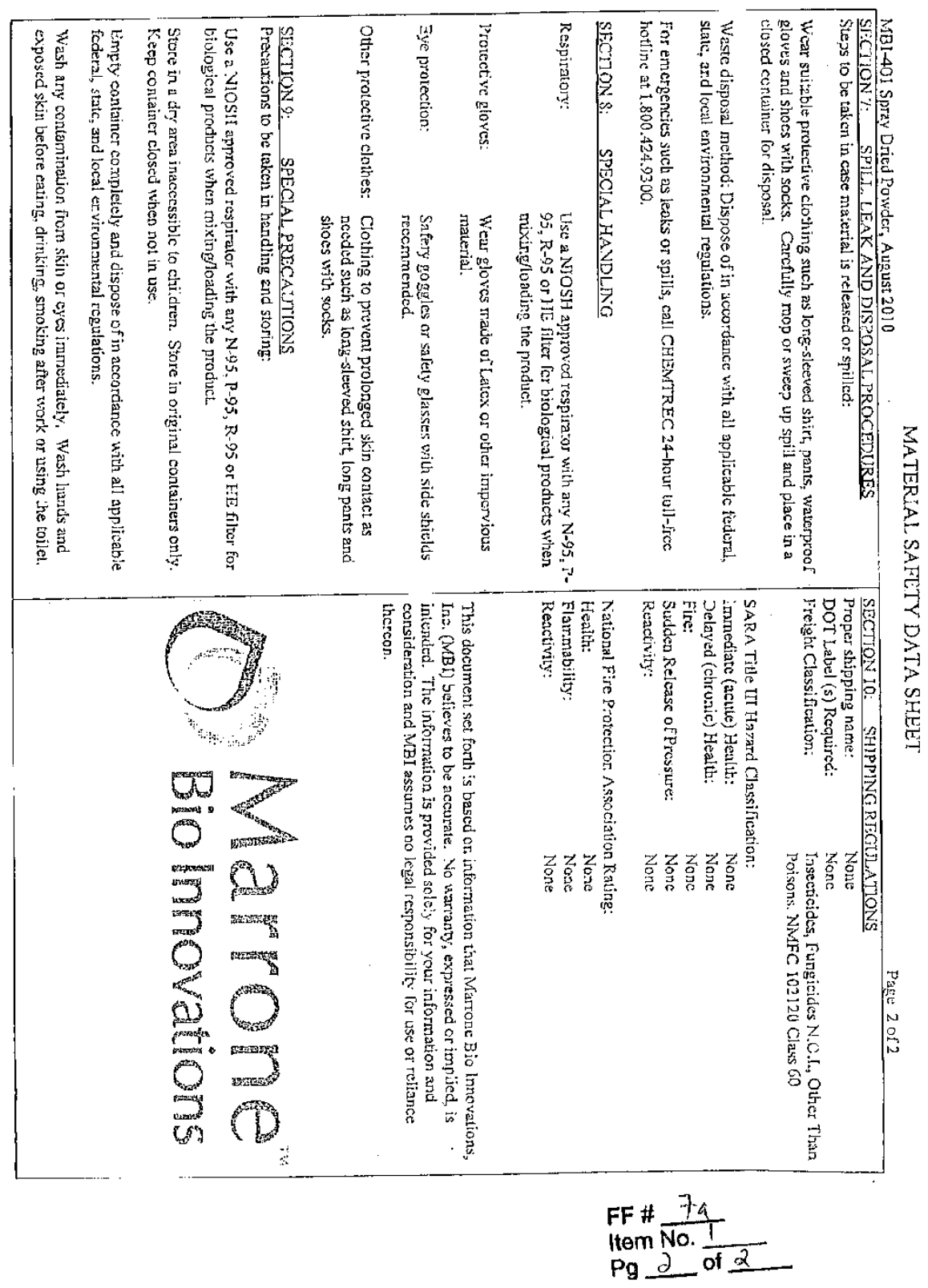




\section{(1) Marrone}

\section{CERTIFICATE OF ANALYSIS}

AEH-13-PSEUDO-05

$\begin{array}{ll}\text { Name of Product: } & \text { MBI-401 SDP } \\ \text { Active Ingredient: } & \begin{array}{l}100 \% \text { Pseudomonas fluorescens strain CL145A cells and spent } \\ \text { fermentation media }\end{array} \\ \text { Pcrcent Active lngredicnt: } & 50 \% \text { by wcight } \\ \text { Viable Cfu/g: } & 0 \text { cfu/g Pseudomonas fluorescens strain CL145A } \\ \text { Lot Number: } & \text { TR 4669-4-(3) } \\ \text { Mussel Bioassay: } & \text { Pass } \\ \text { Appearance: } & \text { Tan powder } \\ \text { Storagc Conditions: } & 4{ }^{\circ} \mathrm{C}, \text { protccted from light } \\ \text { Date of Manufacture: } & 08 \text { February } 2012 \\ \text { Expiration Dale: } & 08 \text { Novernber } 2013\end{array}$

I hereby certify that the above information is true and correct.

Quality Control:

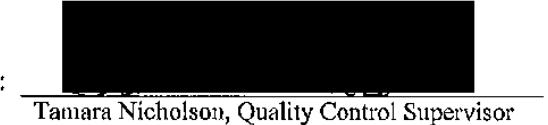

Date: 28 August 2013

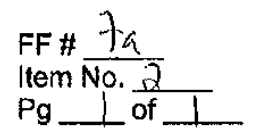

2121 Second Street, Suite B-107

Davis, CA 95618

- Phone: 530-750-2800 


\section{Study Number AEH-13-PSEUDO-05}

Test Material Requirements for Enclosure treatments

Date $7 / 25 / 13 \quad$ initials $\sqrt{6}$
Location L. Durimg Exp. Date_ $7 / 26 / 13$
TR4669-4-3 (run 4 sample 3)

\begin{tabular}{|c|c|c|c|c|c|c|c|}
\hline \multicolumn{8}{|c|}{$1^{\prime \prime}=\quad 0.0254$ meters } \\
\hline \multicolumn{3}{|c|}{ Enclosures $=56^{\prime \prime}$ square $=$} & \multicolumn{3}{|c|}{2.02322 square meters } & \multirow[b]{2}{*}{$\begin{array}{l}\text { Active } \\
\text { Req. } \\
\text { (g) }\end{array}$} & \multirow[b]{2}{*}{$\begin{array}{c}\text { Product } \\
\text { Req. } \\
\text { (g) }\end{array}$} \\
\hline $\begin{array}{c}\text { Enclosure } \\
\text { ID }\end{array}$ & $\begin{array}{c}\text { Treatment } \\
\mathrm{mg} / \mathrm{L}\end{array}$ & $\begin{array}{c}\text { Depth } \\
\text { (inches) }\end{array}$ & $\begin{array}{c}\text { Depth } \\
\text { (meters) }\end{array}$ & $\begin{array}{l}\text { Enclosure } \\
\text { Vol. (m3) }\end{array}$ & $\begin{array}{c}\text { Enc. Vol } \\
\text { (L) }\end{array}$ & & \\
\hline 1 & 100 & 53 & 1.35 & 2.72 & 2723.66 & 272.37 & 544.73 \\
\hline 2 & 50 & 51 & 1.30 & 2,62 & 2620.88 & 131.04 & 262.09 \\
\hline 3 & 0 & 54 & 1.37 & 2.78 & 2775.05 & 0.00 & 0.00 \\
\hline 4 & 50 & 54 & 1.37 & 2.78 & 2775.05 & 138.75 & 277.51 \\
\hline 5 & 50 & 54 & 1.37 & 2.78 & 2775.05 & 138.75 & 277.51 \\
\hline 6 & 100 & 53 & 1.35 & 2.72 & 2723.66 & 272.37 & 544.73 \\
\hline 7 & 0 & 53 & 1.35 & 2.72 & 2723.66 & 0.00 & 0.00 \\
\hline 8 & 0 & 54 & 1.37 & 2.78 & 2775.05 & 0.00 & 0.00 \\
\hline 9 & 100 & 55 & 1.40 & 2.83 & 2826.44 & 282.64 & 565.29 \\
\hline
\end{tabular}

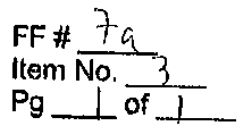


Study Number: AEH-13-PSEUDO-C5

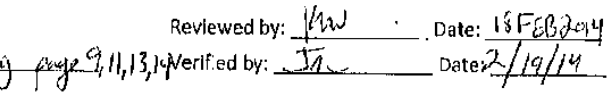

Flle Folder: 7a

Lab book/pgs: $\underline{\ell-10 \hat{y}}$

Test Chemical Stock Preparation

Test Chemical: Pseudomonas fluroescens strain 145A

Test Chemical Lot $\#:$ T2 $4664-4-3$ M 20 in ans

Test Location: Lake Darlina, Alexandrka, su

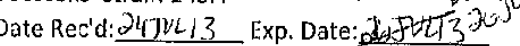
Instruments Used: $B A L 3 ; B H L 4$

Weights of Chemlcal Samples:

\begin{tabular}{|c|c|c|c|c|}
\hline Sample ID & Sample Weight & Comments & Date & Initlals \\
\hline Aneilitizal $\neq 1$ & 2.00 & $B \times 4 \mid$ of 2 & $25 \mathrm{Mm}^{3}$ & Hev \\
\hline Andyhal tha & 2.00 & $B a x \operatorname{lof} 2$ & $\uparrow$ & \\
\hline Stuck 1 & 277.53 & 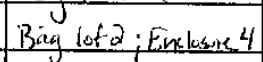 & & \\
\hline Stock 2 & 27.5 .59 & Bug bifo, Enclosen 5 & & \\
\hline Stock 3 & 26.2 .09 & Bug lof $2:$ Enclusere 2 & & \\
\hline Stock 4 & 544.73 & Bag lof 2 Enclasure 1 & & \\
\hline Stick S & 563.27 & Bue lof a Encking & $\sqrt{1}$ & 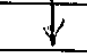 \\
\hline Slock 6 & 544.61 & Bing of 2; Enclisure & 25,213 & Eno \\
\hline & 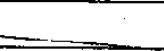 & & . & \\
\hline & & & & \\
\hline
\end{tabular}

NOTE: Chemical samples to be stored refrigerated until used for stock preparation.

Stock Solution Preparation:

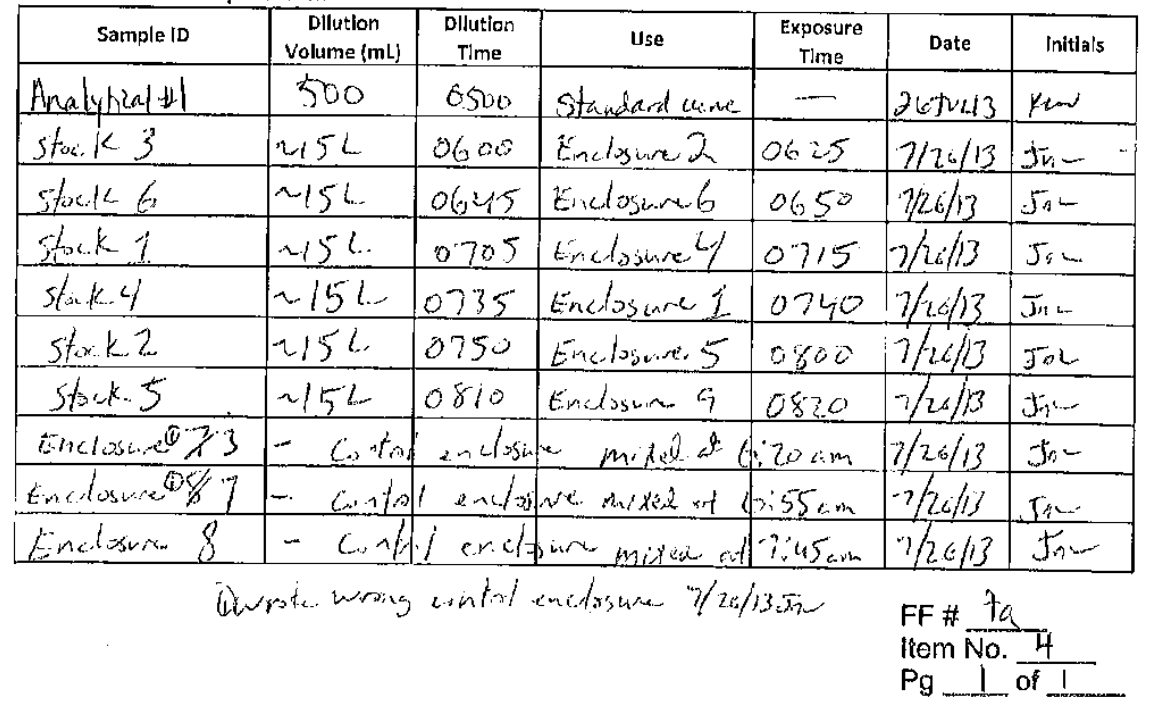


AEH-13-PSEUDO-05

\title{
CHEMICAL LOG BOOK
}

\author{
MBI-401 SDP
}

Marrone Bio Innovations

Davis, California

\section{LOT NUMBER: TR4669-4-3}

(Run 4; Sample 3)

Container 1 of 2

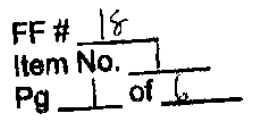


SIGNATURE PAGE

AEH-13-PSEUDO.05

All personnel rraking an entry in this log must fill out the form below in accordance with SOP GEN 009.

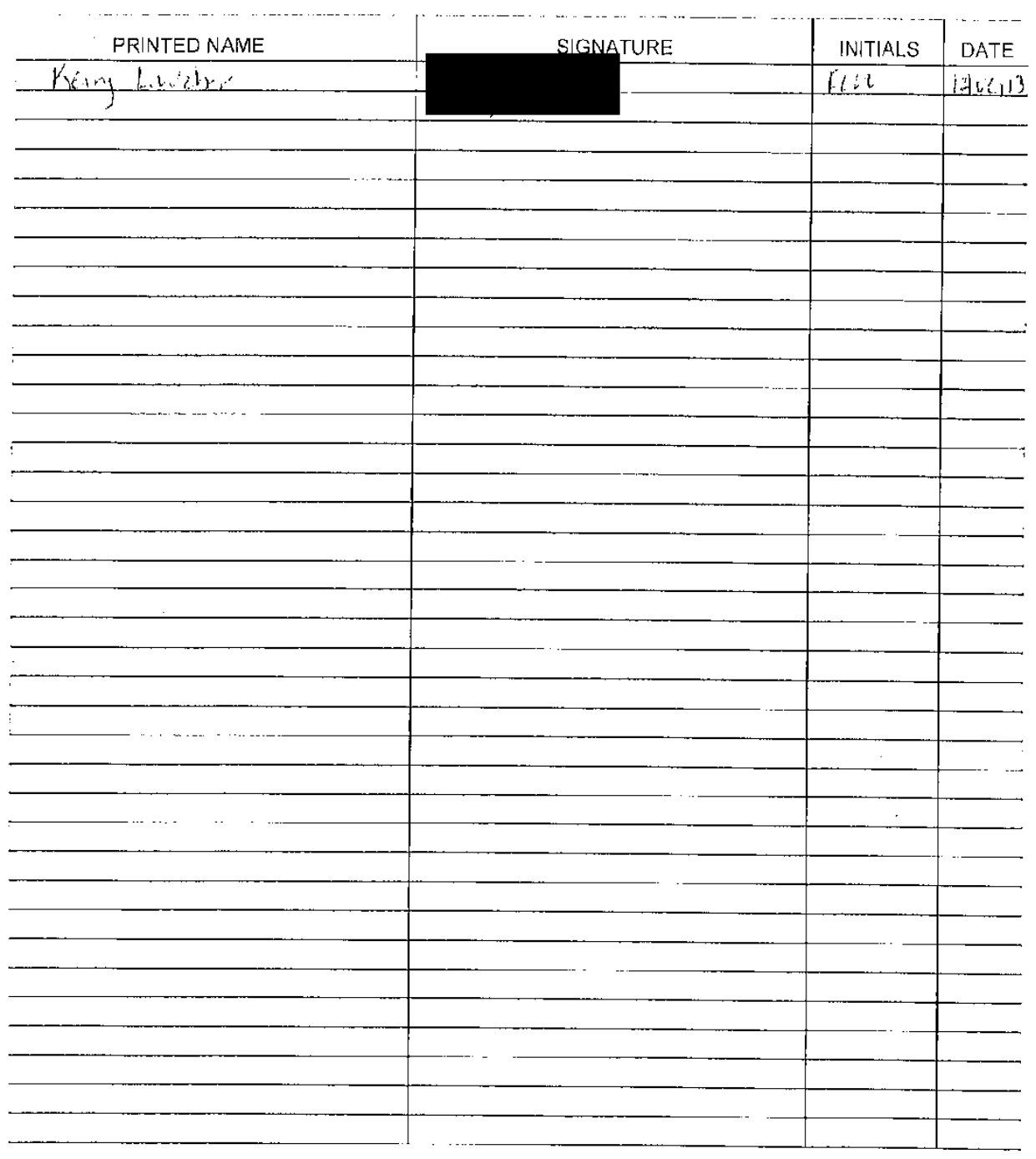

"This Use and Maintenance Log Book has been inspected and fourd to be in compliance with SOP GEN OOg Inspected and sealed on

by

Qualily Assurance Unit

$$
\text { Page } 2 \text { of } 6
$$




\section{DORIABAL}

1대-13.PSEUDO-05

SOP NO. GEN 012.3

Page 5 of 7

JOIm GEN 012.3a

Page I of 1

TEST CHEMICAL DATA FORM

Test Chemical (Chemical Name) of -tTh5A M M

Circle one: Test Article Control Article

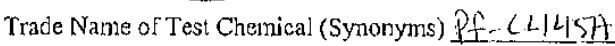

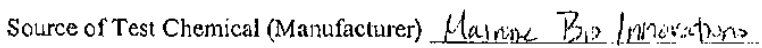

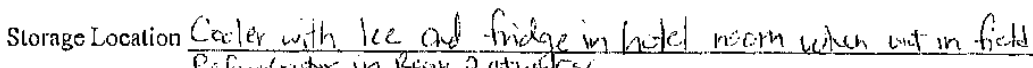
Efingtrutor in Risin atwatesc.

Date Received Jutul 3 Date Opened $25 \mathrm{TH} 13$

Expiration Date

(5 years unless otherwise stated)

Test Chemical Lot Number $124669-4-3$

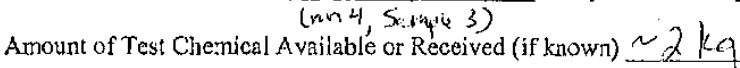
Purity of Chemical to $6 \%$

Initial Mass (with cover on) of Test Chemical and Container 2046.77

Characterization of Tesı Chemical: Color Tah

Physical State: liquid solid $X$

Chemical Abstract Service Nunber N/A

Solid Form: powder $x$ crystal _ pellet

Manufacturer Certificate of Analysis Yes $(2)$ No

Acditional Comments about the Test Chemical:

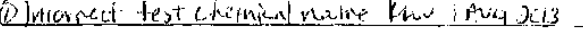

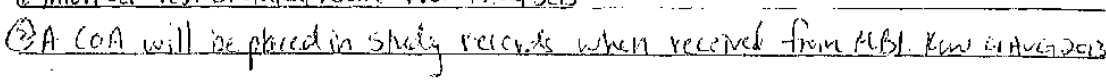

Sample Placed in Archives: Yes _ No X (Entries should also be made on Form GEN 012.b)

Archive Location_N N P _ Material Safaly Data Sheet Available: Yes X_ No _.

Signature of Study Director or designee initjating Test Chemical Use Log and date:

Signature

Date Ut Avis?

Page 3 of 6 
AEH-13-PSEUDO-05

Study Number: AEH-13-PSEUDO-05

Study Title: Efficacy of Pseudomonas fluorescens. (Pf-CL145A) SDP for controlling zebra mussels within field enclosures

Test Chemical Lot/Use:

Zequanox test chemical (lot \#401P120724C) was brought to the test location for use in the study based an preliminary results from pre-test activity tests conducted by the New York State Museum Field Research Laboratory (NYSM-FRL). While at the field test site (Lake Darling; Alexandria, MN) on 23 JUL 2013, NYSM-FRL staff called and indicated the test chemical lot $\$ 401 P 120724 \mathrm{C}$ did not pass initial efficacy testing and it was therefore not used for the study. Marrone Bio Innovations shipped $4 \mathrm{~kg}$ of replacement test chemical (lot TR4660-4-3; run 4, sample 3) to the field location via overnight UPS. The new lot of test chemical was weighed based on the calculated amounts needed for the proper treatment level. The data sheet with individual samples weights can be found in File Folder 7. A copy of the data sheet is bound in the chemical logbooks and the data were transcribed to the chemical logbooks that were created upon returning to UMESC. Three analytical aliquots were weighed. The first analytical aliquot was spilled and therefore discarded. A sample of the test material will be shipped overnight to NYSM-FRI. to verify post-exposure activity.

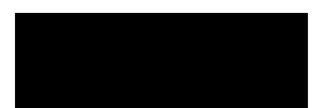

7 Kcrry t. Weber, Fisheries Biologist

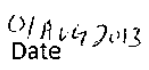

Page 4 of 6 
AEH-13-PSEUDO-05

Study Number: AEH-13-PSEUDO-05

Flle Folder:

Lab book/pgs:

Reviewed by:

Verifiec by:

Date:

Date:

\section{Test Chemical Stock Preparation}

Test Chemical: Pseudomonas fluroescens strain 145A

Test Chemical Lot H: -TR 46.69-4-3

Date Rec'd: $24 \mathrm{~T} W L 13$ Exp. Date:

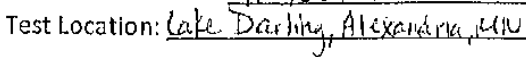
Instruments Used: BAL 3 . B A H L

Welghts of Chemical Samples:

\begin{tabular}{|c|c|c|c|c|}
\hline Sample ID & Sample Welght & Comments & Date & Inltials \\
\hline Anet lytial $\neq 1$ & 2.00 & $B$ a 1 of 2 & $25 \mathrm{~min}$ & Hav \\
\hline Andytical +2 & 2.00 & $B 09 \operatorname{lf} 2$ & $\hat{\uparrow}$ & \\
\hline Stuck & 277.53 & Bag lot 2 ; Enclisure 4 & & \\
\hline Stock 2 & 277.59 & Buy hT2, Enclure 5 & & \\
\hline Stock 3 & 262.09 & Big lof 2 ; Encluse 2 & & \\
\hline Stuck 4 & 544.73 & Brag lof 2 Enckas-orl & & \\
\hline Stok S & 563.27 & Bu lef2i Enckuses & $\sqrt{1}$ & $\downarrow$ \\
\hline Slock 6 & 544.61 & Bes dof 2: Encloweche & 2530213 & Yno \\
\hline & & 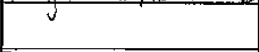 & & \\
\hline & & & & \\
\hline
\end{tabular}

NOTE: Chemical samples to be stored refrigerated until used for stock preparation.

Stock Solutlon Preparation:

\begin{tabular}{|c|c|c|c|c|c|c|}
\hline Sample ID & $\begin{array}{c}\text { Dllution } \\
\text { Volume }(\mathrm{mL})\end{array}$ & $\begin{array}{c}\text { Dilution } \\
\text { Time }\end{array}$ & Use & $\begin{array}{l}\text { Exposure } \\
\text { Time }\end{array}$ & Date & Intials \\
\hline Apralytial $\#$ & 500 & 0500 & Standaral unve & $=$ & $26+243$ & For \\
\hline stock 3 & 452 & 0600 & Enclxure 2 & 062.5 & $2 / 26 / 13$ & $I_{n_{i}}$ \\
\hline stor 126 & -152 & $06 \div 5$ & Enclojuret & 06,50 & $726 / 3$ & $5 \pi^{2}$ \\
\hline sfok ? & -151 & 0705 & Erictojiare L & 0.715 & $7 / 26 / 13$ & Jin \\
\hline $56 . k 4$ & 2151 & 0735 & Erodosure it & 0740 & $7 / 2,1) 3$ & $J_{i} \ldots$ \\
\hline$s t a x+2$ & -156 & $3750^{\circ}$ & Enclogione 5 & 01900 & $7 / 6,03$ & Jal \\
\hline $5 f-x-5$ & $2 / 52$ & 0810 & Encloswe 4 & 0820 & $2 / 22 / 13$ & $5 i+$ \\
\hline$\left\{\pi<1 x, x^{0} \geq 3\right.$ & - cosis & $i n \operatorname{losin}$ & $-m_{1} \lambda_{0} d-y^{t}$ & $20 \mathrm{~cm}$ & $1 / 26 / 13$ & th- \\
\hline 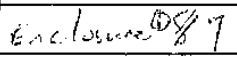 & $-\quad(\ldots, \ldots)$ & $12+4 / j$ & 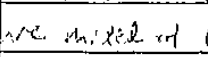 & .550 .1 & $-7 / 26 / 13$ & TrO \\
\hline Freloswar 8 & $-c a k$ & $j$ const: & ines millea & 3.45 rpin & $01 / 26 / 13$ & Jin \\
\hline
\end{tabular}

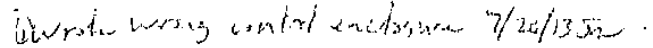

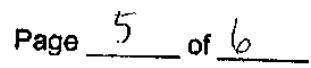




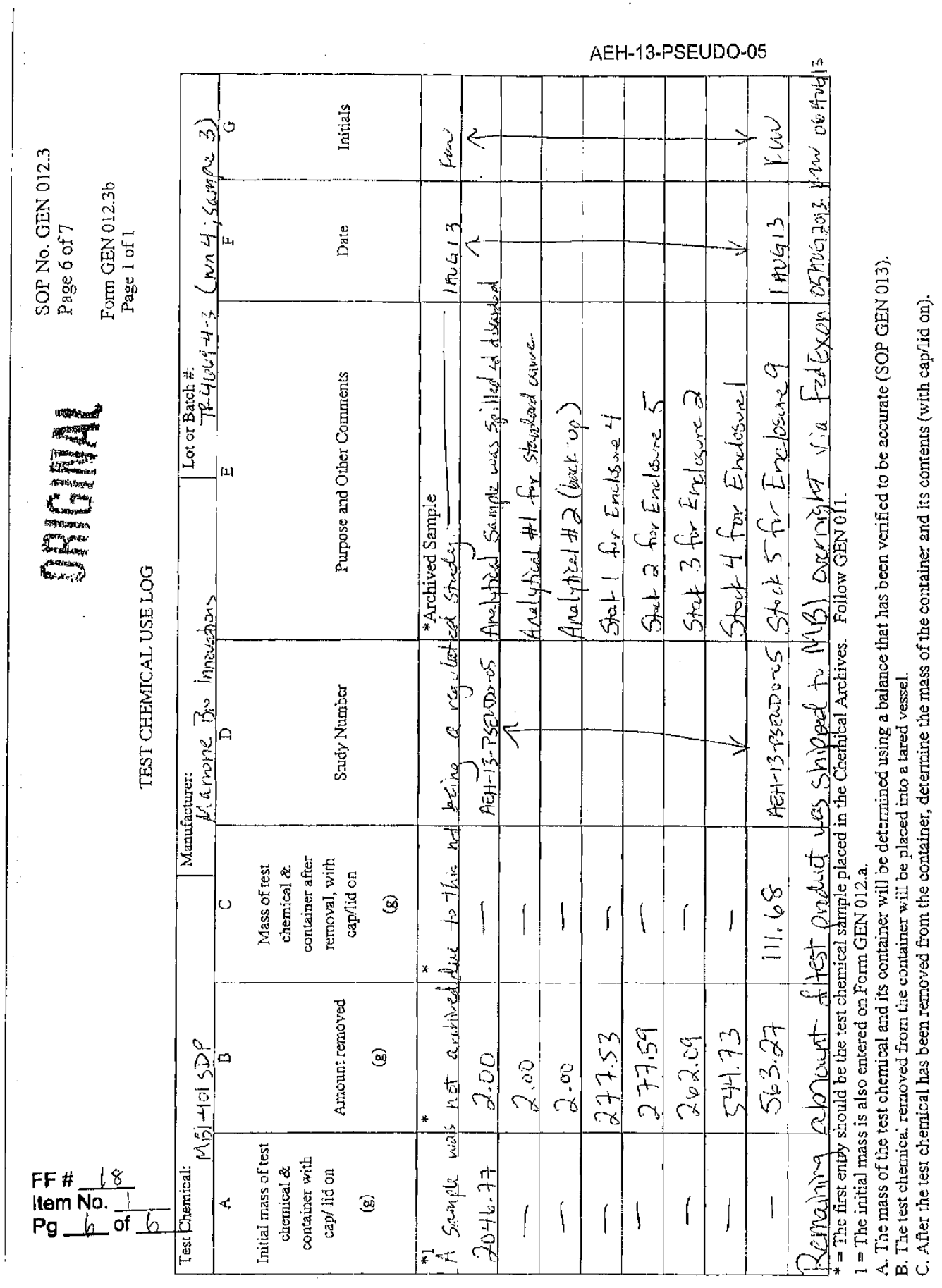




\section{CHEMICAL LOG BOOK}

MBI-401 SDP

Marrone Bio Innovations

Davis, California

LOT NUMBER: TR4669-4-3

(Run 4; Sample 3)

Container 2 of 2

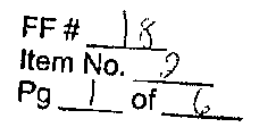


Ail personnel making an entry in this log must fill out the form below in accordance with SOP GEN 009 .

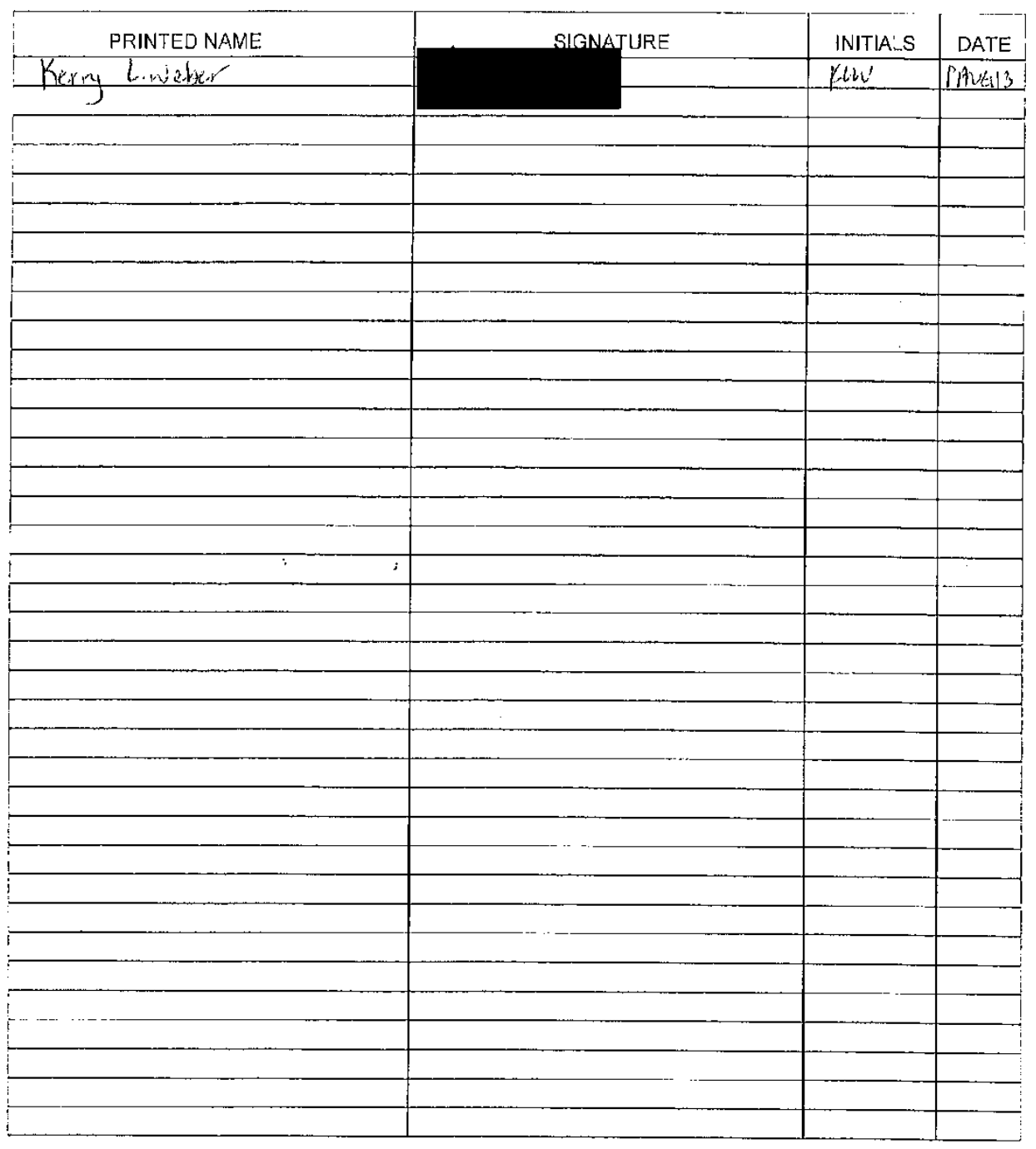

"This Use and Maintenance Log Book has been inspected and found to be in compliance with SOP GEN 009 .

by

Quality Assurance Ụit

Page 2 of 6 


\section{DMGIIALE}

AEH-13-PSEUDO-05

SOP NO. GEN 012.3

Page 5 of 7

Form GEN 012.3a

TEST CHEMICAL DATA FORM

Page 1 of 1

Test Chemical (Chemical Name) $H(6 L-401 \leq D P$

Circle one: (Test Article Control Atticle

Trade Name of Test Chemical (Synonyms) if C L 1459

Source of Test Chemical (Manufacturet) Marmue. Bo Irravitions,

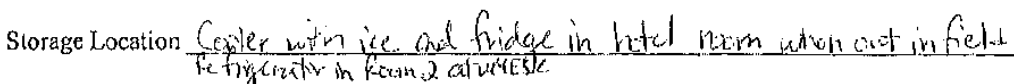

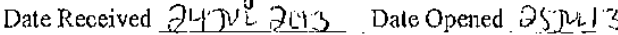

Expiration Dale

(5 years unless otherwise stated)

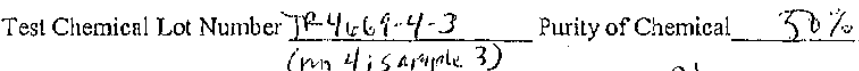

Amount of 'Test Chemical Available or Received (if known) $\simeq 2 k-$

Initial Mass (with cover on) of 'Test Chemical and Container 2045,39

Characterization of Test Chemical:

Color

Tak

Physical State: liquid solid $x$

Chemical Abstract Service Number U/A

Solid Iorm: powder $\underline{x}$ crystal _. pellet

Manufacturer Certificate of Analysis Yes No

Additional Comments about the Test Chemical:

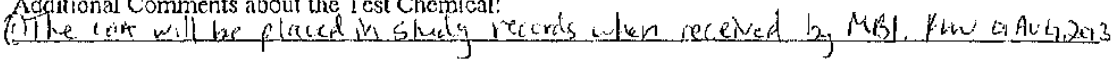

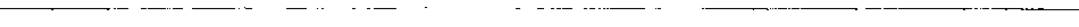

Sample Placed in Arclives: Yes _.. No X (Entries should also be made on Jom GEN 012.b)

Archive Location ‥ Material Safety Data Sheet Available: YesX_ No _

Signature of Study Director or designee initiating Test Chemical Use Log and date:

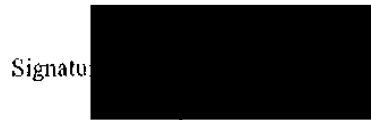

- Date Q Avatis

Page 3 of 6 
AEH-13-PSEUDO-05

Study Number: AEH-13-PSEUDO-05

Study Title: Efficacy of Pseudomonas fluorescens (Pf-CL.145A) SDP for controlling zebra mussels with in field enclosures

\section{Test Chenrical Lot/Use:}

Zequanox test chemical (lot \#401P12072AC) was brought to the test location for use in the study based on preliminary results from pre-test activity tests conducted by the New York State Niuseum Field Research Laboratory (NYSM-FRL). White at the field test site (Lake Dar!ing; Alexandria, MN) on $23 \mathrm{JUL}$ 2013, NYSM-FRL. staff called and indicated the tesl chemical lot \$401P120724C did not pass initial efficacy testing and it was therefore not used for the study. Marrone Bio Innovations shipped $4 \mathrm{~kg}$ of replacement test chemical (lot TR4660-4-3; run 4, sample 3) to the field location via overnight UPS. The new lot of test chemical was waighed based on the calculated amounts nceded for the proper treatment level. The data sheet with individual samples weights can be found in File Folder 7. A copy of the data sheet is bound in the chemical logbooks and the data were transcribed to the chemical logbooks that were created upon returning to UMESC. Three analytical aliquots were weighed. The first analytical aliquot was spilled and thercfore discarded. A sample of the test materiai will be shipped overnight to NYSM-FRL to verify post-exposure activity.

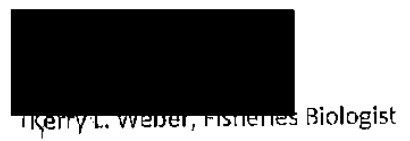


Study Number: AEH-13-PSEUDO-05 ille Folder:

Lab book/pgs:

Reviewed by: Date: vertfied by: ___ _ _ _ _ _ _ _

\section{Test Chemical Stock Preparation}

Test Chemical: Pseudomonos fluroescens strain 145A Test Chemical Lot \#: TR $46699-4-3$ Date Rec'd: $347 V / 3$ Exp. Date:

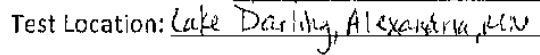
Instruments Used: BHil. 3; BHLL

Weights of Chemical Samples:

\begin{tabular}{|c|c|c|c|c|}
\hline Sample ID & Sample Welght & Comments & Date & Inttlals \\
\hline Anerlutian * 1 & 2.00 & $B x, 1$ of 2 & $25 \pi 43$ & Fen \\
\hline Amblyoul +2 & 2.00 & Bog of a & $\hat{\imath}$ & \\
\hline Sluck 1 & 277.53 & Ban lot 2 Finksore 4 & & \\
\hline Stock 2 & 274.59 & Bug kf 2 , Enilosur. 5 & & \\
\hline Stock 3 & 26.2 .09 & Big lat $2 ;$ Enclusere 2 & & \\
\hline Sinck 4 & 544.73 & Bog if 2 , Enekss-ar i & & \\
\hline Stock 5 & 563.27 & Bro letz; Enclusue? & $\sqrt{1}$ & $\Psi$ \\
\hline Stock 6 & 544.61 & 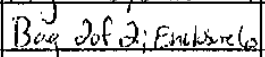 & $25 \times 43$ & 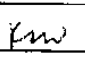 \\
\hline & & & & \\
\hline & & & & \\
\hline
\end{tabular}

NOTE: Chemlcal samples to be stored refrigerated until used for stock preparation.

Stock Solution Preparation:

\begin{tabular}{|c|c|c|c|c|c|c|}
\hline Sample ID & $\begin{array}{c}\text { Dilution } \\
\text { Volume }(\mathrm{mL}) \\
\end{array}$ & $\begin{array}{c}\begin{array}{c}\text { Dilution } \\
\text { Time }\end{array} \\
\end{array}$ & Use & $\begin{array}{c}\text { Exposure } \\
\text { TImee } \\
\end{array}$ & Date & Inltials \\
\hline Ancialyharlyl & 500 & 0500 & Standand une & - & $26+143$ & $k+2$ \\
\hline stock 3 & 2152 & 0600 & End xine 2 & 0625 & $2 / 20 / 13$ & $\operatorname{In}_{n}$ \\
\hline stocke 6 & $215 i$ & 0425 & Endojint 6 & $065^{\circ}$ & $726 / 13$ & $\sqrt{11}$ \\
\hline stoki & 251 & 0705 & Einctyouresel & 0715 & $2 / 2: / 3$ & Sin \\
\hline stixky & 2151 & 0735 & Eractoswe 1 & 0740 & $7 / 26 / 13$ & $J_{i 1} \ldots$ \\
\hline stok $k 2$ & +1.56 & 2750 & Exalosw 5 & $0 \% 00$ & $7 / 26 / 13$ & JaL \\
\hline $5 t a k+5$ & 2152 & $0 \% 10$ & Enclaswin G & $0 \times 20$ & $7 / 26 / 3$ & $J_{i}$ \\
\hline $\operatorname{sinc}(x+2)=3$ & - costro & $2-1$ chosen & mindes of & $20 \mathrm{~cm}$ & $2 / 26 / 13$ & 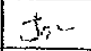 \\
\hline tat loswa $0 \%$ & $-\quad c \cdot 11$ & $2 \pi / 3$ & we milel ed & $3.55 \mathrm{~cm}$ & $-7 / 26 / 17$ & $\operatorname{Tr}$ \\
\hline Eratsuns & $-c \times v$ & $i \cos \theta$ & bure mnigese & $\because, 4 S_{\text {c.n }}$ & $17 / 2<13$ & $J_{1}^{2}$ \\
\hline
\end{tabular}

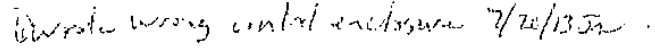

Page 5 of 6 


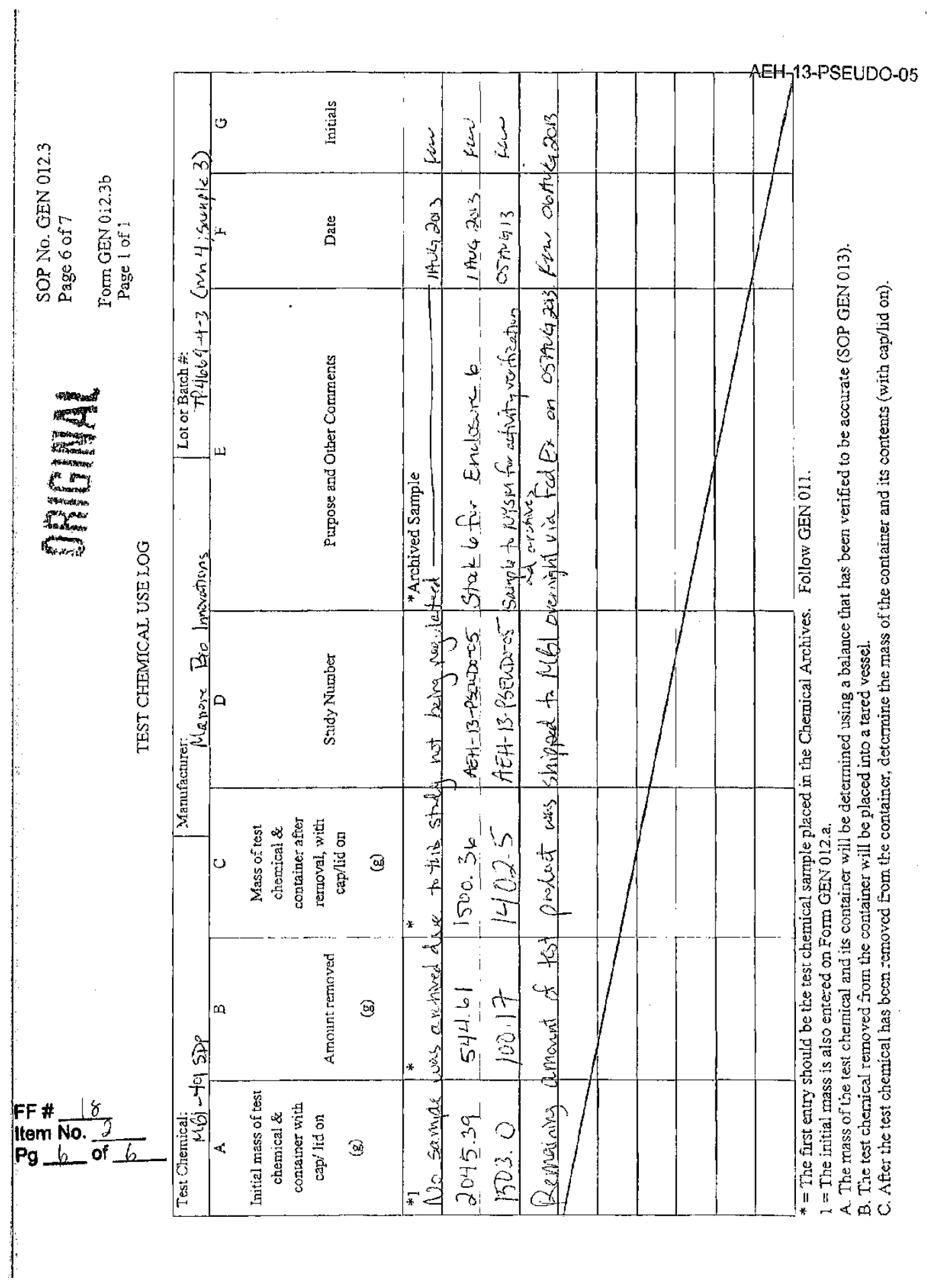




\section{NYSM Post-Treatment Product Validation Assay MBI-401 SDP TR-4669-4-(3)}

(USGS Study \#AEH-13-PSEUDO-05 Field trials at Darling Lake in 2013)

Date product received from USGS: 2013/08/08

Date of start of test: 2013/08/08

AEH-13-PSEUDO-05

BACKGROUND: As standard protocol for the USEPA project, each time a batch of Zequanox product is used in a test a UMESC, a portion of the product is bioassayed by the NYSM to validate toxicity post-treatment.

- MBI-40I SDP TR-4669-4-(3)

- USGS Study \#AEH-13- PSEUDO-05 Field trials at Darling Lake in 2013

PURPOSE: Post-test product validation of MBI-401 SDP TR-4669-4-(3) used in AEH-13-PSEUDO05 Field Trials at Darling I ake in 2013.

MATERIALS AND METHODS:

Preparation of product for testing:

Product was shipped under cold conditions and held in the laboratory refrigerator at $4^{\circ} \mathrm{C}$ until use.

Within 30 min of treatment application, prepare treatment stock of MBI-401 formulated product:

MBI-401 SDP TR-4669-4-(3) (SDP - 50\% active ingredient): $3 \mathrm{~g}$ of the powder was added slowly to a beaker with dilution water with stirring on a stir plate for even suspension and then the total volume was adjusted to $30 \mathrm{ml}$ with dilution water. The suspension was transferred to a $50 \mathrm{ml}$ centrifuge and stored in refrigerator until ready to use. The suspension, when evenly dispersed was $100 \mathrm{mg}$ product $/ \mathrm{ml}$ or $50 \mathrm{mg}$ a.i. $/ \mathrm{ml}$. For $200 \mathrm{ppm}$ a.i. treatments in testing jars, $2 \mathrm{ml}$ were added to each testing jar $(500 \mathrm{ml})$.

Cambridge CF (Standard for Positive Controls):

As an efficacy standard, we used $P f$-CL $145 \mathrm{~A}$ killed $\mathrm{CF}$ that was maintained at $-80^{\circ} \mathrm{C}$ (Cambridge CF). Since its production in 2005 , this material has been valuable as a reference standard. The Cambridge CF was produced in 2005 (2005-0027) in 100-L batches 10, 11 and 12 and E-beamed to kill the cells. The solution, at $110 \mathrm{mg} / \mathrm{mil}$ dry weight, is stored in $1 \mathrm{~cm}$ thick sheets in the Cambridge ultrafreezer at $-80^{\circ} \mathrm{C}$. A section of the sheet was broken off and weighed to determine volume (ca. $1 \mathrm{~g}=1 \mathrm{ml}$ ).

For this bioassay, a positive control suspension was produced on $06 / 18 / 2012$ from the frozen blocks described above and dispensed into multiple $50 \mathrm{ml}$ centrifuge tubes for single-use treatment of bioassays. The dry weight of the material was $68.15 \mathrm{mg} / \mathrm{ml}$; therefore $1.5 \mathrm{ml}$ was added to each testing jar to treat at $200 \mathrm{pp}$.

Mussel collections:

Mussels were scraped from stibstrates (rocks) in the field and brought back to the lab in coolers. Mussels were placed in pint-sized jars and placed in aquaria filled with unchlorinated tap water with aeration and filtration (Whisper filters) in the laboratory at $20^{\circ} \mathrm{C}$. One day p:ior to treatment, mussels werc carefully examined and placed into testing jars and allowed to attach overnight.

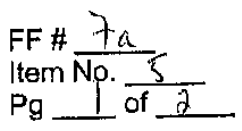


AEH-13-PSEUDO-05

MBI-401 SDP TR4669-4-3 Darling field trials Validation (Darling Field Tr:al)

Mussel collection and handling:

\begin{tabular}{|c|c|c|c|c|}
\hline Species & Collection site & Collection date & Date in lab $\left(20^{\circ} \mathrm{C}\right)$ & Picked for test \\
\hline $\begin{array}{c}\text { Zebra } \\
\text { mussels }\end{array}$ & $\begin{array}{c}\text { Hedges Lake } \\
\text { (Washington County) }\end{array}$ & $07 / 09 / 2013$ & $07 / 09 / 2013$ & $08 / 07 / 2013$ \\
\hline
\end{tabular}

Experimental design:

For validation of efficacy the following treatments were set up:

Zebra mussels ( 25 mussels/jar)

3 - Untreated Control (A, B, C)

3- 200 ppm (a.i.) Cambridge CF Positive Control (Pf-CL145A killed cells) (A, B, C)

$3-200 \mathrm{ppm}$ (a.i.) MBI-401 SDP TR-4669-4-(3) (A, B, C)

Total should be 9 jars.

Testing jar bioassay protocol:

On the day prior to treatment $(08 / 07 / 2013)$ mussels were carefully examined and 25 mussels placed into each testing jar containing ca. $100 \mathrm{ml}$ aerated hard water and allowed to attach overnight. The next morning (08/08/2013), unattached nussels were removed and replaccd with attached mussels from an extra glass Petri dish. Water was replaced with $500 \mathrm{ml}$ fresh aerateci hard water.

After at least one hour, the treatment was applied. The oplical density of each jar was measured in duplicate ( $\mathrm{A}_{660 \text { nn }}$ Genesys Spectrophotometer).

After 24 hrof treatment, mussel mortality was checked and mussels were transferred to square plastic dishes with frcsh aerated hard water. Mortality was checked and recorded each day with water replacements, for an additional 6 days ( 7 days total). On the final day of mortality checks, 20 mussels were measured from the untreated controls using a caliper.

Results:

Mussel length: Zebra mussels $16.63 \pm 3.80 \mathrm{~mm}$.

Optical density of treatments:

\begin{tabular}{|c|c|}
\hline Treatment & Mean $( \pm S D)$ OD $\left(A_{660 \mathrm{~nm}}\right)$ \\
\hline \hline Untreated Control & $0.002 \pm 0.002$ \\
\hline Cambridge CF (Positive Control) & $0.198 \pm 0.009$ \\
\hline Field trial product MBI-401 SDP TR4669-4-(3) & $0.212 \pm 0.008$ \\
\hline
\end{tabular}

Zebra mussel mortality: Mussels were treated in triplicate testing jars $(500 \mathrm{ml})$ at $20^{\circ} \mathrm{C}$ for $24 \mathrm{hr}$ and mortality was recorded for a total of 7 days.

\begin{tabular}{|c|c|}
\hline Treatment & $\begin{array}{c}\text { Mean \% mortality } \\
( \pm \mathrm{SD})\end{array}$ \\
\hline \hline Untreated Control & $1.3 \pm 2.2 \%$ \\
\hline Cambridge CF (Positive Control) & $100.0 \pm 0.0 \%$ \\
\hline Ficld trial product MBI-401 SDP TR4669-1-(3) & $96.0 \pm 6.9 \%$ \\
\hline
\end{tabular}

MOBI-401 SDP TR4669-4-(3) PASSED the post-test bionssay validation (96\% mortality). Untreated control mortality was $1 \%$.

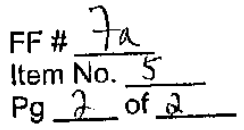




\section{(0) Marrone Mio Innovations $^{m}$}

\section{CERTIFICATE OF ANALYSIS AEH-13-PSEUDO-05}

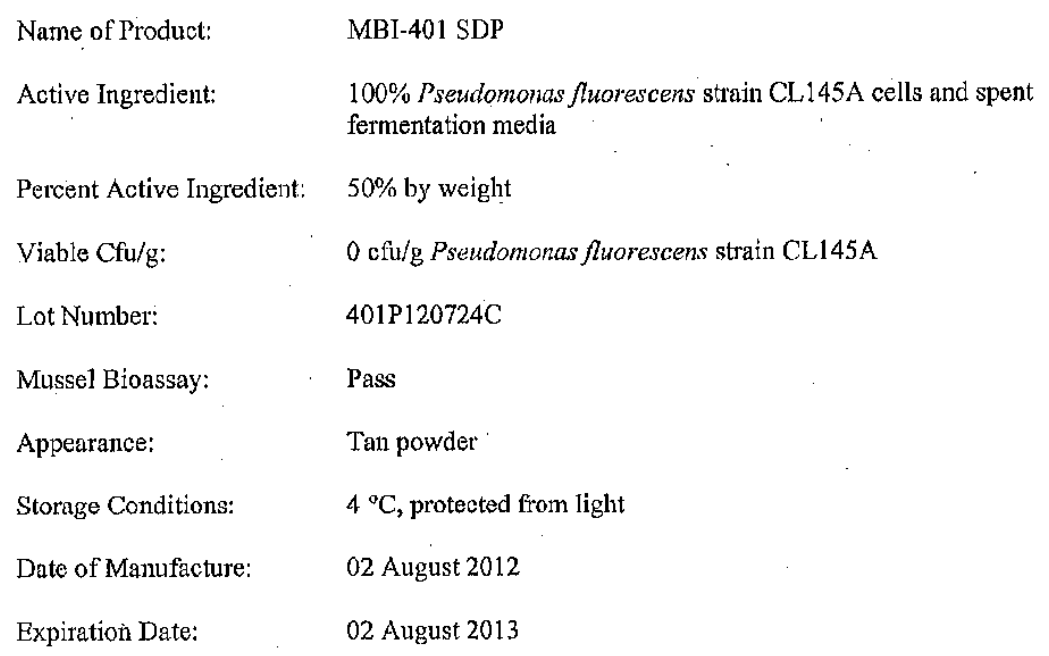

I hereby certify that the above information is true and correct.

Quality Control:
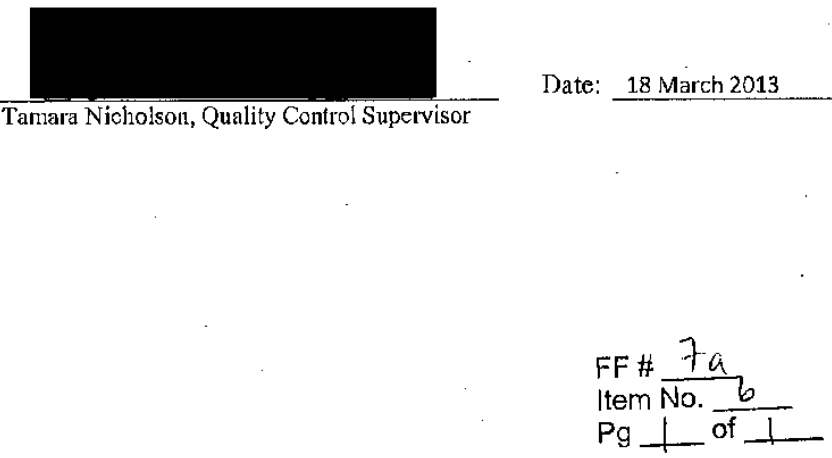


\begin{tabular}{|c|c|c|c|}
\hline Study Number: AFH-1. & E.UDO-05 & Revlewed by: Why & Date: $18 \mathrm{FEB}) \mathrm{A}$ \\
\hline File Folder: $7 a$ & Lab book/pgs: e-log papte 89 & Verifled by: $\sqrt{5}$ & Date: $2 / 1<_{2} / / c_{1}$ \\
\hline
\end{tabular}

\section{Test Chemical Stock Preparation}

Test Chemical: Pseudomonas fiuroescens strain 145A

Test Chemical Lot \#: 401 P120724C

Date Rec'd: $\{1020013$ Exp. Date:02 Hu 92013

Test Location: Lake Dauling, Alexandrio, MN Instruments Used:BALl - Sar lunus Top loader

hudel LC340.of (SN30.333\%20)

Weights of Chemical Samples:

BAx 2-Sarton's Analytxert

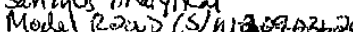

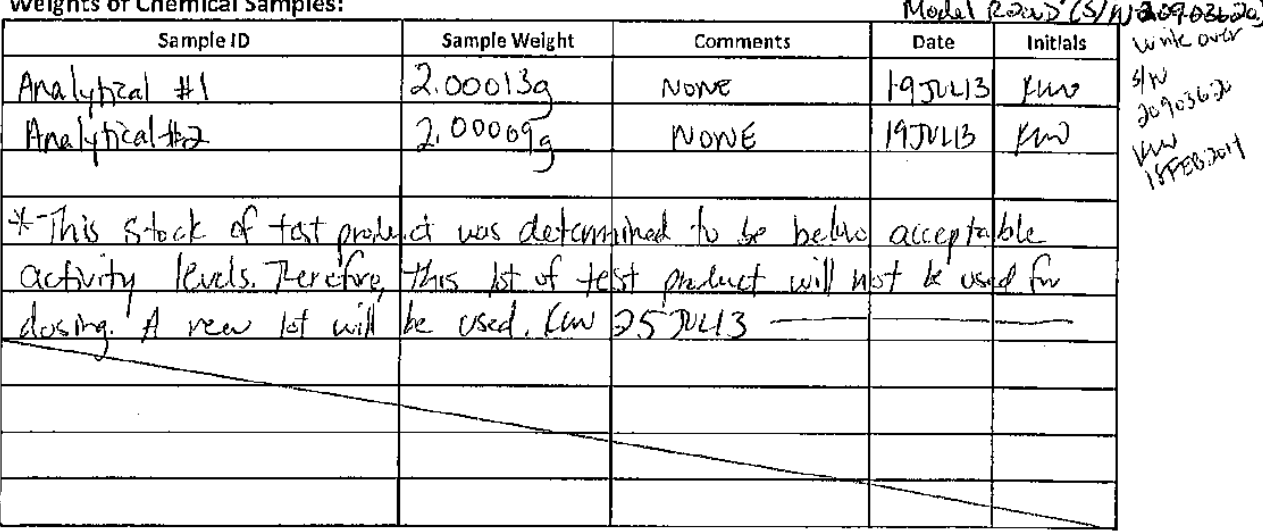

NOTE: Chemical samples to be stored refrigerated until used for stock preparation.

Stock Solution Preparation:

\begin{tabular}{|c|c|c|c|c|c|c|}
\hline Sample ID & $\begin{array}{c}\text { Dllution } \\
\text { Volume }(m 1)\end{array}$ & $\begin{array}{c}\text { Dilution } \\
\text { Time }\end{array}$ & Use & $\begin{array}{c}\text { Exposure } \\
\text { TIme }\end{array}$ & Date & Initials \\
\hline & & & & & & \\
\hline & & & & & & \\
\hline & & & & & & \\
\hline & & & & & & \\
\hline & & & & & & \\
\hline & & & & & & \\
\hline & & & & & & \\
\hline & & & & & & \\
\hline & & & & & & \\
\hline
\end{tabular}

FF H Ta.

ltem No. 7

$\mathrm{Pg} \_1$ of 1 


\section{NYSM Pre-Treatment Product Validation Assay MBI-401 SIPP120724C (FAILED)}

(USGS Study \#AEH-13-PSEUDO-05 Field trials at Darling Lake in 2013)

Date product received from MBI: 2013/07/10

Date of start of test: $2013 / 07 / 10$

AEH-13-PSEUDO-05

BACKGROUND: As standard prolocol for the USEPA project, each time a batch of Zequanox product is used in a test a UMESC, a portion of the product is bioassayed by the NYSM to validate toxicity pre-treatment

- MBI-401 SDP 120724C

- USGS Study \#AEH-13- PSEUDO-05 Field trials at Darling Lake in 2013

PURPOSE: Pre-test product validation of MBI-401 SDPP $120724 \mathrm{C}$ to be used in AEH-13-PSEUDO05 Field Trials at Darling Lake in 2013.

\section{MATERIALS AND METHODS:}

Preparation of product for testing:

Product was shipped under cold conditions and held in the laboratory refrigerator at $1^{\circ} \mathrm{C}$ until use. Within 30 min of treatment application, prepare treatment stock of MBI-40I formulated product:

MBI-401 SDP P120724C (SDP - 50\% active ingredient): $3 \mathrm{~g}$ of the powder was added slowly to a beaker with dilution water with stirring on a stir plate for even suspension and then the total volume was adjusted to $30 \mathrm{ml}$ with dilution water. The suspension was transferred to a $50 \mathrm{ml}$ centrifuge and stored in refrigerator until ready to use. The suspension, when evenly dispersed was $100 \mathrm{mg}$ product $/ \mathrm{ml}$ or $50 \mathrm{mg}$ a.i./.ml. For $200 \mathrm{ppm}$ a.i. treatments in testing jars, $2 \mathrm{ml}$ were added to each testing jar $(500 \mathrm{ml})$.

Cambridge Cf (Standard for Positive Controls):

As an efficacy standard, we used $P f$-CL145A killed CF that was maintained at $-80^{\circ} \mathrm{C}$ (Cambridge $C F$ ). Since its production in 2005 , this material has been valuable as a reference standard. The Cambridge CF was produced in 2005 (2005-0027) in 100-L batches 10,11 and 12 and E-beamed to kill the cells. The solution, at $110 \mathrm{mg} / \mathrm{ml}$ dry weight, is stored in $1 \mathrm{~cm}$ thick sheets in the Cambridge ultrafreezcr at $-80^{\circ} \mathrm{C}$. A section of the sheet was broken off and weighed to determinc volume (ca. $1 \mathrm{~g}=1 \mathrm{ml}$ ).

For this bioassay, a positive control suspension was produced on $06 / 18 / 2012$ from the frozen blocks described above and dispensed into multiple $50-\mathrm{ml}$ centrifuge tubes for single-use treatment of bioassays. The dry weight of the material was $68.15 \mathrm{mg} / \mathrm{ml}$; therefore $1.5 \mathrm{ml}$ was added to each testing jar to treat at $200 \mathrm{ppm}$.

Mussel collections:

Mussels were scraped from substrates (rocks) in the field and brought back to the lab in coolets. One day prior to treatment, mussels were carefully examined and placed into testing jar's and allowed to attach overnight.

Mussel collection and handling:

\begin{tabular}{|c|c|c|c|c|}
\hline Spccics & Collection site & Collcction date & Date in lab $\left(20^{\circ} \mathrm{C}\right)$ & Picked for test \\
\hline \hline $\begin{array}{c}\text { Zebra } \\
\text { mussels }\end{array}$ & $\begin{array}{c}\text { Hedges Lake } \\
\text { (Washington County) }\end{array}$ & $07 / 09 / 2013$ & $07 / 09 / 2013$ & $07 / 09 / 2013$ \\
\hline
\end{tabular}

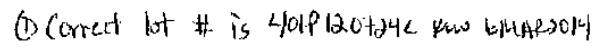

FF \# 70 Item No. $\frac{8}{8}$ $\mathrm{Pg} \perp$ of $\partial$ 
Experimental design:

For validation of efficacy the following treatments were set up:

AEH-13-PSEUDO-05

Zebra mussels (25 mussels/jar):

3 - Untreated Control (A, B, C)

3 - 200 ppm (a.i.) Cambridge CF Positive Control ( $P f$-CL145A killed cells) (A, B, C)

$3-200 \mathrm{ppm}$ (a.i.) MBI-401 SDPP120724C (A, B, C)

Total should be 9 jars.

Testing jar bioassay protocol:

On the day prior to treatment $(07 / 09 / 2013)$ mussels were carefully examined and 25 mussels placed into each testing jar containing ca. $100 \mathrm{ml}$ aerated hard water and allowed to attach overnight. The next morning (07/10/2013), unattached mussels were removed and replaced with attached mussels from an extra glass Petri dish. Water was replaced with $500 \mathrm{ml}$ fresh acrated hard water.

After at least one hour, the treatment was applied. The optical density of each jar was measured in duplicate ( $\mathrm{A}_{660 \mathrm{n} n \mathrm{I}}$ Genesys Spectrophotometer).

After $24 \mathrm{hr}$ of treatment, mussel mortality was checked and mussels were transferred to square plastic dishes with fresh aerated hard water. Mortality was checked and recorded each day with water replacements, for an additional 14 days ( 15 cays tota1). This bioassay was terminated after $>10 \%$ mortality was achieved in the untreated controls. On the final day of mortality checks, 20 mussels were measured from the untreated controls using a caliper.

\section{Results:}

Mussel length: Zebra mussels $18.59 \pm 3.05 \mathrm{~mm}$.

Optical densily of treatments:

\begin{tabular}{|c|c|}
\hline Treatment & Mean $( \pm S D)$ OD $\left(A_{660 \mathrm{~mm}}\right)$ \\
\hline \hline Untreated Control & $0.003 \pm 0.004$ \\
\hline Cambridgc CF (Positiyc Control) & $0.200 \pm 0.003$ \\
\hline Field trial product MBI-40LLDDP P120724C & $0.246 \pm 0.003$ \\
\hline
\end{tabular}

Zebra mussel mortality: Mussels were treated in triplicate testing jars $(500 \mathrm{ml})$ at $20^{\circ} \mathrm{C}$ for $24 \mathrm{hr}$ and mortality was recorded for a total of 15 days.

\begin{tabular}{|c|c|}
\hline Treatment & $\begin{array}{c}\text { Mean \% mortality } \\
( \pm S D)\end{array}$ \\
\hline \hline Untreated Control & $10.6 \pm 8.4 \%$ \\
\hline Cambridge CF (Positive Control) & $100.0 \pm 0.0 \%$ \\
\hline Ficld trial product MBI-40 SSDP P120724C & $68.0 \pm 8.0 \%$ \\
\hline
\end{tabular}

MBI-401 SDR'P120724C FAILED the pre-test validation bioassay ( $68 \%$ mortality). Untreated control mortality was $11 \%$. Subsequent to this product's failure, MBI sent us a new lot (TR4669-4-(3)) for use in the Darling Lake field triak. Lot TR4669-4-(3) passed validation.

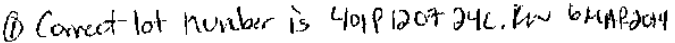

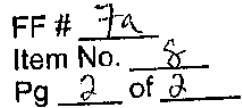




\title{
CHEMICAL LOG BOOK
}

\author{
MBl-401 SDP
}

Marrone Bio Innovations

Davis, California

\section{LOT NUMBER: 401P120724C}

Container 1 of 1

$$
\begin{aligned}
& \text { FF \# } \frac{15}{\text { Nom No. } \frac{3}{4}} \\
& \mathrm{Pg} \perp 1
\end{aligned}
$$




\section{SIGNATURE PAGE}

AEH-13-PSEUDO-05

Al: personnel making an entry in this log must fill out the form below in accordance with SOP GEN 009.

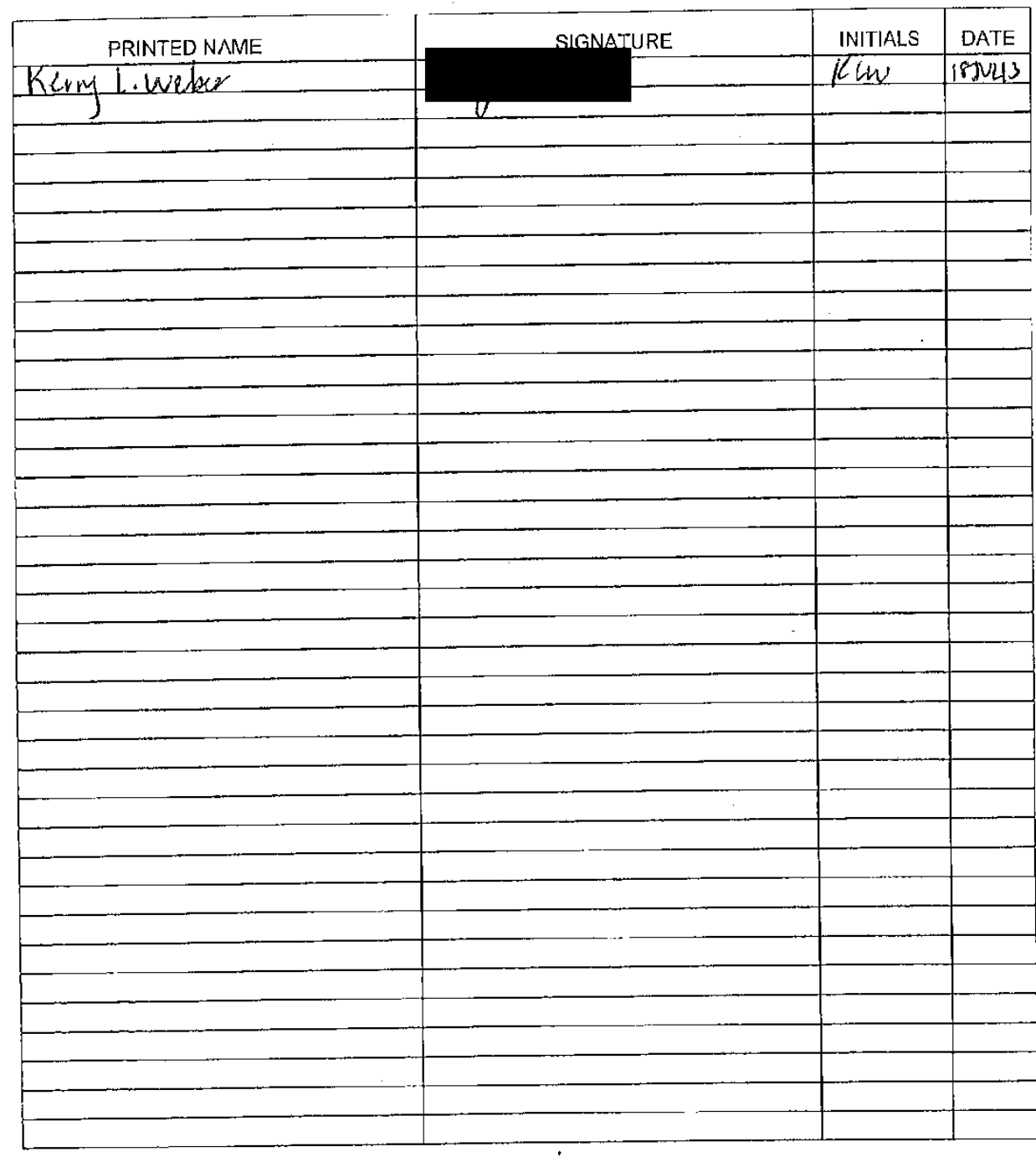

"This Use and Maintenance Log Book has been inspected and found to be in compliance with SOP GEN 009. Inspected and sealed on $\frac{5 / \mathrm{C} / 8,2043}{\text { Date }}$ by Quality Assurance thit

Page 2 of 4 
SOP No. GEN 012.3

Page 5 of 7

TEST CEEMICAL DATA YORM

Form GEN 012.3a

Page 1 of 1

Test Chemical (Chemical Name) MbL-401 SDP

Circle one: Test Atticlo Control Asticle

Trade Name of Test Chemical (Syronyms) if - L L / $45 A$

Source of Test Chemical (Manufacturer) Marne Bo Invevatoins

storage Location Locked refingerator $f_{0} w_{2 n} 2$

Date Received $18 \pi \mathrm{VL}_{2} \mathrm{al} 3$ Date Opened

Expiration Date 2 Aula 2013

( $S$ years unless othierwise stated)

Tesl Chemical Lot Number $401 P 120724 \mathrm{C}$ Purity of Chemical $\quad 50 \%$

Amount of Test Chemical Available or Received (if known)

Initial Mass (with cover on) of Test Chemical and Container $\quad 5150.39$

Characterization of Test Chemical; color $\frac{T a h n}{\text { Physical State: liquid _. solid } X}$

Chemical Abstract Service Number _ N

Solid form: powcer $X$ crystal _ pellet

Manufacturer Certificnte of Analysis Yes $X$ No

Additional Comments aboul the Test Chemical:

Sample Placed in Archives: Yes _ No X (Entries should also be made on Yorm GEN 0 12.b)

Archive Location ..._______ Material Safety Data Sheet Available: Yes $X$ No

Signature of Study Director or dlesignee iniliating Test Chemical Use Log and date:

Signature

Date $18+4213$

Page 3 of 4 


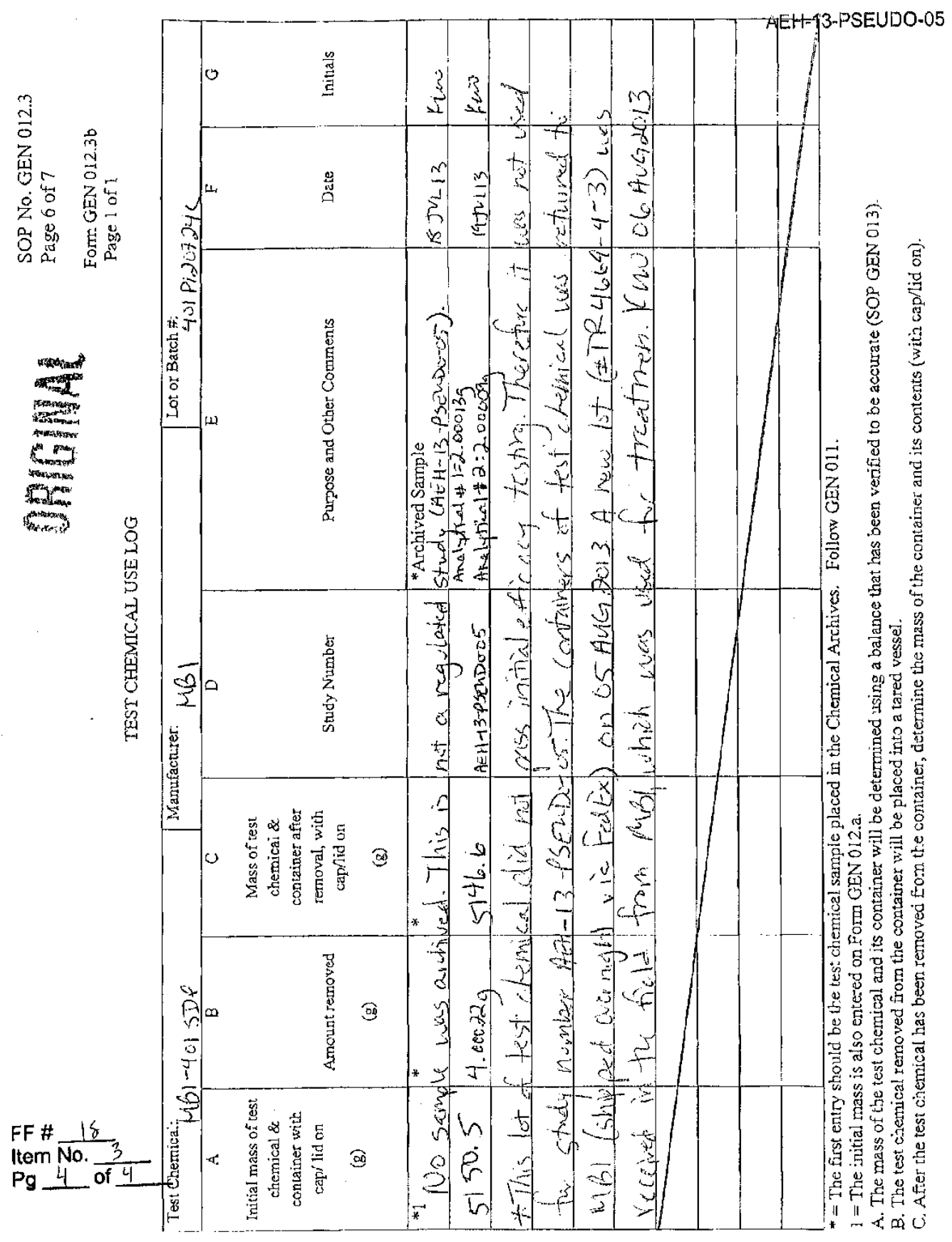




\section{Appendix 5. Test Animal Information}

\begin{tabular}{clcc}
\hline $\begin{array}{c}\text { Item } \\
\text { number }\end{array}$ & \multicolumn{1}{c}{ Item description } & $\begin{array}{c}\text { Number } \\
\text { of } \\
\text { pages }\end{array}$ & $\begin{array}{c}\text { Report } \\
\text { page } \\
\text { number }\end{array}$ \\
\hline 1 & Special permit (No. 18804) for taking and possession of mussels & 2 & 127 \\
2 & Prohibited Invasive Species Permit (No. 290) & 1 & 129 \\
3 & Collection Report for Special Permit 18804 & 2 & 130 \\
4 & Mussels Species Identification datasheet & 9 & 132 \\
5 & Unionid Mussel Weight and Length - Data Summary & 6 & 141 \\
6 & Zebra Mussel Lengths Pre-exposure Assessment - Data Summary & 10 & 147 \\
7 & Zebra Mussel Lengths Post-exposure Assessment - Data Summary & 11 & 157 \\
\hline
\end{tabular}




\title{
STATE OF MINNESOTA \\ DEPARTMENT OF NATURAL RESOURCES \\ DIVISION OF ECOLOGICAL AND WATTCR RESOURCES \\ 500 LAFAYTTTE ROAD, BOX 25 \\ ST. PAUI, MN 551.55
}

SPECIAL PERMIT NO. 18804

(Taking and poskession of mussels)

July 22, 2013

TO WHOM IT MAY CONCFRN:

Under the authority of Minn. Statutes, Section 97A.401 and Minn. Rules, Part 6212.1400, permission is hereby granted to:

\author{
James Linoma \\ C.S. Geologleal Survey \\ Lpper Midwest Envirommental Sciences Center \\ 2630 Fanta Reed Road \\ LaCrosse, WI 54603
}

to collect and possess up to 250 individuais of each of the following species: Fatmucket (Lampsitis siliquoidea), Fragile Papershcll (Leptodea fragilis), and Giant Floater (Pyganodon grandis) for the purpose of research, subject to the following conditions:

1. Kerry Wcbcr, Jeremy Wise, Hugh McMath, and Denise Mayer are considered Subpermittees under this permit. 'The Permittee and Subpermittees must possess a copy of this permit while conducting permitted activities. Assistants may carry out activities allowed by this permit under the direct on-site supervision of the Permittee or Subpermittees.

2. Collection of mussels will be limited to Lake Darling, Douglas County. Collection will be conducted only by hand via. wading, snorkeling, or scuba diving.

3. Any mussels retained under this permit will be destroyed upon completion of research. No mussels will be returned to the wild. Humane mothods will be used in taking tissue samples and/or euthanizing the mussels.

4. Before conducting work under this permit in state waters, the Permittee must decontaminate any ecluipment that has been used in any waters in Minncsota or other locations that are infested with aquatic invasive species. When leaving any waters, the Permittee must remove any aquatic plants and animals (e.g., snails, zooplankton) from equipment, and drain water from watercraft and all equipment used. If permitled activities :equire live transport, the Permittee must either bring uninfested surface water or ground waler to the collection site for specimen transportation, or obtain a Permit to Appropriate/Transport Infested Waters. If permilted activities include the collection of any prohibited invasive species, the Permittec must obtain a Probibited Invasive Species Permit. See the attached for further information on aquatic invasive species and related permits.

5. It is the Permittce's responsibility to accuire any additional permits that may be required related to the collection, possession, or transportation of mussels under this perıuit, invluding any permits required to transport specimens across state or international borders. This permit does not allow collection of any endangered or threateneć specjes.

6. A report must be submitted by mail or cmail to the Minnesota Findangered Species Coordinator (Richard J. Baker, Division of Ecological and Water Resources, Box 25, 500 Lafayette Road, St Paul, MN 55155; 651/259-5073; richard.baker(alstate, nnn.us) by January 31, 2014 describing the results of work authorized under this permit. Copies of other reports and publications resulting from permitted aclivities shall be submitted as they become available.

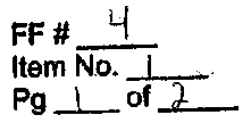


MNDNR Special Permit No. 18804

Permittee: l,uoma

AEH-13-PSEUDO-05

\section{Page 2}

This permit is effective inmedintely, and through December 31,2013 , but may be revoked at any time.

Failure to sulbinisererts olideliver specimens as required may be grounds for denial of future permit requesis.

James Japs

$7 / 22 / 2013$

EMPS Section Mránagcr, Division of Ecological and Water Resources

Date

I hereby certify that $T$ have read and understand the provision of this permit and understand that this permit is not walidumlass it is signed by me.

Permittye

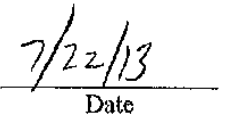

C: $\quad$ Richard J. Baker, Endangered Species Coordinator, Div. Ecological and Water Resources Peter Buesseler, Regional Manager, Div. Ecological and Water Resources

Stacey Sharp, Regional Manager, Div. Enforcement

Christine IIerwig, Regional Nongame Specialist, Div. Ecological and Water Resources Mike Davis, Malacologist, Div. Ecological and Watcr Resources

Bernard Sietman, Malacologist, Div. Ecological and Water Resources

Don Pereira, Reseaxch Manager, Div. Fish and Wildlife

Gary Montz, Research Scientist, Div. Ecological and Water Resources Permit Clerk, Div. Fish and Wildlife

FF \# 4

Item No. I

$\mathrm{Pg}$ \& of 2 


\section{Prohibited Invasive Species Permit \\ MN Department of Natural Resources, Division of Ecological and Water Resources} 1200 Warner Road, St. Paul Minnesota 55106-6793

Permiftee: James A. Luoma and Designeos

USGS-Upper Midwest Environmental Sciences Center

2630 Fanta Reod Road

La Crosse, W/ 54603

Location: Lake Darling (DOW\# 21008000)

Specles: Zebra mussel (Drelssena polymorpha)

Scope:

Permlssion is hereby granted to possess and transport zebra mussels that may be part of a research project on Lake Darling In Douglas County. Zebra mussels can be used to calculate populations that are adhering to native mussels. The permittee and all designees shail have a copy of this permit in their possession while conducting authorlzed activities.

\section{Condillons:}

Confinement and Disposal: The permiltee shall take all reasonable measures to prevent the spread of zebra mussels to other waters during the actlvitles authorlzed by thls permit. All materlals potentlally contalning zebra mussels shall be transported over the road In covered, water-tight containers to a laboratory setting.

Decontamination of Equipment: All equlpment used in movement of potentially contaminated materlals shall be decontaminated at an onsite locatlon as outlined in the permlt application. When not transporting materlals between wher, equipment shall be clean and free of infested material prior to leaving the slte.

Inspections: Facilities for hoiding prohibited Invaslve specles of wild animals and aquatlo plants for research, exhibition, education, control, or disposal purposes identlifed in this permit are subject to inspection at any reasonable time by the commissioner of naturat resources or a designated employee.

Revocation: Permits lssued under this part may be revoked by the commissioner if the permittee does not comply with the conditions of thls permit or Minnesola rules 6216.0100 to 6216.0600 , or if revocation is necessary to protect the interest of the public, to protect native plant and animal populations in the state, or to otherwise protect
the state's natural resources.

Transferabllity: This permit is not transferable.

Disclaimer of Llabillty: No llability is assumed by the State or any of its officers, egents, or employees by lssuance of this prohiblted invasive species permit, or for any act or omission of the permittee, or by any prohlbled
specles in possession of the permittes.

Expiration date: This permlt is valld from July 22, 2013 through September 15, 2013

Issued by:

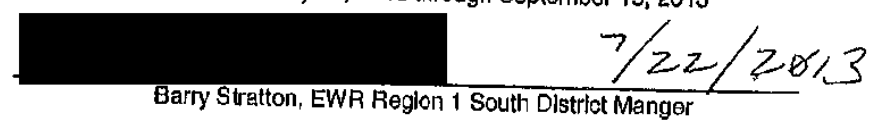

I hereby cerlily that thave read and understand the provisions of this permil and understand this permit is not valld untoss stgned by permitie

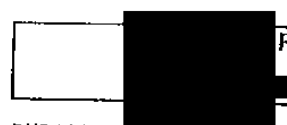

c:

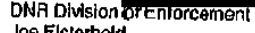
Joe Elsterhold

\section{$\square$}

miltee Signalure
Capt. Greg Salo Mark Panwetter
$1 / 2.2 / 3$

Bary Stratton
Ann Plerce

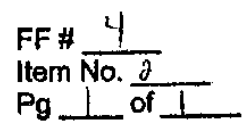




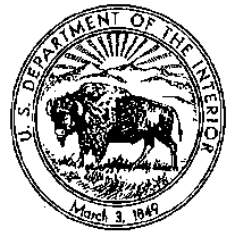

\author{
United States Department of the Interior \\ U.S. GEOLOGICAL SURVEY \\ Biological Resources Division \\ Upper Midwest Environmental Sciences Center \\ 2630 Fanta Reed Road \\ La Crosse, Wisconsin 54603
}

November 18,2013

Richard Baker, Minnesota Endangered Species Coordinator

Minnesota Department of Natural Resources

AEH-13-PSEUDO-05

Division of Ecological and Water Resources

Box 25

500 Lafayette Road

St. Paul, MN 55144

RF: Collection Report for Special Permit 18804

Dear Mr. Baker:

Below you will find a coflection and disposition report of activities conducted under special permit number 18804, issued to the James Luoma, USGS IJpper Midwest Environtnental Sciences Center on July 22, 2013.

If you need additional information regarding this report, please contact myself at 608.781 .6391 or (jluoma@usgs.gov). Thank you for your assistance with this permit process.

Sincerely,

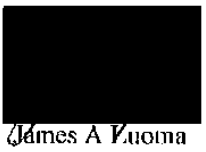

Research Fisheries Biologist

FF \# 4

Item No. $\frac{3}{2}$

cc: Randy Hines

Collection, Use and Disposition Report. Special Pernil \#. 18804 


\begin{tabular}{|c|c|c|c|c|}
\hline \multicolumn{5}{|c|}{$\begin{array}{c}\text { Special Permit \# 18804, Report of collection, use and disposition } \\
\text { U.S. Geological Survey } \\
\text { Upper Midwest Environmental Sciences Center } \\
2630 \text { Fanta Reed Road } \\
\text { LaCrossc, WI 54603 }\end{array}$} \\
\hline Species & Source & $\begin{array}{c}\text { Number } \\
\text { of } \\
\text { Animals }\end{array}$ & Use & Disposítion \\
\hline L. siliquoidea & $\begin{array}{l}\text { Lake } \\
\text { Darling }\end{array}$ & 229 & L. Darling Zequanox exposures & Euthanized after assessment \\
\hline L. siliquoidea & $\begin{array}{l}\text { Lake } \\
\text { Darling }\end{array}$ & 1 & L. Darling Zequanox exposures & $\begin{array}{l}\text { Lnrecovered, identification from } \\
\text { plotograph }\end{array}$ \\
\hline L. fragilis & $\begin{array}{l}\text { Lake } \\
\text { Darling }\end{array}$ & 12 & L. Darling Zecuanox exposures & Euthanized after assessment \\
\hline L. fragilis & $\begin{array}{l}\text { Lake } \\
\text { Darling }\end{array}$ & 1 & L. Darling Zequanox exposures & $\begin{array}{l}\text { Unrecovered, identification from } \\
\text { photograph }\end{array}$ \\
\hline
\end{tabular}

AEH-13-PSEUDO-05

FF \# 4

item No. 3

$\mathrm{Pg} 2$ of 2

Collection, Lse and Disposition Report. Special ?ermit $\div 18804$ 
Study Number: AEH-13-PSEUDO-05 Flle Folder: $7 \mathrm{~b}$

Lab book/pgs: t-log pang $\partial$

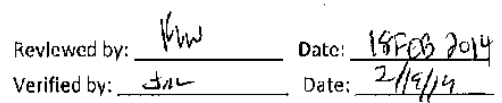

\section{Mussel Species Identification}

\begin{tabular}{|c|c|c|c|c|c|}
\hline \multicolumn{3}{|c|}{$\begin{array}{l}\text { Exposure Date: } 26 \text { JUL } 2013 \\
\text { Test Chemical: Pf-CL145A }\end{array}$} & \multicolumn{3}{|c|}{$\begin{array}{l}\text { Test Location: Lake Darling, Alexandria, MN } \\
\text { Lot \#: TR4669-4-(3) }\end{array}$} \\
\hline $\begin{array}{c}\text { Enclosure } \\
\text { ID } \\
\end{array}$ & Tag ID & $\begin{array}{l}\text { Mussel } \\
\text { Species }\end{array}$ & Comments & Date & Initial \\
\hline 1 & C379 & FAM & . & ispowis & $\not 12$ \\
\hline 1 & C358 & FAM & & 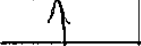 & A \\
\hline 1 & $\mathrm{C} 414$ & $P A M$ & & & \\
\hline 1 & $\mathrm{C} 237$ & $P A M$ & & & \\
\hline 1 & C221 & $F P M$ & Dend dum assossinent & & \\
\hline 1 & $\mathrm{C} 312$ & FAM & Dead durith asigesswent & & \\
\hline 1 & $\mathrm{C} 292$ & FAM & J & & \\
\hline 1 & $\mathrm{C} 350$ & FAM & & & \\
\hline 1 & $C 380$ & FAM & & & \\
\hline 1 & $\mathrm{C} 233$ & PAM & & & \\
\hline 1 & C377 & FAM & . & & \\
\hline 1 & $\mathrm{C} 424$ & FAM & & & \\
\hline 1 & C31. & FAM & & & \\
\hline 1 & $\mathrm{C} 263$ & FAM & & & \\
\hline 1 & $\mathrm{C} 363$ & FPS & & & \\
\hline 1 & C309 & FAM & & & \\
\hline 1 & C208 & Fner & & & \\
\hline 1 & $\mathrm{C} 228$ & Fapy & & & \\
\hline 1 & $\mathrm{C} 266$ & EAM & & & \\
\hline 1 & C195 & PPTM & & & \\
\hline 1 & $\mathrm{C} 360$ & FAR & & & \\
\hline 1 & $\mathrm{C} 359$ & $\angle A A M$ & Dead duning assessyment & & \\
\hline 1 & C302 & FNM & y & & \\
\hline 1 & $\mathrm{C} 207$ & EPS & & & \\
\hline 1 & C345 & PAM & & & \\
\hline 1 & $\mathrm{C} 245$ & FAM & & $\sqrt{\prime}$ & $\sqrt{2}$ \\
\hline 1. & $\mathrm{C} 329$ & FAM & & 18 nor 13 & Haw \\
\hline
\end{tabular}

FF $7 \mathrm{7b}$

Item No.

$\mathrm{Pg} \perp$ of 9 


\begin{tabular}{|c|c|c|}
\hline Study Nu mber: AEH-13-PSEUDO-05 & Reviewed by: KNW & Date: ISFEB 2014 \\
\hline File Folder: $7 l_{2}$ & Verifted by: $\sqrt{3} \mathrm{~L}$ & Date: $2 / 11 / / 4$ \\
\hline
\end{tabular}

Lab book/pgs: e-log pige 21

\section{Mussel Species Identification}

\begin{tabular}{|c|c|c|c|c|c|}
\hline \multicolumn{3}{|c|}{$\begin{array}{l}\text { Exposure Date: } 26 \text { JUL } 2013 \\
\text { Test Chemical: Pf_CL1.4.5A }\end{array}$} & \multicolumn{3}{|c|}{$\begin{array}{l}\text { Test Location: Lake Darling, Alexandria, MN } \\
\text { Lot \#: TR4669-4-(3). }\end{array}$} \\
\hline \begin{tabular}{|c|} 
Enclosure \\
ID \\
\end{tabular} & Tag ID & $\begin{array}{l}\text { Mussel } \\
\text { Species }\end{array}$ & Comments & Date & Initials \\
\hline 2 & $\mathrm{C} 321$ & Eptin & & $15 k 013$ & Vla \\
\hline 2 & $\mathrm{C} 276$ & $F A M$ & & $\hat{A}$ & 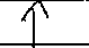 \\
\hline 2 & $\mathrm{C} 249$ & & & & \\
\hline 2 & C310 & & & & \\
\hline 2 & $\mathrm{C} 242$ & & & & \\
\hline 2 & $C 406$ & & & & \\
\hline 2 & $\mathrm{C} 375$ & & & & \\
\hline 2 & $\mathrm{C} 232$ & & & & \\
\hline 2 & $\mathrm{C} 436$ & & Deal durim arsessivere) & & \\
\hline 2 & $C 293$ & & ) & & \\
\hline 2 & $\mathrm{C} 419$ & & & & \\
\hline 2 & $\mathrm{C} 342$ & & & & \\
\hline 2 & $\mathrm{C} 304$ & PAM & & & \\
\hline 2 & $\mathrm{C} 187$ & PATM & & & \\
\hline 2 & $\mathrm{C} 332$ & FAM & & & \\
\hline 2 & $\mathrm{C} 217$ & & & & \\
\hline 2 & $\mathrm{C} 203$ & PPM & & & \\
\hline 2 & C331 & & & & \\
\hline 2 & $\mathrm{C} 298$ & & & & \\
\hline 2 & $\mathrm{C} 317$ & $=A M$ & & & \\
\hline 2 & $\mathrm{C} 408$ & PAM & & & \\
\hline 2 & $\mathrm{C} 188$ & & & & \\
\hline 2 & $\mathrm{C} 395$ & An & Dead durity assessiment & & \\
\hline 2 & $\mathrm{C} 410$ & $P M^{M}$ & $j$ & & \\
\hline 2 & $\mathrm{C} 278$ & & & & \\
\hline 2 & $\mathrm{C} 219$ & & & $\sqrt{i}$ & $\sqrt{\prime}$ \\
\hline 2 & $\mathrm{C} 235$ & Efim & & 18 turvis & Kiw \\
\hline
\end{tabular}

Page 2 of 9 
Study Number: AEH-13-PSEUDO-05 File Folder: $7 !$

lab book/pgs: e-lig page $x$
Reviewec by: hw

Verifted by: Jis vate: is Fer zolu Date: $2 / / 4 / / 4$

Mussel Species Identification

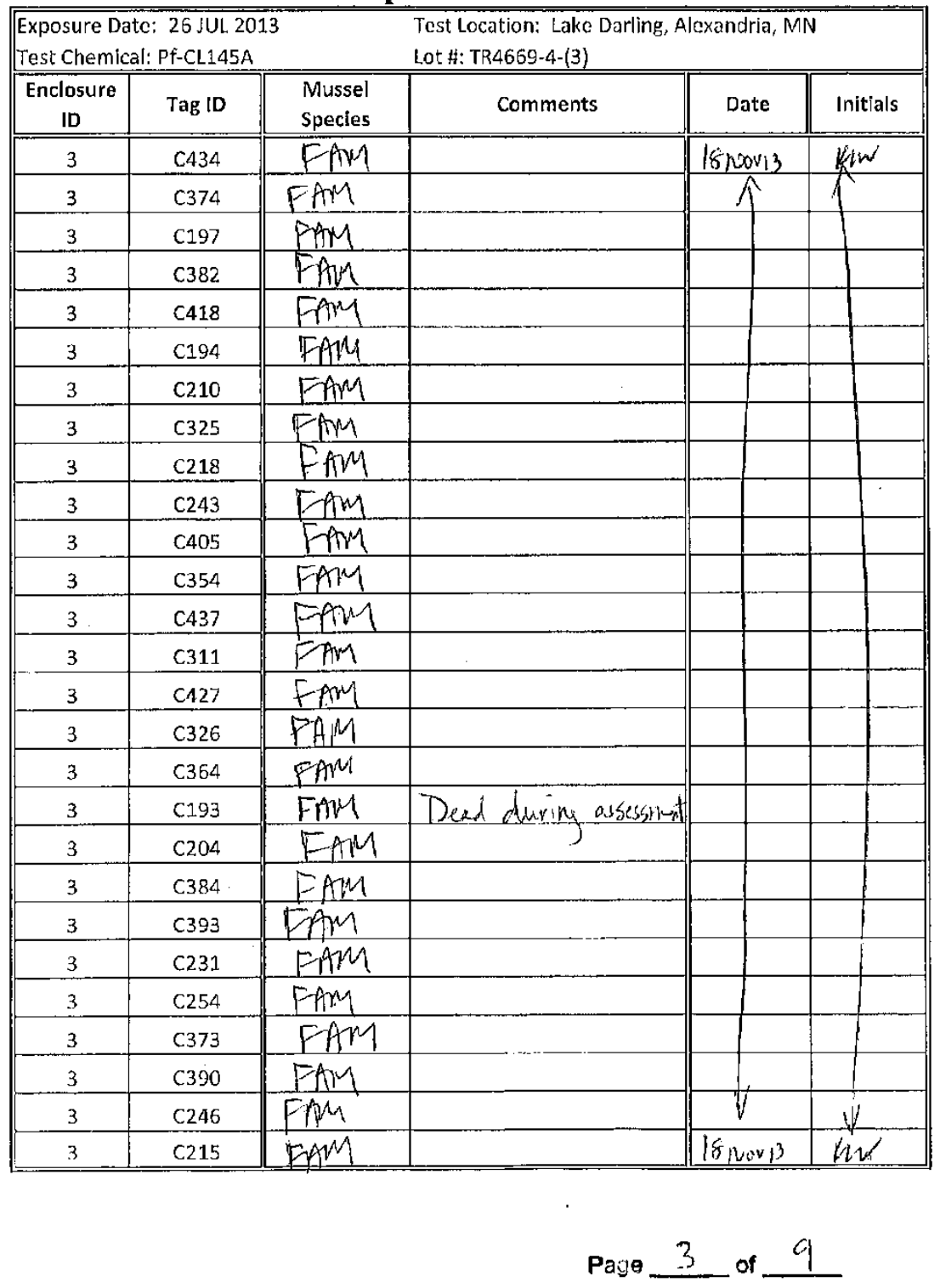


Study Number: AEII-13-PSEUDO-05 File Folder: 76

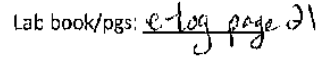

Reviewed by: $1 w^{2}$ Date: 1850 2014

Verlfled by: $\sqrt{n}$ Date: $2 / 1 / 14$

\section{Mussel Species Identification}

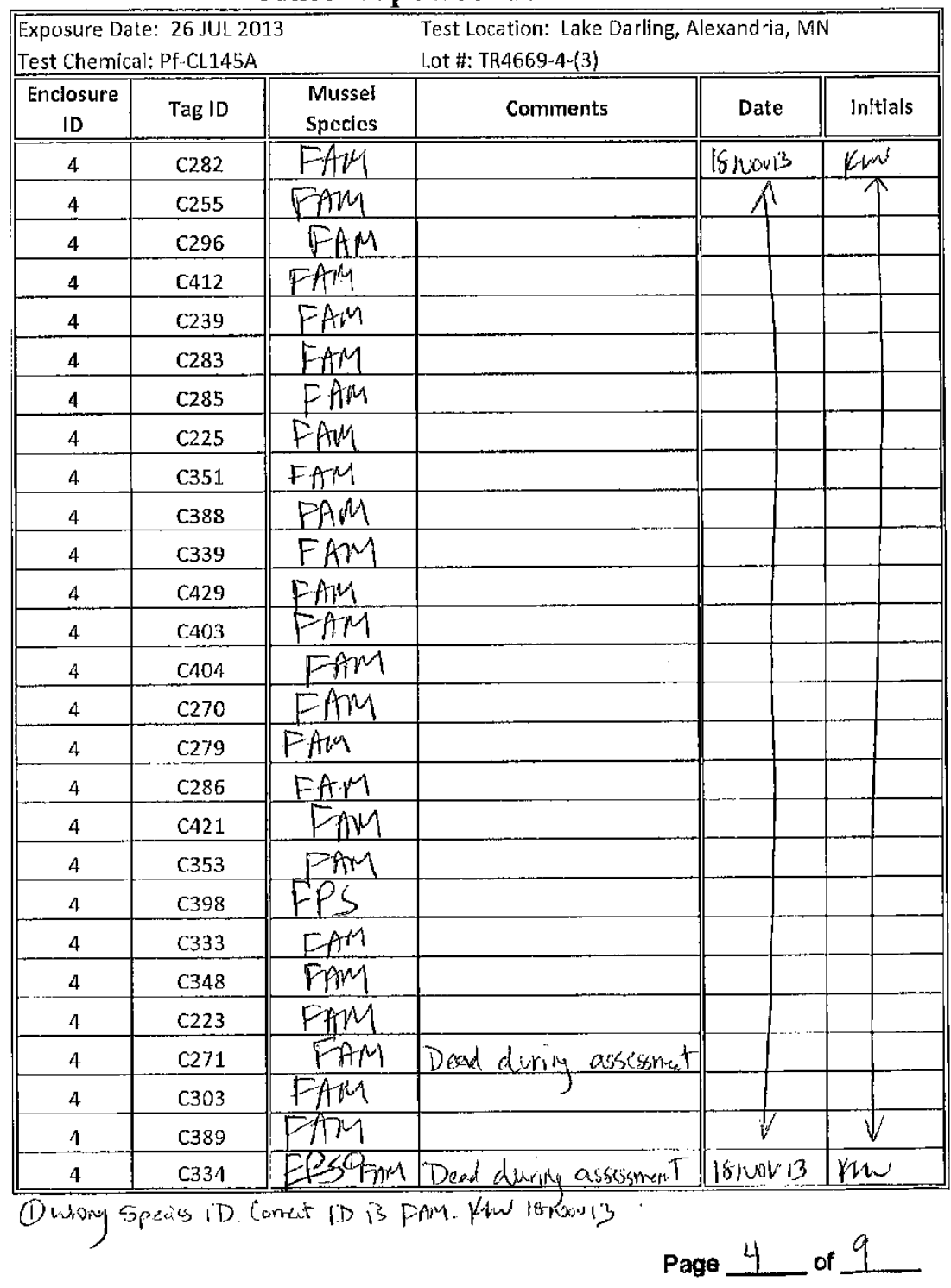


Study Number: AEH-13-PSEUDO-0S Fle Folder: 76

Lab nook/pgs: $c-\log$ page $d$

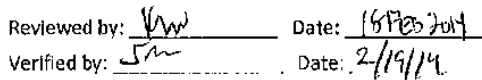

Mussel Species Identification

\begin{tabular}{|c|c|c|c|c|c|}
\hline \multicolumn{3}{|c|}{$\begin{array}{l}\text { Exposure Date: } 26 \text { JUL } 2013 \\
\text { Test Chemical: Pf-CL.145A }\end{array}$} & \multicolumn{3}{|c|}{$\begin{array}{l}\text { Test Location: Lake Darling, Alexandra, MN } \\
\text { Lot H: TR4669-4-(3) }\end{array}$} \\
\hline \begin{tabular}{|c|}
$\begin{array}{c}\text { Enclosure } \\
\text { ID }\end{array}$ \\
\end{tabular} & Tag ID & $\begin{array}{l}\text { Mussel } \\
\text { Species }\end{array}$ & Comments & Date & Initials \\
\hline 5 & $\mathrm{C} 256$ & PAM & & 8 (1)ovis & How \\
\hline 5 & C407 & FAM & & 9 & $\hat{\imath}$ \\
\hline 5 & C.190 & PAM & & & \\
\hline 5 & $\mathrm{C} 284$ & FAPM & & & \\
\hline 5 & $\mathrm{C} 238$ & EAM & & & \\
\hline 5 & $\mathrm{C} 240$ & FAM & & & \\
\hline 5 & $\mathrm{C} 244$ & $A+2$ & & & \\
\hline 5 & $C 253$ & FAM & Ded durity ascrosment & & \\
\hline 5 & $\mathrm{C} 335$ & 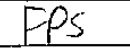 & 1 & & \\
\hline 5 & C300 & FAM & & & \\
\hline 5 & C352 & $M$ & & & \\
\hline 5 & $\mathrm{C} 294$ & $M$ & & & \\
\hline 5 & $\mathrm{C} 320$ & FAM & & & \\
\hline 5 & $\mathrm{C} 433$ & FAM & & & \\
\hline 5 & $\mathrm{C} 330$ & FAM & & & \\
\hline 5 & $\mathrm{C} 368$ & FAM & & & \\
\hline 5 & $\mathrm{C} 392$ & PAM & & & \\
\hline 5 & $\mathrm{C} 236$ & FAM & & & \\
\hline 5 & $\mathrm{C} 397$ & Fin & & & \\
\hline 5 & C259 & FAM & & & \\
\hline 5 & $C 365$ & PAn & & & \\
\hline 5 & $\mathrm{C} 290$ & PAM & & & \\
\hline 5 & $\mathrm{C} 425$ & FAM & & & \\
\hline 5 & $\mathrm{C} 313$ & EAM & & & \\
\hline 5 & $C 214$ & PBY & & & \\
\hline 5 & C431. & $F A M$ & & $\sqrt{ }$ & V \\
\hline 5 & $\mathrm{C} 265$ & Fin & & $18100 v 13$ & $f w$ \\
\hline
\end{tabular}


Study Number: AEH-13-PSEUDO-05 Flle Folder: 76

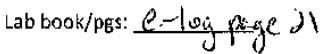

Reviewed by: Ww Date: 18 FEB 204

Verified by: Sin Date: $2 / 1 c^{2} / 4$

Mussel Species Identification

\begin{tabular}{|c|c|c|c|c|c|}
\hline \multicolumn{3}{|c|}{$\begin{array}{l}\text { Exposure Date: } 26 \text { JUL } 2013 \\
\text { Test Chemical: Pf-CL145A }\end{array}$} & \multicolumn{3}{|c|}{$\begin{array}{l}\text { Test Location: Lake Darling, Alexandria, MN } \\
\text { Lot \#: TR4669-4 }(3)\end{array}$} \\
\hline \begin{tabular}{|c|} 
Enclosure \\
ID
\end{tabular} & Tag ID & $\begin{array}{l}\text { Musse! } \\
\text { Species }\end{array}$ & Comments & Date & Initials \\
\hline 6 & $\mathrm{C} 297$ & EAM & & 18 inow & nnt \\
\hline 6 & $\mathrm{C} 362$ & FPS & & 象 & $\hat{\imath}$ \\
\hline 6 & C385 & FAM & & & \\
\hline 6 & $\mathrm{C} 250$ & PAM & & & \\
\hline 6 & $C 280$ & FAM & Dead during assessmuit & & \\
\hline 6 & $\mathrm{C} 423$ & FAM & & & \\
\hline 6 & $\mathrm{C} 356$ & Unknown & Not refovered & & \\
\hline 5 & $\mathrm{C} 200$ & FAM & & & \\
\hline 6 & C396 & FAM & Deed during assossment & & \\
\hline 6 & $\mathrm{C} 281$ & FAM & & & \\
\hline 6 & $\mathrm{C} 322$ & FAM & & & \\
\hline 6 & $\mathrm{C} 251$ & FAM & Dead deving assis,ment & & \\
\hline 6 & $\mathrm{C} 313$ & $F A M$ & & & \\
\hline 6 & $\mathrm{C} 252$ & EAM & & & \\
\hline 6 & $\mathrm{C} 308$ & FAM & & & \\
\hline 6 & $\mathrm{C} 277$ & FNM & & & \\
\hline 6 & $\mathrm{C} 336$ & FPS & & & \\
\hline 5 & $\mathrm{C} 402$ & FAMA & & & \\
\hline 6 & $\mathrm{C} 315$ & Ffm & & & \\
\hline 6 & $\mathrm{C} 213$ & FAM & Peadduring asscssiment & & \\
\hline 6 & $\mathrm{C} 205$ & FAM & & & \\
\hline 6 & $\mathrm{C} 426$ & FAM & & & \\
\hline 5 & $\mathrm{C} 378$ & FAM & & & \\
\hline 6 & $\mathrm{C} 383$ & EATM & & & \\
\hline 6 & $\mathrm{C} 372$ & FAM & & & \\
\hline 6 & C307 & FPS & Dodducing assessmont & $\sqrt{\prime}$ & $\sqrt{7}$ \\
\hline 5 & $\mathrm{C} 316$ & FMM & & $18700 \times 13$ & $\ln w$ \\
\hline
\end{tabular}

Page 6 of 9 
Study Number: AEH-13.PSEUDO-05 File folder: $t$ th Lab book/pes: e-log page di
Reviewed by: 6 W

verifled by: Jin

Date: Ifrop 2014 Date: $2 / 6 / / 4$

\section{Mussel Species Identification}

\begin{tabular}{|c|c|c|c|c|c|}
\hline \multicolumn{3}{|c|}{$\begin{array}{l}\text { Exposure Date: } 26 \mathrm{JUL} \text {. } 2013 \\
\text { Tes: Chemical: Pf-CL145A }\end{array}$} & \multicolumn{3}{|c|}{$\begin{array}{l}\text { Test Location: Lake Darling, Alexandria, MN } \\
\text { Lot \#: TR4669-4-(3) }\end{array}$} \\
\hline $\begin{array}{c}\text { Enclosure } \\
\text { ID }\end{array}$ & Tag ID & $\begin{array}{l}\text { Mussel } \\
\text { Species }\end{array}$ & Comments & Date & Initials \\
\hline 7 & $\mathrm{C} 357$ & FAM & & $1500 \times 13$ & $k n$ \\
\hline 7 & $\mathrm{C} 299$ & FAM & Dead duritg assessment & 7 & 1 \\
\hline 7 & $\mathrm{C} 273$ & FAM & & & \\
\hline 7 & $\mathrm{C} 257$ & FAM & & & \\
\hline 7 & $\mathrm{C} 301$ & FAM & Be-at otwing a assessint! & & \\
\hline 7 & $\mathrm{C} 349$ & FAM & & & \\
\hline 7 & $\mathrm{C} 355$ & EAM & & & \\
\hline 7 & C361 & $P A M$ & & & \\
\hline 7 & $\mathrm{C} 401$ & FAM & & & \\
\hline 7 & $\mathrm{C} 248$ & PAM & Dead denty assesstiwent & & \\
\hline 7 & $C 260$ & FAM & & & \\
\hline 7 & $\mathrm{C} 413$ & FAM & & & \\
\hline 7 & C306 & FAM & & & \\
\hline 7 & $\mathrm{C} 275$ & FAM & & & \\
\hline 7 & $\mathrm{C} 268$ & FNM & 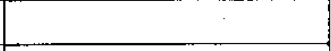 & & \\
\hline 7 & $\mathrm{C} 247$ & FAM & & & \\
\hline 7 & $\mathrm{C} 261$ & $F A M$ & & & \\
\hline 7 & C394 & FPS & & & \\
\hline 7 & $\mathrm{C} 272$ & FAM & & & \\
\hline 7 & $\mathrm{C} 234$ & FAM & & & \\
\hline$y$ & $\mathrm{C} 319$ & FAM & & & \\
\hline 7 & $\mathrm{C} 417$ & FAM & & & \\
\hline 7 & C2.12 & FAM & & & \\
\hline 7 & $\mathrm{C} 370$ & FAM & & & \\
\hline 7 & $\mathrm{C} 347$ & FAM & & & \\
\hline 7 & C289 & FPAY & Dead durity assessiment & $\sqrt{ }$ & 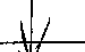 \\
\hline 7 & C192. & $E A M$ & & $1800 \mathrm{VB}$ & kw \\
\hline
\end{tabular}

(1) WL Dead during assessment klw 181200203

Page 7 of 9 
Study Number: AEH-13-PSEUDO-05 File Folder: 76 ,

Lab book/pgs: e-log parge of
Revicwed by: $\frac{610}{\sqrt{n}}$ Date: $\frac{18 F E S 2014}{2 / 19 / / 4}$

\section{Mussel Species Identification}

\begin{tabular}{|c|c|c|c|c|c|}
\hline \multicolumn{3}{|c|}{$\begin{array}{l}\text { Exposure Date: } 26 \text { JUL } 2013 \\
\text { Test Chemical: Pf-CL145A }\end{array}$} & \multicolumn{3}{|c|}{$\begin{array}{l}\text { Test Location: Lake Darling, Alexandria, MN } \\
\text { Lot \#: TR4669-4-(3) }\end{array}$} \\
\hline \begin{tabular}{|c|} 
Enclosure \\
ID \\
\end{tabular} & Tag ID & $\begin{array}{l}\text { Mussel } \\
\text { Species }\end{array}$ & Comments & Date & Initials \\
\hline 8 & C369 & FAr & & 18 Novi3 & WL \\
\hline 8 & $\mathrm{C} 189$ & FAM & & $A$ & 4 \\
\hline 8 & $\mathrm{C} 4.15$ & TAM & & & \\
\hline 8 & $\mathrm{C} 269$ & FAM & & & \\
\hline 8 & $\mathrm{C} 271$ & FAM & & & \\
\hline 8 & C191 & PAM & & & \\
\hline 8 & $\mathrm{C} 186$ & PPM & & & \\
\hline 8 & $\mathrm{C} 211$ & FAM & & & \\
\hline 8 & C337 & FAM & & & \\
\hline 8 & C399 & EAM & & & \\
\hline 8 & C366 & FAM & & & \\
\hline 8 & C435 & FAM & & & \\
\hline 8 & C196 & FAM & & & \\
\hline 8 & C428 & PAM & & & \\
\hline 8 & $\mathrm{C} 222$ & Faply & & & \\
\hline 8 & C391 & FAM & & & \\
\hline 8 & C198 & PAM & & & \\
\hline 8 & $\mathrm{C} 230$ & FAl & & & \\
\hline 8 & C199 & SMM & & & \\
\hline 8 & $\mathrm{C} 216$ & FAM & & & \\
\hline 8 & $\mathrm{C} 264$. & FAM & & & \\
\hline 8 & $\mathrm{C} 241$ & EAm & & & \\
\hline 8 & $\mathrm{C} 324$ & FAM & & & \\
\hline 8 & C387 & FAM & Deed during assessinest & & \\
\hline 8 & $\mathrm{C} 340$ & FAM & Dzad dunty assessineivt & & \\
\hline 8 & $\mathrm{C} 209$ & FAM & & 7 & V \\
\hline 8 & $\mathrm{C} 220$ & FAr & & $18 N u v 13$ & in \\
\hline
\end{tabular}


Study Number: AEH-13-PSEUDO-C5 File Folder: 7 t

Lab book/pgs: exloy payjed
Revlewed by: How _... Date: iffer 2oly

verifled by: $5 n$ Date: $2 / 19 / 4$

Mussel Species Identification

\begin{tabular}{|c|c|c|c|c|c|}
\hline \multicolumn{3}{|c|}{$\begin{array}{l}\text { Exposure Date: } 26 \text { JUL } 2013 \\
\text { Test Chemical: Pf-CL145A }\end{array}$} & \multicolumn{3}{|c|}{$\begin{array}{l}\text { Test Location: Lake Darling, Alexandria, MN } \\
\text { Lot H: TRA669・4 } 4(3)\end{array}$} \\
\hline $\begin{array}{c}\text { Enclosure } \\
\text { ID }\end{array}$ & Tag ID & $\begin{array}{l}\text { Mussel } \\
\text { Species } \\
\end{array}$ & Comments & Date & Initials \\
\hline 9 & $\mathrm{C} 327$ & $F A M$ & & $18100 \mathrm{v} 3$ & the \\
\hline 9 & C.338 & FAM & & $A$ & 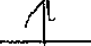 \\
\hline 9 & C323 & Unknown & Wit recovered & & \\
\hline 9 & C305 & FAM & & & \\
\hline 9 & $\mathrm{C} 227$ & AM & & & \\
\hline 9 & $\mathrm{C} 229$ & EAM & & & \\
\hline 9 & $\mathrm{C} 376$ & PAM & & & \\
\hline 9 & $\mathrm{C} 206$ & FAYM & & & \\
\hline 9 & $\mathrm{C} 411$ & FAM & Dead derity assessment & & \\
\hline 9 & $\mathrm{C} 262$ & FAM & & & \\
\hline 9 & $\mathrm{C} 226$ & AM & 2oed durity assesumin & & \\
\hline 9 & C 344 & & & & \\
\hline 9 & C346 & FAM & & & \\
\hline 9 & $\mathrm{C} 422$ & FAM. & & & \\
\hline 9 & $\mathrm{C} 420$ & FAM & & & \\
\hline 9 & $\mathrm{C} 202$ & FAM & Deod durita assessment & & \\
\hline 9 & $\mathrm{C} 430$ & FAM & "Dead durith assessind & E & \\
\hline 9 & $\mathrm{C} 409$ & FAM & & & \\
\hline 9 & $\mathrm{C} 295$ & PAM & & & \\
\hline 9 & $\mathrm{C} 287$ & FAM & & & \\
\hline 9 & $\mathrm{C} 258$ & FAM & Dead devring assesciment & & \\
\hline 9 & $\mathrm{C} 367$ & FAM & & & \\
\hline 9 & C386 & FPS & & & \\
\hline 9 & C328 & FAM & & & \\
\hline 9 & $\mathrm{C} 416$ & FAM & Dendannz assusintan] & & \\
\hline 9 & C381 & PAY & & $\Downarrow$ & $\sqrt{4}$ \\
\hline 9 & $\mathrm{C} 432$ & Fap & & 18 wivis & $k_{n}$ \\
\hline
\end{tabular}

FF \# $7 \mathrm{~b}$

$\mathrm{Pg} \underline{\mathrm{g}}$ of 9 


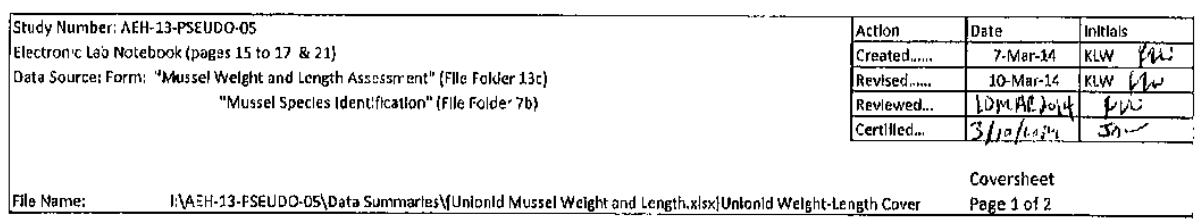

Unionid Mussel Welght and Length

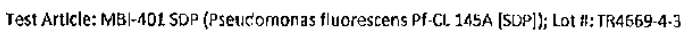

Exposure Date: July 26, 2013

Assessment Dates; August 13-15, 2013

Data Explanatlon:

Fach of the 9 enclosures contalned 22 unlonld $m$. Jssels with adhe'l 8 zebra mussels and 5 unionid rrussels with zebra mussels removed (l.e., used to assess Inltial zekra mussel density). During the post-exposure assessment (August 13.15, 2013), the unionld mussels were yelghed before and after zelbra mussel removal. Shell length was measured on each unionid mussel, anc specles conll'med from shell morphology. Fatmucket (lampsills sillouoldeo; FAM) was the do nlnate specles whlie fraglle papershell (Leptodea frog/lis ; FPS) was rare (1.e, 231 FAM versus 12 FPS). Weight and length data for fragile papershell mussels were analyzed separate y. Data exclusions, including welghts of unlunld mortalltles ard we'ght and length of unrecovered untonids can be frund on page 2 of thls data summary.

Tag ID $=$ Hallprint shelffish tag glued to the natlve mussel

Welght $(g)$ ) Welght of unlonld mussel after zebra mussel removal durling the post-exposure assessment (August 13-15, 2013)

Sheil Length (mm) = Length of unionid mussel after zebra mussel removal durling the post-exposure assessment (August 13-15, 2013)

Data Summan:

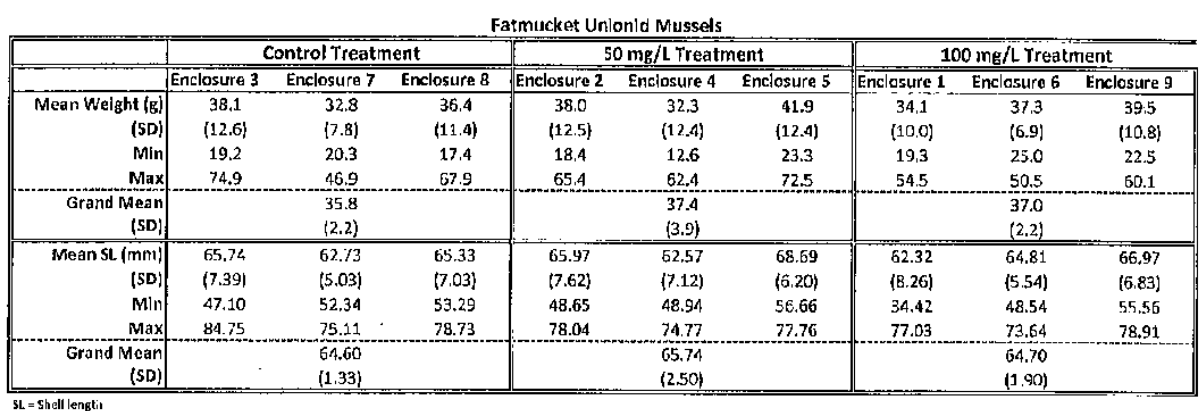

\begin{tabular}{|c|c|c|}
\hline & Welght (k) & St (nmm) \\
\hline Mean & 30.3 & 65.90 \\
\hline (50) & (18.9) & $\{11.62\}$ \\
\hline MIn & 12.6 & 52.28 \\
\hline Max & 33,0 & 95.64 \\
\hline
\end{tabular}

Fille :older $7 \mathrm{~b}$

Item Number of 1 . 


\begin{tabular}{|c|c|}
\hline Study Number: AEH-13-PSEUDO-05 & Coversheet (cont.) \\
\hline Electronic Lab Notebook (pages :5 10 17 \& 21) & Рaze 2 of 2 \\
\hline Data Source: Form: "Mussel Welght and Lenglh Assessnxent" (Flle fulder 13c) & \\
\hline "Mussel Specles Udentificat|on' \{Flle Folder $7 \mathrm{~b}$ ) & \\
\hline
\end{tabular}

\section{Unionid Mussel Weight and Length}

Test Article: MBI-401 5DP (Pseudomonas fluorescens Pf-CL 145A [SDP); lct it: TR4669-4-3

Exposure Date: Joly 26, 2013

Assessment Dates: August 13-15, 2013

Data Exclustons:

The following unlonld mussels were mortalitles from the assessment (August 13 to 15, 2013). The welght data for these mussels will not be used within the unionld welght analysls and will be denoted with "." wilthin the dataset. The length data for these mussels will be used wilthin the unlonld eng:h analysls: Test Enclosure $1(100 \mathrm{mg} / \mathrm{L}): \quad$ Test Enclosure $6(100 \mathrm{mg} / \mathrm{L}): \quad$ Test Enclosure $8\{0 \mathrm{mg} / \mathrm{L})$

\begin{tabular}{|c|c|c|}
\hline C312 & $\mathrm{C} 213$ & $C 340$ \\
\hline C359 & C251 & 0387 \\
\hline Test Enclosure $2(50 \mathrm{mg} / \mathrm{L}):$ & $\mathrm{C} 280$ & Test Enclostre $9(100 \mathrm{mg} / \mathrm{L})$ : \\
\hline C436 & C307 & $\mathrm{C} 202$ \\
\hline Test Enclosure $3(0 \mathrm{mg} / \mathrm{l})$ : & C396 & 0226 \\
\hline C193 & Test Enclosure $7(0 \mathrm{mg} / \mathrm{L})$ : & $\mathrm{C} 258$ \\
\hline Test tniclosure $\mathrm{a}(50 \mathrm{~m} / \mathrm{L} / \mathrm{s}$ : & $C 248$ & C430 \\
\hline $\mathrm{C} 271$ & C289 & C411 \\
\hline 634 & 0299 & C416 \\
\hline
\end{tabular}

The following mussels were not recovered for the assessment (August 13 to 15,2013 ) and were removed from the unlonid mussel weight and leng:h analysis: Test Enclosure $5(100 \mathrm{mg} / \mathrm{l}$ ) Test Enclosure $9(100 \mathrm{mg} / \mathrm{L})$

2 of 6 


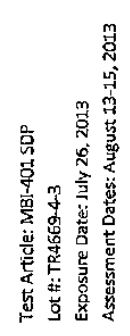
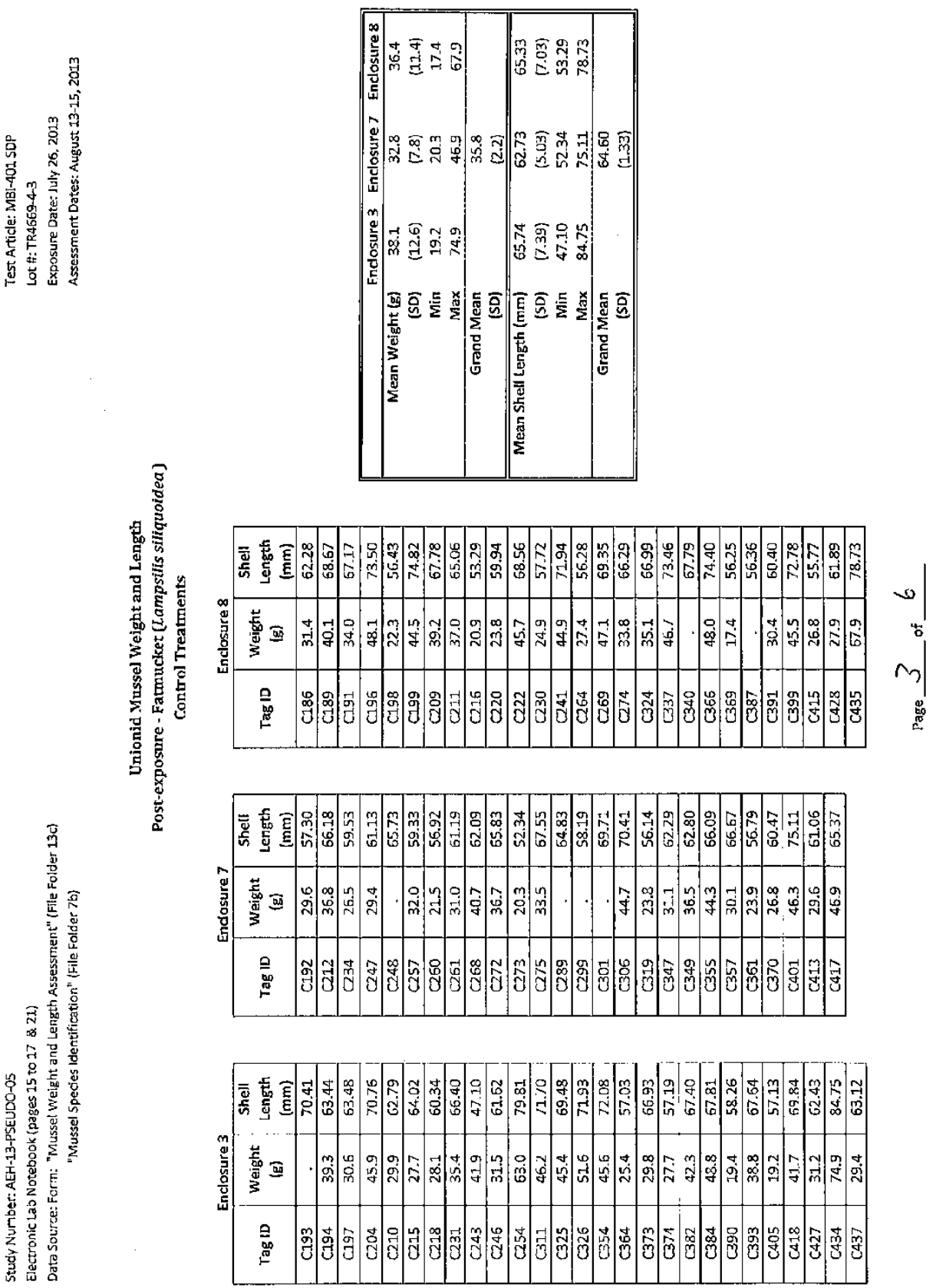


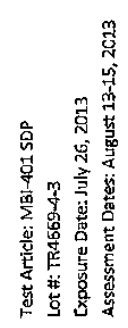
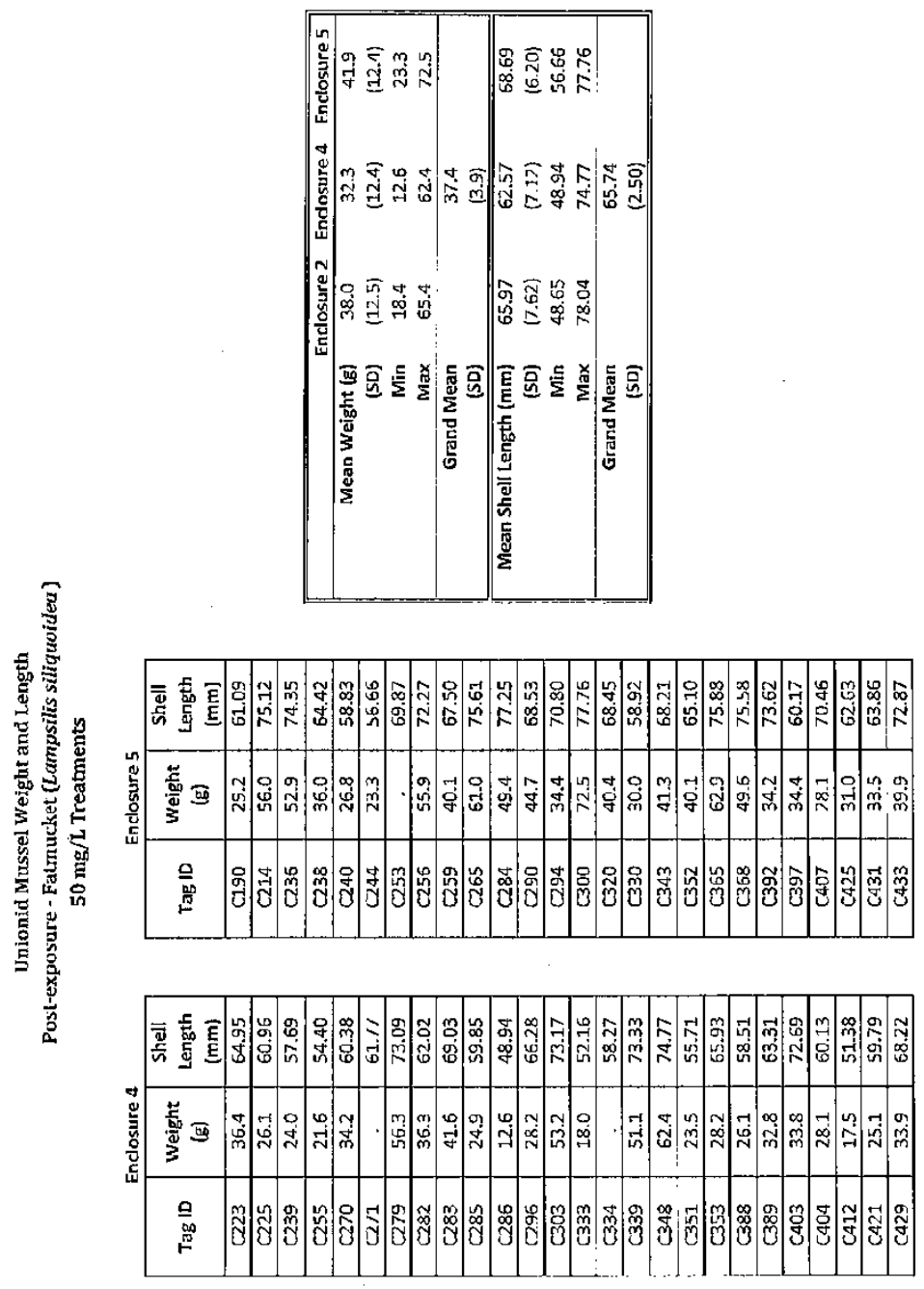

象

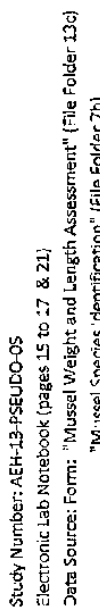

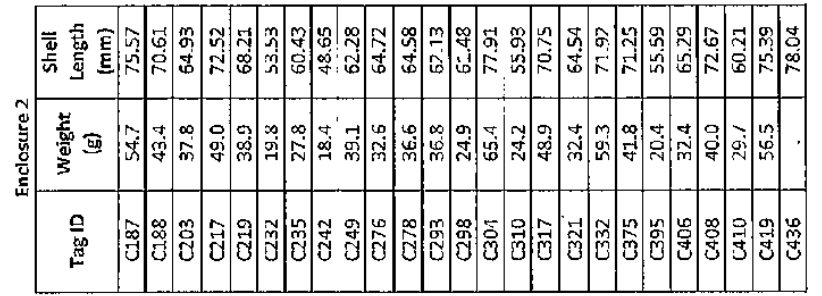



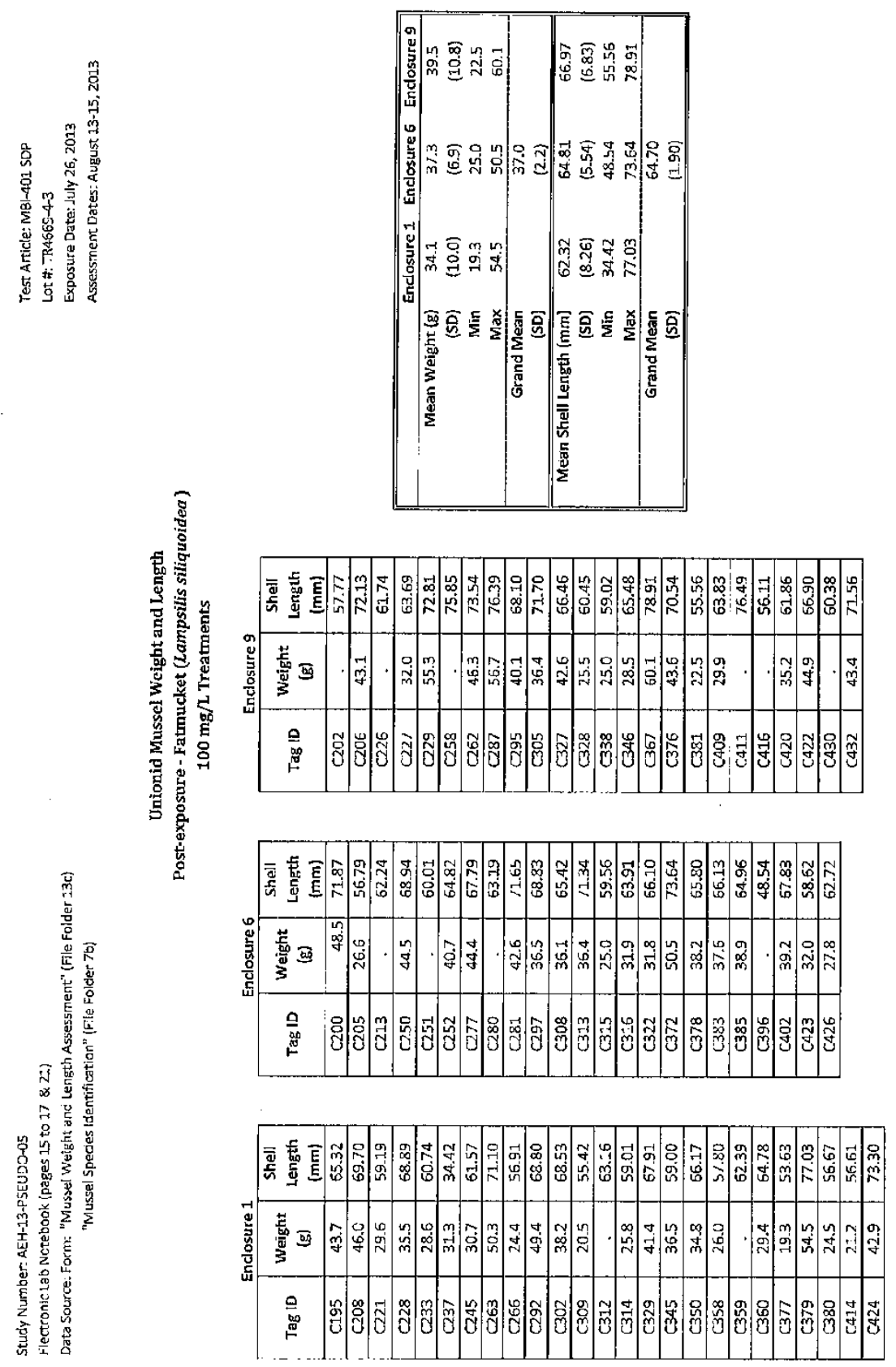
Stucy Number: AEH-13-PSEUDO-05

Electronlc Lab Notebook (pages 15 to 17 \& 21)

Data Source: Form: "Mussel Welght and Length Assessment" (Flle Folder 13c)

"Mussel Species Identlfication" (File Folder $7 \mathrm{~b}$ )
Test Art|cle: MBI-401 SDP

Lot \#: TR4669-4-3

Exposure Date: July 26, 2013

Assessmen: Dates: August 13-15, 2013

Unionid MusseI Weight and Length

Post-exposure - Fragile papershell (Leptodea fragilis)

All Treatments

\begin{tabular}{|c|c|c|c|c|}
\hline Enclosure ID & $\begin{array}{c}\text { Treatment } \\
\text { Level }(\mathrm{mg} / \mathrm{L})\end{array}$ & $\operatorname{Tag} 10$ & Weight $\{\mathrm{g}\}$ & $\begin{array}{l}\text { Shell Length } \\
(\mathrm{mm})\end{array}$ \\
\hline 7 & 0 & C394 & 27.5 & 66.90 \\
\hline 2 & 50 & C331 & 23.2 & 63.74 \\
\hline 2 & 50 & $\mathrm{C} 342$ & 40.5 & 74.08 \\
\hline 4 & 50 & C398 & 15.7 & 54.72 \\
\hline 5 & 50 & C335 & 12.6 & 53.34 \\
\hline 1 & 100 & $\mathrm{C} 207$ & 83.0 & 95.64 \\
\hline 1 & 100 & C363 & 31.7 & 72.23 \\
\hline 6 & 100 & C307 & . & 60.81 \\
\hline 6 & 100 & C336 & 18.2 & 52.28 \\
\hline 6 & 100 & C362 & 39.1 & 74,40 \\
\hline 9 & 100 & C344 & 25.5 & 63.94 \\
\hline \multirow[t]{5}{*}{9} & 100 & $\mathrm{C} 386$ & 16.1 & 58.71 \\
\hline & & Mean & 30.3 & 65.90 \\
\hline & & $(S D)$ & (18.9) & $(11.62)$ \\
\hline & & Min & 1.2 .6 & 52.28 \\
\hline & & Max & 83.0 & 95,64 \\
\hline
\end{tabular}

\footnotetext{
File Folder $7 b$

Item Number 2
} 


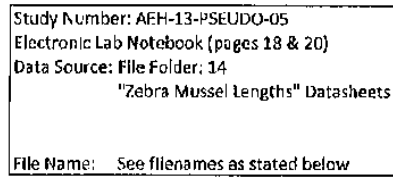

File Name: See flienames as stated below

Zebra Mussel Lengths

Pre-exposure Assessment

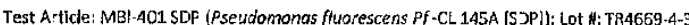
Exposure Date: July 26, 2013

Assessment Dates: August 13-15, 2013

Overall Data Summary:

\begin{tabular}{|c|ccc|ccc|ccc|}
\cline { 2 - 9 } \multicolumn{1}{c|}{} & \multicolumn{3}{c|}{ Control } & \multicolumn{3}{c|}{ 50 mg/h } & \multicolumn{3}{c|}{$100 \mathrm{mg} / \mathrm{L}$} \\
\hline Grand Mean & 12.04 & 11.95 & 12.11 & $\mathbf{1 2 . 6 3}$ & 11.65 & 11.90 & 11.32 & 11.53 & 10.82 \\
(SD) & $(0.88)$ & $(0.76)$ & $(0.02)$ & $(0.17)$ & $(0.40)$ & $(0.74)$ & $(0.15)$ & $(0.33)$ & $10.41\}$ \\
Min & 6.03 & 6.11 & 6.08 & 6.07 & 6.01 & 5.19 & 6.01 & 6.00 & 6.01 \\
Max & 27.43 & 25.70 & 26.49 & 19.72 & 25.28 & 27.82 & 23.56 & 27.95 & 17.38 \\
\hline
\end{tabular}

Data Explanatlon:

Flve unionid mussels with adhering zebra mussels were collected Irom each test enclosure for use In estlmatlng the pre-exposure zebra mussel colonlzatlon density. All zebra mussels were removed, sorted into live/dead and preserved $1 n 70 \%$ ethanol for enumeration and length measurement. Live zebra mussels removed from 3 unlonids from each treatment replicate were neasured for length; mussels < $6 \mathrm{~nm}$ were removed from analysls.

Pre-exposure Control [0 mg/L] Test Enclosure Zebra Mussel Length Jata:

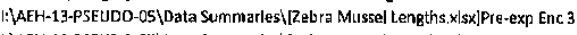

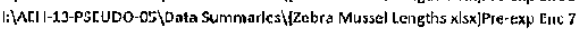

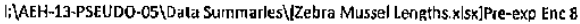

Pre-exposure $50 \mathrm{mg} / \mathrm{L}$ Test Enclosure Zebra Mussel Length Data:

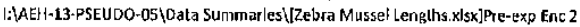
I: AEH-13-PSEUOO-05 Data Summar|es:|Lebbra Mussel Lengths.xisx] PTe-exp Enc 4

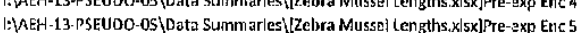

Pre-exposure Benthic Injectlon Test Enclosure Zebra Mussel Length Data:

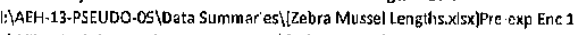

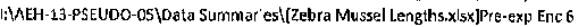

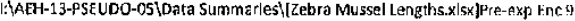

Data anomailes and deviatlons:

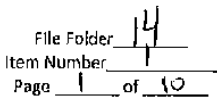




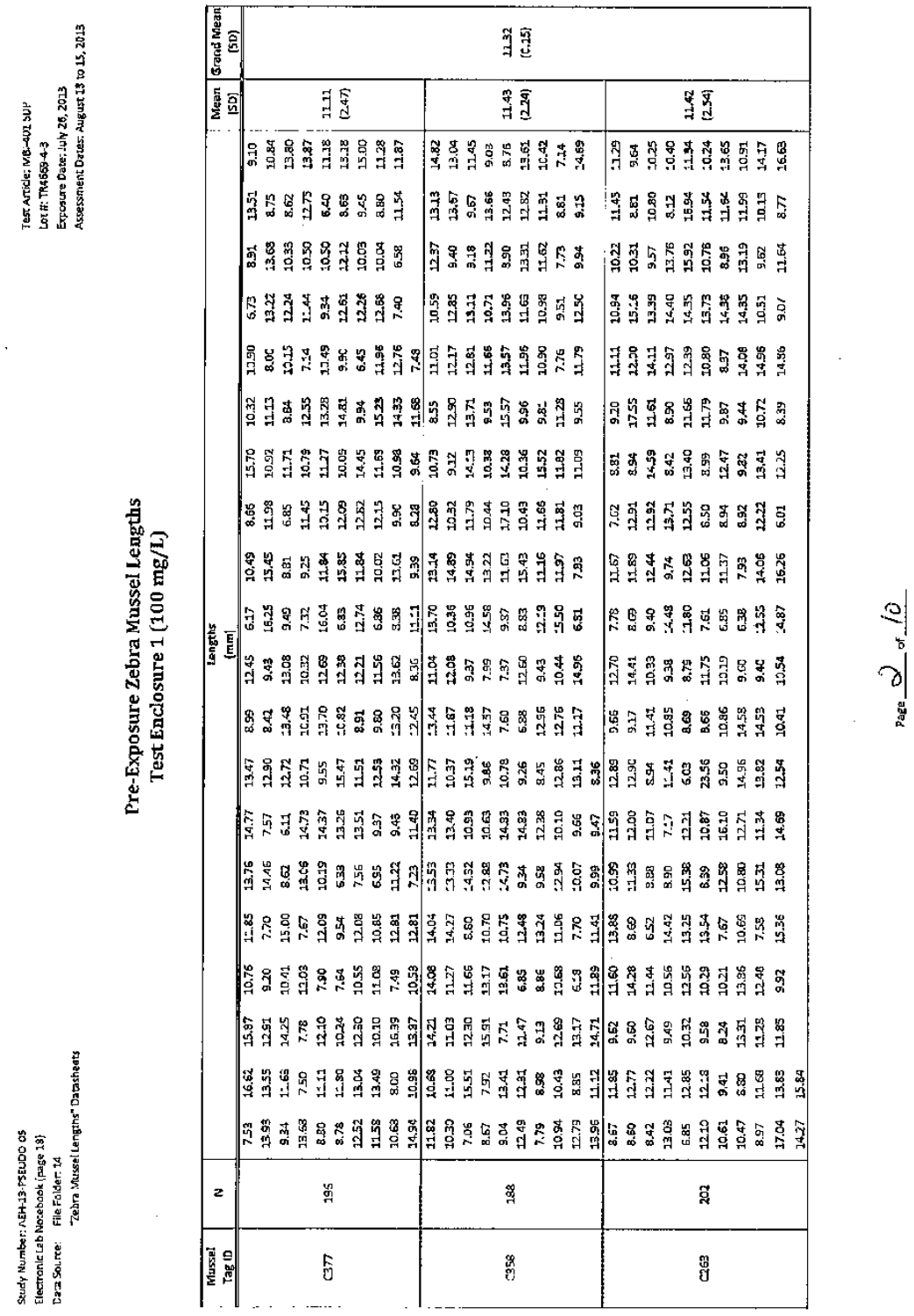




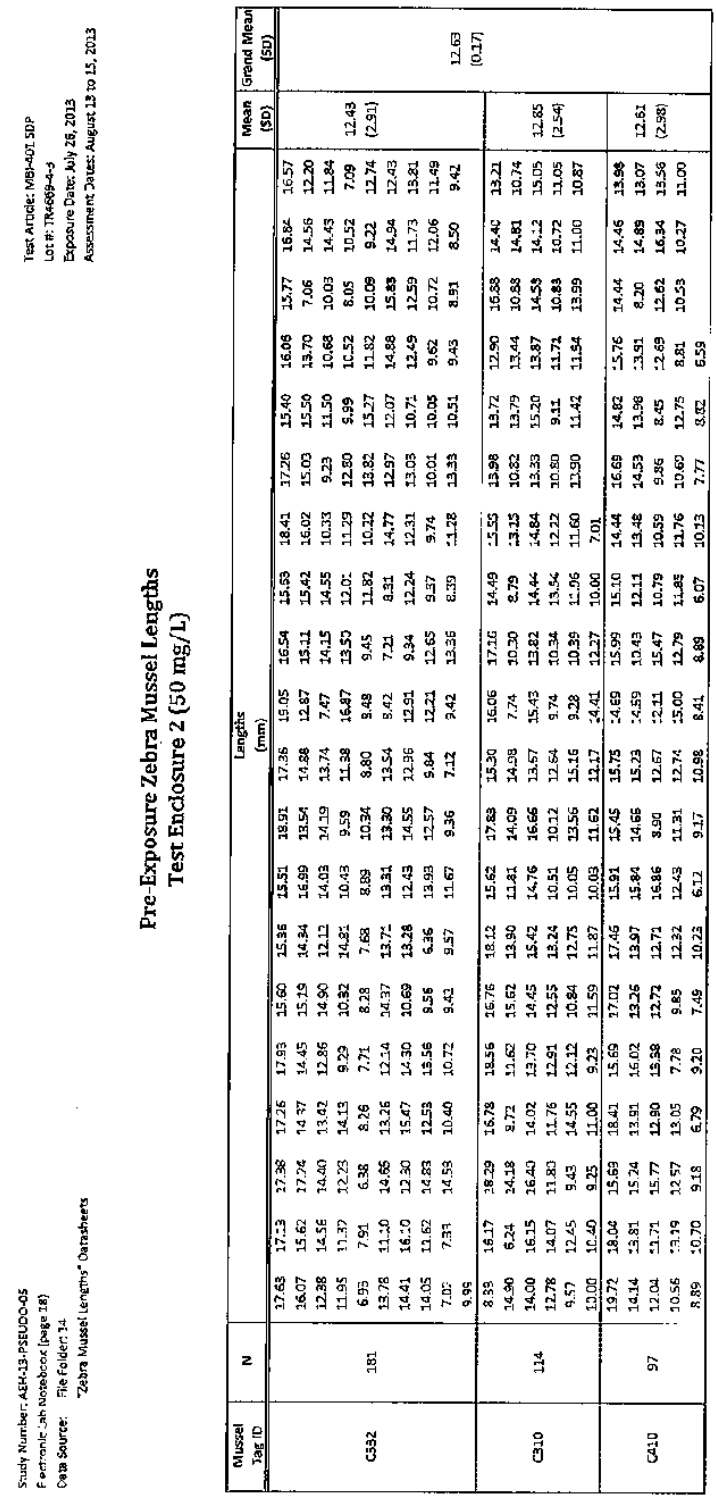




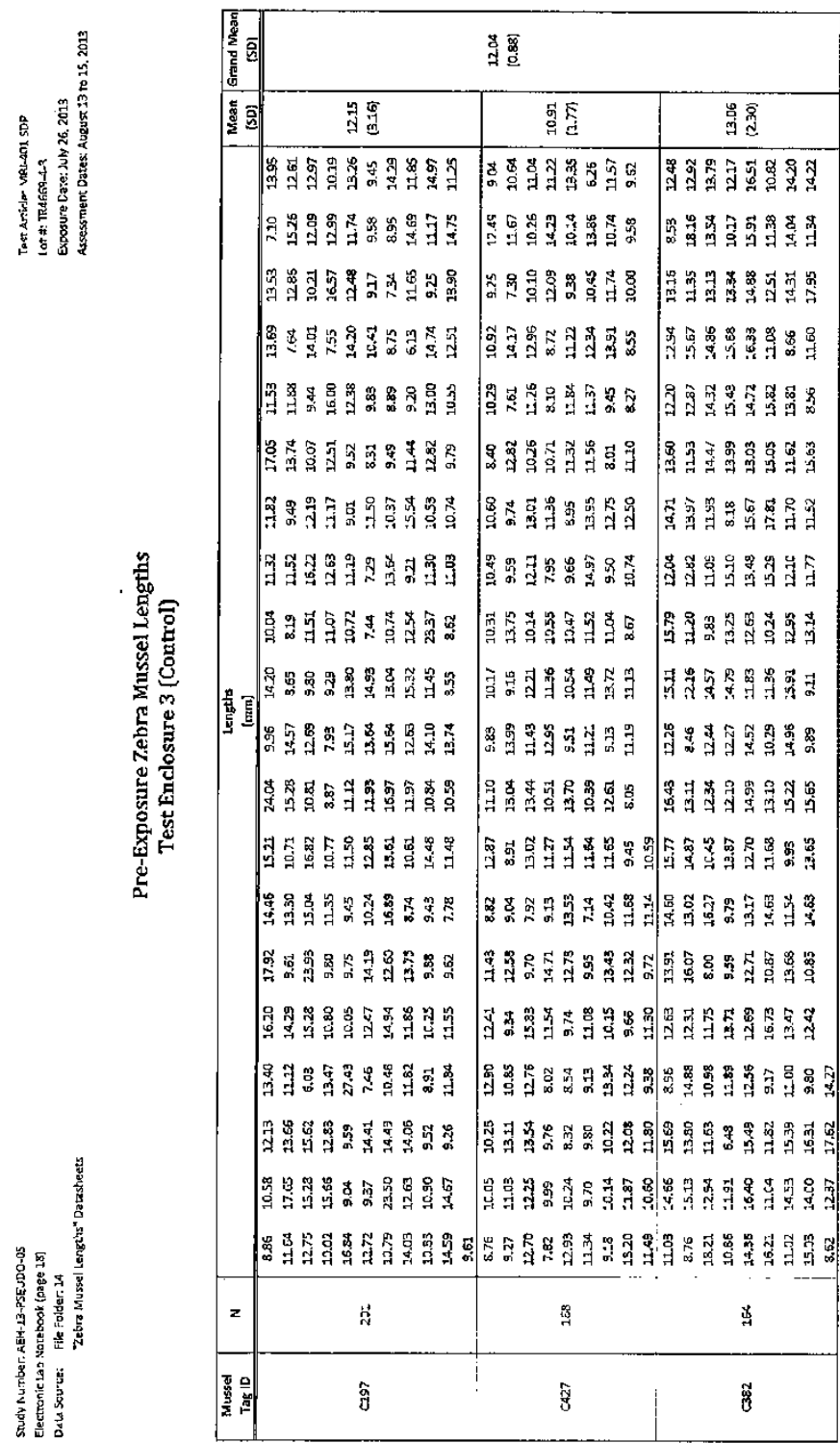

2 


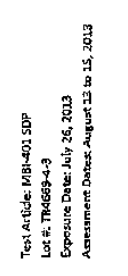

\begin{tabular}{|c|c|c|c|}
\hline 递 & \multicolumn{3}{|l|}{ 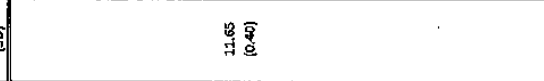 } \\
\hline \begin{tabular}{|l|l|l|} 
\\
\end{tabular} & 牙等 & 줄 & 重量 \\
\hline & 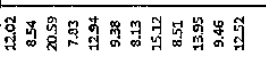 & 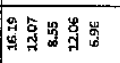 & 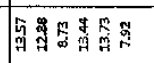 \\
\hline & 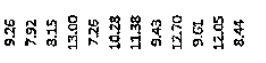 & 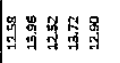 & 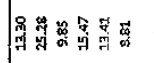 \\
\hline & 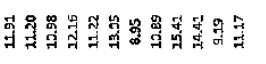 & 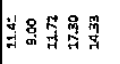 & 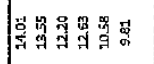 \\
\hline & 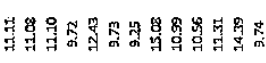 & 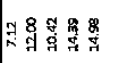 & 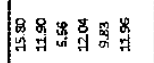 \\
\hline & 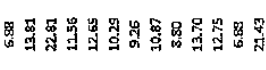 & 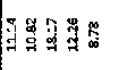 & 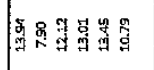 \\
\hline & 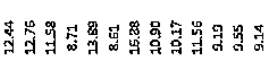 & 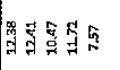 & 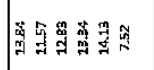 \\
\hline & 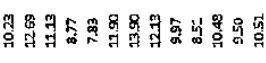 & 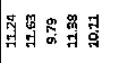 & 解 \\
\hline & 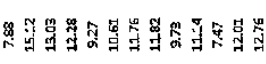 & 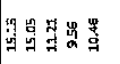 & 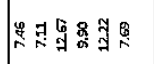 \\
\hline & 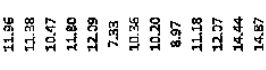 & 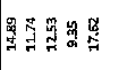 & 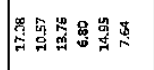 \\
\hline & 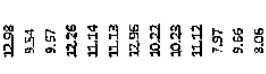 & 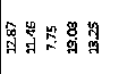 & 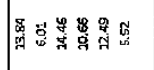 \\
\hline & 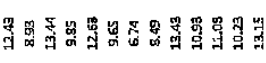 & 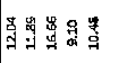 & 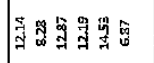 \\
\hline & 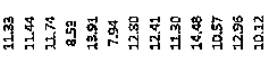 & 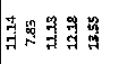 & 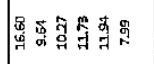 \\
\hline & 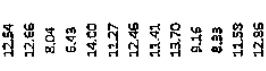 & 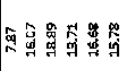 & 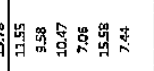 \\
\hline & 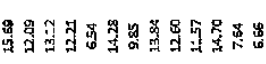 & 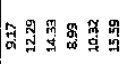 & 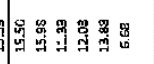 \\
\hline & 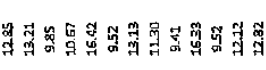 & 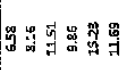 & 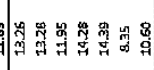 \\
\hline & 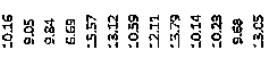 & 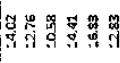 & 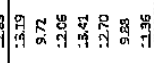 \\
\hline & 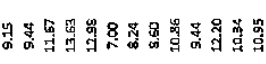 & 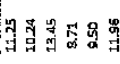 & 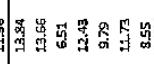 \\
\hline & 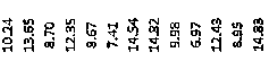 & & 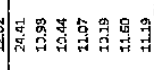 \\
\hline & 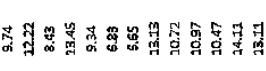 & 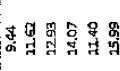 & 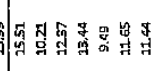 \\
\hline & 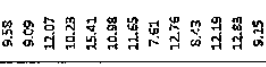 & 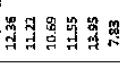 & 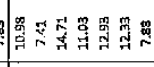 \\
\hline$=$ & $\hat{s}$ & 兽 & 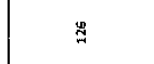 \\
\hline 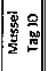 & 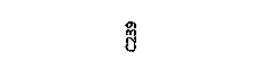 & ฮี & 鰨 \\
\hline
\end{tabular}

e!

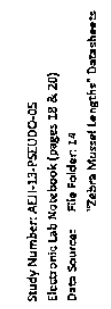




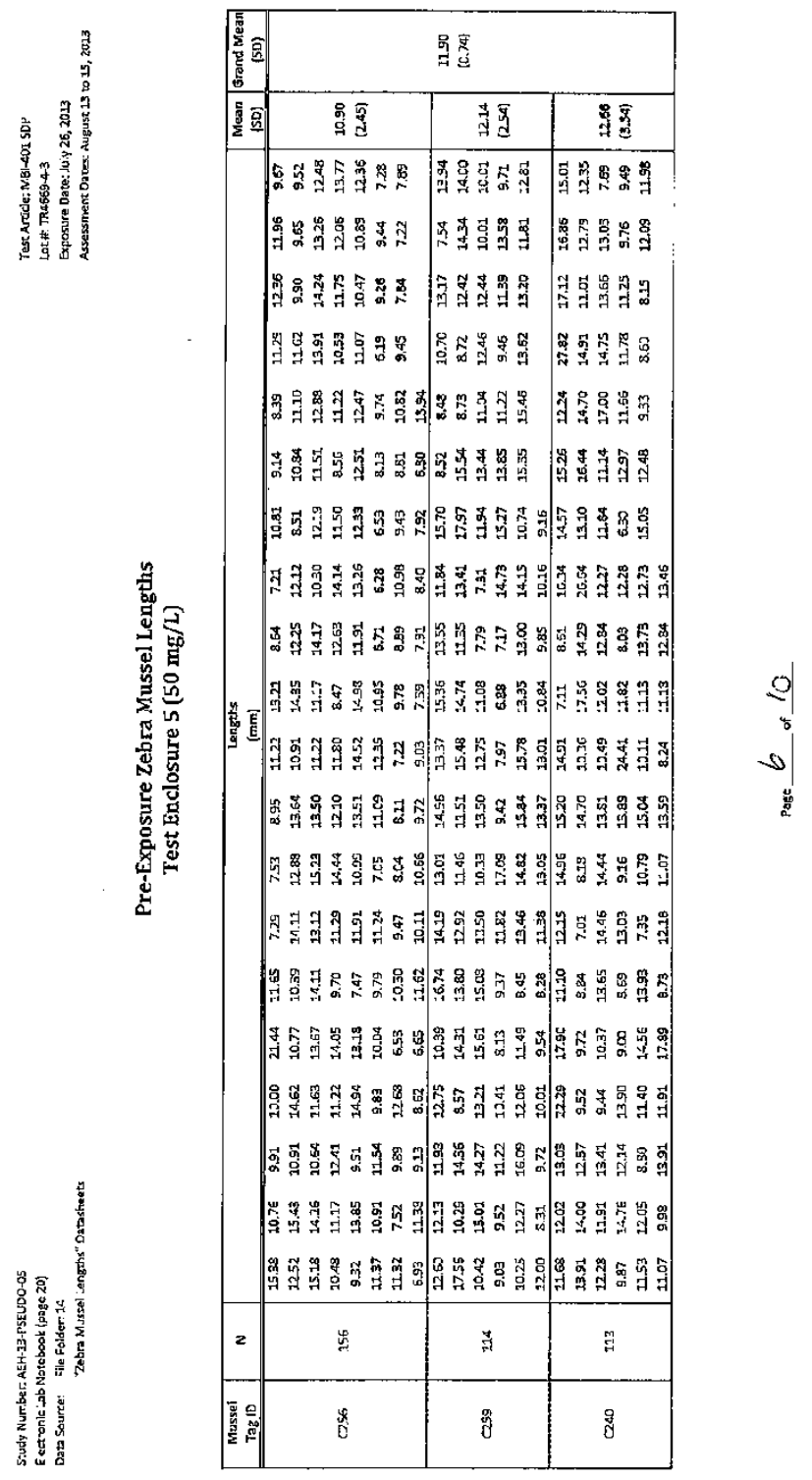




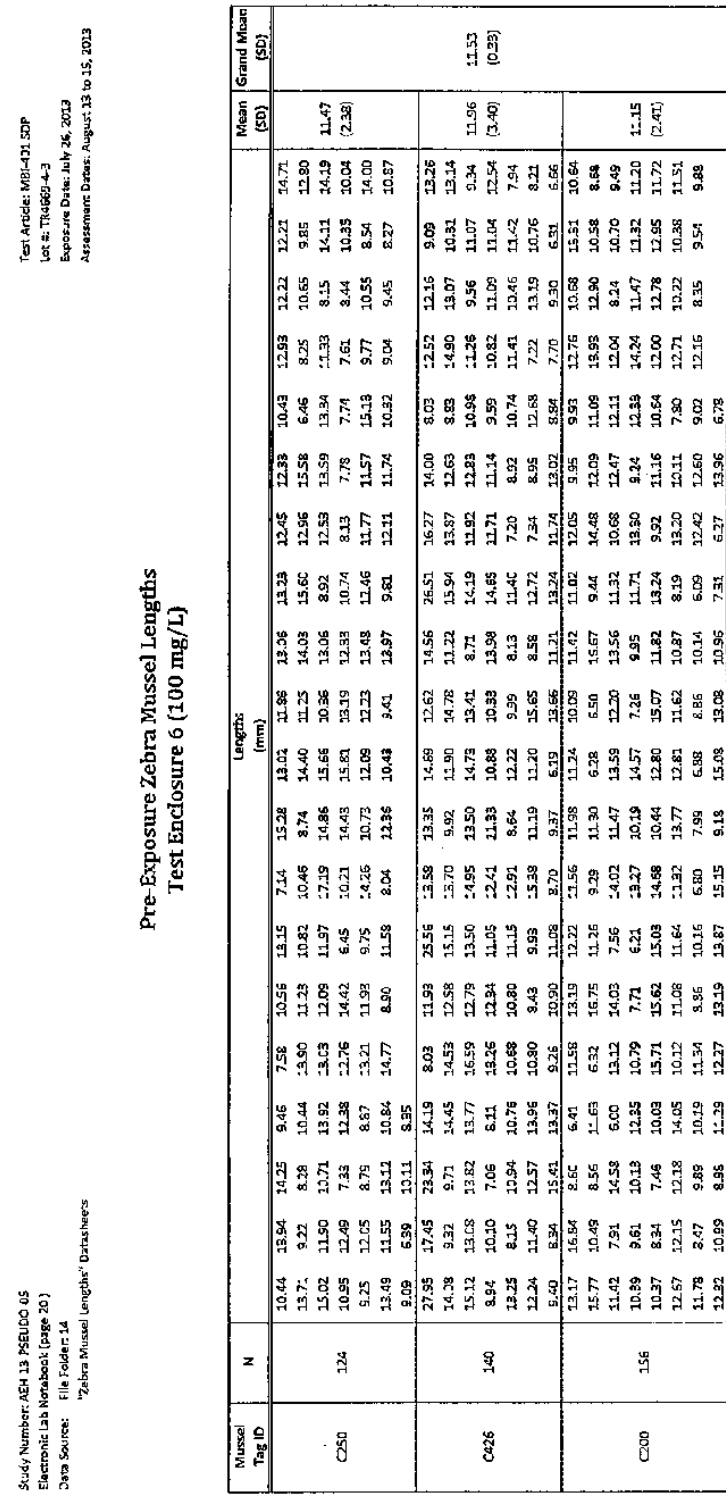

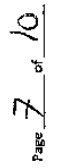




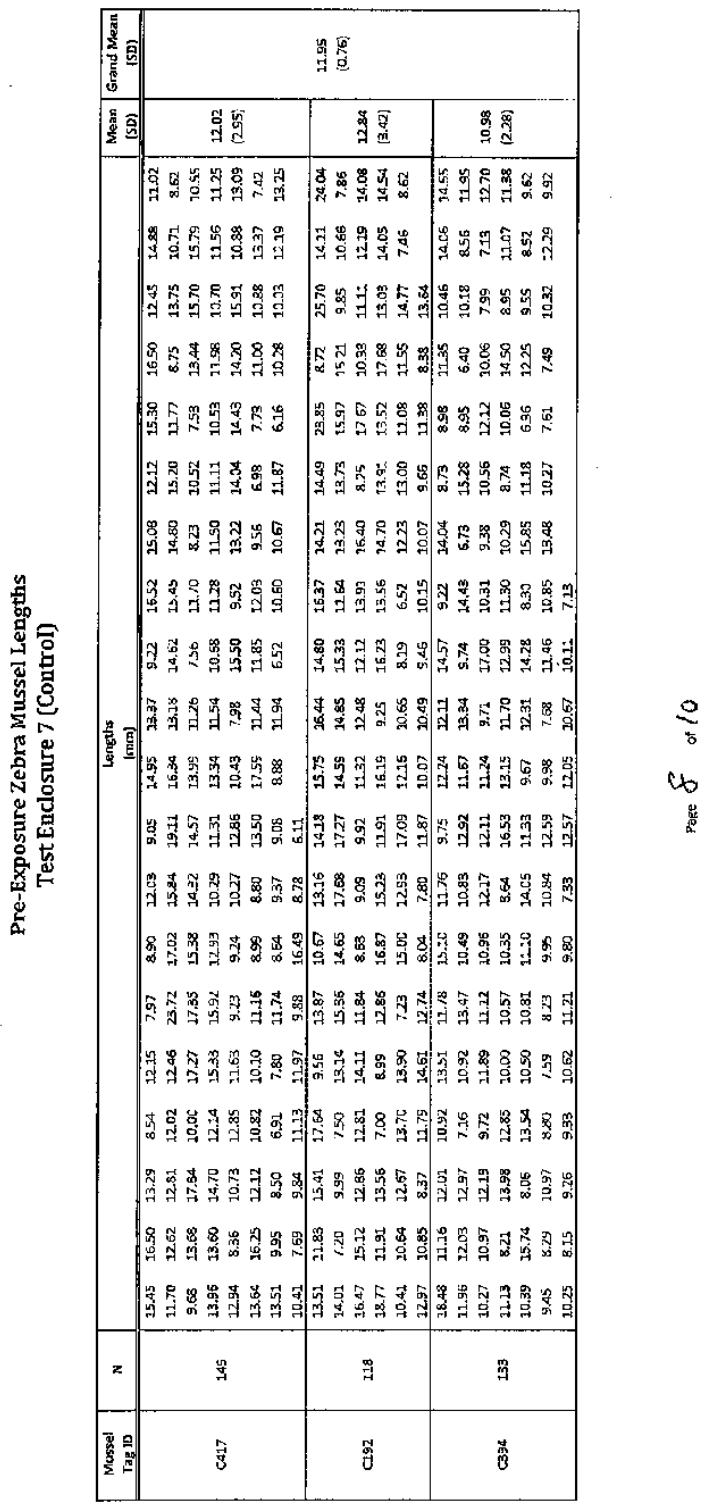




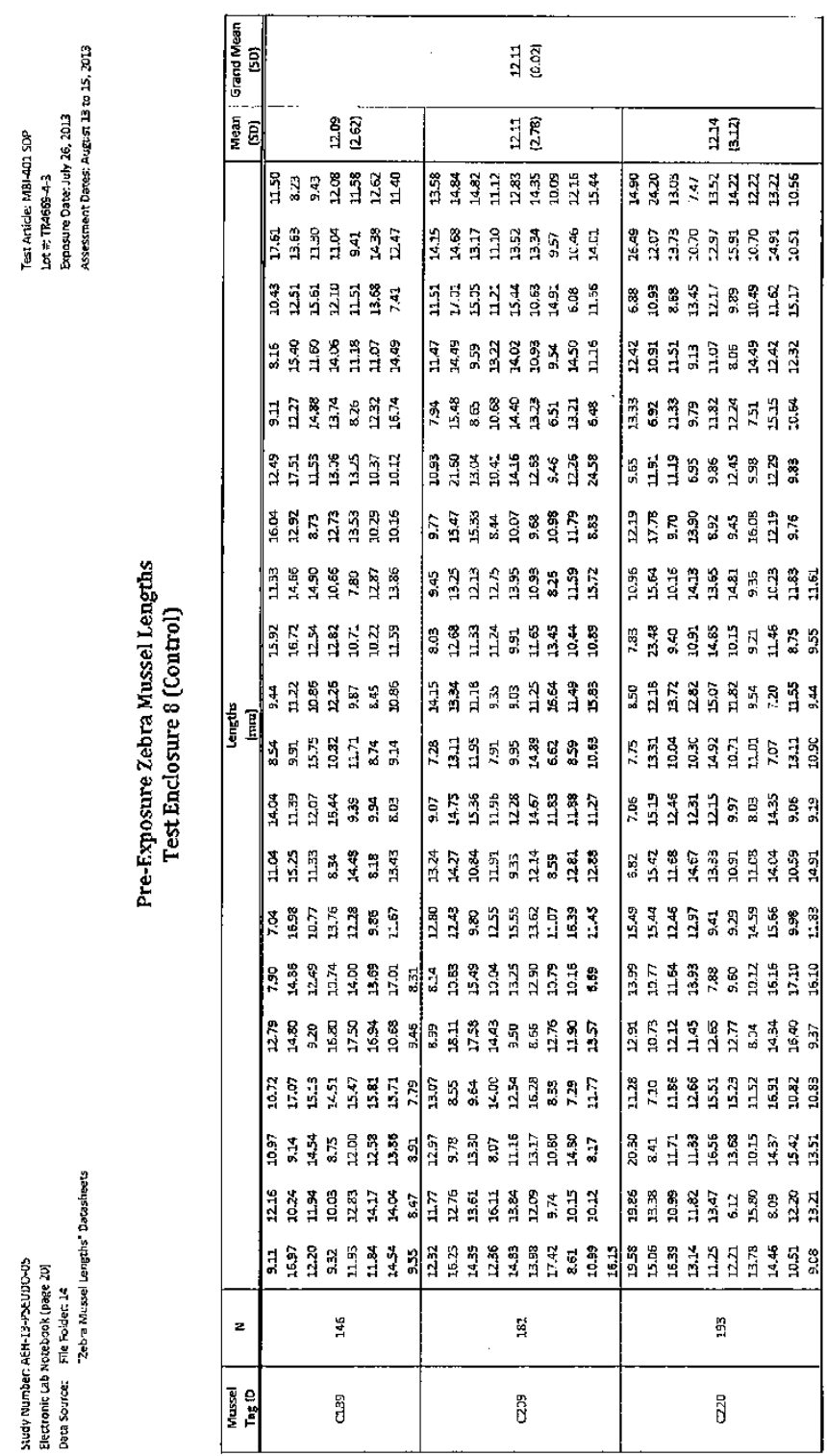

a 


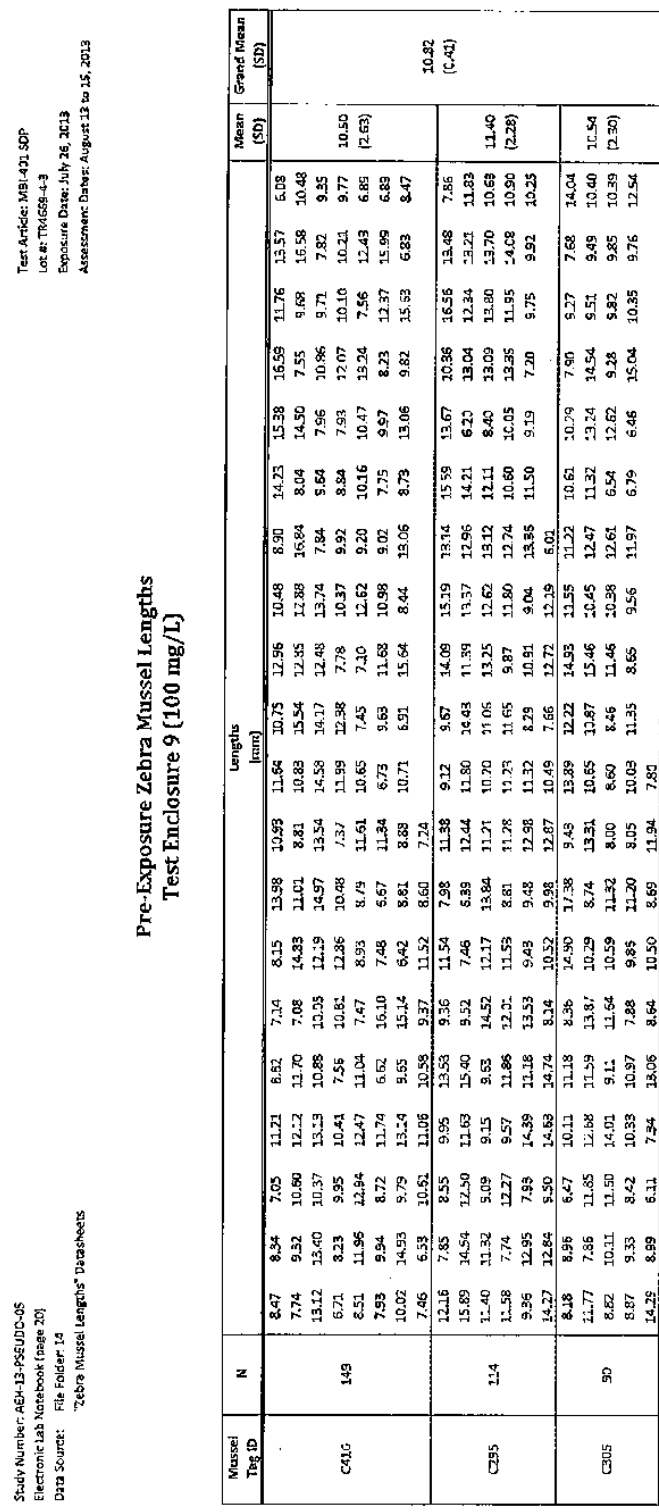




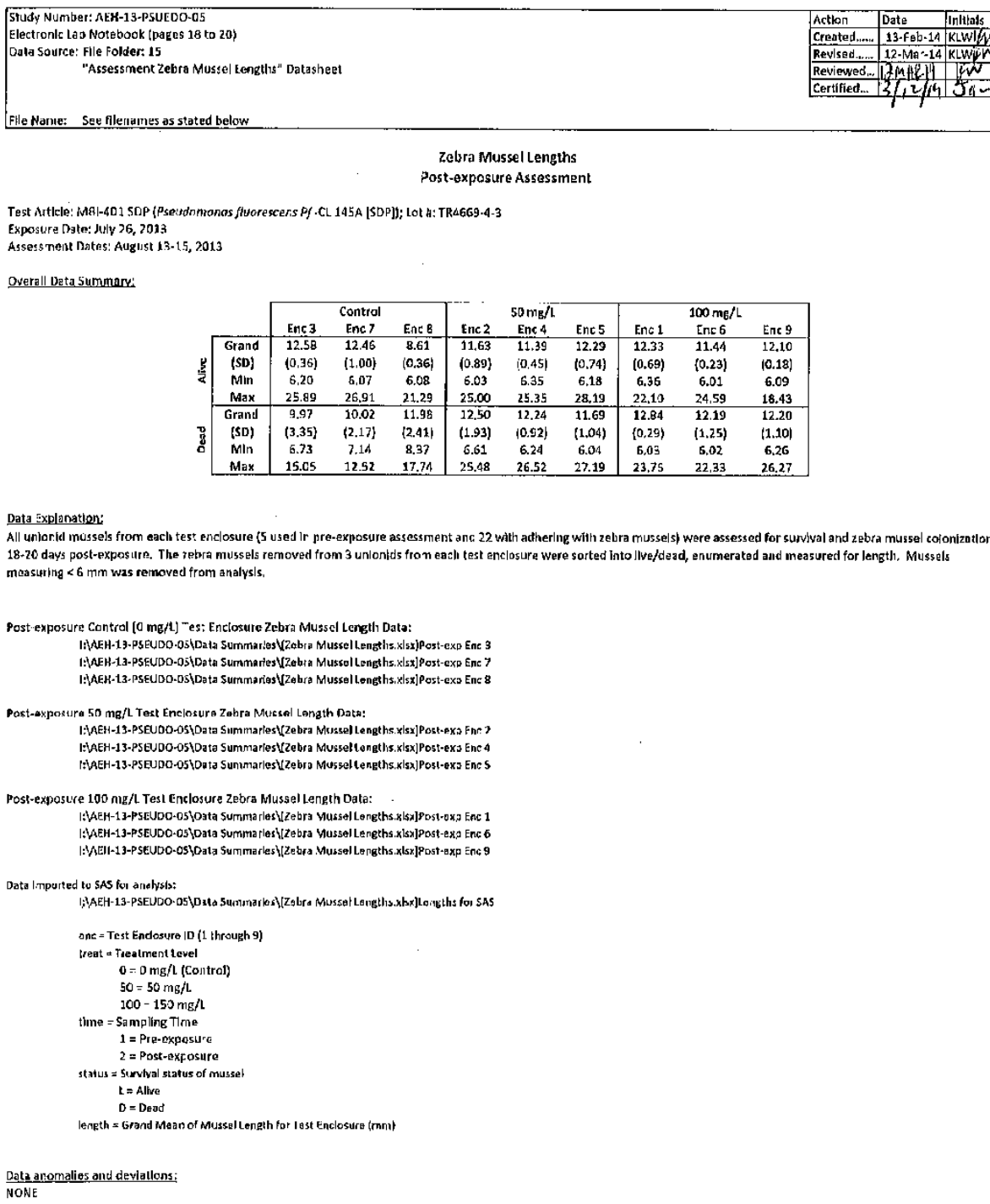

\begin{tabular}{|c|c|c|c|c|c|c|c|c|c|c|}
\hline & \multicolumn{3}{|c|}{ Control } & \multicolumn{3}{|c|}{$50 \mathrm{mg} / \mathrm{L}$} & \multicolumn{3}{|c|}{$100 \mathrm{mg} / \mathrm{L}$} \\
\hline & & Enc 3 & Enc 7 & Enc B & Enc 2 & Enc 4 & Ent 5 & Enc 1 & $\operatorname{Enc} 6$ & Enc 9 \\
\hline & Grand & 12.58 & 12.46 & 8.61 & 11,63 & 11.39 & 12.29 & 12.33 & 11.44 & 12.10 \\
\hline & (SD) & $(0,36)$ & $\{1,00\}$ & $(0,36)$ & (0.89) & {$[0,45\}$} & $(0.74)$ & $(0.69)$ & $\{0.23\}$ & (0.18) \\
\hline & Min & 6.20 & 6,07 & 6.08 & 6.03 & 6.35 & 6.18 & 6.35 & 6.01 & 6.09 \\
\hline & $\operatorname{Max}$ & 25.89 & 26,91 & 21.29 & 25.00 & 25.35 & 28.19 & 22,19 & 24,59 & 18.43 \\
\hline & Grand & 9.97 & 10.02 & 11.98 & 12.50 & 12.24 & 11.69 & 12.84 & 12.19 & 12.20 \\
\hline & (SD) & (3.35) & $\{2.17\}$ & $\{2.41\}$ & (1.93) & (0.52) & $(1,04)$ & $\{0.29\}$ & $(1.25)$ & $(1.10)$ \\
\hline & Mln & 6.73 & 7.16 & 8.37 & 6.61 & 6.24 & 6.04 & 6,03 & 5,02 & 6.26 \\
\hline & Max & 15.05 & 12.52 & 17.74 & 25.48 & 26.52 & 27.19 & 23,75 & 22,33 & 26.27 \\
\hline
\end{tabular}

Data Explanation:

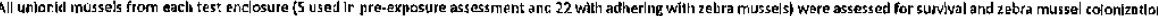
$18-20$ days post-expos ure. The sebra mussels removed from 3 unlonids fro neasil test enclosure were sorted into llve/dead, enumerated and measured for length, Mussels measuring $<6 \mathrm{~mm}$ was removed from analysis

Post exposure Control (0 me/t)"es: Enclosure Zebra Musscl Length Dato:

A:MEH-13.PSEUDO-0STData SUmm

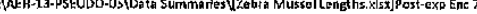

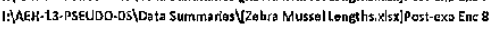

Past-axposura $50 \mathrm{mg} / \mathrm{L}$ Test Enclosura Zabra Mussal Langth Data:

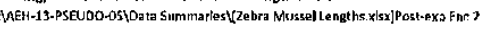

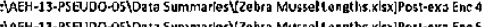

Post-expostre $100 \mathrm{mg} / \mathrm{L}$ Tesi Enclosure Zebra Mussel Length Data:

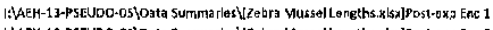

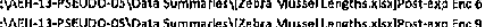

Data Impurted to SAS for anehysli:

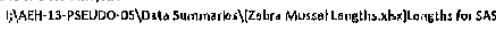

one = Test Endosure ID (1 through 9)

treat a Tiealment tevel

$0=0 \mathrm{mg} / \mathrm{L}$ (Contro

$50=50 \mathrm{mg} / \mathrm{L}$

$1=$ Pre-expasure

$2=$ Post-axfosure

status = Sunvival status of musset

$t=$ Alve

length s Grand Mearrof Mussel Leneth for lest Enclosure (mnnt)

Qata anomalies and deviallons:

NONE 


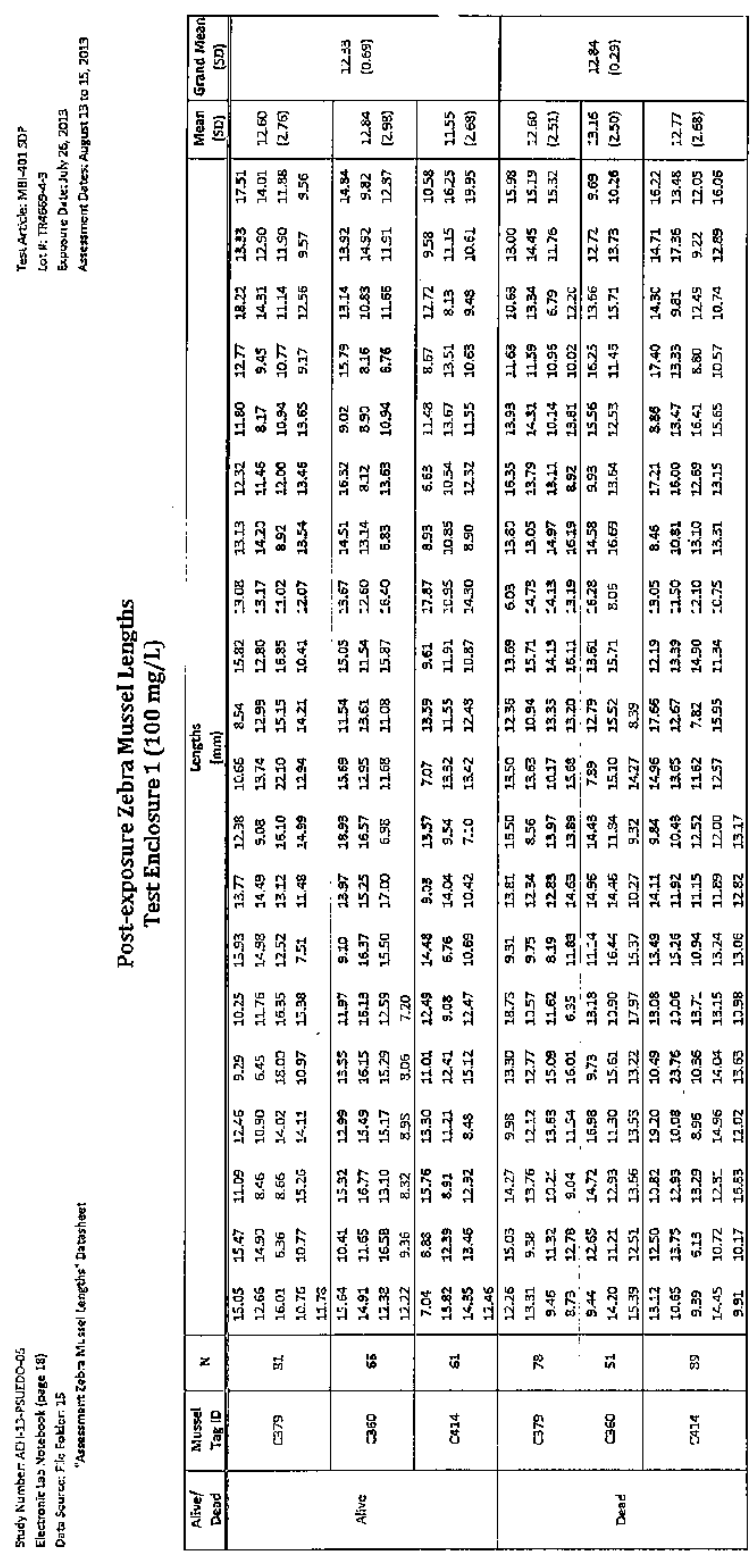

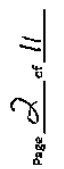




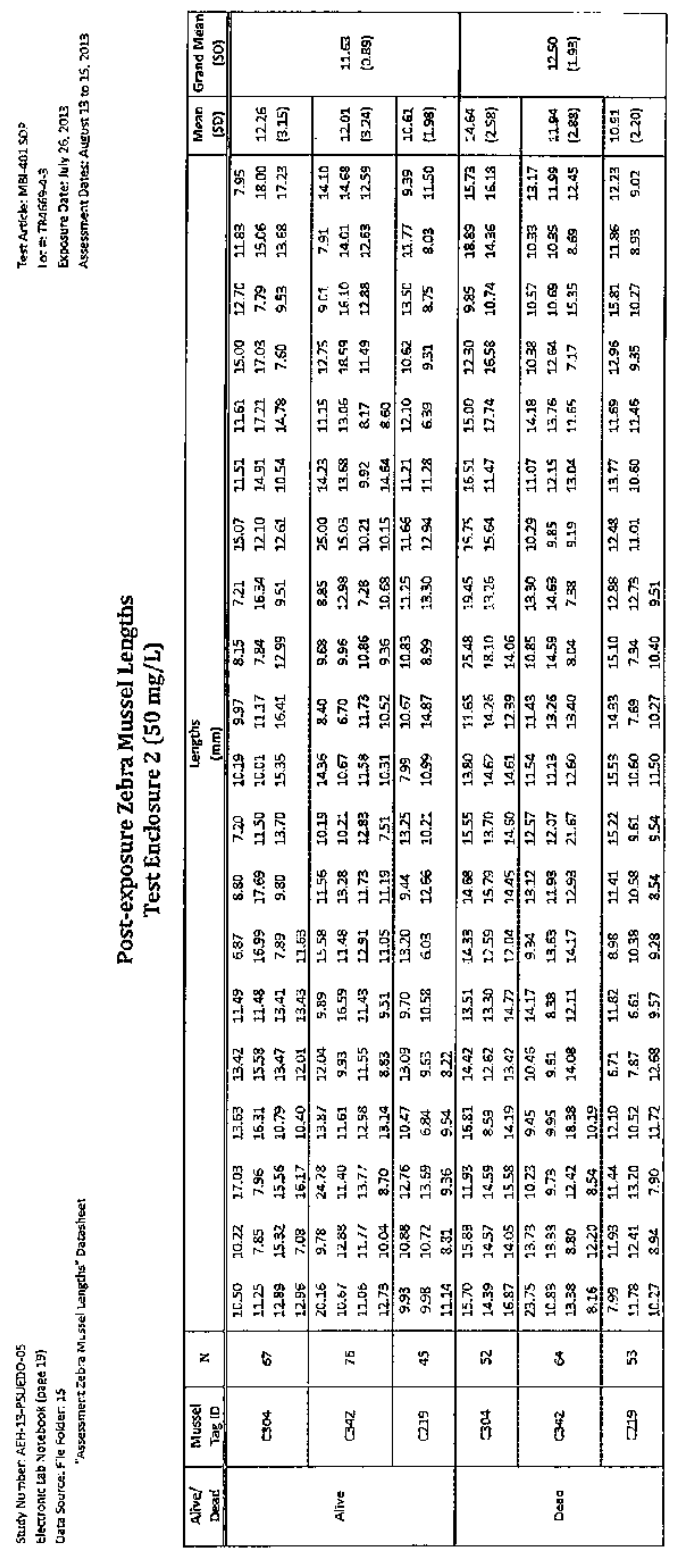

$=$ 


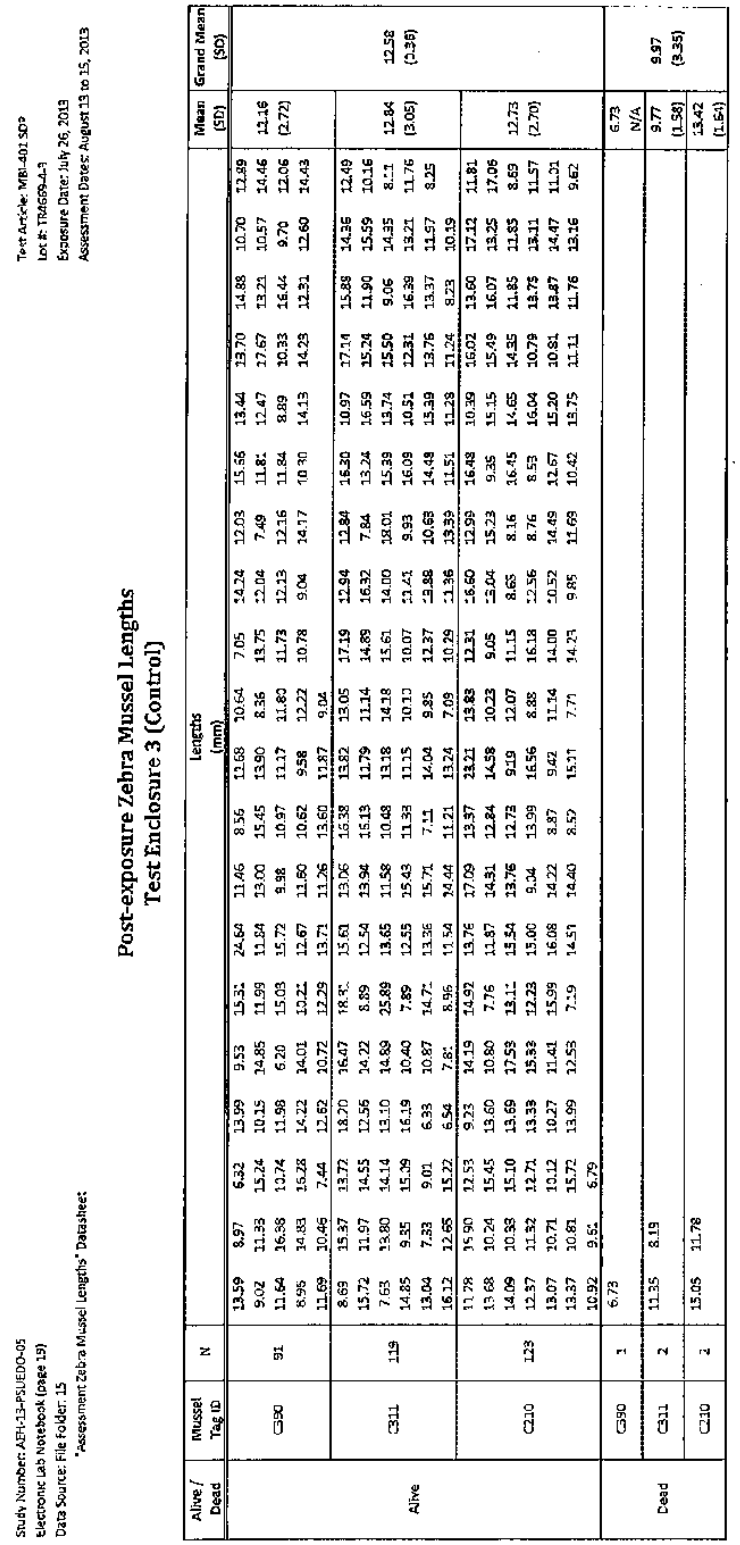

$=$ 


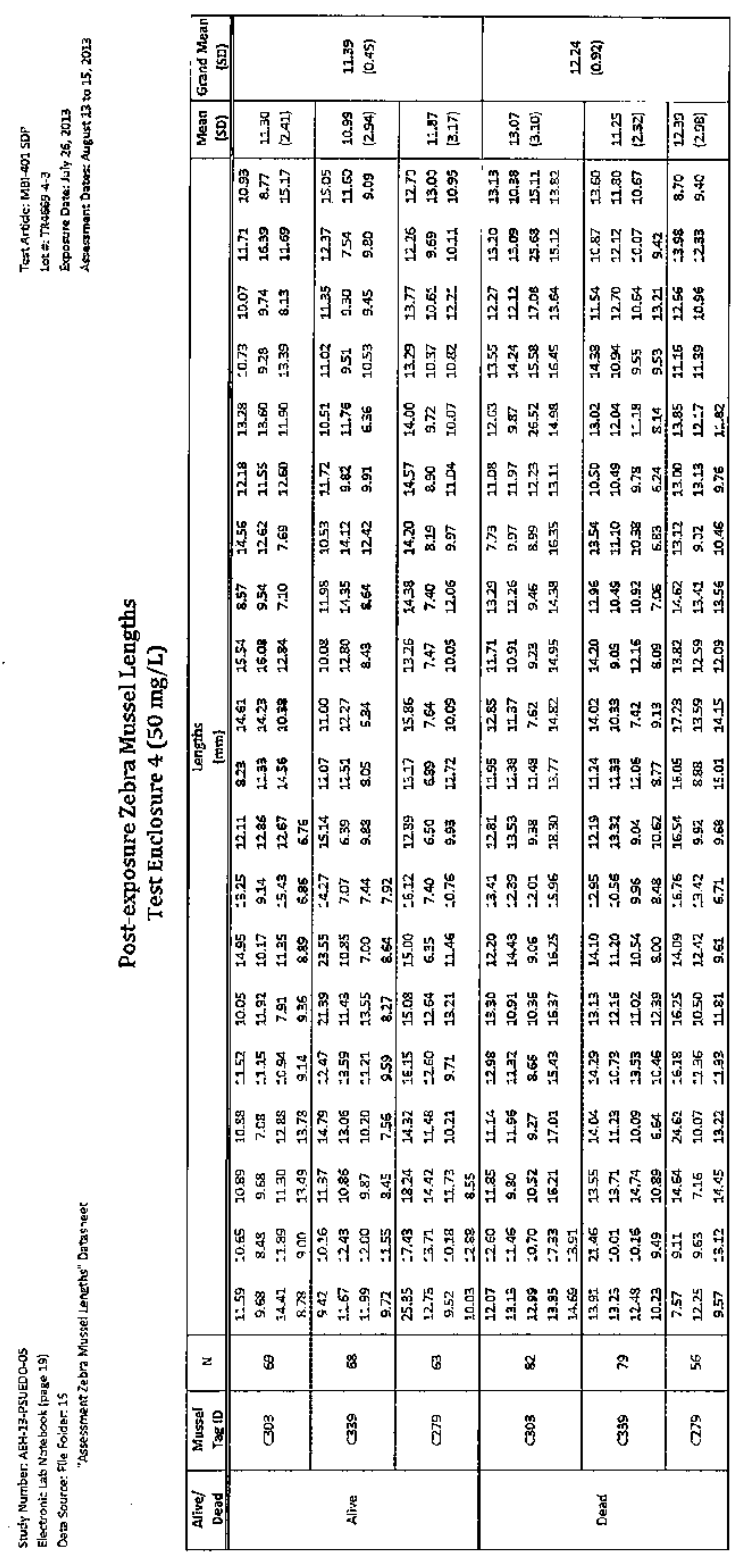

$=$ 


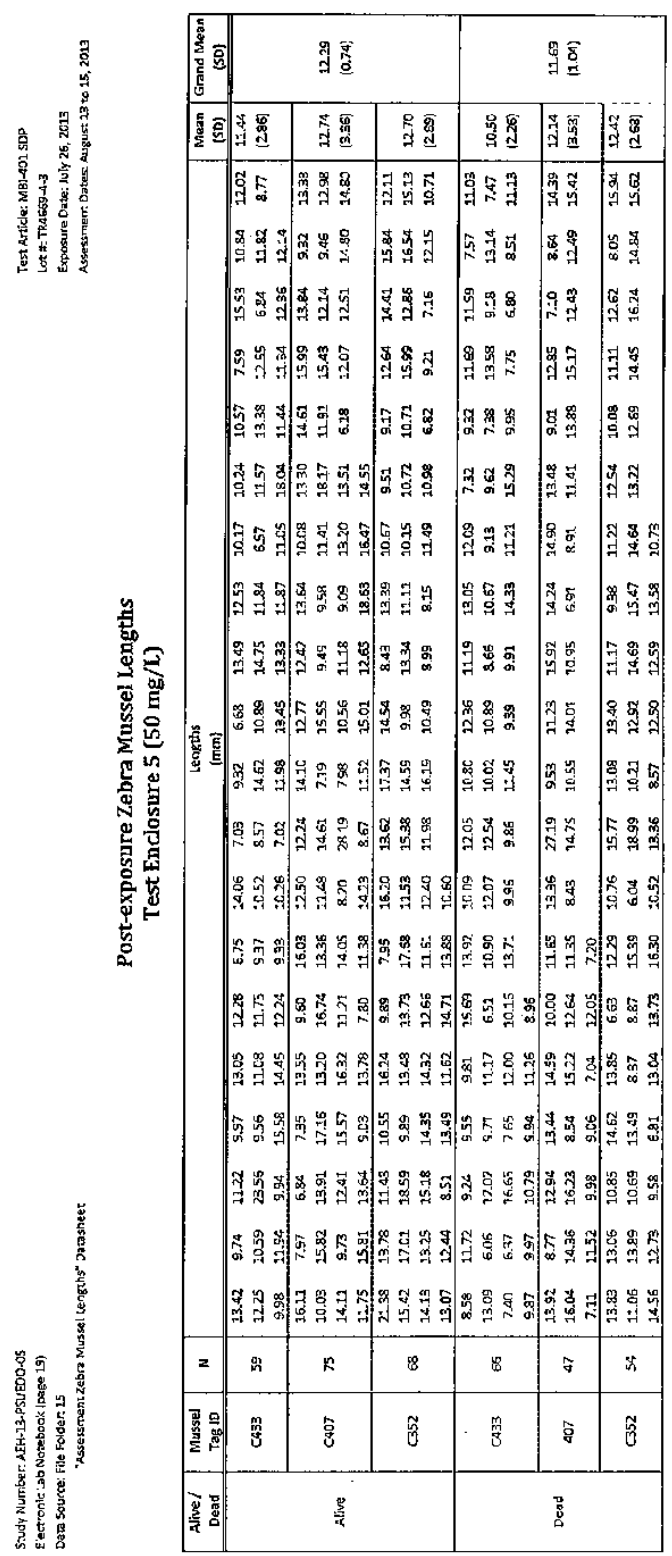

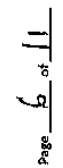




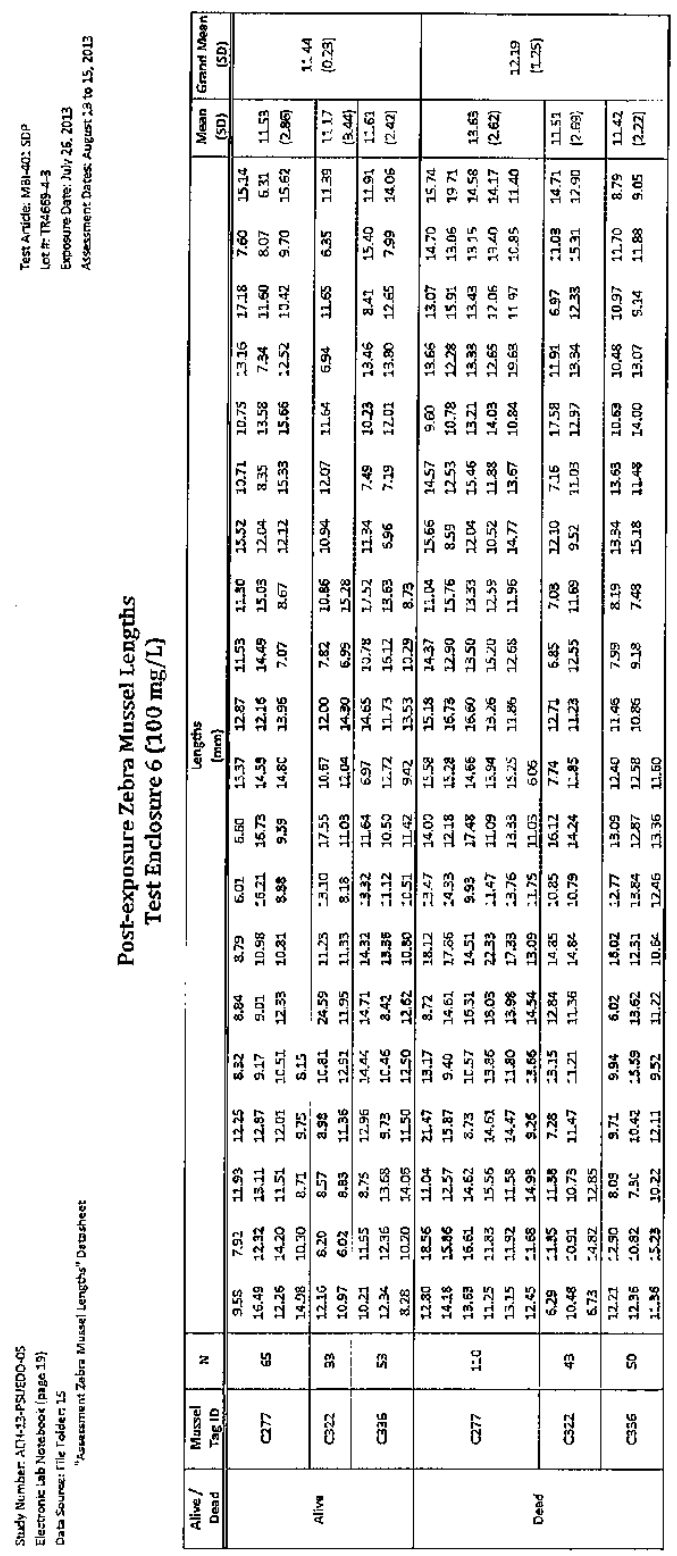

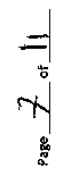




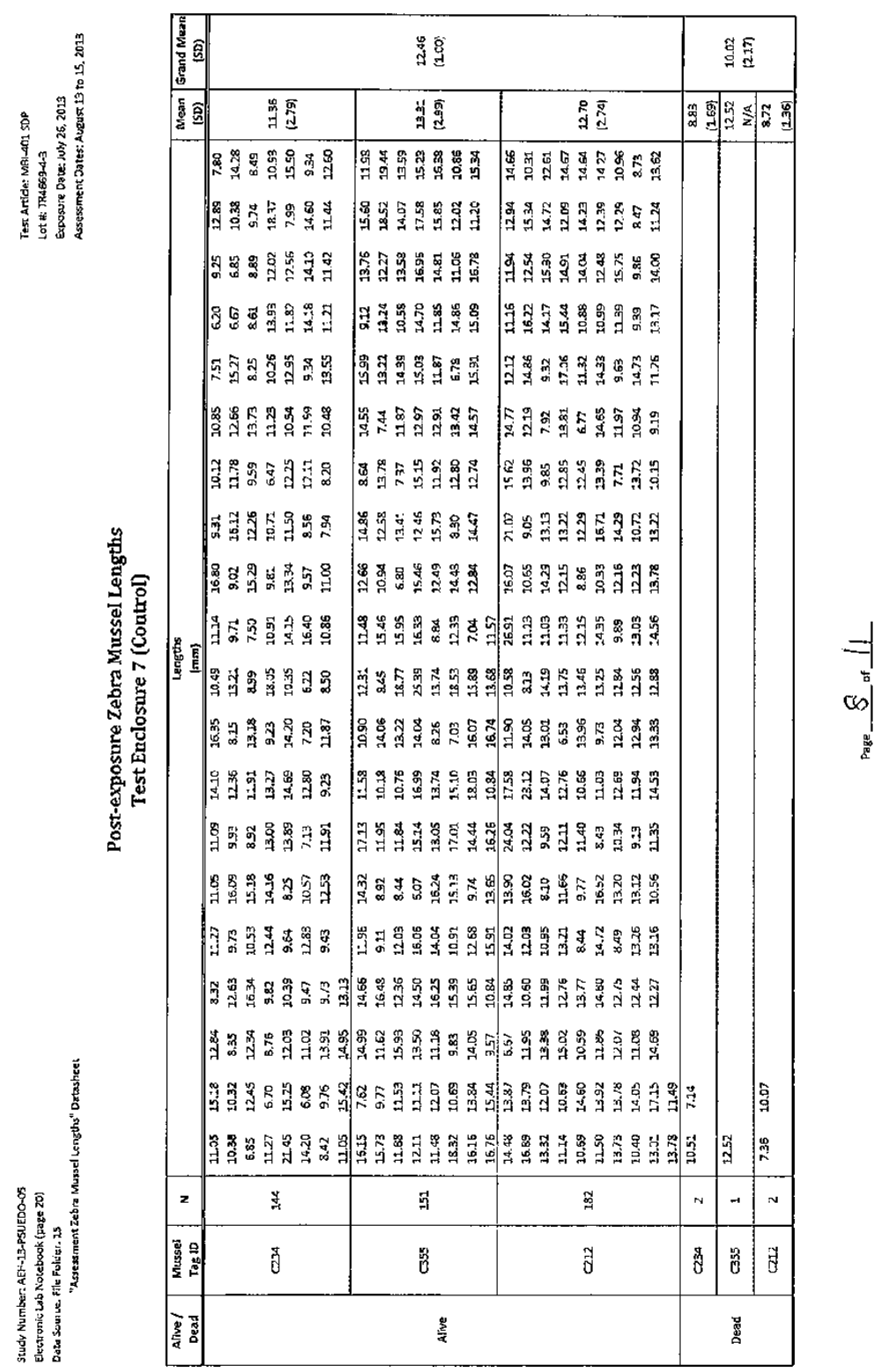




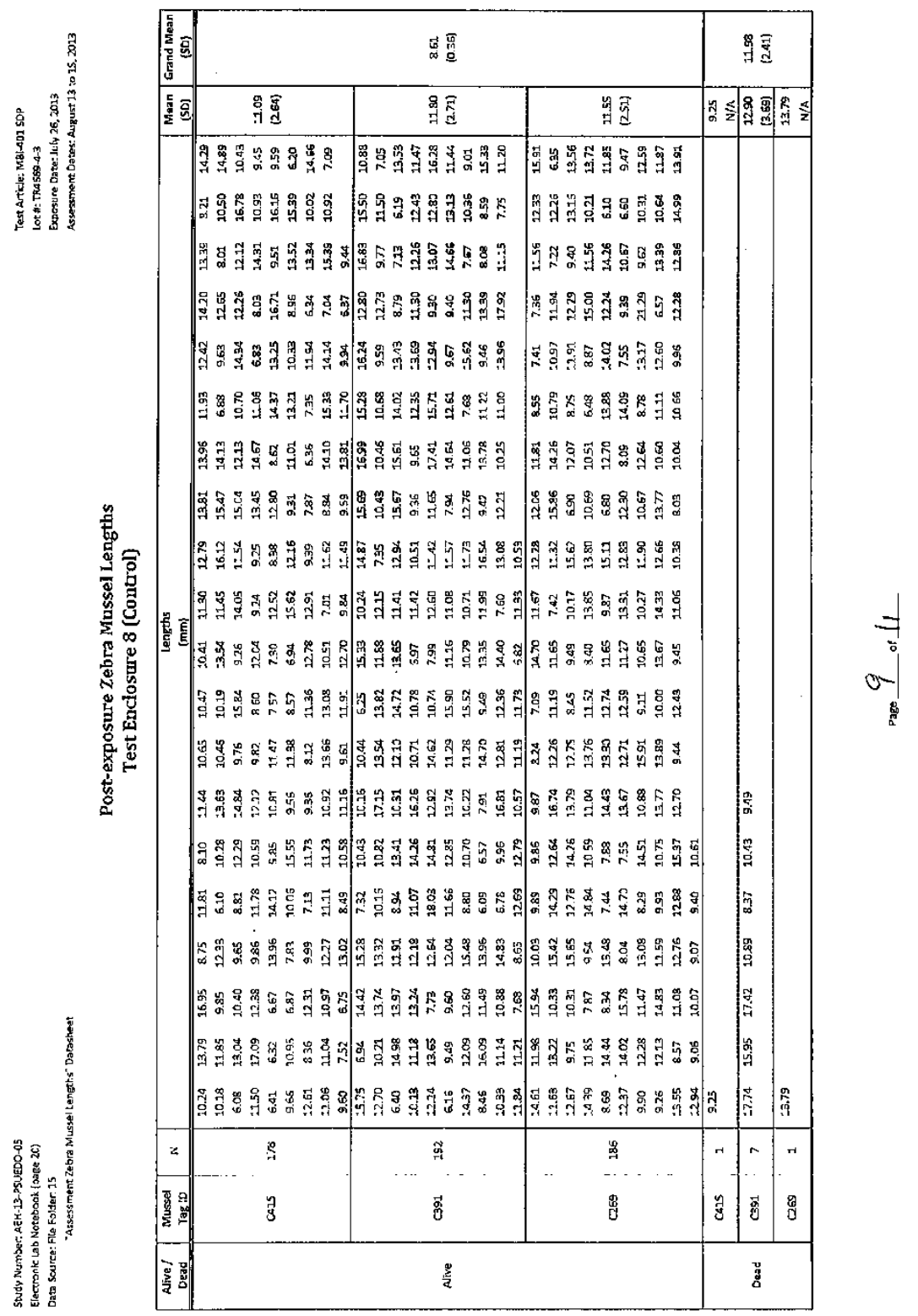




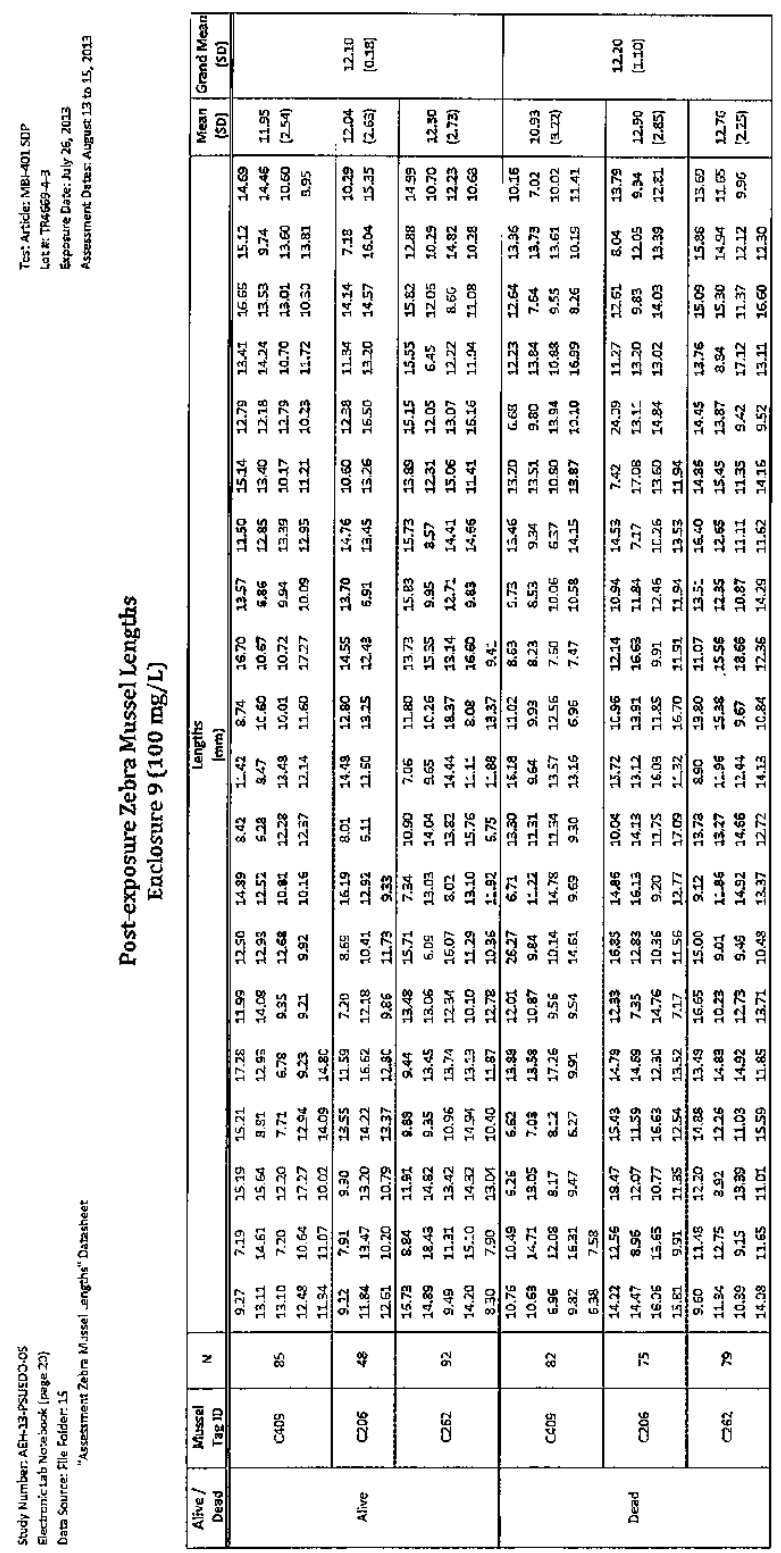

1
71
0
0
$\vdots$
$\vdots$ 


$\begin{array}{cccc}\text { enc } & \text { treat } & \text { time } & \text { length } \\ 1 & 100 & 1 & 11.32 \\ 2 & 50 & 1 & 12.63 \\ 3 & 0 & 1 & 12.04 \\ 4 & 50 & 1 & 11.65 \\ 5 & 50 & 1 & 11.90 \\ 6 & 100 & 1 & 11.53 \\ 7 & 0 & 1 & 11.95 \\ 8 & 0 & 1 & 12.11 \\ 9 & 100 & 1 & 10.82 \\ 1 & 100 & 2 & 12.33 \\ 2 & 50 & 2 & 11.63 \\ 3 & 0 & 2 & 12.58 \\ 4 & 50 & 2 & 11.39 \\ 5 & 50 & 2 & 12.29 \\ 6 & 100 & 2 & 11.44 \\ 7 & 0 & 2 & 12.46 \\ 8 & 0 & 2 & 8.61 \\ 9 & 100 & 2 & 12.10\end{array}$

AEH-13-PSEUDO-05

FF \# 15

Item No. I

$\mathrm{Pg} \ldots 11$ of 11 


\section{Appendix 6. Water Quality}

\begin{tabular}{clcc}
\hline $\begin{array}{c}\text { Item } \\
\text { number }\end{array}$ & \multicolumn{1}{c}{ Item description } & $\begin{array}{c}\text { Number } \\
\text { of } \\
\text { pages }\end{array}$ & $\begin{array}{c}\text { Report } \\
\text { page } \\
\text { number }\end{array}$ \\
\hline 1 & Pre-Exposure Period Water Chemistry - Data Summary & 2 & 169 \\
2 & Exposure Period Water Chemistry - Data Summary & 2 & 171 \\
3 & Ammonia - Data Summary & 3 & 173 \\
4 & Temperature Data Loggers (HOBOs) - Data Summary & 4 & 176 \\
5 & Report of Analysis - Ammonia Report from Water Quality Laboratory at UMESC & 1 & 180 \\
\hline
\end{tabular}




\begin{tabular}{|c|c|c|c|c|}
\hline \multirow{2}{*}{\multicolumn{2}{|c|}{ Study Number: AER-13-PSEUDO.05 }} & Actior & Date & miltals \\
\hline & Electronle Lab Notebook ipage 12; & Ereated...... & $5: \$ e p-13$ & KLWtiv \\
\hline \multirow[t]{3}{*}{ Data Source: } & FHe Folder: 11 & Revised, in. & 19-Feb-14 & $\mathrm{xi} w\left(\mathrm{w}^{\mathrm{w}}\right.$ \\
\hline & Forms: "Water quallty" datasheet (pre-exposure for DO, pH, te.nperatures; "Alkallhily" datasheet: & Reviewed... & $149583 \mathrm{M}$ & Yow \\
\hline & "ConJuetivky and Hardness dalasheet & Centiffied.... & $2 / 15 / / 14$ & 15,5 \\
\hline File Hame: & I:AEEH-19.PSEULO-05|Data Sumunarles\}(Waier Chem.xilsx|Pra-Exposure Water Chem & & & \\
\hline
\end{tabular}

Pre-Exposure Period Water Chemistry

Test Article: MBI-401 SDP (Pseudorronas fluorescees Pf-CL. 145A [SDP]) Lot H: TRA669-4-3

Exposure Date: July 26, 201

Assessinent Jates: August 13-15, 2013

Data Explanatlon:

Pre-Exposure Period Water Chemlstry- Dissolved onygen, pht, and temperature were measured prlor to exposure initlatlon in each test erlclosure, Akallnily, hardness, and conductivity were

measured from water samples collected in the vicinity of each test enclosure.

Data anomilles and devlations

NONE

=lle Foker $\frac{\mid 1}{1}$



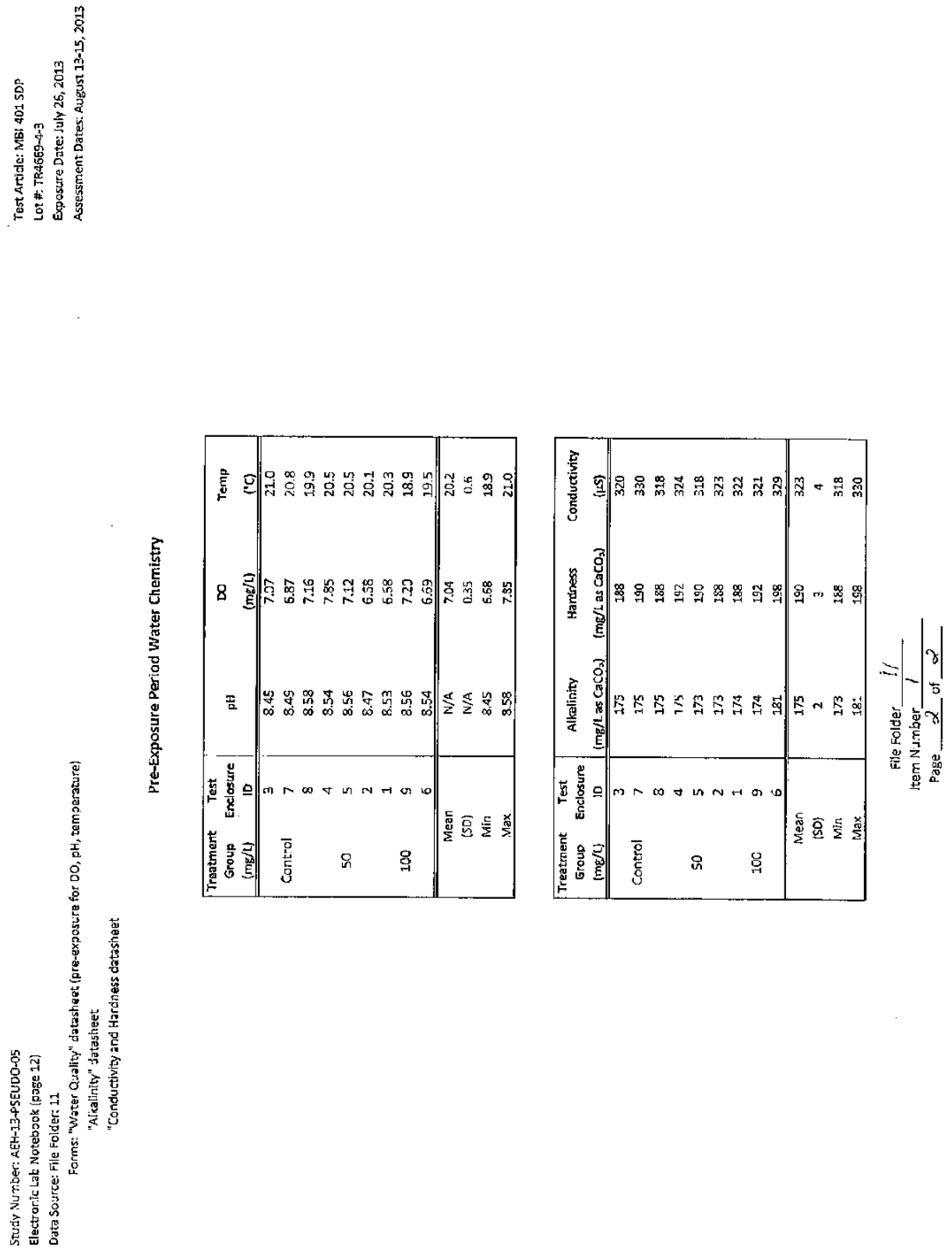


\begin{tabular}{|c|c|c|c|c|}
\hline \multicolumn{2}{|c|}{$\begin{array}{l}\text { Study Number: AEH-13-PSEUDO-05 } \\
\text { Electronic Lab Notebook (page 12) }\end{array}$} & Action & Jate & \begin{tabular}{|l} 
Initlals \\
KLiN itins
\end{tabular} \\
\hline \multirow[t]{3}{*}{ Data Source: } & Flle Folder: 11 & \begin{tabular}{|l} 
Greaticd....... \\
Revised...
\end{tabular} & \begin{tabular}{|l|l}
$5-\mathrm{Sep}-13$ \\
$19 \cdot \mathrm{Fel}-14$
\end{tabular} & $\mid \begin{array}{ll}\alpha L N \mid W \\
K L W\end{array}$ \\
\hline & Forms: "Water Quality" datasheet (4h and Bh') & Revieved... & jallabilu & even \\
\hline & & Certifled... & $1.2 / 19 / 13$ & $\sqrt{1}-$ \\
\hline File Name: & 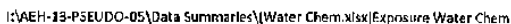 & & & \\
\hline
\end{tabular}

Exposure Perlod Water Chemlstry

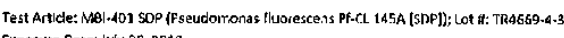

Ekposure Da:e: July 26, 2013

Assessment Dates: August 13-15, 201

Data Explanation:

Exposure Period Water Chemls:ry - Olssolvgd oxygen, $\mathrm{pK}$ and tempersture measurements from the center of all test enclosures at 4 and gh.

Data anomalles and deviatlons

NONE

Hille ralder 41

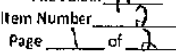


Study vurber: A:H-13-35FUDO-05

Elect-onic Lab Notebook (page 12)

Data Source: Flle Folder: 11

Test Article: MBI \&C1 SDP

Lot it: TRA669-<-3

Exposure Date: July 26, 2013

Forms: "Water Quality" datasheet (4n and 8h)

Assessmient Dates: August 13-15, 2013

Exposure Water Chemistry

\begin{tabular}{|c|c|c|c|c|c|c|c|}
\hline \multirow{2}{*}{$\begin{array}{c}\text { Treatment } \\
\text { Group } \\
\text { (mig/L) } \\
\end{array}$} & \multirow{2}{*}{$\begin{array}{c}\text { Test } \\
\text { Enclesure Io }\end{array}$} & \multicolumn{3}{|c|}{ A Hour } & \multicolumn{3}{|c|}{8 Hour } \\
\hline & & $\begin{array}{c}\mathrm{DO} \\
(\mathrm{mg} / \mathrm{L})\end{array}$ & $\mathrm{pH}$ & $\begin{array}{c}\text { Tenip. } \\
\left({ }^{\circ} \mathrm{C}\right)\end{array}$ & $\begin{array}{c}\text { Do } \\
\text { [mg/L })\end{array}$ & $\mathrm{pH}$ & $\begin{array}{l}\text { Temp. } \\
\text { ("C) }\end{array}$ \\
\hline \multirow{3}{*}{ Control } & 3 & 6.48 & 8.50 & 23.2 & 5.83 & 8.40 & 23.0 \\
\hline & 7 & 6.34 & 8.46 & 22.6 & 6.49 & 8.46 & 23.0 \\
\hline & 8 & 6,64 & 8.45 & 22.9 & 6.77 & 8.49 & 22.9 \\
\hline & 6.49 & $\mathrm{~N} / \mathrm{A}$ & 22.9 & 6.36 & $\mathrm{~N} / \mathrm{A}$ & 23.0 \\
\hline & (SD) & 0.12 & $\mathrm{~N} / \mathrm{A}$ & 0.2 & 0.39 & $N / A$ & 0.0 \\
\hline \multicolumn{2}{|c|}{ Min } & 6.34 & 8.45 & 22.6 & 5.83 & 8,40 & 22.9 \\
\hline \multicolumn{2}{|c|}{$\operatorname{Max}$} & 6.64 & 8.50 & 23.2 & 6.77 & 8.49 & 23,0 \\
\hline \multirow{3}{*}{50} & 4 & 5.96 & 8.38 & 23.3 & 5.67 & 8.35 & 23.0 \\
\hline & 5 & 6.15 & 8,36 & 23.1 & 5.68 & 8.36 & 23,2 \\
\hline & 2 & 5.73 & 8.35 & 23.0 & 5.54 & 8.25 & 23.1 \\
\hline & 5.95 & $N / A$ & 23.1 & 5,63 & $\mathrm{~N} / \mathrm{A}$ & 23.1 \\
\hline & (SD) & 0.17 & N/A & 0.1 & 0.06 & $\mathrm{~N} / \mathrm{A}$ & 0.1 \\
\hline \multicolumn{2}{|r|}{ Min } & 5.73 & 8.35 & 23.0 & 5.54 & 8.25 & 23.0 \\
\hline \multicolumn{2}{|c|}{ Max } & 6.15 & 8.38 & 23.3 & 5.58 & 8.35 & 23.2 \\
\hline \multirow{3}{*}{100} & 1 & 5.68 & 8.16 & 23.1 & 5.24 & 8.20 & 23.2 \\
\hline & 9 & 6.12 & 8.28 & 23.1 & 5.60 & 8.23 & 22.8 \\
\hline & 6 & 5.75 & 8.24 & 22.7 & 5.24 & 8.18 & 23.2 \\
\hline \multicolumn{2}{|c|}{ Mean } & 5.85 & $\mathrm{~N} / \mathrm{A}$ & 23.0 & 5.36 & $\mathrm{~N} / \mathrm{A}$ & 23.1 \\
\hline \multirow{2}{*}{\multicolumn{2}{|c|}{$\begin{array}{l}\text { (SD) } \\
\text { Min }\end{array}$}} & 0.19 & N/A & 0.2 & $0.1 \%$ & $\mathrm{~N} / \mathrm{A}$ & 0.2 \\
\hline & & 5.68 & 8.16 & 22.7 & 5.24 & 8.18 & 22.8 \\
\hline \multicolumn{2}{|c|}{$\operatorname{Max}$} & 6.12 & 8.28 & 23.1 & 5.60 & 8.23 & 23.2 \\
\hline \multicolumn{2}{|c|}{ Grand Mean } & 6.09 & $\mathrm{~N} / \mathrm{A}$ & 23.0 & 5.78 & $\mathrm{~N} / \mathrm{A}$ & 23.0 \\
\hline \multicolumn{2}{|c|}{ [SD] } & 0.33 & N/A & 0.2 & 0.49 & $N / A$ & 0.1 \\
\hline \multicolumn{2}{|c|}{ Mln } & 5.68 & 8.16 & 22.6 & 5.24 & 8.18 & 22.8 \\
\hline \multicolumn{2}{|c|}{ Max } & 6.64 & 8.50 & 23.3 & 6.77 & 8.49 & 23.2 \\
\hline
\end{tabular}

Flle Folder II

Item Number $\frac{2}{2}$ 


\begin{tabular}{|c|c|c|c|}
\hline Study Number: AEF-13-PSEUDO-05 & Action & Date & |nitials \\
\hline Electronic Lab Notebook (page 12) & Created...... & $11-F e b-14$ & KI.WWin' \\
\hline TAN Data Source: LTRMP Report (File Folder 11) & Revised...... & 19-Feb-14 & KLW $\mathrm{LWW}$ \\
\hline \multirow[t]{2}{*}{$\mathrm{pH}$ and Temperature Data Source: $8 \mathrm{~h}$ Water Quality Datasheet (File Folder 11) } & Reviewed... & 96214 & Ww \\
\hline & Certlfied....... & $2 / 16 i / 4$ & Jan \\
\hline
\end{tabular}

\section{Ammonia}

Test Article: MBI-401 SDP (Pseudomonas fluorescens Pf-CL 145A [SDP]); Lot \#: TR4669-4-3

Exposure Date: July 26, 2013

Assessment Dates: August 13-15, 2013

Data Explanation:

1) Water samples were collected upon exposure termination, acldified with $10 \%$ sulfuric acld, and analyzed for total ammonia nitrogen (TAN) by the $4500-\mathrm{NH}_{3}$ G. Automated Phenate Method (Standard Methods for the Fxamination of Water and Wastewater, 21st Edltion, 2005) usIng a Technicon Autoanalyzer II.

2) Un-lonlzed ammonia fractions were calculated according to the formula in Emerson et al. (1975) using $p-1$ and temperature observed at the time of sample collection.

Data Anomalies and Deviatlons:

NONE

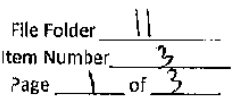



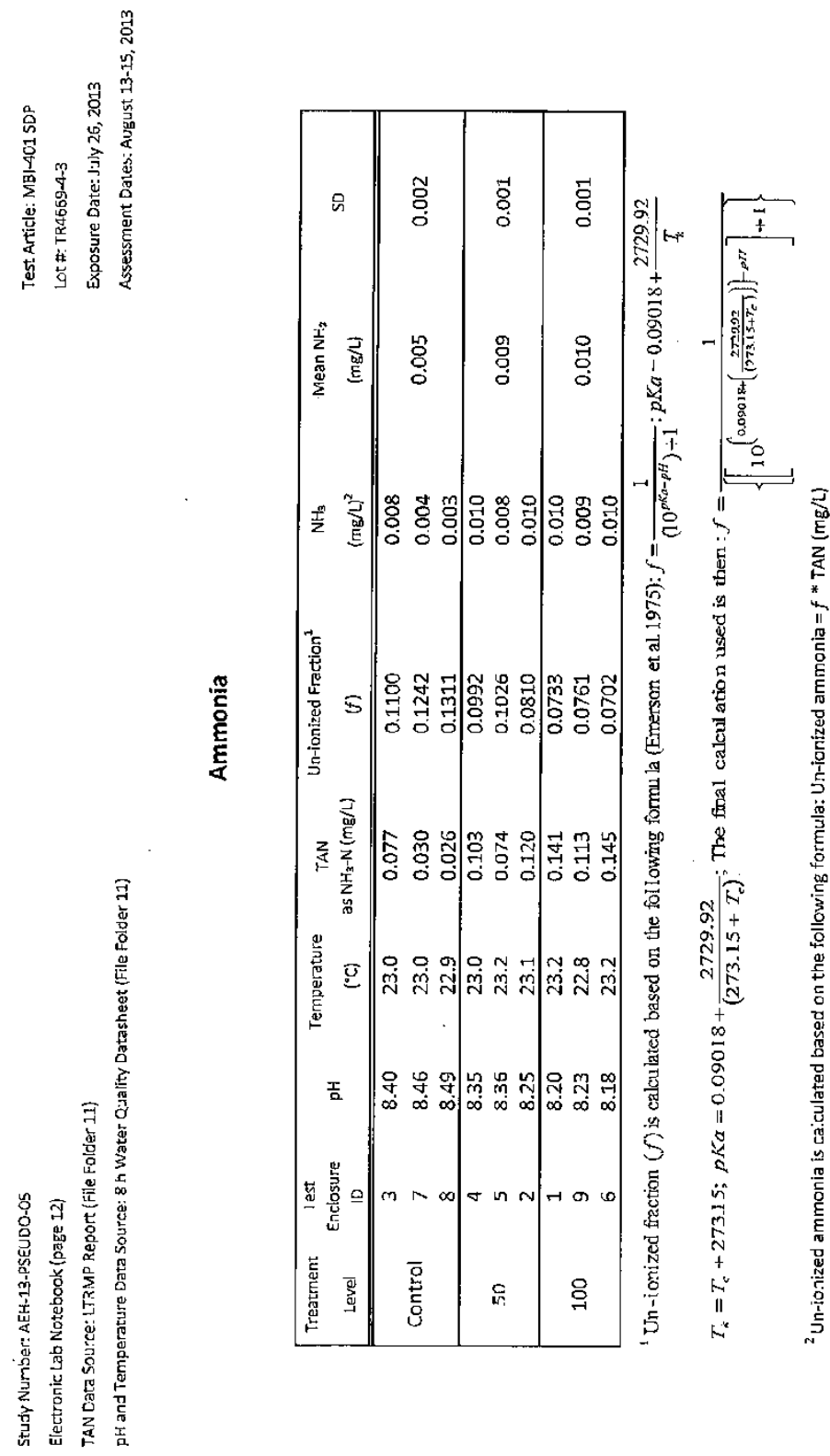

2 


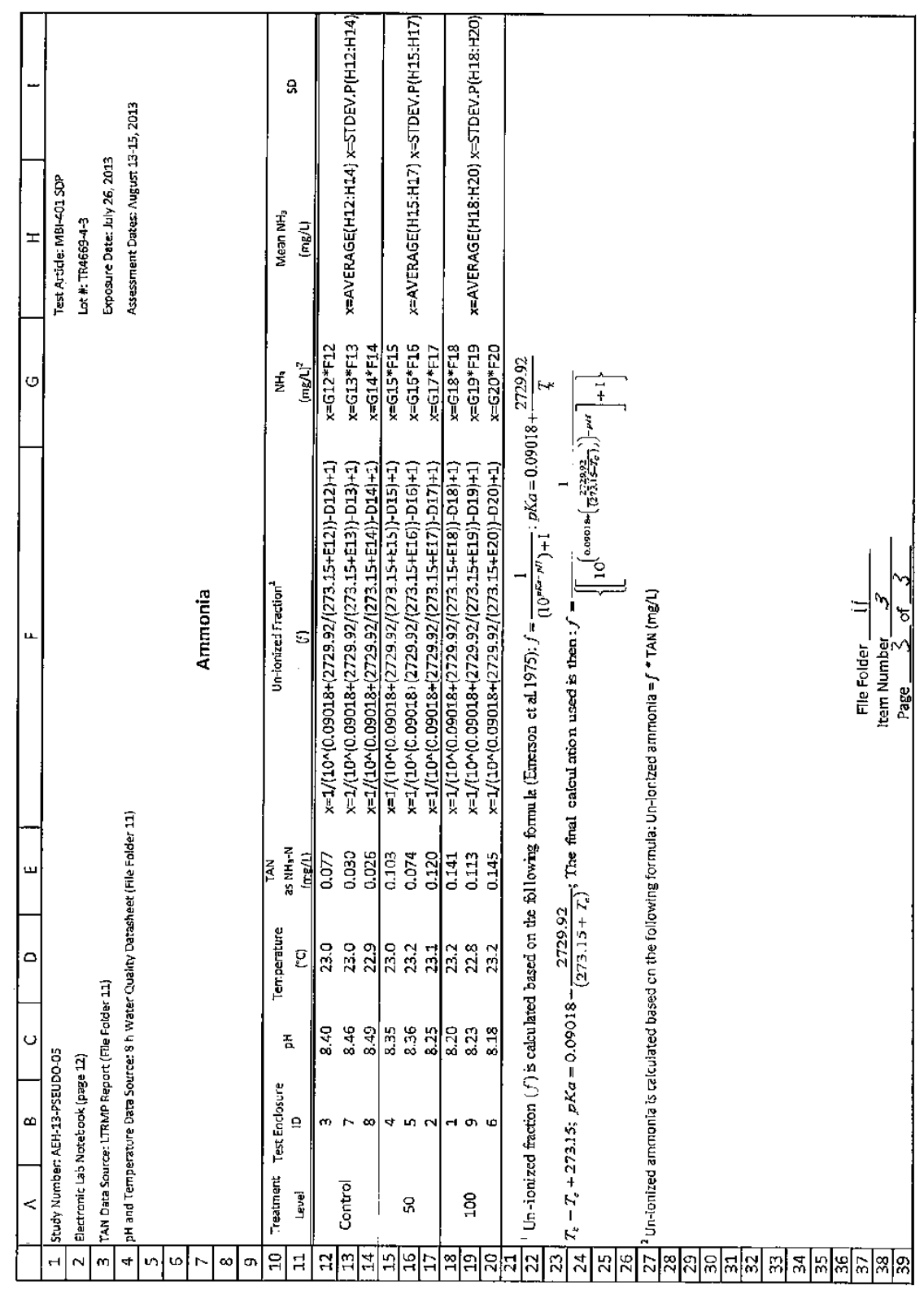




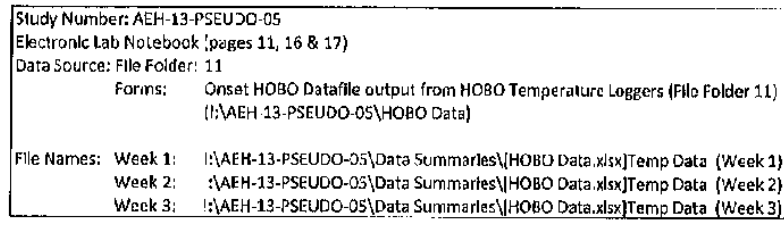

Temperature Data Loggers (HOBOs)

Test Article: MBi 401 SOP (Psevdononas fituorescens Pf-Q 145A [SDP]); Lot H; TR $\angle 669-4-3$ Exposure Date: July 26, 2013

Assessment Dates: August 13 to 15, 2013

DataExplantatlon:

On July 26, 2013 a $H O B O$ temperature loggers were programmed to record water temperature every three hours (eight times a day) and attacined to the retention barrlers. The HOBO temperature loggers were removed and temperature data werc offloaded during the post-expcsure unionld mussel assessment (August 13 2013 for test enclosures 1,2 and 3 ; August $-4,2013$ for test enclosures 4, 6 and 7; August 15, 2013 for test enclosures 5,8 and 9). Mean (ally temperature ("C) and 2013 for test enclosures 1,2 and 3 ; August $: 4,2013$ for test enclosures 4,6 and 7 ; August 15,2013 for test enclosuro
standard devlation were calculated from oally temperature measurements from all HOBO temperature loggers.

\begin{tabular}{|c|c|c|c|c|c|}
\hline $\begin{array}{l}\text { Data Logger } \\
\text { Number } \\
\end{array}$ & $\begin{array}{c}\text { Serial } \\
\text { Number }\end{array}$ & $\begin{array}{c}\text { Test Enclosure } \\
1 \mathrm{D}\end{array}$ & $\begin{array}{l}\text { Treatment } \\
\text { Level (mg/L) }\end{array}$ & $\begin{array}{l}\text { Ceployment } \\
\text { Date }\end{array}$ & $\begin{array}{l}\text { TermInation } \\
\text { Date }\end{array}$ \\
\hline 1 & 10020132 & $\overline{11}$ & 100 & $25-\int u t-13$ & $13-\mathrm{Au}-13$ \\
\hline 2 & 10020138 & 2 & 50 & $25-5,1-13$ & 13-Aug-13 \\
\hline 3 & 10020139 & 3 & Control & 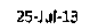 & 13-Aug-13 \\
\hline 4 & 10020141 & 4 & 50 & $25-\mid ;: 181 \cdot 13$ & 14-Aug-13 \\
\hline 5 & 10020142 & 5 & 50 & $25-\{|u|-13$ & $15-A d g-13$ \\
\hline 6 & 10020133 & 6 & 100 & 25-Jul-13 & 14.A.dg-13 \\
\hline 7 & 10020131 & 7 & Control & $25-\mathrm{Jul}-13$ & $14-A\lrcorner g-13$ \\
\hline 8 & 10020134 & 8 & Control & $25-J u l-13$ & 15-Aug-13 \\
\hline 9 & 10020136 & 9 & 100 & $25-\mathrm{Jul} \cdot 13$ & 15-Aug-13 \\
\hline
\end{tabular}

\begin{tabular}{|c|c|c|c|c|c|}
\hline \multicolumn{5}{|c|}{ Mean } & \\
\hline Date & (SO) & $\mathrm{N}$ & Date & $\begin{array}{l}\text { Mean } \\
\text { (SDD }\end{array}$ & $\mathrm{N}$ \\
\hline $7 / 26 / 2013$ & $\begin{array}{l}23.86 \\
(0.35)\end{array}$ & 9 & $B / 5 / 2013$ & $\begin{array}{l}22,69 \\
(0,29)\end{array}$ & 9 \\
\hline $7 / 27 / 2013$ & $\begin{array}{l}22,96 \\
0.321\end{array}$ & 9 & $8 / 6 / 2013$ & $\begin{array}{r}22.59 \\
(\mathbf{0} .55)\end{array}$ & 9 \\
\hline $7 / 28 / 2313$ & $\begin{array}{l}22.26 \\
(0.33)\end{array}$ & 9 & $8 / / 2013$ & $\begin{array}{l}227 \\
(0.62)\end{array}$ & 9 \\
\hline $7 / 29 / 2013$ & $\begin{array}{l}22.35 \\
0.50\}\end{array}$ & 9 & $8 / 8 / 2013$ & $\begin{array}{l}23.03 \\
(0.57)\end{array}$ & 9 \\
\hline $7 / 30 / 2013$ & $\begin{array}{l}22.54 \\
(0.76)\end{array}$ & 9 & $8 / 9 / 2013$ & $\begin{array}{l}22,73 \\
\{0,33\}\end{array}$ & 9 \\
\hline $7 / 31 / 2013$ & $\begin{array}{l}22.69 \\
\{0.35]\end{array}$ & 马 & $8 / 10 / 2013$ & $\begin{array}{l}23.04 \\
(0.80)\end{array}$ & 9 \\
\hline $8 / 1 / 2 C 13$ & $\begin{array}{l}22.89 \\
(0.63)\end{array}$ & 9 & $8 / 11 / 2013$ & $\begin{array}{l}23.39 \\
(0.54)\end{array}$ & 9 \\
\hline $8 / 2 / 2 C_{13}$ & $\begin{array}{l}23.00 \\
(0.45)\end{array}$ & 9 & $8 / 12 / 2013$ & $\begin{array}{l}23.33 \\
(0.52)\end{array}$ & 9 \\
\hline $8 / 3 / 2013$ & $\begin{array}{c}22.82 \\
(0.53)\end{array}$ & 9 & $8 / 13 / 2013$ & $\begin{array}{l}23.34 \\
(0.61)\end{array}$ & 6 \\
\hline $8 / 4 / 2013$ & $\begin{array}{l}72.78 \\
(0.78)\end{array}$ & g & $8 / 14 / 2013$ & $\begin{array}{l}23.51 \\
(0.72\}\end{array}$ & 3 \\
\hline
\end{tabular}

Date anomallics and deviations:

Tempe ature readings from the first and last days of recorder deployment (july 25, 2013 lor all test enclosures and after August 12, 2013 for test enclosures 1, 2 and 3, after August 13, 2013 for test enclosures 4,6 and 7 and after August 14,2013 for test enclosures 5, 8 and 9) were omitted from data analysis. The temperature recorders may have sampled amblent als temperazure durlng these time polnts. These entire days will be omitted as diurnal temperature fluctuation skew the mean dally temperature. The mean temperature for August 13,2013 was calculated using 6 data loggers $(H 4,5,6,7,8$ and 9$)$. The ncan tenipcrature for August 14, 2013 was calcalated using only 3 data loggers $(f 5,8$ end 9 ).

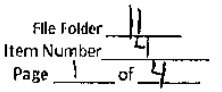




\begin{tabular}{|c|c|c|c|c|c|c|c|c|c|c|}
\hline 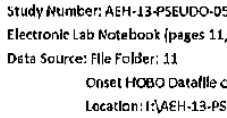 & detrom & $\begin{array}{l}\text { Tempe } \\
\text { Oata }\end{array}$ & Loggers & Folder 1 & & & & $\begin{array}{l}\text { Test Artlc| } \\
\text { Lot Al: TRA } \\
\text { Exposure } \\
\text { Assessmet }\end{array}$ & $\begin{array}{l}\text { M81 401 SOP } \\
\text { J-4-3 } \\
\text { le: Nly 2B, } 2 \\
\text { Dates: Alegus }\end{array}$ & 3 to 15,2013 \\
\hline & & cosure & $d$ and & -еxpos & $\begin{array}{l}\text { toldln } \\
\text { k } 1\end{array}$ & lad Wa & empe & & & \\
\hline & & & & & agger $N$ & & & & & \\
\hline Date & 1. & 2 & 3 & 4 & 5 & 6 & 7 & 8 & 9 & Mean \\
\hline Tlme \{GMT-05:00\} & & & & & eratur & & & & & (SD) \\
\hline $07 / 26 / 20130000 ; 0 \mathrm{CC}$ & 24.5 & 24.5 & 24.5 & 24.4 & 24.4 & 24.5 & 24.4 & 24.4 & 24,5 & \\
\hline |0/26/2013 3:00:00 & 24.1 & 24.1 & 24.2 & 24,0 & 24.1 & 24.2 & 24.0 & 24.0 & 24.2 & \\
\hline |07/26/2013 6:00:00 & 29.7 & 29.7 & 23.8 & 23.6 & 23.8 & 23.8 & 23.6 & 23.6 & 23.8 & \\
\hline 07/26/2013 9:00:00 & 29,6 & 23.6 & 23.6 & 23,5 & 23.6 & 23.6 & 23.5 & 23.5 & 29.6 & 23.9 \\
\hline $07 / 26 / 2013$ 12:00:00 & 23.6 & 23.7 & 23.8 & 23.6 & 23.7 & 23.7 & 23.7 & 23.7 & 23.7 & (C.4) \\
\hline |07/26/2013 15:c0:00 & 23.8 & 24.1 & 24.2 & 23.6 & 23.7 & 24.2 & 24.0 & 23.8 & 23.8 & \\
\hline $07 / 26 / 2013$ 18:00:00 & 24.3 & 24.1 & 24.2 & 23.8 & 24.0 & 24.4 & 24.1 & 24.4 & 24.1 & \\
\hline 07/25/2013 21:00:00 & 23.5 & 23,4 & 23.4 & 23.3 & 23.4 & 23.5 & 23.3 & 29.4 & 23.5 & \\
\hline $07 / 27 / 20130: 00: 00$ & 23.3 & 23.3 & 23.2 & 23.1 & 23.2 & 23.3 & 23.1 & 23.2 & 23.2 & \\
\hline $07 / 27 / 2019$ 3:00:00 & 23.0 & 23.1 & 23.0 & 22.9 & 23.0 & 23.1 & 22.9 & 23.0 & 23.0 & \\
\hline $07 / 27 / 20136: 00: 00$ & 22.8 & 22.8 & 22.8 & 22.5 & 22.8 & 22.8 & 22.7 & 22.8 & 22.8 & \\
\hline $07 / 27 / 201 \exists 9: 00 ; 00$ & 23.3 & 23.1 & 23,4 & 22.7 & 22.9 & 23,3 & 23.2 & 23.0 & 22.9 & 23.0 \\
\hline 07/27/2013 12:00.00 & 23.5 & 23.2 & 29.3 & 22.5 & 27.9 & 24.1 & 23.2 & 23.1 & 23.1 & (0.3) \\
\hline $07 / 27 / 201315: 0000$ & 23.2 & 23.0 & 23.1 & 22.7 & 22.9 & 23.1 & 23.1 & 23.0 & , 23.0 & \\
\hline $07 / 27 / 201318: 00.00$ & 23.2 & 23.0 & 23.1 & 22,7 & 22,9 & 23.3 & 23.0 & 23.3 & 23.0 & \\
\hline $07 / 27 / 201921: 0000$ & 22.4 & 22.3 & 222.4 & 22.2 & 223. & 22.3 & 22.2 & 22.3 & 22.3 & \\
\hline $07 / 28 / 20130: 00: 00$ & 22.1 & 22.1 & 22.1 & 22.0 & 22.1 & 22.1 & 22.0 & 22.0 & 22.1 & \\
\hline $37 / 28 / 20133: 60<00$ & 22.0 & 22.0 & 22.0 & 21,9 & 22.0 & 22.0 & 21.9 & 22.0 & 22.0 & \\
\hline $37 / 28 / 20196: 00: 00$ & 219 & 21.9 & 21.9 & 21.7 & 21.9 & 21.9 & 21.8 & 21.9 & 21.9 & \\
\hline 3/28/2013 9:00:c0 & 22.4 & 22.2 & 22.4 & 21.9 & 22.0 & 22.4 & 22.4 & 22.1 & 22.1 & 22.3 \\
\hline $97 / 28 / 201312: 00: 00$ & 22.9 & 22.6 & 22.7 & 22.0 & 22.2 & 23,2 & 22.5 & 22.4 & 22,4 & 10.3\} \\
\hline 27/28/2013 15:00:00 & 22.8 & 22.6 & 22,6 & 22.1 & 22,2 & 22.4 & 22.5 & 22.8 & 22.4 & \\
\hline 07/28/2013 18:00:00 & 22.9 & 22.6 & 22.7 & 22.2 & 22,4 & 22.8 & 22.6 & 22.9 & 22.6 & \\
\hline $07 / 28 / 201321: 00: 03$ & 22.2 & 22.2 & 22.2 & 22.0 & 22.2 & 22.2 & 22.1 & 22.3 & 22.2 & \\
\hline $07 / 29 / 20130 ; 00: 00$ & 22.1 & 22.1 & 22.1 & 22.0 & 22.1 & 22.1 & 22.0 & 22.0 & 22.1 & \\
\hline 07/29/2013 3;c0:00 & 22,0 & 220 & 22.0 & 21,8 & 22.0 & 22.0 & 21.9 & 21.9 & 22.0 & \\
\hline $07 / 29 / 20136 ; 00: 00$ & 218 & 21.7 & 21.8 & 21,6 & 22.8 & 21.7 & 21.6 & 21. & 21.7 & \\
\hline $07 / 29 / 20139: 00: 00$ & 22.6 & 22.1 & 22.5 & 21.8 & 22.0 & 22.6 & 22.5 & 22.2 & 22.0 & 22.3 \\
\hline 07/29/2013 12:00:00 & 23.3 & 23.2 & 23.6 & 22.6 & 22.9 & 23.9 & 23,3 & 23.1 & 23.0 & 10.5) \\
\hline 07/29/2013 15:00:00 & 23.1 & 23.0 & 23.0 & 22.6 & 22.8 & 22.9 & 22.8 & 22.8 & 22,9 & \\
\hline 07/29/2013 18:05:00 & 22.6 & 22.4 & 22.6 & 22.3 & 22,1 & 22.5 & 22.3 & 22.4 & 22.5 & \\
\hline $07 / 29 / 201321: 03: 00$ & .223. & 223 & 22.3 & .22.1 & 22.2 & 22.3 & 22.1 & 22.2 & 22.2 & \\
\hline $07 / 30 / 20130: 00: 00$ & 22.1 & 22,0 & 22.0 & $2 \div 8$ & 22.0 & 22,0 & 21.9 & 22.0 & 22,0 & \\
\hline 07/30/2013 3:00:00 & 22,0 & 22.0 & 22.0 & $2 . .8$ & 21,9 & 22,0 & 21,9 & 21.5 & 22.0 & \\
\hline 07/30/2013 6:00:00 & 21.9 & 21,9 & 21.9 & $2 i .7$ & 21.8 & 21.9 & 21.8 & 21.9 & 21.9 & \\
\hline |07/30/20139:00:00 & 22.0 & 22.0 & 22.0 & 21.8 & 21.9 & 22.0 & 21.9 & 22,0 & 22.0 & 22.5 \\
\hline $07 / 30 / 201312: 00: 00$ & 22,9 & 22,3 & 22.4 & 21.9 & 22.0 & 22.4 & 22.4 & 22,3 & 22.5 & (0.B) \\
\hline $07 / 30 / 201315: 00: 00$ & 24.3 & 23.7 & 24.2 & 23.2 & 23.2 & 23.6 & 23.8 & 23,5 & 23.4 & \\
\hline 07/30/2013 18:00:00 & 23.7 & 23.6 & 23.7 & 23.4 & 23.5 & 23.6 & 23.5 & 23.6 & 23.6 & \\
\hline $07 / 30 / 2013210000$ & .23 .2 & .23 .2 & 23.1 & 23.0 & 29.1 & 23,1 & 23.0 & 23.1 & 23.2 & \\
\hline $07 / 31 / 2013000000$ & 22.8 & 22.8 & 22,8 & 22.5 & 22.8 & 22.8 & 22.7 & 22.5 & 22.9 & \\
\hline $07 / 31 / 20133: 00: 00$ & 22.6 & 22.6 & 37.5 & 22.4 & 22.6 & 22.6 & 22.4 & 22.5 & 22,6 & \\
\hline $07 / 31 / 20136: 00: 00$ & 22.3 & 22.3 & 22,2 & 22.1 & 22.3 & 22.2 & 22.1 & 22.2 & 22.3 & \\
\hline $07 / 31 / 20139: 00: 00$ & 22.6 & 22.3 & 22.4 & 22.0 & 22.2 & 22.4 & 22.3 & 22.3 & 22.3 & 22.7 \\
\hline $07 / 31 / 201312: 00: 00$ & 23.7 & 23.3 & 23.4 & 22.7 & 22.9 & 23.5 & 23.3 & 23.2 & 23.1 & 10.3\} \\
\hline $07 / 31 / 201315: 00: 00$ & 23.3 & 23.1 & 23.0 & 22,6 & 22.8 & 22.9 & 23.0 & 23.1 & 22.9 & \\
\hline |07/31/2013 18:00;00 & 23.1 & 22.9 & 22.9 & 22.5 & 22.1 & 23.0 & 22.8 & 23.3 & 22.8 & \\
\hline $07 / 31 / 201321: 00: 00$ & 22.5 & 22.6 & 225 & 224 & 22.5 & 22.5 & .22 .4 & 22.5 & 22.5 & \\
\hline $08 / 01 / 20130: 5000$ & 22.2 & 22.3 & 22.3 & 22.1 & 22.3 & 22.3 & 22,2 & 22.2 & 22.2 & \\
\hline $00 / 01 / 20133 ; 0000$ & 22.2 & 22.2 & 22.2 & 22.0 & 22.2 & 22.3 & 22.1 & 22.2 & 22.3 & \\
\hline $08 / 01 / 20136 ; 00.00$ & 22,1 & 22,2 & 22.1 & 22.0 & 22,1 & 22.2 & 220 & 22,1 & 22.1 & \\
\hline $08 / 01 / 20139.00 .00$ & 23,0 & 22,6 & 22.8 & 22.2 & 22.4 & 22.3 & 22.8 & 22.6 & 22.4 & 22.9 \\
\hline 08/01/2013 12:00:00 & 24.0 & 23.5 & 23,5 & 22.9 & 23.2 & 23.3 & 23.9 & 23.2 & 23.2 & [2.5] \\
\hline 08/01/2013 15:00:00 & 24.0 & 23.7 & 23.5 & 25.2 & 23.4 & 23.5 & 23.5 & $23 . \bar{\varepsilon}$ & 23.5 & \\
\hline 08/01/2013 18:00:00 & 24.1 & 23.9 & 23.8 & 25.4 & 23.7 & 23,3 & 23.7 & $21 . \mathrm{C}$ & 23.7 & \\
\hline $08 / 01 / 201321: 00: 00$ & 23.3 & 23.2 & 23.1 & $2 \pi .9$ & 23.1 & 23.1 & 23.0 & z3.1 & 23.2 & \\
\hline
\end{tabular}

Page 2 or 4 


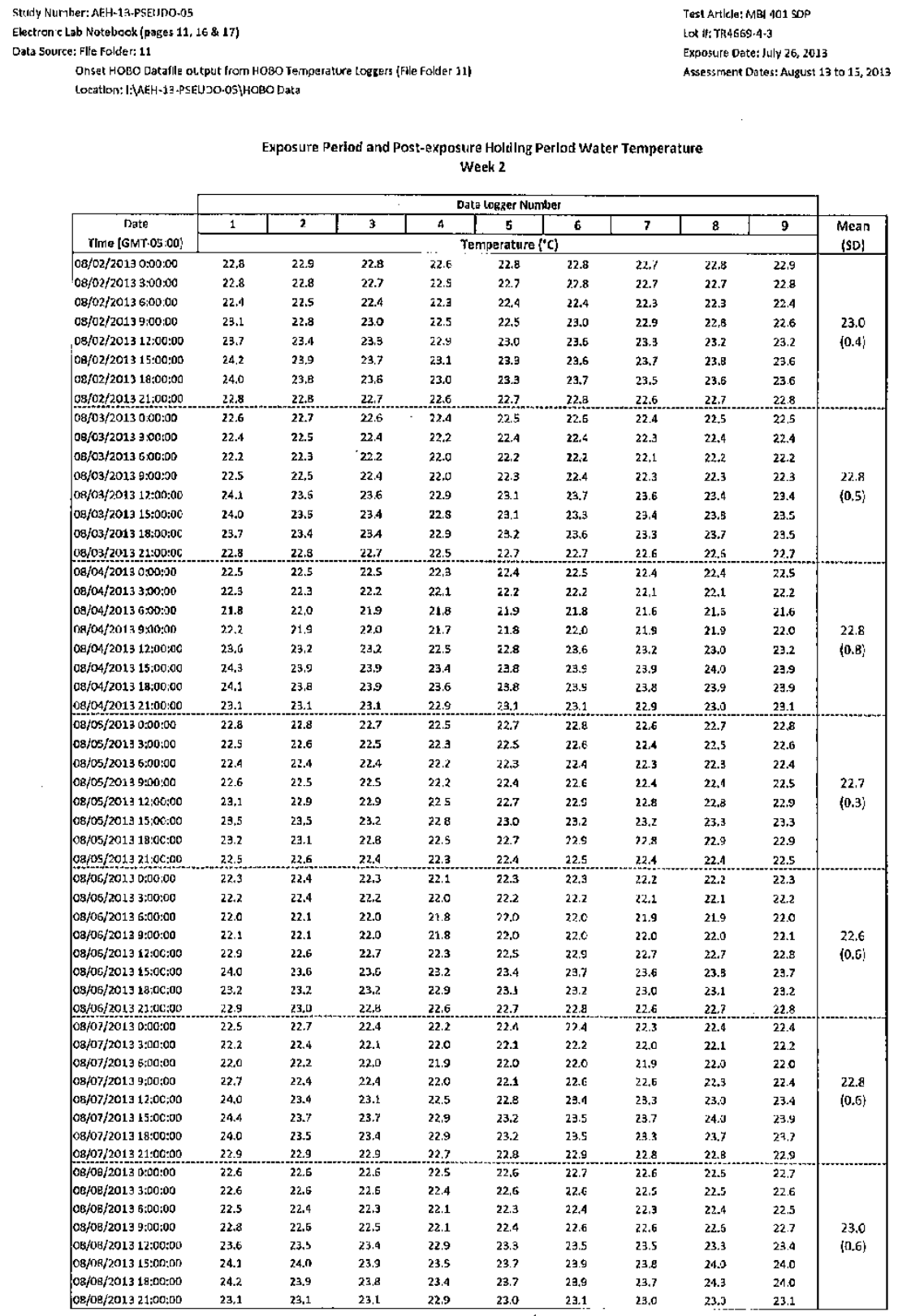

Page 3 or 4 


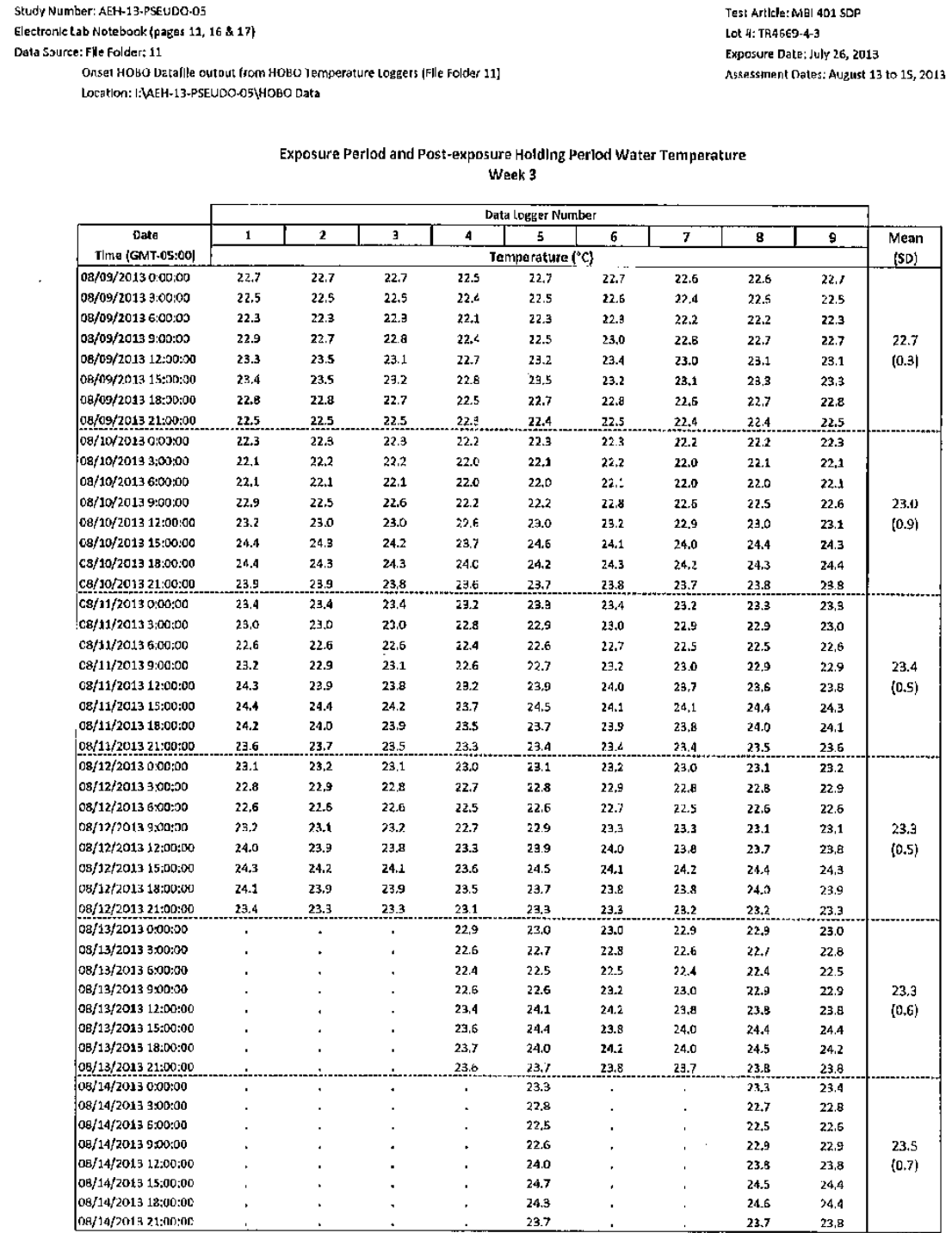

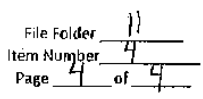




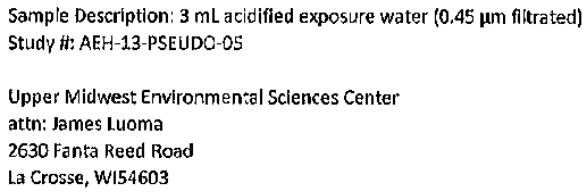

REPORT OF ANALYSIS

Sample Code

E1T8HC100

E2T8HC5O

E3T8HCO

E4T8HC5O

E5T\&HCSO

E6T8HC100

E7TRHCO

ERT8HCO

E9T8HC100

USGS

Water Quallty Laboratory

Laboratory Dlrector

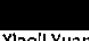

Xlaoll Yuan
Water Quality Laboratory

Upper Midwest Environmental Sciences Cente

USGS

2630 Fanta Reed Road

La Crosse, WI 54603

Analyzing Date:08/8/2013

Report Date: 08/13/2013

$$
\begin{array}{r}
0.141 \\
0.12 \\
0.077 \\
0.103 \\
0.074 \\
0.145 \\
0.03 \\
0.026 \\
0.113
\end{array}
$$

FF \# II

Item No. 10

$\mathrm{Pg} \perp$ of 1 


\section{Appendix 7. Spectrophotometric Summary and SAS Output, Program, and Log}

\begin{tabular}{clrc}
\hline $\begin{array}{c}\text { Item } \\
\text { number }\end{array}$ & \multicolumn{1}{c}{ Item description } & $\begin{array}{c}\text { Number } \\
\text { of } \\
\text { pages }\end{array}$ & $\begin{array}{c}\text { Report } \\
\text { page } \\
\text { number }\end{array}$ \\
\hline 1 & Spectrophotometric Data - Data Summary & 2 & 182 \\
2 & SAS output for Standard Curve Linear Regression and sample concentrations & 11 & 184 \\
3 & SAS program for Standard Curve Linear Regression and sample concentrations & 2 & 195 \\
4 & SAS log for Standard Curve Linear Regression and sample concentrations & 4 & 197 \\
\hline
\end{tabular}




\begin{tabular}{|c|c|c|c|}
\hline Study NLriber: AEH-13-PSEUDO-05 & Action & Date & Initials \\
\hline Electronlc Lab Notebook (page 14) & Created...... & 5-Sep-2013 & KLW/Wh \\
\hline Data Source: File folder: 12 & Revised....... & 19-Feb-2014 & KLW $M N$ \\
\hline Forms: "Spectroplıotometric Data" datasheet & \begin{tabular}{|l} 
Reviewed... \\
Certiffied.......
\end{tabular} & $\frac{1961 / 14}{2 / 1 / 4}$ & $\frac{4 w^{2}}{5 n^{2}}$ \\
\hline
\end{tabular}

\section{Spectrophotometric Data}

Test Article: MBI-401 SDP (Pseudomonas fluorescens Pf-CL 14SA (SDP]); Lot H: TR4669-4-3

Exposure Date: July 26, 2013

Assessment Dates: August 13-15, 2013

Data Explanatlon:

1) A linear regresslon curve was created uslng the absorbances $(660 \mathrm{~nm})$ ' rom known concentrattons $(25,50,100$ and $200 \mathrm{mg} / \mathrm{L})$ of $\mathrm{MBl}-40 \mathrm{SOP}$.

2) Standard checks were performed at $A$ hour by co $\mathrm{Mparing}$ the $25,50,100$ and $200 \mathrm{mg} / \mathrm{L}$ dilutlons used to create the llnear regresslon to the llinear regresslon

3) Data codes used within SAS

enc $=$ Test Enclosure 10 \{1 through 9$\}$

thero $=$ theoret lal concentratior $\{25,50,100$ and $200 \mathrm{mg} / \mathrm{L}$

time $=$ sample tIme $(1,4$ and $8 \mathrm{~h}$ post-dosing initlation $\}$

abs $=$ ineasured absorbance $5^{t}$ saniple

conc $=$ concentration $(\mathrm{mg} / \mathrm{L})$

4) Infornatlon that is not relevant to a sample (I.E., test enclosure ID for standards) or that will be calculated by SAS (i.e., predlcted concentratlon for standard checks and samples) is denoted by a "." for inpet Into SAS.

Data Analysis Methods:

1) A linear regression equatior was fit uslng the SAS Prockeg proceciure using the absorbances $\{660 \mathrm{~nm}\}$ of 3 replicate dllutions of $25,50,100$ and $200 \mathrm{mg} / \mathrm{L}$ MBI-401 SDP 2) Standard checks and treatment sample concertiations were predicted from the regresslon analysis

Data Analysisi

1) The following mean treatment concentratlons were determined in SAS:

3a) Mean concentration for easch individual test enclosure over the entire exposure perioc

3b) Mean concentration for each t'eatment group at each sampling time

Flle Names:

Spectropho:ometric Data for SAS Input

I: AEH-13-PSEUDO-05YData SUmmaries\LAmnonla Data.xlsx]Ammonia Coversheet

5AS Program/Code

I:YAEH-13-PSEUJO-05\SAS and sas output'spec analysls program

SAS Leg

I:ATH-13-PSEUDO-0SISAS and sas output\}spec analysis log

SAS Output

I:'AEH-13-MBICRADA-01\SAS\spec analysis output

Data Anomalles and Deviatlons

NONE

File Folder: 12

$\left.{ }_{\text {Page }}\right|^{\text {of }-2}$ 


\begin{tabular}{|c|c|c|c|c|}
\hline enc & thero & time & abs & conc \\
\hline . & 25 & 0 & 0.030 & 25 \\
\hline . & 50 & 0 & 0.063 & 50 \\
\hline . & 100 & 0 & 0.121 & 100 \\
\hline . & 200 & 0 & 0.241 & 200 \\
\hline . & 25 & 0 & 0.030 & 25 \\
\hline . & 50 & 0 & 0.060 & 50 \\
\hline . & 100 & 0 & 0.122 & 100 \\
\hline . & 200 & 0 & 0.241 & 200 \\
\hline . & 25 & 0 & 0.031 & 25 \\
\hline . & 50 & 0 & 0.062 & 50 \\
\hline , & 100 & 0 & 0.121 & 100 \\
\hline . & 200 & 0 & 0.240 & 200 \\
\hline . & 25 & 4 & 0.025 & . \\
\hline . & 50 & 4 & 0.061 & . \\
\hline . & 100 & 4 & 0.120 & . \\
\hline . & 200 & 4 & 0.239 & . \\
\hline 3 & 0 & 1 & 0.000 & . \\
\hline 7 & 0 & 1 & 0.001 & . \\
\hline 8 & 0 & 1 & 0.000 & . \\
\hline 2 & 50 & 1 & 0.045 & . \\
\hline 4 & 50 & 1 & 0.049 & . \\
\hline 5 & 50 & 1 & 0.048 & . \\
\hline 1 & 100 & 1 & 0.093 & . \\
\hline 6 & 100 & 1 & 0.095 & . \\
\hline 9 & 100 & 1 & 0.092 & . \\
\hline 3 & 0 & 4 & 0.000 & $\cdot$ \\
\hline 7 & 0 & 4 & -0.001 & . \\
\hline 8 & 0 & 4 & 0.001 & . \\
\hline 2 & 50 & 4 & 0.040 & . \\
\hline 4 & 50 & 4 & 0.046 & . \\
\hline 5 & 50 & 4 & 0.045 & . \\
\hline 1 & 100 & 4 & 0.095 & . \\
\hline 6 & 100 & 4 & 0.090 & . \\
\hline 9 & 100 & 4 & 0.090 & . \\
\hline 3 & 0 & 8 & -0.003 & . \\
\hline 7 & 0 & 8 & -0.001 & . \\
\hline 8 & 0 & 8 & -0.001 & . \\
\hline 2 & 50 & 8 & 0.038 & . \\
\hline 4 & 50 & 8 & 0.040 & . \\
\hline 5 & 50 & 8 & 0.040 & . \\
\hline 1 & 100 & 8 & 0.084 & , \\
\hline 6 & 100 & 8 & 0.087 & . \\
\hline 9 & 100 & 8 & 0.085 & , \\
\hline
\end{tabular}

AEH-13-PSEUDO-05

FF \# 12

Item No. I

$\mathrm{Pg} 2$ of 2 


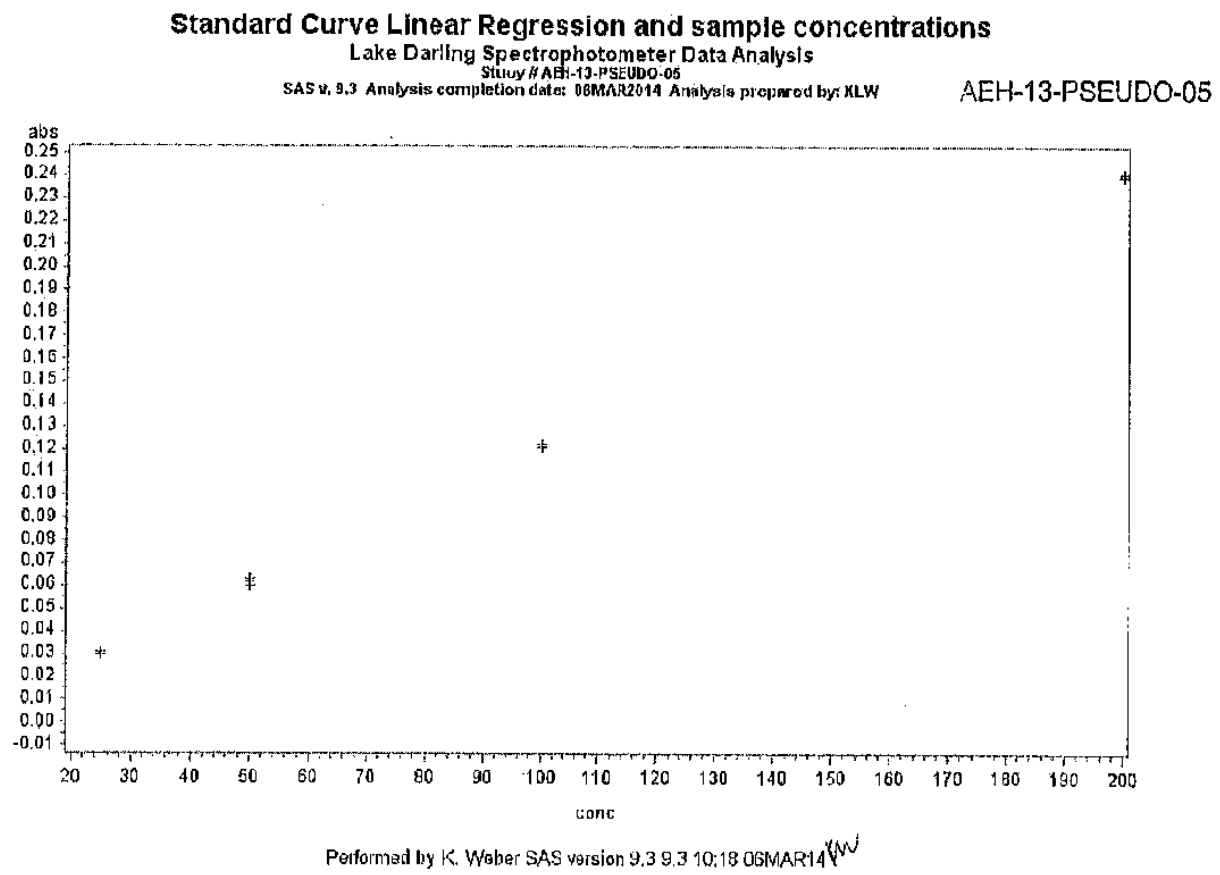

$F F \# 12$

Item No. 3

$\mathrm{Pg} \perp$ of II 


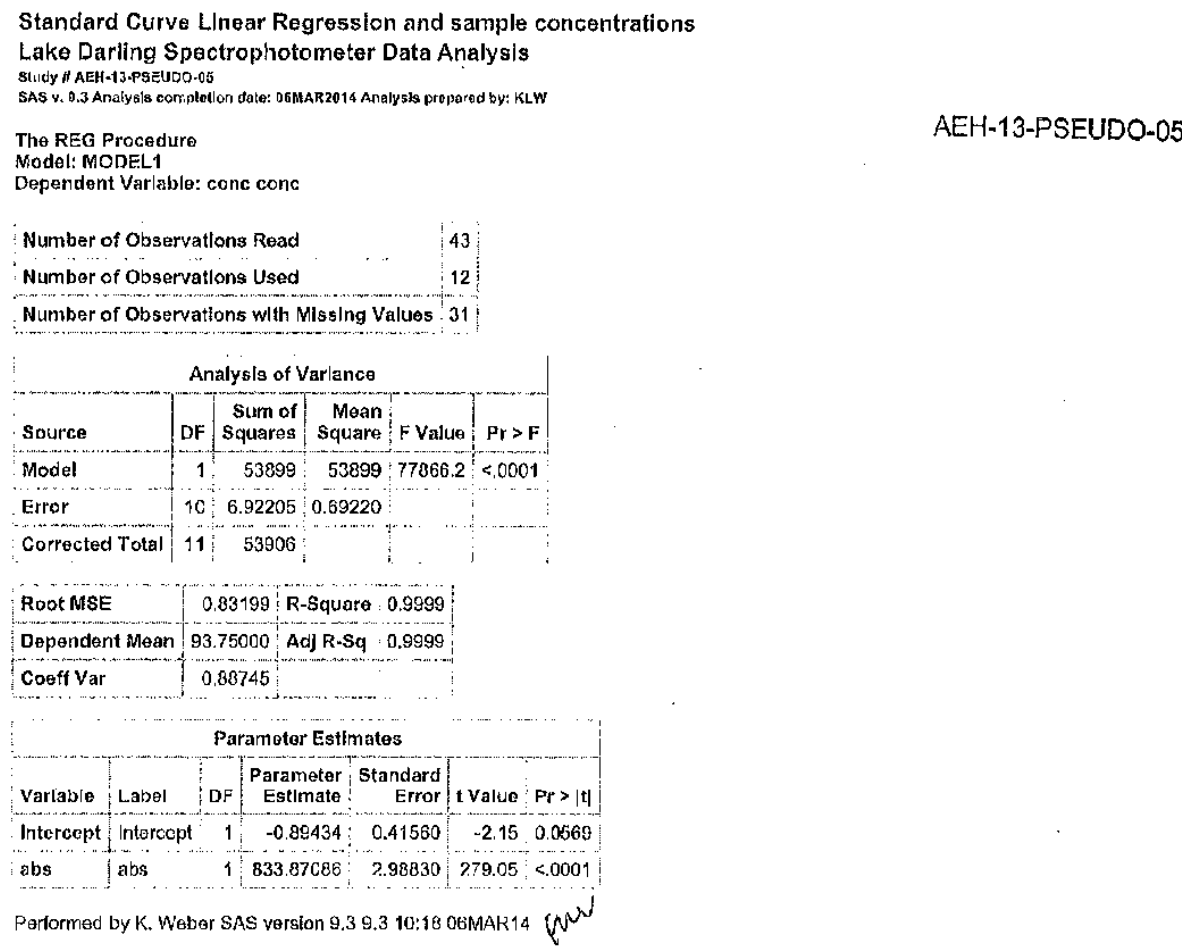




\section{Standard Curve Linear Regression and sample concentrations}

Lake Darling Spectrophotometer Data Analysis

Study \# AEH-13-PSEUDO-05

SAS Y, 0,3 Analysts colnploilon da10: 06MAR2014 Analya is proparod by: KLW WN

AEH-13-PSEUDO-05

\section{The REG Procedure}

MOdel: MODEL.1

Dependent Variable: conc conc

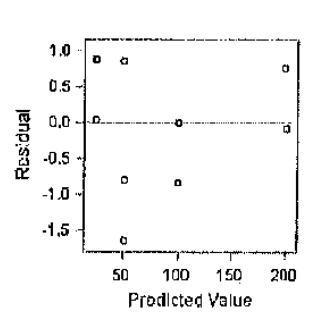

Fit Dlagnostics for cone
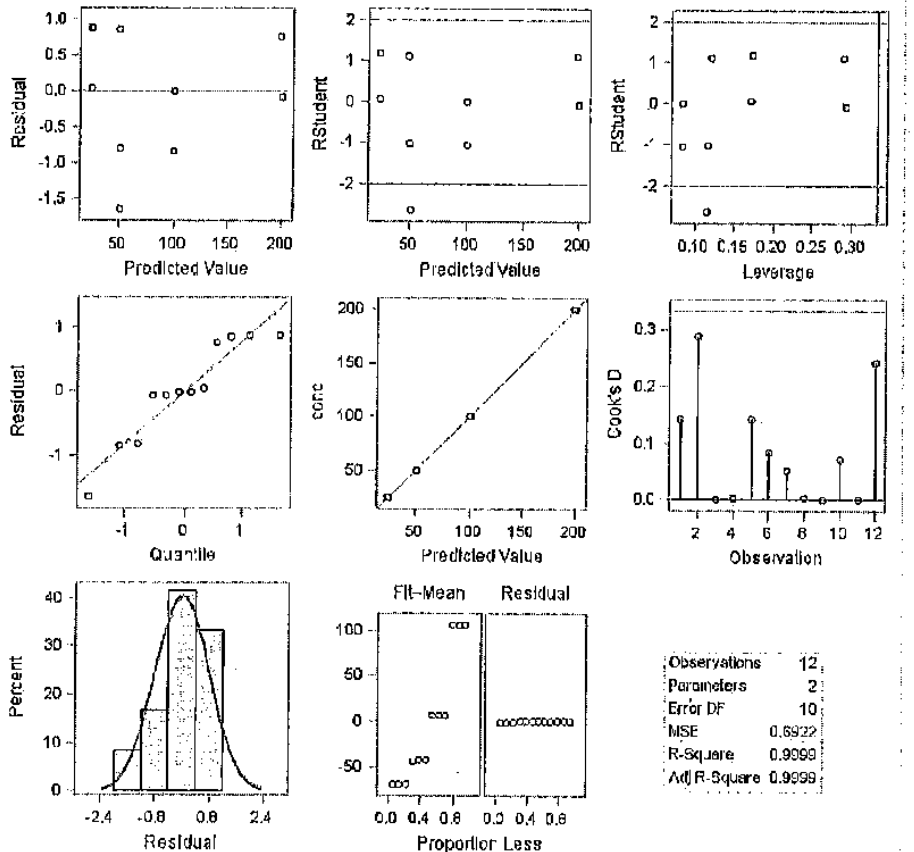

Proporllor Less 


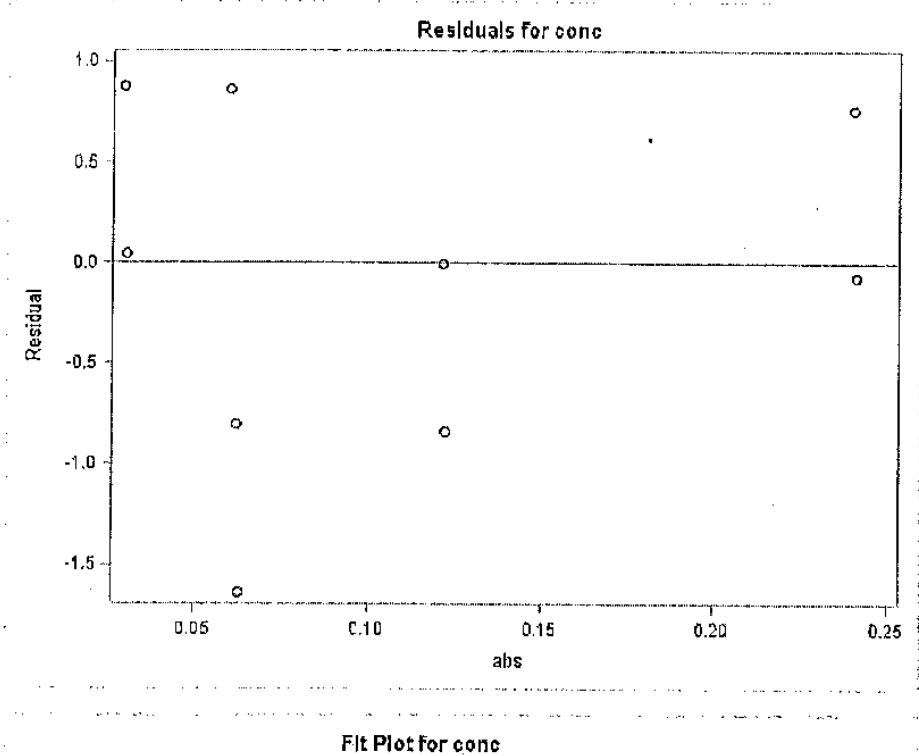

AEH-13-PSEUDO-05

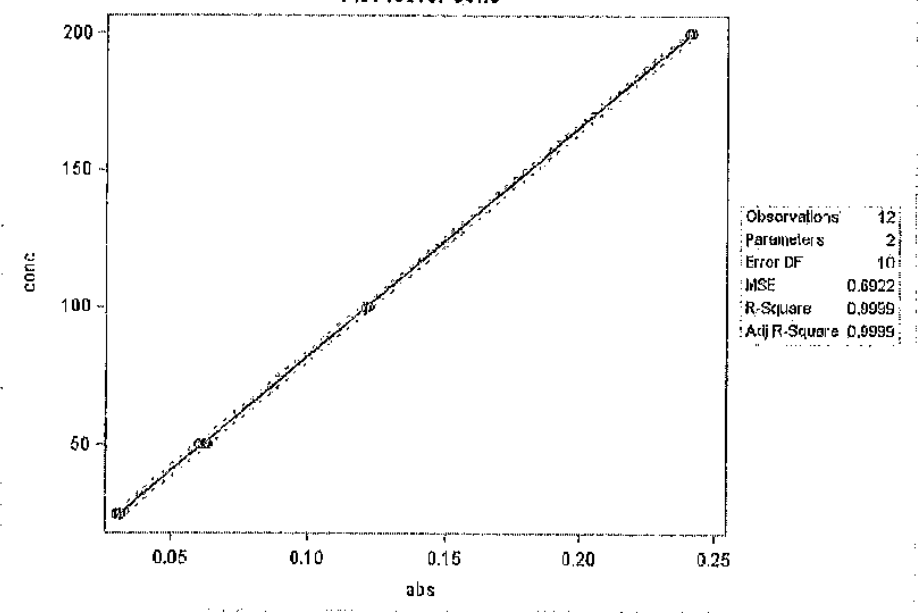

Performed by $K$. Weber SAS version 9.3 9.3 10:18 06MAR14 W' 


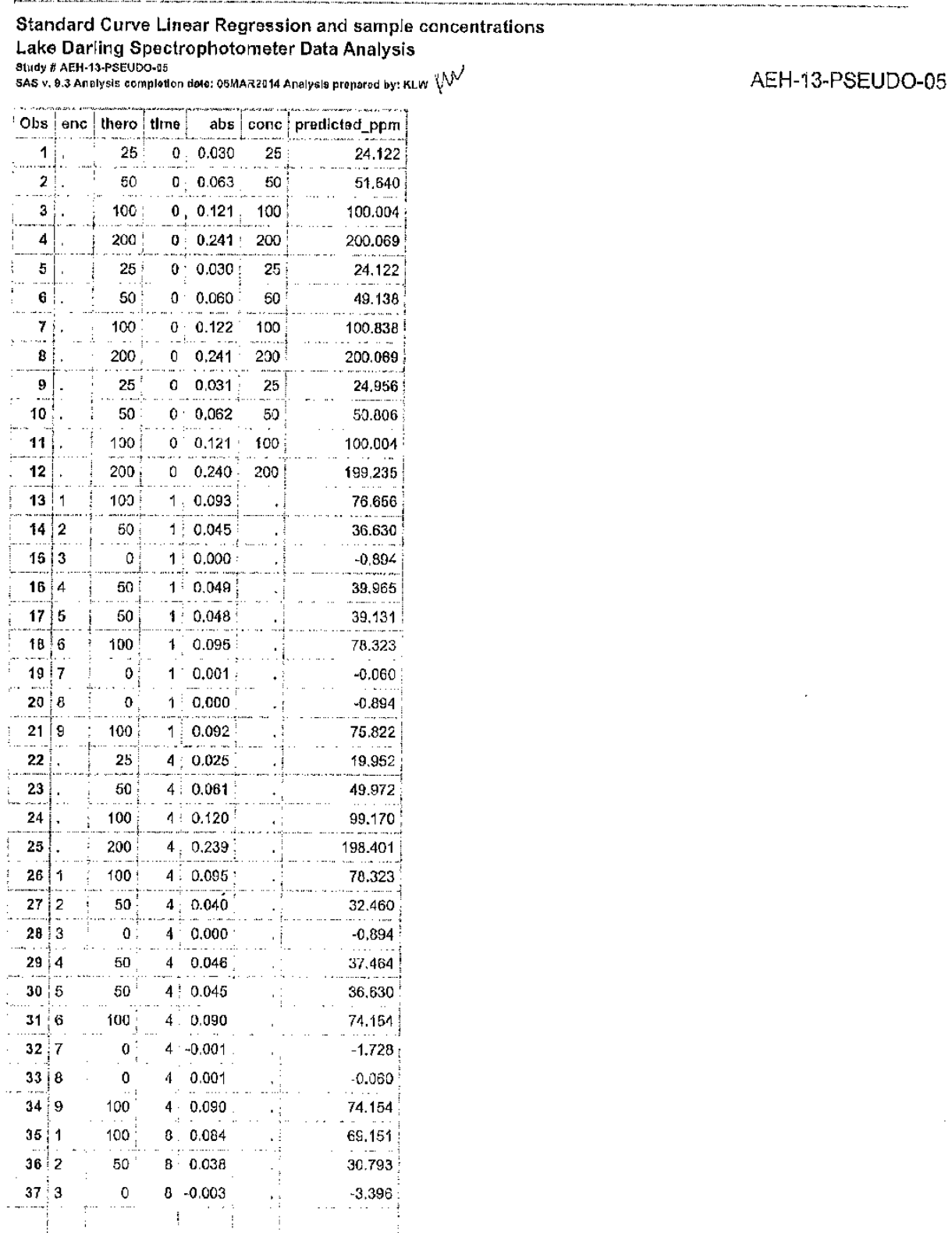




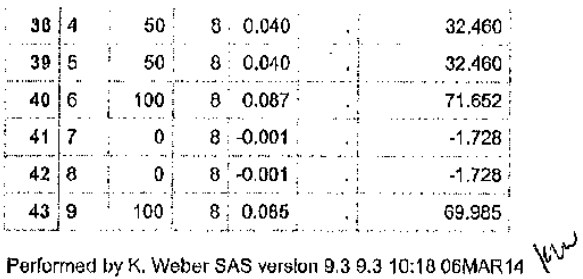


Mean treatment concentration by enclosure for all exposure sampling times Individual enclosure means

The MEANS Procedure

enc $=1$ thero $=100$

Analysis Varlable : predicted_ppm Predicted Value of conc

Mean Std Dev $\quad$ Lower $95 \%$ Upper $95 \%$

74.70994 .8860

enc $=2$ thero $=50$

Analysis Varlable : predlcted_ppm Predicted Value of conc

Lower $95 \%$ Upper 95\%

Mean Std Dev CL for Mean CL for Mean

$33.2944: 3.0086 \quad 25.8256$ ! 40.7631

enc $=3$ thero $=0$

Analysis Variable : predicted_ppm Predicted \begin{tabular}{|c|c|c|} 
& Value of conc \\
& ............. & \\
Lower $95 \%$ & Upper $95 \%$
\end{tabular} \begin{tabular}{|l|l|l|l} 
Mean & Std Dev & Lower $95 \%$ & Upper $95 \%$ \\
Co Mean & CL for Mean
\end{tabular} \begin{tabular}{ll|l|l|l|}
\hline$-1.7282: 1.4443$ & -5.3161 & 1.8596
\end{tabular}

enc 04 thero 50

Analysis Varlable : predicted_ppm Predleted Value of cone
[- Lower $95 \%$ Upper $95 \%$

Mean Sid Dev $\begin{array}{r}\text { Lower } 95 \% \\ \text { CL for Mean }\end{array}$ CL for Mean $95 \%$ $\begin{array}{llll}36.6298 & 3.8213 & 27.1373 & 46.1224\end{array}$

enc $=5$ thero $=50$

: Analysis Varlable : predicted_ppm Predicted Value of cone
Lower $95 \%$ Uppor $95 \%$ Mean Std Dev CL for Mean CL far Mean $\begin{array}{lllll}36.0739 & 3.3700 & 27.7023 & 44.4456\end{array}$

enc $=6$ thero $=100$

Analysis Varlable : predicted_ppm Predicted Value of conc
Lower $95 \%$ Upper $95 \%$

Mean Std Dev CL for Mean CL for Mean

$\begin{array}{lllll}74.7099 & 3.3709 & 66.3383 & 83.0816\end{array}$

enc $=7$ thero $=0$

Analysls Varlable : predicted_ppm Predlcted 


\begin{tabular}{|c|c|c|c|}
\hline \multicolumn{4}{|c|}{ Value of conc } \\
\hline Mean & Std Dev & $\begin{array}{l}\text { Lower } 95 \% \\
\mathrm{CL} \text { for Mean }\end{array}$ & $\begin{array}{l}\text { Upper } 95 \% \\
\text { CL for Mean }\end{array}$ \\
\hline-1.1723 & 0.9629 & -3.5642 & 1.2196 \\
\hline
\end{tabular}

Analysis Varlable : predicted_ppm Predicted

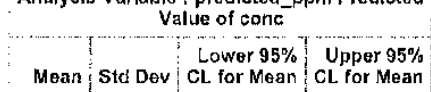

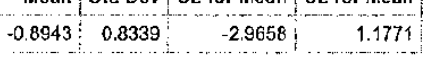

enc $=9$ thero $=100$

Analysis Variable ; predicted_ppm Predlcted Value of conc

Maan Std Dev Lower 95\% Upper $95 \%$ CL for Mean CL for Mean

$\begin{array}{llllllll}73.3202 & 3.0066 & 65.8514 & 80.7889\end{array}$

Peformed by K. Weber SAS version 9.39 .3 10:18 06MAR14 W 


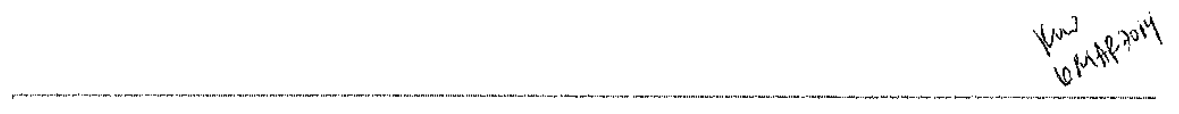

Mean treatment concentration by treatment group at each exposure sampling time

Tho MEANS Procoduro

therowo time $=1$

Analysls Variable : precicted_ppm Predlcted

\begin{tabular}{|c|c|c|c|}
\hline Mean & Std Dev & $\begin{array}{r}\text { Lower } 95 \% \\
C L \text { for Mean }\end{array}$ & $\begin{array}{l}\text { Upper } 95 \% \\
\text { CL for Mean }\end{array}$ \\
\hline-0.6164 & 0.4814 & -1.8123 & 0.5796 \\
\hline
\end{tabular}

thero $=0$ time $=4$

Analysls Varlable : prodicted_ppm Predlcted

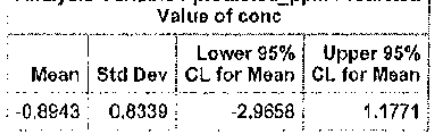

thero $=0$ t/me $=8$

Analysls Varlable : predicted_ppm Predicted Value of conc

Value of conc
Lower $95 \%$ Upper $95 \%$ Mean Std Dev CL for Mean $\quad$ CL for Mean

\begin{tabular}{rr|r|r|}
-2.2841 & 0.9679 & -4.6760 & 0.1078 \\
\hline
\end{tabular}

thero $=50$ tlune $=1$

Analysis Variable : predicted_ppm Pred/cted Value of cone
\[ \text { Lower } 95 \% \]
Upper $95 \%$

\begin{tabular}{ll|l:l} 
Mean & Std Dev & LL for $95 \%$ & Upper $95 \%$ \\
CL & CL for Mean
\end{tabular}

$\begin{array}{llll}38.5765 & 1.7358 & 34.2636 & 42.8876\end{array}$

thero $=50$ time $=4$

Analysls Varlable : predicted_ppm Predlcted \begin{tabular}{c|c|c|} 
Value of cone \\
\hline Lower $96 \%$ & Upper 95\% \\
\hline CL &
\end{tabular} Mean Std Dov CL for Mean CL for Mean $\begin{array}{lllll}35.5180 & 2.6805 & 28.8592 & 42.1768\end{array}$

thero $=50$ time $=8$

Analysis Variable : prodicted_ppm Predlcted \begin{tabular}{c} 
Value of conc \\
\hline Lower $95 \%$ \\
Uper $95 \%$
\end{tabular} Mean Std Dev CL for Mean CL for Mean \begin{tabular}{llll|}
31.3046 & 0.9629 & 29.5127 & 34.2965 \\
\hline
\end{tabular}

thero $=100$ time $=1$

Analys|s Variabie ; predicted_ppm Predlcted Value of cont 


\begin{tabular}{|c|c|c|c|}
\hline Mean & Std Dev & $\begin{array}{l}\text { Lower } 95 \% \\
\text { CL for MEan }\end{array}$ & $\begin{array}{l}\text { Upper } 95 \% \\
\text { CL for Mean }\end{array}$ \\
\hline 76.8336 & 1.2738 & 73.7694 & 80.0978 \\
\hline \multicolumn{4}{|c|}{ thero $=100$ tlme $=4$} \\
\hline \multicolumn{4}{|c|}{$\begin{array}{l}\text { Analygls Varlable ; predlcted_ppm Predlcted } \\
\text { Value of conc }\end{array}$} \\
\hline Mean & Std Dev & $\begin{array}{l}\text { Lower } 95 \% \\
\text { CL- for Mean }\end{array}$ & $\begin{array}{l}\text { Upper } 95 \% \\
\text { CL for Mean }\end{array}$ \\
\hline 75.5438 & 2.4072 & 69.5641 & 81.5236 \\
\hline \multicolumn{4}{|c|}{ thero $=100$ thm $m=8$} \\
\hline \multicolumn{4}{|c|}{$\begin{array}{l}\text { Analysis Varlable : predicted_ppm Predicted } \\
\text { Value of conc }\end{array}$} \\
\hline Mean & Std Dev & $\begin{array}{l}\text { Lower } 95 \% \\
\text { CL for Mean }\end{array}$ & $\begin{array}{r}\text { Upper } 95 \% \\
\text { CL. for Mean }\end{array}$ \\
\hline 70.2626 & 1.2738 & 67.0984 & 73.4268 \\
\hline
\end{tabular}




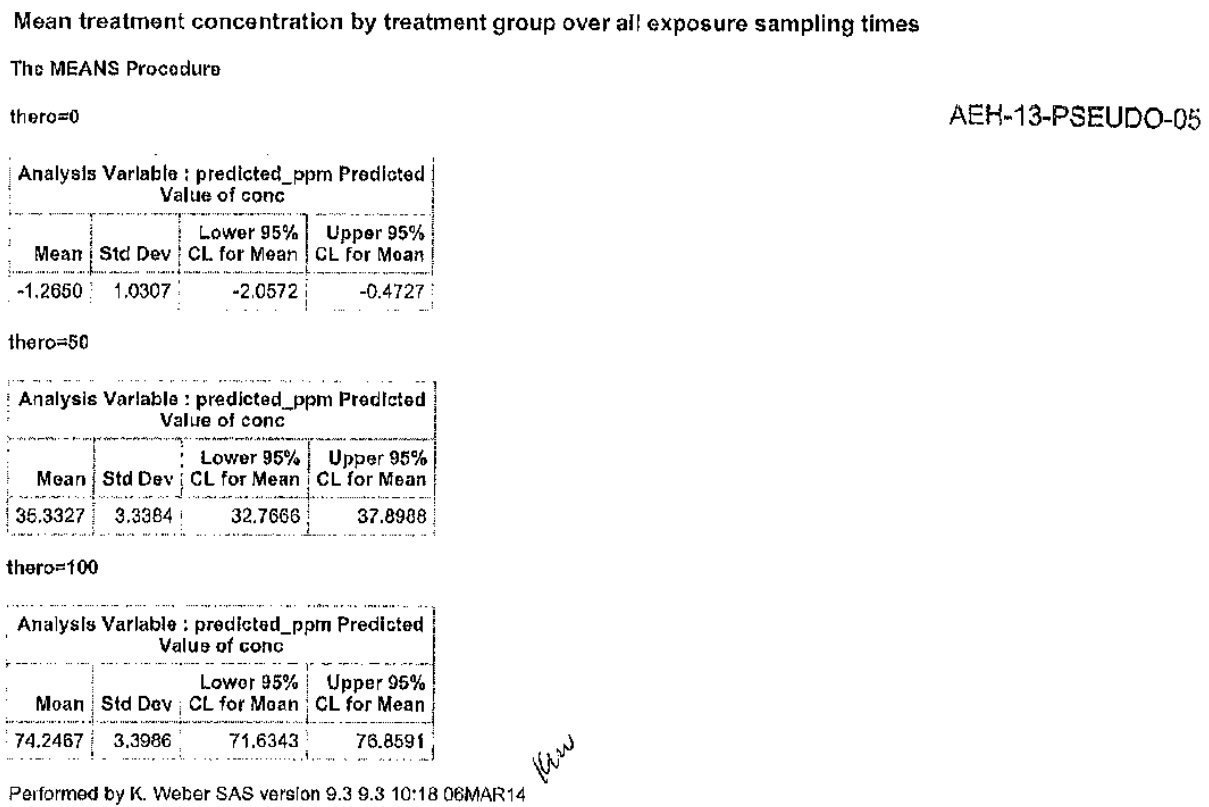

FF \# 12

Item No. 3

$\mathrm{Pg}$ II of II

file://C:UUscrsłklwebertsashtml,htm 


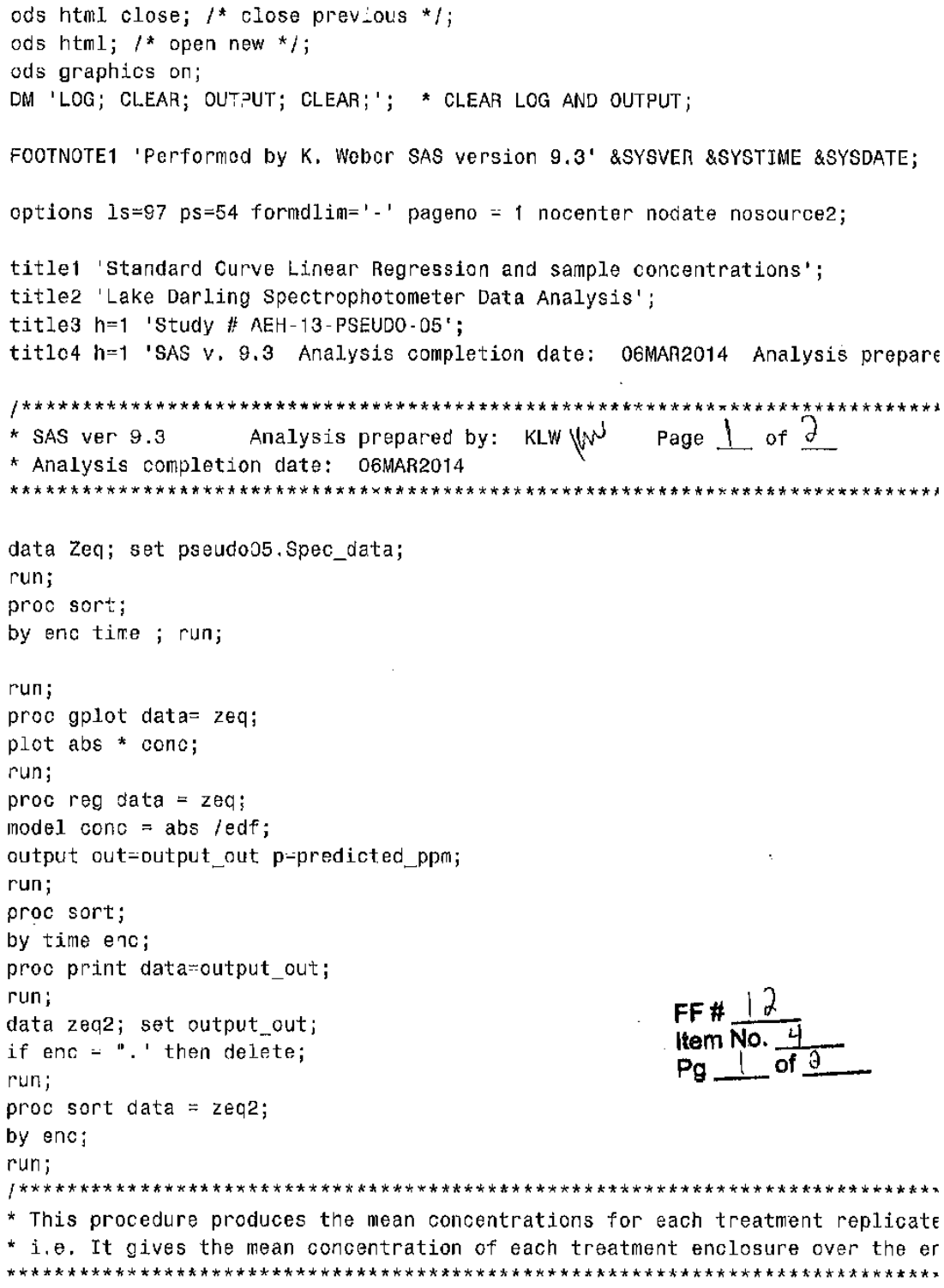




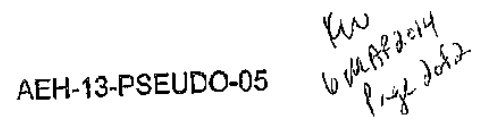

title1 "Mean treatment concentration by enclosure for all exposure sampling t: title2 "Individual enclosure means";

proc means data $=$ zeq2 mean std Ic1m uclm $f w=8$;

by enc thero;

var predicted_ppm;

run;

proc sort;

by thero time;

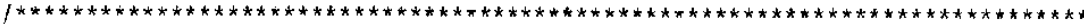

* This procedure produces the mean concentrations for each treatment group at

* i.e. It gives the mean concentration of the three control, 50ppm, \& 100ppm t

* at each sample time over the entire exposure

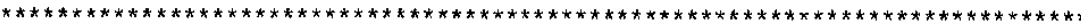

title1 "Mean treatment concentration by treatment group at each exposure samp? proc means data = zeq2 mean std $1 \mathrm{clm}$ uclm $\mathrm{fw}=8$;

by thero time;

var predicted_ppm;

run;

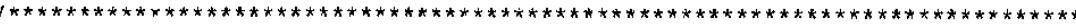

* This procedure produces the mean concentrations for each treatment group ove

* i.e. It gives the mean concentration of the three control, 50ppm, \& 100ppm $t$

* over the entire exposure

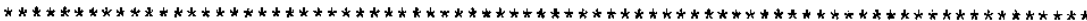

title1 "Mean treatment concentration by treatment group over all exposure samp proc means data = zeq2 mean std $1 \mathrm{clm}$ uclm $\mathrm{fw}_{\mathrm{w}}=8$;

by thero;

var predicted_ppm

run;

quit;

run;

FF \# 12

Item No. 4

$\mathrm{Pg} 2$ of $\underline{2}$ 


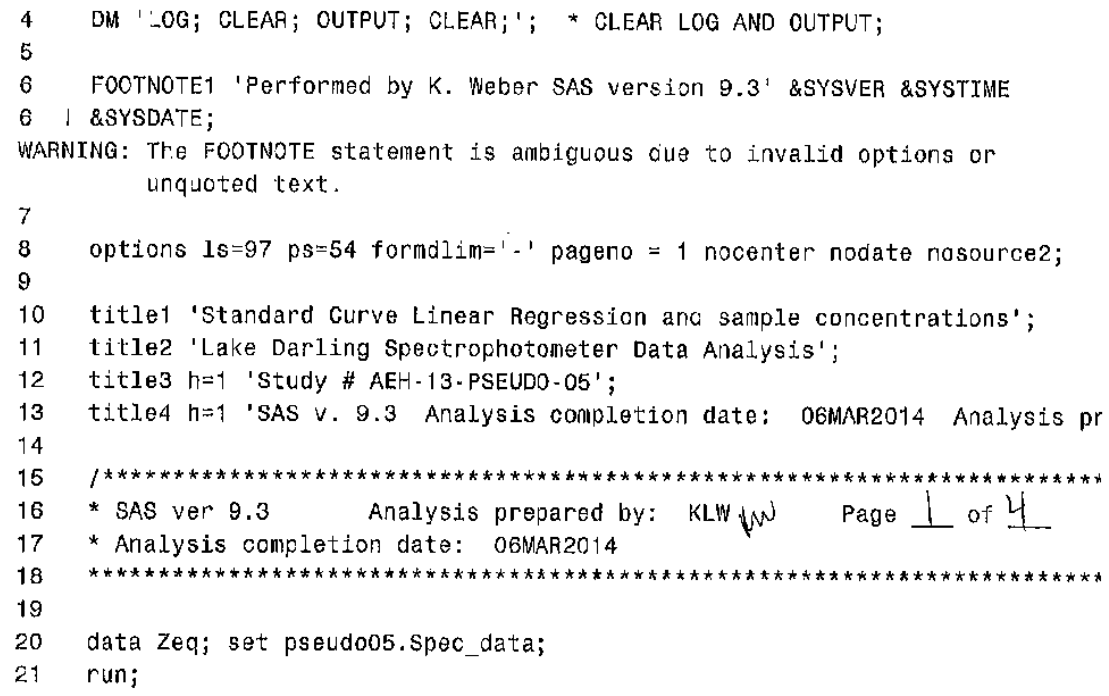

NOTE: There were 43 observations read from the data set PSEUDO05.SPEC_DATA.

NOTE: The data set WORK.ZEQ has 43 ubservations and 5 variables.

NOTE: DATA statement used (Total process time):

real time $\quad 0.02$ seconds

cpu time $\quad 0.03$ seconds

22 proc sort;

23 by enc time; run;

NOTE: There were 43 observations read from the data set wORK.ZEQ.

NOTE: The data set WORK.ZEQ has 43 observations and 5 variables.

NOTE: PROCEDURE SORT used (Total process time):

real time $\quad 0.01$ seconds

cpu time $\quad 0.01$ seconds

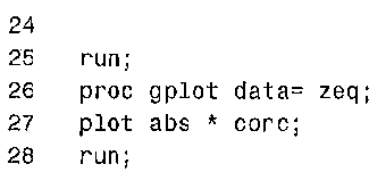

NOTE: 31 observation(s) contained a MISSING value for the abs * conc request.

FF \# 12

Item No. 5

$\mathrm{Pg} \ldots 1$ of 4 


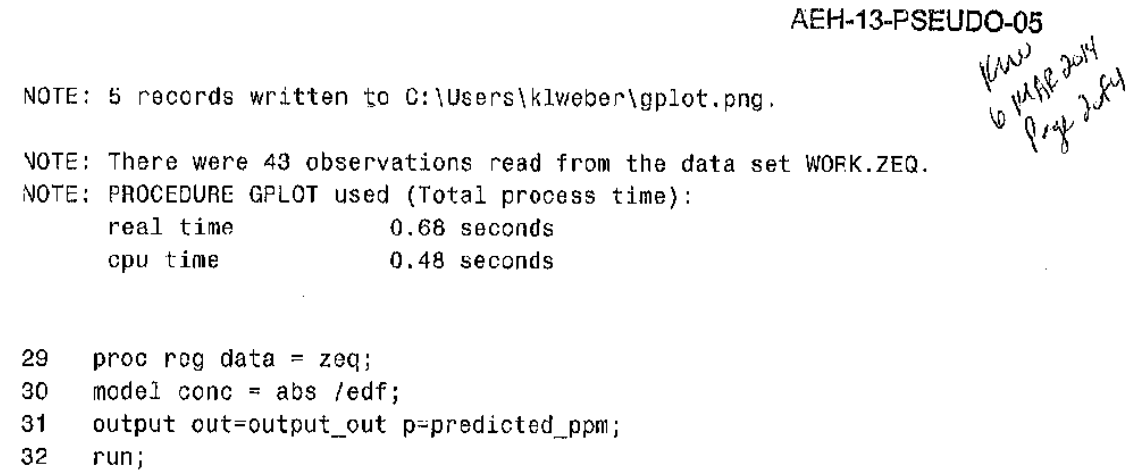

NOTE: The data set WORK.OUTPUT OUT has 43 observations and 6 variables.

NOTE: PROCEDURE REG used (Total process time):

real time $\quad 5.07$ seconds

cpu time $\quad 0.54$ seconds

33 proc sort;
31 by time enc;

NOTE: There were 43 observations read from the data set WORK. OUTPUT OUT. NOTE: The data set WORK.OUTPUT_OUT has 43 observations and 6 variables.

NOTE: PROCEDURE SORT used (Total process time):

real time $\quad 0.01$ seconds

cpu time $\quad 0.01$ seconds

35 proc print data=output_out;

36 run;

NOTE: There were 43 observations read from the data set WORK, OUTPUT OUT. NOTE: PROCEDURE PRINT used (Total process time):
real time
0.09 seconds
cpu time
0.03 seconds

37 data zeq2; set output_out;

38 if enc $=$ "." then delete;

39 run

NOTE: There were 43 observations read from the data set wORK, OUTPUT OUT. NOTE: The data set WORK.ZEQ2 has 27 observations and 6 variablos.

NOTE: DATA statomont usod (Total process time):

Page 2 of 4 


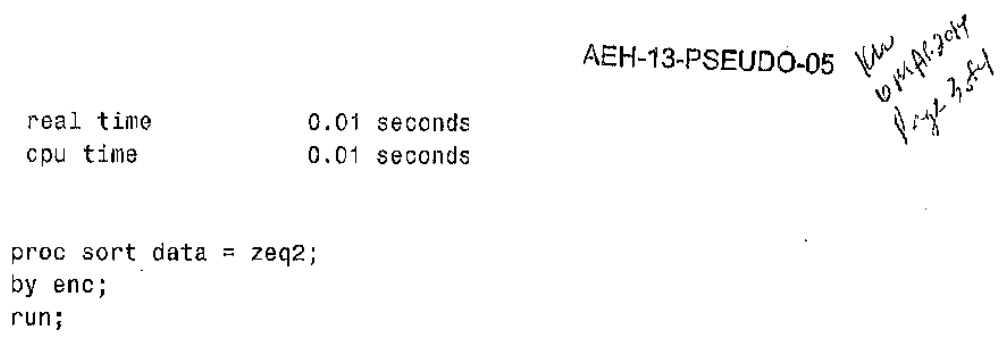

NOTE: There were 27 observations read from the data set WORK.ZEQ2.

NOTE: The data set WORK.ZEQ2 has 27 observations and 6 varíables.

VOTE: PROCEDURE SORT used (Total process time):
real time
0.01 seconds

cpu time

0.01 seconds

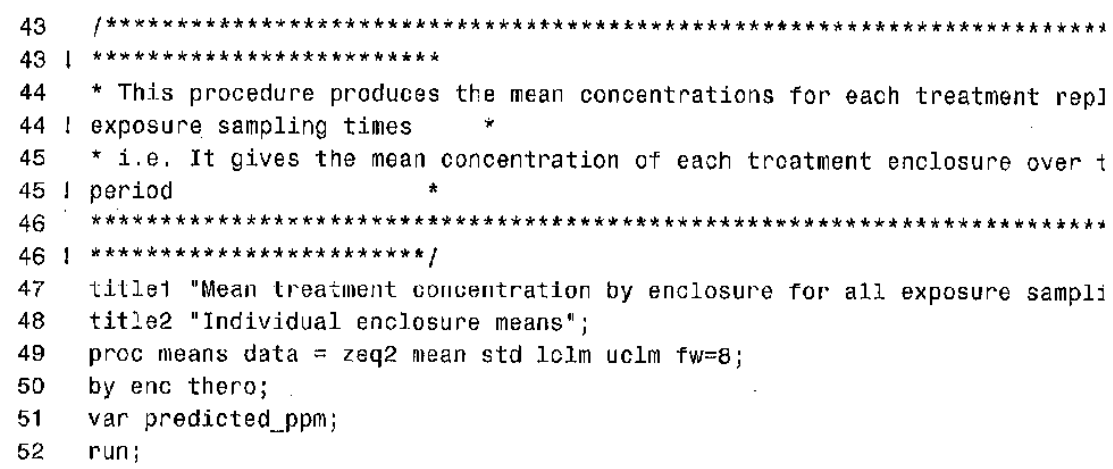

* This procedure produces the mean concentrations for each treatment gro 56 ! tine

* i.e. It gives the mean concentration of the threc control, 50ppn, \& 100 57 I replicates *

58 * at cach sample time over the entire exposure 581

Page 3 of 4 


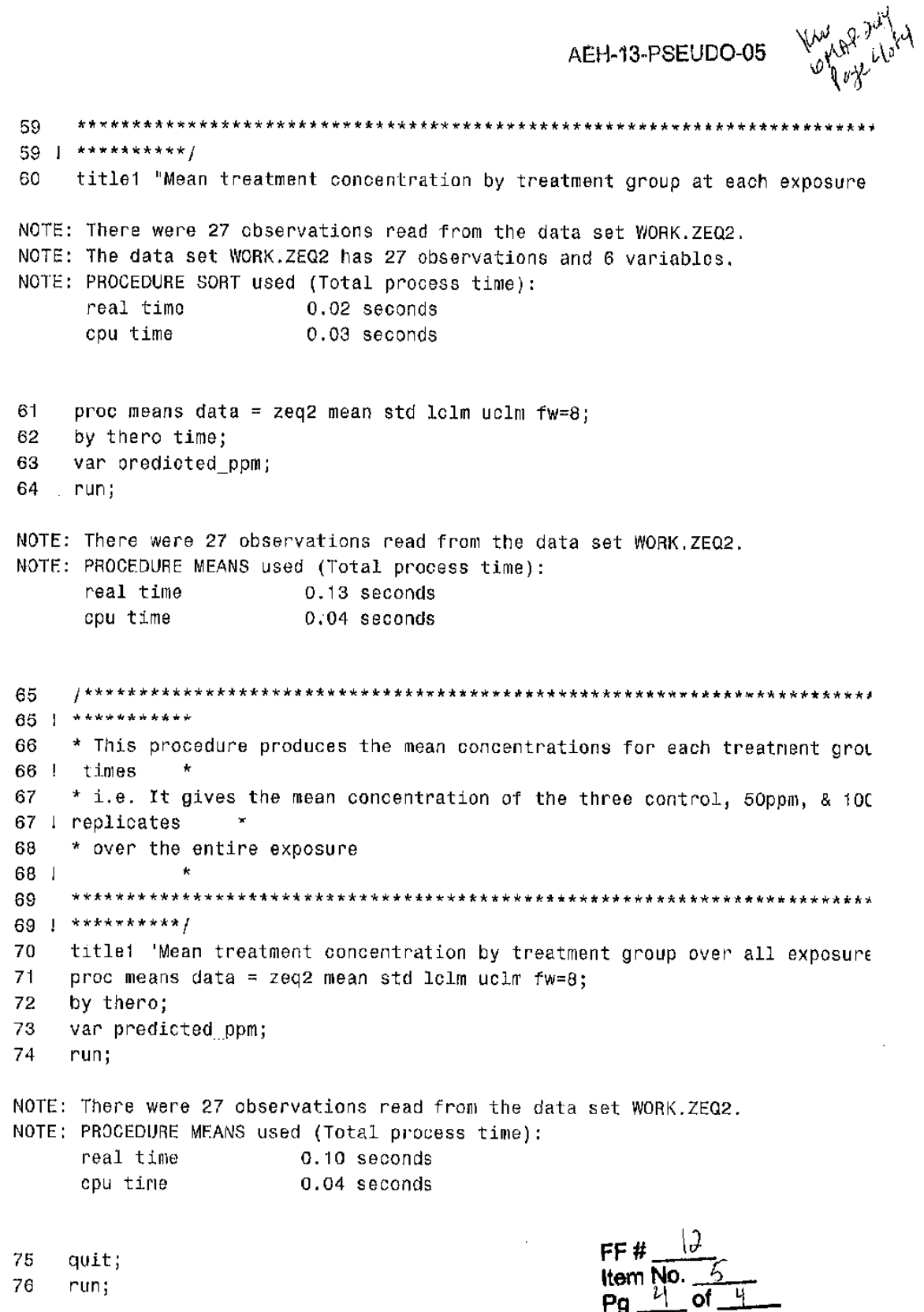




\section{Appendix 8. Zebra Mussel Density Association Summary Data Analysis}

\begin{tabular}{|c|c|c|c|}
\hline $\begin{array}{l}\text { Item } \\
\text { number }\end{array}$ & Item description & $\begin{array}{c}\text { Number } \\
\text { of } \\
\text { pages }\end{array}$ & $\begin{array}{l}\text { Report } \\
\text { page } \\
\text { number }\end{array}$ \\
\hline 1 & Zebra Mussel Density Associations - Data Summary & 15 & 202 \\
\hline
\end{tabular}




\begin{tabular}{|c|c|c|c|c|}
\hline Study Number: AEH-1 & 13-PSEUDO-05 & Action & Date & Initiels \\
\hline Electronic Lab Notebc & ook (pages $9,10,15$ to 18 \& 21 to 22 ) & Created..... & $12-5 e+13$ & KLW \\
\hline Data Source Forms: ' & "Initial Assessment of Native and Zebra Mussels" (Fi e Folcer 13a) & Revlsed..... & $26-$ Mar-14 & KLW HW \\
\hline & "Mussel linitlal Assessment" (Flle Folder 13b) & Revlewed.... & $78 x^{4}+14$ & buts \\
\hline & "Mussel Welght and Length Assessment" (File Jolder 13c) & Ceriflied... & 5) Lolit & $\frac{\pi}{57}$ \\
\hline File NeTRe: IVIACH-1: & "Mussel Surifval Assessment" IFlle Folder 19c| & & Coversheet & \\
\hline
\end{tabular}

Zebra Mussel Density Associations

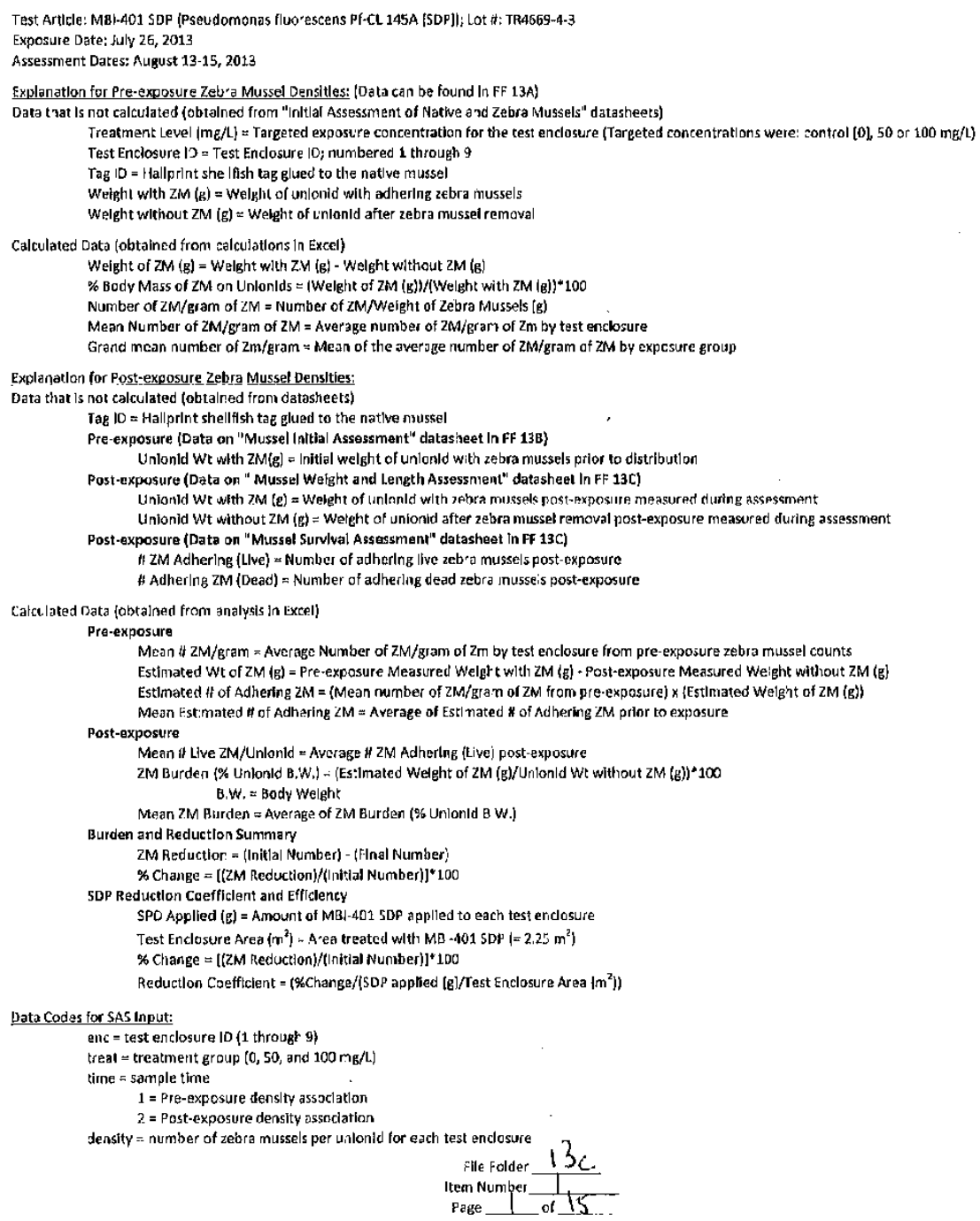




\begin{tabular}{|c|c|c|}
\hline \multirow{2}{*}{\multicolumn{2}{|c|}{$\begin{array}{l}\text { Study Number: AEH-13-PSEUDO-05 } \\
\text { Electronlc Lab Notebook (pages } 9,10,15 \text { to } 18 \& 21 \text { to } 22 \text { ) }\end{array}$}} & Coversheet (cont.) \\
\hline & $k$ (pages $9,10,15$ to $18 \& 21$ to 22$)$ & Page 2 of 2 \\
\hline \multirow[t]{4}{*}{ Oata Source Forms: } & "Inlthal Assessment of Native and Zebra Mussels" (file Fofder 13a) & \\
\hline & "Mussel Inlttal Assessrient" (File Folder 19b) & \\
\hline & "Mussel Welght and Length Assessnient" (Flle Folder 13c) & \\
\hline & "Mussel Survival Assessment" (t-ile Folder 13c) & \\
\hline
\end{tabular}

Zebra Mussel Density Assoclations

Test Article: M81-401 SDF (Pseudomonas fluorescens Pf-CL 145A [SDP]); Lot A: TRM669-4-3

Exposure Date; July 26, 2013

Assessment Dates: August 13-15, 2013

The follovitng unionid mussels were mortalitles fram the assessment (August 13 to 15, 2013). These data will not be used within the post-exposure density assoclation analysls:

\begin{tabular}{|c|c|c|}
\hline Test Endosure $1(100 \mathrm{mg} / \mathrm{l})$ : & Test Enclosure 6 (100 mg/Ll: & Test Enclosure $8(0 \mathrm{mg} / \mathrm{h})$ : \\
\hline C312 & $\mathrm{C} 213$ & C340 \\
\hline C359 & C251 & C38) \\
\hline Test Enclosu'e $2(50 \mathrm{mg} / \mathrm{L})$ : & $C 280$ & Test Enclosure $9(100 \mathrm{mg} / \mathrm{L})$ : \\
\hline C436 & C307 & C70\% \\
\hline Test Enclosure $3\{0 \mathrm{mg} / \mathrm{L}\}$ : & C396 & $\mathrm{C} 226$ \\
\hline c193 & Test Enclosure $7(0 \mathrm{mg} / \mathrm{l})$ : & C258 \\
\hline Test Enclosuie $4(50 \mathrm{mg} / \mathrm{L})$ : & C24B & C430 \\
\hline $0: 11$ & C289 & C411 \\
\hline C334 & C299 & CA16 \\
\hline Test Enclosure $5(50 \mathrm{mg} / \mathrm{l})$ : & $c 301$ & \\
\hline
\end{tabular}

The fullowi'ig mussels were not recovered during the assessment :August 13 10 15, 2013).

Test Enclosure 6 (: $: 0 \mathrm{C} \mathrm{mg} /$ L): $\quad$ Test Enclosure $9(100 \mathrm{mg} / \mathrm{L})$ :

C356 - Induded In pre-exposure zebra mussel density analysls C323-Removed from zebra mussel density analysis

The fol owing inussek were used to estinate the pre-exposure de nsity of zebra mussels adhering to unlorids. These data were analyzed separately tPre-exposure 7elra M:Issel Density Assoclations).

rest Enclosure $1(100 \mathrm{mg} / \mathrm{L})$ :

C2C8

$\mathrm{C} 263$

C350

$\mathrm{C} 358$
$\mathrm{C} 37$

Test Enclusure 2 (50 $\mathrm{me} / \mathrm{h})$

C310

C332

C375

C419

Test Enclosure $3(0 \mathrm{mg} / \mathrm{l})$ :

C197

C254

C.382

C427

\begin{tabular}{|c|}
\hline \multirow{2}{*}{$\begin{array}{l}\text { Test Enclosure } 4 \text { (50 mg/L): } \\
\text { C225 }\end{array}$} \\
\hline \\
\hline C239 \\
\hline $\mathrm{C} 2 \mathrm{~g} 2$ \\
\hline C333 \\
\hline$C 348$ \\
\hline Test Enclosure 5 ( $50 \mathrm{mg} / \mathrm{L}$ ): \\
\hline C238 \\
\hline C240 \\
\hline $\mathrm{C} 256$ \\
\hline C259. \\
\hline C343 \\
\hline Test Enclosure $6(100 \mathrm{rmg} / \mathrm{L})$ : \\
\hline $\mathrm{C} 200$ \\
\hline C250 \\
\hline$c 356$ \\
\hline C402 \\
\hline C426 \\
\hline
\end{tabular}

Page 2 of 15

Test Enclosure $7(0 \mathrm{mg} / \mathrm{h}):$
C192
C261
C361
C394
C417
C189
C191
C209
C220
C337
Test Enclusure $(0 \mathrm{mg} / \mathrm{mg} / \mathrm{t})$
C295
C305
C367
C411
C416


Study Number: AEH-13-PSEUDO- 0.5

Electronlc Lab Notebock (pages $10 \& 21$ to 22)

Dat: Source: Forms: "Inttial Assessment of Na:lve and Zebra Mussels" (fille foider 19a)
Test Article: MB1 .401 SDP Lot H: TR4669-<-3

Exposure Date: July 26, 2013

Assessment Dates August i3-15, 2013

Zebra Mussel Density Associations

Prc-exposure Unionid Mussels

\begin{tabular}{|c|c|c|c|c|c|c|c|c|c|c|}
\hline $\begin{array}{c}\text { Treatment } \\
\text { Level } \\
\text { (mg/L) }\end{array}$ & $\begin{array}{c}\text { Test } \\
\text { Enclosure } \\
\text { ID }\end{array}$ & Tag ID & $\begin{array}{c}\text { Welght } \\
\text { w/zM (g) }\end{array}$ & $\begin{array}{c}\text { Welght } \\
\text { w/o ZM } \\
\text { (g) }\end{array}$ & $\begin{array}{c}\text { Number } \\
\text { of Zebra } \\
\text { Mussels } \\
\text { (Live) }\end{array}$ & $\begin{array}{c}\text { Welght of } \\
\text { ZM (g) }\end{array}$ & $\begin{array}{l}\text { \% Body Mass } \\
\text { of ZM on } \\
\text { Unionle's }\end{array}$ & $\begin{array}{l}\text { Number of } \\
\mathrm{ZM} / \mathrm{gram} \text { of } \\
\mathrm{ZM}\end{array}$ & $\begin{array}{c}\text { Mean } \\
\text { Number of } \\
\mathrm{ZM} / \mathrm{gram} \text { of } \\
\mathrm{ZM} \text { (SD) }\end{array}$ & $\begin{array}{l}\text { Grand Mean } \\
\text { Number of } \\
2 \mathrm{M} / \mathrm{g} \text { ram of } \mathrm{ZM} \\
\text { [SD] }\end{array}$ \\
\hline \multirow{15}{*}{ Contral } & \multirow{5}{*}{3} & $\mathrm{C} 197$ & 87.49 & 29.51 & 201 & 57,98 & 196.48 & 3,47 & & \multirow{15}{*}{$\begin{array}{c}3.82 \\
(0.20)\end{array}$} \\
\hline & & C254 & 129.20 & 64.98 & 270 & 64.22 & 98.83 & 4.20 & 4.11 & \\
\hline & & C382 & 95.43 & 41.40 & 164 & 54,03 & 130.51 & 3,04 & $(0.37)$ & \\
\hline & & $\mathrm{C} 427$ & 60.56 & 30.47 & 168 & 30.09 & 98.75 & 5.58 & & \\
\hline & & C434 & 121.23 & 75,00 & 196 & 46.23 & 61.64 & 4.24 & & \\
\hline & \multirow{5}{*}{7} & $\mathrm{C} 192$ & 72.08 & 30.89 & 118 & 41.19 & 133.34 & 2.86 & \multirow{5}{*}{$\begin{array}{c}3.67 \\
(0.73)\end{array}$} & \\
\hline & & C261 & 66.57 & 30.12 & 123 & 36.45 & 121.02 & 3.37 & & \\
\hline & & C361 & 67.80 & 24.88 & 152 & 42.92 & 172.51 & 3.54 & & \\
\hline & & C394 & 53.13 & 26.77 & 133 & 26,36 & 98.47 & 5.05 & & \\
\hline & & CA17 & 91.02 & 48.99 & 149 & 42,03 & 85.79 & 3.55 & & \\
\hline & \multirow{5}{*}{8} & $\mathrm{C1} 189$ & 81.34 & 41.04 & 146 & 40.30 & 98.20 & 3.62 & \multirow{5}{*}{$\begin{array}{c}3.69 \\
(0.55)\end{array}$} & \\
\hline & & C191 & 85.90 & 35.24 & 164 & 50.66 & 143.76 & 3.24 & & \\
\hline & & $\mathrm{C} 209$ & 92.00 & 41.12 & 181 & 50.88 & 123.74 & 3.56 & & \\
\hline & & C22.0 & 82.76 & 24,20 & 193 & 58.56 & 241.98 & 3.30 & & \\
\hline & & C337 & 91.54 & 46.67 & 213 & 44.87 & 96.14 & 4.75 & & \\
\hline \multirow{15}{*}{50} & \multirow{5}{*}{2} & C310 & 60.52 & 23.64 & 114 & 36.89 & 156.05 & 3.09 & \multirow{5}{*}{$\begin{array}{c}3.55 \\
(0.87)\end{array}$} & \multirow{15}{*}{$\begin{array}{c}3.78 \\
10.201\end{array}$} \\
\hline & & C332. & 109.88 & 57.36 & 181 & 52.52 & 91.56 & 3.45 & & \\
\hline & & C375 & 74.17 & 42.09 & 94 & 32.08 & 76.22 & 2.93 & & \\
\hline & & C410 & 60.95 & 28.90 & \$7 & 32,05 & 110,90 & 3.03 & & \\
\hline & & C419 & 111,41 & 56.12 & 291 & 55.29 & 98.52 & 5.26 & & \\
\hline & \multirow{5}{*}{4} & $\mathrm{C} 225$ & 56.62 & 27.72 & 108 & 28.90 & 104.26 & 3.74 & \multirow{5}{*}{$\begin{array}{c}4.03 \\
(0.65)\end{array}$} & \\
\hline & & C239 & 76.10 & 22.10 & 257 & 54.00 & 244.34 & 4.76 & & \\
\hline & & C282 & 64.49 & $35.00^{\circ}$ & 88 & 29,49 & 84.26 & 2.98 & & \\
\hline & & C333 & 48.70 & 17.33 & 126 & 31.37 & 181.02 & 4,02 & & \\
\hline & & C348 & 116.97 & 61.39. & 260 & 55.58 & 90.54 & 4.68 & & \\
\hline & \multirow{5}{*}{ s } & $\mathrm{C} 238$ & 85.28 & 34.40 & 229 & 50.88 & 147,91 & 4.50 & \multirow{5}{*}{$\begin{array}{c}3.7 \\
(0.68)\end{array}$} & \\
\hline & & $\mathrm{C} 2 \mathrm{NO}$ & 67.22 & 28.16 & 113 & 39.06 & 138.71 & 2.89 & & \\
\hline & & $\mathrm{C} 256$ & 87.84 & 53.99 & 156 & 33,85 & 62.70 & 4,61 & & \\
\hline & & $\mathrm{C} 259$ & 70.93 & 38.85 & 114 & 32.08 & 82.57 & 3.55 & & \\
\hline & & C343 & 63,45 & 39.03 & 80 & 21.42 & 62.57 & 3.28 & & \\
\hline \multirow{15}{*}{100} & \multirow{5}{*}{1} & C208 & 88.81 & 45.45 & 172 & 43.36 & 95.40 & 3.97 & \multirow{5}{*}{$\begin{array}{c}4.29 \\
(0.35)\end{array}$} & \multirow{15}{*}{$\begin{array}{l}n .16 \\
(0.10)\end{array}$} \\
\hline & & $\mathrm{C} 263$ & 98.82 & 49.74 & 202 & 19.08 & 98.6\% & 4.12 & & \\
\hline & & C350 & 70.50 & 34.33 & 110 & 36,17 & 105.36 & 3.04 & & \\
\hline & & C358 & 68.17 & 25.09 & 188 & 43.08 & 171,70 & 4.36 & & \\
\hline & & $\mathrm{C} 377$ & 52,12 & 19.35 & 196 & 32.77 & 169.35 & 5.98 & & \\
\hline & & $\mathrm{C} 200$ & 85.77 & 50.54 & 156 & 35.13 & 69.37 & 4.44 & & \\
\hline & & C250 & 71,05 & 42.35 & 124 & 28.70 & $67 . \%$ & 4.32 & 4.04 & \\
\hline & 6 & C356 & 59.64 & 25.73 & 148 & 33.91 & 131.79 & 4.36 & $(0.42)$ & \\
\hline & & $\mathrm{C} 102$ & 111.07 & 40.37 & 262 & 70.70 & 175.13 & 3.71 & & \\
\hline & & C426 & 70.01 & 28.66 & 140 & 41.35 & 144.28 & 3.39 & & \\
\hline & \multirow{5}{*}{9} & C295 & 67.10 & 39.75 & 114 & 27.35 & 68.81 & 4.17 & \multirow{5}{*}{$\begin{array}{c}4.13 \\
(0.61)\end{array}$} & \\
\hline & & C30s & 56.29 & 34.93 & so & 21,36 & 61.15 & 4.21 & & \\
\hline & & C367 & 123.87 & 60.76 & 249 & 63.11 & 103.87 & 3.35 & & \\
\hline & & C411 & 111.65 & 48.10 & 204 & 63.55 & 132.12 & 3.21 & & \\
\hline & & C416 & 53.28 & 24,17 & 149 & 29.11 & 120.64 & 5.12 & & \\
\hline
\end{tabular}

page 3 of 15 


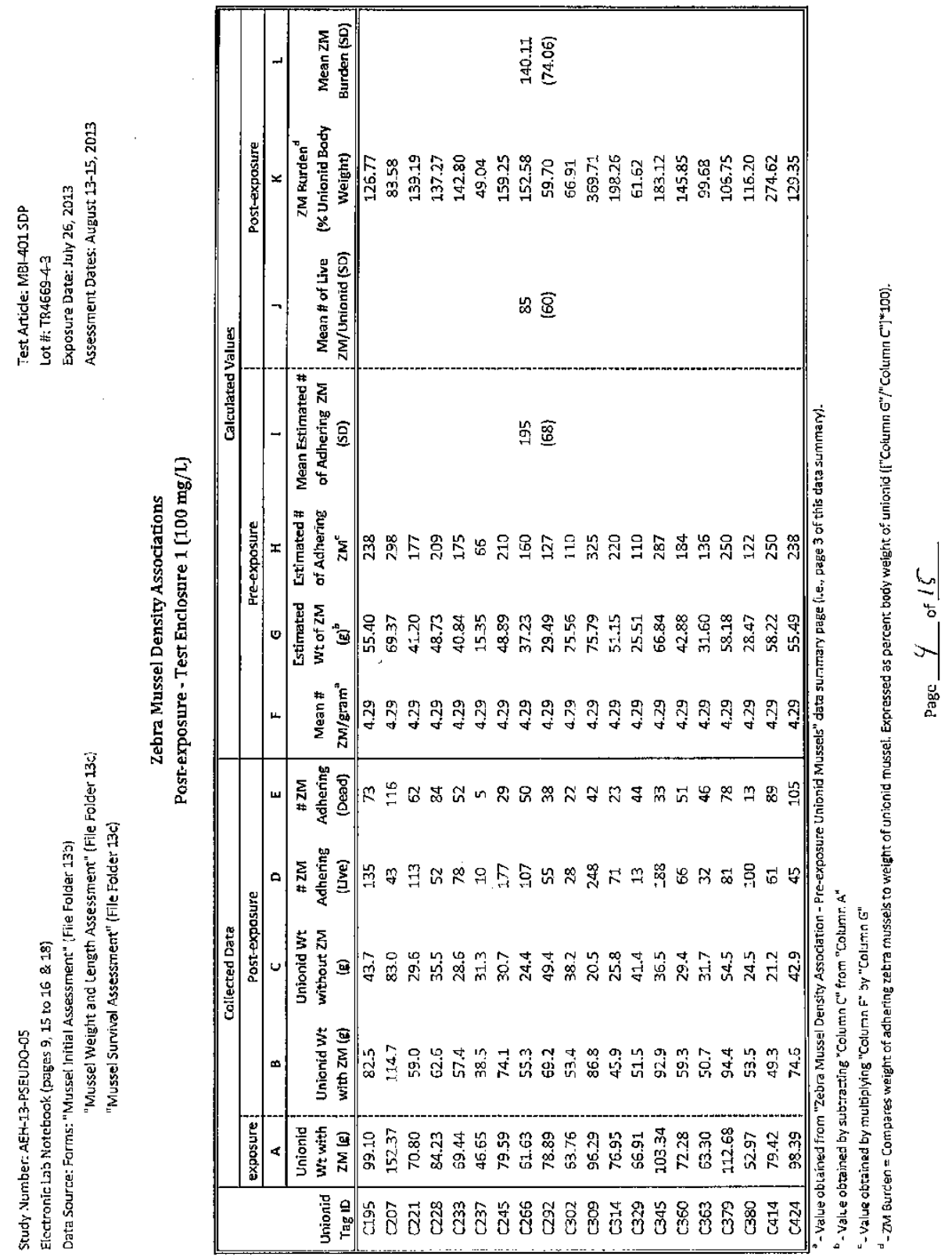




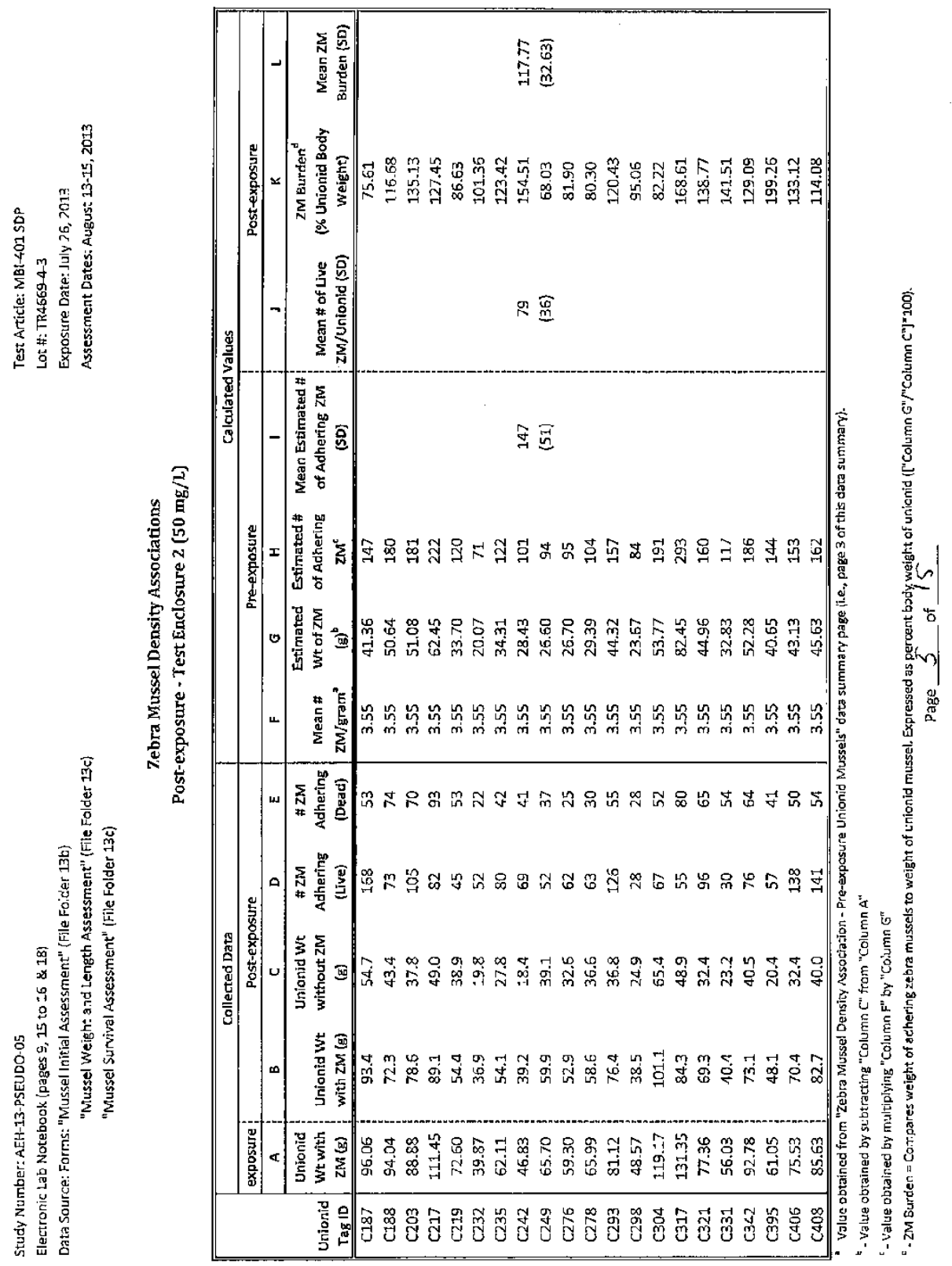




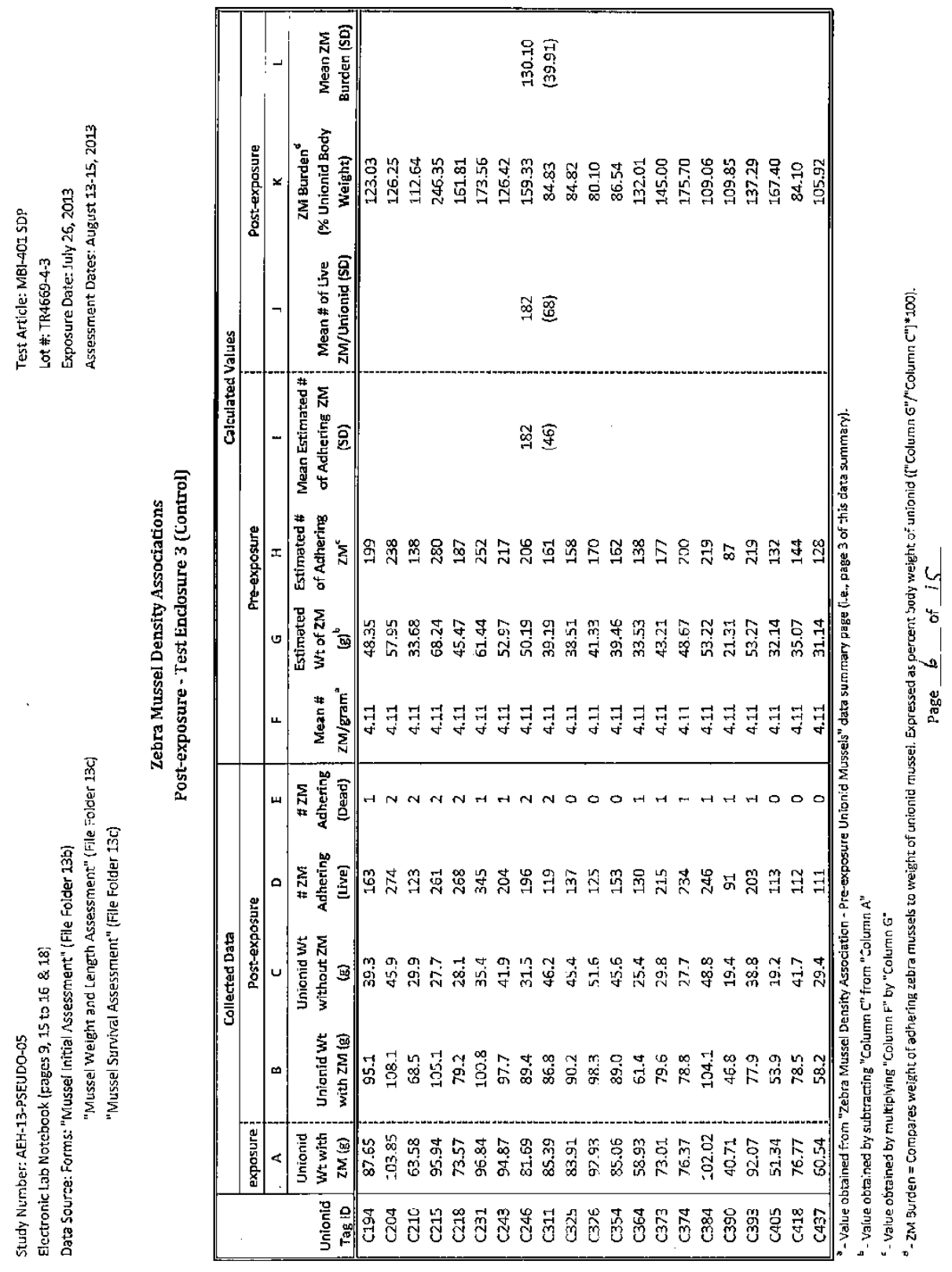




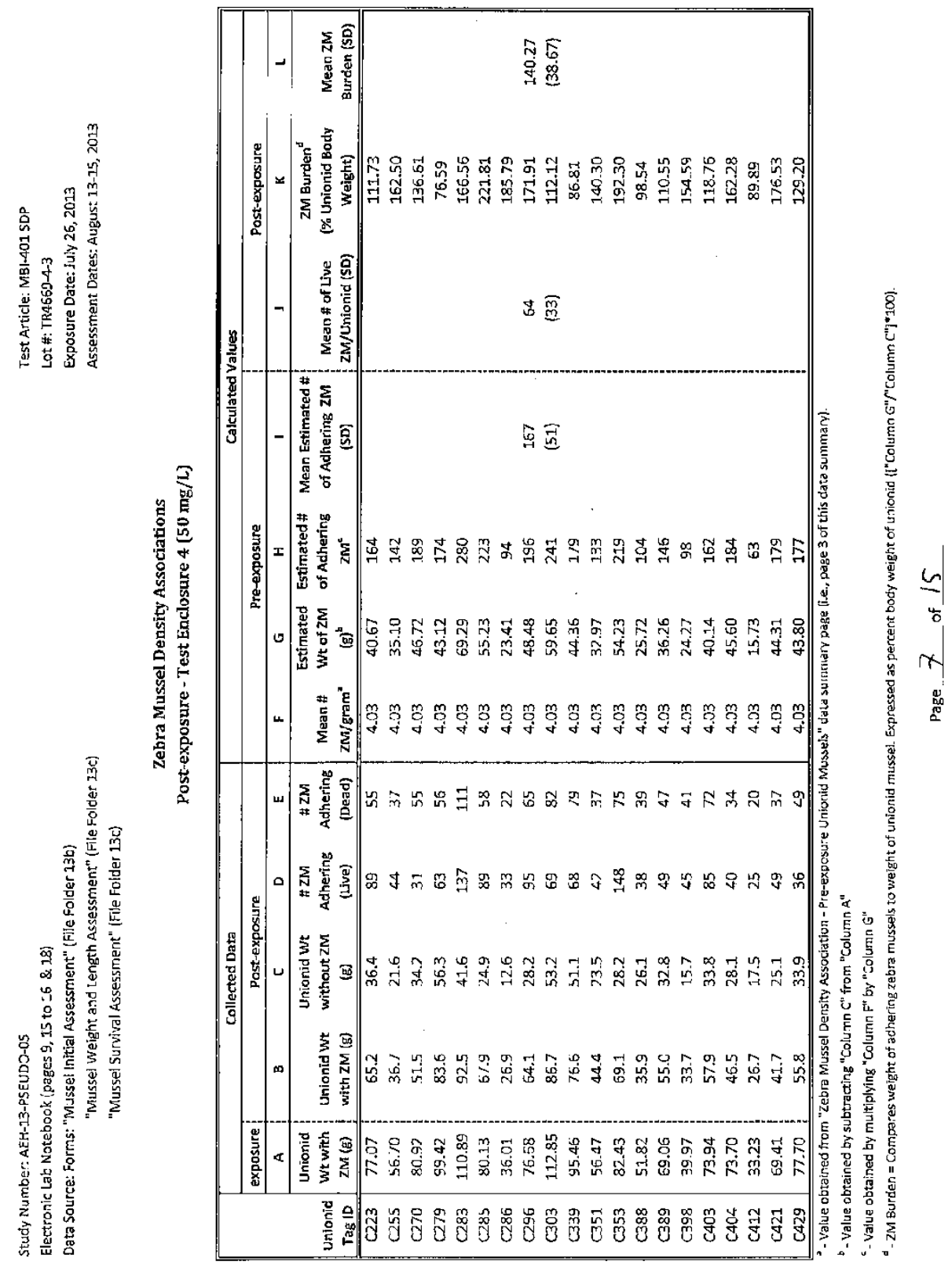




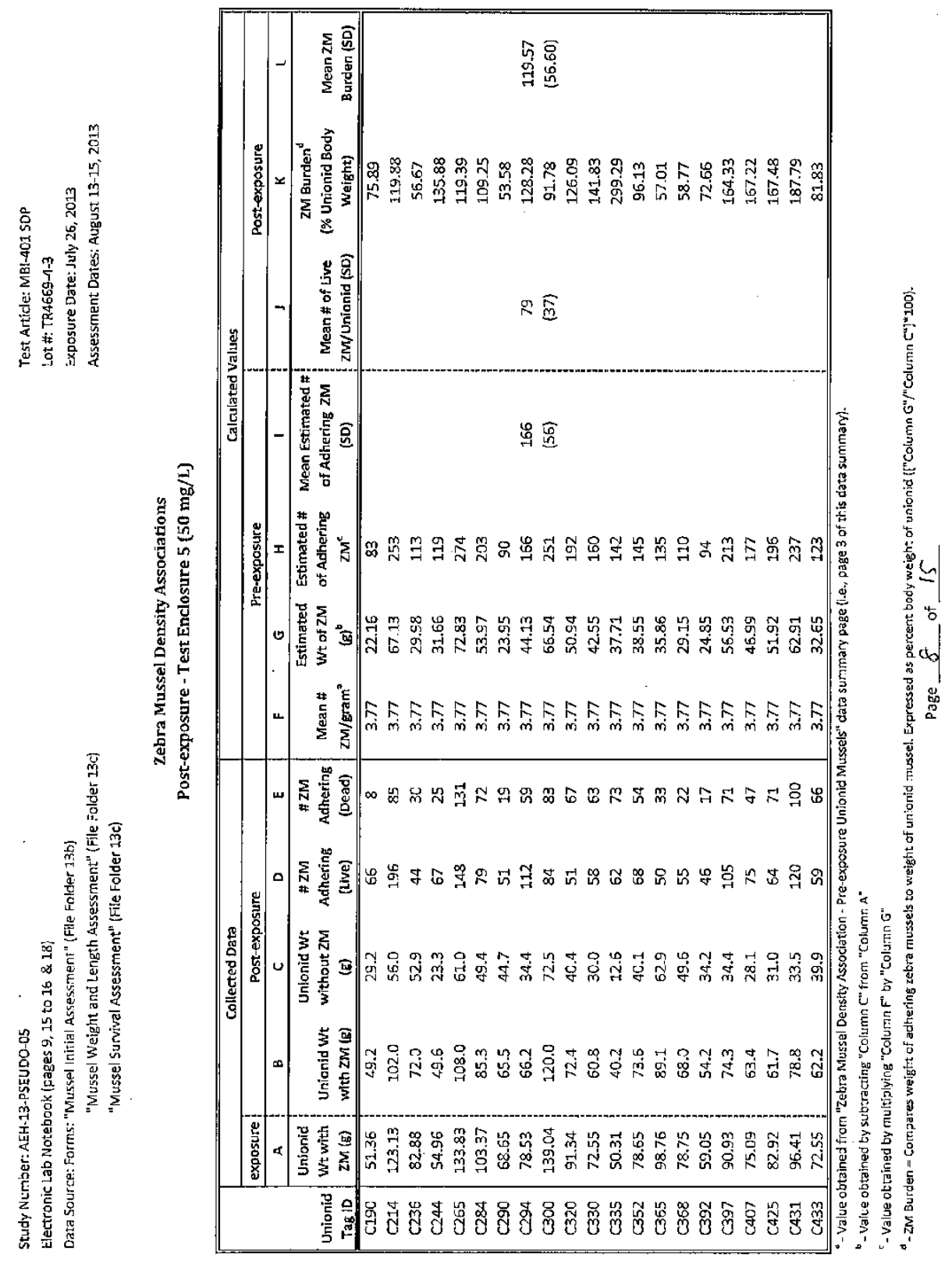




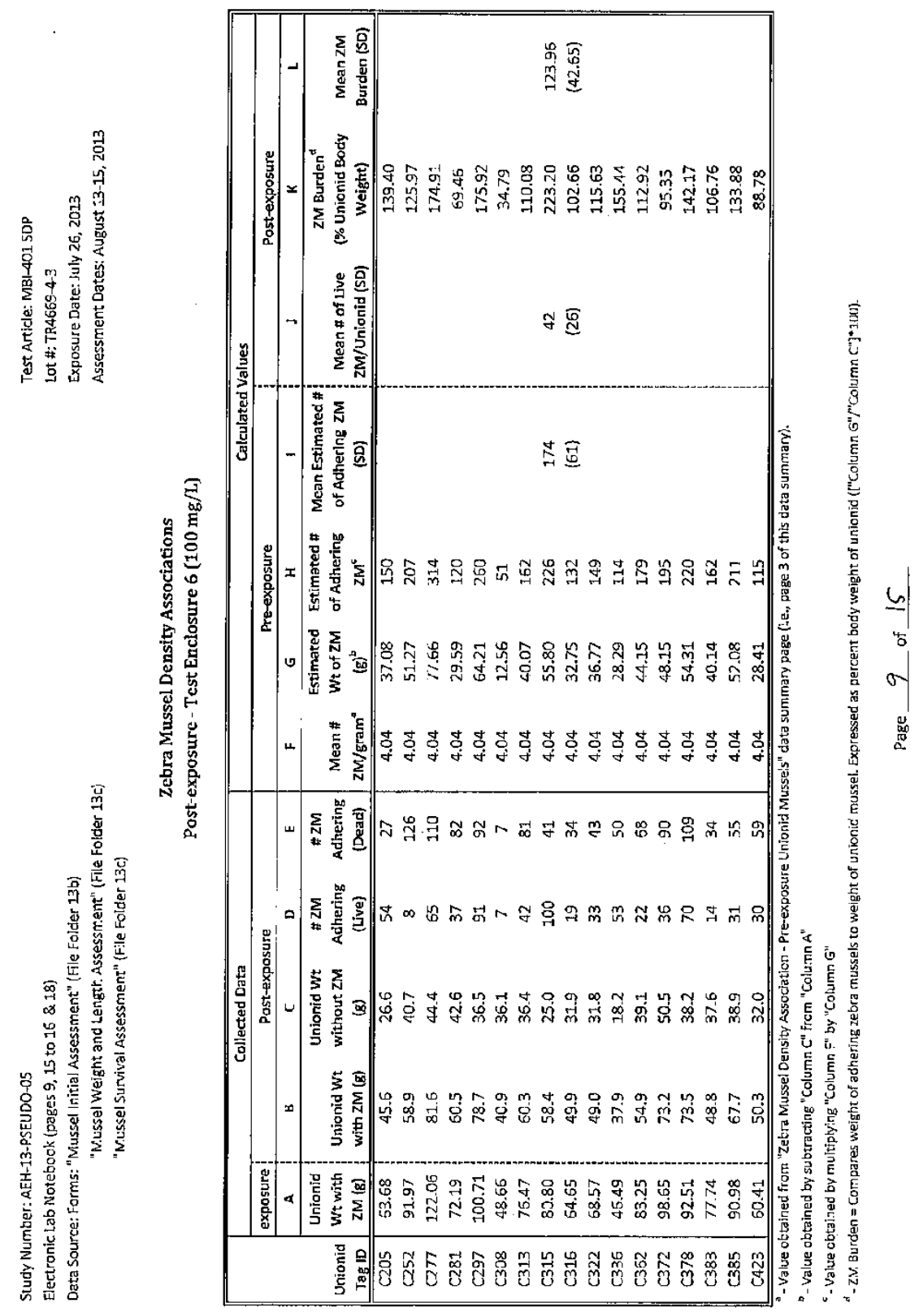




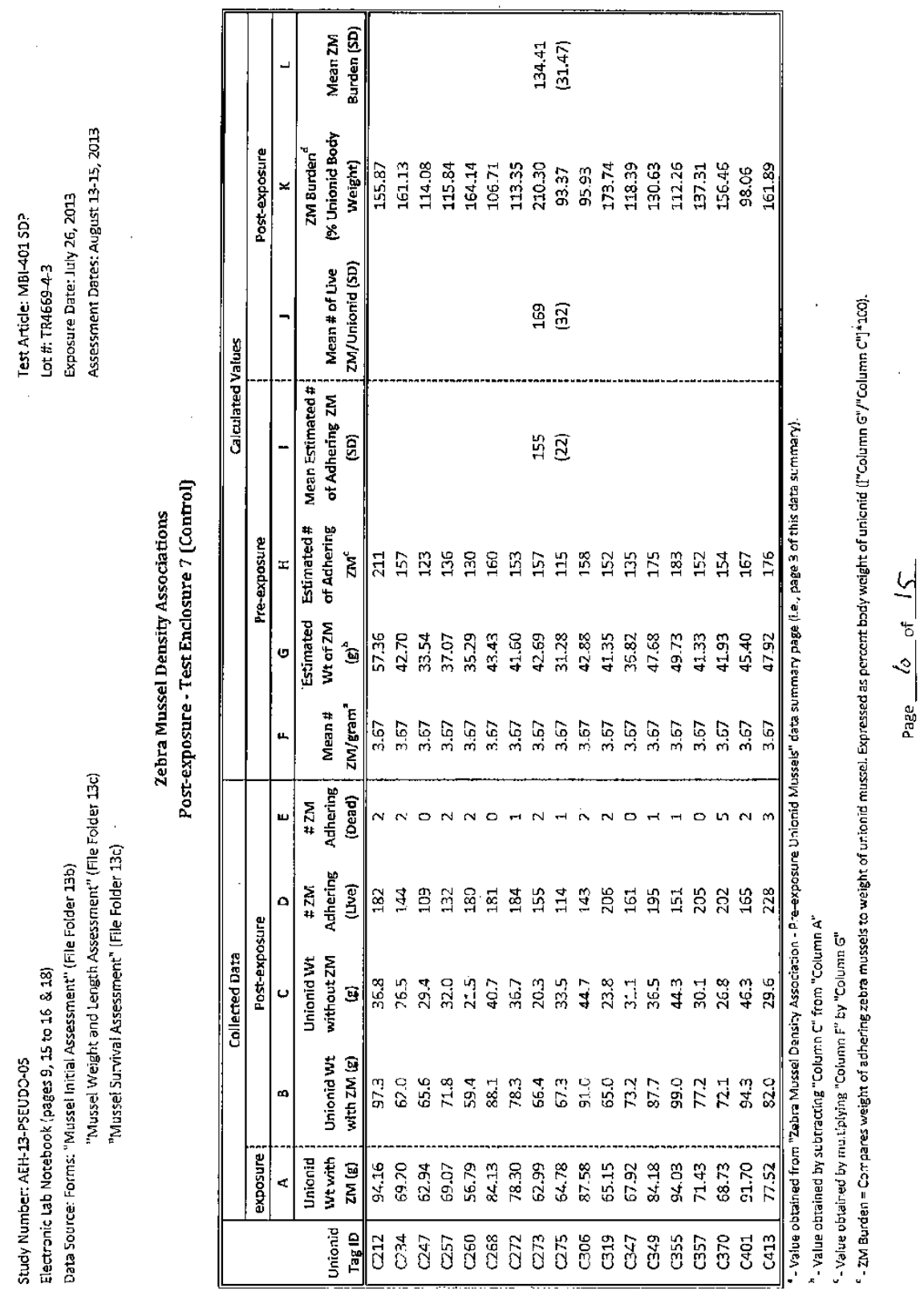




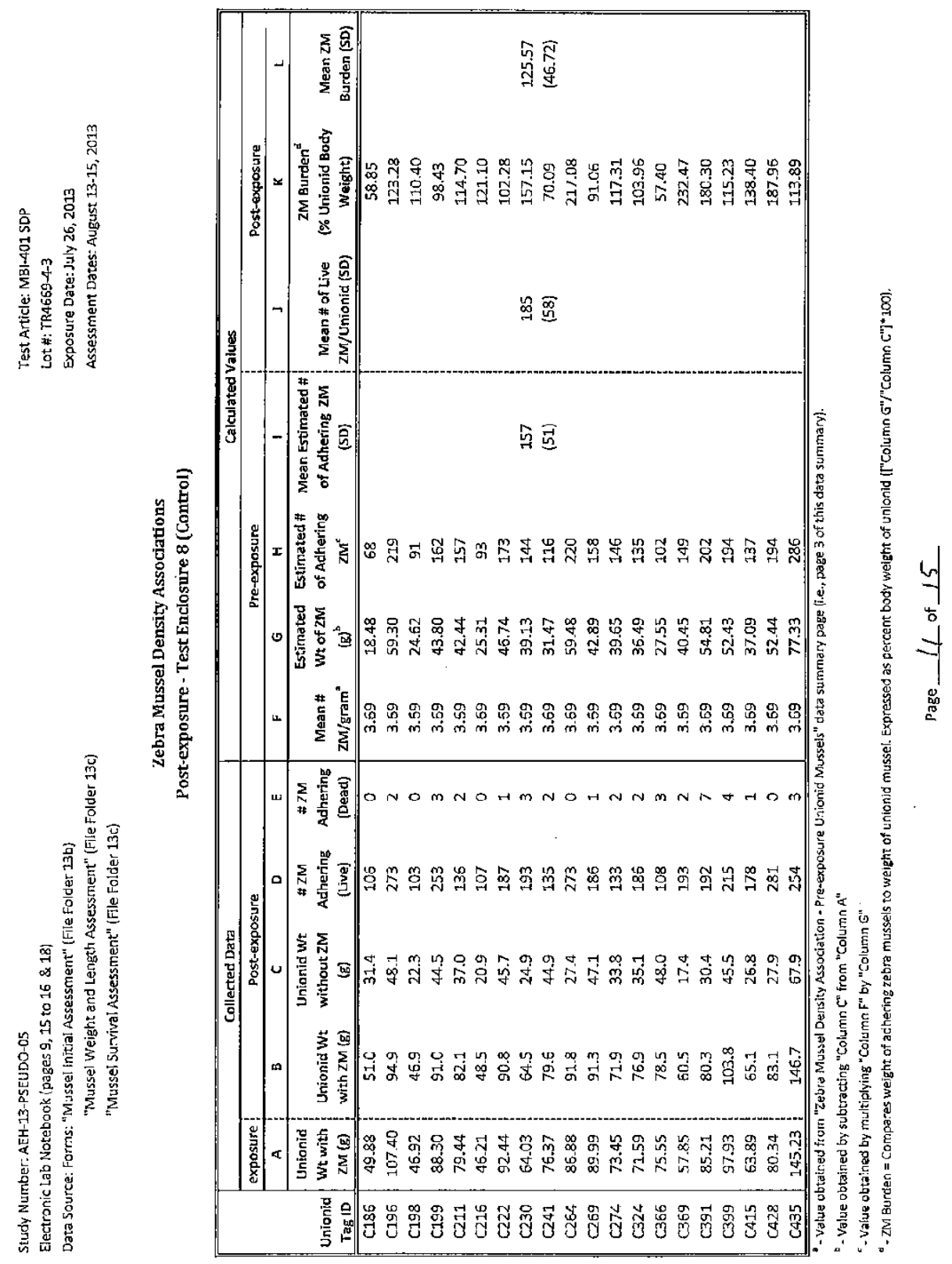




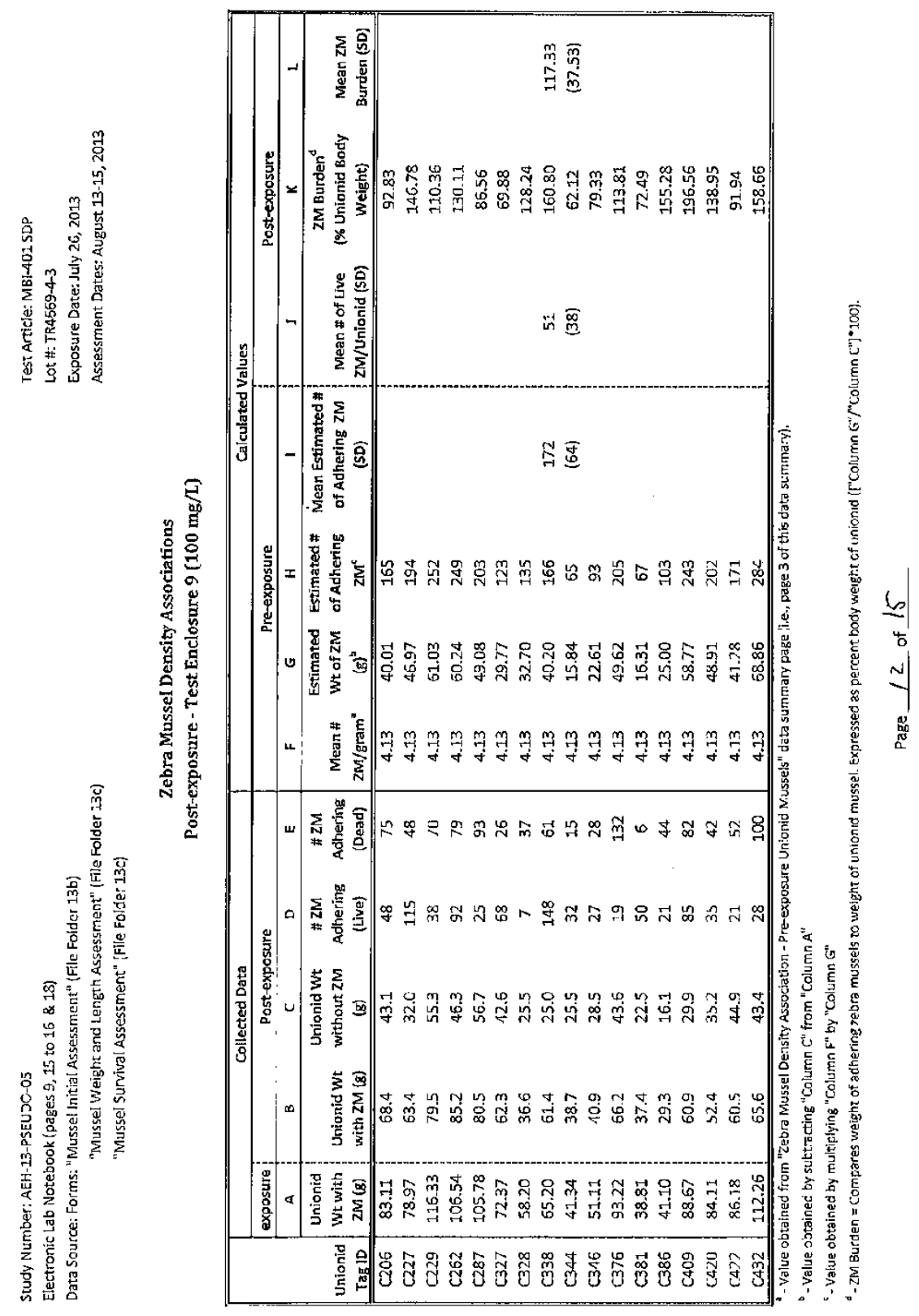



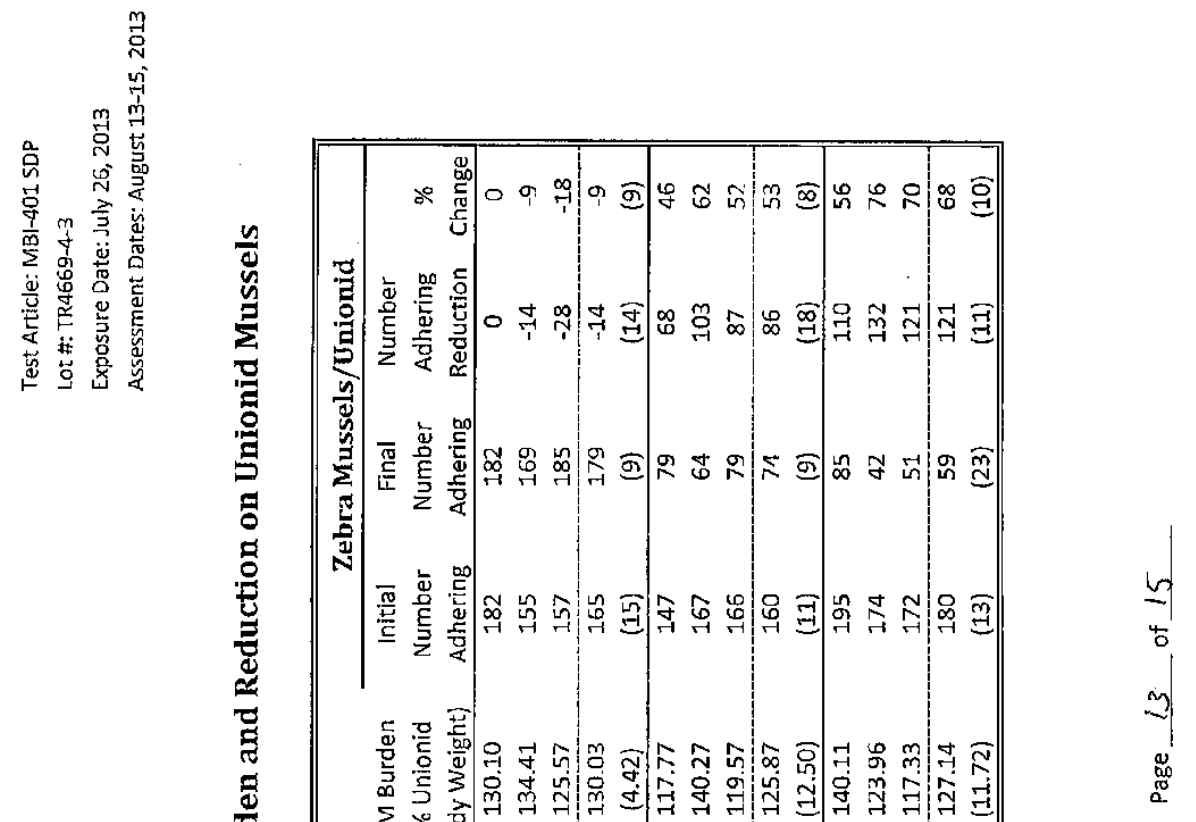

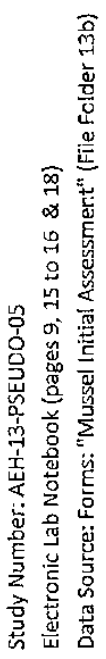



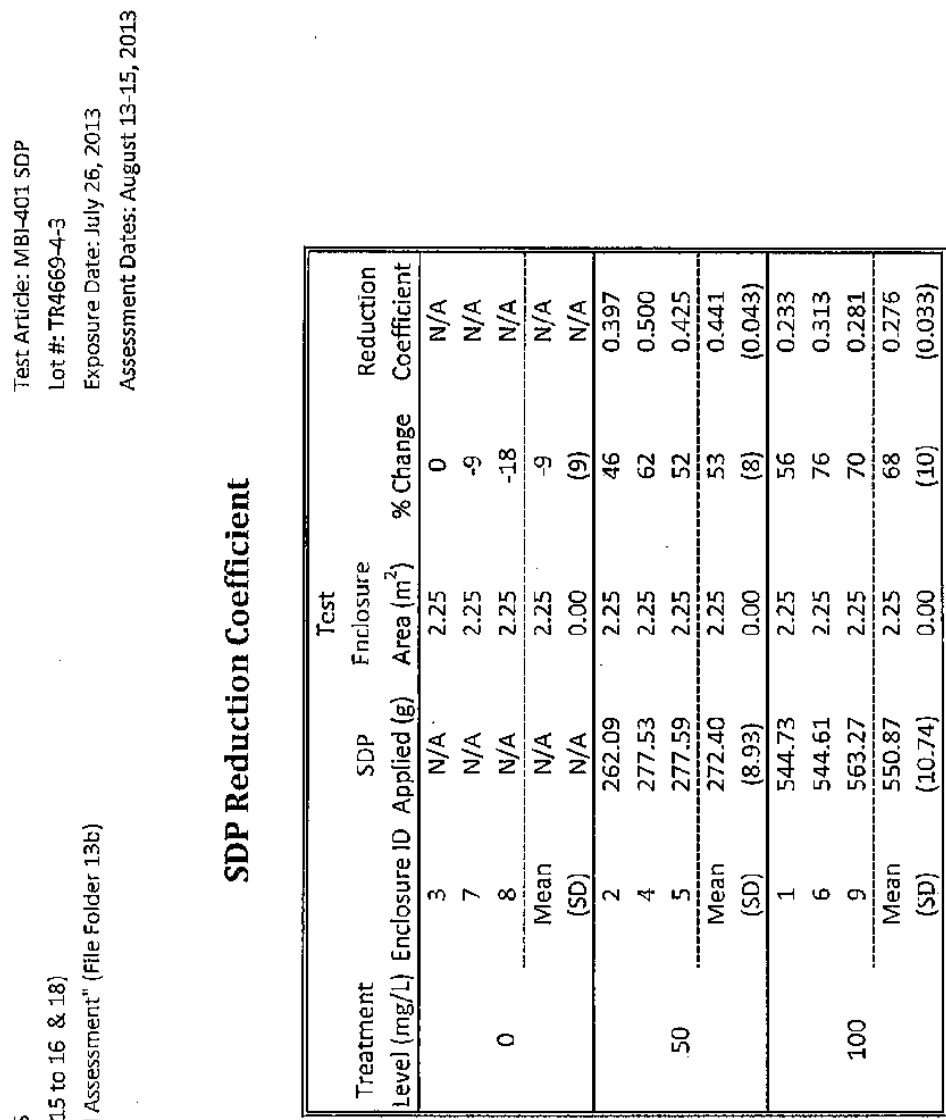

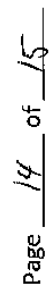

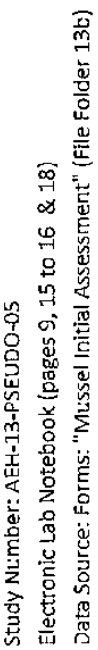




$\begin{array}{cccc}\text { enc } & \text { treat } & \text { time } & \text { density } \\ 1 & 100 & 1 & 195 \\ 1 & 100 & 2 & 85 \\ 2 & 50 & 1 & 147 \\ 2 & 50 & 2 & 79 \\ 3 & 0 & 1 & 182 \\ 3 & 0 & 2 & 182 \\ 4 & 50 & 1 & 167 \\ 4 & 50 & 2 & 64 \\ 5 & 50 & 1 & 166 \\ 5 & 50 & 2 & 79 \\ 6 & 100 & 1 & 174 \\ 6 & 100 & 2 & 42 \\ 7 & 0 & 1 & 155 \\ 7 & 0 & 2 & 169 \\ 8 & 0 & 1 & 157 \\ 8 & 0 & 2 & 185 \\ 9 & 100 & 1 & 172 \\ 9 & 100 & 2 & 51\end{array}$

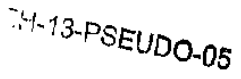

FF \# $\frac{13 c}{\mathrm{No} \cdot \frac{1}{15}}$
$\mathrm{Pg}{ }_{15}$ of 155 


\section{Appendix 9. Unionid Mussel Survival Assessment Summary}

\begin{tabular}{cccc}
\hline $\begin{array}{c}\text { Item } \\
\text { number }\end{array}$ & Item description & $\begin{array}{c}\text { Number } \\
\text { of } \\
\text { pages }\end{array}$ & $\begin{array}{c}\text { Report } \\
\text { page } \\
\text { number }\end{array}$ \\
\hline 1 & Unionid Mussel Survival - Data Summary & 5 & 218 \\
\hline
\end{tabular}


Study Number: AEH-13.PSEUDO-05

Electronle Lab Notebook (pages 9, 10,15 to 17 \& 21)

Data Source: Forms: "Mussel Specles identffication ' (Flle Folder 7b) "Inlttal Assessment of Natlve a,d Zebra Musse s" (File Folder 13a)

"Mussel Welgit and Length Assessment" (File iolder 13c

\begin{tabular}{|c|c|c|}
\hline Action & Uate & Initials \\
\hline Created....... & 20-Feb-14 & KLW K KW \\
\hline Revised...... & 6-Mar-14 & KLW YWL \\
\hline Kevlewed... & GMARU & $n x^{2}$ \\
\hline Certlfied... & $3 / 6 / / 4$ & 312 \\
\hline
\end{tabular}

Flle Name: See fllenames as stated below.

Unionld Mussel Survival

Test Artic E: M81-401 SJP (Pseudomonas fluorescens Pf-CL 145A [SDP]); L.ot it: TR4669-4-3

Exposure Date: July 26, 20:3

Assessment Dates: August 13-15, 2013

Data Explanation:

1) Unlonid mussel survival status was determined 18 to 20 days post-exposure (August 13-:5, 2013).

2) Data codes used withln SAS

enc $=$ Test Enclosure $10\{1$ through 9\}

treal $=$ treatment level $(0,50$ and 100$)$

$0=$ control $(0 \mathrm{mg} / \mathrm{L})$

$50=50 \mathrm{mg} / \mathrm{L}$.

$100=100 \mathrm{mg} / \mathrm{L}$

group = zebra mussel assessment time

1 = pre-exposure (l.e., 45 Lnionld mussels used to determine zebra mussel density associations)

$2=$ post-exposure

tag $=$ Hallprirt shellfish tag glued to the natlve mussel

specles = unionid species

FAM = Fatmucket (Lampsilis sillquoidea )

FPS $=$ Fraglle Papershe I (Leptodea frogilis )

UNK $=$ Unknown

status = status of unionid mussel during post-expcsure assessment

$L=L$ Ive

$=$ Dead

$\mathrm{U}=$ Unrecovered

Data Anomalles and Deviations:

NONE

File Folder $\frac{13 c}{2}$ 

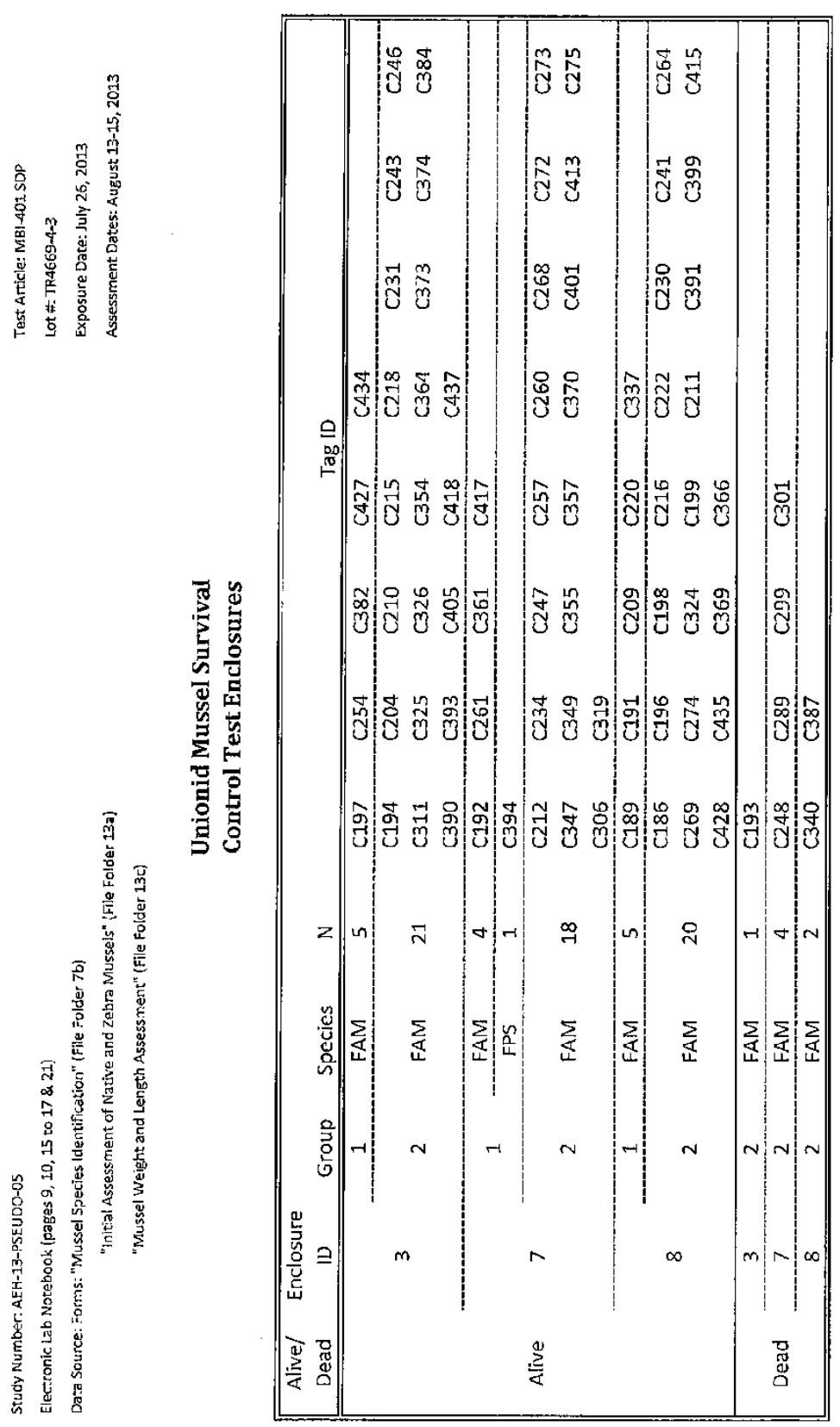

$\gamma \mid$ 

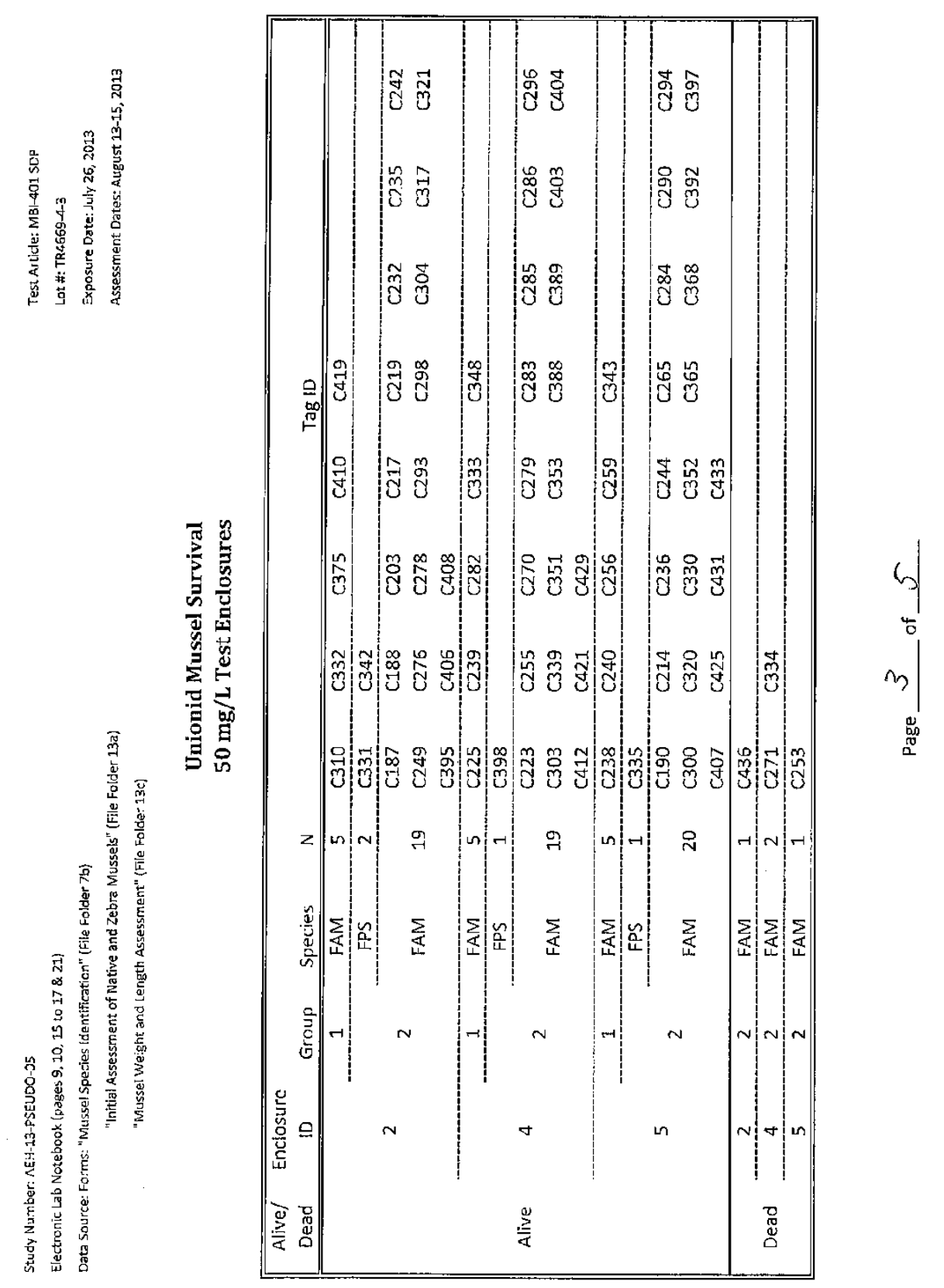

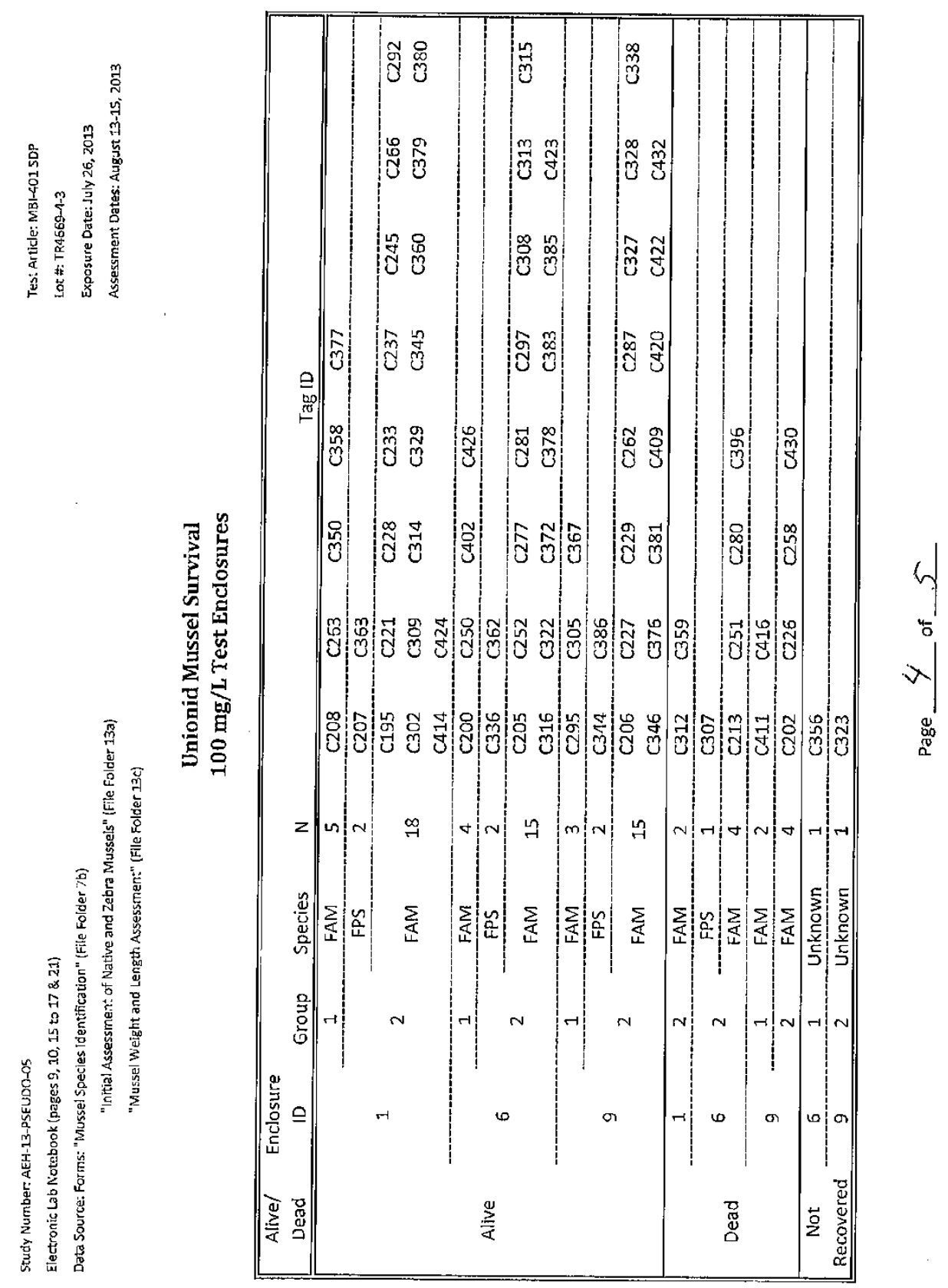


\begin{tabular}{|c|c|c|c|c|c|c|c|c|}
\hline enc & treat & group & species & tot & dead & unrec & tdead & \\
\hline 1 & 100 & 1 & FAM & 5 & 0 & 0 & 0 & \\
\hline 1 & 100 & 2 & FAM & 20 & 2 & 0 & 2 & \\
\hline 1 & 100 & 1 & FPS & 0 & 0 & 0 & 0 & \\
\hline 1 & 100 & 2 & FPS & 2 & 0 & 0 & 0 & \\
\hline 2 & 50 & 1 & FAM & 5 & 0 & 0 & 0 & \\
\hline 2 & 50 & 2 & FAM & 20 & 1 & 0 & 1 & \\
\hline 2 & 50 & 1 & FPS & 0 & 0 & 0 & 0 & AEH-13-PSEUDO-05 \\
\hline 2 & 50 & 2 & FPS & 2 & 0 & 0 & 0 & \\
\hline 3 & 0 & 1 & FAM & 5 & 0 & 0 & 0 & \\
\hline 3 & 0 & 2 & FAM & 22 & 1 & 0 & 1 & \\
\hline 3 & 0 & 1 & FPS & 0 & 0 & 0 & 0 & \\
\hline 3 & 0 & 2 & FPS & 0 & 0 & 0 & 0 & \\
\hline 4 & 50 & 1 & FAM & 5 & 0 & 0 & 0 & \\
\hline 4 & 50 & 2 & FAM & 21 & 2 & 0 & 2 & \\
\hline 4 & 50 & 1 & FPS & 0 & 0 & 0 & 0 & \\
\hline 4 & 50 & 2 & FPS & 1 & 0 & 0 & 0 & \\
\hline 5 & 50 & 1 & FAM & 5 & 0 & 0 & 0 & \\
\hline 5 & 50 & 2 & FAM & 21 & 1 & 0 & 1 & \\
\hline 5 & 50 & 1 & FPS & 0 & 0 & 0 & 0 & \\
\hline 5 & 50 & 2 & FPS & 1 & 0 & 0 & 0 & \\
\hline 6 & 100 & 1 & FAM & 5 & 0 & 1 & 1 & \\
\hline 6 & 100 & 2 & FAM & 19 & 4 & 0 & 4 & \\
\hline 6 & 100 & 1 & FPS & 0 & 0 & 0 & 0 & \\
\hline 6 & 100 & 2 & FPS & 3 & 1 & 0 & 1. & \\
\hline 7 & 0 & 1 & FAM & 4 & 0 & 0 & 0 & \\
\hline 7 & 0 & 2 & FAM & 22 & 4 & 0 & 4 & \\
\hline 7 & 0 & 1 & FPS & 1 & 0 & 0 & 0 & \\
\hline 7 & 0 & 2 & FPS & 0 & 0 & 0 & 0 & \\
\hline 8 & 0 & 1 & FAM & 5 & 0 & 0 & 0 & \\
\hline 8 & 0 & 2 & FAM & 22 & 2 & 0 & 2 & \\
\hline 8 & 0 & 1 & FPS & 0 & 0 & 0 & 0 & \\
\hline 8 & 0 & 2 & FPS & 0 & 0 & 0 & 0 & \\
\hline 9 & 100 & 1 & FAM & 5 & 2 & 0 & 2 & \\
\hline 9 & 1.00 & 2 & FAM & 20 & 4 & 1 & 5 & \\
\hline 9 & 100 & 1 & FPS & 0 & 0 & 0 & 0 & \\
\hline 9 & 100 & 2 & FPS & 2 & 0 & 0 & 0 & \\
\hline
\end{tabular}

FF \# 132

Item No. 2

$\mathrm{Pg} \_5$ of 5 


\section{Appendix 10. Statistical Analysis, Including SAS Programs, Outputs, and Logs for Unionid Mussel Survival, Zebra Mussel Colonization Density Associations, and Zebra Mussel Test Animal Lengths}

\begin{tabular}{clrc}
\hline $\begin{array}{c}\text { Item } \\
\text { number }\end{array}$ & \multicolumn{1}{c}{ Item description } & $\begin{array}{c}\text { Number } \\
\text { of } \\
\text { pages }\end{array}$ & $\begin{array}{c}\text { Report } \\
\text { page } \\
\text { number }\end{array}$ \\
\hline 1 & SAS output for unionid mussel survival & 11 & 224 \\
2 & SAS program for unionid mussel survival & 3 & 235 \\
3 & SAS log for unionid mussel survival & 6 & 238 \\
4 & SAS output for zebra mussel density & 23 & 244 \\
5 & SAS program for zebra mussel density & 2 & 267 \\
6 & SAS log for zebra mussel density & 4 & 269 \\
7 & SAS output for zebra mussel lengths & 23 & 273 \\
8 & SAS program for zebra mussel lengths & 2 & 296 \\
9 & SAS log for zebra mussel lengths & 4 & 298 \\
\hline
\end{tabular}


Efflescy of Pseudomonas fluorescens (PF.CL.145A) SDP for controillng zebra mussols within field enclesures

Unionid Mussal Survival

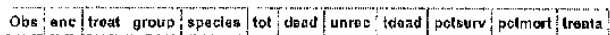

AEH-13-PSEUDO-05

\begin{tabular}{|c|c|c|c|c|c|c|c|c|c|c|c|}
\hline 1 & 2 & 50 & 1 & FAM & 6 & 0 & 0 & D & 100.000 & 0.0000 & A \\
\hline 2 & 4) & 50 & 1 & FAM & 5 & 0 & 0 & 0 & 100.000 & 0.0000 & A \\
\hline 3 & 5 & 60 & 1 & FAM & 5 & 0 : & 0 & 0 & $100.003:$ & 0.0000 & A \\
\hline 4 & 1 & 100 & 1 & FAM & 6 & 0 & 0 & 0 & 160.003 & 0.0000 & in \\
\hline 5 & 8 & 100 & 1. & AM & 5 & 0 & 1 & 1 & 80.000 & 20.0000 & $\theta$ \\
\hline 8 & 9 & 100 & 1 & FAM & 5 & 2 & 0 & 2 & 80,000 & 40,0000 & $\theta$ \\
\hline 7 & 3 & 0 & 1 & FAM & 5 & 0 & D & 0 & 100.000 & 0.0000 & c \\
\hline 8 : & $?$ & 0 & 1 & FAM & 4 & a & 0 & 0 & 100.000 & 0,0000 & c \\
\hline 9 & a & 0 & 1 & FAM & 6) & 0 & (5) & 0 & 100.000 ; & 0.0000 & c \\
\hline 10 & 2 & 50 & 2 & FAM & 20 & 1 & 0 & 1 & $95000^{\circ}$ & 5.0000 & A \\
\hline 11 & 4 & 60 & 2 & FAM & 21 & 2 & 0 & 2 & 90.476 & 9.5238 & A \\
\hline 12 & 5 & 50 & 2 & FAIA & 21 & 1 & 0 & 1 & 95,230 & 4.7619 & A \\
\hline 13 & 1 & 100 & $2 !$ & IFAlA & 20 & 2 & 0 & 2 & 90.000 & 10.0000 & $B$ \\
\hline 14 & 6 & 100 & 2 & FAM & 19 & $4 !$ & 0 & 4 & 78.947 & 21,0526 & $B$ \\
\hline 15 & $\mathrm{~g}$ & 100 & 2 & FAM & 20 & 4 & $1:$ & 5 & 76.000 & 25,0000 & B \\
\hline 16 & 3 & 0 & 2 & FAM & 22 & 1 & 0 & 1 & 95.455 & 4.5455 & 0 \\
\hline 17 & 7 & 0 & $2:$ & FAM & 2 & $A$ & 0 & 4 & 81.818 & 18.1818 & 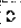 \\
\hline 10 & 8 & 0 & 2 & FAM & 22 & $2:$ & 0 & 2 & 90.939 & 9.0909 & 10 \\
\hline 10 & 7 & 0 & 3 & FPS & 1 & c & 0 , & a. & 100.030 & 0.0000 & $\mathrm{c}$ \\
\hline 20 & 2 & 50 & 2. & FPS & 2 & $c$ & 0 & 0 & 100.000 & 0.0000 & A \\
\hline 21 & 4 & 60 & 2 & FPS & 1 & 0 & 0 & 0 & 103.000 & 0.0000 & A \\
\hline 22 & 5 & 60 & 2 & FPS & $1:$ & 0 & 0 & 0 & 103.000 & 0,0000 & A \\
\hline 23 & 1 & 100 & 2 & FPS & 2 & 0 & 0 & 0 & 100.000 & 0.0000 & $B$ \\
\hline 24 & 6 & 100 & 2 & FPS & 3 & 1 & 0 & 1 & 66.687 & 33.3333 & B \\
\hline 25 & 9 & $100^{\circ}$ & 2 & FPS & 2 & of & 0 & 0 & wou,dou & 0,0000 & B \\
\hline
\end{tabular}

File Folder:

Item Number:

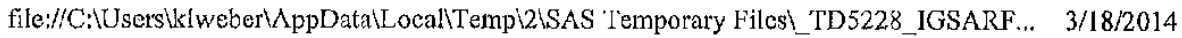


AEH-13-PSEUDO-05

\begin{abstract}
Wean Suıvlyal by Bpecles (gortad hy Iroatmen: lovpl and group)
\end{abstract}
The MEANS Procodure

specles=FAM

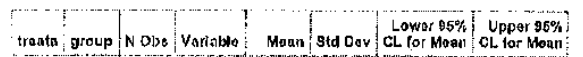

A 1.3 palsurv 100.0 .

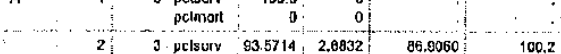

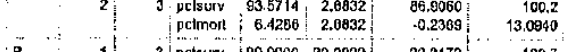

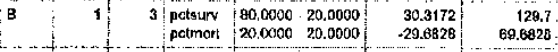

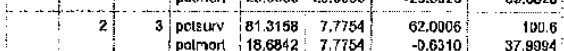

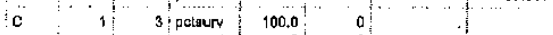

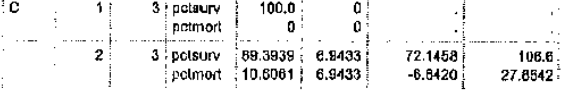

spacies=FPS

spaties=FFP

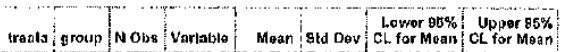

$\begin{array}{lllllll} & \text { A } & 2 & 3 \text { polsuiv } & 100.0 & 0\end{array}$

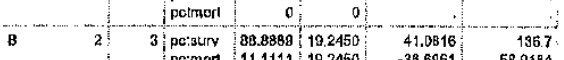

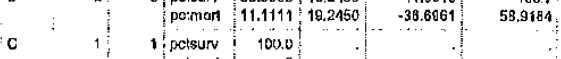

Pérormed by K. Weber SAS version 9.3 13:31 18MAR1A W" 
AEH-13-PSEUDO-05

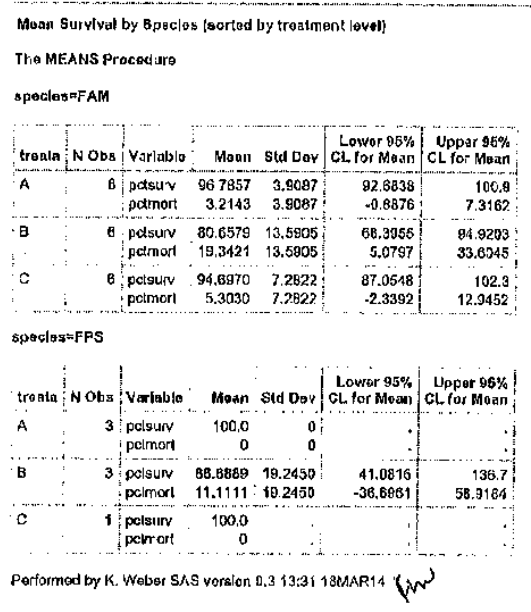

file:/C:IUsersłklweberhppData!LocaIYIempไ2\SAS Temporary Files\_TD5228_IGSARF... 3/18/2014 


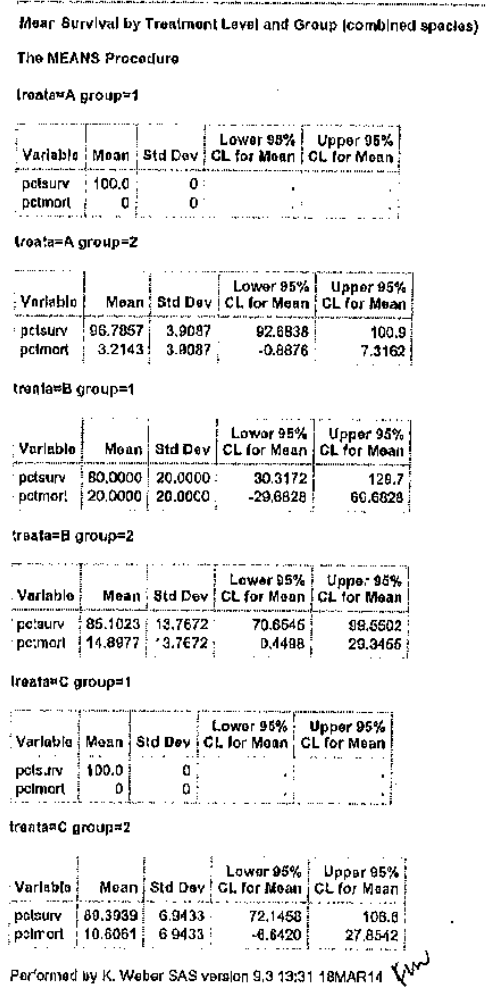


Mokn Survival hy Traatment (combined spocles anu group)

The MEANS Procedure

treatazA

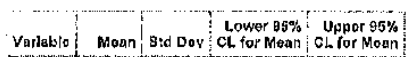

\begin{tabular}{l|l|l|l|l|}
\hline petsurv & 97.8671 & 3.4830 & 95.1793 & 100.6
\end{tabular}

\begin{tabular}{r|r|rrr}
\hline peimor & 2.1429 & $3.4830^{\circ}$ & -0.5344 & 4.8202 \\
\hline
\end{tabular}

treata=B

Vnrtable Mean Std Dev Cower 05\% CLpor 95\%

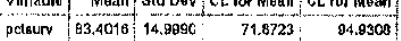

\begin{tabular}{l|l|l|l|l|}
\hline peImorl & 16.5989 & 14.9890 & $5.0692 \mid$ & $28.12 n$ \\
\hline
\end{tabular}

treata $=0$

Vardabto Maan std Dev| Cowar 95\% uppor 95\%

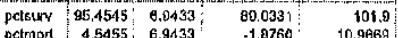

Patamed by $K$. Wober SAS version 9.3 13:31 194AAR14 "W

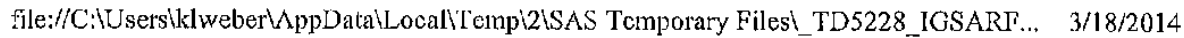




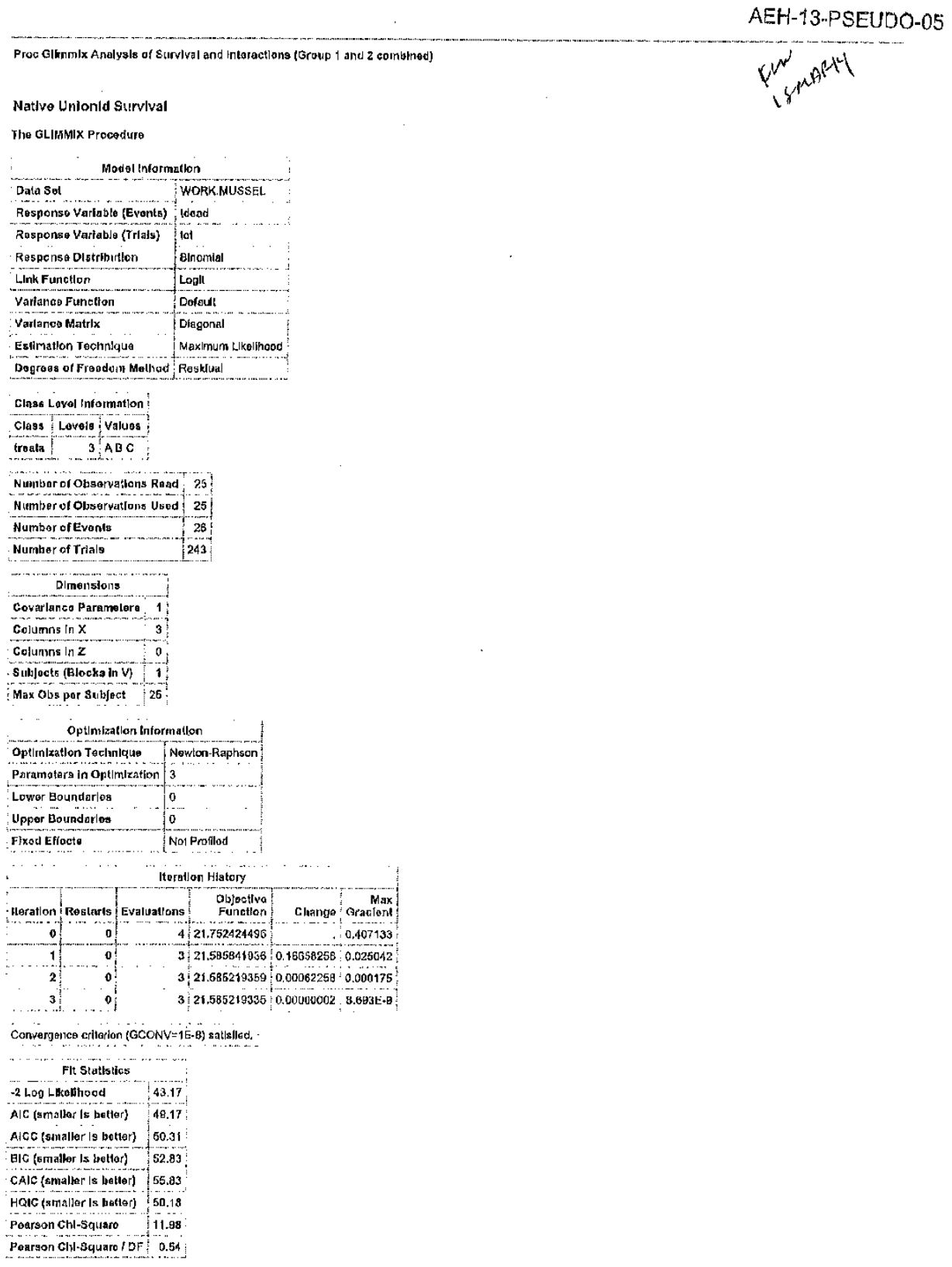

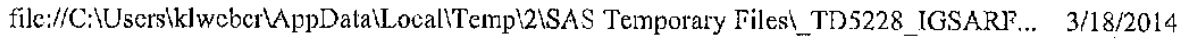




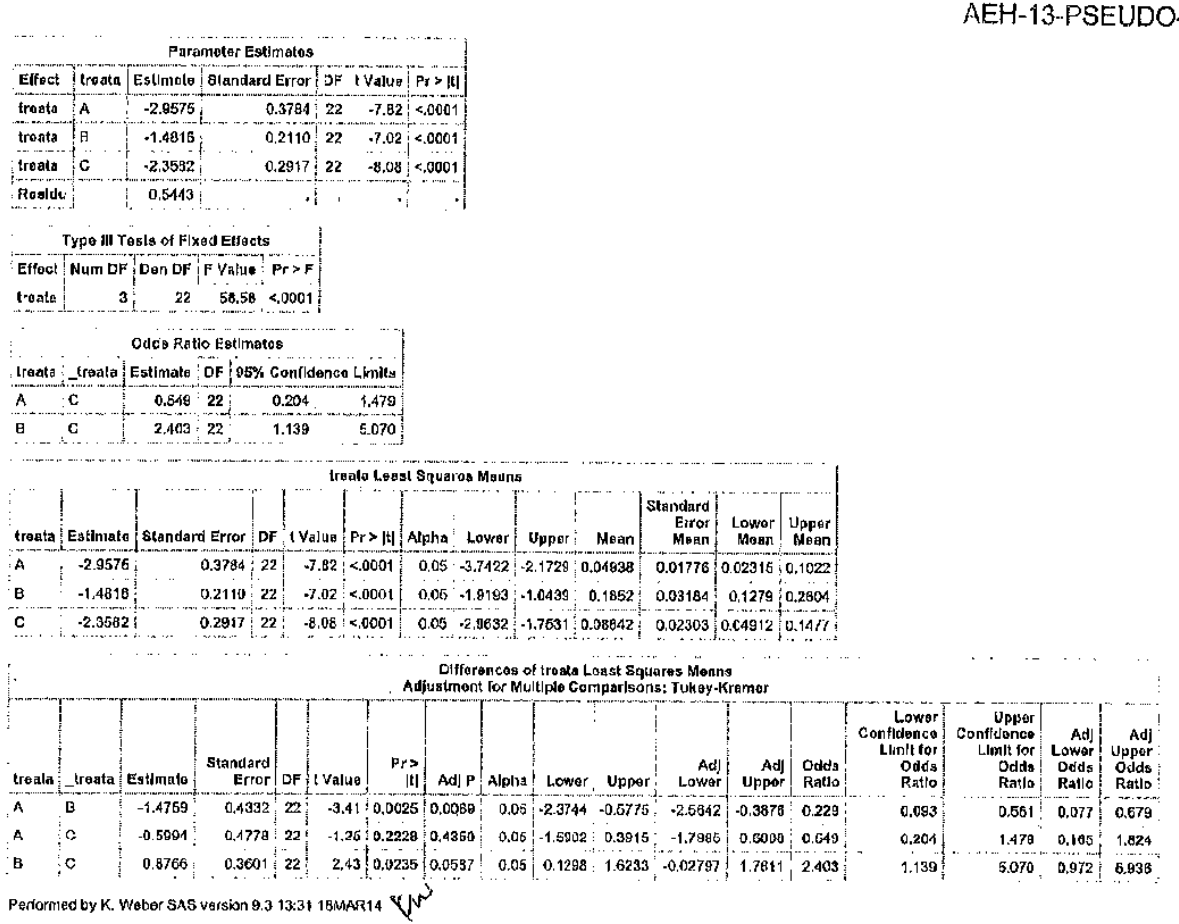

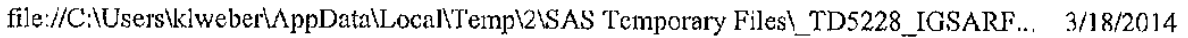


AEH-13-PSEUDO-05

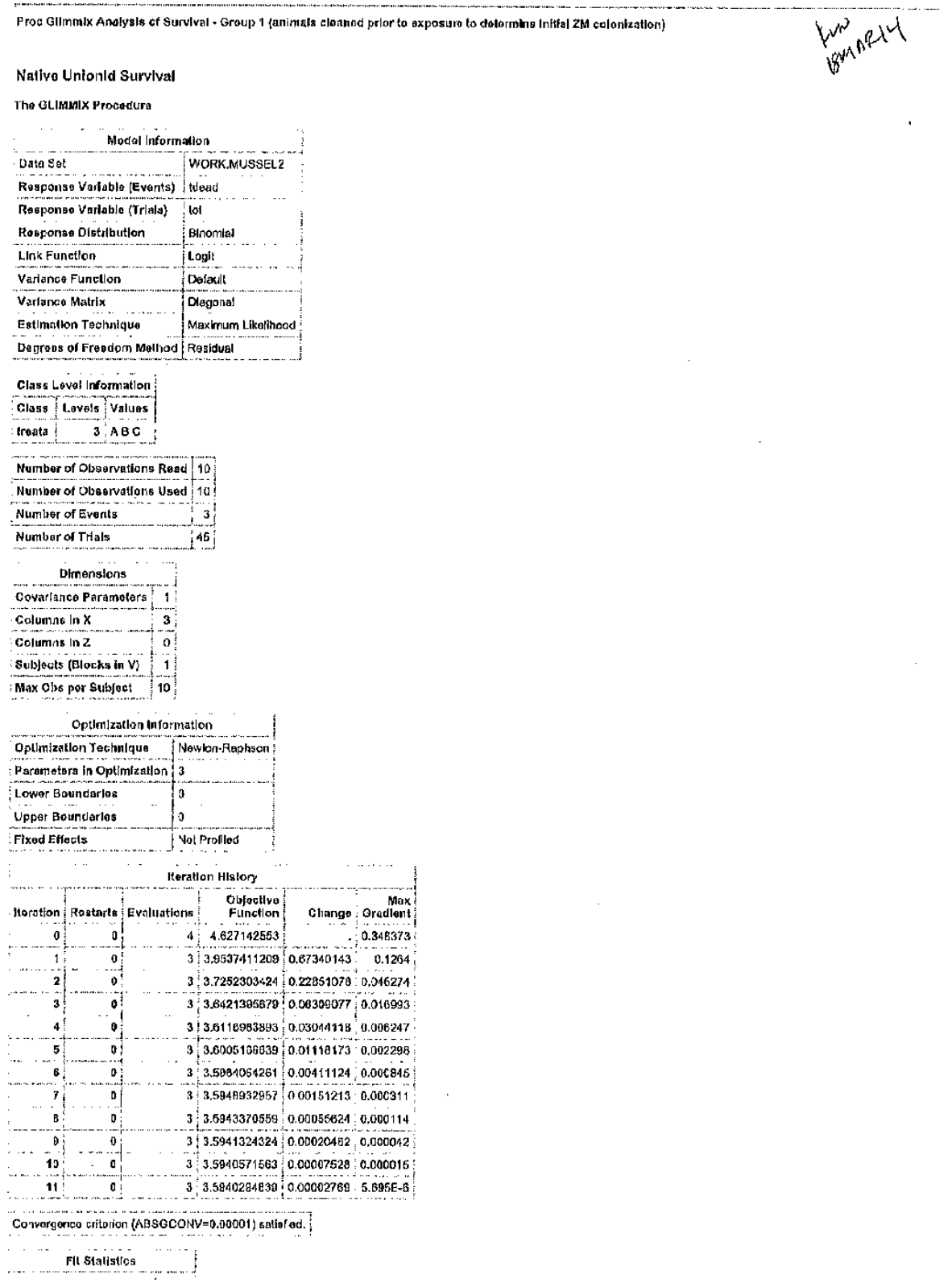

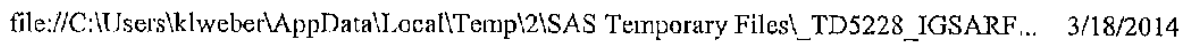




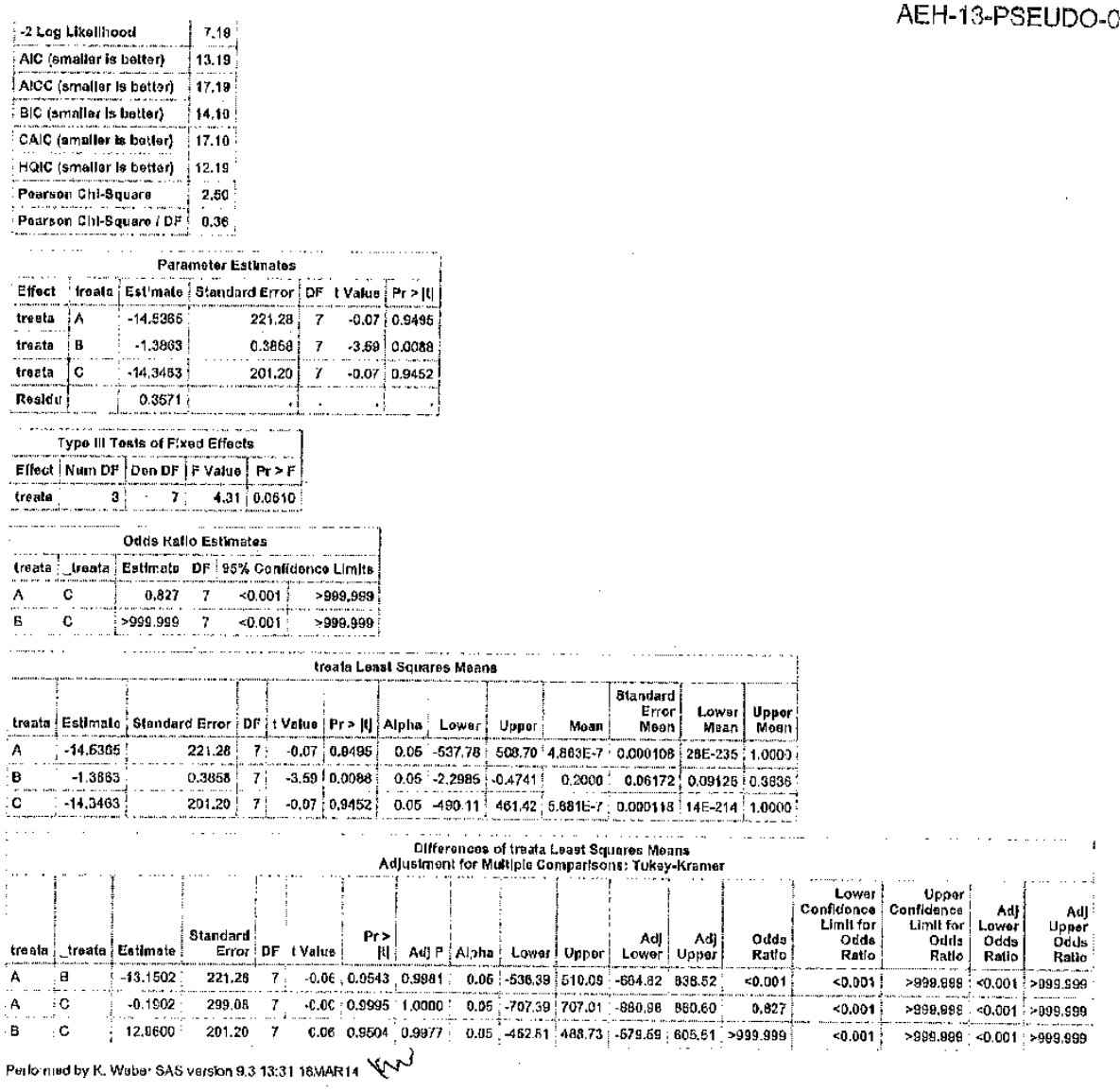

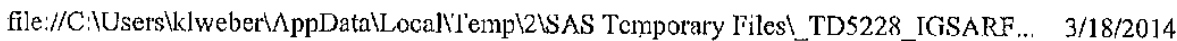


AEH-13-PSEUDO-05

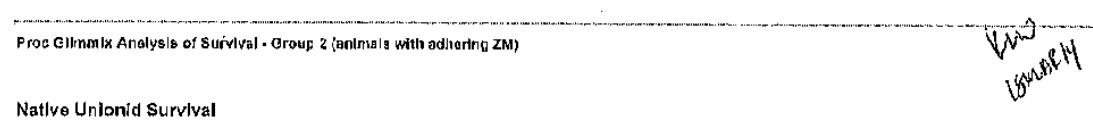

The GLIMMIX Procedure

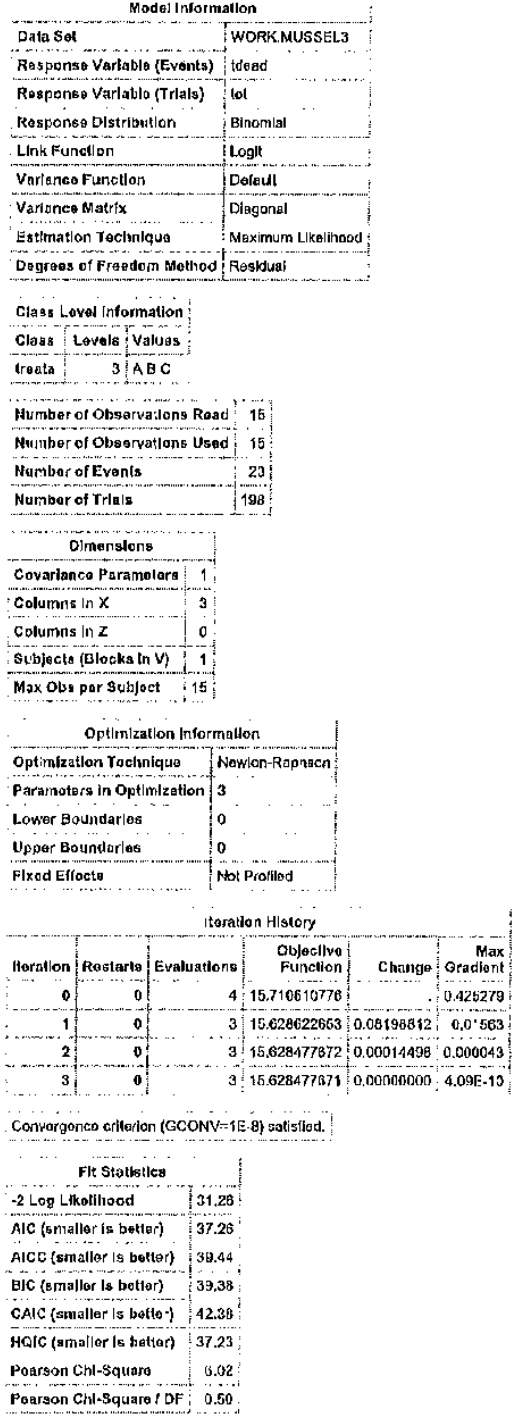

file://C:UUsersiklweberLAppDatalLocaltTempl2LSAS Temporary Files!_TDS228_IGSARF... 3/18/2014 


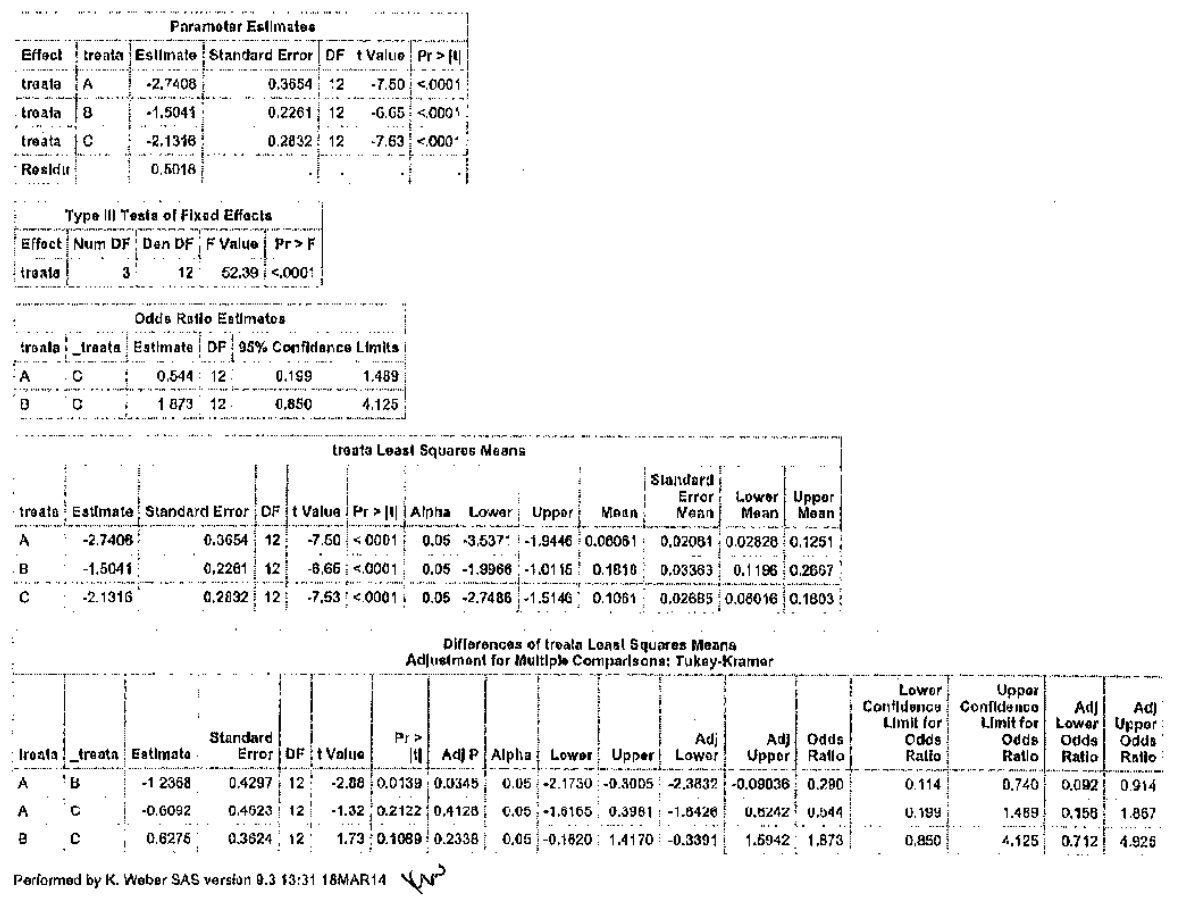

File Folder: 16

Item Number:

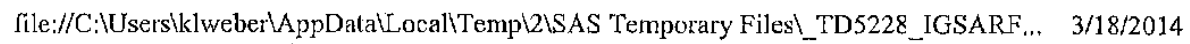


dM 'Log; clear; output; clear;'; * clear log and outPut;

FOOTNOTE1 'Performed by K. Weber SAS version ' \&SYSVER \&SYSTIME \&SYSDATE;

options $1 s=97$ ps=54 formdlim=' - ' pageno $=1$ nocenter nodate nosource 2 ;

title1 h=2 'Efficacy of Pseudomonas fluorescens (Pf-CL145A) SDP';

title2 $h=2$ 'for contro'ling zebra mussels within field enclosures';

title3 $h=2$ 'Unionid Mussel Survival';

title4 h=1.5'Study Nun'ber AEH-13-PSEUDO-05';

title5 $h=1$ 'SAS $\vee .9 .3$ Analysis completion date: 18MAR14 Analysis prepared

l********************************************************************************

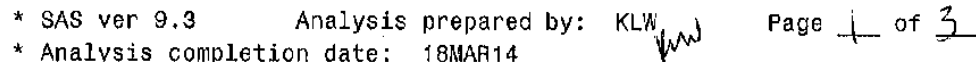

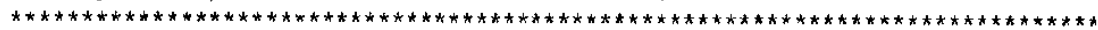

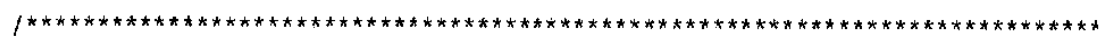

* Variable Names:

* enc $=$ enclosure ID (1 through 9)

* treat $=$ treatment level; $0=$ Control $[0 \mathrm{mg} / \mathrm{L}], 50=50 \mathrm{mg} / \mathrm{L}, 100=100 \mathrm{mg} / \mathrm{L}$

* group $=$ zebra mussel asssessment time

* $\quad 1=$ pre-exposure (i.e., zebra mussels from 45 unionid mussels used

* $\quad 2=$ post-exposure

* species $=$ unionid mussel species

* $\quad$ FAM $=$ Fatmucket (Lampsilis siliquoidoa)

* $\quad$ FPS $=$ Fragi.l papershell 1 (Leptodea fragilis)

* $\quad$ UNK $=$ Unknown (i.e., mussel unrecovered)

* tot $=$ total number of unionid mussels

* dead = rumber of dead unionid mussels at post-exposure assessment

* unrec $=$ number of inrecovered unionid mussel.s from the post-exposure assessn

* tdead $=$ number of dead unionids + number of unrecovered unionid mussels

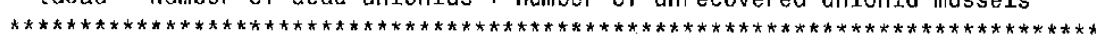

data mussel; set Pseudoos.Unionid_survival;

if tot $=0$ then delete;

petsurv $=($ tot - tdead $) /$ tot $*\{00$;

pctmort $=\mathrm{tdead} /$ tot $* 100$;

if treat $=0$ then treata $=$ ' $\mathrm{C}$ ';

if treat $=50$ then treata $={ }^{\prime} \mathrm{A}$;

If treat $=100$ then treata $={ }^{\prime} \mathrm{B}^{\prime}$;

run;

proc sort data=mussel; by species group treata; run;

FF \# ib

Item No. 3

proc print data=mussel; title4 $\mathrm{h}=1$ 'all data'; run;

$\mathrm{Pg} \perp$ of 3 
Title1 $\mathrm{h}=2$ 'Mean Survival by Species (sorted by treatment level and group)'; proc means data = mussel mean std $1 \mathrm{clm}$ uclm $\mathrm{fw}=8$;

by species;

class treata group;

var petsurv petmort;

run;

Title1 h=2 'Mean Survival by Species (sorted by treatment level)';

proc means data $=$ mussel mean $s t d ~ 1 c 1 m u c l m ~ f w=8$;

by species;

class treata;

var petsurv petmort;

run;

proc sort data=mussel; by treata group; run;

Title1 h=2 'Mean Survival by Treatment Level and Group (combined species)';

proc means data $=$ nussel mean std $101 m$ ucln $f w=8$;

by treata group;

var petsurv petmort;

run;

Title1 $h=2$ Mean Survival by Treatment (combined species and group)';

proc means data $=$ mussel mean std $101 n$ uclm $f_{w}=8$;

by treata;

var potsurv pctmort;

run:

Title1 $\mathrm{h}=2$ 'Proc Glimmix Analysis of Survival and Interactions (Group 1 and 2 proc glimmix data = mussel;

title4 'Native Unionid Survival';

c1.ass treata;

model tdead $/$ tot $=$ treata $/ d=$ bin link = logit noint $s$ or;

lsmeans treata/pdiff cl ilink or adjust=tukey;

random residual_;

run;

data mussel2; set mussel;

if group -2 then delete;

Title1 $h=2$ 'Proc Glimntix Analysis of Survival - Group 1 (animals cleaned prior proc glinmix data = mussel2;

title4 'Native Unionid Survival';

class treata:

model tdead/tot = treata $/ d=$ bin link = logit noint s or; lsmeans treata/pdiff ol ilink or adjust=tukey;

randon_residual_;

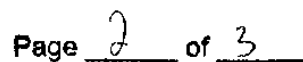


AEH-13-PSEUDO-05

run;

data mussel3; set mussel;

if group = 1 then delete;

Titlel $h=2$ 'Proc Glimmix Analysis of Survival - Group 2 (animals with adherinc proc glimmix data = mussel3;

title4 'Native Unionid Survival';

class treata;

model tdead $/$ tot $=$ treata $/ \mathrm{d}=$ bin link = logit noint $\mathrm{s}$ or;

lsmeans troata/pdiff cl ilink or adjust=tukey;

random _residual_;

run;

quit;

FF \# 16

ttem No. 2

$\mathrm{Pg} 3$ of 3 


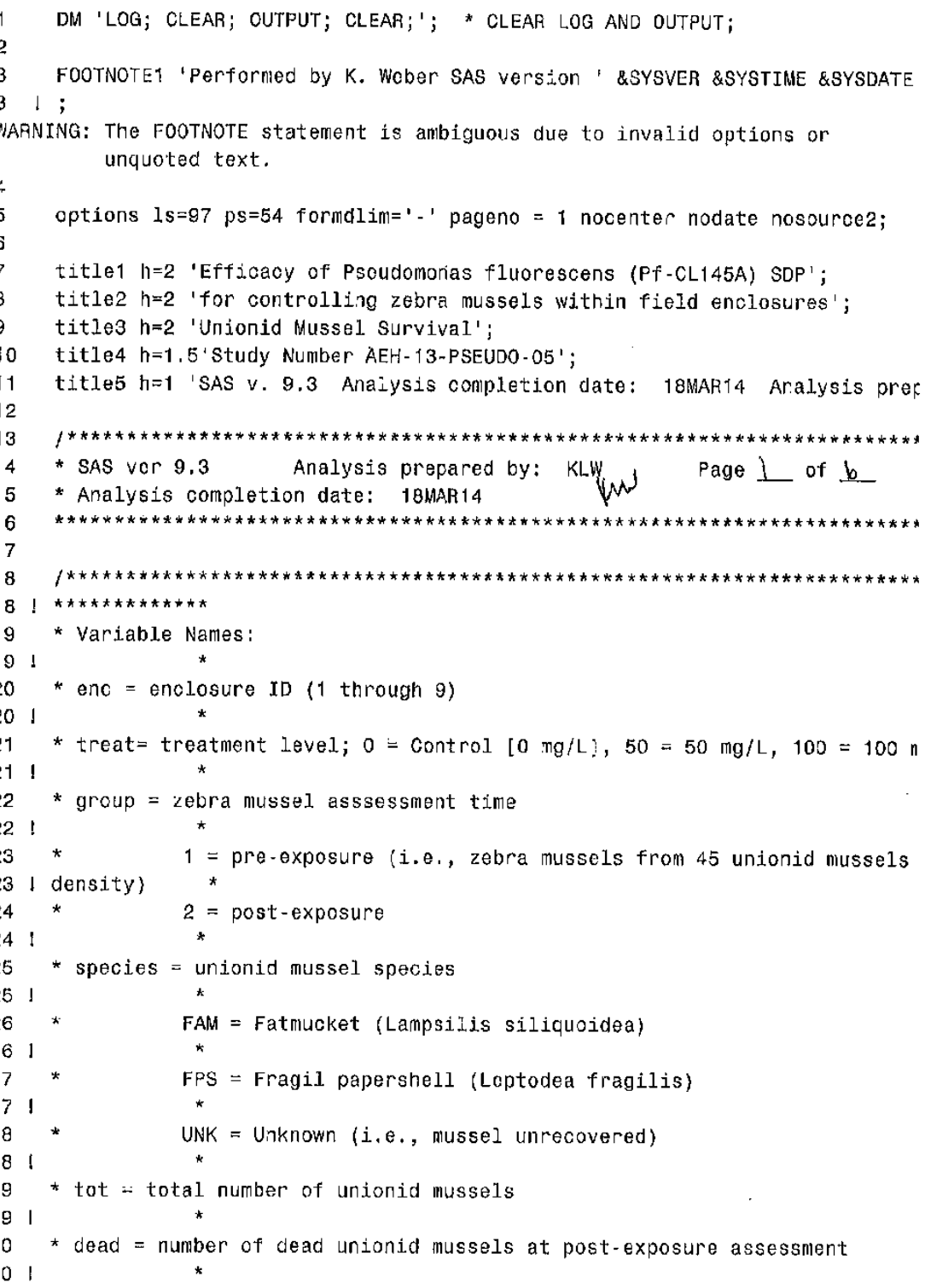




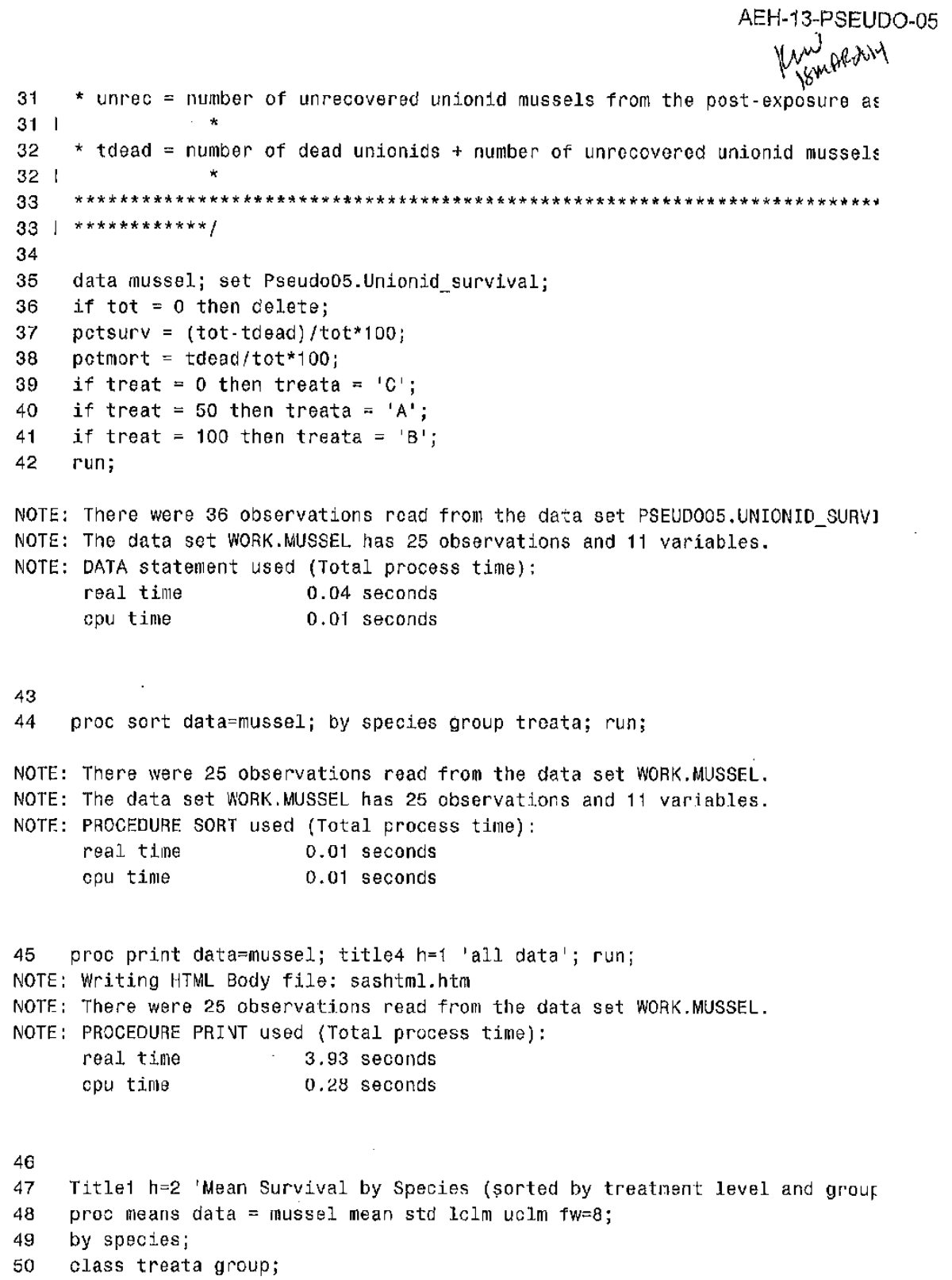

Page 2 of 6 
AEH-13-PSEUDO-05

51 var pctsurv potmort;

52 run;

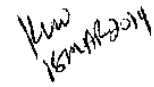

NOTE: There were 25 observatiors read from the data set WORK. MUSSEL.

NOTE: PROCEDURE MEANS used (Total process time):

$\begin{array}{ll}\text { real time } & 0.12 \text { seconds } \\ \text { cpu time } & 0.07 \text { seconds }\end{array}$

Titlel $h=2$ 'Mean Survival by Species (sorted by treatment level)'; proc means data = mussel mean std lcim uc.lm fw $=8$;

by species;

class treata;

var potsurv potmort;

run;

NOTE: There were 25 observations read from the data set WORK. MUSSEL

NOTE: PROCEDURE MEANS used (Total process time):
real time
0.10 seconds

cpu time

0.06 seconds

60

61 proc sort data=mussel; by treata group; run;

NOTE: There were 25 observations read from the data set WORK.MUSSEI..

NOTE: The data set WORK.MUSSEL has 25 observations and 11 variables.

NOTE: PROCEDURE SORT used (Total process time):
real time
0.00 seconds

cpu ;ime

0.00 seconds

62 Title1 $\mathrm{h}=2$ 'Mean Survival by Treatment Level, and Group (combined species)

63 proc means data = nussel mean std lclm uclr fw=8;

64 by treata group;

65 var petsurv petmort;

66 run;

NOTE: There vere 25 observations read from the data set WORK. NUSSEL

NOTE: PROCEDURE MEANS used (Total process time):
real time
0.10 seconds
cpu tine
0.06 seconds

67

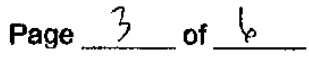




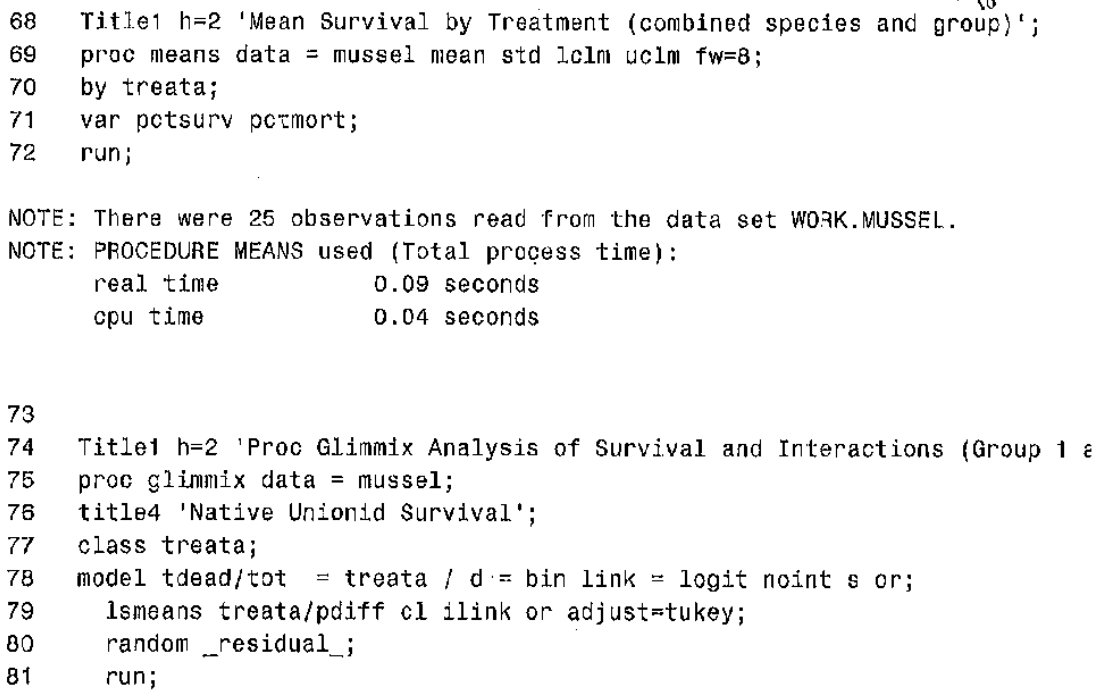

NOTE: The nodel does not contain an intorcept. Columns of $X$ are scaled only ar NOTE: Convergence oriterion ( $G C O N V=1 E-8$ ) satisfied.

NOTE: PROCEDURE GLIMMIX used (Total process time):
real time
0.29 seconds
cpu time
0.20 seconds

82

data mussol2; set mussel;

if group $=2$ then delete:

Title1 h=2 'Proc Glinmix Analysis of Survival - Group 1 (animals cleaned

86 ( to determine initial $\mathrm{ZM}$ colonization)';

NOTE: There were 25 observations read from the data set WORK. MUSSEL.

NOTE: Tho data set WORK.MUSSEL2 has 10 observations and 11 variables.

NOTE: DATA statement used (Total process time):
real time
0.01 seconds
cpu time
0.01 seconds

Page 4 of 6 
ргос glimmix data = mussel2;

title4 'Native Unionid Survival';

AEH-13-PSEUDO-05

class treata

model tdead/tot = treata $/ \mathrm{d}=$ bin link = logit noint $\mathrm{s}$ or;

lsmeans treata/pdiff cl ilink or adjust=tukey;

random _residual_;

run;

NOTE: The model doos not contain an intercept. Columns of $X$ are scaled onIy ar NOTE: Convergence criterion (ABSGCONV=0.00001) satisfied.

NOTE: PROCEOURE GLIMMIX used (Total process time):
real time
0.26 seconds

cpu time

0.15 seconds

94
95
96
97
98

data mussel3; set mussel;

if group $=1$ then delete;

Title1 $h=2$ 'Proc Glimmix Analysis of Survival - Group 2 (animals with adr

NOTE: There vere 25 observations read from the data set WORK.MUSSEL,

NOTE: The data set WORK.MUSSEL 3 has 15 observations and 11 variables.

NOTE: DATA statement used (Total process time):

real time $\quad 0.01$ seconds

cpu time $\quad 0.01$ seconds

99 proc glimmix data = musse I3;

100 title4 'Native Lnionid Survival';

101 class treata;

102 model tdead $/$ tot $=$ treata $/ \mathrm{d}=$ bin link = logit noint $s$ or;

103 lsmeans treata/pdiff cl ilink or adjust=tukey;

104 random residual_;

105 run;

NOTE: The model does not contain an intercept, Columns of $x$ are scaled only ar NOTE: Convergence criterion (GCONV=1E-8) satisfied.

NOTE: PROCEDURE GL.TMMIX used (Total process time):
real time
0.29 seconds

Page 5 of 6 
opu time

0.20 seconds

106 quit;
AEH-13-PSEUDO-05

wivis
FF \# 16

Item No. $\frac{3}{\mathrm{Pg}_{\mathrm{L}} \mathrm{b}}$ 
Efficacy of Pseudomonas fluorescens (Pf-CL145A) SDP

for controlling zebra mussels within field enclosures

Zebra Mussel Colonization Changes

SAS v. 9.3 Analysis completion date: 25MAR14 Analysis prepared by: KLW

Obs enc treat time density

\begin{tabular}{|r|r|r|r|r|}
\hline 1 & 3 & 0 & 1 & 182 \\
\hline 2 & 3 & 0 & 2 & 182 \\
\hline 3 & 7 & 0 & 1 & 155 \\
\hline 4 & 7 & 0 & 2 & 169 \\
\hline 5 & 8 & 0 & 1 & 157 \\
\hline 6 & 8 & 0 & 2 & 185 \\
\hline 7 & 2 & 50 & 1 & 147 \\
\hline 8 & 2 & 50 & 2 & 79 \\
\hline 9 & 4 & 50 & 1 & 167 \\
\hline 10 & 4 & 50 & 2 & 64 \\
\hline 11 & 5 & 50 & 1 & 166 \\
\hline 12 & 5 & 50 & 2 & 79 \\
\hline 13 & 1 & 100 & 1 & 195 \\
\hline 14 & 1 & 100 & 2 & 85 \\
\hline 15 & 6 & 100 & 1 & 174 \\
\hline 16 & 6 & 100 & 2 & 42 \\
\hline 17 & 9 & 100 & 1 & 172 \\
\hline 18 & 9 & 100 & 2 & 51 \\
\hline & & &
\end{tabular}

Performed by K. Weber SAS version 9.3 08:43 25MAR14 ( $w$ )

File Folder: 16

Item Number: 4 


\begin{tabular}{|c|c|c|c|}
\hline \multicolumn{4}{|c|}{$\begin{array}{l}\text { ZM Density: } \\
\text { Expressed as \#ZM per unionid; pre- and post-exposure } \\
\text { Mean Density on all unionids/test enclosure/sampling event } \\
\text { Study Number AEH-13-PSEUDO-05 }\end{array}$} \\
\hline \multicolumn{4}{|c|}{$\begin{array}{l}\text { The UNIVARIATE Procedure } \\
\text { Variable: density (density) } \\
\text { treat }=0 \\
\text { time }=1\end{array}$} \\
\hline \multicolumn{4}{|c|}{ Moments } \\
\hline$N$ & 3 & Sum Weights & 3 \\
\hline Mean & 164.666667 & Sum Observations & 494 \\
\hline Std Deviation & 15.0443788 & Variance & 226.333333 \\
\hline Skowness & 1,69767732 & Kurtosis & \\
\hline Uncorrected SS & 81798 & Corrected SS & 452.666667 \\
\hline Coeft Variation & 9.13626243 & Std Error Mean & 8.68587615 \\
\hline
\end{tabular}

\begin{tabular}{|c|c|c|c|}
\hline \multicolumn{4}{|c|}{ Basic Statistical Measures } \\
\hline \multicolumn{2}{|c|}{ Location } & \multicolumn{2}{|l|}{ Variability } \\
\hline Mean & 164.6667 & Std Deviation & 15.04438 \\
\hline Median & 157.0000 & Variance & 226.33333 \\
\hline \multirow[t]{2}{*}{ Mode } & & Range & 27,00000 \\
\hline & & Interquartile Range & 27.00000 \\
\hline
\end{tabular}

\begin{tabular}{|c|c|c|c|c|c|c|}
\hline \multicolumn{6}{|c|}{ Tests for Location: Mu0 $=0$} & \\
\hline \multirow{2}{*}{$\begin{array}{l}\text { Test } \\
\text { Student's } t\end{array}$} & \multicolumn{3}{|c|}{ Statistic } & \multicolumn{2}{|c|}{ p Value } & \\
\hline & $t$ & \multicolumn{2}{|c|}{18.95798} & $\mathrm{Pr}>|t|$ & \multicolumn{2}{|l|}{0.0028} \\
\hline Sign & $\mathbf{M}$ & \multicolumn{2}{|r|}{1.5} & $\operatorname{Pr}>=|M|$ & 0.2500 & \\
\hline Signed Rank & $\mathbf{S}$ & \multicolumn{2}{|r|}{3} & $\operatorname{Pr}>=|\mathbf{S}|$ & 0.2500 & \\
\hline \multicolumn{7}{|c|}{ Tests for Normality } \\
\hline \multicolumn{3}{|l|}{ Test } & \multicolumn{2}{|c|}{ Statistic } & \multicolumn{2}{|c|}{ p Value } \\
\hline \multicolumn{3}{|l|}{ Shapiro-Wilk } & $w$ & 0.805228 & $8 \quad \operatorname{Pr}<W$ & 0.1270 \\
\hline \multicolumn{3}{|c|}{ Kolmogorov-Smirnov } & $D$ & 0.361502 & $2 \mathrm{Pr}>\mathrm{D}$ & 0.1217 \\
\hline \multicolumn{3}{|c|}{ Cramer-von Mises } & W-Sq & 0.076265 & $5 \quad \mathrm{Pr}>\mathrm{W}-\mathrm{Sq}$ & 0.1619 \\
\hline \multicolumn{3}{|c|}{ Anderson-Darling } & A-Sq & 0.418086 & $6 \mathrm{Pr}>\mathrm{A}-\mathrm{Sq}$ & 0.1028 \\
\hline \multicolumn{7}{|c|}{ Quantiles (Definition 5) } \\
\hline
\end{tabular}




\begin{tabular}{|l|r|}
\hline Quantile & Estimate \\
\hline $100 \%$ Max & 182 \\
\hline $99 \%$ & 182 \\
\hline $95 \%$ & 182 \\
$90 \%$ & 182 \\
\hline $75 \%$ Q3 & 182 \\
\hline $50 \%$ Median & 157 \\
\hline $25 \%$ Q1 & 155 \\
\hline $10 \%$ & 155 \\
\hline $5 \%$ & 155 \\
$1 \%$ & 155 \\
\hline $0 \%$ Min & 155 \\
\hline
\end{tabular}

Extreme Observations

Lowest Highest

Value Obs Value Obs

\begin{tabular}{llll|l}
155 & 2 & 155 & 2 \\
\hline & 157 &
\end{tabular}

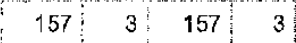

\begin{tabular}{llllll}
182 & 1 & 182 & 1 \\
\hline
\end{tabular}

Performed by K. Weber SAS version 9.3 08:43 25MAR14 $\mathrm{W}^{3}$ 
AEH-13-PSEUDO-05

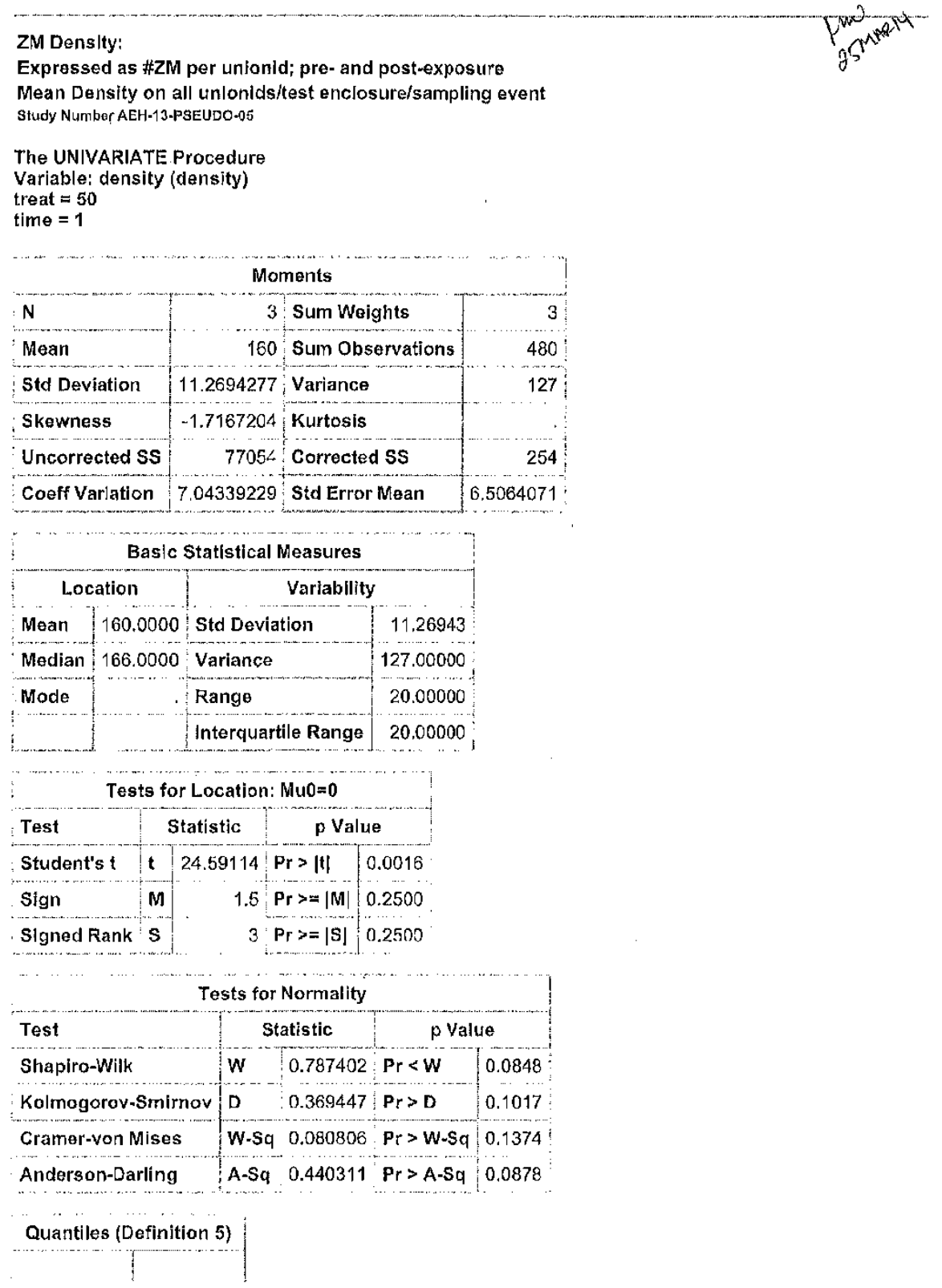




\begin{tabular}{|l|r|}
\hline Quantile & Estimate \\
\hline $100 \%$ Max & 167 \\
\hline $99 \%$ & 167 \\
\hline $95 \%$ & 167 \\
\hline $90 \%$ & 167 \\
\hline $75 \%$ Q3 & 167 \\
\hline $50 \%$ Median & 166 \\
\hline $25 \%$ Q1 & 147 \\
\hline $10 \%$ & 147 \\
\hline $5 \%$ & 147 \\
\hline $1 \%$ & 147 \\
\hline $0 \%$ Min & 147 \\
\hline
\end{tabular}

Extreme Observations

Lowest Highest

Value Obs Value Obs

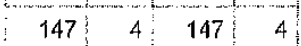

166 6 6166 6

\begin{tabular}{l|lll:l}
167 & 5 & 167 & 5
\end{tabular}

Porformed by K. Weber SAS version 9.3 08:43 25MAR14 $W^{W^{3}}$ 


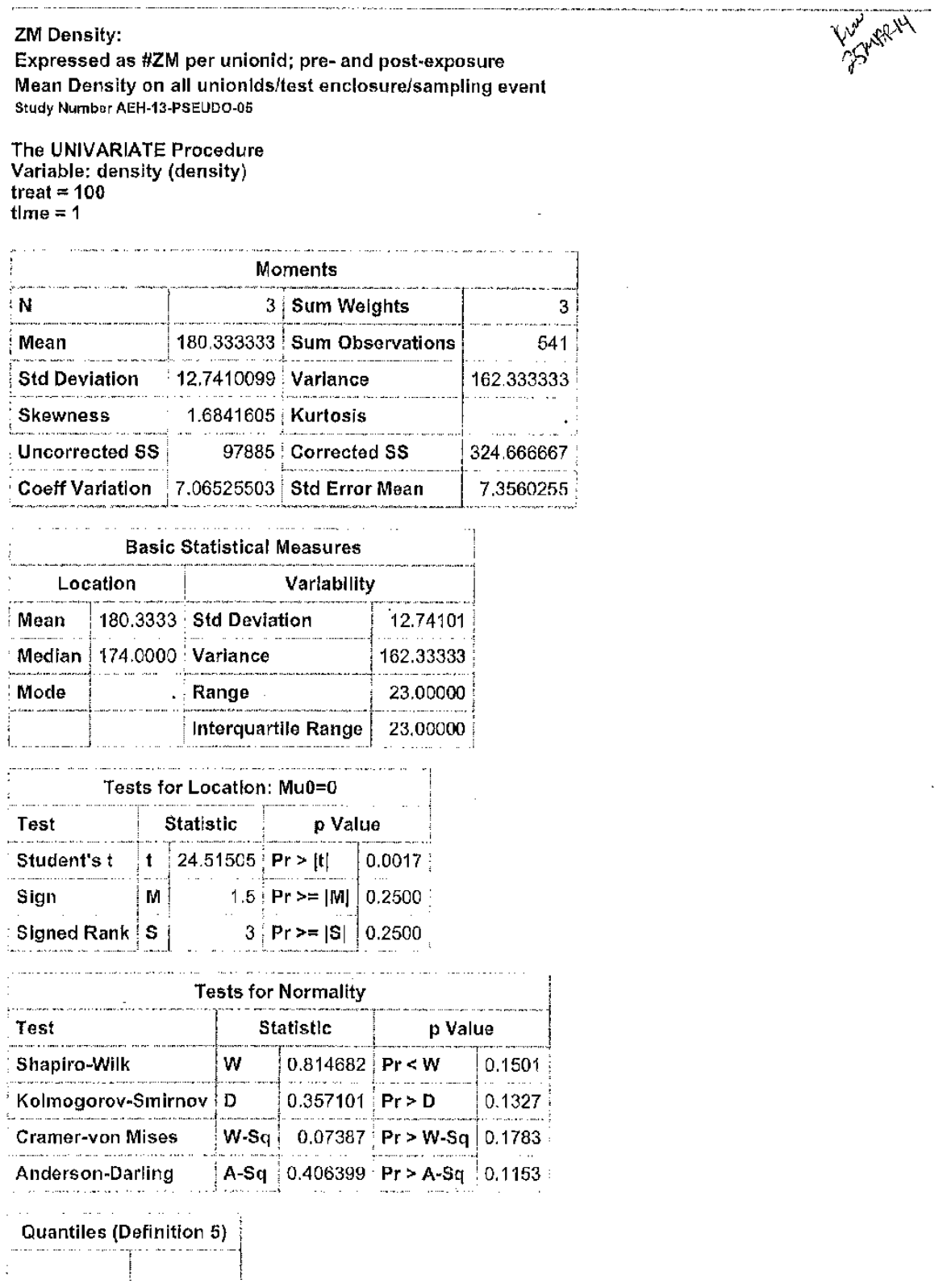




\begin{tabular}{|l|r|}
\hline Quantile & Estimate \\
\hline $100 \%$ Max & 195 \\
\hline $99 \%$ & 195 \\
\hline $95 \%$ & 195 \\
$90 \%$ & 195 \\
\hline $75 \%$ a3 & 195 \\
\hline $50 \%$ Median & 174 \\
$25 \%$ Q1 & 172 \\
\hline $10 \%$ & 172 \\
\hline $5 \%$ & 172 \\
\hline $1 \%$ & 172 \\
\hline $0 \%$ Min & 172 \\
\hline
\end{tabular}

\begin{tabular}{|c|c|c|c|}
\hline \multicolumn{4}{|c|}{ Extreme Observations } \\
\hline \multicolumn{2}{|c|}{ Lowest } & \multicolumn{2}{|c|}{ Highest } \\
\hline Value & Obs & Value & Obs \\
\hline 172 & $\theta$ & 172 & 9 \\
\hline 174 & 8 & 174 & 8 \\
\hline 195 & 7 & 195 & 7 \\
\hline
\end{tabular}

Jerformed by K. Weber SAS version 9.3 08:43 25MAR14 $w^{w^{3}}$ 


\section{ZM Density:}

Expressed as \#ZM per unionid; pre- and post-exposure

Mean Density on all unlonids/test enclosure/sampling event

Study Number AEH-13.PSEUDO-05

The UNIVARIATE Procedure

Q-Q Plot for density

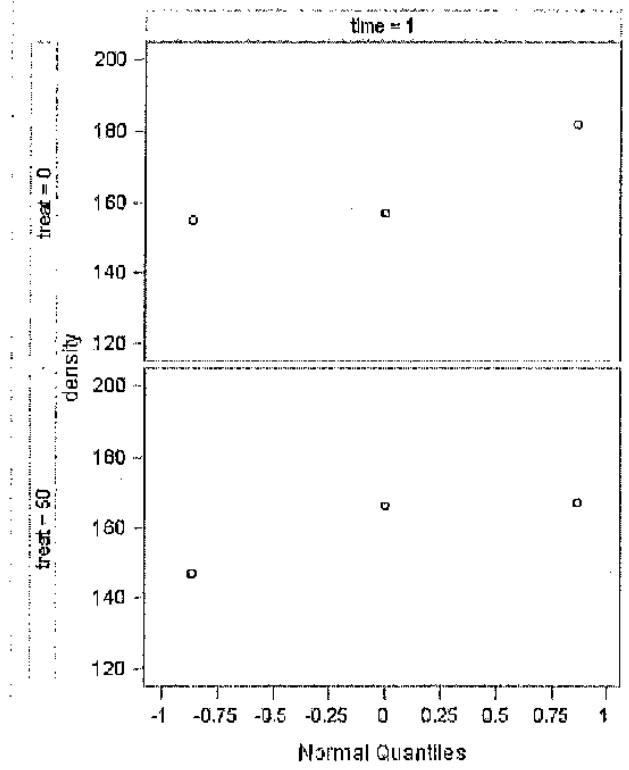




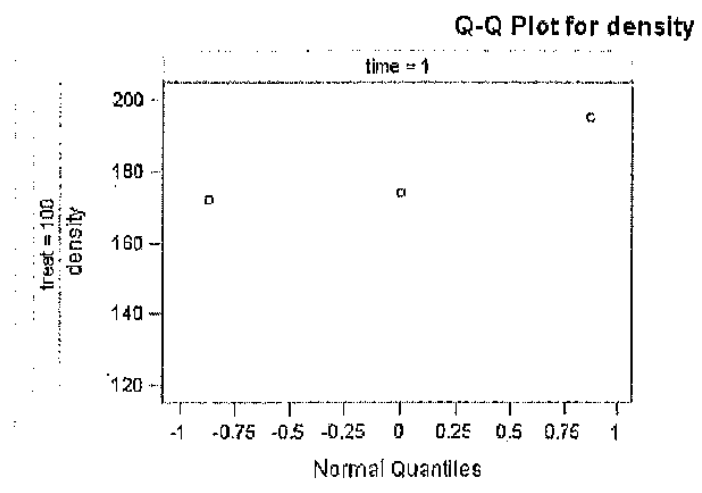

Performed by K. Weber SAS version 9,3 08:43 25MAR14 $W^{3}$ 

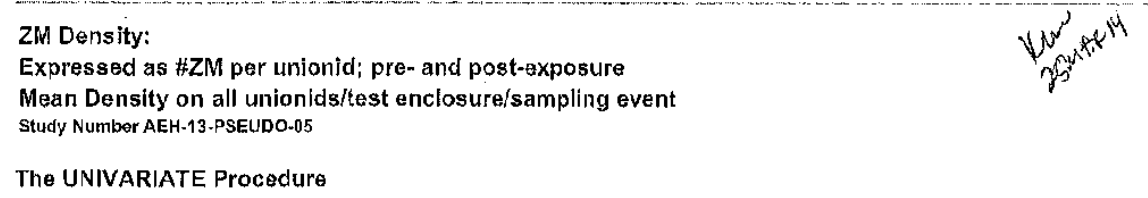

The UNIVARIATE Procedure

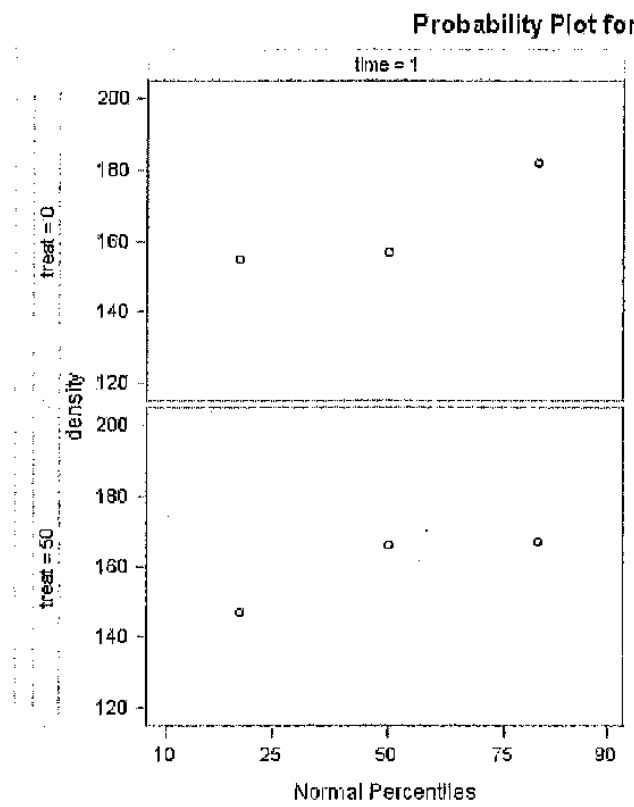


SAS Output

Page 11 of 23

AEH-13-PSEUDO-05

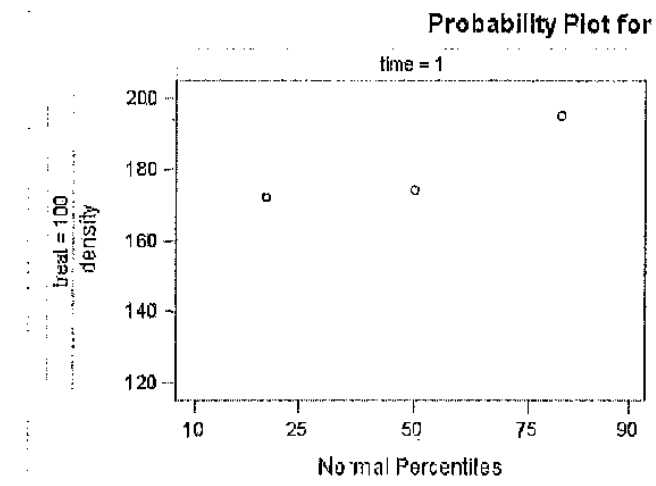

Perormed by K. Weber SAS version 9.3 08:43 25MAR14 W $\mathrm{W}^{3}$

file:/C:Usersiklweberisashtml.htm 


\section{AEH-13-PSEUDO-05}

\section{Density: Bartletts test of homogeneity of variance}

Expressed as \#ZM per unionid; pre- and post-exposure

Mean Density on all unionids/test enclosure/sampling event

Study Numbor AEH-13-PSEUDO-05

The GLM Procedure

\begin{tabular}{|l|l|}
\hline Class Level Information \\
\begin{tabular}{|c|r|r} 
Class & Levels & Values \\
\hline time & 2 & 12 \\
\hline
\end{tabular}
\end{tabular}

\begin{tabular}{ll|l} 
Number of Observations Read 18 \\
\hline
\end{tabular}

Number of Observations Used 18

Performed by K. Weber SAS version 9.3 08:43 25MAR14 $W^{w^{3}}$ 
AEH-13-PSEUDO-05

Density: Bartletts test of homogeneity of variance

Expressed as \#ZM per unionid; pre- and postexposure

Mean Density on all unionids/test enclosure/sampling event

Study Numbor AEH-13-PSEUDO-05

The GLM Procedure

Dependent Variable: density density

\begin{tabular}{|c|c|c|c|c|c|}
\hline Source & $\mathrm{DF}$ & Sum of Squares & Mean Square & F Valuo & $\mathrm{Pr}>\mathrm{F}$ \\
\hline Model & 1 & 18624.50000 & 18624.50000 & 10.48 & 0.0052 \\
\hline Error & 16 & 28446.00000 & 1777.87500 & & \\
\hline Corrected Total & 17 & 47070.50000 & & & \\
\hline
\end{tabular}

R-Square Coeff Var [Root MSE density Mean

\begin{tabular}{ll|l|r|r|r|}
0.395672 & 30.96562 & 42.16486 & 136.1667
\end{tabular}

\begin{tabular}{|l|r|r|r|r|r}
\hline Source & DF & Type I SS & Mean Square & $F$ Value & $P r>F$ \\
\hline time & $1: 18624.50000$ & 18624.50000 & 10.48 & 0.0052
\end{tabular}

\begin{tabular}{lll|l|l|l|l|l|l} 
Source & DF & Type III SS & Mean Square & $F$ Value & $P r>F$ \\
\hline
\end{tabular}

\begin{tabular}{|l|l|l|l|l|l|l|l|l}
\hline time & $1: 18624.50000$ & 18624.50000 & 10.48 & 0.0052
\end{tabular}


AEH-13-PSEUDO-05

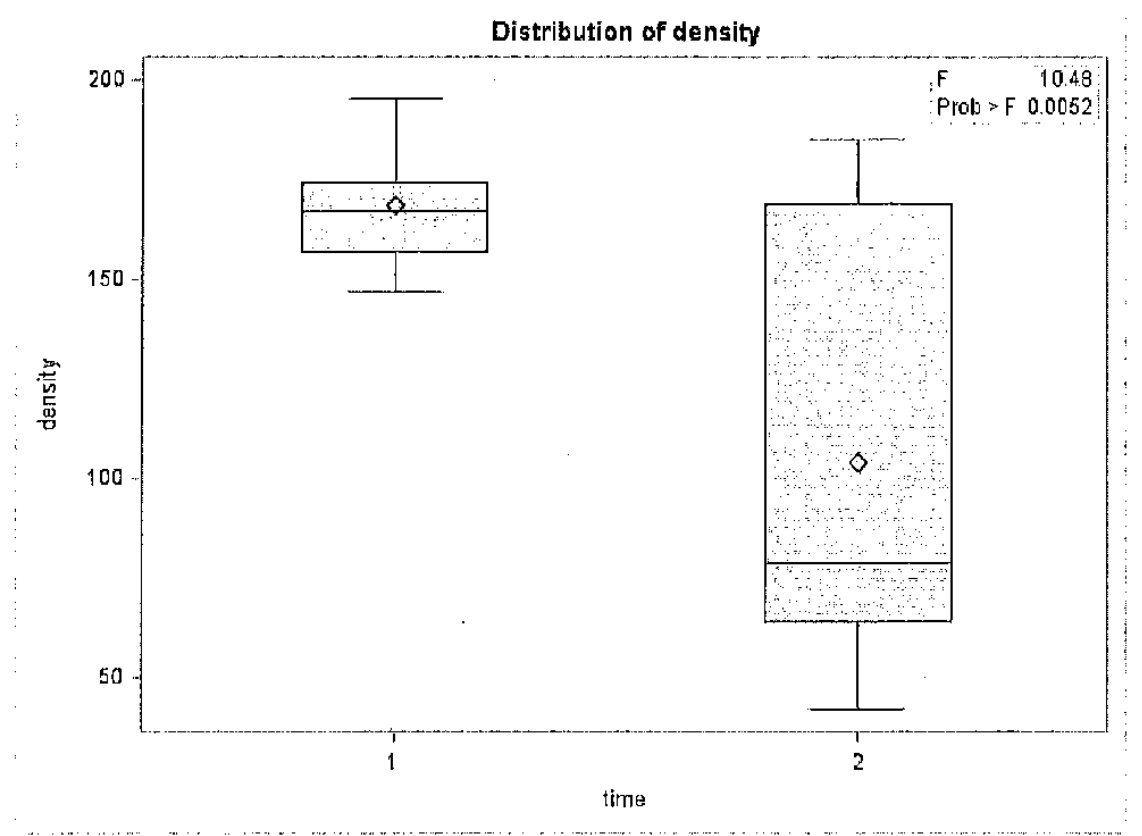

Performed by K. Weber SAS versiun 9.3 08:43 25MAR14 w 
AEH-13-PSEUDO-05

Density: Bartletts test of homogeneity of variance

Expressed as \#ZM per unionid; pre- and post-exposure

Mean Density on all unionids/test enclosure/sampling ovent

Study Number AEH-13.PSEUDO-05

The GLM Procedure

Bartlett's Test for Homogeneity of density Variance

Source DF Chi-Square $\mathrm{Pr}>\mathrm{Ch} / \mathrm{Sq}$

\begin{tabular}{|l|r|r|r|}
\hline time & 1 & 11.1896 & 0.0008
\end{tabular}

Performed by $\mathrm{K}$. Weber SAS version 9.3 08:43 25MAR14 $\mathrm{VN}^{\mathrm{N}}$ 
Density: Bartletts test of homogeneity of variance

Expressed as \#ZM per unionid; pre- and post-exposure

Mean Density on all unionids/test enclosure/sampling event

Study Number AEH-13-PSEUDO-05

The GLM Procedure

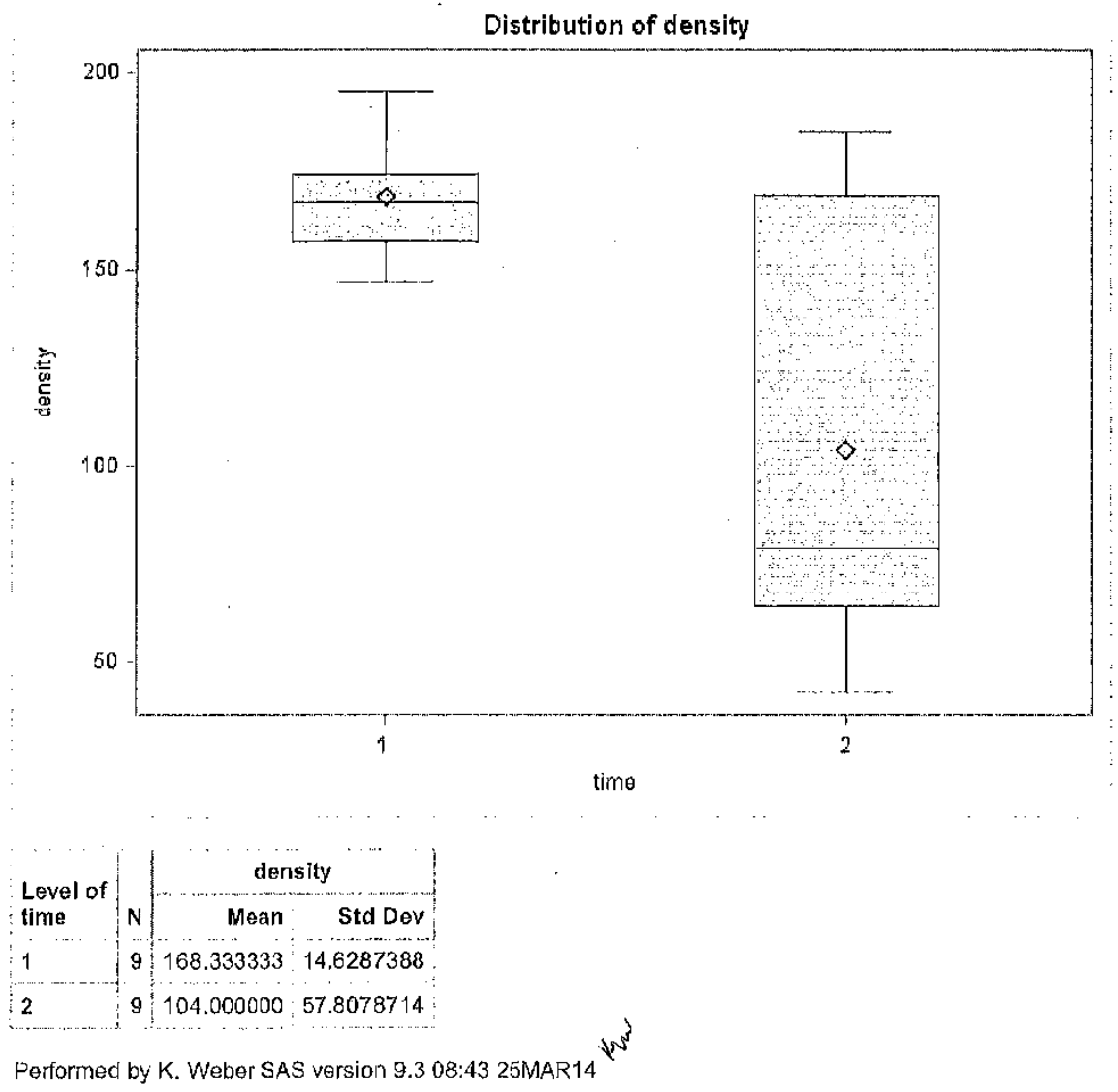

file://C:IUserstklwebertsashtml.htm 


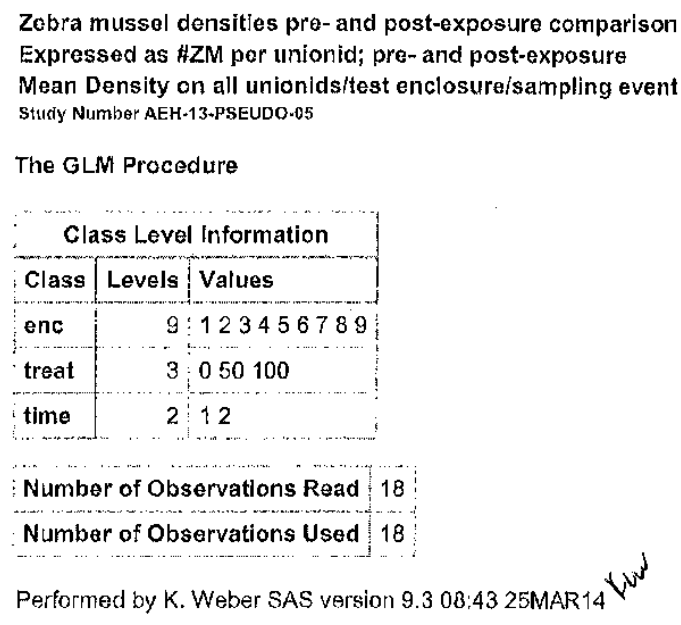


Zebra mussel dens|ties pre- and post-exposure comparison Expressed as \#ZM per unionid; pre- and post-exposure Mean Density on all unlonids/test enclosure/sampling event Study Number AEH-13.PSEUDO-05

\section{The GLM Procedure}

Dependent Variable: density density

\begin{tabular}{|l|r|r|r|r|}
\hline Source & DF & Sum of Squares & Mean Square & F Value \\
\hline Model & 6 & 378460.3333 & 63076.7222 & $321.46<.0001$ \\
\hline Error & 12 & 2354.6667 & 196.2222 & \\
\hline Uncorrected Total & 18 & 380815.0000 & & \\
\hline
\end{tabular}

\begin{tabular}{|c|c|c|c|}
\hline$R-S c$ & Coeff Var & Root MSE & density Mean \\
\hline & 10.2873 & 14.00793 & 1000 \\
\hline
\end{tabular}

\begin{tabular}{|c|c|c|c|c|c|}
\hline Source & $D F$ & Type I SS & Mean Square & F Value & $\operatorname{Pr}>\mathrm{F}$ \\
\hline treat & & 345110,8333 & 115036.9444 & 586.26 & $<, 0001$ \\
\hline time & 1 & 18624.5000 & 18624.5000 & 94.92 & $<, 0001$ \\
\hline treat*time & 2 & 14725.0000 & 7362.5000 & 37.52 & $<.0001$ \\
\hline Source & $D F$ & Type III SS & Mean Square & F Value & $\mathrm{Pr}>\mathrm{F}$ \\
\hline treat & 2 & 11366 & 5683.16667 & 28.96 & $<.0001$ \\
\hline $\operatorname{tim} \theta$ & 1 & 18624.50000 & 18624.50000 & 94.92 & $<.0001$ \\
\hline treat*time & 2 & 14725.00000 & 7362.50000 & 37.52 & $<.0001$ \\
\hline
\end{tabular}

\begin{tabular}{|c|c|c|c|c|c|}
\hline Parameter & Estimate & & Standard Error & t Value & $|\operatorname{Pr}>| t \mid$ \\
\hline treat 0 & 178.6666667 & $\mathrm{~B}$ & 8.08748462 & 22.09 & $<.0001$ \\
\hline treat 50 & 74.0000000 & $B$ & $8 . C 8748462$ & 9.15 & $<.0001$ \\
\hline treat 100 & 59.3333333 & $B$ & 8.08748462 & 7.34 & $<.0001$ \\
\hline time 1 & 121.0000000 & $B$ & 11.43743043 & 10.58 & $<.0001$ \\
\hline time 2 & 0.0000000 & $B$ & & & \\
\hline treat time 01 & -135.0000000 & $\mathrm{~B}$ & 16.17496923 & -8.35 & $<.0001$ \\
\hline treat & 0.0000000 & $B$ & & & \\
\hline treat*time 501 & -35.0000000 & $B$ & 16.17496923 & -2.16 & 0.0514 \\
\hline treat*time 502 & 0.0000000 & $B$ & & & \\
\hline treat*time 1001 & 0.0000000 & $B$ & & & \\
\hline ne 1002 & 0.0000000 & $B$ & & & \\
\hline
\end{tabular}


AEH-13-PSEUDO-05

Note: The $X^{\prime} X$ matrix has been found to be singular, and a generalized inverse was used to solve the normal equalions. Terms whose estimates are followed by the letter ' $B$ ' are not uniquely estimable.

Porformed by K. Wobor SAS version 9.3 08:43 25MAR14 W 
Zebra mussel densities pre- and post-exposuro comparison Expressed as \#ZM per unionld; pre- and post-exposure Mean Density on all unionids/test enclosure/sampling event Study Nurmber AEH-13-PSEUDO-05

\section{The GLM Procedure}

\begin{tabular}{|c|c|c|c|c|c|}
\hline Observation & Observed & Predicted & Residual & $95 \%$ Confidence Limi & an Predicted \\
\hline 1 & 82.0000000 & 164.6666667 & 17.3333333 & 147,0455514 & 182.2877819 \\
\hline $2 \vdots$ & 182.0000000 & 178.66666667 & 3.3333333 & 161.0455514 & 196.2877819 \\
\hline 3 & 155.0000000 & 164.6666667 & -9.6666667 & 147.0455514 & 182.2877819 \\
\hline 4 & 169.0000000 & 178.6666667 & -9.6666667 & 161.0455514 & 196.2877819 \\
\hline 5 & 157.0000000 & 164.6666667 & -7.6666667 & $147.04555 ; 4$ & 182.2877819 \\
\hline 6 & 185.0000000 & 178.6666667 & 6.3333333 & 161.0455514 & 196.2877819 \\
\hline 7 & 147.0000000 & 160.0000000 & -13.0000000 & 142.3788848 & 177.6211152 \\
\hline 8 & 79.0000000 & 74.0000000 & 5.0000000 & 56.3788848 & 81.6211152 \\
\hline 9 & 167,0000000 & 160.0000000 & 7.0000000 & 142.3788848 & 177.6211152 \\
\hline 10 & 64.0000000 & 74.0000000 & -10.0000000 & $56.37888<8$ & 91.6211152 \\
\hline 11 & 166.0000000 & 160.0000000 & 6.0000000 & 142,3788848 & 177.6211152 \\
\hline 12 & 79.0000000 & 74,0000000 & 5.0000000 & 56.3788848 & 91.6211152 \\
\hline 13 & 195.0000000 & 180.3333333 & 14.6666667 & 162.7122181 & 197.9544486 \\
\hline 14 & 85.0000000 & 59.3333333 & 25.6666667 & 41.7122181 & 76.9544486 \\
\hline 15 & 174.0000000 & 180.3333333 & -6.3333333 & 162.7122181 & 197.9544486 \\
\hline 16 & 42,0000000 & 59,3333333 & -17.3333333 & 41.7122181 & 76.9544486 \\
\hline 17 & 172,0000000 & 180.3333333 & -8.3333333 & 162.7122181 & 197.9544486 \\
\hline 18 & 51.0000000 & 59.3333333 & -8.3333333 & 41.7122181 & 76.9544486 \\
\hline
\end{tabular}

\begin{tabular}{l|r|}
\hline Sum of Residuals & -0.000000 \\
\hline Sum of Squared Residuals & 2354.666667 \\
\hline Sum of Squared Residuals - Error SS & -0.000000 \\
\hline PRESS Statistic & 5298.000000 \\
\hline First Order Autocorrelation & 0.230653 \\
\hline Durbin-Watson D & 1.381606 \\
\hline Performed by K. Weber SAS version 9.3 08:43 25MAR14 W
\end{tabular}


Zebra mussol censities pre- and post-exposure comparison Expressed as \#ZM per unionid; pre- and post-exposure Mean Density on all unjonids/test enclosure/sampling event Study Number AEH-13-PSEUDO-05

The GLM Procedure

Least Squares Means

Adjustment for Multiple Comparisons: Tukey

\begin{tabular}{|c|c|c|c|}
\hline treat & time & density LSMEAN & LSMEAN Number \\
\hline 0 & 1 & 164.666667 & 1 \\
\hline 0 & 2 & 178.666667 & 2 \\
\hdashline 50 & 1 & 160.000000 & 3 \\
\hline 50 & 2 & 74.000000 & 4 \\
\hline 100 & 1 & 180.333333 & 5 \\
\hline 100 & 2 & 59.333333 & 6 \\
\hline
\end{tabular}

\begin{tabular}{|c|c|c|c|c|c|c|}
\hline & \multicolumn{6}{|c|}{$\begin{array}{l}\text { Least Squares Means for effect treat*time } \\
\operatorname{Pr}>|t| \text { for H0: LSMean }(\hat{i})=L S M e a n(j) \\
\text { Dependent Variable: density }\end{array}$} \\
\hline$i / j$ & 1 & 2 & 3 & 4 & 5 & 6 \\
\hline 1 & & 0.8175 & 0.9982 & $<, 0001$ & 0.7432 & $<.0001$ \\
\hline 2 & 0.8175 & & 0.5950 & $<, 0001$ & 1.0000 & $<.0001$ \\
\hline 3 & 0.9982 & 0.5950 & & $<.0001$ & 0.5124 & $<.0001$ \\
\hline 4 & $<.0001$ & $<.0001$ & $<.0001$ & & $<.0001$ & 0.7889 \\
\hline 5 & 0.7432 & 1.0000 & 0.5124 & $<.0001$ & & $<.0001$ \\
\hline 6 & $<.0001$ & $<.0001$ & $<, 0001$ & 0.7889 & $<.0001$ & \\
\hline
\end{tabular}

\begin{tabular}{|l|l|l|l|l|}
\hline treat & time & density LSMEAN & $95 \%$ Confidence Limits \\
\hline 0 & 1 & 164.666667 & 147.045551 & 182.287782 \\
\hline 0 & 2 & 178.666667 & 161.045551 & 196.287782 \\
\hline 50 & 1 & 160.000000 & 142.378885 & 177.621115 \\
\hline 50 & 2 & 74.000000 & 56.378885 & 91.621115 \\
\hline 100 & 1 & 180.333333 & 162.712218 & 197.954449 \\
\hline 100 & 2 & 59.333333 & 41.712218 & 76.954449 \\
\hline
\end{tabular}

\begin{tabular}{|c|c|c|c|}
\hline & & ares & Means for Effect treat ${ }^{\star} t \mid m e$ \\
\hline i & $\mathrm{j}$ & $\begin{array}{r}\text { Difference Between } \\
\text { Means }\end{array}$ & $\begin{array}{l}\text { Simultaneous } 95 \% \text { Confidence Limits } \\
\text { for LSMean(i) } \text { LSMean(j) }\end{array}$ \\
\hline & 2 & -14.000000 & -52.417204 \\
\hline
\end{tabular}




\begin{tabular}{|r|r|r|r|r|}
\hline 1 & 3 & 4666667 & -33.750537 & 43.083870 \\
\hline 1 & 4 & 90.666667 & 52.249463 & 129.083870 \\
\hline 1 & 5 & -15.666667 & -54.083870 & 22.750537 \\
\hline 1 & 6 & 105.333333 & 66.916130 & 143.750537 \\
\hline 2 & 3 & 18.666667 & -19.750537 & 57.083870 \\
\hline 2 & 4 & 104.666667 & 66.249463 & 143.083870 \\
\hline 2 & 5 & -1.666667 & -40.083870 & 36.750537 \\
\hline 2 & 6 & 119.333333 & 80.916130 & 157.750537 \\
\hline 3 & 4 & 86.000000 & 47.582796 & 124.417204 \\
\hline 3 & 5 & -20.333333 & -58.750537 & 18.083870 \\
\hline 3 & 6 & 100.6666667 & 62.249463 & 139.083870 \\
\hline 4 & 5 & -106.333333 & -144.750537 & -67.916130 \\
\hline 4 & 6 & 14.666667 & -23.750537 & 53.083870 \\
\hline 5 & 6 & 121.000000 & 82.582796 & 159.417204 \\
\hline
\end{tabular}

Performed by $K$. Weber SAS version 9.3 08:43 25MAR14 $W^{3}$ 
Mean zebra mussel densities pre- and post-exposure by treatment level

Expressed as \#ZM per unionid; pre-and post-exposure

Mean Density on all unionlds/test enclosure/sampling event

Study Numbar AEH-13-PSEUD0-05

The MEANS Procedure

treat $=0$

\begin{tabular}{|c|c|c|c|c|c|}
\hline \multicolumn{6}{|c|}{ Analysis Variable : density density } \\
\hline time & N Obs & Mean & Std Dev & $\begin{array}{l}\text { Lower } 95 \% \\
\text { CL for Mean }\end{array}$ & $\begin{array}{r}\text { Upper } 95 \% \\
\text { CL for Mean }\end{array}$ \\
\hline 1 & 3 & 164.7 & 15.0444 & 127.3 & 202.0 \\
\hline 2 & 3 & 178.7 & 8.5049 & 157.5 & 199.8 \\
\hline
\end{tabular}

treat $=50$

\begin{tabular}{|c|c|c|c|c|c|}
\hline \multicolumn{6}{|c|}{ Analysis Variable : density density } \\
\hline timo & N Obs & Mean & Std Dev & $\begin{array}{l}\text { Lower } 95 \% \\
\text { CL for Mean }\end{array}$ & $\begin{array}{r}\text { Upper } 95 \% \\
\text { CL for Mean }\end{array}$ \\
\hline 1 & 3 & 160,0 & 11.2684 & 132,0 & 188.0 \\
\hline 2 & 3 & 74.0000 & 8.6603 & 52.4867 & 95.5133 \\
\hline
\end{tabular}

treat $=100$

\begin{tabular}{|c|c|c|c|c|c|}
\hline \multicolumn{6}{|c|}{ Analysis Variable : density density } \\
\hline time & N Obs & Mean & Std Dev & $\begin{array}{l}\text { Lower } 95 \% \\
\text { CL for Mean }\end{array}$ & $\begin{array}{r}\text { Upper } 95 \% \\
\text { CL for Mean }\end{array}$ \\
\hline 1 & 3 & 180.3 & 12.7410 & 148.7 & 212.0 \\
\hline 2 & 3 & 9.3333 & 22.6789 & 2.9958 & 115.7 \\
\hline
\end{tabular}

Performed by K. Wober SAS version 9.3 08:43 25MAR 14

File Folder: 16

Item Number: 
ods html clcse; /* close previous */;

ods html; $/ *$ open new */;

DM 'LOG; ClEAR; OUTPUT; CLEAR;'; * CLEAR LOG AND OUTPUT;

FOOTNOTE1 'Performed by $K$. Weber SAS version ' \&SYSVER \&SYSTIME \&SYSDATE;

options $1 \mathrm{~s}=97$ ps=54 formdlim='. ' pageno $=1$ nocenter nodate nosource2;

title1 $h=2$ 'Efficacy of Pseudomonas fluorescens (Pf - -CL145A) SDP';

title2 $h=2$ 'for controlling zebra mussels within field enclosures';

title3 $h=2$ 'Zebra Mussel Colonization Changes';

title4 $h=2$ 'SAS $\vee$. 9.3 Analysis completion date: 25 MAR14 Analysis prepared $k$

/

* SAS ver $9.3 \quad$ Analysis prepared by: KLW fw Page 1 of 2
* Analysis completion date: 25MAR2014

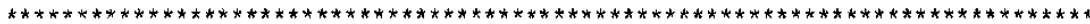

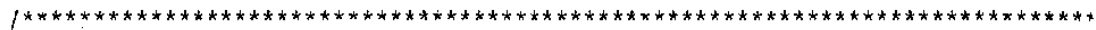

* Variable Names:

* enc = test enclosure ID (1 through 9)

* treat $=$ treatinent level; $0=$ Control $[0 \mathrm{mg} / \mathrm{L}], 50=50 \mathrm{mg} / \mathrm{L}, 100=100 \mathrm{mg} / \mathrm{L}$

* time = sample time; $1=$ pre-exposure; $2=$ post $-\theta x p o s u r e$

* denisty= density of live zebra mussels on the unionid (\# ZM/unionid)

*******************************************************************************

data mussel; set Pseudo05.Density_mean_num;

run;

proc sort;

by treat enc; run;

proc print; run;

data mussel2; set mussel;

if time $=2$ then delete;

proc univariate data=mussel2 normal notabcontents;

title1 $h=$ ?. ' $Z M$ Density: ';

title2 $h=2$ 'Expressed as \#ZM per unionid; pre- and post-exposure';

title3 $h=2$ 'Mean Density on all unionids/test enclosure/sampling event';

title4 $h=1.5$ 'Study Number AEH-13-PSEUDO-05';

class treat time;

var density;

probplot density.

qqplot density;

run;

FF\# 16

Item No. 5 
AEH-13-PSEUDO-05

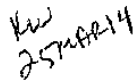

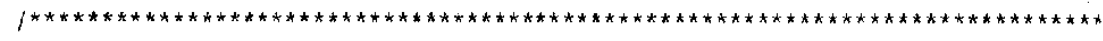

* This analysis determines the variability among treatments

ods graphics on;

proc glm data= mussel;

title1 $h=2$ Density: Bartletts test of homogeneity of variance';

title2 $h=2$ 'Expressed as \#ZM per unionid; pre- and post-exposure';

title3 $h=2$ 'Mean Dersity on all unionids/test enclosure/sampling event';

title4 $h=1.5$ 'Study Number AEH-13-PSEUDO-05';

class time;

model density=time;

moans time / HOVTEST=BARTLETT; run;

Ods graphics off;

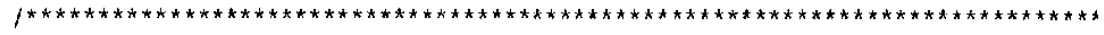

* This analysis compares the test enclosure densities by treatment and time

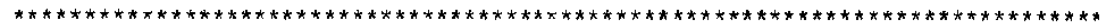

title1 $h=2$ 'Zebra mussel densities pre-and post-cxposuro comparison";

title2 $h=2$ 'Expressed as \#ZM per unionid; pre-and post-exposure';

title3 $h=2$ 'Mean Density on all unionids/test enclosure/sampling event';

title4 $h=4.5$ 'Study Number AEH-13-PSEUDO-05';

proc glm data = mussel;

class enc treat time;

model density $=$ treat $\mid$ time / noint solution cli clm;

lsmeans treat*time / pdiff=all cl;

run;

quit;

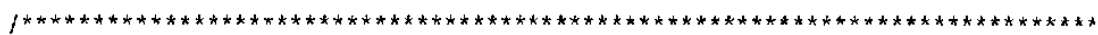

* This analysis determines the densities by treatment level pre- and post-expc

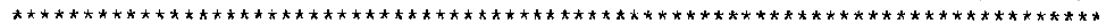

title1 $h=2$ 'Mean zebra mussel densities pre-and post-exposure by treatinent le title2 $h=2$ 'Expressed as HZM per unionid; pro-and post-exposure';

titlo3 $h=2$ 'Mean Density on all unionids/test enclosure/sampling event';

title4 $h=1.5$ 'Study Number AEll-13-PSEUD0-05';

proc means cata = mussel mean std $l \mathrm{clm}$ uclm $\mathrm{fw}_{\mathrm{w}}=8$;

by treat;

class time;

var density;

run;

FF \# W

Item No.

$\mathrm{Pg} 2 \overrightarrow{\text { of } 2}$ 
DM 'LOG; ClEAr; OUtPut; Clear;'; * CleAR LOG AND OUTPUT;

FOOTNOTE1 'Performed by K. Weber SAS version ' \&SYSVER \&SYSTIME \&SYSDATE ;

WARNING: The FOOTNOTE statement is ambiguous oue to invalid options or unquoted text.

options $1 s=97$ ps=54 formd $1 \mathrm{~lm}=\mathrm{I}^{\prime} \cdot$ pageno $=1$ nocenter nodate nosounce2;

titlc1 $h=2$ 'Efificacy of Pseudiomonas fluorescens (Pf-CL145A) SDP';

title2 $h=2$ 'for controlling zebra mussels within field enclosures';

title3 $h=2$ 'Zebra Mussel Colonization Changes';

title4 $h=2$ 'SAS v. 9.3 Analysis completion ciate: 25MAR14 Analysis prepe

/

* SAS ver 9.3 Analysis prepared by: KLW Page 1 of 4

* Analysis complotion date: 25MAR2014 WW

$* * * * * * * * * * * * * * * * * * * * * * * * * * * * * * * * * * * * * * * * * * * * * * * * * * * * * * * * * * * * * * * * * * * * * * * * * *$

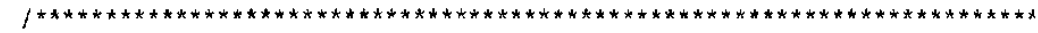

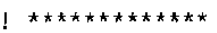

* Variable Names:

1

*. enc $=$ test enclosure ID (1 through 9$)$

!

* treat $=$ treatment level; $0=$ Control. $[0 \mathrm{mg} / \mathrm{L}], 50=50 \mathrm{mg} / \mathrm{L}, .100=100 \mathrm{n}$ !

* time $=$ sample time; 1 = pre-exposure; 2 = post-exposure

1

* denisty $=$ density of live zebra mussels on the unionid (\# ZM/unionid)

1

$|* * * * * * * * * * * *|$

data mussel; set Pseudo05.Density_mean_num;

run;

NOTE: There were 18 observations read fron the data set PSEUDO05.DENSITY_MEAN_ NOTE: The data set WORK.MUSSEL has 18 observations and 4 variables.

NOTE: DATA statement used (Total process time):

real time $\quad 0.03$ seconds

cpu time $\quad 0.03$ seconds

30 proc sort;

FF\# 业

Item Nó. 6

$\mathrm{Pg} \perp$ of 4 
31 by treat enc; run;

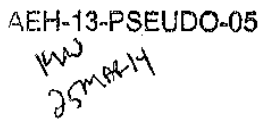

NOTE: There were 18 observations read from the data sct WORK. WUSSEL,

NOTE: The data sot WORK.MUSSEL has 18 observations and 4 variables.

NOTE: PROCEDURE SOR- used (Total process time):
real time
0.01 seconds
cpu time
0.01 seconds

32 proc print; run;

NOTE: There were 18 observations read from the data set WORK.MUSSEL.

NOTE: PROCEDURE PRINT used (Total process time):
real time
0.08 seconds
cpu tine
0.03 seconds

33

data musse12; set mussel;

if time $=2$ then delete;

NOTE: There were 18 observations read from the data set WORK. MUSSEL.

NOTE: The data set WORK.MUSSEL2 has 9 observations and 4 variables.

NOTE: DATA statement used (Total process time):
real time
0.01 seconds
cpu tine
0.00 seconds

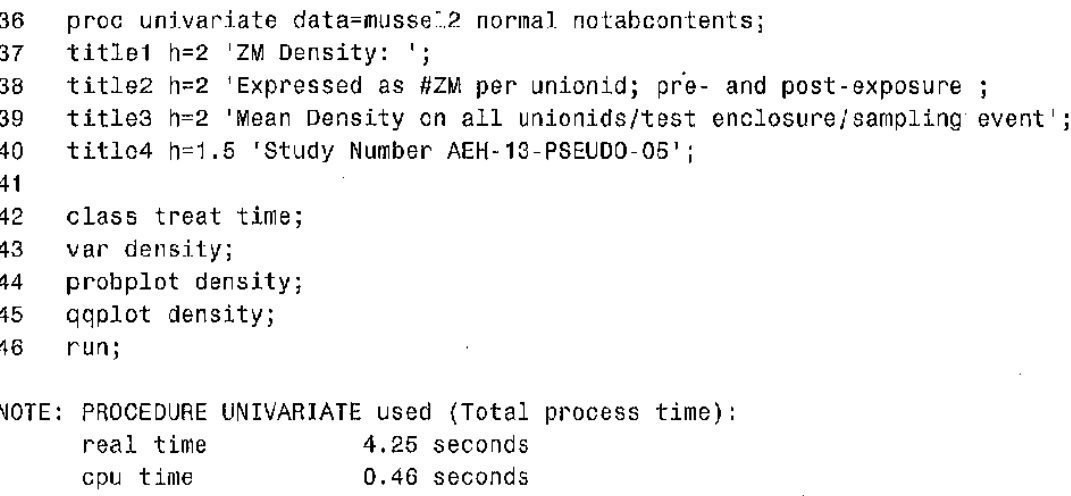


AEH-13-PSEUDO-05

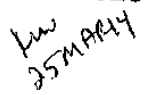

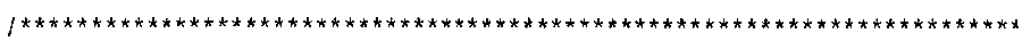

* This analysis determines the variability among treatments

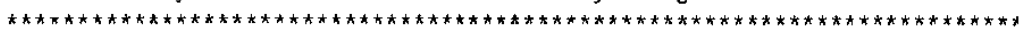

ods graphics on;

proc glm data= mussel;

tit_e1 $h=2$ 'Density: Bartletts test of homogeneity of variance';

title2 $h=2$. 'Expressed as $\# Z N$ per unionid; pre-and post-exposure;

title3 $h=2$ 'Mean Density on all unionids/test enclosune/sampling event';

title4 $\mathrm{h}=1.5$ 'Study Number AEH-13-PSEUDO-05';

class time;

model density=time;

means time / HOVTEST-BARTLETT; run;

ods graphics off;

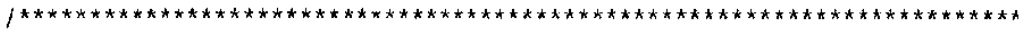

* This analysis compares the test enclosure densities by treatment and $t$

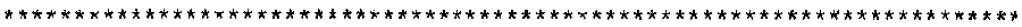

title1 $h=2$ "Zebra mussel densities pre-and post-exposure comparison";

title2 h=2 'Expressed as HZM per unionid; pre-and post-exposure';

title3 $h=2$ 'Mean Density on all unionids/test enclosure/sampling event';

title4 $h=1.5$ 'Study Number AEH-13-PSEUDO-05';

NOTE: PROCEDURE GLIM used (Total process $t$ ime):

real time 0.85 seconds

cpu time $\quad 0.26$ seconds

proc glm data = mussel;

class enc treat time;

nodel density = treat $/$ time / noint solution cli clm;

lsmeans treat*tine / pdiff=all cl;

run;

NOTE: Due to the presence of CLASS variables, an intorcopt is implicitly fitte been corrected for the mean.

76 quit;

NOTE: PROCEDURE GLM used (Total process time):

real time

0.33 seconds

cpu time

0.17 seconds

77

Page 3 of 4 


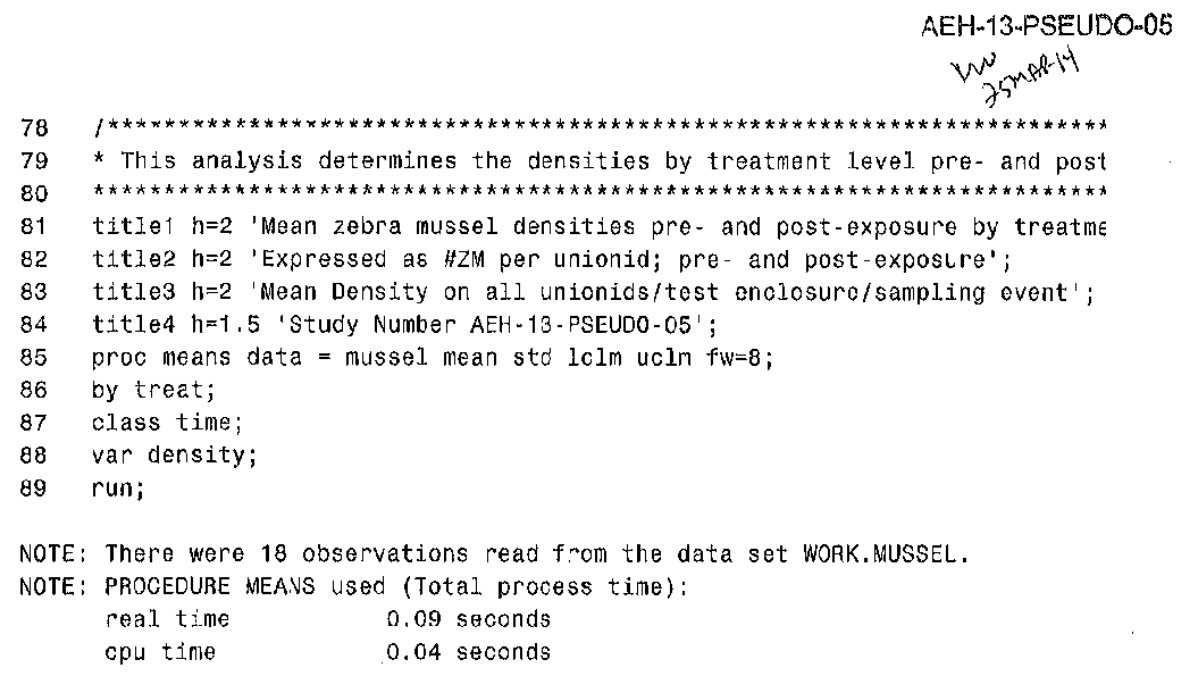

FF \# 16

Item Nó. 6

$\mathrm{Pg} 4$ of 4 
Efficacy of Pseudomonas fluorescens (Pf-CL145A) SDP

AEH-13-PSEUDO-05 for controlling zebra mussels within fiold enclosuros

Zebra Mussel Length Analysis

study Number AEH-13.PSEUDO-05

SAS v. 9.3 Analys $/ 8$ completion data: 18MAR14 Analysis propared by: KLW

Obs enc treat time length

$\begin{array}{llllllll}1 & 3 & 0 & 1 & 12.0407\end{array}$

$\begin{array}{lllllllll}2 & 3 & 0 & 2 & 12,5773\end{array}$

\begin{tabular}{l|l|l|l|l}
3 & 7 & 0 & 1 & 11.9452
\end{tabular}

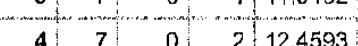

\begin{tabular}{l|l|l|lllll}
5 & 8 & 0 & 1 & 12.1144 \\
\hline
\end{tabular}

\begin{tabular}{ll|l|l|l|l|}
6 & 8 & 0 & 2 & 8.6073
\end{tabular}

\begin{tabular}{l|l|l|l|l}
7 & 2 & 50 & 1 & 12.6307
\end{tabular}

\begin{tabular}{lll|l|l|l|l}
8 & 2 & 50 & 2 & 11.6271
\end{tabular}

\begin{tabular}{l|l|l|l|l}
9 & 4 & 50 & 1 & 11.6470
\end{tabular}

\begin{tabular}{l|l|l|l|l|l|l}
10 & 4 & 50 & 2 & 11.3896
\end{tabular}

\begin{tabular}{l|l|l|l|l|}
11 & 5 & 50 & 1 & 11.8969
\end{tabular}

\begin{tabular}{l|l|l|l|l|l|l|l|l|l|}
12 & 5 & 50 & 2 & 12.2949
\end{tabular}

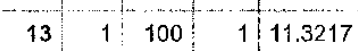

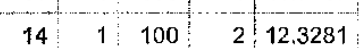

\begin{tabular}{l|l|l|l|l|l|l|l|l|l|l|l|l|}
15 & 6 & 100 & 1 & 11.5253
\end{tabular}

$\begin{array}{llllllll}16 & 6 & 100 & 2 & 11.4374\end{array}$

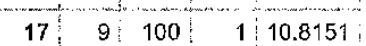

\begin{tabular}{l|l|l|l|l|}
18 & 9 & 100 & 2 & 12.0955 \\
\hline
\end{tabular}

Performed by K. Weber SAS version 9.3 14:27 18MAR14

File Folder: 16

Item Number: 
Length of Live Zebra Mussels in Each Treatment

Means of Lengths measured from samples collected from all unionid mussels/test enclosure

Study Number AEH-13-PSEUDO-05

The UNIVARIATE Procedure

Variable: length (length)

treat $=0$

time $=1$

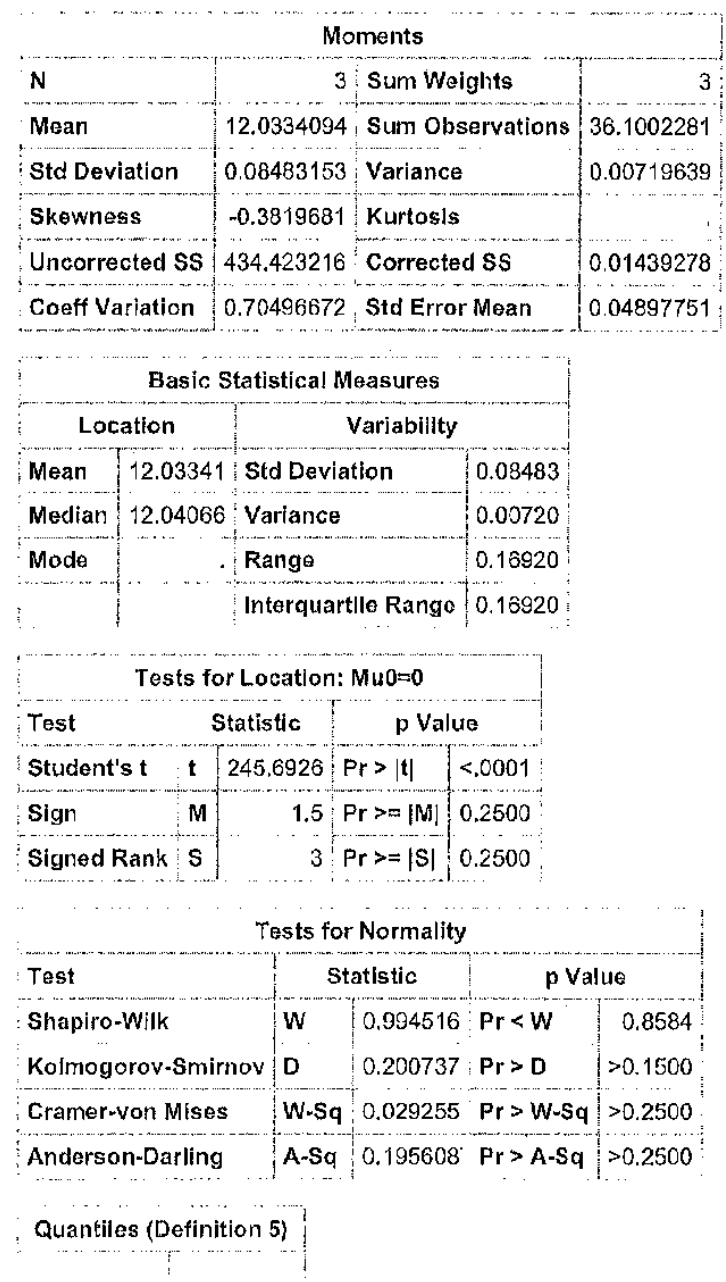




\begin{tabular}{|l|r|}
\hline Quantile & Estimate \\
\hline $100 \% \operatorname{Max}$ & 12.1144 \\
\hline $99 \%$ & 12.1144 \\
\hline $95 \%$ & 12.1144 \\
\hline $90 \%$ & 12.1144 \\
\hline $75 \%$ Q3 & 12.1144 \\
\hline $50 \%$ Median & 12.0407 \\
\hline $25 \%$ Q1 & 11.9452 \\
\hline $10 \%$ & 11.9452 \\
\hline $5 \%$ & 11.9452 \\
\hline $1 \%$ & 11.9452 \\
\hline $0 \%$ Min & 11.9452 \\
\hline
\end{tabular}

Extreme Observations

Lowest Highest

Value Obs Value obs

\begin{tabular}{|l|l|l|l|l|l|l|l|l|}
11.9452 & 2 & 11.9452 & 2
\end{tabular}

\begin{tabular}{|l|l|l|l|l|l|l|l|l|l|}
12.0407 & 1 & 12.0407 & 1
\end{tabular}

\begin{tabular}{|l|l|l|l|}
12.1144 & 3 & 12.1144 & 3 \\
\hline
\end{tabular}

Porformod by K. Wober SAS version 9.3 14:27 18MAR14 $W^{3}$ 
Length of Live Zebra Mussels in Each Treatment (s) Means of Lengths measured from samples collected from all unionid mussels/test enclosure

St:Aly Number AEH.13.PSEUDO-05

The UNIVARIATE Procedure

Varlable: length (length)

treat $=\mathbf{5 0}$

$\operatorname{time}=1$

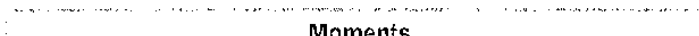

\begin{tabular}{|l|l|l|l|}
\hline \multicolumn{3}{|c|}{ Moments } \\
\hline N & 3 & Sum Weights & 3 \\
\hline Mean & 12.0581995 & Sum Observations & 36.1745986 \\
\hline Std Doviation & 0.51129389 & Variance & 0.26142144 \\
\hline Skewness & 1.27819581 & Kurtosis & \\
\hline Uncorrected SS & 436.723371 & Corrected SS & 0.52284288 \\
\hline Coeff Variation & 4.24021753 : Std Error Mean & 0.29519566 \\
\hline
\end{tabular}

\begin{tabular}{c|c|c|} 
Basic Statistical Measures \\
\hline Location & Variability \\
\hline
\end{tabular}

\begin{tabular}{l|l|l|}
\hline Mean 12,05820 Std Devlation & 0.51129 \\
\hline
\end{tabular}

\begin{tabular}{l|l|l|} 
Medlan 11.89692 & Variance & 0.26142
\end{tabular}

\begin{tabular}{|l|l|l|l}
\hline Mode & Range & 0.26142 \\
\hdashline & 0.98370
\end{tabular}

\begin{tabular}{|l|l|l|}
\hline Mode & Range & 0.98370 \\
\hline & Interquartile Range & 0.98370
\end{tabular}

Tests for Location: MuO=0

\begin{tabular}{l|l|l|}
\hline Tast & Statistic & $p$ Value \\
\hline
\end{tabular}

Student's $t$ t $40.84816 \quad \mathrm{Pr}>|\mathrm{t}| \quad 0.0006$

Sign $\quad M \quad 1.5 \mathrm{Pr}>=|\mathrm{M}| \quad 0.2500$

Signed Rank S $3 \operatorname{Pr}>=|\mathrm{S}|[0.2500$

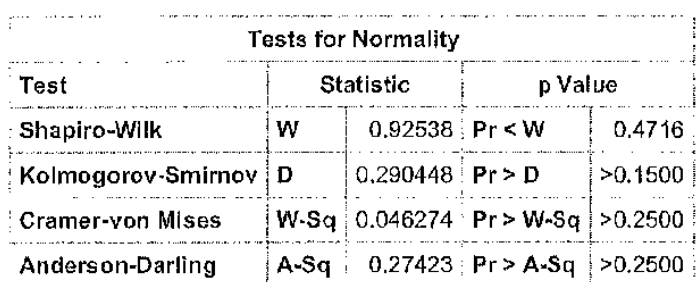

Quantiles (Definition 5) 


\begin{tabular}{|l|r|}
\hline Quantile & Estimate \\
\hline $100 \%$ Max & 12.6307 \\
\hline $99 \%$ & 12.6307 \\
\hline $95 \%$ & 12.6307 \\
\hline $90 \%$ & 12.6307 \\
\hline $75 \%$ Q3 & 12.6307 \\
\hline $50 \%$ Median & 11.8969 \\
\hline $25 \%$ Q1 & 11.6470 \\
\hline $10 \%$ & 11.6470 \\
\hline $5 \%$ & 11.6470 \\
\hline $1 \%$ & 11.6470 \\
\hline $0 \%$ Min & 11.6470 \\
\hline
\end{tabular}

Extreme Observations

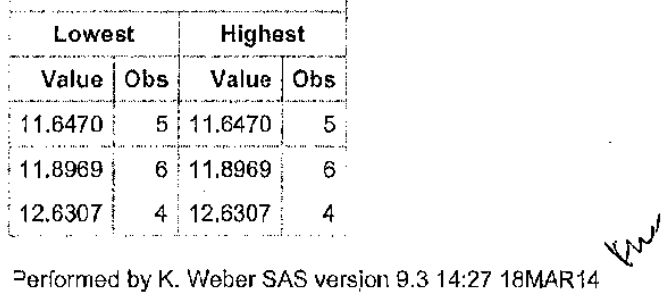




Length of Live Zebra Mussels in Each Treatment
enclosure

Study Number AEH-13.PSEUDO-05

The UNIVARIATE Procedure

Variable: longth (length)

treat $=100$

time $=1$

\begin{tabular}{|c|c|c|c|}
\hline \multicolumn{4}{|c|}{ Moments } \\
\hline N & 3 & Sum Weights & 3 \\
\hline Mean & 11.2207028 & Sum Observations & 33.6621083 \\
\hline Std Deviation & 0.36567833 & Variance & 0.13372064 \\
\hline Skewness & -1.1482579 & Kurtosis & \\
\hline Uncorrected SS & 377.979953 & Corrected SS & 0.26744128 \\
\hline Coeff Variation & 3.25896103 & Std Error Mean & 0.21112448 \\
\hline
\end{tabular}

\begin{tabular}{|c|c|c|c|}
\multicolumn{3}{|c|}{ Basic Statistical Measures } \\
\hline Location & \multicolumn{2}{|c|}{ Variability } \\
\hline Mean & 11.22070 & Std Deviation & 0.36568 \\
\hline Median & 11.32172 & Variance & 0.13372 \\
\hline Mode & Range & 0.71012 \\
\hline & & Interquartile Range & 0.71012 \\
\hline
\end{tabular}

\begin{tabular}{|c|c|c|c|c|}
\hline \multicolumn{5}{|c|}{ Tests fol } \\
\hline Test & & Statistic & p Va & \\
\hline Student's t & $t$ & 53.14733 & $P r>|t|$ & 0.0004 \\
\hline Sign & $M$ & 1.5 & $\operatorname{Pr}>=|M|$ & 0.2500 \\
\hline Signed Rank & $s$ & 3 & $P r>=|S|$ & 0.2500 \\
\hline
\end{tabular}

\begin{tabular}{|c|c|c|c|c|}
\hline \multicolumn{5}{|c|}{ Tests for Normally } \\
\hline \multirow{2}{*}{$\begin{array}{l}\text { Test } \\
\text { Shapiro-Wilk }\end{array}$} & \multicolumn{2}{|c|}{ Statistic } & \multicolumn{2}{|c|}{$\mathrm{p}$ Value } \\
\hline & w & 0.942764 & $\operatorname{Pr}<W$ & 0.5386 \\
\hline Kolmogorov-Smirnov & D & 0.275489 & $\mathrm{Pr}>\mathrm{D}$ & $>0,1500$ \\
\hline Cramer-von Mises & W-sq & 0.041989 & $\mathrm{Pr}>\mathrm{W}-\mathrm{Sq}$ & $>0.2500$ \\
\hline Anderson-Darling & $A-S q$ & 0.254197 & $\mathrm{Pr}>\mathrm{A}-\mathrm{Sq}$ & $>0.2500$ \\
\hline
\end{tabular}




\begin{tabular}{|c|c|}
\hline Quantile & Estimate \\
\hline $100 \%$ Max & 11.5253 \\
\hline $99 \%$ & 11.5253 \\
\hline $95 \%$ & 11.5253 \\
\hline $90 \%$ & 11.5253 \\
\hline $75 \%$ Q3 & 11.5253 \\
\hline $50 \%$ Median & 11.3217 \\
\hline $25 \%$ Q1 & 10.8151 \\
\hline $10 \%$ & 10.8151 \\
\hline $\mathbf{5 \%}$ & 10.8151 \\
\hline $\mathbf{1} \%$ & 10.8151 \\
\hline $0 \%$ Min & 10.8151 \\
\hline
\end{tabular}

: Extreme Observations

Lowest Highest

Value Obs Value Obs

\begin{tabular}{|l|l|l|l|}
\hline 10.8151 & 9 & 10.8151 & 9 \\
\hline
\end{tabular}

\begin{tabular}{|l|l|l|l|l|l|l|l|l|l|l|l|l|l|l|}
\hline 11.3217 & 7 & 11.3217 & 7 \\
\hline
\end{tabular}

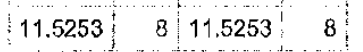

Performed by K. Weber SAS version 9.3 14:27 18MAR14 $W^{3}$ 
Length of Live Zebra Mussels in Each Treatment

Means of Lengths measured from samples collected from all unionid mussels/test enclosure

Study Number AEH-13.PSEUDO-05

The UNIVARIATE Procedure

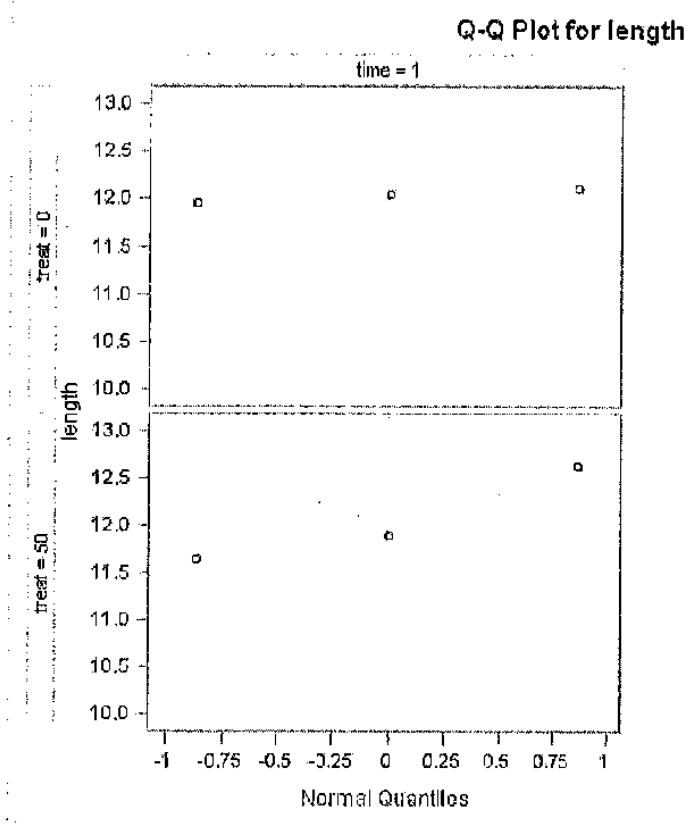




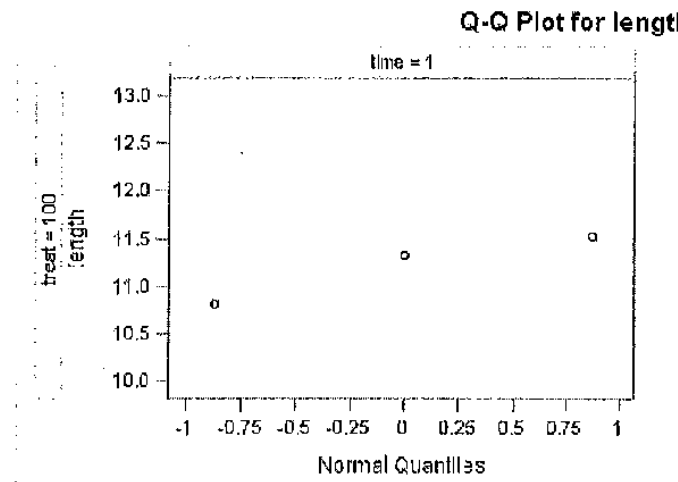

Perlormed by K. Weber SAS version 9.3 14:27 18MAR14 Ww 
AEH-13-PSEUDO-05

Length of Live Zebra Mussels in Each Treatment

Wination

Means of Lengths measured from samples collected from all unionid mussels/test enclosure

Sludy Number AEH-13-PSEUDO-05

The UNIVARIATE Procedure

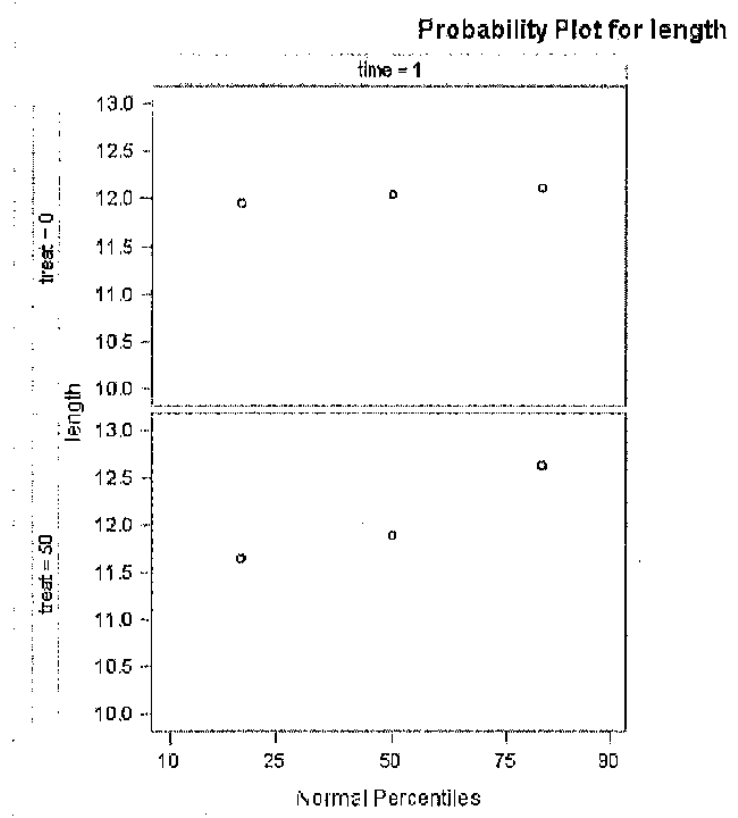




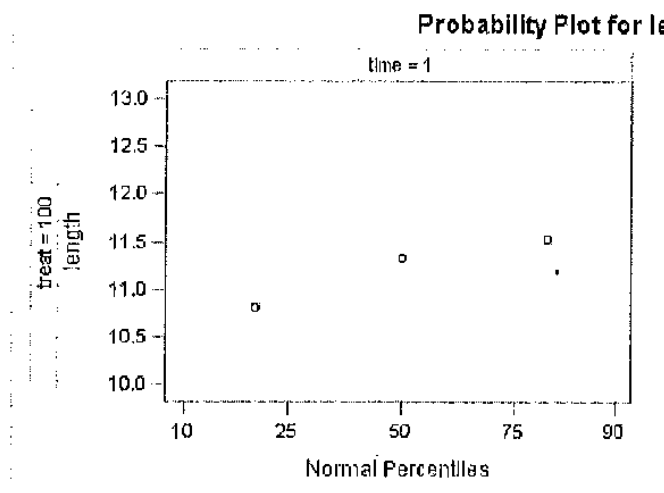

Performed by K. Weber SAS version 9.3 14:27 18MAR14 W $\mathrm{w}^{\mathrm{W}}$ 
AEH-13-PSEUDO-05

\section{ZM Length:Bartietts test of homogeneity of variance}

Length of Live Zebra Mussels in Each Treatment Pre- and Post-exposure

Means of Lengths measured from samples collected from all unionid mussels/test enclosure/sample time

Study Number AEH-13-PSEUDO-05

The GLM Procedure

Class Level Information

Class Levels Values

tlme $\quad 212$

Number of Observations Read 18

Number of Observations Used 18

Performed by K. Weber SAS version 9.3 14:27 18MAR14 W 
AEH-13.PSEUDO-05

ZM Length:Bartletts test of homogeneity of variance Length of Live Zebra Mussels in Each Treatment Pre- and Post-exposure Means of Lengths measured from samples collected from all unionid mussels/test enclosure/sample time Study Number AEH-13.PSEUDO.05

The GLM Procedure

Dependent Varlable: length length

\begin{tabular}{|l|r|r|r|r|r|}
\hline Source & DF & Sum of Squares & Mean Square & F Value & Pr $>$ F \\
\hline Model & 1 & 0.06973195 & 0.06973195 & 0.08 & 0.7823 \\
\hdashline Error & 16 & 14.12736600 & 0.88296037 & & \\
\hdashline Corrected Total & 17 & 14.19709794 & & & \\
\hline
\end{tabular}

R-Square Coeff Var Root MSE length Mean

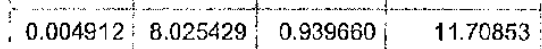

Source DF Type I SS Mean Square $F$ Value $\mathrm{Pr}>\mathrm{F}$

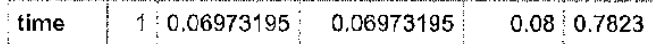

\begin{tabular}{c|c|c|c|c|c|c|}
\hline Source & $D F$ & Type III SS & Mean Square & $F$ Value $P r>F$ \\
\hline
\end{tabular}

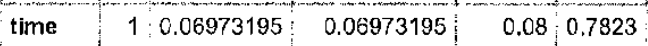


AEH-13-PSEUDO-05

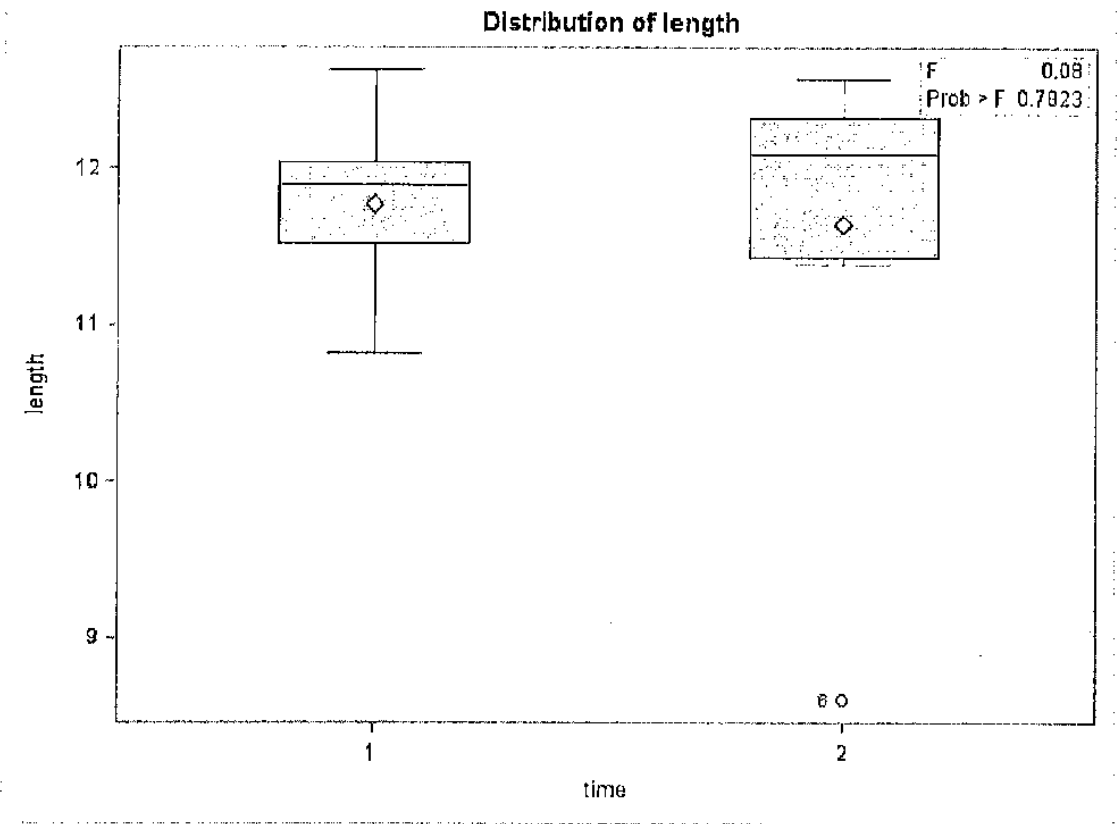

Performed by K. Weber SAS version 9.3 14:27 18MAR14W $W^{3}$ 
ZM Length:Bartletts test of homogeneity of variance

Length of Live Zebra Mussels in Each Treatment Pre- and Post-exposure

Means of Lengths measured from samples collected from all unionid mussels/test

enclosure/sample time

Study Number AEH-13-PSEUDO-05

The GLM Procedure

\begin{tabular}{|c|c|}
$\begin{array}{c}\text { Bartlett's Test for Homogeneity } \\
\text { of length Variance }\end{array}$ \\
\begin{tabular}{|c|c|c|c|}
\hline Source & DF & Chi-Square & $\mathrm{Pr}>\mathrm{Ch} / \mathrm{Sq}$ \\
\hline time & 1 & 4.9312 & 0.0264 \\
\hline
\end{tabular}
\end{tabular}

Performed by K. Weber SAS version $9.314: 27$ 18MAR1 $4 \mathrm{~N}^{\mathrm{N}}$ 
ZM Length:Bartletts test of homogeneity of variance Length of Live Zebra Mussels in Each Treatment Pre- and Post-exposure Means of Lengths measured from samples collected from all unionid mussels/test enclosure/sample time Study Number AEH-13-PSEUDO-05

The GLM Procedure

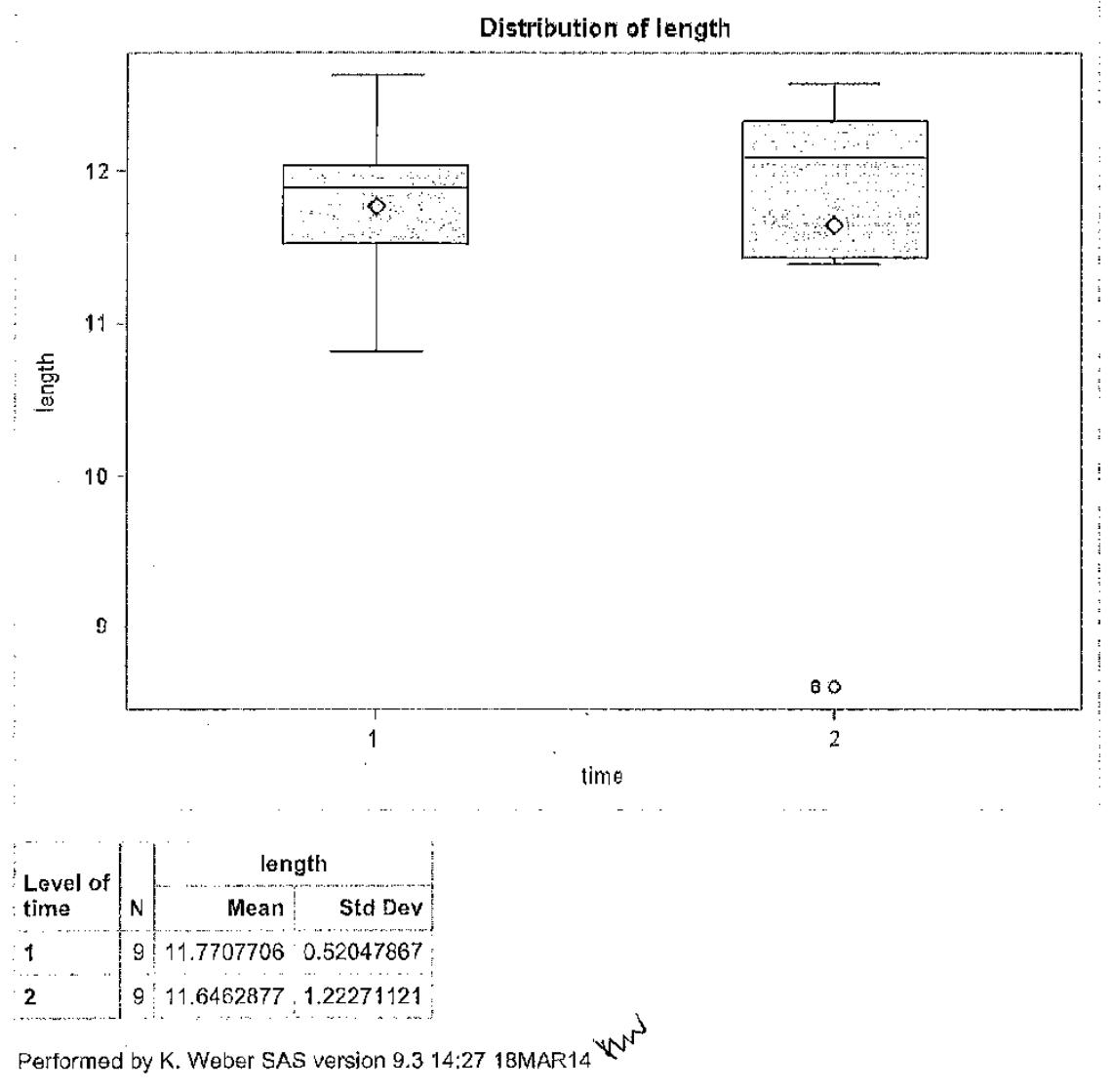


AEH-13-PSEUDO-05

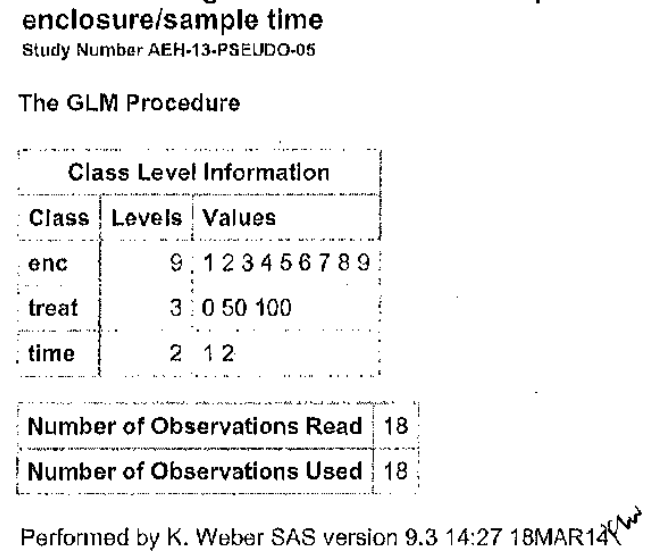


Length of Live Zebra Mussels in Each Treatment Pre- and Post-exposure wivay Means of Lengths measured from samples collected from all unionid mussels/test enclosure/sample time Study Number AEH-13.PSEUDO-05

The GLM Procedure

Dependent Variable: length length

\begin{tabular}{|c|c|c|c|c|c|}
\hline Source & $\mathrm{DF}$ & Sum of Squares & Mean Square & F Value & $\mathrm{Pr}>\mathrm{F}$ \\
\hline Model & 6 & 2469.934665 & 411.655778 & 415.95 & $<, 0001$ \\
\hline Error & 12 & 11.876204 & 0.989684 & & \\
\hline Uncorrected Total & 18 & 2481.810870 & & & \\
\hline
\end{tabular}

\begin{tabular}{c:c|c} 
R-Square Coeff Var Root MSE length Mean \\
0.163477 & 0.496614 & 0.994828 \\
\hline
\end{tabular}

\begin{tabular}{ll|l|l|l|l|l|l|l|l|l|l|l|l|l|l}
0.163477 & 8.496614 & 0.984828 & 11.70853 \\
\hline
\end{tabular}

\begin{tabular}{l|c|c|c|c|c|c|}
\hline Source & DF & Type ISS & Mean Square & $F$ Value & $\mathrm{Pr}>\mathrm{F}$ \\
\hline treat & 3 & 2467.999186 & 822.666395 & 831.24 & $<.0001$ \\
\hdashline time & 1 & 0.069732 & 0.069732 & 0.07 & 0.7952 \\
\hdashline treat*time & 2 & 1.865748 & 0.932874 & 0.94 & 0.4167 \\
\hline
\end{tabular}

\begin{tabular}{|l|r|r|r|r|r|}
\hline Source & DF & Type III SS & Mean Square & F Value & Pr $>$ F \\
\hline treat & 2 & 0.38541418 & 0.19270709 & 0.19 & 0.8256 \\
\hline time & 1 & 0.06973195 & 0.06973195 & 0.07 & 0.7952 \\
\hline treat*time & 2 & 1.86574762 & 0.93287381 & 0.94 & 0.4167 \\
\hline
\end{tabular}

\begin{tabular}{|c|c|c|c|c|c|}
\hline Parameter & Estimate & & Standard Error & t Value & $\mathrm{Pr}>|\mathrm{t}|$ \\
\hline treat 0 & 11.21466092 & $\mathrm{~B}$ & 0.57436448 & 19.53 & $<.0001$ \\
\hline treat 50 & 11.77054328 & B & 0.57436448 & 20.49 & $<.0001$ \\
\hline treat 100 & 11.95365881 & $\mathrm{~B}$ & 0.57436448 & 20.81 & $<.0001$ \\
\hline time 1 & -0.73295605 & $B$ & 0.81227404 & -0.90 & 0.3846 \\
\hline time 2 & 0.00000000 & B & & & \\
\hline treat*time 01 & 1.55170450 & $\mathrm{~B}$ & $1 .\{4872897$ & 1.35 & 0.2017 \\
\hline treattime 02 & 0.00000000 & $\mathrm{~B}$ & & & \\
\hline treatktime 501 & $1.0206: 229$ & $B$ & 1.14872897 & 0.89 & 0.3917 \\
\hline treat*time 502 & 0.00000000 & $\mathrm{~B}$ & & & \\
\hline treat*t 1 me 1001 & 0.00000000 & $\mathrm{~B}$ & & . & \\
\hline treat ${ }^{*}$ time 1002 & 0.00000000 & $\mathrm{~B}$ & · & $\cdot$ & \\
\hline
\end{tabular}




\section{AEH-13-PSEUDO-05}

Note: The X'X matrix has been found to be singular, and a generalized inverse was used to solve the normal equations. Terms whose estimates are followe by the letter ' $\mathrm{B}$ ' are not uniquely estimable.

Performed by K. Weber SAS version 9.31427 18MAR14 W 
AEH-13-PSEUDO-05

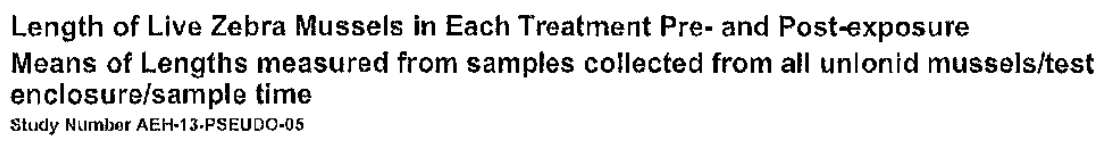

The GLM Procedure

\begin{tabular}{|c|c|c|c|c|c|}
\hline \multirow{2}{*}{$\begin{array}{r}\text { Observation } \\
1\end{array}$} & \multirow{2}{*}{$\begin{array}{r}\text { Observed } \\
12.04066306\end{array}$} & \multirow{2}{*}{\begin{tabular}{|} 
Predicted \\
12.03340937
\end{tabular}} & \multirow{2}{*}{\begin{tabular}{|r} 
Residual \\
0.00725369
\end{tabular}} & \multicolumn{2}{|c|}{$\begin{array}{l}\text { 95\% Confidence Limits for Mean Predicted } \\
\text { Value }\end{array}$} \\
\hline & & & & 10.78197666 & 13.28484208 \\
\hline 2 & 12.57734200 & 11.21466092 & 1.36268108 & 9.96322821 & 12.46609363 \\
\hline 3 & 11.94518391 & 12,03340937 & -0.08822547 & 10.78197666 & 13.28484208 \\
\hline 4 & 12.45929985 & 11.21466092 & 1.24463893 & 9.96322821 & 12.46609363 \\
\hline 5 & 12.11438115 & 12.03340937 & 0.08097178 & 10.78197666 & 13.28484208 \\
\hline $6:$ & 8.60734090 & 11.21466092 & $-2,60732002$ & 9.96322821 & 12,46609363 \\
\hline 7 & 12.63068496 & 12.05819953 & 0.57248543 & 10.80676682 & 13.30963224 \\
\hline 8 & 11.62714409 & 11.77054328 & -0.14339919 & 10.51911057 & 13.02197599 \\
\hline 9 & 11.64698975 & 12,05819953 & -0.41120977 & 10.80676682 & 13.30963224 \\
\hline 10 & 11.38962384 & 11.77054328 & 0.38091944 & 10.51911057 & 13.02197599 \\
\hline 11 & 11.89692387 & 12.05819953 & -0.16127566 & 10.80676682 & 13.30963221 \\
\hline 12 & 12.29486191 & 11.77054328 & 0.52431863 & 10.51911057 & 13.02197599 \\
\hline 13 & 11.32172148 & 11.22070276 & 0.10101872 & 9.96927006 & 12.47213547 \\
\hline 14 & 12.32808047 & 11.95365881 & 0.37442166 & 10.70222610 & 13.20509152 \\
\hline 15 & 11.52525263 & 11.22070276 & $0.3045498 \%$ & 9.96927006 & 12.47213547 \\
\hline 16 & 11.43741649 & 11.95365881 & -0.51624232 & 10.70222610 & 13.20509152 \\
\hline 17 & 10,81513418 & 11.22070276 & -0.40556859 & 9.96927006 & 12.47213547 \\
\hline 18 & 12.09547947 & 11.953658881 & 0.14182066 & $10.70222610^{\circ}$ & 13.20509152 \\
\hline
\end{tabular}

\begin{tabular}{l|c} 
Sum of Residuals & 0.00000000 \\
\hline Sum of Squared Residuals & 11,87620420 \\
\hline Sum of Squared Residuals - Error SS & -0.00000000 \\
\hline PRESS Statistic & 26.72145945 \\
\hline First Order Autocorrelation & -0.12742312 \\
\hline Durbin-Watson D & 2.25314825 \\
\hline
\end{tabular}

Performed by K. Weber SAS version 9.3 14:27 18MAR14 WJ 
Length of Live Zebra Mussels in Each Treatment Pre- and Post-exposure

Means of Lengths measured from samples collected from all unionid mussels/test enclosure/sample time Study Numbar AEH.13-PSEUDO-05

The GLM Procedure

Least Squares Means

Adjustment for Multiple Comparisons: Tukey

\begin{tabular}{|l|l|r|r|}
\hline treat & time & length LSMEAN & LSMEAN Number \\
\hline 0 & 1 & 12.0334094 & 1 \\
\hline 0 & 2 & 11.2146509 & 2 \\
\hline 50 & 1 & 12.0581995 & 3 \\
\hline 50 & 2 & 11.7705433 & 4 \\
\hline 100 & 1 & 11.2207028 & \\
\hline 100 & 2 & 11.9536588 & \\
\hline
\end{tabular}

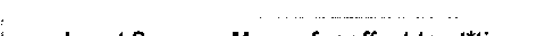
$\mathrm{Pr}>|\mathrm{t}|$ for Ho: LSMean(i)=LSMean (i) Dependent Variable: length

\begin{tabular}{|l|l|l|l|l|l|l|l|}
\hline i/l & 1 & & 2 & 3 & 4 & 5 & 6 \\
\hline 1 & & 0.9066 & 1.0000 & 0.9994 & 0.9091 & 1.0000 \\
\hline 2 & 0.9066 & & 0.8958 & 0.9804 & 1.0000 & 0.9366 \\
\hline 3 & 1.0000 & 0.8958 & & 0.9991 & 0.8985 & 1.0000 \\
\hline 4 & 0.9994 & 0.9804 & 0.9991 & & 0.9813 & 0.9999 \\
\hline 5 & 0.9091 & 1.0000 & 0.8985 & 0.9813 & & 0.9386 \\
\hline 6 & 1.0000 & 0.9366 & 1.0000 & 0.9999 & 0.9386 & \\
\hline
\end{tabular}

treat time length LSMEAN $95 \%$ Confidence Limits

\begin{tabular}{|l|l|l|l|l|}
\hline 0 & 1 & 12.033409 & 10.781977 & 13.284842 \\
\hline 0 & 2 & 11.214661 & 9.963228 & 12.466094 \\
\hline 50 & 1 & 12.058200 & 10.806767 & 13.309632 \\
\hline 50 & 2 & 11.770543 & 10.519111 & 13.021976 \\
\hline 100 & 1 & 11.220703 & 9.969270 & 12.472135 \\
\hline 100 & 2 & 11.953659 & 10.702226 & 13.205092 \\
\hline
\end{tabular}

\begin{tabular}{|c|c|c|c|}
\hline \multicolumn{4}{|c|}{ Least Squares Means for Effect treat*time } \\
\hline $\mathbf{i}$ & $\mathbf{j}$ & $\begin{array}{r}\text { Difference Betweon } \\
\text { Means }\end{array}$ & $\begin{array}{c}\text { Simuitaneous } 95 \% \text { Confidence Limits } \\
\text { for LSMean(i) } L S M \text { ean(j) }\end{array}$ \\
\hline 1 & 2 & 0.818748 & $-1,909600$ \\
\hline
\end{tabular}




\begin{tabular}{|c|c|c|c|c|}
\hline 1 & 3 & -0.024790 & -2.753139 & 2.703558 \\
\hline 1 & 4 & 0.262866 & -2.465483 & 2.991215 \\
\hline 1 & 5 & 0.812707 & -1.916642 & 3.541055 \\
\hline 1 & 6 & 0.079751 & -2.648598 & $2.8080 \mathrm{cg}$ \\
\hline 2 & 3 & -0.843539 & -3.571887 & 1.884810 \\
\hline 2 & 4 & -0.555882 & -3.284231 & 2.172466 \\
\hline 2 & 5 & -0.006042 & -2.734390 & 2.722307 \\
\hline 2 & 6 & -0.738998 & -3.467346 & 1.989351 \\
\hline 3 & 4 & 0.287656 & $-2,440692$ & 3,016005 \\
\hline 3 & 5 & 0.837497 & -1.890852 & 3.565845 \\
\hline 3 & 6 & 0.104541 & -2.623808 & 2.832889 \\
\hline 4 & 5 & 0.549841 & -2.178508 & 3.278189 \\
\hline 4 & 6 & -0.183116 & -2.911464 & 2.545233 \\
\hline 5 & 6 & -0.732956 & -3.461305 & 1.995393 \\
\hline
\end{tabular}

Performed by K. Weber SAS version 9.3 14:27 18MAR14 ww 
AEH-13-PSEUDO-05

\begin{abstract}
Length of Live Zebra Mussels in Each Treatment Pre- and Post-exposure Grand Means of Lengths measured from samples collected from all unionid mussels/test enclosure/sample time
\end{abstract} Study Number AEH-13.PSEUDO-05

The MEANS Procedure

treat $=0$

\begin{tabular}{|c|c|c|c|c|c|}
\hline \multicolumn{6}{|c|}{ Analysis Variable: length length } \\
\hline time & N Obs & Mean & Std Dev & $\begin{array}{r}\text { Lower } 95 \% \\
\text { CL. for Mean }\end{array}$ & $\begin{array}{r}\text { Upper } 95 \% \\
\text { CL for Mean }\end{array}$ \\
\hline 1 & 3 & 12.0334 & 0.0848 & 11.8227 & 12.2441 \\
\hline 2 & 3 & 11.2147 & 2,2588 & 5.6035 & 16.8258 \\
\hline
\end{tabular}

treat $=50$

\begin{tabular}{|c|c|c|c|c|c|}
\hline \multicolumn{6}{|c|}{ Analysis Variable: length length } \\
\hline $\operatorname{tim} \theta$ & N Obs & Mean & Std Dev & $\begin{array}{l}\text { Lower } 95 \% \\
\text { CL for Mean }\end{array}$ & $\begin{array}{l}\text { Upper } 95 \% \\
\text { CL for Mean }\end{array}$ \\
\hline 1 & 3 & 12.0582 & 0.5113 & 10.7881 & 13.3283 \\
\hline 2 & 3 & 11.7705 & 0,4693 & 10.6046 & 12.9365 \\
\hline
\end{tabular}

treat $=100$

\begin{tabular}{|c|c|c|c|c|c|}
\hline \multicolumn{6}{|c|}{ Analysis Variable : length length } \\
\hline time & N Obs & Mean & Std Dev & $\begin{array}{l}\text { Lower } 95 \% \\
\text { CL for Mean }\end{array}$ & $\begin{array}{r}\text { Upper } 95 \% \\
\text { CL for Mea! }\end{array}$ \\
\hline 1 & 3 & 11.2207 & 0.3657 & 10.3123 & 12.1291 \\
\hline 2 & 3 & 11.9537 & 0.4620 & 10.8061 & 13,1012 \\
\hline
\end{tabular}

Performed by K. Weber SAS version $9.314: 27$ 18MARA $4 \mathrm{~W}^{3}$

File Folder: 16

Item Number: 7 


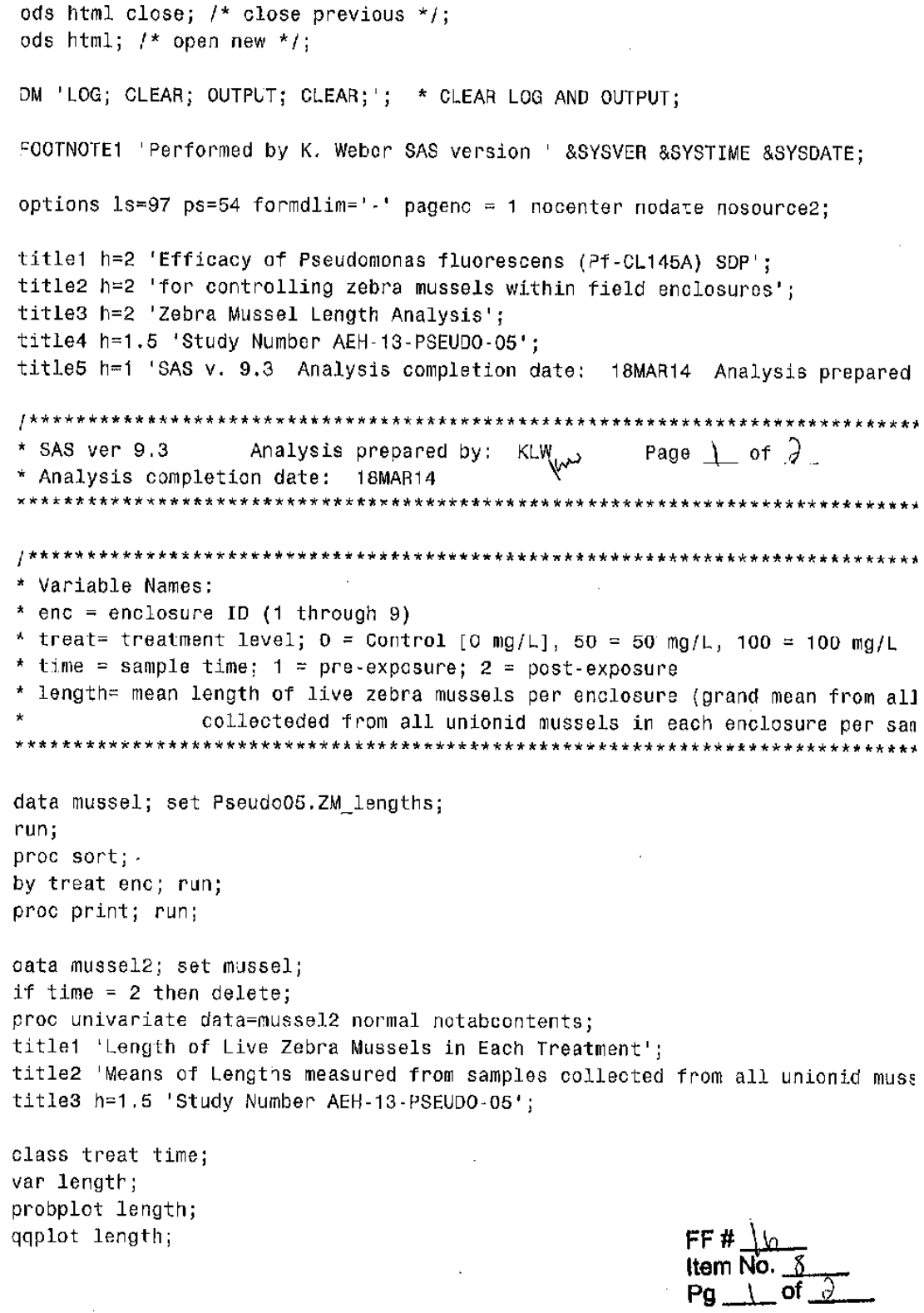

FF \#

Item No. 8

$\mathrm{Pg} \_$of 2 


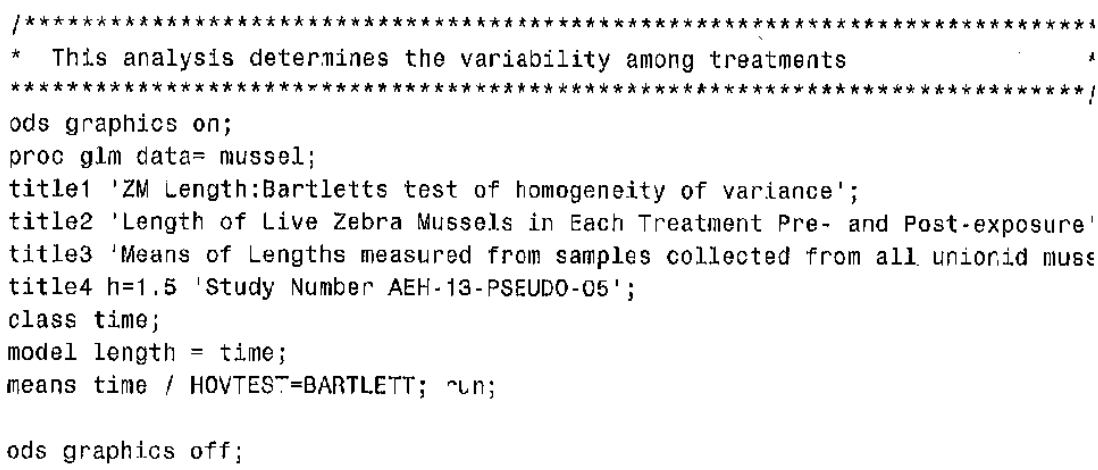

FF \# 16

Item No. 8

$\operatorname{Pg} \partial$ of $\partial$ 
DM 'LOG; ClEAR; OUTPUT; CLEAR;'; * CLEAR LOG AND OUTPU"';

FOOTNOTE1 'Performed by $K$. Weber SAS Version ' \&SYSVER \&SYSTIME \&SYSDATE 1 ;

WARNING: The FOOTNOTE statement is ambiguous due to invalid options on unquoted text.

options $1 s=97$ ps=54 formdlim=' -' pageno = 1 nocenter nodate nosource2;

title1 he2 'Efficacy of Pseudomonas fluorescens (Pf-CL145A) SDP';

title2 $n=2$ 'for controlling zebra mussels within field enclosures';

title3 h=2 'Zebra Mussel Length Analysis';

title4 $h=1.5$ 'Study Number AEH-13-PSEUD0-05';

title5 $h=1$ 'SAS $v .9 .3$ Analysis completion date: 18MAR14 Analysis prer.

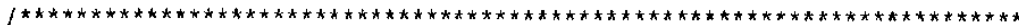

* SAS ver 9.3 Analysis prepared by: KLW Page 1 of 4

* Analysis completion date: 18MAR14. (Ww

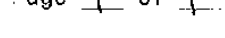

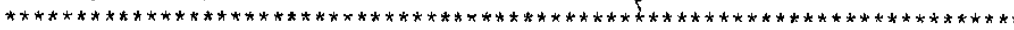

|

$* * * * * * * * * * * * * *$

* Variable Nanes:

* enc $=$ enclosure ID $(1$ through 9$)$

* treat $=$ treatment level; $0=$ Control $[0 \mathrm{mg} / \mathrm{L}], 50=50 \mathrm{mg} / \mathrm{L}, 100=100 n$

* time $=$ sample time; $1=$ pre-exposure; $2=$ post-exposure

1

* length = mean length of live zebra mussels per enclosure (grand mean frc 1 mussels

!

*

data musse1; set Pseudo05.ZM_lengths;

run;

NOTE: There were 18 observations read fron the data set PSEUDO0S.ZM LENGTHS.

NOTE: The data sot WORK.MUSSEL has 18 observations and 4 variables.

NOTE: DATA statement used (Total process time):

real time $\quad 0.03$ seconds

cpu time $\quad 0.01$ seconds

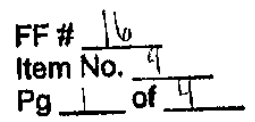


32 proc sort;
33 by troat enc; run;

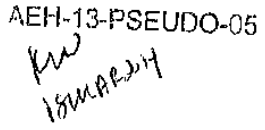

NOTE: There were 18 observations read from the data set WORK. MUSSEL.

NOTE: The data set WORK.MUSSEL has 18 observations and 4 variables.

NOTE: PROCEDURE SORT used (Total process time):

real time $\quad 0.00$ seconds

cpu zime $\quad 0.00$ seconds

34 proc print; run;

NOTE: There were 18 observations read from the data set WORK. MUSSEL,

NOTE: PROCEDUAE PRINT used (Total process time):

real time $\quad 0.07$ seconds

cpu time $\quad 0.01$ seconds

35

36 data musse12; . set mussel;

37 if time $=2$ then delete;

NOTE: Thero were 18 observations read from the data set WOR. MUSSEL.

NOTE: The data set WORK.MUSSEL.2 has 9 observations and 4 variables.

NOTE: DATA statement used (Total process time):
rea] time
0.01 seconds
cpu time
0.01 seconds

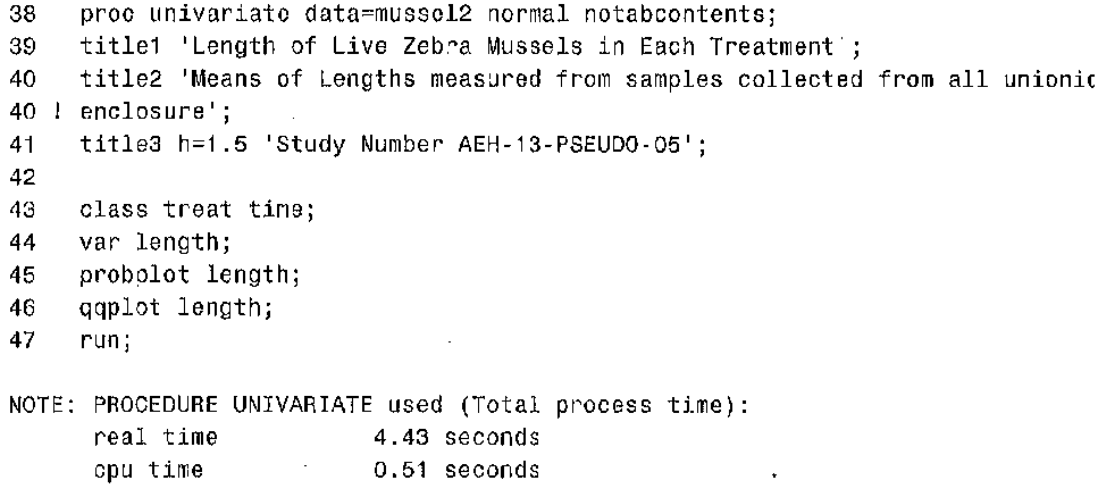




\section{AEH-13-PSEUDO-05}

49

50

2

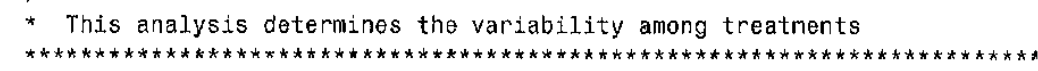

Page 3 of 4 
AEH-13-PSEUDO-05

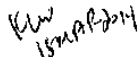

NOTE: Due to the presence of CLASS variables, an intercept is implicitly fitte been corrected for the mean.

79 quit;

NOTE: PROCEDURE GLM used (Total process time):

real time $\quad 0.34$ seconds

cpu time $\quad 0.23$ seconds

80

81

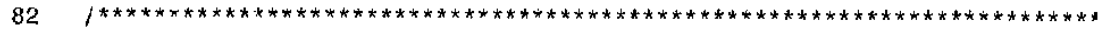

$821 * * * * * * * * * * * * * * *$

83 * This analysis deternines the grand mean zebra mussel lengths among tre 83 I post-exposure *

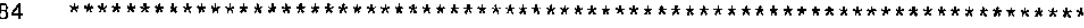

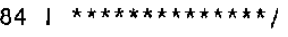

85 title1 'Length of Live Zebra Mussels in Each Treatmen- Pre- and Post-expc 86 title2 'Grand Means of Lengths moasured from samples collected from all i

861 enclosure/sample time';

87 title3 $h=1.5$ 'Study Number AEH-13-PSEUDO-05';

88 proc means data - mussel mean std $1 \mathrm{clm} u c l m$ f $w=8$;

89 by treat;

90 class time;

91 var length;

92 run;

NOTE: There were 18 observations read from the data set WORK. MUSSEL.

NOTE: PROCEDURE MEANS used (Total process time):

real time $\quad 0.10$ seconds

cpu time $\quad 0.01$ seconds

FF \# 16

Item No. $\frac{9}{4}$


ISSN 2331-1258 (online)

http://dx.doi.org/10.3133/2015-1051 